\title{
Study of CP Symmetry Violation in the Charmonium-K*(892) Channel By a Complete Time Dependent Angular Analysis (BaBar Experiment)
}

(in French)

\author{
Stephane T’Jampens
}

SLAC-R-836

Prepared for the Department of Energy

under contract number DE-AC02-76SF00515

Printed in the United States of America. Available from the National Technical Information Service, U.S. Department of Commerce, 5285 Port Royal Road, Springfield, VA 22161. 
This document, and the material and data contained therein, was developed under sponsorship of the United States Government. Neither the United States nor the Department of Energy, nor the Leland Stanford Junior University, nor their employees, nor their respective contractors, subcontractors, or their employees, makes an warranty, express or implied, or assumes any liability of responsibility for accuracy, completeness or usefulness of any information, apparatus, product or process disclosed, or represents that its use will not infringe privately owned rights. Mention of any product, its manufacturer, or suppliers shall not, nor is it intended to, imply approval, disapproval, or fitness of any particular use. A royalty-free, nonexclusive right to use and disseminate same of whatsoever, is expressly reserved to the United States and the University. 


\section{UNIVERSITÉ PARIS XI UFR SCIENTIFIQUE D'ORSAY}

THÈSE

Présentée

Pour obtenir

\section{Le GRADE de DOCTEUR EN SCIENCES de l'UNIVERSITÉ PARIS XI ORSAY}

PAR

\section{Stéphane T'JAMPENS}

Sujet : Étude de la violation de la symétrie $C P$ dans les canaux charmonium- $K^{*}(892)$ par une analyse angulaire complète dépendante du temps (expérience BABAR)

Soutenue le 18 décembre 2002 devant la commission d'examen

M. BONNEAUD Gérard Directeur de thèse

M. CAHN Robert Rapporteur

M. CHARLES Jérôme

M. DunWOOdIE William Membre invité

M. LEFRANÇOIS Jacques Président et rapporteur

M. VERDERI Marc Co-directeur de thèse 
(C) Stéphane T'JAMPENS, 2002

All rights reserved. 
I was born not knowing and have had only little time to change that here and there Richard P. Feynman

To those who have undertaken to study physics for the love of it and who, despite course drillings, demands for fast results, and market pressures, still love their science, have not given up the hope of understanding it better, and dare to ask radical questions. For theirs is the Kingdom of Photons. Mario Bunge 


\section{Remerciements}

Je remercie François Jacquet de m'avoir accueilli au sein du LPNHE (devenu LLR) de l'École Polytechnique dans les meilleurs conditions. Je remercie également Henri Videau, directeur actuel du LLR.

Gérard Bonneaud m'a encadré durant cette thèse tout en respectant ma liberté et mes initiatives. Je le remercie pour son soutien, sa confiance et pour m'avoir permis de longs séjours à SLAC. Je n'oublie pas non plus ses talents de cuisinier.

J'adresse mes remerciements à Jacques Lefrançois et Bob Cahn qui ont assuré la tâche de rapporteurs. Merci pour leurs questions pertinentes, leur lecture approfondie de mon manuscrit. Merci tout spécialement à Bob pour nos nombreuses discussions fructueuses au fur et à mesure des chapitres. Je suis très reconnaissant à l'ensemble des membres du jury pour m'avoir fait l'honneur d'y faire parti.

Je tiens à saluer tous les physiciens du groupe BABAR du LLR (Denis Bernard, François Brochard, Christophe Thiebaux et Georges Vasileiadis) avec qui j'ai eu un contact chaleureux et sympathique.

Je tiens à remercier les membres de BABAR (en particulier Riccardo Faccini, Christos Touramanis, Shahram Rahatlou et Steve Levy).

Enfin, j'exprime ici ma plus grande reconnaissance aux deux personnes qui ont eu le plus grand impact durant ma thèse : Marc Verderi et Bill Dunwoodie. J'ai travaillé en étroite collaboration avec Marc. Je le remercie pour son enthousiasme, pour la possibilité de discuter librement sur n'importe quel sujet, pour ses talents de virtuose en $\mathrm{C}++$, son regard critique et ses remarques pertinentes. Merci à toi pour ton amitié. Bill m'a fait profiter de son expérience dans de nombreux domaines de physique et m'a éclairé sur la physique des résonances. Je le remercie pour la richesse de ses remarques. Une grande partie du travail effectué durant ma thèse leur doit beaucoup. 


\section{Table des matières}

$\begin{array}{ll}\text { Introduction } & 11\end{array}$

1 Violation de $C P$ dans le système des mésons $B \quad 15$

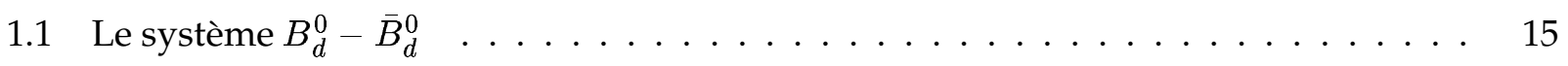

1.1.1 Hamiltonien effectif : approximation de Weisskopf-Wigner . . . . . . . . . 16

1.1.2 Conventions de phase . . . . . . . . . . . . . . . . . . . . 19

1.1 .3 Évolution temporelle . . . . . . . . . . . . . . . . . 19

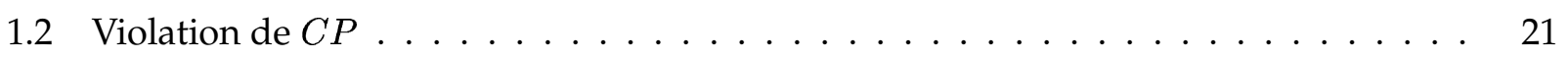

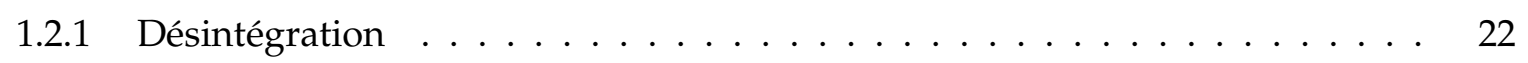

1.2 .2 Mélange . . . . . . . . . . . . . . . . . . 23

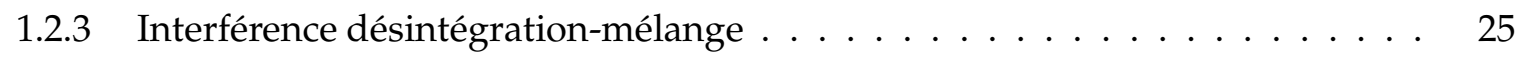

1.3 Violation de $C P$ dans le Modèle Standard . . . . . . . . . . . . . . . 28

1.3.1 Mise en œuvre minimale de la violation de $C P$ : la matrice CKM . . . . . . 29

1.3.2 Propriétés de la matrice CKM . . . . . . . . . . . . . . 34

1.3.3 Prédictions pour le système $B_{d}^{0}-\bar{B}_{d}^{0} \ldots \ldots \ldots \ldots \ldots \ldots$

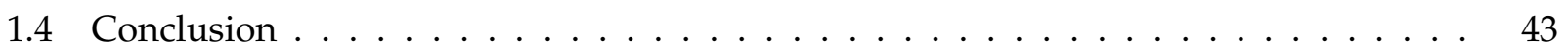

2 Phénoménologie des canaux vecteur-vecteur du type charmonium- $K^{*}(892) \quad 45$

2.1 Amplitude de désintégration non-leptonique . . . . . . . . . . . . . . 45

2.1.1 Amplitude dans l'approximation de factorisation . . . . . . . . . . . . 46

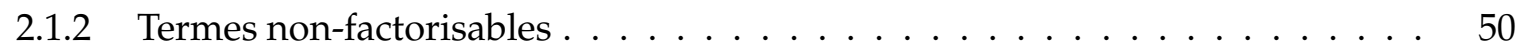

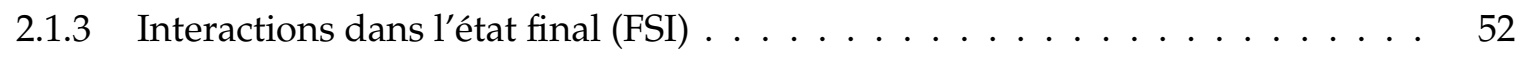

2.1 .4 Tests de la factorisation $\ldots \ldots \ldots \ldots \ldots \ldots \ldots$

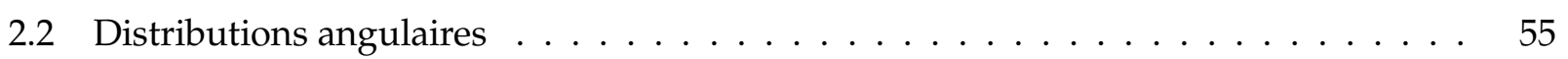

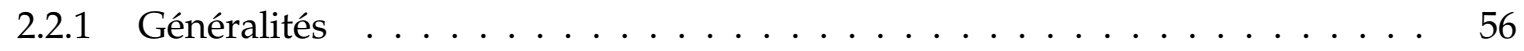

2.2.2 Les canaux $J / \psi K^{*}(892)$ et $\psi(2 S) K^{*}(892) \ldots \ldots \ldots \ldots \ldots \ldots$

2.2 .3 Le canal $\chi_{c 1} K^{*}(892) \ldots \ldots \ldots \ldots \ldots \ldots \ldots \ldots$

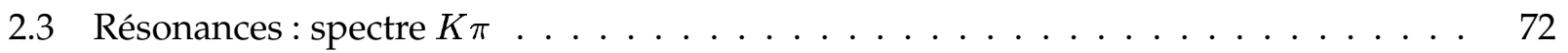

2.3.1 Breit-Wigner relativiste avec facteur de barrière centrifuge . . . . . . . . 72

2.3.2 Ondes $S, P, D$ du système $K \pi \ldots \ldots \ldots \ldots \ldots \ldots \ldots \ldots \ldots$

2.3.3 Distribution angulaire avec ondes $S, P$ pour le système $K \pi \quad \ldots \ldots \ldots$. . . 77 
3 Dispositif expérimental $\quad \mathbf{8 1}$

3.1 Le collisionneur PEP-II . . . . . . . . . . . . . . . . . . . . . . . . . . . . 83

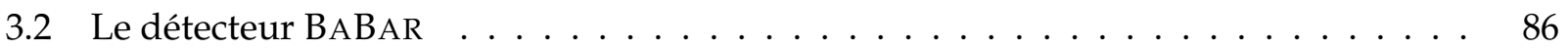

3.2.1 Détecteur de vertex $(\mathrm{SVT}) \ldots \ldots \ldots \ldots \ldots \ldots \ldots$. . . . . . . . . . . 87

3.2 .2 Chambre à dérive $(\mathrm{DCH}) \ldots \ldots \ldots \ldots \ldots \ldots \ldots$

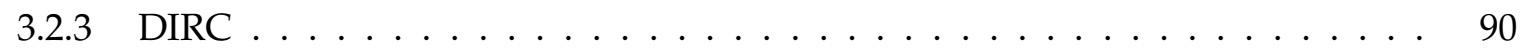

3.2 .4 Calorimètre $(\mathrm{EMC}) \ldots \ldots \ldots \ldots \ldots \ldots$

3.2 .5 Retour de flux instrumenté $($ IFR $) \ldots \ldots \ldots \ldots \ldots$

3.2.6 Système de déclenchement $\ldots \ldots \ldots \ldots \ldots \ldots$. . . . . . . . . 97

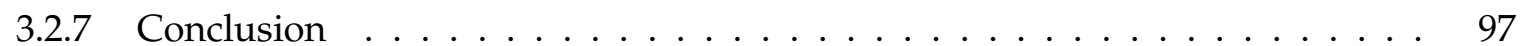

3.3 Sélection des particules finales $\ldots \ldots \ldots \ldots \ldots \ldots \ldots$

3.3.1 Sélection des particules chargées . . . . . . . . . . . . . . . . 98

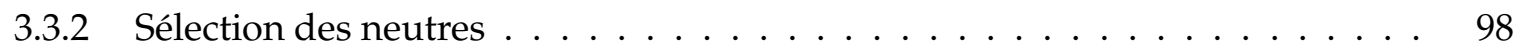

3.3.3 Identification des particules $\ldots \ldots \ldots \ldots \ldots$. . . . . . . . . . . 99

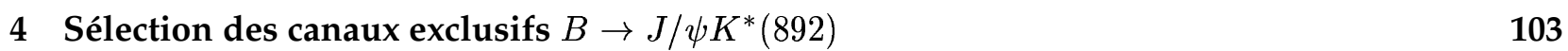

4.1 Reconstruction des mésons $B \ldots \ldots \ldots \ldots \ldots \ldots$. . . . . . . . . . . 104

4.1 .1 Présélection des événements . . . . . . . . . . . . . . . . . . . . . . . . 104

4.1.2 Sélection des mésons intermédiaires $\pi^{0}, K_{S}^{0}, K^{*}$ et $J / \psi \ldots \ldots \ldots \ldots$. . . . 105

4.1 .3 Sélection des mésons $B \ldots \ldots \ldots \ldots \ldots$. . . . . . . . . . 106

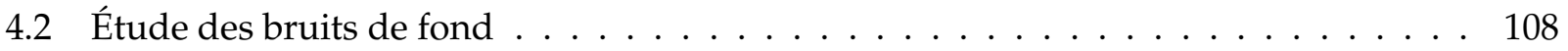

4.2.1 Bruits de fond sans charmonium . . . . . . . . . . . . . . . . . . . . 109

4.2.2 Bruits de fond $B \rightarrow J / \psi\left(\rightarrow \ell^{+} \ell^{-}\right) X \ldots \ldots \ldots \ldots \ldots \ldots$

4.2.3 Bruits de fond provenant du transfert entre les canaux $B \rightarrow J / \psi K^{*}(892) \quad \ldots 109$

4.3 Estimation du nombre d'événements signal f . . . . . . . . . . . . . . . 114

5 Mesure des amplitudes de transversité des canaux $B \rightarrow J / \psi K^{*}(892) \quad 123$

5.1 Ajustement par maximum de vraisemblance . . . . . . . . . . . . . . . 123

5.1.1 Fonction de transfert . . . . . . . . . . . . . . . . . . . . . . . 124

5.1.2 Densité de probabilité normalisée du signal : canaux $B \rightarrow J / \psi K^{*}(892) \ldots \ldots 126$

5.1.3 Traitement des fonctions de transfert . . . . . . . . . . . . . . . . . . 127

5.1.4 Ajustement en présence de bruits de fond . . . . . . . . . . . . . . . . . . . . 129

5.2 Ajustement par pseudo-maximum de vraisemblance . . . . . . . . . . . . . . . 130

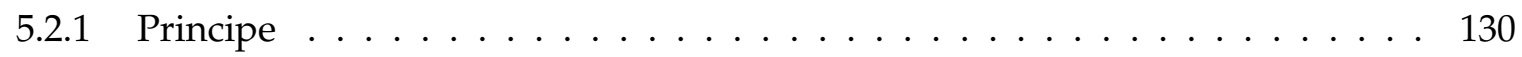

5.2 .2 Le problème des incertitudes . . . . . . . . . . . . . . . . . . . . 131

5.2 .3 Ajustement sur les données . . . . . . . . . . . . . . . . . . . . . 133

5.3 Validations . . . . . . . . . . . . . . . . . . . . . . . . . . 133

5.3.1 Validation sur simulation complète . . . . . . . . . . . . . . 133

5.3 .2 Facteurs de correction d'erreur . . . . . . . . . . . . . . . . . . . . . . 139

5.4 Ajustement sur les données . . . . . . . . . . . . . . . . . . . . . . . . 154

5.5 Étude des incertitudes systématiques . . . . . . . . . . . . . . . . . . . . . . 154

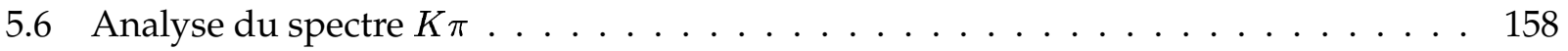

5.7 Conclusion . . . . . . . . . . . . . . . . . . . . . . . . . . 165 
6 Analyse angulaire complète dépendante du temps du canal $B_{d}^{0} \rightarrow J / \psi\left(K_{S}^{0} \pi^{0}\right)^{* 0} \quad 167$

6.1 Échantillons $B_{s a v}, B_{C P}$ et $B^{+} \ldots \ldots \ldots \ldots \ldots \ldots \ldots$

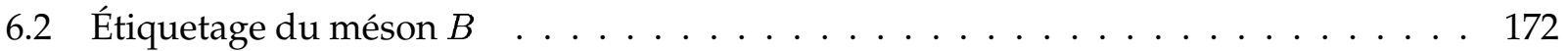

6.2 .1 Algorithme d'étiquetage . . . . . . . . . . . . . . . . . 173

6.2 .2 Performances . . . . . . . . . . . . . . . . . . . . 177

6.3 Mesure de la différence de temps propres . . . . . . . . . . . . . . . . . . . . 182

6.3.1 Reconstruction des vertex . . . . . . . . . . . . . . . . . . . . . . . . 182

6.3 .2 Mesure de $\Delta t \ldots \ldots \ldots \ldots \ldots \ldots \ldots \ldots \ldots$

6.3 .3 Fonction de résolution . . . . . . . . . . . . . . . . . . . . . 187

6.4 Ajustement par maximum de vraisemblance . . . . . . . . . . . . . . . . . . . 189

6.4.1 Ajustement pour l'échantillon $B_{s a v} \ldots \ldots \ldots \ldots$. . . . . . . 191

6.4.2 Ajustement pour le canal $B_{d}^{0} \rightarrow J / \psi\left(K_{S}^{0} \pi^{0}\right)^{* 0} \ldots \ldots \ldots \ldots$. . . . . . 193

6.4 .3 Ajustement global . . . . . . . . . . . . . . . . . . . . 202

6.5 Résultats de l'ajustement . . . . . . . . . . . . . . . . . . . . 202

6.6 Validations . . . . . . . . . . . . . . . . . . . . . . 203

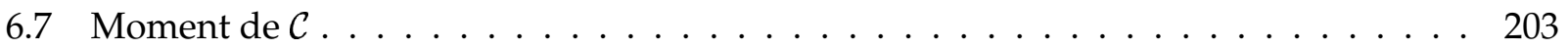

6.8 Étude des incertitudes systématiques . . . . . . . . . . . . . . . . . . 208

6.8 .1 Propriétés du signal . . . . . . . . . . . . . . . . . . . 208

6.8 .2 Propriétés des bruits de fond $: B_{\text {sav }} \ldots \ldots \ldots \ldots \ldots \ldots \ldots$

6.8 .3 Propriétés des bruits de fond $: B_{C P} \ldots \ldots \ldots \ldots \ldots \ldots \ldots \ldots \ldots$

6.8.4 Paramètres externes . . . . . . . . . . . . . . . . . . . . . . 211

6.8 .5 Statistique Monte Carlo . . . . . . . . . . . . . . . . . . . . . . . 211

6.9 Ambiguïté sur les phases fortes et signe de $\cos 2 \beta \ldots \ldots \ldots \ldots \ldots \ldots$

$\begin{array}{ll}\text { Conclusion } & 217\end{array}$

A Remarks on Conventions for the Derivation of Angular Distribution Using the Helicity $\begin{array}{ll}\text { Formalism } & 219\end{array}$

A.1 Introduction . . . . . . . . . . . . . . . . . . . . . . . . 219

A.2 Rotation in Euclidean Three-Dimensional Space . . . . . . . . . . . . . . . 220

A.2.1 Definitions . . . . . . . . . . . . . . . . . . . . . . . . . 220

A.2.2 Representation . . . . . . . . . . . . . . . . . . . . 221

A.2.3 Inversion . . . . . . . . . . . . . . . . . . . . . 221

A.3 Rotation Operator in Hilbert Space $\mathcal{H} \ldots \ldots$. . . . . . . . . . . . 222

A.4 One-Particle Plane-Wave Helicity State . . . . . . . . . . . . . . . . . . . . . . 223

A.5 Two-Particle Plane-Wave Helicity States in the Center-of-Mass Frame . . . . . . . . 225

A.6 Angular Distribution . . . . . . . . . . . . . . . . . . . 225

B Développement de la distribution angulaire de $B \rightarrow \psi\left(\rightarrow \ell^{+} \ell^{-}\right) K^{*}(\rightarrow K \pi)$ en moments 229

C Distribution angulaire de la désintégration $B \rightarrow \chi_{c 1} K^{*}(892)$ en base d'hélicité 233 
D Distribution angulaire de la désintégration $B \rightarrow J / \psi(K \pi)_{S+P+D} \quad 237$

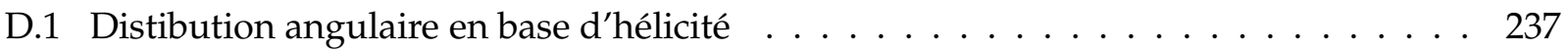

D.2 Distibution angulaire en base de transversité $\ldots \ldots \ldots \ldots \ldots \ldots \ldots$

E Développement en moments des fonctions $\varphi_{l}^{i \rightarrow j}\left(\vec{\omega}^{\prime}\right) \quad 243$ 


\section{Introduction}

Les symétries jouent un rôle fondamental en physique. Elles permettent de contraindre la dynamique d'un système en réduisant le nombre de degrés de liberté. Leur utilisation atteint un point culminant avec les théories de jauge, base du Modèle Standard de la physique des particules, en générant directement la dynamique au lieu de simplement la contraindre. Les symétries sont de différentes natures : il existe les symétries géométriques associées au déplacement du système physique dans l'espace-temps et celles qui n'affectent pas les coordonnées d'espace-temps telles que les symétries internes, les symétries dynamiques, etc. Mais quelle est la signification d'une symétrie pour une loi physique? Empruntons la réponse à R. Feynman [1] : « le mathématicien Hermann Weyl a donné une excellente définition de la symétrie : une chose est symétrique si, après avoir été soumise à une certaine action, son apparence n'est pas modifiée. C'est ce que nous voulons exprimer en disant que les lois physiques sont symétriques : on peut exercer une certaine action sur elles ou sur la manière de les représenter, cela ne changera rien, les résultats restent toujours les mêmes. »

Les symétries par rapport aux translations dans le temps et dans l'espace, ainsi qu'aux rotations dans l'espace, conduisent à des quantités conservées : l'énergie, l'impulsion et le moment cinétique, respectivement. Cette connexion entre symétries continues et quantités conservées est connue sous le nom de théorème de Noether. La théorie mathématique associée aux symétries est la théorie des groupes, dont Eugène Wigner fut l'un des pionniers pour son utilisation en physique. Le groupe associé à la relativité restreinte, un des deux ingrédients de base des théories de champs quantiques, est le groupe de Poincaré [2]. Ce groupe contient les symétries continues précédemment citées, ainsi que la transformation spéciale de Lorentz. Il contient aussi deux symétries discrètes, à opposer à continue, la parité $(P)$ et le renversement du temps $(T)$ [3]. Il existe une troisième symétrie discrète qui ne fait pas partie du groupe de Poincaré : la conjugaison de charge $(C)$. Elle n'existe qu'en physique quantique. Cette symétrie change les nombres quantiques de charge d'une particule tels que la charge électrique, les nombres de saveur (étrangeté, beauté, etc.), la troisième composante de l'isospin, etc., en leurs opposés. Cependant, il convient de garder à l'esprit que $C$ n'est pas une symétrie exacte de la Nature [4]. Ainsi, la conjugaison de charge ne change pas une particule en son antiparticule (comme on le lit trop souvent); pour définir une antiparticule, il faut utiliser la transformation $C P T$ [5].

Ce sont ces symétries discrètes qui sont au cœur de cette thèse, plus précisément la symétrie charge-parité $C P$. Pendant longtemps ces symétries ont été supposées exactes jusqu'à ce qu'en 1956, T. D. Lee et C. N. Yang proposent différentes expériences pour tester la conservation de la parité dans l'interaction faible [6]. Il s'avéra que cette interaction viole la symétrie parité de façon maximale [7] d'où le caractère $V-A$ de cette dernière. Il fut ensuite montré qu'elle viole aussi la 
symétrie de conjugaison de charge de façon maximale [8].

En physique quantique, les opérateurs représentant les transformations $P, C$ et $T$, que l'on note ${ }^{1}$ $\mathcal{P}, \mathcal{C}$ et $\mathcal{T}$, sont des opérateurs unitaires sauf $\mathcal{T}$, qui est un opérateur antiunitaire. La conséquence de ceci est connue sous le nom de dégénérescence de Kramers. Alors que les opérateurs $\mathcal{P}^{2}$ et $\mathcal{C}^{2}$ sont définis à une phase près, l'opérateur $\mathcal{T}^{2}$ ne peut valoir que \pm 1 , correspondant à deux classes identifiées aux bosons et aux fermions [3], respectivement. Il est remarquable d'avoir cette distinction sans introduire le spin. Un autre point important concerne la définition de ces opérateurs. Commençons par $\mathcal{P}$ et $\mathcal{C}$. En mécanique classique, la parité n'affecte que les composantes spatiales sans modifier la composante temporelle. En particulier, elle commute avec une translation temporelle $t \rightarrow t+\Delta t$. Cela implique en mécanique quantique que l'opérateur $\mathcal{P}$ doit commuter avec $\exp (-i \mathcal{H} \Delta t)$, l'opérateur translation dans le temps où $\mathcal{H}$ est l'opérateur hamiltonien. Cela revient à avoir $[\mathcal{P}, \mathcal{H}]=0$, i.e. la parité est conservée. Cela contredit le fait que la parité n'est pas une symétrie de l'interaction faible. En conclusion, il n'existe pas d'opérateur $\mathcal{P}$ représentant la transformation parité [4]. Il en va de même pour l'opérateur $\mathcal{C}$ qui doit satisfaire $[\mathcal{C}, \mathcal{H}]=0$. Mais $C$ n'est pas une bonne symétrie de la Nature. On a donc la même conclusion : il n'existe pas d'opérateur $\mathcal{C}$ représentant la transformation conjugaison de charge. Pour définir ces opérateurs, on se restreint aux interactions forte et électromagnétique, qui sont invariantes sous $P$ et $C$ (jusqu'à preuve du contraire). On utilise alors ces opérateurs pour sonder l'invariance ou non des autres interactions. Autrement dit, on restreint le lagrangien complet aux lagrangiens des interactions forte et électromagnétique pour pouvoir définir les opérateurs $\mathcal{P}$ et $\mathcal{C}$. On teste alors l'effet de ces opérateurs sur les lagrangiens d'autres interactions. S'ils ne sont pas invariants, on dit que ces interactions violent $P$ ou/et $C$ ou que $P$ ou/et $C$ est (sont) violée(s). En conclusion, la parité intrinsèque des particules ne peut leur être assignée qu'à travers des processus forts et électromagnétiques. Quant au nombre quantique associé à la conservation de la conjugaison de charge, il n'est défini que pour des particules ayant toutes leurs charges nulles. Passons maintenant à l'opérateur $\mathcal{T}$. Tout d'abord, il convient de noter qu'il n'existe pas d'opérateur en physique quantique représentant le temps du fait que ce dernier est un «nombre c » [10], c'est-à-dire de façon plus précise le paramètre d'un certain groupe de transformations; ce n'est pas une variable dynamique au même titre que les opérateurs position et impulsion [11]. Que représente l'opérateur $\mathcal{T}$ ? L'opérateur $\mathcal{T}$ transforme la translation dans le temps $t \rightarrow t+\Delta t$ en $t \rightarrow t-\Delta t$. Le renversement du temps est relié à la question de savoir si dans la Nature un processus dans lequel les particules initiales sont remplacées par les particules finales et vice-versa, sans oublier de changer les impulsions et spins des particules en leurs opposés, donne les mêmes résultats.

Après les découvertes de la violation de $P$ et $C$, la symétrie $C P$ était devenue la symétrie fondamentale pour distinguer la droite de la gauche [9]. Cependant, à la plus grande surprise générale fut découvert en 1964 que l'interaction faible violait faiblement la symétrie $C P$ [12]. En vertu du théorème $C P T$ [13], symétrie exacte pour toute théorie quantique des champs locale, la violation de $C P$ implique la violation de $T$. Ainsi, il existe une flèche du temps au niveau microscopique [14]. La violation de $C P$ a une conséquence plutôt intéressante : le projet «Ozma » [15]. Ce projet lancé en 1960 aux États-Unis tenta de répondre à la question suivante : pouvons-nous dialoguer par

\footnotetext{
${ }^{1}$ Les transformations sont représentées par des lettres latines droites alors que les opérateurs représentant ces transformations sont en lettres latines calligraphiques.
} 
ondes radio avec des extraterrestres afin de déterminer, avant de les rencontrer, s'ils sont faits de matière ou d'antimatière? La spectroscopie de leurs atomes serait identique, même si leurs atomes étaient faits d'antiprotons, d'antineutrons et de positons. La violation de la symétrie $C$ ne permet pas de distinguer de manière absolue matière et antimatière, puisqu'appeler un électron de la matière et un positon de l'antimatière est une convention. La violation de $C P$ permet de distinguer matière et antimatière de façon absolue, indépendamment de convention. En observant que les désintégrations des kaons neutres $\left(K_{L}^{0} \rightarrow \pi^{ \pm} \ell^{\mp} \nu\right)$ produisent un peu plus souvent des positons que des électrons, l'électron est alors distingué du positon par nature plutôt que par convention! En ce sens, la symétrie $C P$ est plus fondamentale que la symétrie $C$. De plus, la violation de $C P$ est un des trois ingrédients essentiels pour expliquer la domination de la matière sur l'antimatière dans l'univers [16].

Dans le Modèle Standard avec ses trois générations, la violation de $C P$ est directement reliée au mécanisme de Higgs, à travers les couplages de Yukawa entre les champs de quarks et le champ de Higgs [5] : elle est mise en œuvre de manière minimale, c'est-à-dire sans introduire de nouvelles interactions ou particules, à travers la matrice CKM, présente dans les courants chargés. Le but principal de l'expérience BABAR n'est pas la communication avec d'éventuels mondes extraterrestres mais l'étude de la violation de $C P$ dans le système des mésons $B$. Notre connaissance de la violation de $C P$ dans le Modèle Standard est plus que limitée puisqu'elle n'a été observée que dans le système des kaons neutres et que ces observations ne sont pas suffisamment précises pour infirmer l'origine même du processus de violation de $C P$. On ne sait donc pas si le modèle présent dans le Modèle Standard, à savoir le formalisme CKM, est celui de la Nature. Le système des mésons $B$ présente plusieurs avantages par rapport au système des kaons. De nombreux effets de violation de $C P$ sont prédits du fait de la large variété de modes de désintégration dont certains permettent un accès propre aux paramètres CKM, i.e. quasiment sans incertitudes hadroniques. Les asymétries prédites sont plus larges, de l'ordre de $10 \%$, voire plus. En juillet 2001, les deux expériences BABAR et Belle ont mis en évidence la violation de $C P$ dans le système des mésons $B[17,18], 35$ ans après sa découverte dans le système des kaons neutres.

Le premier chapitre de cette thèse est consacré à la violation de $C P$ dans le système des mésons $B$ en passant en revue le formalisme du système à deux niveaux $B_{d}^{0}-\bar{B}_{d}^{0}$, les diverses manifestations de la violation de $C P$, pour se terminer sur la description de la violation de $C P$ dans le Modèle Standard. Le second chapitre détaille la phénoménologie des canaux étudiés dans cette thèse, à savoir les canaux vecteur-vecteur. Les amplitudes de désintégration non-leptonique sont décrites dans le cadre de l'approximation de factorisation. Vient ensuite une description des interactions dans l'état final. Une caractéristique des canaux vecteur-vecteur est que les observables de ces désintégrations sont accessibles par une analyse angulaire et/ou temporelle des produits de désintégration des mésons vecteurs. Je dérive les distributions angulaires des canaux $J / \psi K^{*}(892), \psi(2 S) K^{*}(892)$ et $\chi_{c 1} K^{*}(892)$ dans le formalisme d'hélicité, puis dans celui de transversité, en insistant sur les différentes conventions utilisées. L'appendice A est entièrement consacré à ces problèmes de conventions. Ce chapitre se termine sur la physique des résonances afin de traiter correctement le spectre $K \pi$, combinaison de la résonance $K^{*}(892)$ mais aussi de résonances plus lourdes, de leurs interférences et d'une production non résonante. Le troisième chapitre décrit le dispositif expérimental, à savoir le collisionneur PEP-II et le détecteur BABAR. Il se termine par une description de la sélection 
des particules finales. Le quatrième chapitre traite de la sélection des canaux $B \rightarrow J / \psi K^{*}(892)$. La mesure des amplitudes de transversité des canaux $B \rightarrow J / \psi K^{*}(892)$ est décrite dans le cinquième chapitre, ingrédient nécessaire à la mesure de $\sin 2 \beta$ et $\cos 2 \beta$ dans le mode $C P, B_{d}^{0} \rightarrow J / \psi\left(K_{S}^{0} \pi^{0}\right)^{* 0}$, détaillée dans le sixième chapitre. Ces deux chapitres se terminent par une discussion des résultats. Une conclusion d'ensemble synthétise le travail effectué durant cette thèse. 


\section{Chapitre 1}

\section{Violation de $C P$ dans le système des mésons $B$}

Ce chapitre est consacré à la violation de $C P$ dans le système des mésons $B$. La section 1.1 traite du système à deux niveaux $B_{d}^{0}-\bar{B}_{d}^{0}$, indépendamment de modèles théoriques. Les diverses manifestations de la violation de $C P$ sont expliquées dans la section 1.2. Enfin, la section 1.3 prend comme cadre théorique le Modèle Standard et présente les propriétés de la matrice CKM ainsi que les prédictions pour le système $B_{d}^{0}-\bar{B}_{d}^{0}$.

\subsection{Le système $B_{d}^{0}-\bar{B}_{d}^{0}$}

Le système considéré est celui des deux mésons neutres, le $B_{d}^{0}$ et son antiparticule, le $\bar{B}_{d}^{0}$. La convention retenue est d'associer comme partenaire d'isospin au méson $B_{u}^{+}$le $B_{d}^{0}$, soit un contenu en quarks $\bar{b} d$. $B_{d}^{0}$ et $\bar{B}_{d}^{0}$ se distinguent par le nombre quantique interne de beauté, $B$, avec la propriété que $\Delta B=0$ pour les interactions forte et électromagnétique et $\Delta B \neq 0$ pour l'interaction faible. Si l'interaction faible n'existait pas, $B_{d}^{0}$ et $\bar{B}_{d}^{0}$ seraient stables avec la même masse $M_{0}$.

La présence de l'interaction faible change la situation considérablement : la beauté n'étant pas conservée par cette interaction, $B_{d}^{0}$ et $\bar{B}_{d}^{0}$ peuvent se désintégrer. De plus, elle permet la transition particule-antiparticule au cours de laquelle le nombre quantique de beauté change de deux unités. Cela signifie que les mésons $B$ neutres peuvent osciller de l'un vers l'autre et vice-versa via des états communs, réels ou virtuels, avant de se désintégrer. C'est le phénomène de mélange. Ce phénomène existe pour tout système de deux particules neutres distinguées par un nombre quantique interne non conservé tel que l'étrangeté, la beauté, etc.

Ainsi, $\left|B_{d}^{0}\right\rangle$ et $\left|\bar{B}_{d}^{0}\right\rangle$ sont états propres de l'hamiltonien des interactions forte et électromagnétique avec une masse commune $M_{0}$ et des saveurs opposées. Ces interactions conservant la saveur, on a $\left\langle\bar{B}_{d}^{0} \mid B_{d}^{0}\right\rangle=0$. En prenant en compte l'interaction faible, $\left|B_{d}^{0}\right\rangle$ et $\left|\bar{B}_{d}^{0}\right\rangle$ ne sont plus états propres de masse, c'est-à-dire qu'ils ne diagonalisent pas l'hamiltonien total du système. L'interaction faible lève la dégénérescence. 


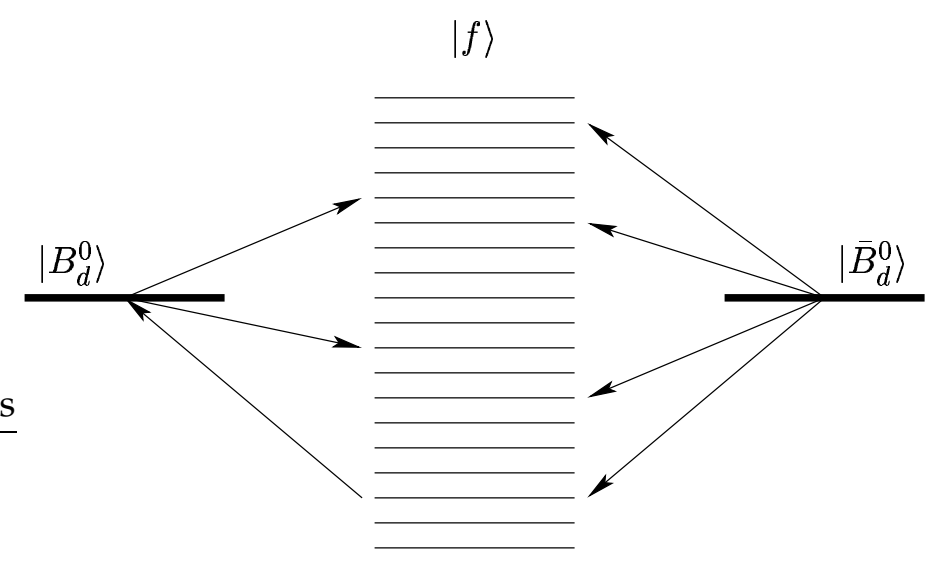

Continuum

FIG. 1.1: Schématisation du système $B_{d}^{0}-\bar{B}_{d}^{0}$ couplé à un continuum d'états, réels ou virtuels, par l'interaction faible.

\subsubsection{Hamiltonien effectif : approximation de Weisskopf-Wigner}

Le formalisme de mélange est basé sur la théorie des perturbations dépendantes du temps d'un système à deux niveaux, $\left|B_{d}^{0}\right\rangle$ et $\left|\bar{B}_{d}^{0}\right\rangle$, couplé à un continuum d'états, $|f\rangle$, par un hamiltonien d'interaction $H_{w}$, vers lesquels $\left|B_{d}^{0}\right\rangle$ et $\left|\bar{B}_{d}^{0}\right\rangle$ peuvent se désintégrer. L'hamiltonien complet du système s'écrit

$$
H=H_{0}+H_{w},
$$

où $H_{0}$, hamiltonien non-perturbé, contient les interactions forte et électromagnétique et $H_{w}$, traité comme une perturbation, contient l'interaction faible qui induit les transitions $B_{d}^{0} \leftrightarrow \bar{B}_{d}^{0}, B_{d}^{0} \rightarrow f$ et $\bar{B}_{d}^{0} \rightarrow f$ (voir Fig. 1.1).

De manière générale, l'état du système $B_{d}^{0}-\bar{B}_{d}^{0}$ couplé à un continuum d'états $|f\rangle$ est

$$
|\psi(t)\rangle=a(t)\left|B_{d}^{0}\right\rangle+b(t)\left|\bar{B}_{d}^{0}\right\rangle+\sum_{f} c_{f}(t)|f\rangle
$$

dont l'évolution temporelle est régie par l'équation de Schrödinger

$$
i \frac{\partial|\psi(t)\rangle}{\partial t}=\mathcal{H}|\psi(t)\rangle
$$

où $\mathcal{H}$ est l'opérateur hamiltonien de dimension infinie. L'évolution d'un tel état est au delà de nos possibilités. Cependant, si [19]

- l'état initial est uniquement la superposition des états propres de saveur : $|\psi(0)\rangle=a(0)\left|B_{d}^{0}\right\rangle+b(0)\left|\bar{B}_{d}^{0}\right\rangle ;$

- nous sommes intéressés uniquement par l'évolution temporelle de $a$ et $b$;

- l'échelle de temps $t$ est beaucoup plus grande que l'échelle de temps de l'interaction forte ; alors la situation se simplifie et l'évolution temporelle du système devient calculable. C'est l'approximation de Weisskopf-Wigner.

Dans cette approximation, on se restreint au système $B_{d}^{0}-\bar{B}_{d}^{0}$ dont l'état s'écrit comme la superposition d'états propres de saveur, $|\psi(t)\rangle=a(t)\left|B_{d}^{0}\right\rangle+b(t)\left|\bar{B}_{d}^{0}\right\rangle$, où $t$ est le temps propre du système 
$B_{d}^{0}-\bar{B}_{d}^{0}$. Il obéit à une équation de Schrödinger effective

$$
i \frac{\partial}{\partial t}\left(\begin{array}{c}
a(t) \\
b(t)
\end{array}\right)=\mathcal{H}_{\mathrm{eff}}\left(\begin{array}{c}
a(t) \\
b(t)
\end{array}\right)
$$

où $\mathcal{H}_{\text {eff, }}$ opérateur hamiltonien effectif, est donné par :

$$
\mathcal{H}_{\mathrm{eff}}=\left(\begin{array}{cc}
\left\langle B_{d}^{0}\left|\mathcal{H}_{\mathrm{eff}}\right| B_{d}^{0}\right\rangle & \left\langle B_{d}^{0}\left|\mathcal{H}_{\mathrm{eff}}\right| \bar{B}_{d}^{0}\right\rangle \\
\left\langle\bar{B}_{d}^{0}\left|\mathcal{H}_{\mathrm{eff}}\right| B_{d}^{0}\right\rangle & \left\langle\bar{B}_{d}^{0}\left|\mathcal{H}_{\mathrm{eff}}\right| \bar{B}_{d}^{0}\right\rangle
\end{array}\right)=\mathbf{M}-\frac{i}{2} \boldsymbol{\Gamma}=\left(\begin{array}{cc}
M_{11}-\frac{i}{2} \Gamma_{11} & M_{12}-\frac{i}{2} \Gamma_{12} \\
M_{12}^{*}-\frac{i}{2} \Gamma_{12}^{*} & M_{22}-\frac{i}{2} \Gamma_{22}
\end{array}\right) .
$$

Les matrices hermitiennes $2 \times 2$, $\mathbf{M}$ et $\boldsymbol{\Gamma}$, sont appelées respectivement les matrices de masse et de désintégration.

L'invariance sous la symétrie $C P T$, i.e. $\left[\mathcal{C P} \mathcal{T}, \mathcal{H}_{\text {eff }}\right]=0$, implique que $M_{11}=M_{22} \equiv M_{0}$ et $\Gamma_{11}=\Gamma_{22} \equiv \Gamma_{0}$, où $M_{0}$ et $\Gamma_{0}$ sont la masse et la largeur de désintégration des états propres de saveur $\left|B_{d}^{0}\right\rangle$ et $\left|\bar{B}_{d}^{0}\right\rangle$. Si l'on suppose l'invariance sous la symétrie $C P$ alors $M_{12}^{*} / \Gamma_{12}^{*}=M_{12} / \Gamma_{12}$ [22] ou, de manière équivalente, $\arg \left(\Gamma_{12} / M_{12}\right)=0$.

Les éléments des matrices $\mathbf{M}$ et $\boldsymbol{\Gamma}$ sont donnés ${ }^{1}$, au deuxième ordre en perturbation, par $[19,23$, 24] $\left(i, j=1,2 ; 1 \equiv B_{d}^{0} ; 2 \equiv \bar{B}_{d}^{0}\right)$

$$
\begin{aligned}
M_{i j} & =M_{0} \delta_{i j}+\left\langle i\left|\mathcal{H}_{w}^{(\Delta B=2)}\right| j\right\rangle+\sum_{f} P\left[\frac{\left\langle i\left|\mathcal{H}_{w}^{(\Delta B=1)}\right| f\right\rangle\left\langle f\left|\mathcal{H}_{w}^{(\Delta B=1)}\right| j\right\rangle}{M_{0}-E_{f}}\right], \\
\Gamma_{i j} & =2 \pi \sum_{f}\left\langle i\left|\mathcal{H}_{w}^{(\Delta B=1)}\right| f\right\rangle\left\langle f\left|\mathcal{H}_{w}^{(\Delta B=1)}\right| j\right\rangle \delta\left(M_{0}-E_{f}\right)
\end{aligned}
$$

où $P$ est la partie principale. Remarquons que

- les éléments diagonaux de $\mathbf{M}$ sont dominés par la masse $M_{0}$ des états $\left|B_{d}^{0}\right\rangle$ et $\left|\bar{B}_{d}^{0}\right\rangle$;

- les éléments non-diagonaux $M_{12}$ et $M_{21}$ représentent les transitions via des états intermédiaires virtuels (partie dispersive) $\bar{B}_{d}^{0} \rightarrow f \rightarrow B_{d}^{0}$ et $B_{d}^{0} \rightarrow f \rightarrow \bar{B}_{d}^{0}$;

- les éléments diagonaux de $\boldsymbol{\Gamma}$ décrivent les désintégrations $B_{d}^{0} \rightarrow f$ et $\bar{B}_{d}^{0} \rightarrow \bar{f}$;

- les éléments non-diagonaux $\Gamma_{12}$ et $\Gamma_{21}$ représentent les transitions $\bar{B}_{d}^{0} \rightarrow f \rightarrow B_{d}^{0}$ et $B_{d}^{0} \rightarrow$ $f \rightarrow \bar{B}_{d}^{0}$ via des états intermédiaires réels (partie absorptive), vers lesquels $B_{d}^{0}$ et $\bar{B}_{d}^{0}$ peuvent se désintégrer.

L'opérateur hamiltonien effectif $\mathcal{H}_{\text {eff }}$ n'est pas hermitien : ses états propres ${ }^{2}$ (états propres de masse)

$$
\begin{aligned}
& \left|B_{a}\right\rangle=p\left|B_{d}^{0}\right\rangle+q\left|\bar{B}_{d}^{0}\right\rangle, \quad\left|p^{2}\right|+\left|q^{2}\right|=1 \\
& \left|B_{b}\right\rangle=p\left|B_{d}^{0}\right\rangle-q\left|\bar{B}_{d}^{0}\right\rangle
\end{aligned}
$$

${ }^{1}$ Le terme $\left\langle i\left|\mathcal{H}_{w}^{(\Delta B=2)}\right| j\right\rangle$ dans $M_{i j}$ est nul dans le Modèle Standard du fait qu'aucun processus impliquant $H_{w}^{(\Delta B=2)}$ ne peut avoir lieu au premier ordre. À ne pas confondre avec les processus $\Delta B=2$ qui sont une succession de deux transitions $H_{w}^{(\Delta B=1)}: B_{d}^{0} \stackrel{\Delta B=1}{\longrightarrow} f \stackrel{\Delta B=1}{\longrightarrow} \bar{B}_{d}^{0}$ ou $\bar{B}_{d}^{0} \stackrel{\Delta B=-1}{\longrightarrow} f \stackrel{\Delta B=-1}{\longrightarrow} B_{d}^{0}$. Le modèle « superfaible », qui définit les processus $\Delta F=2(F=S, B, \ldots)$ comme unique source de violation de $C P$ (ce qui implique l'absence de violation de $C P$ directe), autorise un tel terme par la présence d'une nouvelle interaction [20]. Cependant, des mesures récentes de $\Re e\left(\epsilon^{\prime} / \epsilon\right)$ [21] concluent à de la violation de $C P$ directe dans le système des kaons et donc excluent ce modèle. Dans le système des mésons $B$, toute manifestation de violation de $C P$ directe ou toute différence dans les asymétries d'états propres de $C P$ (au signe près) exclurait ce modèle.

${ }^{2}$ On suppose l'invariance sous la symétrie $C P T$. 
ne sont pas orthogonaux ${ }^{3}$. Les valeurs propres correspondantes (complexes) sont

$$
\begin{aligned}
\mu_{a} & =M_{a}-\frac{i}{2} \Gamma_{a}=\left(M_{0}-\frac{i}{2} \Gamma_{0}\right)+\frac{q}{p}\left(M_{12}-\frac{i}{2} \Gamma_{12}\right), \\
\mu_{b} & =M_{b}-\frac{i}{2} \Gamma_{b}=\left(M_{0}-\frac{i}{2} \Gamma_{0}\right)-\frac{q}{p}\left(M_{12}-\frac{i}{2} \Gamma_{12}\right)
\end{aligned}
$$

avec

$$
\left(\frac{q}{p}\right)^{2}=\frac{M_{12}^{*}-\frac{i}{2} \Gamma_{12}^{*}}{M_{12}-\frac{i}{2} \Gamma_{12}}
$$

conduisant à deux solutions :

$$
\frac{q}{p}= \pm \sqrt{\frac{M_{12}^{*}-\frac{i}{2} \Gamma_{12}^{*}}{M_{12}-\frac{i}{2} \Gamma_{12}}} .
$$

Choisir le signe moins plutôt que le signe plus revient à interchanger les indices $a \leftrightarrow b$ des états propres de masse (eqs. (1.8) et (1.9)).

Les équations (1.10) et (1.11) conduisent, pour les différences de masse et de largeur, à

$$
\begin{aligned}
& \Delta M \equiv M_{b}-M_{a}=\Re e\left(\mu_{b}-\mu_{a}\right)=-2 \Re e\left[\frac{q}{p}\left(M_{12}-\frac{i}{2} \Gamma_{12}\right)\right], \\
& \Delta \Gamma \equiv \Gamma_{b}-\Gamma_{a}=-2 \Im m\left(\mu_{b}-\mu_{a}\right)=4 \Im m\left[\frac{q}{p}\left(M_{12}-\frac{i}{2} \Gamma_{12}\right)\right],
\end{aligned}
$$

qui sont reliées par :

$$
\begin{aligned}
(\Delta M)^{2}-\frac{1}{4}(\Delta \Gamma)^{2} & =4\left|M_{12}\right|^{2}-\left|\Gamma_{12}\right|^{2} \\
\Delta M . \Delta \Gamma & =4 \Re e\left(M_{12} \Gamma_{12}^{*}\right) .
\end{aligned}
$$

Le rapport $q / p$ est donné par :

$$
\frac{q}{p}=-\frac{1}{2} \frac{\Delta M-\frac{i}{2} \Delta \Gamma}{M_{12}-\frac{i}{2} \Gamma_{12}}=-2 \frac{M_{12}^{*}-\frac{i}{2} \Gamma_{12}^{*}}{\Delta M-\frac{i}{2} \Delta \Gamma}
$$

La conservation de la symétrie $C P$ implique que $|q / p|=1$, les différences de masse et de largeur se réduisant alors à $[22,24]$

$$
|\Delta M|=2\left|M_{12}\right|, \quad|\Delta \Gamma|=2\left|\Gamma_{12}\right| .
$$

Pour le moment, les indices $a$ et $b$ des équations (1.8) et (1.9) n'ont pas de sens physique. Les signes de $\Delta M$ et $\Delta \Gamma$ sont arbitraires. Cependant, leur signe relatif a un sens physique : il indique si l'état le plus lourd a la durée de vie la plus longue ou la plus courte [22, 24]. Le changement des indices $a \leftrightarrow b$ implique le changement $q / p \rightarrow-q / p$, lié avec $\Delta M \rightarrow-\Delta M$ et $\Delta \Gamma \rightarrow-\Delta \Gamma$. Ainsi, le signe de $q / p$, ainsi que celui de $\Delta \Gamma$, n'ont de sens que par rapport à celui de $\Delta M$. Par convention, nous choisissons $\Delta M>0$, c'est-à-dire $M_{b}>M_{a}$. Une fois cette convention adoptée ${ }^{4}$, il devient

\footnotetext{
${ }^{3}\left\langle B_{b} \mid B_{a}\right\rangle=|p|^{2}-|q|^{2} \neq 0$. C'est une mesure de la violation de $C P$ dans une dynamique $\Delta B=2$, appelée violation de $C P$ dans le mélange.

${ }^{4} \mathrm{On}$ a vu qu'exprimer les états propres de masse explicitement en termes des états propres de saveur implique des conventions. Ce choix est sans influence sur les observables si l'on s'en tient à la convention choisie, aux risques sinon d'aboutir à des contradictions ou des conclusions erronées.
} 
légitime de se poser la question ${ }^{5}$ de savoir si $\Gamma_{b}>\Gamma_{a}$ ou $\Gamma_{a}>\Gamma_{b}$ [27].

\subsubsection{Conventions de phase}

L'ambiguïté de signe ci-dessus (cf. eq. (1.13)) n'est en fait qu'un cas particulier d'une ambiguïté plus générale. En mécanique quantique, les kets sont définis à une phase près sans conséquence pour les observables. $\left|\bar{B}_{d}^{0}\right\rangle$ est relié à $\left|B_{d}^{0}\right\rangle$ par une transformation $C P$ :

$$
\mathcal{C P}\left|B_{d}^{0}\right\rangle=\eta_{B}\left|\bar{B}_{d}^{0}\right\rangle \quad \text { avec } \quad\left|\eta_{B}\right|=1
$$

Adopter un autre choix de phase, tel que par exemple changer $\mathcal{C P}\left|B_{d}^{0}\right\rangle=\left|\bar{B}_{d}^{0}\right\rangle$ en $\mathcal{C P}\left|B_{d}^{0}\right\rangle=e^{i \alpha_{B}}\left|\bar{B}_{d}^{0}\right\rangle$ change les éléments non-diagonaux de $\mathbf{M}$ et $\boldsymbol{\Gamma}$ :

$$
M_{12}-\frac{i}{2} \Gamma_{12} \rightarrow e^{i \alpha_{B}}\left(M_{12}-\frac{i}{2} \Gamma_{12}\right)
$$

ainsi que $q / p$ :

$$
\frac{q}{p} \rightarrow e^{-i \alpha_{B}} \frac{q}{p},
$$

laissant le produit $(q / p) \cdot\left(M_{12}-\frac{i}{2} \Gamma_{12}\right)$ invariant, comme il se doit, étant donné que les valeurs propres de l'hamiltonien effectif (qui sont des observables ${ }^{6}$ ), dépendent de ce produit. Il convient aussi de remarquer que $q / p$ n'est pas une observable, seul son module ayant une signification physique.

\subsection{3 Évolution temporelle}

Seuls les états propres de masse ont une évolution exponentielle :

$$
\left|B_{a, b}(t)\right\rangle=e^{-i \mu_{a, b} t}\left|B_{a, b}(0)\right\rangle
$$

où $t$ est évalué dans le repère du méson $B$ au repos ${ }^{7}$ (temps propre). Les états propres de saveur étant des combinaisons linéaires des états propres de masse, ils exhibent des phénomènes d'oscillation.

Un état pur $\left|B_{d}^{0}\right\rangle$ (ou $\left|\bar{B}_{d}^{0}\right\rangle$ ) au temps propre $t=0$ évolue en un mélange de $\left|B_{d}^{0}\right\rangle$ et $\left|\bar{B}_{d}^{0}\right\rangle$ :

$$
\begin{aligned}
\left|B_{d}^{0}(t)\right\rangle & =\frac{1}{2 p}\left(\left|B_{a}(t)\right\rangle+\left|B_{b}(t)\right\rangle\right)=g_{+}(t)\left|B_{d}^{0}\right\rangle+\frac{q}{p} g_{-}(t)\left|\bar{B}_{d}^{0}\right\rangle, \\
\left|\bar{B}_{d}^{0}(t)\right\rangle & =\frac{1}{2 q}\left(\left|B_{a}(t)\right\rangle-\left|B_{b}(t)\right\rangle\right)=\frac{p}{q} g_{-}(t)\left|B_{d}^{0}\right\rangle+g_{+}(t)\left|\bar{B}_{d}^{0}\right\rangle,
\end{aligned}
$$

où

$$
g_{ \pm}(t)=\frac{1}{2}\left(e^{-i \mu_{a} t} \pm e^{-i \mu_{b} t}\right)
$$

\footnotetext{
${ }^{5}$ Dans le cas de la conservation de la symétrie $C P$, il devient légitime de se poser aussi la question de savoir si l'état le plus lourd est de parité $C P$ intrinsèque paire ou impaire [27].

${ }^{6}$ notamment à travers $\Delta M$ et $\Delta \Gamma$.

${ }^{7} B_{a}$ et $B_{b}$ ont des masses différentes et donc des repères au repos différents. Pourtant, dans l'approximation de Weisskopf-Wigner, le temps $t$ est le temps mesuré dans le repère au repos donné par la masse commune $M_{0}$ des interactions forte et électromagnétique.
} 
et

$$
\left|g_{ \pm}(t)\right|^{2}=\frac{e^{-\Gamma_{0} t}}{2}\left[\cosh \frac{\Delta \Gamma t}{2} \pm \cos (\Delta M t)\right]
$$

avec $\Gamma_{0}=\left(\Gamma_{a}+\Gamma_{b}\right) / 2$.

La probabilité qu'un méson $B_{d}^{0}$ au temps $t=0$ soit un méson $B_{d}^{0}$ au temps $t$ est égale à la probabilité qu'un méson $\bar{B}_{d}^{0}$ au temps $t=0$ soit un méson $\bar{B}_{d}^{0}$ au temps $t$ :

$$
\operatorname{Prob}\left(B_{d}^{0} \text { à } t \mid B_{d}^{0} \text { à } t=0\right)=\operatorname{Prob}\left(\bar{B}_{d}^{0} \text { à } t \mid \bar{B}_{d}^{0} \text { à } t=0\right)=\left|g_{+}(t)\right|^{2} .
$$

La situation est différente pour les probabilités $\operatorname{Prob}\left(\bar{B}_{d}^{0}\right.$ à $t \mid B_{d}^{0}$ à $\left.t=0\right)$ et $\operatorname{Prob}\left(B_{d}^{0}\right.$ à $t \mid \bar{B}_{d}^{0}$ à $\left.t=0\right)$ :

$$
\operatorname{Prob}\left(\bar{B}_{d}^{0} \text { à } t \mid B_{d}^{0} \text { à } t=0\right)=\left|\frac{q}{p}\right|^{2}\left|g_{-}(t)\right|^{2}, \quad \operatorname{Prob}\left(B_{d}^{0} \text { à } t \mid \bar{B}_{d}^{0} \text { à } t=0\right)=\left|\frac{p}{q}\right|^{2}\left|g_{-}(t)\right|^{2},
$$

qui ne sont égales que si la symétrie $C P$ est conservée dans le mélange, i.e. $|q / p|=1$.

En notant $A_{f}=\left\langle f\left|\mathcal{T}^{(\Delta B=1)}\right| B_{d}^{0}\right\rangle, \bar{A}_{f}=\left\langle f\left|\mathcal{T}^{(\Delta B=1)}\right| \bar{B}_{d}^{0}\right\rangle$ et les paramètres complexes $\lambda_{f}$ et $\bar{\lambda}_{f}$ par

$$
\lambda_{f} \equiv \frac{q}{p} \frac{\bar{A}_{f}}{A_{f}}, \quad \bar{\lambda}_{f} \equiv \frac{1}{\lambda_{f}}
$$

on obtient

$$
\begin{aligned}
\Gamma\left(B_{d}^{0}(t) \rightarrow f\right) & =\left|\left\langle f\left|\mathcal{T}^{(\Delta B=1)}\right| B_{d}^{0}(t)\right\rangle\right|^{2} \\
& =\left|A_{f}\right|^{2}\left[\left|g_{+}(t)\right|^{2}+\left|\lambda_{f}\right|^{2}\left|g_{-}(t)\right|^{2}+2 \Re e\left(\lambda_{f} g_{+}^{*}(t) g_{-}(t)\right)\right], \\
\Gamma\left(B_{d}^{0}(t) \rightarrow \bar{f}\right) & =\left|\left\langle\bar{f}\left|\mathcal{T}^{(\Delta B=1)}\right| B_{d}^{0}(t)\right\rangle\right|^{2} \\
& =\left|\bar{A}_{\bar{f}}\right|^{2}\left|\frac{q}{p}\right|^{2}\left[\left|g_{-}(t)\right|^{2}+\left|\bar{\lambda}_{\bar{f}}\right|^{2}\left|g_{+}(t)\right|^{2}+2 \Re e\left(\bar{\lambda}_{\bar{f}} g_{+}(t) g_{-}^{*}(t)\right)\right], \\
\Gamma\left(\bar{B}_{d}^{0}(t) \rightarrow f\right) & =\left|\left\langle f\left|\mathcal{T}^{(\Delta B=1)}\right| \bar{B}_{d}^{0}(t)\right\rangle\right|^{2} \\
& =\left|A_{f}\right|^{2}\left|\frac{p}{q}\right|^{2}\left[\left|g_{-}(t)\right|^{2}+\left|\lambda_{f}\right|^{2}\left|g_{+}(t)\right|^{2}+2 \Re e\left(\lambda_{f} g_{+}(t) g_{-}^{*}(t)\right)\right], \\
\Gamma\left(\bar{B}_{d}^{0}(t) \rightarrow \bar{f}\right) & =\left|\left\langle\bar{f}\left|\mathcal{T}^{(\Delta B=1)}\right| \bar{B}_{d}^{0}(t)\right\rangle\right|^{2} \\
& =\left|\bar{A}_{\bar{f}}\right|^{2}\left[\left|g_{+}(t)\right|^{2}+\left|\bar{\lambda}_{\bar{f}}\right|^{2}\left|g_{-}(t)\right|^{2}+2 \Re e\left(\bar{\lambda}_{\bar{f}} g_{+}^{*}(t) g_{-}(t)\right)\right],
\end{aligned}
$$

avec

$$
g_{+}^{*}(t) g_{-}(t)=\frac{e^{-\Gamma_{0} t}}{2}\left[\sinh \frac{\Delta \Gamma t}{2}+i \sin (\Delta M t)\right] .
$$

Ces expressions donnent la probabilité qu'un état qui est à l'instant $t=0$ un $\left|B_{d}^{0}\right\rangle$ ou un $\left|\bar{B}_{d}^{0}\right\rangle$ se désintègre à l'instant $t$ dans l'état final $|f\rangle$ ou $|\bar{f}\rangle$.

Si $|f\rangle$ est un état propre de $C P$, on a

$$
|\bar{f}\rangle \equiv \mathcal{C P}|f\rangle=\eta_{C P}(f)|f\rangle, \quad\left(\eta_{C P}(f)= \pm 1\right)
$$


où $\eta_{C P}(f)$ est la parité $C P$ intrinsèque de $f$. Cela implique

$$
\bar{A}_{\bar{f}}=\eta_{C P}(f) \bar{A}_{f}, \quad A_{\bar{f}}=\eta_{C P}(f) A_{f}, \quad \lambda_{f}=\lambda_{\bar{f}}=\frac{1}{\bar{\lambda}_{f}}=\frac{1}{\bar{\lambda}_{\bar{f}}} .
$$

Les équations d'évolution temporelle (1.31) et (1.32) ((1.33) et 1.34)) deviennent identiques.

\subsection{Violation de $C P$}

Afin de mettre en évidence la conservation ou la violation d'une symétrie, il convient de comparer des paires de processus reliés par la dite symétrie. Dans le cas de la symétrie $C P$, nous sommes donc intéressés par des paires de processus de désintégration qui sont reliés par une transformation $C P$. Soient $f$ et $\bar{f}$ les états finals conjugués de $C P$ :

$$
\mathcal{C P}|f\rangle=e^{i \alpha_{f}}|\bar{f}\rangle, \quad \mathcal{C P}|\bar{f}\rangle=e^{-i \alpha_{f}}|f\rangle
$$

Les états de saveur sont aussi définis par une transformation $C P$ :

$$
\mathcal{C P}\left|B_{d}^{0}\right\rangle=e^{i \alpha_{B}}\left|\bar{B}_{d}^{0}\right\rangle
$$

Les phases $\alpha_{f}$ et $\alpha_{B}$ sont arbitraires. Si $|f\rangle$ est un état propre de $C P$ alors $e^{i \alpha_{f}}=\eta_{C P}(f)= \pm 1$, suivant que $f$ est de parité $C P$ intrinsèque paire ou impaire.

Les amplitudes $A_{f}$ et $\bar{A}_{\bar{f}}$, conjuguées de $C P$ et décrivant les processus $B_{d}^{0} \rightarrow f$ et $\bar{B}_{d}^{0} \rightarrow \bar{f}$, peuvent s'écrire comme les sommes de différentes contributions :

$$
\begin{aligned}
A_{f} & =\left\langle f\left|\mathcal{T}^{(\Delta B=1)}\right| B_{d}^{0}\right\rangle=\sum_{j}\left|A_{j}\right| e^{i \delta_{j}} e^{i \phi_{j}}, \\
\bar{A}_{\bar{f}} & =\left\langle\bar{f}\left|\mathcal{T}^{(\Delta B=1)}\right| \bar{B}_{d}^{0}\right\rangle \\
& =\left\langle\bar{f}\left|(\mathcal{C P})^{\dagger}(\mathcal{C P}) \mathcal{T}^{(\Delta B=1)}(\mathcal{C P})^{\dagger}(\mathcal{C P})\right| \bar{B}_{d}^{0}\right\rangle \\
& =e^{-i\left(\alpha_{B}-\alpha_{f}\right)}\left\langle f\left|(\mathcal{C P}) \mathcal{T}^{(\Delta B=1)}(\mathcal{C P})^{\dagger}\right| B_{d}^{0}\right\rangle \\
& =e^{-i\left(\alpha_{B}-\alpha_{f}\right)} \sum_{j}\left|A_{j}\right| e^{i \delta_{j}} e^{-i \phi_{j}}
\end{aligned}
$$

Deux types de phase $[22,25]$ interviennent dans les amplitudes de désintégration : les phases faibles $\phi_{j}$ sont des paramètres de la partie du lagrangien qui violent $C P$. Elles apparaissent généralement dans le secteur électrofaible de la théorie (dans le Modèle Standard, ces phases proviennent de la matrice $\mathrm{CKM}$ ) et contribuent à $A_{f}$ et $\bar{A}_{\bar{f}}$ avec des signes opposés. Les phases fortes $\delta_{j}$ apparaissent dans les amplitudes de diffusion même si le lagrangien est invariant sous la symétrie $C P$. Elles proviennent en général des effets de rediffusion via des états intermédiaires réels (FSI $\equiv$ Final State Interaction) dus à l'interaction forte (ou électromagnétique) et contribuent à $A_{f}$ et $\bar{A}_{\bar{f}}$ avec le même signe. Seules les phases qui ne changent pas après rephasage des vecteurs d'état (cf. §1.1.2) ont un sens physique et peuvent conduire à la violation de $C P$.

Les effets de la violation de $C P$ se manifestent par des différences de phase entre (au moins) deux amplitudes. Ces phases peuvent être observées uniquement si ces amplitudes contribuent de 
(A)

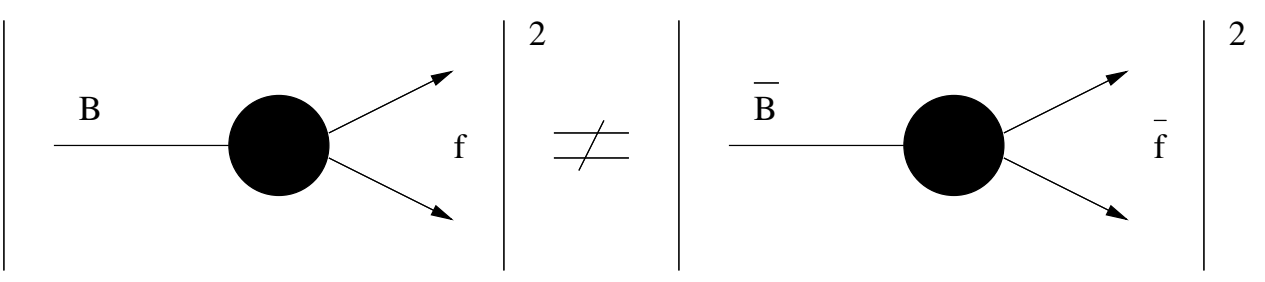

(B)

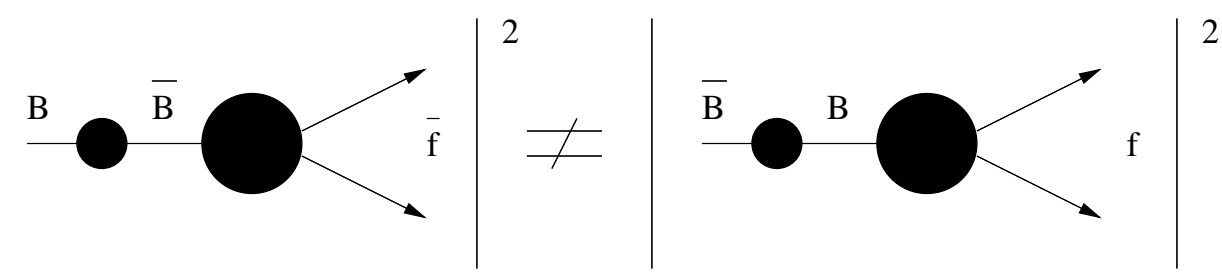

(C)
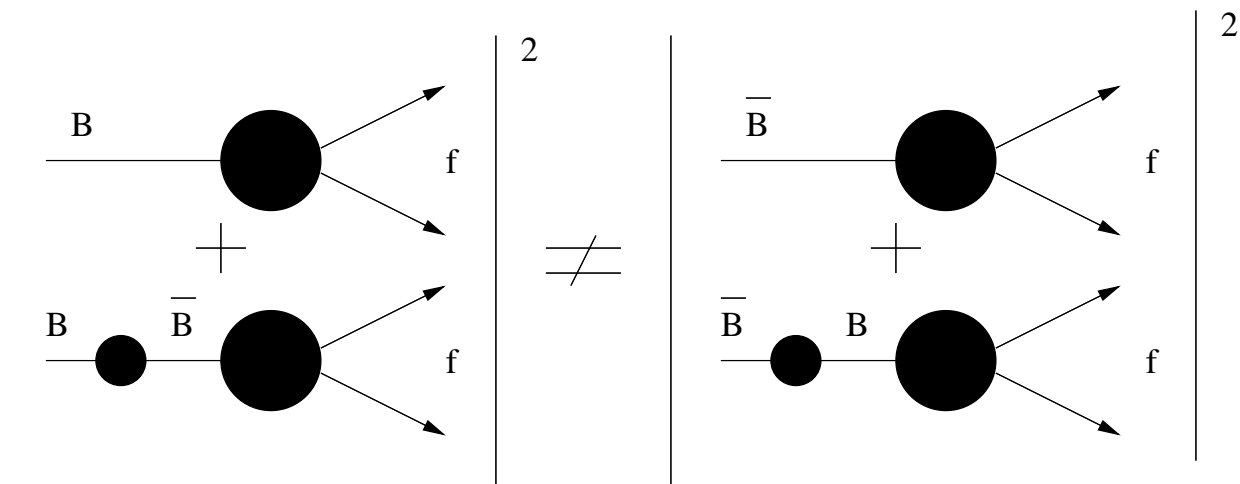

FIG. 1.2: Trois types de violation de $C P$ : (A) violation de $C P$ directe ; (B) violation de $C P$ indirecte ; (C) violation de $C P$ dans l'interférence entre les désintégrations avec et sans mélange.

façon cohérente au même processus et peuvent ainsi interférer. Ces effets sont susceptibles de se manifester de trois façons différentes [23, 25, 26], illustrés par la figure 1.2 :

- violation de $C P$ dans la désintégration, dite aussi violation de $C P$ directe $^{8}$. Cela se produit lorsqu'une amplitude et son conjugué de $C P$ ont des taux de désintégration différents. Ce type de violation de $C P$ n'apparaît que dans les amplitudes $\Delta B=1$;

- violation de $C P$ dans le mélange ou violation de $C P$ indirecte, qui se manifeste dans les amplitudes $\Delta B=2$;

- violation de $C P$ dans l'interférence entre les désintégrations avec et sans mélange qui se produit pour des désintégrations communes à $B_{d}^{0}$ et $\bar{B}_{d}^{0}$.

\subsubsection{Désintégration}

Bien que la définition des phases fortes et faibles dépende de conventions de phase, le rapport

$$
\left|\frac{\bar{A}_{\bar{f}}}{A_{f}}\right|=\left|\frac{\sum_{j}\left|A_{j}\right| e^{i \delta_{j}} e^{-i \phi_{j}}}{\sum_{j}\left|A_{j}\right| e^{i \delta_{j}} e^{i \phi_{j}}}\right|
$$

\footnotetext{
${ }^{8}$ Certains auteurs, en particulier ceux de la référence [20], utilisent le terme de violation de $C P$ directe pour toute violation de la symétrie $C P$ qui n'est pas indirecte, c'est-à-dire qui exclut le modèle superfaible (voir la note en bas de page 1).
} 
a)

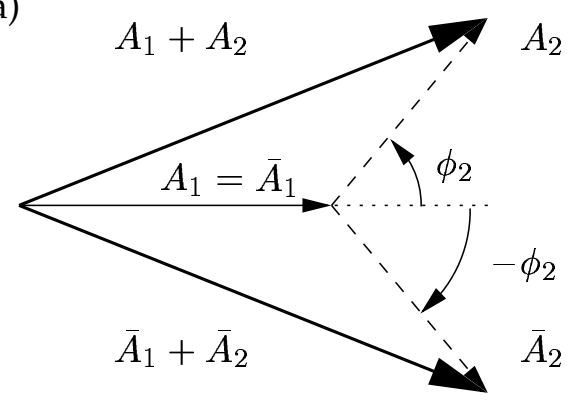

b) $A_{1}+A_{2} \nearrow_{1} A_{2}$

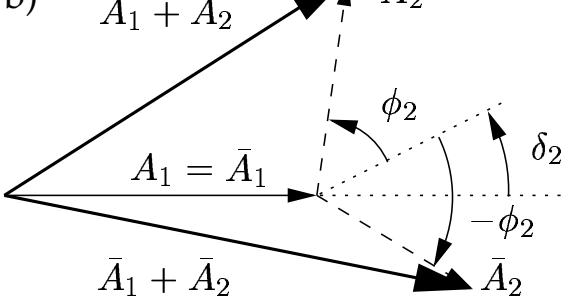

FIG. 1.3: Violation de $C P$ directe avec deux amplitudes $A_{1}$ et $A_{2}$. Les amplitudes $\bar{A}_{1}$ et $\bar{A}_{2}$ sont les amplitudes conjuguées de $C P$ de $A_{1}$ et $A_{2}$. a) Il existe une phase faible relative entre les amplitudes $A_{1}$ et $A_{2}$, mais pas de phase forte. Le processus conjugué de $C P, \bar{A}_{1}+\bar{A}_{2}$, a la même norme que le processus initial, $A_{1}+A_{2}$, et il n'y a pas de violation de $C P$. b) Il y a à la fois une phase forte et une phase faible relatives entre les amplitudes $A_{1}$ et $A_{2}$. La norme de $\bar{A}_{1}+\bar{A}_{2}$ diffère de celle de $A_{1}+A_{2}$, traduisant la violation de la symétrie $C P$.

est indépendant de ces conventions et est donc la quantité physique significative. Quand la symétrie $C P$ est conservée, les phases faibles $\phi_{j}$ sont toutes égales. En effet, dans le cas où la symétrie $C P$ est conservée, i.e. $\left[\mathcal{C P}, \mathcal{T}^{(\Delta B=1)}\right]=0$, l'amplitude $\bar{A}_{\bar{f}}$ est reliée à l'amplitude $A_{f}$ par (cf. eqs. (1.40) et (1.41)) :

$$
\bar{A}_{\bar{f}}=\left\langle\bar{f}\left|\mathcal{T}^{(\Delta B=1)}\right| \bar{B}_{d}^{0}\right\rangle=e^{-i\left(\alpha_{B}-\alpha_{f}\right)}\left\langle f\left|\mathcal{T}^{(\Delta B=1)}\right| B_{d}^{0}\right\rangle=e^{-i\left(\alpha_{B}-\alpha_{f}\right)} A_{f} .
$$

Ainsi, la condition de violation de $C P$ dans la désintégration se traduit par :

$$
\left|\frac{\bar{A}_{\bar{f}}}{A_{f}}\right| \neq 1 \quad \Longrightarrow \text { violation de } C P
$$

et résulte donc de l'interférence d'amplitudes de désintégration conduisant au même état final. Il est à noter que ce type de violation de $C P$ requiert qu'au moins deux termes de (1.40) aient des phases fortes et faibles différentes. En effet, on a

$$
\left|A_{f}\right|^{2}-\left|\bar{A}_{\bar{f}}\right|^{2}=-2 \sum_{i, j}\left|A_{i}\right|\left|A_{j}\right| \sin \left(\phi_{i}-\phi_{j}\right) \sin \left(\delta_{i}-\delta_{j}\right)
$$

La figure 1.3 illustre la situation de violation de $C P$ directe dans le cas de deux amplitudes. Ce type de violation de $C P$ est le seul accessible aux mésons $B$ chargés. Dans le cas des mésons $B$ neutres, la violation de $C P$ directe entre en général en compétition avec les deux autres types de violation de $C P$.

La violation de $C P$ directe met en jeu des effets de QCD à longue distance, à travers $A_{i}$ et $\delta_{i}$, affectant ainsi la détermination des phases faibles.

\subsubsection{Mélange}

On a vu que les mésons neutres peuvent se mélanger via des canaux communs :

$$
B_{d}^{0} \leftrightarrow f \leftrightarrow \bar{B}_{d}^{0}
$$



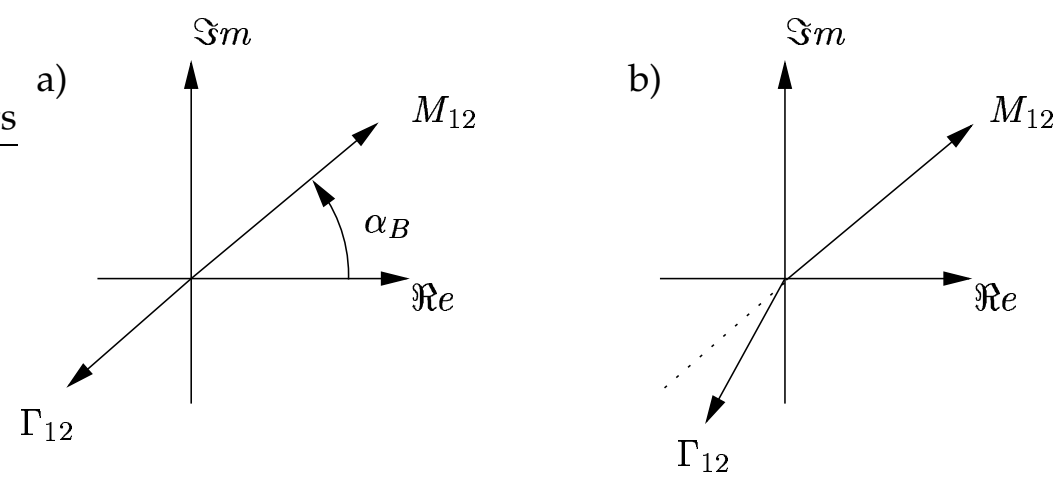

FIG. 1.4: Violation de $C P$ dans le mélange. a) Les amplitudes $M_{12}$ et $\Gamma_{12}$ sont colinéaires de sorte que $\Im m\left(M_{12} \Gamma_{12}^{*}\right)=0$, il n'y a donc pas de violation de $C P$. L'orientation générale de $M_{12}$ et $\Gamma_{12}$ dépend de conventions de phase (cf. la discussion $\S 1.1 .2)$ b) $M_{12}$ et $\Gamma_{12}$ pointent dans des directions différentes, il y a violation de $C P$.

La quantité physique reliée au mélange est $q / p$ mais dépend de conventions de phase. La quantité physique pertinente car indépendante de ces conventions est

$$
\left|\frac{q}{p}\right|^{2}=\left|\frac{M_{12}^{*}-\frac{i}{2} \Gamma_{12}^{*}}{M_{12}-\frac{i}{2} \Gamma_{12}}\right| .
$$

Quand la symétrie $C P$ est conservée, les états propres de masse sont aussi états propres de $C P$. Cela se traduit par

$$
\frac{M_{12}^{*}}{\Gamma_{12}^{*}}=\frac{M_{12}}{\Gamma_{12}}
$$

soit $|q / p|=1$ ( $q / p$ est alors une phase pure). Ainsi, l'équation (1.47) implique que

$$
\left|\frac{q}{p}\right| \neq 1 \quad \Longrightarrow \text { violation de } C P \text { dans le mélange. }
$$

Cette condition est équivalente à

$$
\Im m\left(M_{12} \Gamma_{12}^{*}\right)=\left|M_{12}\right|\left|\Gamma_{12}\right| \sin \left(\theta_{M_{12}}-\theta_{\Gamma_{12}}\right) \neq 0
$$

en écrivant $M_{12}=\left|M_{12}\right| e^{i \theta_{M_{12}}}$ et $\Gamma_{12}=\left|\Gamma_{12}\right| e^{i \theta_{\Gamma_{12}}}$. Elle exprime que pour avoir violation de $C P$, il faut que les amplitudes $M_{12}$ et $\Gamma_{12}$ ne soient pas colinéaires dans le plan complexe et que ni $M_{12}$ ni $\Gamma_{12}$ ne doivent être nulles (voir Fig. 1.4). Il est remarquable que cette condition soit aussi simple, étant donnée la complexité des différents états intermédiaires intervenant dans $M_{12}$ et $\Gamma_{12}$. La violation de $C P$ dans le mélange a pour origine l'interférence entre l'amplitude totale des états intermédiaires virtuels, $M_{12}$, et l'amplitude totale des états intermédiaires réels, $\Gamma_{12}$ (voir Fig. 1.5).

Dans le système des mésons $B$, on s'attend à un effet très petit, de l'ordre de $10^{-2}$ [25]. En effet, les canaux de désintégration communs à $B_{d}^{0}$ et $\bar{B}_{d}^{0}$, décrits par $\Gamma_{12}$ et responsables de la différence $\Delta \Gamma$, sont connus pour avoir des rapports d'embranchement de l'ordre de $10^{-3}$, voire moins. Ainsi, bien que $\Delta \Gamma$ n'ait pas encore été mesuré, on peut prétendre avec sûreté que $|\Delta \Gamma| / \Gamma_{0}=\mathcal{O}\left(10^{-2}\right)$, et cela indépendamment du modèle théorique. De plus, la valeur mesurée de $\Delta M / \Gamma_{0}=0.755 \pm 0.015$ 


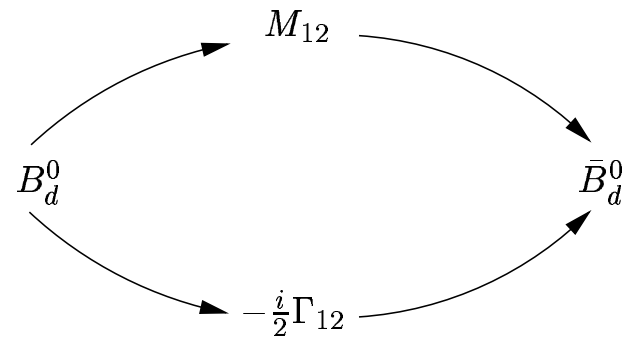

a)

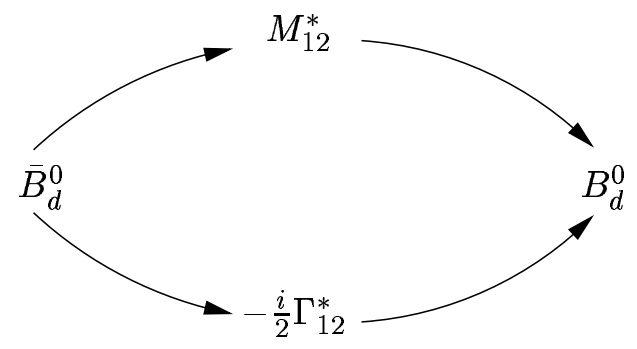

b)

FIG. 1.5: Violation de $C P$ dans le mélange. L'interférence se produit entre l'amplitude totale des états intermédiaires hors couche de masse $\left(M_{12}\right)$ et l'amplitude totale des états intermédiaires sur couche de masse $\left(\Gamma_{12}\right)$.

[28] implique, indépendamment du modèle théorique, que

$$
|\Delta \Gamma| \ll \Delta M
$$

Il s'ensuit que $\left|\Gamma_{12}\right| \ll\left|M_{12}\right|$, et donc au premier ordre en $\Gamma_{12} / M_{12}$, on obtient de l'équation (1.18) que

$$
\frac{q}{p} \simeq-\frac{M_{12}^{*}}{\left|M_{12}\right|}\left[1-\frac{1}{2} \Im m\left(\frac{\Gamma_{12}}{M_{12}}\right)\right] .
$$

Les équations (1.16) et (1.17) impliquent, à une approximation de l'ordre de $10^{-2}$, que

$$
\Delta M \simeq 2\left|M_{12}\right|, \quad \Delta \Gamma \simeq 2 \frac{\Re e\left(M_{12} \Gamma_{12}^{*}\right)}{\left|M_{12}\right|} .
$$

Pour prédire $|q / p|$ dans un modèle donné, il est nécessaire de calculer $\Gamma_{12}$ et $M_{12}$. Cela implique de larges incertitudes hadroniques, en particulier dans les modèles d'hadronisation pour $\Gamma_{12}$. Ainsi, même si un effet de violation de $C P$ indirecte est mesuré, il sera difficile de le relier à des paramètres de la matrice CKM.

\subsubsection{Interférence désintégration-mélange}

Considérons le cas où $B_{d}^{0}$ et $\bar{B}_{d}^{0}$ se désintègrent dans le même état final $f$, pas nécessairement avec le même taux. Les états finals propres de $C P$ tombent dans cette catégorie. Nous ne traiterons que ce cas là, pour les autres cas, voir par exemple les références [29, 30].

En désignant par $A_{f_{C P}}=\left\langle f_{C P}\left|\mathcal{T}^{(\Delta B=1)}\right| B_{d}^{0}\right\rangle$ et $\bar{A}_{f_{C P}}=\left\langle f_{C P}\left|\mathcal{T}^{(\Delta B=1)}\right| \bar{B}_{d}^{0}\right\rangle$ avec $\left|\bar{f}_{C P}\right\rangle \equiv \mathcal{C P}\left|f_{C P}\right\rangle=$ $\eta_{C P}(f)\left|f_{C P}\right\rangle\left(\eta_{C P}(f)= \pm 1\right)$, on peut montrer que la quantité

$$
\lambda_{f_{C P}} \equiv \frac{q}{p} \frac{\bar{A}_{f_{C P}}}{A_{f_{C P}}}=\eta_{C P}(f) \frac{q}{p} \frac{\bar{A}_{\bar{f}_{C P}}}{A_{f_{C P}}}
$$

est indépendante de conventions de phase et donc significative (la dépendance de $q / p$ et de $\bar{A}_{f_{C P}} / A_{f_{C P}}$ en conventions de phase s'annule). La condition de violation de $C P$ dans l'interférence désintégrationmélange est

$$
\lambda_{f_{C P}} \neq 1 \Longrightarrow \text { violation de } C P \text {. }
$$

Il est à noter que la violation de $C P$ dans la désintégration $\left(\left|\bar{A}_{\bar{f}_{C P}} / A_{f_{C P}}\right| \neq 1\right)$ et aussi dans le 


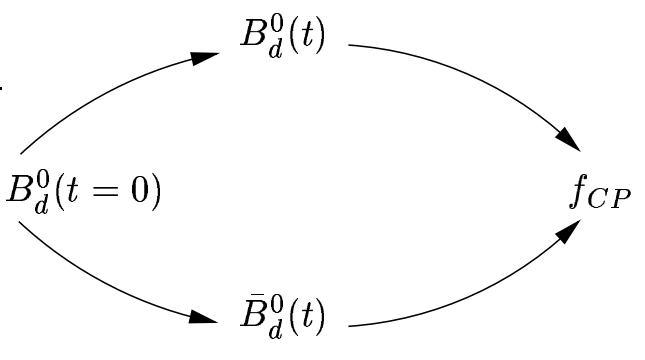

a)

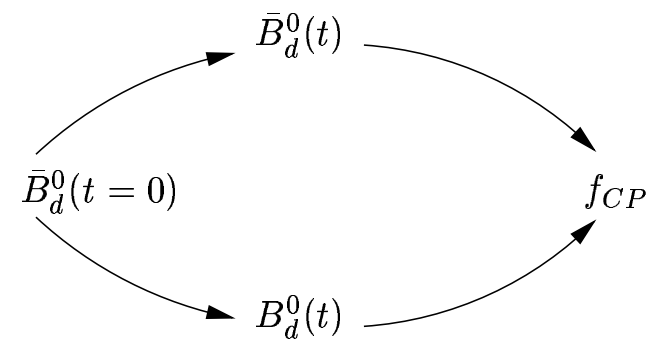

b)

FIG. 1.6: a) Violation de $C P$ dans l'interférence entre la désintégration, au temps $t$, directe $B_{d}^{0} \rightarrow f_{C P}$, et celle après mélange, $B_{d}^{0} \rightarrow \bar{B}_{d}^{0} \rightarrow f_{C P}$, pour un méson $B$ initialement $B_{d}^{0}$. b) idem mais pour un méson $B$ initialement $\bar{B}_{d}^{0}$.

mélange $(|q / p| \neq 1)$ vérifient (1.55), à travers $\left|\lambda_{f_{C P}}\right| \neq 1$. Cependant, le cas particuliers où $|q / p|=1$ et $\left|\bar{A}_{\bar{f}_{C P}} / A_{f_{C P}}\right|=1$ avec violation de $C P$ :

$$
\left|\lambda_{f_{C P}}\right|=1, \quad \Im m \lambda_{f_{C P}} \neq 0
$$

représente la situation théorique la plus favorable. Dans ce cas, $\lambda_{f_{C P}}$ est une phase pure et peut être calculée sans incertitudes hadroniques. Dans le Modèle Standard, cette phase est directement reliée aux paramètres CKM.

En définissant l'asymétrie

$$
\mathcal{A}_{f_{C P}}(t)=\frac{\Gamma\left(B_{d}^{0}(t) \rightarrow f_{C P}\right)-\Gamma\left(\bar{B}_{d}^{0}(t) \rightarrow f_{C P}\right)}{\Gamma\left(B_{d}^{0}(t) \rightarrow f_{C P}\right)+\Gamma\left(\bar{B}_{d}^{0}(t) \rightarrow f_{C P}\right)}
$$

où, d'après l'équation (1.24), on a

$$
\begin{aligned}
\Gamma\left(B_{d}^{0}(t) \rightarrow f_{C P}\right) & \equiv\left|\left\langle f_{C P}\left|\mathcal{T}^{(\Delta B=1)}\right| B_{d}^{0}(t)\right\rangle\right|^{2} \\
& =\left|\left\langle f_{C P}\left|\mathcal{T}^{(\Delta B=1)}\right| B_{d}^{0}\right\rangle g_{+}(t)+\left\langle f_{C P}\left|\mathcal{T}^{(\Delta B=1)}\right| \bar{B}_{d}^{0}\right\rangle \frac{q}{p} g_{-}(t)\right|^{2}
\end{aligned}
$$

Cette expression montre l'interférence entre la désintégration directe $B_{d}^{0} \rightarrow f_{C P}$ (premier terme) et celle après mélange $B_{d}^{0} \rightarrow \bar{B}_{d}^{0} \rightarrow f_{C P}$ (deuxième terme), pour un méson $B$ initialement $B_{d}^{0}$.

En prenant en compte que, pour le système des mésons $B,|\Delta \Gamma| \ll \Gamma_{0}$ et $|q / p| \simeq|p / q| \simeq 1$, on obtient

$\left.\Gamma \stackrel{(-)}{B_{d}^{0}}(t) \rightarrow f_{C P}\right)=\left|A_{f_{C P}}\right|^{2} \frac{e^{-\Gamma_{0} t}}{2}\left[1+\left|\lambda_{f_{C P}}\right|^{2} \stackrel{(-)}{+}\left(1-\left|\lambda_{f_{C P}}\right|^{2}\right) \cos (\Delta M t) \stackrel{(+)}{-} 2 \Im m \lambda_{f_{C P}} \sin (\Delta M t)\right]$

conduisant à l'asymétrie ${ }^{9}$

$$
\mathcal{A}_{f_{C P}}(t)=\frac{1-\left|\lambda_{f_{C P}}\right|^{2}}{1+\left|\lambda_{f_{C P}}\right|^{2}} \cdot \cos (\Delta M t)-\frac{2 \Im m \lambda_{f_{C P}}}{1+\left|\lambda_{f_{C P}}\right|^{2}} \cdot \sin (\Delta M t)
$$

où le premier terme signe la violation de $C P$ dans la désintégration $(|q / p|=1)$. Pour $\left|\lambda_{f_{C P}}\right|=1$

\footnotetext{
${ }^{9}$ Le signe - devant $\Im m \lambda_{f_{C P}}$ provient de notre définition de $\Delta M \equiv M_{b}-M_{a}$. Si on avait choisi $\Delta M \equiv M_{a}-M_{b}$, on aurait un signe $+[22]$. Cependant, il convient de remarquer que le résultat final, i.e. après avoir exprimé $\lambda_{f_{C P}}$, est indépendant du choix de convention fait sur $\Delta M[23,31]$.
} 


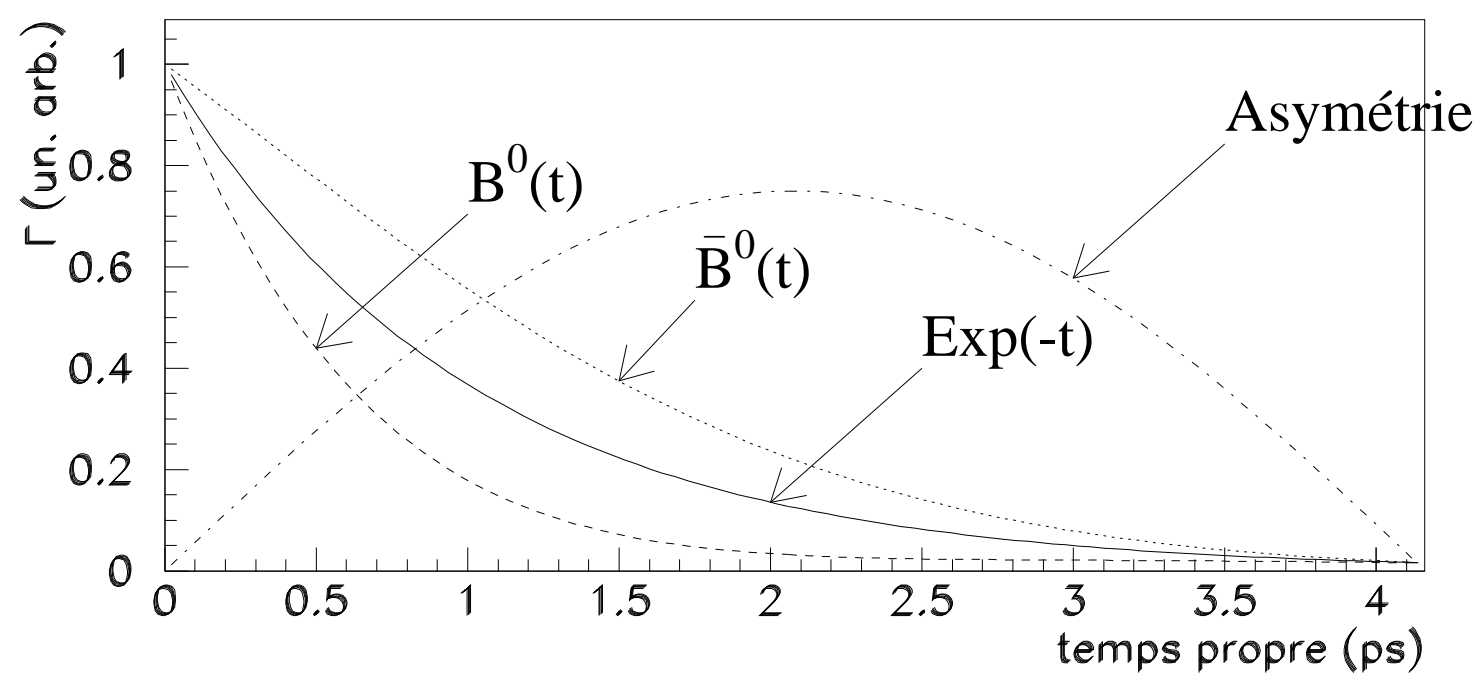

FIG. 1.7: Asymétrie $C P$ provenant de l'interférence désintégration-mélange, dans le cas particulier $\left|\lambda_{f_{C P}}\right|=1$. Les différentes courbes représentent les taux de désintégration en fonction du temps propre, $\Gamma\left(B_{d}^{0}(t) \rightarrow f_{C P}\right)$ et $\Gamma\left(\bar{B}_{d}^{0}(t) \rightarrow\right.$ $f_{C P}$ ) pour un méson $B$ étant à $t=0$ un $B_{d}^{0}$ et un $\bar{B}_{d}^{0}$, respectivement. L'asymétrie est une sinusoïde en fonction du temps, dont la valeur maximale est $\Im m \lambda_{f_{C P}}$ prise égale à 0.75 .

(voir Fig. 1.7), on a

$$
\mathcal{A}_{f_{C P}}(t)=-\Im m \lambda_{f_{C P}} \sin (\Delta M t)
$$

Les modes « propres » avec $\left|\lambda_{f_{C P}}\right|=1$ sont ceux dominés par une seule phase faible $\phi_{f_{C P}}$, soit

$$
\frac{\bar{A}_{f_{C P}}}{A_{f_{C P}}} \simeq e^{-2 i \phi_{f_{C P}}}
$$

Enfin, si on considère deux états propres de $C P, f_{C P}$ et $g_{C P}\left(f_{C P} \neq g_{C P}\right)$, alors la conservation de la symétrie $C P$ implique [26]

$$
\eta_{C P}(f) \lambda_{f_{C P}}-\eta_{C P}(g) \lambda_{g_{C P}}=\frac{q}{p}\left(\frac{\bar{A}_{\bar{f}_{C P}}}{A_{f_{C P}}}-\frac{\bar{A}_{\bar{g}_{C P}}}{A_{g_{C P}}}\right)=0 .
$$

Toute valeur non-nulle établirait l'existence de violation ${ }^{10}$ de $C P$ dans les processus $\Delta B=1$. Pour cette raison, ce type de violation de $C P$ est parfois appelé violation de $C P$ directe [28], bien que dans ce cas aucune différence non-triviale de phases fortes ne soit nécessaire.

En conclusion, des trois types de violation de $C P$ possibles dans le système des mésons $B$ (voir la table 1.1), le plus favorable est celui de l'interférence entre les désintégrations avec et sans mélange, avec les modes dominés par une seule phase faible, du fait de la quasi-absence d'incertitudes hadroniques, au contraire des deux autres types de violation de $C P$ qui les rendent plus difficiles à interpréter.

\footnotetext{
${ }^{10}$ Ce type de violation de $C P$ est relié à $\epsilon^{\prime}$ dans le système des kaons neutres.
} 
ТАВ. 1.1: Résumé des différents types de violation de $C P$ avec leur observable et incertitudes hadroniques respectives.

\begin{tabular}{|l||c|c|}
\hline Type de violation de $C P$ & Observable & Incertitudes hadroniques \\
\hline \hline Désintégration & $\left|\bar{A}_{\bar{f}} / A_{f}\right|$ & importantes \\
Mélange & $|q / p|$ & importantes \\
Interférence désintégration-mélange & $\Im m \lambda_{f_{C P}}$ & faibles si $\left|\lambda_{f_{C P}}\right|=1$ \\
\hline
\end{tabular}

\subsection{Violation de $C P$ dans le Modèle Standard}

Le Modèle Standard [32, 33, 34] est la théorie minimale qui décrit tous les phénomènes connus de physique des particules, c'est-à-dire des quarks, des leptons et de leurs interactions. Du fait de ses impressionnants succès, il convient de situer la violation de $C P$ dans ce cadre théorique afin de mettre en lumière des déviations possibles dues à de la nouvelle physique.

Le Modèle Standard est une théorie quantique des champs renormalisable construite sur le principe d'invariance locale de jauge, dont le groupe de jauge est $S U(3)_{C} \otimes S U(2)_{L} \otimes U(1)_{Y}$. Ce groupe de jauge inclut le groupe de symétrie de l'interaction forte, $S U(3)_{C}$, et le groupe de symétrie de l'interaction électrofaible, $S U(2)_{L} \otimes U(1)_{Y}$. Le Modèle Standard peut être décomposé en trois secteurs :

- le secteur de jauge, qui contient les bosons médiateurs des interactions. Il est composé de huit gluons, bosons de jauge de l'interaction forte, et des particules $\gamma, W^{ \pm}$et $Z^{0}$ qui sont les quatre bosons de jauge de l'interaction électrofaible. Les gluons sont de masse nulle, électriquement neutres et portent un nombre quantique de couleur. Il y a huit gluons car ils apparaissent avec huit configurations de couleurs différentes. Étant colorés, les gluons interagissent non seulement avec les quarks mais aussi entre-eux. Les bosons de l'interaction faible, $W^{ \pm}$et $Z^{0}$, sont massifs et interagissent entre-eux. Le photon, $\gamma$, est de masse nulle, sans charge électrique et n'interagit pas avec lui-même ;

- le secteur fermionique, qui se compose des quarks et des leptons (particules de matière), est organisé en trois familles de propriétés identiques sauf pour les masses. Le contenu en particules de chaque famille est :

$$
\begin{aligned}
1^{\text {ère famille }} & \left(\begin{array}{c}
\nu_{e} \\
e^{-}
\end{array}\right)_{L}, e_{R}^{-},\left(\begin{array}{c}
u \\
d
\end{array}\right)_{L}, u_{R}, d_{R} ; \\
2^{\text {ème famille }} & \left(\begin{array}{c}
\nu_{\mu} \\
\mu^{-}
\end{array}\right)_{L}, \mu_{R}^{-},\left(\begin{array}{c}
c \\
s
\end{array}\right)_{L}, c_{R}, s_{R} ; \\
3^{\text {ème famille }} & \left(\begin{array}{c}
\nu_{\tau} \\
\tau^{-}
\end{array}\right)_{L}, \tau_{R}^{-},\left(\begin{array}{c}
t \\
b
\end{array}\right)_{L}, t_{R}, b_{R} ;
\end{aligned}
$$

et les antiparticules correspondantes. Les spineurs droit ou gauche ${ }^{11}$ sont définis à l'aide de

\footnotetext{
${ }^{11}$ Les spineurs de Dirac sont des états propres de la chiralité, dont l'opérateur est $\gamma_{5}$. Chaque spineur de chiralité donné est un mélange de deux états d'hélicité. Dans la limite des masses nulles, la chiralité est égale à l'hélicité : un fermion de chiralité +1 est d'hélicité droite (Right en anglais), et un fermion de chiralité -1 est d'hélicité gauche (Left en anglais). C'est la raison du choix des indices $R$ et $L$ pour les spineurs [22, 38].
} 
l'opérateur chiralité, $\gamma_{5}$, par

$$
e_{L}^{-}=\frac{1}{2}\left(1-\gamma_{5}\right) e^{-}, \quad e_{R}^{-}=\frac{1}{2}\left(1+\gamma_{5}\right) e^{-},
$$

et se transforment en doublets et singlets de $S U(2)_{L}$, respectivement. Les neutrinos sont pris de masse nulle du fait de l'absence de neutrinos droits ;

- le secteur scalaire, qui n'a pas encore reçu de confirmation expérimentale. Le fait que les bosons de jauge de l'interaction faible soient massifs, $M_{W^{ \pm}, Z^{0}} \neq 0$, indique que $S U(2)_{L} \otimes U(1)_{Y}$ n'est pas une symétrie du vide. À l'opposé, le photon étant de masse nulle signifie que $U(1)_{e m}$ est une bonne symétrie du vide. Ainsi, le mécanisme de brisure spontanée de symétrie dans le Modèle Standard doit être

$$
S U(3)_{C} \otimes S U(2)_{L} \otimes U(1)_{Y} \rightarrow S U(3)_{C} \otimes U(1)_{e m}
$$

et a reçu le nom de mécanisme de Higgs. Une conséquence de ce mécanisme est la prédiction d'une nouvelle particule : le boson de Higgs, qui doit être une particule scalaire neutre. Cette particule n'a pas encore été observée.

Le point fondamental est que la symétrie $C P$ est brisée explicitement ${ }^{12}$ dans toute théorie des champs qui contient des constantes complexes de couplage dans le lagrangien, qui ne peuvent être absorbées par une redéfinition des champs de la théorie ${ }^{13}$. Or, tout lagrangien basé sur une pure théorie de jauge est invariant sous la symétrie $C P$ [35]. Dans le Modèle Standard ${ }^{14}$, la violation de $C P$ est directement reliée au mécanisme de Higgs, à travers les couplages de Yukawa entre les champs de quarks et le champ de Higgs [5] : elle est mise en œuvre à travers la matrice CKM [37], présente dans les courants chargés.

\subsubsection{Mise en œuvre minimale de la violation de $C P$ : la matrice CKM}

Commençons par analyser le lagrangien électrofaible pour déceler les sources possibles de violation de $C P$. Le lagrangien électrofaible peut être écrit comme la somme de trois contributions ${ }^{15}$ $[22,33]$ :

$$
\mathcal{L}_{E W}=\mathcal{L}_{\text {cinetique }}+\mathcal{L}_{\text {Higgs }}+\mathcal{L}_{\text {Yukawa }}
$$

Le premier terme est le lagrangien de jauge d'énergie cinétique des quarks et leptons défini par

$$
\mathcal{L}_{\text {cinetique }}=\bar{E}_{L}^{I}(i \not D) E_{L}^{I}+\bar{\ell}_{R}^{I}(i \not D) \ell_{R}^{I}+\bar{Q}_{L}^{I}(i \not D) Q_{L}^{I}+\bar{U}_{R}^{I}(i \not D) U_{R}^{I}+\bar{D}_{R}^{I}(i \not D) D_{R}^{I}
$$

où la prescription de couplage minimale, $\not \partial \rightarrow \not D$, est effectuée. Rappelons que l'invariance locale de jauge implique que toutes les particules sont de masse nulle, car les composantes droite et gauche ayant des nombres quantiques différents, un terme de masse (qui est de la forme $m_{\Psi} \bar{\Psi}_{L} \Psi_{R}$ ) violerait

\footnotetext{
${ }^{12}$ À opposer à spontanément.

${ }^{13}$ Cela signifie que la présence de termes complexes dans un lagrangien n'implique pas nécessairement la violation de $C P$.

${ }^{14}$ Nous ne traiterons pas la possibilité de violation de $C P$ dans l'interaction forte. Pour un compte-rendu sur le problème de la violation de $C P$ dans l'interaction forte, voir la référence [36].

${ }^{15}$ Nous omettons le terme cinétique ainsi que le terme d'interaction avec le champ de Higgs des bosons de jauge. Ils sont sans conséquence sur la violation de $C P$.
} 
cette invariance. Il faut utiliser le mécanisme de Higgs pour donner une masse aux particules. Les champs fermioniques sont définis dans la base d'interaction (spineurs avec l'indice $I$ ) par

$$
Q_{L}^{I}=\left(\begin{array}{c}
U^{I} \\
D^{I}
\end{array}\right)_{L}, E_{L}^{I}=\left(\begin{array}{c}
\nu_{\ell}^{I} \\
\ell^{I}
\end{array}\right)_{L} ; \quad U_{R}^{I}, D_{R}^{I}, \ell_{R}^{I}
$$

où $U^{I}=(u, c, t, \ldots)^{I}, D^{I}=(d, s, b, \ldots)^{I}$ et $\ell^{I}=\left(e^{-}, \mu^{-}, \tau^{-}, \ldots\right)^{I}$ désignent les états propres de saveur des quarks de type « up » et de type « down » et les leptons chargés, respectivement. $\not D \equiv$ $D^{\mu} \gamma_{\mu}$, où $D^{\mu}$ est la dérivée covariante donnée par :

$$
D^{\mu}=\partial^{\mu}-i g W_{a}^{\mu} T_{a}-i g^{\prime} Y B^{\mu}
$$

$g$ et $g^{\prime}$ sont les deux constantes de couplage pour $S U(2)_{L}$ et pour $U(1)_{Y}$, respectivement. Les champs de jauge de $S U(2)_{L}$ sont $W_{a}^{\mu}(a=1,2,3)$ et celui de $U(1)_{Y}$ est $B^{\mu}$. Enfin, $Y$ est l'hypercharge faible et $T_{a}(a=1,2,3)$ sont les générateurs de $S U(2)_{L}$ définis à l'aide des matrices de Pauli, $\tau_{a}, \operatorname{par} T_{a} \equiv \tau_{a} / 2$.

De l'équation (1.65), on extrait le lagrangien d'interaction des quarks et leptons :

$$
\begin{aligned}
\mathcal{L}_{i n t} & =\mathcal{L}_{C C}+\mathcal{L}_{N C}, \\
\mathcal{L}_{C C} & =\frac{g}{\sqrt{2}}\left(J_{\mu}^{+} W^{+\mu}+J_{\mu}^{-} W^{-\mu}\right), \\
\mathcal{L}_{N C} & =e J_{\mu}^{e m} A^{\mu}+\frac{g}{\cos \theta_{W}}\left(J_{\mu}^{3}-\sin ^{2} \theta_{W} J_{\mu}^{e m}\right) Z^{\mu} .
\end{aligned}
$$

Les champs $W^{ \pm \mu}, A^{\mu}$ et $Z^{\mu}$ sont définis par :

$$
W^{ \pm \mu}=\frac{1}{\sqrt{2}}\left(W_{1}^{\mu} \mp i W_{2}^{\mu}\right), \quad\left(\begin{array}{c}
A^{\mu} \\
Z^{\mu}
\end{array}\right)=\left(\begin{array}{cc}
\cos \theta_{W} & \sin \theta_{W} \\
-\sin \theta_{W} & \cos \theta_{W}
\end{array}\right)\left(\begin{array}{c}
B^{\mu} \\
W_{3}^{\mu}
\end{array}\right),
$$

et les courants chargés et neutres par :

$$
\begin{aligned}
J_{\mu}^{+} & =\bar{U}_{L}^{I} \gamma_{\mu} D_{L}^{I}+\bar{\nu}_{L}^{I} \gamma_{\mu} \ell_{L}^{I} \\
J_{\mu}^{3} & =\frac{1}{2}\left[\bar{U}_{L}^{I} \gamma_{\mu} U_{L}^{I}-\bar{D}_{L}^{I} \gamma_{\mu} D_{L}^{I}+\bar{\nu}_{L}^{I} \gamma_{\mu} \nu_{L}^{I}-\bar{\ell}_{L}^{I} \gamma_{\mu} \ell_{L}^{I}\right] \\
J_{\mu}^{e m} & =\frac{2}{3}\left(\bar{U}_{L}^{I} \gamma_{\mu} U_{L}^{I}+\bar{U}_{R}^{I} \gamma_{\mu} U_{R}^{I}\right)-\frac{1}{3}\left(\bar{D}_{L}^{I} \gamma_{\mu} D_{L}^{I}+\bar{D}_{R}^{I} \gamma_{\mu} D_{R}^{I}\right)-\left(\bar{\ell}_{L}^{I} \gamma_{\mu} \ell_{L}^{I}+\bar{\ell}_{R}^{I} \gamma_{\mu} \ell_{R}^{I}\right)
\end{aligned}
$$

$\theta_{W}$ est l'angle de mélange de Weinberg, relié à $g, g^{\prime}$ et $e$ par ${ }^{16}$

$$
\cos \theta_{W}=\frac{g}{\sqrt{g^{2}+g^{\prime 2}}}, \quad \sin \theta_{W}=\frac{g^{\prime}}{\sqrt{g^{2}+g^{\prime 2}}} \quad \text { et } \quad e=g \sin \theta_{W} .
$$

$\mathcal{L}_{\text {cinetique, }}$ exprimé dans la base d'interaction, est invariant sous la symétrie $C P$.

Le deuxième terme de $\mathcal{L}_{E W}$ est le lagrangien du champ scalaire de Higgs défini par :

$$
\mathcal{L}_{\text {Higgs }}=\left(D^{\mu} \Phi\right)^{\dagger}\left(D_{\mu} \Phi\right)-V(\Phi)
$$

${ }^{16} e=-|e|$ 
avec

$$
V(\Phi)=-\mu^{2} \Phi^{\dagger} \Phi+\lambda\left(\Phi^{\dagger} \Phi\right)^{2}, \quad \Phi=\left(\begin{array}{c}
\phi^{+} \\
\phi^{0}
\end{array}\right) .
$$

$D^{\mu}$ est la dérivée covariante définie par l'équation (1.67) $\operatorname{avec}^{17} Y=1 / 2, \lambda$ et $\mu^{2}$ sont des constantes positives ${ }^{18}$. Dans le Modèle Standard, il n'y a qu'un doublet de Higgs ce qui implique que $\mathcal{L}_{\text {Higgs }}$ est invariant ${ }^{19}$ sous la symétrie $C P$. Certaines extensions du Modèle Standard introduisent plusieurs doublets de Higgs qui conduisent à des sources de violation de $C P$ supplémentaires [22, 23, 25].

Il ne reste que $\mathcal{L}_{\text {Yukawa }}$ pour être une source de violation de $C P$. La forme du lagrangien de Yukawa qui couple le champ de Higgs aux quarks et aux leptons est contrainte par l'invariance de jauge de $S U(2)_{L}$, mais cette condition ne contraint pas sa structure en saveur :

$$
\mathcal{L}_{\text {Yukawa }}=-\sum_{i, j=1}^{n_{g}}\left[\lambda_{i j}^{D}\left(\bar{Q}_{L i}^{I} \cdot \Phi\right) D_{R j}^{I}+\lambda_{i j}^{U}\left(\bar{Q}_{L i}^{I} \cdot \tilde{\Phi}\right) U_{R j}^{I}+\lambda_{i j}^{\ell}\left(\bar{E}_{L i}^{I} \cdot \Phi\right) \ell_{R j}^{I}+\text { h.c. }\right],
$$

où $\tilde{\Phi}$ est le champ conjugué de $\Phi$, i.e. $\tilde{\Phi} \equiv i \tau_{2} \Phi$. $n_{g}$ est le nombre de familles ou générations $\left(n_{g}=3\right.$ dans le Modèle Standard). $\lambda_{i j}^{D}$, $\lambda_{i j}^{U}$ et $\lambda_{i j}^{\ell}$ sont des matrices $n_{g} \times n_{g}$ complexes, générales, c'est-à-dire pas nécessairement symétriques ou hermitiennes. Ce caractère arbitraire des couplages $\lambda_{i j}^{D}$, $\lambda_{i j}^{U}$ et $\lambda_{i j}^{\ell}$ est responsable de la plupart des paramètres libres du Modèle Standard. Puisque dans le Modèle Standard les neutrinos n'ont pas de couplage de Yukawa, du fait de l'absence de neutrinos droits, ils sont prédits de masse nulle. Les couplages de Yukawa sont la seule source possible de violation de $C P$ dans le Modèle Standard.

Après brisure spontanée de la symétrie de jauge, le champ de Higgs devient, en jauge unitaire :

$$
\Phi=\left(\begin{array}{c}
\phi^{+} \\
\phi^{0}
\end{array}\right) \rightarrow \frac{1}{\sqrt{2}}\left(\begin{array}{c}
0 \\
v+h
\end{array}\right)
$$

où $v$ est la valeur moyenne dans le vide du champ de Higgs, $\langle 0|\Phi| 0\rangle=v$, et $h$ le champ correspondant à la particule de Higgs. Le lagrangien de Yukawa s'écrit alors

$$
\mathcal{L}_{\text {Yukawa }}=-\sum_{i, j=1}^{n_{g}}\left[\lambda_{i j}^{D} \bar{D}_{L i}^{I} D_{R j}^{I}+\lambda_{i j}^{U} \bar{U}_{L i}^{I} U_{R j}^{I}+\lambda_{i j}^{\ell} \bar{\ell}_{L i}^{I} \ell_{R j}^{I}+h . c .\right] \frac{v}{\sqrt{2}}\left(1+\frac{h}{v}\right) .
$$

Les termes proportionnels à $v$ couplent les composantes droite et gauche des champs de quarks et de leptons et génèrent ainsi les termes de masse. Les matrices (complexes) de masse sont définies par:

$$
M_{i j}^{D} \equiv \frac{v}{\sqrt{2}} \lambda_{i j}^{D}, \quad M_{i j}^{U} \equiv \frac{v}{\sqrt{2}} \lambda_{i j}^{U}, \quad M_{i j}^{\ell} \equiv \frac{v}{\sqrt{2}} \lambda_{i j}^{\ell} .
$$

Les couplages de Yukawa sont la source des matrices de masse. Autrement dit, les paramètres complexes sont maintenant contenus dans les matrices de masse.

Pour déterminer le spectre en masse des quarks et des leptons, il est nécessaire de diagonaliser

\footnotetext{
${ }^{17}$ On a choisi de définir l'opérateur charge électrique $Q$ par $Q=T_{3}+Y$.

${ }^{18} \mathrm{Il}$ est à noter que le mécanisme de Higgs est un mécanisme ad hoc. La vraie dynamique derrière le mécanisme de Higgs est inconnue.

${ }^{19}$ L'hermicité de $\mathcal{L}_{\text {Higgs }}$ implique que tous les paramètres du potentiel de Higgs sont réels.
} 
les matrices de masse $M_{i j}^{\chi}(\chi=U, D, \ell)$. On définit pour cela six matrices unitaires telles que

$$
V_{L}^{\chi} M^{\chi} V_{R}^{\chi \dagger}=M_{\text {diag }}^{\chi}
$$

où $M_{\text {diag }}^{\chi}$ est une matrice diagonale et réelle $\left(\left(M_{\text {diag }}^{\chi}\right)_{i j}=m_{i}^{\chi} \delta_{i j}\right)$. Les champs de quarks et de leptons exprimés dans la base des états propres de masse (spineurs sans l'indice $I$ ) sont alors donnés par

$$
\begin{aligned}
D_{L i}=\left(V_{L}^{D}\right)_{i j} D_{L j}^{I}, & D_{R i}=\left(V_{R}^{d}\right)_{i j} D_{R j}^{I}, \\
U_{L i}=\left(V_{L}^{U}\right)_{i j} U_{L j}^{I}, & U_{R i}=\left(V_{R}^{u}\right)_{i j} U_{R j}^{I}, \\
\ell_{L i}=\left(V_{L}^{\ell}\right)_{i j} \ell_{L j}^{I}, & \ell_{R i}=\left(V_{R}^{\ell}\right)_{i j} \ell_{R j}^{I} .
\end{aligned}
$$

En réexprimant le lagrangien de Yukawa (cf. eq. (1.80)) en termes des champs physiques, c'est-àdire dans la base des états propres de masse, on obtient :

$$
\mathcal{L}_{\text {Yukawa }}^{\text {phys }}=-\sum_{i, j=1}^{n_{g}}\left[M_{\text {diag }, i j}^{D} \bar{D}_{L i} D_{R j}+M_{\text {diag }, i j}^{U} \bar{U}_{L i} U_{R j}+M_{\text {diag }, i j}^{\ell} \bar{\ell}_{L i} \ell_{R j}+\text { h.c. }\right]\left(1+\frac{h}{v}\right) .
$$

Il n'y a aucune trace des matrices unitaires $V_{L}^{\chi}$ et $V_{R}^{\chi}$. En conséquence, les interactions du champ de Higgs avec les fermions conservent $P, C$ et donc $C P$ ainsi que la saveur.

Il est important de souligner que les propriétés de symétrie d'un lagrangien sont à rechercher sur le lagrangien physique et non pas sur le lagrangien exprimé en termes de champs non physiques. Il faut donc analyser les autres termes du lagrangien $\mathcal{L}_{E W}$ qui ont besoin d'être réexprimés en fonction des champs physiques, c'est-à-dire $\mathcal{L}_{\text {cinetique }}$. Tout terme de la forme $\bar{\Psi}_{L}^{I} \gamma_{\mu} \Psi_{L}^{I}$ ou $\bar{\Psi}_{R}^{I} \gamma_{\mu} \Psi_{R}^{I}\left(\Psi=U, D, \nu_{\ell}, \ell\right)$ sera inchangé dans le passage de la base d'interaction à la base des états propres de masse. En effet, on a par exemple

$$
\bar{U}_{L}^{I} \gamma_{\mu} U_{L}^{I}=\bar{U}_{L} V_{L}^{U} \gamma_{\mu} V_{L}^{U \dagger} U_{L}=\bar{U}_{L} \gamma_{\mu} U_{L}, \quad\left(V_{L}^{U} V_{L}^{U \dagger}=1\right)
$$

En conséquence, le lagrangien de cinétique pure (les termes de l'équation (1.65) avec $\not$ ) ainsi que le lagrangien d'interaction impliquant les courants neutres $\left(\mathcal{L}_{N C}\right)$ seront inchangés. Cela implique l'absence de courant neutre changeant la saveur à l'ordre des arbres (FCNC $\equiv$ Flavor Changing Neutral Current), connue sous le nom de mécanisme de GIM [39]. C'est une conséquence du fait que, premièrement, chaque famille de quarks et de leptons est complète, c'est-à-dire que chaque quark ou lepton de type « up » a son partenaire de type « down ${ }^{20}$ et deuxièmement, que tous les fermions de charge $(Q)$ et de chiralité ( $R$ ou $L$ ) données ont la même valeur de $T_{3}$, la troisième composante de l'isospin faible [41]. Aux ordres supérieurs, du fait des échanges de courants chargés virtuels changeant la saveur, des transitions impliquant des courants neutres changeant la saveur sont autorisées mais fortement supprimées par la petitesse de la constante de Fermi. Les courants neutres sont donc invariants sous la symétrie $C P$ et conservent la saveur.

\footnotetext{
${ }^{20} \mathrm{C}^{\prime}$ est la motivation qui a conduit Glashow, Iliopoulos et Maiani à postuler l'existence d'un quatrième quark, le quark $c$ (non découvert à cette époque) afin d'éliminer les effets du courant neutre changeant l'étrangeté, très contraint par les mesures expérimentales. Une autre contrainte sur les familles est l'annulation des anomalies, qui impose que le nombre de familles de quarks et de leptons soit identique [33, 40].
} 

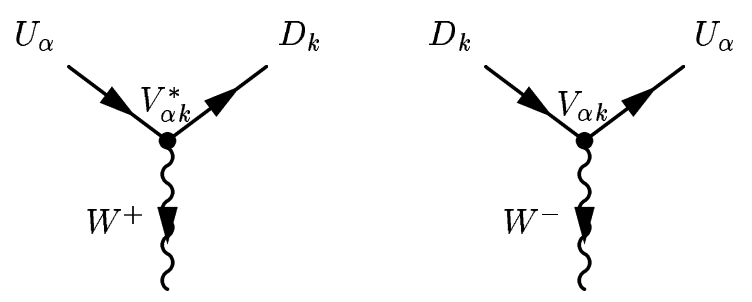

FIG. 1.8: Éléments de la matrice CKM apparaissant aux vertex des courants faibles chargés, où $U_{\alpha}\left(D_{k}\right)$ vaut pour $u, c, t, \ldots(d, s, b, \ldots)$.

Pour les courants chargés, la situation est assez différente, on trouve

$$
J_{\mu}^{+}=\bar{U}_{L} \gamma_{\mu} V_{C K M} D_{L}+\bar{\nu}_{L} \gamma_{\mu} \ell_{L}
$$

où

$$
V_{C K M}=V_{L}^{U} V_{L}^{D \dagger}
$$

est la matrice CKM [37], responsable du mélange entre les quarks via les bosons chargés de l'interaction faible. Elle est unitaire par construction et s'écrit

$$
V_{C K M}=\left(\begin{array}{cccc}
V_{u d} & V_{u s} & V_{u b} & \ldots \\
V_{c d} & V_{c s} & V_{c b} & \ldots \\
V_{t d} & V_{t s} & V_{t b} & \ldots \\
\vdots & \vdots & \vdots & \ddots
\end{array}\right)
$$

Par convention, $V_{C K M}$ opère sur les états propres de masse des quarks de type «down » de charge $-1 / 3$. Les termes diagonaux font intervenir les transitions dans une même famille alors que les termes non-diagonaux font intervenir les transitions entre familles. Les amplitudes des processus dans lesquels un $W^{-}$est émis $\left(D_{k} \rightarrow W^{-} U_{\alpha}\right.$ ou $\left.\bar{U}_{\alpha} \rightarrow W^{-} \bar{D}_{k}\right)$ ou un $W^{+}$est annihilé $\left(D_{k} W^{+} \rightarrow U_{\alpha}\right.$ ou $\left.\bar{U}_{\alpha} W^{+} \rightarrow \bar{D}_{k}\right)$ sont proportionnelles à $V_{\alpha k}$, alors que ceux où un $W^{+}$est émis $\left(U_{\alpha} \rightarrow W^{+} D_{k}\right.$ ou $\left.\bar{D}_{k} \rightarrow W^{+} \bar{U}_{\alpha}\right)$ ou un $W^{-}$est annihilé $\left(U_{\alpha} W^{-} \rightarrow D_{k}\right.$ ou $\left.\bar{D}_{k} W^{-} \rightarrow \bar{U}_{\alpha}\right)$ sont proportionnelles à $V_{\alpha k}^{*}$ (cf. Fig. 1.8).

Dans le secteur leptonique, du fait que les neutrinos sont pris de masse nulle, la matrice unitaire de passage entre la base d'interaction et la base des états propres de masse $V_{L}^{\nu}$ est arbitraire. On peut donc choisir $V_{L}^{\nu}$ de sorte que $V_{L}^{\nu} V_{L}^{\ell \dagger}=1$. Cependant, des mesures récentes [42] semblent confirmer l'oscillation des neutrinos et donc le fait que les neutrinos sont massifs. Dans ce cas ${ }^{21}$, $V_{L}^{\nu} V_{L}^{\ell \dagger} \neq 1$. La situation est alors plus compliquée pour les neutrinos que pour les quarks [22, 23, 44]. Les neutrinos étant de charge électrique nulle, ils possèdent des termes de masse de Dirac et/ou de Majorana. Dans le Modèle Standard avec un doublet de Higgs, seuls les termes de masse de Dirac sont possibles.

En conclusion, après avoir exprimé le lagrangien électrofaible dans la base des états propres de masse, la seule source possible de violation de $C P$ dans le secteur électrofaible du Modèle Standard est la matrice de mélange des quarks présente dans les courants chargés, i.e. la matrice CKM. Il

\footnotetext{
${ }^{21}$ La matrice de mélange dans le secteur leptonique est appelée la matrice MNS [43].
} 
convient de souligner que la matrice CKM n'est pas une paramétrisation ad hoc des transitions entre familles de quarks mais fait partie intégrante du Modèle Standard en étant directement reliée à la génération de la masse des quarks.

\subsubsection{Propriétés de la matrice CKM}

\section{Nombre de paramètres indépendants}

Le fait que les matrices de masse et donc aussi $V_{C K M}$ contiennent des phases complexes ne signifie pas nécessairement qu'elles vont générer des conséquences observables comme la violation de $C P$. Déterminons le nombre de paramètres physiques indépendants contenus dans la matrice $V_{C K M}$. Une matrice unitaire de dimension $n_{g} \times n_{g}$ contient $n_{g}^{2}$ paramètres. Tous ces paramètres ne sont pas physiquement significatifs. En effet, pour $n_{g}$ familles de quarks, $2 n_{g}-1$ phases peuvent être absorbées dans la définition des champs de quarks

$$
U_{L k} \rightarrow e^{i \phi_{k}^{U}} U_{L k}, \quad D_{L k} \rightarrow e^{i \phi_{k}^{D}} D_{L k}
$$

conduisant à

$$
V_{C K M} \rightarrow\left(\begin{array}{ccc}
e^{-i \phi_{1}^{U}} & \ldots & 0 \\
\vdots & \ddots & \vdots \\
0 & \ldots & e^{-i \phi_{n_{g}}^{U}}
\end{array}\right) V_{C K M}\left(\begin{array}{ccc}
e^{i \phi_{1}^{D}} & \ldots & 0 \\
\vdots & \ddots & \vdots \\
0 & \ldots & e^{-i \phi_{n_{g}}^{D}}
\end{array}\right)
$$

Puisqu'une phase globale est sans effet, $2 n_{g}-1$ phases relatives peuvent être enlevées de $V_{C K M}$ de cette façon. Au final, $V_{C K M}$ contient $\left(n_{g}-1\right)^{2}$ paramètres physiques indépendants. Parmi ces $\left(n_{g}-\right.$ $1)^{2}$ paramètres, $n_{g}\left(n_{g}-1\right) / 2$ sont des angles de mélange (angles d'Euler caractérisant une matrice de rotation dans un espace (euclidien) de dimension $n_{g}$ ) ce qui conduit à $\left(n_{g}-1\right)^{2}-n_{g}\left(n_{g}-1\right) / 2=$ $\left(n_{g}-1\right)\left(n_{g}-2\right) / 2$ phases indépendantes. Les résultats pour $n_{g}=2,3$ et 4 familles sont donnés dans la table 1.2. La principale conclusion est qu'il est nécessaire d'avoir au moins trois familles pour que la matrice $V_{C K M}$ contienne une phase complexe non-triviale. Dans le cas de deux familles, on retrouve la matrice de Cabibbo [45] et la symétrie $C P$ est conservée. Le fait que la symétrie $C P$ soit conservée avec deux familles a une conséquence importante. Cela implique que la violation de $C P$ dans le secteur des kaons sera petite, comme observée, même si la phase complexe dans la matrice $\mathrm{CKM}$ avec trois familles est importante. En effet, en première approximation, les désintégrations de kaons ne contiennent que des éléments de la matrice CKM provenant des deux premières familles, les quarks $b$ et $t$ n'intervenant pas. C'est uniquement dans les diagrammes d'ordres supérieurs $q^{\prime}$ 'interviennent les quarks des trois familles et conduisent à la violation de $C P$. On s'attend donc à des effets de violation de $C P$ beaucoup plus importants dans le système des mésons $B$ qui font intervenir les quarks des trois familles dès l'ordre des arbres.

Avant d'aller plus loin, examinons l'effet du rephasage des champs de quarks sur les autres termes du lagrangien $\mathcal{L}_{E W}^{\text {phys }}$. Le seul terme qui en est modifié est $\mathcal{L}_{\text {Yukawa }}^{\text {phys }}$ parce que les champs gauches et droits sont connectés et seuls les champs gauches sont rephasés. Cependant, afin que $\mathcal{L}_{\text {Yukawa }}^{\text {phys }}$ reste inchangé, il suffit de redéfinir chaque champ droit avec la même phase que le champ gauche correspondant. 
TAB. 1.2: Degrés de liberté de la matrice $V_{C K M}$ en fonction du nombre de familles de quarks.

\begin{tabular}{|c||ccc|}
\hline$n_{g}$ (familles) & $\begin{array}{c}\text { param. indep. } \\
\left(n_{g}-1\right)^{2}\end{array}$ & $\begin{array}{c}\text { angles } \\
n_{g}\left(n_{g}-1\right) / 2\end{array}$ & $\begin{array}{c}\text { phases } \\
\left(n_{g}-1\right)\left(n_{g}-2\right) / 2\end{array}$ \\
\hline 2 & 1 & 1 & 0 \\
3 & 4 & 3 & 1 \\
4 & 9 & 6 & 3 \\
\hline
\end{tabular}

\section{Conditions pour avoir violation de $C P$}

La symétrie $C P$ n'est pas nécessairement violée dans le Modèle Standard avec ses trois générations. Si deux quarks de type « down » ou " up » étaient dégénérés, un angle de mélange et une phase pourraient être enlevés de $V_{C K M}$. Ainsi, la violation de $C P$ requiert

$$
m_{t} \neq m_{c} \neq m_{u}, \quad m_{b} \neq m_{s} \neq m_{d}
$$

De même, si la valeur de n'importe quel angle de mélange vaut 0 ou $\pi / 2$, la symétrie $C P$ est conservée. Enfin, si la phase vaut 0 ou $\pi$, il n'y a pas de violation de $C P$. Toutes ces conditions peuvent se résumer en une seule [46], indépendante de conventions de phase. En définissant le commutateur $\left(C_{J}\right)$ des matrices de masse dans la base d'interaction (cf. eq. (1.81)) par :

$$
i C_{J} \equiv\left[M^{U} M^{U \dagger}, M^{D} M^{D \dagger}\right]
$$

on peut montrer que [5]

$$
\operatorname{det} C_{J}=-2 J\left(m_{t}^{2}-m_{c}^{2}\right)\left(m_{c}^{2}-m_{u}^{2}\right)\left(m_{t}^{2}-m_{u}^{2}\right)\left(m_{b}^{2}-m_{s}^{2}\right)\left(m_{s}^{2}-m_{d}^{2}\right)\left(m_{b}^{2}-m_{d}^{2}\right) .
$$

La quantité $J$, appelée l'invariant de Jarlskog, est définie en utilisant la condition d'unitarité de $V_{C K M}$ :

$$
\Im m\left[V_{\alpha j} V_{\alpha k}^{*} V_{\beta k} V_{\beta j}^{*}\right]=J \sum_{\gamma, l=1}^{3} \epsilon_{\alpha \beta \gamma} \epsilon_{j k l},
$$

où les indices grecs (latins) valent pour les quarks de type " up » ("down ») et $\epsilon_{a b c}$ est le tenseur totalement antisymétrique. La condition générale sur les matrices de masse en base d'interaction pour avoir violation de $C P$ est

$$
\operatorname{det} C_{J} \neq 0 \Longleftrightarrow \text { violation } \operatorname{de} C P \text {. }
$$

Dans le cas où aucune masse de quarks « up » ou « down » n'est dégénérée (cf. eq (1.93)), cette condition se réduit à

$$
J \neq 0 \Longleftrightarrow \text { violation de } C P \text {. }
$$




\section{Paramétrisations}

Plusieurs paramétrisations existent pour la matrice CKM [5]. Nous nous contenterons de deux paramétrisations, les plus utilisées : la paramétrisation standard [48], recommandée par le Particle Data Group [28] et la paramétrisation de Wolfenstein [50].

La paramétrisation standard est donnée par :

$$
\begin{gathered}
V_{C K M}=\left(\begin{array}{ccc}
V_{u d} & V_{u s} & V_{u b} \\
V_{c d} & V_{c s} & V_{c b} \\
V_{t d} & V_{t s} & V_{t b}
\end{array}\right)= \\
\left(\begin{array}{ccc}
c_{12} c_{13} & s_{12} c_{13} & s_{13} e^{-i \delta_{13}} \\
-s_{12} c_{23}-c_{12} s_{23} s_{13} e^{i \delta_{13}} & c_{12} c_{23}-s_{12} s_{23} s_{13} e^{i \delta_{13}} & s_{23} c_{13} \\
s_{12} s_{23}-c_{12} c_{23} s_{13} e^{i \delta_{13}} & -c_{12} s_{23}-s_{12} c_{23} s_{13} e^{i \delta_{13}} & c_{23} c_{13}
\end{array}\right),
\end{gathered}
$$

où $c_{i j}=\cos \theta_{i j}, s_{i j}=\sin \theta_{i j}$ et $i, j$ sont des indices de familles allant de 1 à $n_{g}=3$. $\delta_{13}$ est l'unique phase physique responsable de la violation de $C P$. Dans cette paramétrisation, l'invariant de Jarlskog est donné par :

$$
J=c_{12} c_{23} c_{13}^{2} s_{12} s_{23} s_{13} \sin \delta_{13},
$$

et montre explicitement que pour être non nul, tous les angles de mélange doivent être différents de 0 ou $\pi / 2$ et la phase différente de 0 ou $\pi$.

Les modules de la matrice CKM sont déterminés de trois façons différentes [51] :

- mesures directes reliées aux processus du Modèle Standard à l'ordre des arbres. Sont mesurés actuellement $\left|V_{u d}\right|,\left|V_{u s}\right|,\left|V_{u b}\right|,\left|V_{c d}\right|,\left|V_{c s}\right|,\left|V_{c d}\right|$ et $\left|V_{t b}\right|$;

- utilisation de l'unitarité de la matrice CKM qui relie différents éléments de cette matrice. Ces relations servent à contraindre $\left|V_{t d}\right|,\left|V_{t s}\right|,\left|V_{t b}\right|$ et $\left|V_{c s}\right|$;

- mesures indirectes reliées aux processus du Modèle Standard à l'ordre des boucles. $\left|V_{t b} V_{t d}\right|$ est contraint de cette façon en utilisant la mesure de $\Delta M_{B_{d}}$ et la limite sur $\Delta M_{B_{s}}$.

On obtient alors pour les modules les niveaux de confiance à 90\% suivants [28] :

$$
\left|V_{C K M}\right|=\left(\begin{array}{ccc}
0.9741-0.9756 & 0.219-0.226 & 0.0025-0.0048 \\
0.219-0.226 & 0.9732-0.9748 & 0.038-0.044 \\
0.004-0.014 & 0.037-0.044 & 0.9990-0.9993
\end{array}\right)
$$

Les éléments de la diagonale sont proches de $1,\left|V_{u s}\right|$ et $\left|V_{c d}\right|$ sont de l'ordre de $20 \%,\left|V_{c b}\right|$ et $\left|V_{t s}\right|$ sont de l'ordre de $4 \%,\left|V_{u b}\right|$ et $\left|V_{t d}\right|$ sont de l'ordre de $1 \%$. La matrice CKM possède donc une hiérarchie : ces éléments sont de plus en plus petits au fur et à mesure que l'on s'éloigne de la diagonale. Les différentes valeurs des modules de la matrice CKM permettent de conclure que $|J| \sim 10^{-5}$, loin de la valeur maximale que peut atteindre $J, 1 /(6 \sqrt{3}) \approx 0.1$, ce qui donne une idée de la suppression due à la hiérarchie de la matrice CKM [49].

En utilisant le fait que $\left|V_{u b}\right|=s_{13} \simeq 0.003$ ( $c_{13}$ est donc proche de l'unité), on peut négliger les 
termes proportionnels à $s_{13}$ devant des termes proches de 1 , ce qui donne

$$
V_{C K M} \approx\left(\begin{array}{ccc}
c_{12} & s_{12} & s_{13} e^{-i \delta_{13}} \\
-s_{12} c_{23} & c_{12} c_{23} & s_{23} \\
s_{12} s_{23}-c_{12} c_{23} s_{13} e^{i \delta_{13}} & -c_{12} s_{23} & c_{23}
\end{array}\right) .
$$

Dans cette approximation, seuls $V_{u b}$ et $V_{t d}$ sont complexes, tous les autres termes sont réels. En utilisant la hiérarchie observée, on peut développer la matrice CKM, comme le remarqua Wolfenstein [50], en puissance du paramètre $\lambda \equiv s_{12}=\sin \theta_{C} \simeq\left|V_{u s}\right| \simeq 0.22$, où $\theta_{C}$ est l'angle de Cabibbo :

$$
V_{C K M}=\left(\begin{array}{ccc}
1-\frac{\lambda^{2}}{2} & \lambda & A \lambda^{3}(\rho-i \eta) \\
-\lambda & 1-\frac{\lambda^{2}}{2} & A \lambda^{2} \\
A \lambda^{3}(1-\rho-i \eta) & -A \lambda^{2} & 1
\end{array}\right)+\mathcal{O}\left(\lambda^{4}\right)
$$

Dans cette paramétrisation approximative, les quatre paramètres indépendants sont $A, \lambda, \rho$ et $\eta$. L'invariant de Jarlskog vaut $J \simeq A^{2} \lambda^{6} \eta$, il y a donc violation de $C P$ si $\eta \neq 0$. En utilisant la paramétrisation de Wolfenstein, il convient de garder à l'esprit que c'est une approximation valable à $\mathcal{O}\left(\lambda^{4}\right) \approx 10^{-3}$ et que l'unitarité n'est assurée que jusqu'à $\mathcal{O}\left(\lambda^{3}\right)$.

Afin d'obtenir une meilleure précision, c'est-à-dire un développement à des ordres supérieurs, il convient d'utiliser une paramétrisation exacte avec un schéma de développement en série de $\lambda$. Un tel développement, qui se base sur la paramétrisation standard, définit les paramètres $(A, \lambda, \rho, \eta)$ $\operatorname{par}^{22}[52,53]$ :

$$
s_{12} \equiv \lambda, \quad s_{23} \equiv A \lambda^{2}, \quad s_{13} e^{-i \delta_{13}} \equiv A \lambda^{3}(\rho-i \eta),
$$

à tous les ordres en $\lambda$ et non pas jusqu'à $\mathcal{O}\left(\lambda^{4}\right)$. En effectuant les changements dans la paramétrisation standard, la matrice CKM exprimée en fonction de $(A, \lambda, \rho, \eta)$ satisfait exactement l'unitarité. En développant à l'ordre supérieur, les éléments de la matrice CKM sont donnés par :

$$
\begin{aligned}
V_{u d} & =1-\frac{1}{2} \lambda^{2}-\frac{1}{8} \lambda^{4}+\mathcal{O}\left(\lambda^{6}\right), \\
V_{u s} & =\lambda+\mathcal{O}\left(\lambda^{7}\right), \\
V_{u b} & =A \lambda^{3}(\rho-i \eta) \\
V_{c d} & =-\lambda+\frac{1}{2} A^{2} \lambda^{5}[1-2(\rho+i \eta)]+\mathcal{O}\left(\lambda^{7}\right), \\
V_{c s} & =1-\frac{1}{2} \lambda^{2}-\frac{1}{8} \lambda^{4}\left(1+4 A^{2}\right)+\mathcal{O}\left(\lambda^{6}\right), \\
V_{c b} & =A \lambda^{2}+\mathcal{O}\left(\lambda^{8}\right), \\
V_{t d} & =A \lambda^{3}[1-\bar{\rho}-i \bar{\eta}]+\mathcal{O}\left(\lambda^{7}\right), \\
V_{t s} & =-A \lambda^{2}+\frac{1}{2} A \lambda^{4}[1-2(\rho+i \eta)]+\mathcal{O}\left(\lambda^{6}\right), \\
V_{t b} & =1-\frac{1}{2} A^{2} \lambda^{4}+\mathcal{O}\left(\lambda^{6}\right),
\end{aligned}
$$

\footnotetext{
${ }^{22}$ Une autre définition des paramètres $(A, \lambda, \rho, \eta)$ est donnée dans [54], utilisant une autre paramétrisation exacte pour $V_{C K M}$. Ainsi, les expressions des éléments de la matrice CKM peuvent différer suivant le schéma de développement en série de $\lambda$ utilisé.
} 
avec

$$
\bar{\rho}=\rho\left(1-\frac{\lambda^{2}}{2}\right), \quad \bar{\eta}=\eta\left(1-\frac{\lambda^{2}}{2}\right) .
$$

Aucune observable physique ne peut dépendre d'une paramétrisation donnée. Une seule paramétrisation doit être utilisée avec cohérence afin d'éviter qu'un autre choix de phase n'entre en conflit.

\section{Unitarité de la matrice CKM et triangles d'unitarité}

La matrice CKM est unitaire sous l'hypothèse de l'universalité du couplage faible, i.e. $g^{\ell}=g^{\nu}=$ $g^{U}=g^{D}$ (c'est ce qui explique la présence d'un unique groupe de jauge, $S U(2)_{L}$, pour toutes les familles). Cette unitarité, ingrédient essentiel dans l'absence de FCNC, conduit à deux types de relations :

$$
\begin{aligned}
& \sum_{j} V_{\alpha j} V_{\beta j}^{*}=\delta_{\alpha \beta} ; \quad \alpha, \beta=u, c, t ; j=d, s, b ; \\
& \sum_{\alpha} V_{\alpha j} V_{\alpha k}^{*}=\delta_{j k} ; \quad \alpha=u, c, t ; j, k=d, s, b .
\end{aligned}
$$

Les relations (1.116) avec $\alpha=\beta$ et (1.117) avec $j=k$ ne contiennent aucune phase et donc n'apportent aucune information sur la violation de $C P$. Elles permettent de tester l'unitarité de la matrice $V_{C K M}$. Notons que l'unitarité est une propriété fondamentale de toute théorie des champs. Tester l'unitarité de la matrice CKM ne signifie pas rechercher des violations de l'unitarité, mais des violations des conséquences de l'unitarité avec trois familles. De telles violations impliqueraient simplement la présence de physique au delà du Modèle Standard. Les relations (1.116) avec $\alpha \neq \beta$ et (1.117) avec $j \neq k$ ont pour origine l'orthogonalité de différentes lignes et colonnes de la matrice CKM. Elles peuvent être représentées géométriquement par six triangles dans le plan complexe [47], qui ont tous la même aire

$$
A_{\triangle}=\frac{|J|}{2}
$$

et le signe de $J$ donne le sens des côtés des triangles. Le rephasage des champs de quarks modifie l'orientation des triangles dans le plan complexe mais leurs formes restent inchangées. Autrement dit, les angles et les côtés des triangles sont indépendants de conventions de phase. Analysons la forme de ces six triangles en utilisant la paramétrisation de Wolfenstein :

$$
\begin{array}{rlll}
\mathcal{O}(\lambda)+\mathcal{O}(\lambda)+\mathcal{O}\left(\lambda^{5}\right) & = & 0, & \left(1,2^{*}\right) \\
\mathcal{O}\left(\lambda^{3}\right)+\mathcal{O}\left(\lambda^{3}\right)+\mathcal{O}\left(\lambda^{3}\right) & = & 0, & \left(1,3^{*}\right) \\
\mathcal{O}\left(\lambda^{4}\right)+\mathcal{O}\left(\lambda^{2}\right)+\mathcal{O}\left(\lambda^{2}\right) & = & 0, & \left(2,3^{*}\right)
\end{array}
$$

où $\left(i, j^{*}\right)$ correspond aux produits des lignes (ou des colonnes) $i$ et $j$ (complexe conjuguée) de la matrice CKM (cf. eq. (1.99)). Quatre des six triangles ont un côté très petit devant les deux autres, ces triangles sont très aplatis. Les deux triangles définis par l'équation (1.119) et l'équation (1.121), à partir des colonnes de la matrice CKM, sont reliés aux désintégrations des kaons et des mésons $B_{s}$, respectivement. Cela explique la petitesse de la violation de $C P$ dans le système des kaons. Seuls deux triangles ont des côtés de tailles comparables. Un de ces deux triangles, donné par la relation 


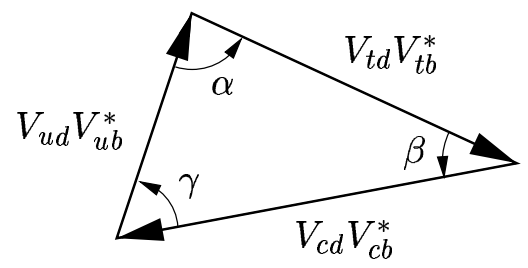

a)

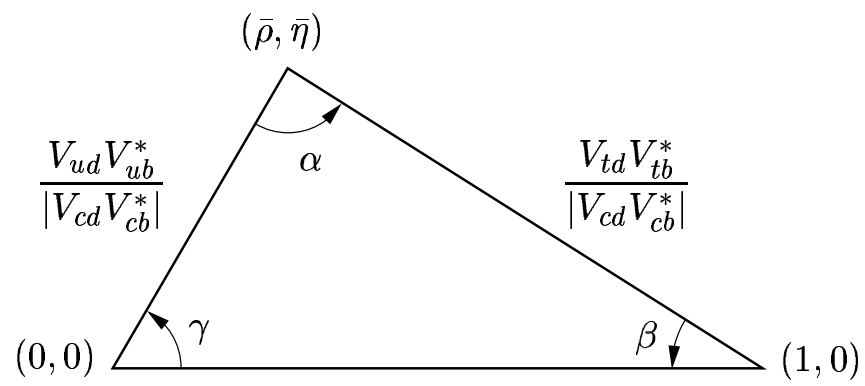

b)

FIG. 1.9: Triangle d'unitarité. a) Représente la condition d'orthogonalité entre la première et la troisième colonne de la matrice CKM ; l'orientation du triangle est arbitraire et dépend de conventions de phase. b) Montre une version redimensionnée du triangle où chaque côté est divisé par $\left|V_{c d} V_{c b}^{*}\right|$ afin que la base soit de longueur unité. La base est aussi alignée sur l'axe des réels par choix d'une convention de phase. Le sommet du triangle a pour coordonnées $(\bar{\rho}, \bar{\eta})$. Toutes les amplitudes violant la symétrie $C P$ dans le Modèle Standard sont proportionnelles à l'aire du triangle.

(unitarité entre les colonnes 1 et 3)

$$
V_{u d} V_{u b}^{*}+V_{c d} V_{c b}^{*}+V_{t d} V_{t b}^{*}=0
$$

a un grand intérêt du point de vue phénoménologique puisqu'il contient les éléments les moins connus de la matrice CKM. Il est relié aux désintégrations des mésons $B$; ainsi de larges effets de violation de $C P$ sont attendus dans le système des mésons $B$. Ce triangle est souvent référencé dans la littérature comme le triangle d'unitarité. Le triangle d'unitarité redimensionné est obtenu à partir de (1.122) en choisissant une convention de phase de sorte que $V_{c d} V_{c b}^{*}$ soit réel et en divisant chaque côté par $\left|V_{c d} V_{c b}^{*}\right|$ (voir Fig. 1.9). Ce triangle a pour coordonnées ${ }^{23}(0,0),(1,0)$ et $(\bar{\rho}, \bar{\eta})$, où $\bar{\rho}$ et $\bar{\eta}$ sont définis par (1.115). Les côtés et les angles de ce triangle sont définis par $[24,53,55]$ :

$$
\begin{gathered}
R_{u} \equiv \frac{V_{u d} V_{u b}^{*}}{\left|V_{c d} V_{c b}^{*}\right|} \simeq \sqrt{\bar{\rho}^{2}+\bar{\eta}^{2}}, \quad R_{t} \equiv \frac{V_{t d} V_{t b}^{*}}{\left|V_{c d} V_{c b}^{*}\right|} \simeq \sqrt{(1-\bar{\rho})^{2}+\bar{\eta}^{2}}, \\
\alpha \equiv \arg \left[-\frac{V_{t d} V_{t b}^{*}}{V_{u d} V_{u b}^{*}}\right], \quad \beta \equiv \arg \left[-\frac{V_{c d} V_{c b}^{*}}{V_{t d} V_{t b}^{*}}\right], \quad \gamma \equiv \arg \left[-\frac{V_{u d} V_{u b}^{*}}{V_{c d} V_{c b}^{*}}\right] .
\end{gathered}
$$

L'unitarité implique que le triangle soit fermé, i.e. $\alpha+\beta+\gamma=\pi[2 \pi]$. Il est parfois avantageux de travailler dans une convention de phase où les éléments de la matrice CKM, $V_{u d}, V_{c s}, V_{c b}$ et $V_{t b}$ sont

${ }^{23}$ Notons que $\bar{\rho} \simeq \rho$ et $\bar{\eta} \simeq \eta$ à environ $3 \%$ de précision. 
réels positifs et $V_{c d}<0$ de sorte que ${ }^{24}$ [22]

$$
V_{u b}=\left|V_{u b}\right| e^{-i \gamma}, \quad V_{t d}=\left|V_{t d}\right| e^{-i \beta}
$$

\section{Conclusion}

Dans le Modèle Standard avec ses trois générations, les effets de la violation de $C P$ sont décrits par une unique phase présente dans la matrice CKM. En principe, toutes les observables impliquant la violation de $C P$ sont calculables en termes de ce seul paramètre, ce qui implique qu'elles ne sont pas indépendantes entre-elles. En pratique, les effets de l'interaction forte doivent être contrôlés avant que de tels calculs soient effectués.

Supposons que la matrice $V_{C K M}$ permette de décrire correctement toutes les observations présentes et futures. Cela signifie-t-il que la violation de $C P$ soit comprise? La réponse est NON. Dans ce cas, nous avons uniquement un moyen économique et phénoménologique pour décrire la violation de $C P$ dans le Modèle Standard. La matrice $V_{C K M}$ étant directement reliée aux matrices de masse, seule la compréhension de l'origine de la masse des quarks permettra de comprendre la violation de $C P$.

Enfin, rien ne dit que le modèle CKM est celui de la Nature. La matrice CKM est la mise en œuvre minimale pour la violation de $C P$ car elle ne nécessite aucune particule ou interaction au delà de celles déjà connues ou nécessaires pour comprendre l'interaction électrofaible. Cependant, la violation de $C P$ dans la Nature possède peut-être plusieurs sources indépendantes. Il convient donc de tester la violation de $C P$ dans de nombreux systèmes différents.

\subsubsection{Prédictions pour le système $B_{d}^{0}-\bar{B}_{d}^{0}$}

\section{Mélange}

Dans le Modèle Standard, $M_{12}$ est décrit, à l'ordre le plus bas, par des diagrammes en boîte représentés par la figure 1.10. Ils représentent une bonne approximation puisque l'on s'attend à ce que les contributions à longue distance soient négligeables. Du fait de la hiérarchie de la matrice CKM et que $m_{u, c} \ll m_{t}, M_{12}$ est dominé par l'échange des quarks top, les contributions des quarks $u$ et $c$ étant totalement négligeables (l'amplitude $M_{12}$ est proportionnelle à $m_{i}^{2}$ pour $m_{i} \gg m_{W}$ avec $m_{i}$ la masse du quark interne). $M_{12}$ implique alors une somme sur les états intermédiaires virtuels dominés par $t+\bar{t}[53,56,57]$ :

$$
M_{12} \approx-\frac{G_{F}^{2} M_{W}^{2} \eta_{B} m_{B} B_{B} f_{B}^{2}}{12 \pi^{2}}\left(V_{t d}^{*} V_{t b}\right)^{2} S_{0}\left(m_{t}^{2} / m_{W}^{2}\right) e^{-2 i \alpha_{B}}
$$

où $G_{F}$ est la constante de Fermi, $m_{W}$ est la masse du boson $W$ et $m_{B}$ la masse du méson $B_{d}^{0}$. $B_{B} f_{B}^{2}$ paramétrise l'élément de matrice hadronique $\left\langle B_{d}^{0}\left|(\bar{d} b)_{V-A}(\bar{d} b)_{V-A}\right| \bar{B}_{d}^{0}\right\rangle \cdot \eta_{B}$ provient de corrections QCD et est de l'ordre de l'unité. La fonction $S_{0}$ est définie par [58] :

$$
S_{0}(x)=\frac{4 x-11 x^{2}+x^{3}}{4(1-x)^{2}}-\frac{3}{2} \frac{x^{3} \ln x}{(1-x)^{3}} .
$$

\footnotetext{
${ }^{24} \grave{A}$ noter que tel est le cas de l'approximation de Wolfenstein.
} 

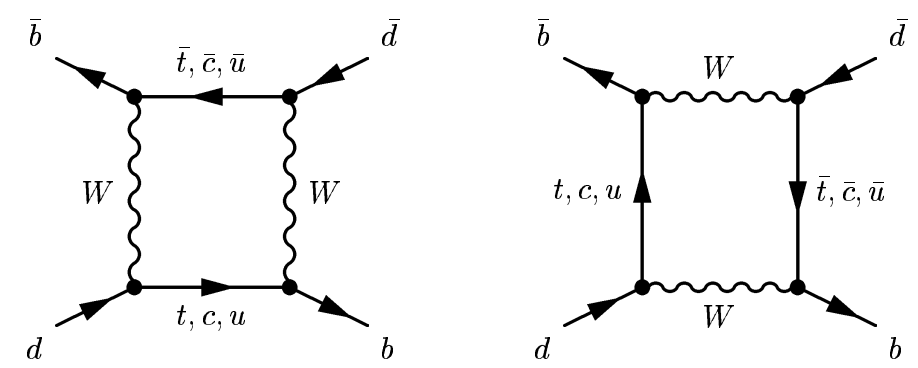

FIG. 1.10: Diagrammes en boîte pour l'oscillation $B_{d}^{0}-\bar{B}_{d}^{0}$ dans le Modèle Standard.

$\Gamma_{12}$ est calculé à partir d'une coupure des diagrammes en boîte [23,31] (avec échange des quarks $u$ et $c$ uniquement), impliquant des états intermédiaires réels et donc de la physique à longue distance. La coupure des diagrammes en boîte est donc une médiocre approximation pour $\Gamma_{12}$ mais donne correctement la suppression due aux divers paramètres électrofaibles, tels que le couplage faible. Les calculs du rapport $\Gamma_{12} / M_{12}$ aboutissent à [31, 53]

$$
\frac{\Gamma_{12}}{M_{12}} \approx-\frac{3 \pi}{2} \frac{1}{S_{0}\left(m_{t}^{2} / m_{W}^{2}\right)} \frac{m_{b}^{2}}{m_{W}^{2}}\left(1+\frac{8 m_{c}^{2}}{3 m_{b}^{2}} \frac{V_{c b} V_{c d}^{*}}{V_{t b} V_{t d}^{*}}\right) .
$$

On a donc $\left|\Gamma_{12} / M_{12}\right|=\mathcal{O}\left(m_{b}^{2} / m_{t}^{2}\right) \ll 1$ car $S_{0}\left(m_{t}^{2} / m_{W}^{2}\right)=\mathcal{O}\left(m_{t}^{2} / m_{W}^{2}\right)$, ce qui confirme l'estimation $\left|\Gamma_{12} / M_{12}\right| \lesssim 10^{-2}$, faite dans la section 1.2.2, indépendamment de modèles théoriques.

L'équation (1.128) permet d'estimer la violation de $C P$ dans le mélange en développant $|q / p|$ en puissance de $\Gamma_{12} / M_{12}$ :

$$
1-\left|\frac{q}{p}\right|=\frac{1}{2} \Im m\left(\frac{\Gamma_{12}}{M_{12}}\right)+\mathcal{O}\left(\left|\frac{\Gamma_{12}}{M_{12}}\right|^{2}\right)=\frac{4 \pi}{S_{0}\left(m_{t}^{2} / m_{W}^{2}\right)} \frac{m_{c}^{2}}{m_{W}^{2}} \frac{J}{\left|V_{t b} V_{t d}^{*}\right|^{2}} \sim 10^{-3},
$$

où $J$ est l'invariant de Jarlskog. Cela confirme la faiblesse de la violation de $C P$ attendue dans le mélange. Ainsi, à une bonne approximation, le rapport $q / p$ est une phase pure :

$$
\frac{q}{p} \simeq-\frac{M_{12}^{*}}{\left|M_{12}\right|} \propto \frac{V_{t d} V_{t b}^{*}}{V_{t d}^{*} V_{t b}}
$$

\section{Désintégrations $b \rightarrow c \bar{c} s$}

Considérons dans le cadre du Modèle Standard les amplitudes $A_{f}$ décrivant la désintégration d'un méson $B$ dans l'état final $f$. La description par diagrammes de quarks [59] est très intuitive mais ne conduit pas à des résultats exacts [60] : après avoir calculé l'hamiltonien effectif gouvernant la transition en utilisant l'OPE (Operator Product Expansion), il faut évaluer les éléments de matrice hadronique. Pour évaluer ces éléments, il est nécessaire de choisir un modèle (p. ex. BSW [61]) pour prendre en compte les effets de QCD non-perturbative et les résultats dépendent alors du modèle. Nous utilisons l'approximation du quark spectateur, où nous supposons que le méson $B$ se désintègre par la désintégration du quark $b($ ou $\bar{b}$ ) sans intervention de l'autre quark du méson. Nous nous restreignons aux états finals impliquant une transition $b \rightarrow c \bar{c} s$.

Les divers diagrammes contribuant à l'amplitude $A(c \bar{c} s)$ sont représentés par la figure 1.11. 
a)

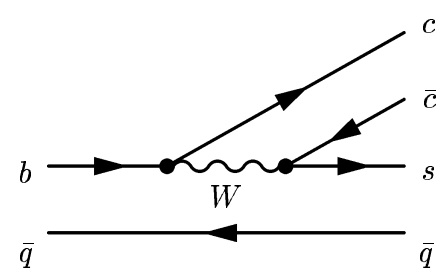

c)

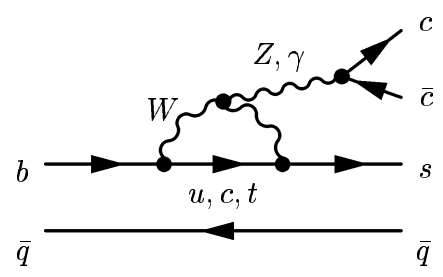

b)

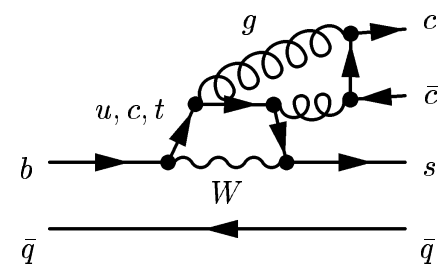

d)

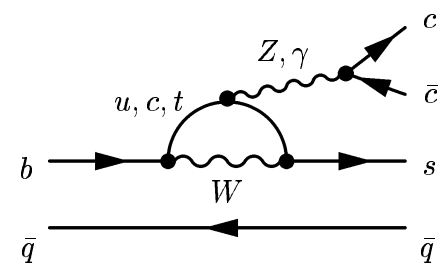

FIG. 1.11: Diagrammes de Feynman contribuant à la désintégration $b \rightarrow c \bar{c} s:$ a) diagramme à l'ordre des arbres; b) pingouin gluonique; c,d) pingouins électrofaibles. À noter que pour les diagrammes pingouins gluoniques, au moins deux gluons sont nécessaires pour le $\chi_{c 1}(1 P)$ et au moins trois gluons pour le $J / \psi$ et le $\psi(2 S)$.

L'amplitude $A(c \bar{c} s)$ est la somme de la contribution à l'ordre des arbres $\left(T_{c \bar{c} s}\right)$ et de celle des diagrammes pingouins $\left(P_{s}^{q}\right)$ gluoniques et électrofaibles [26, 53] :

$$
A(c \bar{c} s)=V_{c b} V_{c s}^{*}\left(T_{c \bar{c} s}+P_{s}^{c}-P_{s}^{t}\right)+V_{u b} V_{u s}^{*}\left(P_{s}^{u}-P_{s}^{t}\right)
$$

où on a utilisé la relation d'unitarité $V_{t b} V_{t s}^{*}=-V_{u b} V_{u s}^{*}-V_{c b} V_{c s}^{*}$. Le diagramme dominant $T_{c \bar{c} s}$ est supprimé de couleur : les quarks $s$ et $\bar{c}$ provenant de $W$ sont séparés dans des mésons différents. Les quarks $c$ et $\bar{c}$, dont la couleur initiale est indépendante, doivent avoir des couleurs compatibles pour former un méson incolore, d'où la suppression de couleur (il en va de même pour le quark $s$ et le quark spectateur). Ce regroupement suivant les facteurs CKM permet d'estimer l'importance de la violation de $C P$ dans la désintégration:

$$
V_{c b} V_{c s}^{*} \simeq A \lambda^{2}, \quad V_{u b} V_{u s}^{*} \simeq A \lambda^{4}(\rho-i \eta) .
$$

Seuls les diagrammes pingouins avec un quark intermédiaire $u$ ont une phase différente par rapport au diagramme dominant mais ils sont supprimés de Cabibbo. De plus, indépendamment des éléments de matrice hadronique et des facteurs CKM, les diagrammes pingouins sont supprimés par un facteur de boucle par rapport au diagramme à l'ordre des arbres. Une grossière estimation pour le rapport entre le diagramme pingouin gluonique et le diagramme à l'ordre des arbres est [62]

$$
\left|\frac{P_{g}}{T}\right| \sim \frac{\alpha_{s}^{2(3)}\left(m_{b}\right)}{12 \pi} \ln \frac{m_{t}^{2}}{m_{b}^{2}}=\mathcal{O}(0.01)(\mathcal{O}(0.001)),
$$

où l'exposant de $\alpha_{s}$ représente le nombre de gluons échangés (cf. Fig. 1.11). Enfin, le fait qu'il faille au moins deux ou trois gluons suivant le méson charmonium (voir Fig. 1.11) pour les diagrammes 
pingouins gluoniques rend les pingouins électrofaibles du même ordre de grandeur :

$$
\left|\frac{P_{E W}}{P_{g}}\right| \sim \frac{\alpha_{w}\left(m_{b}\right)}{\alpha_{s}^{2(3)}\left(m_{b}\right)} \frac{m_{t}^{2} / m_{Z}^{2}}{\ln \left(m_{t}^{2} / m_{b}^{2}\right)}=\mathcal{O}(0.1)(\mathcal{O}(1)) .
$$

En conclusion, l'amplitude $A(c \bar{c} s)$ est dominée par une unique phase faible. Le Modèle Standard prévoit une quasi-absence $(\leq 1 \%[22,25])$ de violation de $C P$ directe dans ces désintégrations. Elles permettent d'accéder aux paramètres CKM avec de très faibles incertitudes hadroniques (voir eq. (1.62)).

Prenons comme exemple de transition $b \rightarrow c \bar{c} s$ la désintégration $B_{d}^{0} \rightarrow J / \psi K_{S}^{0}$. À une bonne approximation, on peut considérer le $K_{S}^{0}$ comme un état propre de $C P$ avec la valeur propre +1 , ainsi la valeur propre de l'état final est $\eta_{C P}\left(J / \psi K_{S}^{0}\right)=-1$. Du fait que $B_{d}^{0} \rightarrow J / \psi K^{0}$ et $\bar{B}_{d}^{0} \rightarrow$ $J / \psi \bar{K}^{0}$, il est nécessaire de prendre en compte le mélange $K^{0}-\bar{K}^{0}$ pour que $B_{d}^{0}$ et $\bar{B}_{d}^{0}$ puissent atteindre le même état final. Cela conduit à

$$
\lambda_{f_{C P}}=\eta_{C P}(f)\left(\frac{q}{p}\right)\left(\frac{\bar{A}_{\bar{f}_{C P}}}{A_{f_{C P}}}\right)\left(\frac{q}{p}\right)_{K}=-\left(\frac{V_{t d} V_{t b}^{*}}{V_{t d}^{*} V_{t b}}\right)\left(\frac{V_{c b} V_{c s}^{*}}{V_{c b}^{*} V_{c s}}\right)\left(\frac{V_{c s} V_{c d}^{*}}{V_{c s}^{*} V_{c d}}\right)=-e^{-2 i \beta} .
$$

Plusieurs approximations ont été utilisées pour aboutir à ce résultat : on a négligé la violation de $C P$ indirecte dans le système des mésons $B(|q / p|=1)$ et le système des kaons ${ }^{25}\left(|q / p|_{K}=1\right)$, ainsi que la violation de $C P$ dans la désintégration $\left(\left|\bar{A}_{\bar{f}_{C P}} / A_{f_{C P}}\right|=1\right)$. On a donc $\left|\lambda_{f_{C P}}\right|=1+\mathcal{O}\left(10^{-2}\right)$. La remarquable caractéristique de ce résultat est de pouvoir exprimer l'asymétrie $C P$ (eq. (1.61)) directement en termes de paramètres $\mathrm{CKM}$, sans connaissance des éléments de matrice hadronique.

Les modes vecteur-vecteur $b \rightarrow c \bar{c} s$ ont une structure en amplitude similaire, mais, puisque les deux particules vecteurs peuvent avoir un moment angulaire relatif pair ou impair, l'état final n'est pas un état propre de $C P$. Les deux états de parité $C P$ différente peuvent être séparés par une analyse angulaire des produits de désintégration des particules vecteurs [63]. Ces modes étant le sujet de cette thèse, ils sont détaillés dans le chapitre suivant.

\subsection{Conclusion}

Le système des mésons $B$ se prête particulièrement bien à l'étude de la violation de $C P$ où de larges asymétries $C P$ sont attendues du fait de la présence de quarks des trois familles dès les diagrammes à l'ordre des arbres. L'unique phase non-triviale présente dans la matrice CKM est la source de la violation de $C P$ dans le Modèle Standard. Elle conduit à des effets observables à travers l'interférence d'amplitudes. Les modes les plus favorables sont ceux dominés par une unique phase faible car ils permettent une détermination propre de paramètres CKM.

\footnotetext{
${ }^{25}$ L'asymétrie $C P$ dans le cas de la transition $b \rightarrow c \bar{c} s$ étant prédite de l'ordre de 1 alors que la violation de $C P$ dans le mélange pour les kaons est de l'ordre de $10^{-3}$, elle peut être négligée. Pour cette raison, on prend $|q / p|_{K}=1$.
} 


\section{Chapitre 2}

\section{Phénoménologie des canaux vecteur-vecteur du type charmonium- $K^{*}(892)$}

Parmi les centaines désintégrations non-leptoniques du méson $B$ en deux corps, les désintégrations ${ }^{1} B \rightarrow \psi K^{*}(892)\left(\psi=J / \psi, \psi(2 S), \chi_{c 1}\right)$ ont beaucoup d'intérêt pour plusieurs raisons. Le méson $B$ étant une particule de spin 0 et les mésons vecteurs des particules de spin 1 , le moment angulaire relatif, $L$, entre les deux mésons vecteurs peut prendre les valeurs $L=0,1,2$. Ainsi, la désintégration faible $B \rightarrow \psi K^{*}(892)$ est décrite par trois ondes partielles : $S, P$ et $D$. C'est la richesse de ces canaux : la présence de ces trois ondes induit des termes d'interférence qui génèrent plus d'observables que les désintégrations $B \rightarrow P P$ ou $B \rightarrow P V$ ( $P$ =particule pseudo-scalaire, $V=$ particule vecteur). Du point de vue de l'étude de la violation de $C P$, en plus d'être sensible à $\sin 2 \beta$, l'interférence des ondes $S$ et $D$ avec l'onde $P$ permet un accès à $\cos 2 \beta$ [64]. Ces interférences permettent de tester différents modèles de désintégration non-leptonique du méson $B$ (factorisation, facteurs de forme, etc.), les interactions dans l'état final (FSI) avec la mesure des phases relatives des amplitudes. De plus, la présence de violation de $C P$ directe pour les désintégrations $B \rightarrow \psi P(V)$ est une signature propre de nouvelle physique, elle nécessite néanmoins des phases fortes différentes. Il est encore possible de signer de la nouvelle physique avec $B \rightarrow \psi V$ même si les phases fortes sont égales [65]. Enfin, une autre signature de nouvelle physique peut être détectée avec $B_{d}^{0} \rightarrow \psi K^{* 0}(892), K^{* 0}(892) \rightarrow K^{+} \pi^{-}$en étudiant les transitions interdites de saveur [66].

\subsection{Amplitude de désintégration non-leptonique}

L'étude des désintégrations non-leptoniques d'un méson $B$ [68] est beaucoup plus complexe que celle des désintégrations leptoniques ou semileptoniques, du fait que toutes les particules peuvent interagir via l'interaction forte. La dynamique des désintégrations non-leptoniques, dans lesquelles n'apparaissent que des hadrons dans l'état final, est fortement influencée par les interactions de couleur confinantes entre les quarks. La désintégration du méson $B$ par l'interaction faible crée un singlet de couleur $q_{1} \bar{q}_{2} q_{3} \bar{q}_{4}$, les quatre quarks interagissant fortement les uns avec les autres

\footnotetext{
${ }^{1}$ Les mésons $J / \psi$ et $\psi(2 S)$ sont des mésons vecteurs, le méson $\chi_{c 1}$ est un méson vecteur axial.
} 
dans un milieu coloré composé de gluons et de paires de quark-antiquark. On ne peut pas dire que $q_{1} \bar{q}_{2}$ est un méson et que $q_{3} \bar{q}_{4}$ est un autre méson. Cependant, le système évolue pour donner dans l'état final deux mésons distants spatialement et donc orthogonaux. Pendant le temps de l'interaction, un quark donné ne sait pas avec quel antiquark il formera un méson, ce qui se traduit par un recouvrement des fonctions d'onde des deux mésons [69]. Les désintégrations non-leptoniques des mésons lourds sont un laboratoire idéal pour tester le caractère non-perturbatif, longue distance de QCD.

Un traitement théorique complet des désintégrations non-leptoniques n'est pas encore à portée de main, des approches phénoménologiques sont utilisées à la place. Il existe différents niveaux de sophistication : on commence par décrire l'amplitude dans l'hypothèse de factorisation. On considère ensuite les effets des termes non-factorisables avec ou sans prise en compte des interactions dans l'état final.

\subsubsection{Amplitude dans l'approximation de factorisation}

Le fait que les produits de désintégration d'un méson $B$ sont assez énergétiques implique que l'hadronisation de ces produits n'intervient qu'une fois qu'ils ont parcouru une certaine distance l'un de l'autre $[70,71]$. La raison est qu'une fois que les quarks se sont regroupés en singlet de couleur, les gluons mous sont inefficaces pour les réarranger. C'est le phénomène connu sous le nom de transparence de couleur ${ }^{2}$ [70]. On s'attend alors à ce que l'amplitude décrivant la désintégration du méson $B$ en deux mésons puisse s'exprimer comme le produit de deux courants hadroniques indépendants, le premier décrivant la formation d'un méson provenant du quark $b$ converti et du quark léger spectateur et l'autre décrivant la production d'un méson avec l'hadronisation d'un $W$ virtuel. C'est l'hypothèse de factorisation. Cette description est supposée valide pour les diagrammes « spectateur externe » (autorisés de couleur) où l'énergie importante, emportée par le $W$, permet aux produits de désintégration du $W$ en hadrons légers d'être bien séparés du système contenant le quark spectateur. Rien ne permet de dire si cette hypothèse est valide dans le cas des diagrammes « spectateur interne » (supprimés de couleur) ainsi que dans le cas impliquant des hadrons lourds dans l'état final. Il convient donc de tester l'approximation de factorisation expérimentalement pour en obtenir une meilleure compréhension.

À l'ordre des arbres, la désintégration faible non-leptonique, $\bar{b} \rightarrow \bar{c} c \bar{s}$, est décrite dans le Modèle Standard par un diagramme avec échange d'un unique $W$ (Fig. 2.1 (a)). Étant donné que $m_{b}$ est beaucoup plus petit que $M_{W}$, on utilise une interaction effective à quatre quarks (interaction de Fermi) comme approximation de la désintégration faible. La théorie complète est alors décrite par un hamiltonien effectif qui n'est qu'une manière pratique de paramétrer les effets de basse énergie :

$$
\mathcal{H}_{\mathrm{eff}}=\frac{G_{F}}{\sqrt{2}} V_{c s} V_{c b}^{*}(\bar{c} s)_{V-A}(\bar{b} c)_{V-A}+\mathcal{O}\left(\frac{k^{2}}{M_{W}^{2}}\right)
$$

\footnotetext{
${ }^{2}$ L'argument présenté n'est supposé valable que dans le cas où les mésons qui n'interviennent pas dans le facteur de forme contenant le quark spectateur sont légers $\left(K, \pi\right.$, etc.), tel que $B_{d}^{0} \rightarrow D^{-} \pi^{+}$. Il est possible qu'il soit encore applicable dans le cas du $\psi$ car la taille transverse du $\psi$ devient très petite dans la limite des quarks lourds, le recouvrement avec le système $\left(B K^{*}\right)$ est petit [68]. Seule la confrontation avec l'expérience permet de dire si la factorisation est un concept utile pour ces désintégrations.
} 
a)

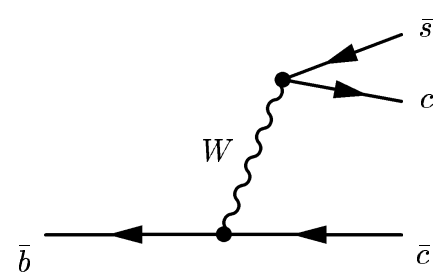

b)

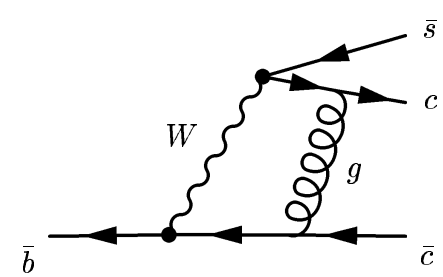

FIG. 2.1: (a) Diagramme à l'ordre des arbres de la désintégration faible $\bar{b} \rightarrow \bar{c} c \bar{s}$. (b) Un diagramme de correction radiative QCD.

où $k$ est le quadrivecteur énergie-impulsion du $W, G_{F}$ la constante de Fermi et $\left(\bar{q} q^{\prime}\right)_{V-A}=\bar{q} \gamma_{\mu}(1-$ $\left.\gamma_{5}\right) q^{\prime}$. Les degrés de liberté du boson $W$ ont été enlevés de la théorie ${ }^{3}$. Le résultat est un opérateur local multiplié par une constante de couplage effective, appelée coefficient de Wilson. Cette procédure s'appelle l'Operator Product Expansion (OPE). L'opérateur ne contient pas les degrés de liberté du boson $W$ qui sont cachés dans le coefficient de Wilson.

La prise en compte de l'interaction forte affecte cette image simple de deux manières. Les corrections radiatives QCD à une boucle (Fig. 2.1 (b) et Fig. 2.2) donnent lieu à de nouveaux vertex faibles effectifs, introduisant un nouvel opérateur. De plus, les forces de confinement à longue distance sont responsables de la liaison des quarks dans les états asymptotiques hadroniques. On aboutit alors à l'hamiltonien effectif ${ }^{4}$

$$
\mathcal{H}_{\mathrm{eff}}=\frac{G_{F}}{\sqrt{2}} V_{c s} V_{c b}^{*}\left[C_{1}(\mu) O_{1}(\mu)+C_{2}(\mu) O_{2}(\mu)\right]
$$

où les opérateurs ${ }^{5} O_{1}$ et $O_{2}$ (appelés opérateurs courant-courant) sont donnés par ( $\alpha, \beta$ sont les indices de couleur) :

$$
O_{1} \equiv\left(\bar{c}_{\alpha} s_{\alpha}\right)_{V-A}\left(\bar{b}_{\beta} c_{\beta}\right)_{V-A}, \quad O_{2} \equiv\left(\bar{c}_{\alpha} s_{\beta}\right)_{V-A}\left(\bar{b}_{\beta} c_{\alpha}\right)_{V-A}
$$

et $C_{1}$ et $C_{2}$ sont les coefficients de Wilson. En l'absence de corrections QCD, on a simplement $C_{1}=1$ et $C_{2}=0$. Les corrections QCD ont introduit une échelle arbitraire $\mu$ dans le problème, permettant de séparer les effets à courte distance, inclus dans les coefficients de Wilson, des effets à longue distance, inclus dans les éléments de matrice hadronique des opérateurs locaux à quatre quarks $O_{i}$ (qui contiennent les phénomènes d'hadronisation, de rediffusion dans l'état final, etc.) :

$$
\text { courte distance }<\mu^{-1}<\text { longue distance. }
$$

Cette échelle est typiquement $\mu=\mathcal{O}\left(m_{b}\right)$. Elle correspond à l'échelle de masse des effets d'hadronisation dominés par la masse du quark $b$, et non pas à l'échelle à laquelle QCD devient nonperturbative (qui est $\mu \approx 1 \mathrm{GeV}$ ). Ainsi, il existe deux régimes pour les éléments de matrice ha-

\footnotetext{
${ }^{3}$ Souvent référencé comme « integrating out » dans la littérature.

${ }^{4}$ On néglige les diagrammes pingouins (cf. la discussion § 1.3.3), qui ajouteraient les opérateurs $O_{3}$ à $O_{6}$ (diagrammes pingouins QCD) et $O_{7}$ à $O_{10}$ (diagrammes pingouins électrofaibles) à $\mathcal{H}_{\text {eff }}$ [64]. La prise en compte de ces opérateurs ne modifie pas de manière significative les prédictions pour les taux d'embranchement mais est essentielle pour l'évaluation des asymétries de violation de $C P$ directe.

${ }^{5}$ La définition opposée existe dans la littérature (pour des raisons historiques) : $O_{2} \leftrightarrow O_{1}[53,73]$.
} 

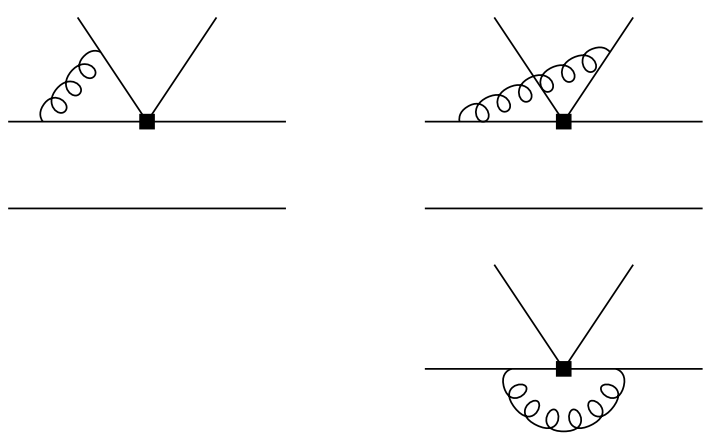
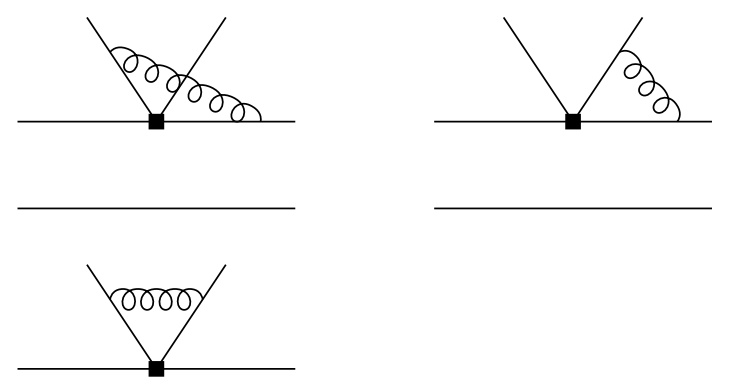

FIG. 2.2: Corrections radiatives QCD à une boucle (ordre $\mathcal{O}\left(\alpha_{s}\right)$ ) [72]. Le carré noir symbolise l'opérateur à quatre quarks $O_{i}(i=1,2)$ (le dernier diagramme est l'équivalent dans la théorie effective du diagramme de la figure 2.1 (b) dans la théorie complète).

dronique : un régime perturbatif avec échange de gluons durs et un régime non-perturbatif avec échange de gluons mous [73, 74]. Les coefficients de Wilson sont calculés en égalant le Modèle Standard et la théorie effective à $\mu=M_{W}$ (matching). On obtient alors $C_{i}\left(M_{W}\right)$. L'évolution perturbative de $C_{i}(\mu)$ est ensuite calculée avec l'aide des techniques du groupe de renormalisation (RGE) jusqu'à l'échelle $\mu=\mathcal{O}\left(m_{b}\right)$ [73]. À cette échelle, les coefficients de Wilson valent ${ }^{6}$ [73] (voir la table XXII de cette référence avec $\Lambda \frac{(5)}{M S}=225 \mathrm{MeV}$, schéma de renormalisation NDR) :

$$
C_{1}=1.082, C_{2}=-0.185
$$

L'identité de Fierz

$$
\left(\bar{\psi}_{1} \psi_{2}\right)_{V-A}\left(\bar{\psi}_{3} \psi_{4}\right)_{V-A}=\left(\bar{\psi}_{1} \psi_{4}\right)_{V-A}\left(\bar{\psi}_{3} \psi_{2}\right)_{V-A}
$$

et la relation de fermeture des matrices de Gell-Mann $\lambda^{a}$,

$$
2 T_{i k}^{a} T_{j l}^{a}=-\frac{1}{N_{c}} \delta_{i k} \delta_{j l}+\delta_{i l} \delta_{j k}, \quad T^{a}=\frac{\lambda^{a}}{2}
$$

permettent de réexprimer l'hamiltonien effectif en termes des opérateurs singlet de couleur $\tilde{O}_{2}$ et octet de couleur $\tilde{O}_{2, \text { oct }}$ définis par :

$$
\tilde{O}_{2} \equiv\left(\bar{c}_{\alpha} c_{\alpha}\right)_{V-A}\left(\bar{b}_{\beta} s_{\beta}\right)_{V-A}, \quad \tilde{O}_{2, o c t} \equiv \sum_{a=1}^{8}\left(\bar{c}_{\alpha} \frac{\lambda_{\alpha \beta}^{a}}{2} c_{\beta}\right)_{V-A}\left(\bar{b}_{\gamma} \frac{\lambda_{\gamma \delta}^{a}}{2} s_{\delta}\right)_{V-A} .
$$

Dans la relation (2.7), $N_{c}=3$ représente le nombre de couleurs. Le facteur de couleur $1 / N_{c}$ provient de la suppression de couleur pour former des singlets de couleur après la transformation de Fierz. Cette transformation est motivée pour décrire la désintégration $B \rightarrow \psi K^{*}(892)$ puisque la résonance $\psi \in\left[J / \psi, \chi_{c 1}, \psi(2 S)\right]$ est reliée à l'opérateur $\left(\bar{c}_{\alpha} c_{\alpha}\right)_{V-A}$. L'amplitude de désintégration pour $B_{q} \rightarrow \psi K^{*}(892)\left(\left(q, K^{*}\right) \in\left[\left(d, K^{* 0}\right) ;\left(u, K^{*+}\right)\right]\right)$ s'écrit, après avoir effectué une transformation de

\footnotetext{
${ }^{6}$ Les coefficients de Wilson dépendent de l'échelle de renormalisation $\mu$, mais aussi du schéma de renormalisation.
} 
Fierz des opérateurs courant-courant [97, 64] :

$$
\begin{aligned}
& \mathcal{A}\left(B_{q}\left(p_{B}\right) \rightarrow \psi\left(p_{\psi}, \epsilon_{\psi}\right) K^{*}\left(p_{K^{*}}, \epsilon_{K^{*}}\right)\right)= \\
& \quad \frac{G_{F}}{\sqrt{2}} V_{c s} V_{c b}^{*} a_{2}(\mu)\left[\left\langle\psi K^{*}\left|\tilde{O}_{2}(\mu)\right| B_{q}\right\rangle+\kappa\left\langle\psi K^{*}\left|\tilde{O}_{2, o c t}(\mu)\right| B_{q}\right\rangle\right],
\end{aligned}
$$

où $a_{2}(\mu)=C_{2}(\mu)+C_{1}(\mu) / N_{c}, \kappa=2 C_{1}(\mu) / a_{2}(\mu) . \epsilon_{\psi}$ et $\epsilon_{K^{*}}$ correspondent aux polarisations des mésons vecteurs $\psi$ et $K^{*}$, respectivement. $p_{B}, p_{\psi}$ et $p_{K^{*}}$ sont les quadrivecteurs énergie-impulsion des mésons $B, \psi$ et $K^{*}$.

Afin de traiter les éléments de matrice hadronique, les champs en interaction d'un des courants sont remplacés par les champs asymptotiques libres. L'amplitude faible se factorise en insérant un état du vide (VIA = Vacuum-Insertion Approximation) et est déterminée par l'élément de matrice hadronique de l'autre courant [77]. Avec ce remplacement des champs en interaction par les champs asymptotiques, on ne tient aucun compte des interactions QCD entre les courants, c'est-à-dire que l'on néglige les interactions dans l'état final (FSI). Les éléments de matrice hadronique peuvent alors s'écrire comme une somme de contributions factorisables et non-factorisables (nf) :

$$
\begin{aligned}
\left\langle\psi K^{*}\left|\tilde{O}_{2}\right| B_{q}\right\rangle & =\left\langle\psi\left|(\bar{c} c)_{V-A}\right| 0\right\rangle\left\langle K^{*}\left|(\bar{b} s)_{V-A}\right| B_{q}\right\rangle+\left\langle\psi K^{*}\left|\tilde{O}_{2}\right| B_{q}\right\rangle_{\mathrm{nf}}, \\
\left\langle\psi K^{*}\left|\tilde{O}_{2, o c t}\right| B_{q}\right\rangle & =\left\langle\psi K^{*}\left|\tilde{O}_{2, o c t}\right| B_{q}\right\rangle_{\mathrm{nf}} .
\end{aligned}
$$

Puisque $|\psi\rangle$ est un état singlet de couleur, les éléments de matrice hadronique factorisés des opérateurs octet de couleur donnés par :

$$
\sum_{a=1}^{8}\left\langle\psi\left|\left(\bar{c} \frac{\lambda^{a}}{2} c\right)_{V-A}\right| 0\right\rangle\left\langle K^{*}\left|\left(\bar{b} \frac{\lambda^{a}}{2} s\right)_{V-A}\right| B_{q}\right\rangle
$$

sont nuls. La figure 2.2 illustre des corrections radiatives QCD factorisables ( 2 ème ligne) et nonfactorisables ( $1^{\text {ère }}$ ligne). Les contributions non-factorisables correspondent à l'échange de gluons entre les systèmes $\left(B_{q} K^{*}\right)$ et $\psi$. Les corrections factorisables correspondent à l'échange de gluons intervenant soit dans le facteur de forme de $B_{q} \rightarrow K^{*}$ ou dans la constante de désintégration $f_{\psi}$.

Dans le cadre de la factorisation (dite naïve [75]), seuls les termes factorisables sont retenus. À noter que dans cette approche, les corrections radiatives QCD non-factorisables à des échelles plus grandes que $\mu$ sont prises en compte dans les coefficients de Wilson alors qu'elles sont négligées pour des échelles plus petites que $\mu$ dans les éléments de matrice hadronique. L'approximation de factorisation brise la «dualité » (équivalence) de la description quark-hadron ${ }^{7}$ de l'amplitude [69, 78], puisqu'éliminer la contribution de l'octet de couleur revient à omettre différentes contributions dans les descriptions quark-hadron. Enfin, dans le cadre de l'approximation de factorisation, le couplage effectif $a_{2}$ est universel, c'est-à-dire indépendant des processus considérés ${ }^{8}$.

Les éléments de matrice hadronique sont ensuite exprimés en termes des facteurs de forme

\footnotetext{
${ }^{7}$ L'idée est que, puisque les quarks et gluons n'existent pas asymptotiquement à l'état libre, l'état final partonique se fragmente en hadrons — «s'hadronise » — avec une probabilité égale à 1 [76].

${ }^{8} \mathrm{Il}$ existe trois classes de processus : les désintégrations autorisées de couleur gouvernées par le coefficient $a_{1}=$ $C_{1}+C_{2} / N_{c}$, les désintégrations supprimées de couleur gouvernées par le coefficient $a_{2}$ et une troisième classe provenant de l'interférence des deux précédents types de désintégration gouvernée par une combinaison de $a_{1}$ et $a_{2}$.
} 
semileptoniques $[61,68]$ :

$$
\begin{aligned}
\left\langle\psi\left|(\bar{c} c)_{V-A}\right| 0\right\rangle= & \epsilon_{\psi}^{* \mu} m_{\psi} f_{\psi} \\
\left\langle K^{*}\left|(\bar{b} s)_{V-A}\right| B_{q}\right\rangle= & \frac{2 i}{m_{B}+m_{K^{*}}} \epsilon_{\mu \nu \rho \sigma} \epsilon_{K^{*}}^{* \nu} p_{B}^{\rho} p_{K^{*}}^{\sigma} V\left(q^{2}\right)-\left[-\frac{\left(\epsilon_{K^{*}}^{*} q\right)}{m_{B}+m_{K^{*}}}\left(p_{B}+p_{K^{*}}\right)_{\mu} A_{2}\left(q^{2}\right)\right. \\
& \left.+\left(m_{B}+m_{K^{*}}\right) \epsilon_{K^{*} \mu}^{*} A_{1}\left(q^{2}\right)-2 m_{K^{*}} \frac{\epsilon_{K^{*}}^{*} \cdot q}{q^{2}} q_{\mu}\left(A_{3}\left(q^{2}\right)-A_{0}\left(q^{2}\right)\right)\right] \\
\left\langle\psi K^{*}\left|\tilde{O}_{2(, o c t)}\right| B_{q}\right\rangle_{\mathrm{nf}}= & m_{\psi} f_{\psi}\left[\frac{2 i}{m_{B}+m_{K^{*}}} \epsilon_{\mu \nu \rho \sigma} \epsilon_{\psi}^{* \mu} \epsilon_{K^{*}}^{* \nu} p_{B}^{\rho} p_{K^{*}}^{\sigma} V_{(o c t)}^{n f}\left(q^{2}\right)-\left\{\left(m_{B}+m_{K^{*}}\right) \times\right.\right. \\
& \left.\left.\left(\epsilon_{\psi}^{*} \cdot \epsilon_{K^{*}}^{*}\right) A_{1(, o c t)}^{n f}\left(q^{2}\right)-\frac{\left(\epsilon_{\psi}^{*} \cdot\left(p_{B}+p_{K^{*}}\right)\right)\left(\epsilon_{K^{*}}^{*} \cdot q\right)}{m_{B}+m_{K^{*}}} A_{2(, o c t)}^{n f}\left(q^{2}\right)\right\}\right]
\end{aligned}
$$

où $q_{\mu}=\left(p_{B}-p_{K^{*}}\right)_{\mu}=\left(p_{\psi}\right)_{\mu}$, ce qui donne $q^{2}=m_{\psi}^{2}$. ̀̀ noter que $\epsilon_{\psi} \cdot p_{\psi}=\epsilon_{\psi} \cdot q=0$. Le paramètre $f_{\psi}$ décrit la constante de désintégration du $\psi$, qui peut être déterminée à partir d'un taux de désintégration du $\psi$ (p. ex. pour $\psi=J / \psi, J / \psi \rightarrow e^{+} e^{-}$, elle vaut $f_{J / \psi}=395 \mathrm{MeV}$ [28]).

En ce qui concerne les facteurs de forme $A_{i}\left(q^{2}\right)$ et $V\left(q^{2}\right)$ pour $B_{q} \rightarrow K^{*}$, plusieurs modèles phénoménologiques ${ }^{9}$ existent $[79,80,81]$ : le modèle de Bauer-Stech-Wirbel (BSW) [61], une version modifiée du modèle de BSW (BSWII ou NSRX) [82], le modèle d'Isgur et al. (ISGW) [83], le modèle de Casalbuoni et al. et de Deandrea et al. (CDDFGN) [84], le modèle de Ali et al. (HQS = Heavy Strange Quark) [85], le modèle de Jaus-Wyler (JW) [86], le modèle d'Orsay (Orsay)[87], le modèle de Cheng-Tseng (CT) [88], le modèle de Ball-Braun (BB) [89], le modèle de Melikhov-Stech (MS) [90], le modèle de Cheng-Cheung-Hwang (LF) [91], le modèle de Neubert-Stech (NS) [68], le modèle de Yang (Yang) [92], le modèle de Gourdin-Keum-Pham (YYK) [93]. Les facteurs de forme sont évalués, soit à $q^{2}=0$ (pour la plupart), soit à $q^{2}=q_{\max }^{2}$, puis sont extrapolés à $q^{2}=m_{\psi}^{2}$ suivant la dépendance en $q^{2}$ des différents modèles.

Dans l'approche phénoménologique BSW [61], les constantes $a_{1}$ et $a_{2}$ ne sont pas calculées perturbativement. Elles sont considérées comme des paramètres libres et ajustées sur les données en utilisant une ou plusieurs désintégrations. Cette procédure assure que, pour les désintégrations prises en compte dans l'ajustement, les termes non-factorisables sont automatiquement absorbés. Les valeurs ainsi obtenues pour $a_{1}$ et $a_{2}$ sont considérées comme universelles à toutes les désintégrations. Cela signifie que les termes non-factorisables des désintégrations prises en compte dans l'ajustement sont supposés universels.

\subsubsection{Termes non-factorisables}

La factorisation naïve souffre de plusieurs inconvénients. Tout d'abord, elle brise l'invariance d'échelle des amplitudes, qui sont des quantités physiques et doivent donc être indépendantes de l'échelle $\mu$. Les constantes de désintégration des mésons ainsi que les facteurs de forme étant des quantités mesurables, elles sont indépendantes de l'échelle $\mu$, alors que les coefficients de Wilson en dépendent. Les amplitudes de désintégration, étant le produit des deux quantités, dépendent donc de l'échelle $\mu$. L'espoir est que la factorisation fournisse une approximation utile si les coefficients de Wilson sont évalués à une échelle $\mu$ convenable, le point de factorisation. Le paramètre $a_{2}$ est particulièrement sensible au choix du point de factorisation du fait des signes différents et de l'ampli-

\footnotetext{
${ }^{9}$ À noter qu'il n'existe pas de mesures des facteurs de forme $B_{q} \rightarrow K^{*}$.
} 
tude des coefficients de Wilson $C_{1}$ et $C_{2}$. De plus, les contributions des amplitudes non-factorisables peuvent s'avérer importantes. Cela est particulièrement le cas pour les amplitudes supprimées de couleur : les contributions des termes non-factorisables dominants sont proportionnelles au coefficient de Wilson $C_{1}$, qui est de l'ordre de l'unité, à comparer au coefficient $a_{2}=\mathcal{O}(0.1)$. Ainsi, même une faible contribution non-factorisable aura un effet significatif sur les amplitudes supprimées de couleur.

Afin de pallier ces inconvénients, les effets non-factorisables sont paramétrés (plutôt qu'ignorés) de différentes manières. Il existe deux façons équivalentes d'introduire ces contributions nonfactorisables [94] :

- soit elles sont ajoutées à l'amplitude factorisée avec $N_{c}=3$ (voir eqs. (2.9), (2.10) et (2.11)) $[68,95,96,97]$ :

$$
a_{2}(\mu) \rightarrow a_{2}^{\text {eff }}=a_{2}(\mu)\left(1+\epsilon_{1}(\mu)+\kappa \epsilon_{8}(\mu)\right)
$$

avec

$$
\epsilon_{1}(\mu)=\left\langle\psi K^{*}\left|\tilde{O}_{2}(\mu)\right| B_{q}\right\rangle_{\mathrm{nf}}, \quad \epsilon_{8}(\mu)=\left\langle\psi K^{*}\left|\tilde{O}_{2, o c t}(\mu)\right| B_{q}\right\rangle_{\mathrm{nf}} .
$$

Le terme $\epsilon_{1}$ est généralement négligé, étant de l'ordre $1 / N_{c}^{2}[68]$;

- soit elles sont décrites en termes de $N_{c} \rightarrow N_{c}^{\text {eff }}$, le nombre de couleur effectif $[98,99,100]$. La dépendance des éléments de matrice hadronique à l'échelle $\mu$ est extraite avant d'appliquer l'approximation de factorisation. Puisque $O_{i}(\mu)$ est l'opérateur à quatre quarks renormalisé à l'échelle $\mu$, son élément de matrice hadronique est relié à celui à l'ordre des arbres par :

$$
\left\langle O_{i}(\mu)\right\rangle=g(\mu)\left\langle O_{i}\right\rangle^{\text {tree }}
$$

ce qui conduit à

$$
\left\langle\mathcal{H}_{\mathrm{eff}}\right\rangle=\sum_{i} C_{i}(\mu) g(\mu)\left\langle O_{i}\right\rangle^{\text {tree }}=C_{i}^{\mathrm{eff}}\left\langle O_{i}\right\rangle^{\text {tree }}
$$

où les coefficients de Wilson effectifs sont indépendants du schéma et de l'échelle de renormalisation. À noter qu'il est nécessaire d'inclure les corrections radiatives QCD pour les coefficients de Wilson et pour les éléments de matrice hadronique au même ordre.

$\mathrm{C}^{\prime}$ est ce qu'on appelle la factorisation généralisée ${ }^{10}$. Cela revient à considérer que les corrections non-factorisables dépendent du processus de désintégration (ce qui limite le pouvoir de prédiction de la factorisation généralisée). De plus, afin de pouvoir définir le paramètre $a_{2}^{\text {eff }}$ pour les désintégrations $B \rightarrow V V$, les termes non-factorisables doivent être identiques pour chaque onde partielle, i.e. indépendants de la polarisation des mésons vecteurs. Aucun argument physique ne permet de justifier une telle considération. Ces corrections non-factorisables permettent de restaurer l'indépendance de l'amplitude à l'échelle $\mu$. Enfin, dans cette approche, les paramètres des termes nonfactorisables $\left(\epsilon_{1}, \epsilon_{8}, N_{c}^{\text {eff }}\right)$ sont considérés réels. Dans la première approche, les interactions dans l'état final (FSI) sont négligées, les amplitudes sont réelles. Dans la seconde approche, le fait de prendre en compte les corrections radiatives QCD à une boucle des éléments de matrice hadronique introduit des coefficients de Wilson effectifs complexes, permettant de traiter une partie des FSI (voir le paragraphe suivant). Les amplitudes sont cependant "relativement réelles », puisque

\footnotetext{
${ }^{10}$ Pour une critique de la factorisation généralisée, voir la référence [101].
} 


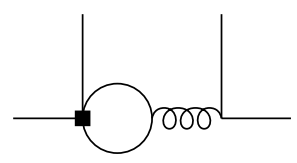

FIG. 2.3: Diagramme de type pingouin intervenant dans le mécanisme BSS.

ces corrections sont faibles.

Afin d'avoir un certain pouvoir de prédiction, les termes non-factorisables sont supposés universels et ajustés sur les données, comme dans l'approche BSW. Il convient de noter que dans une telle approche, les résultats obtenus dépendent du choix du facteur de forme utilisé. À titre d'exemple, une valeur de $\left|a_{2}^{\text {eff }}\right|$ obtenue avec $B \rightarrow J / \psi K^{(*)}$ et $B \rightarrow \psi(2 S) K^{(*)}$ [68] en utilisant deux modèles de facteur de forme donne $\left|a_{2}^{\text {eff }}\right|=0.21 \pm 0.01$ et $\left|a_{2}^{\text {eff }}\right|=0.29 \pm 0.01$, respectivement. Le résultat dépend donc fortement du modèle utilisé pour le facteur de forme. De plus, le paramètre $a_{2}$, calculé perturbativement, est plus sensible à l'échelle et au schéma de renormalisation que le paramètre $a_{1}$ [102]. Le fait de changer l'échelle ou le schéma de renormalisation implique que des contributions précédemment incluses dans les éléments de matrice hadronique de certains opérateurs peuvent se retrouver dans les coefficients de Wilson [22, 73].

\subsubsection{Interactions dans l'état final (FSI)}

La présence d'interactions dans l'état final implique la rupture de l'approximation de factorisation [105]. Par définition, ces interactions se manifestent dans une région d'espace-temps où les particules de l'état final sont déjà formées mais interagissent encore fortement pendant l'éloignement l'une de l'autre. La compréhension des interactions dans l'état final est cruciale pour l'étude de la violation de $C P$. Elles interviennent dans la violation de $C P$ directe et rendent l'extraction des phases faibles délicate [106]. Le canal provenant de la désintégration faible du méson $B$ est rediffusé vers des canaux différents portant les mêmes nombres quantiques via l'interaction forte (ou électromagnétique), incluant des canaux qui n'étaient pas à l'origine couplés à la désintégration faible. Ces rediffusions se manifestent soit au niveau des quarks, soit au niveau des hadrons, souvent référencées comme les FSI courte distance et longue distance, respectivement, bien qu'il soit impossible de les séparer clairement. Les FSI peuvent être de deux sortes : les FSI molles (soft scattering), dues à des effets non-perturbatifs longue distance et donc probablement mieux décrites en termes de hadrons plutôt qu'en termes de quarks [107] ou les FSI dures (hard scattering), qui sont interprétées comme l'interaction entre constituants ponctuels, les quarks et gluons de QCD [108].

Les phases fortes sont générées par les FSI. Les FSI courte distance (FSI dures) proviennent des corrections radiatives QCD à une boucle des éléments de matrice hadronique [109, 110], qui se groupent en deux classes : les diagrammes pingouins (Fig. 2.3), où la partie imaginaire provient de la partie absorptive (états intermédiaires physiques) avec les quarks $u$ et $c$ (le quark $t$ ne donne pas de partie absorptive, uniquement dispersive), appelé mécanisme BSS [74, 108] et les corrections QCD des vertex (voir Fig. 2.2). Puisque $m_{b}>2 m_{c}, 2 m_{u}$, les quarks $u$ et $c$ rediffusés peuvent être sur leur couche de masse. De plus, l'annihilation $c \bar{c}(u \bar{u})$ crée un gluon dur, justifiant l'utilisation de QCD perturbative. Il convient de noter que les diagrammes pingouins mis en jeu ne 
sont pas les diagrammes pingouins intervenant dans les opérateurs $O_{3}, \ldots, O_{10}$ [111]. Les coefficients de Wilson $C_{3}, \ldots, C_{10}$ de ces opérateurs sont petits et contiennent les contributions courte distance des quarks virtuels $u, c, t$ (partie dispersive). Les pingouins du mécanisme BSS sont générés à une échelle $\mu$ plus petite et corrigent les éléments de matrice hadronique des opérateurs $O_{i}[98,99,100,109]$. Bien que les corrections radiatives QCD à une boucle des éléments de matrice hadronique produisent des phases fortes pour tous les opérateurs $O_{i}$, les phases dominantes proviennent des diagrammes pingouins [109] du mécanisme BSS. Cette procédure produit des phases fortes à partir des FSI courte distance. La formation de hadrons n'introduit pas de phases, puisque les amplitudes sont calculées avec des modèles basés sur l'approximation de factorisation [112]. En factorisant les éléments de matrice hadronique, toute l'information sur la valeur du quadrivecteur énergie-impulsion de transfert, $k$, du gluon dans le diagramme type pingouin (Fig. 2.3) est perdue. De simples considérations cinématiques au niveau des quarks impliquent que $k^{2}$ se trouve dans l'intervalle $1 / 4 \lesssim k^{2} / m_{b}^{2} \lesssim 1 / 2$ [74,113]. Les coefficients de Wilson effectifs sont en général évalués à $k^{2}=m_{b}^{2} / 2$.

Les FSI longue distance sont plus difficiles à estimer car elles proviennent d'effets non-perturbatifs probablement mieux décrits en termes de hadrons. Les FSI sont supposées être de moins en moins importantes avec l'augmentation de la masse du quark se désintégrant. On peut montrer que cela vaut pour les FSI dures, puisque les particules dans l'état final émergent avec une impulsion telle qu'elles ont aucune chance de rediffuser. Cela est faux pour les FSI molles [114]. De plus, l'analyse des rediffusions élastiques montre que les phases fortes provenant des FSI molles sont principalement générées par des processus inélastiques [114]. Ainsi, le grand nombre d'états intermédiaires possibles rend la détermination des phases fortes pour des processus exclusifs quasi impossible. La phase forte d'un méson $B$ se désintégrant en deux mésons provenant de la diffusion inélastique méson-méson est typiquement de l'ordre de $20^{\circ}$ [115].

Les FSI, en plus de produire une phase forte, modifient l'amplitude finale, conduisant à une modification du taux de désintégration par rapport aux calculs faits dans l'approximation de factorisation [116].

\subsubsection{Tests de la factorisation}

Les canaux $B_{q} \rightarrow \psi K^{*}(892)$ permettent différents tests de l'approximation de factorisation. Plusieurs modèles phénoménologiques prédisent la fraction de polarisation longitudinale, notée $\Gamma_{L} / \Gamma$, ainsi que le rapport de production vecteur sur pseudo-scalaire, $R_{\psi} \equiv \Gamma(B \rightarrow \psi V) / \Gamma(B \rightarrow \psi P)$ $[81,87,93,95,97,117]$. De plus, des informations supplémentaires peuvent être obtenues en mesurant la phase des amplitudes de désintégration, puisque toute phase relative non-triviale indique des interactions dans l'état final et la rupture de l'approximation de factorisation.

L'amplitude $\mathcal{A}\left(B_{q} \rightarrow \psi K^{*}(892)\right)$ en base d'hélicité (voir appendice A) s'écrit (cf. eq. (2.9)) :

$$
H_{\lambda}=\mathcal{A}\left(B_{q}\left(p_{B}\right) \rightarrow \psi\left(p_{\psi}, \epsilon_{\psi}(\lambda)\right) K^{*}\left(p_{K^{*}}, \epsilon_{K^{*}}(\lambda)\right)\right)=\left\langle\psi(\lambda) K^{*}(\lambda)\left|\mathcal{H}_{\mathrm{eff}}\right| B_{q}\right\rangle
$$

avec $\lambda=0, \pm 1$. On se place dans le repère du méson $B$ au repos. Puisque le méson $B$ est une particule de spin 0 , les mésons vecteurs ont la même hélicité. Les polarisations des mésons vecteurs 
ainsi que les quadrivecteurs énergie-impulsion sont donnés par [118] :

$$
\begin{gathered}
\epsilon_{\psi}^{\mu}( \pm)=\frac{1}{\sqrt{2}}(0, \mp 1,-i, 0), \quad \epsilon_{K^{*}}^{\mu}( \pm)=\frac{1}{\sqrt{2}}(0, \pm 1,-i, 0), \\
\epsilon_{\psi}^{\mu}(0)=\frac{1}{m_{\psi}}\left(|\vec{p}|, 0,0, E_{\psi}\right), \quad \epsilon_{K^{*}}^{\mu}(0)=\frac{1}{m_{K^{*}}}\left(-|\vec{p}|, 0,0, E_{K^{*}}\right), \\
p_{B}^{\mu}=\left(m_{B}, 0,0,0\right), \quad p_{\psi}^{\mu}=\left(E_{\psi}, 0,0,|\vec{p}|\right), \quad p_{K^{*}}^{\mu}=\left(E_{K^{*}}, 0,0,-|\vec{p}|\right),
\end{gathered}
$$

où $|\vec{p}|$ est l'impulsion commune des mésons vecteurs, i.e.

$$
2|\vec{p}| m_{B}=\sqrt{\left(m_{B}^{2}-m_{K^{*}}^{2}-m_{\psi}^{2}\right)^{2}-4 m_{K^{*}}^{2} m_{\psi}^{2}} .
$$

En utilisant les expressions des éléments de matrice hadronique en fonction des facteurs de forme (cf. eqs. (2.13)-(2.15)), on obtient pour les amplitudes d'hélicité ${ }^{11}$ (avant d'introduire les FSI) :

$$
\begin{aligned}
H_{0} & =\frac{G_{F}}{\sqrt{2}} V_{c s} V_{c b}^{*} a_{2} f_{\psi} m_{\psi}\left(m_{B}+m_{K^{*}}\right) A_{1}\left(q^{2}\right)\left(a \xi_{1}-b x \xi_{2}\right) \\
H_{ \pm 1} & =\frac{G_{F}}{\sqrt{2}} V_{c s} V_{c b}^{*} a_{2} f_{\psi} m_{\psi}\left(m_{B}+m_{K^{*}}\right) A_{1}\left(q^{2}\right)\left(-\xi_{1} \pm c y \xi_{V}\right) .
\end{aligned}
$$

Les paramètres (réels) $a, b, c, x, y, \xi_{1}, \xi_{2}$ et $\xi_{V}$ introduits dans les équations (2.25) et (2.26) sont définis par [81] :

$$
\begin{gathered}
a=\frac{m_{B}^{2}-m_{K^{*}}^{2}-m_{\psi}^{2}}{2 m_{K^{*}} m_{\psi}}, \quad b=\frac{2|\vec{p}|^{2} m_{B}^{2}}{m_{K^{*}} m_{\psi}\left(m_{B}+m_{K^{*}}\right)^{2}}, \quad c=\frac{2|\vec{p}| m_{B}}{\left(m_{B}+m_{K^{*}}\right)^{2}}, \\
x=\frac{A_{2}\left(m_{\psi}^{2}\right)}{A_{1}\left(m_{\psi}^{2}\right)}, \quad y=\frac{V\left(m_{\psi}^{2}\right)}{A_{1}\left(m_{\psi}^{2}\right)} \\
\xi_{1}=1+\frac{A_{1}^{\mathrm{nf}}\left(m_{\psi}^{2}\right)}{A_{1}\left(m_{\psi}^{2}\right)}+\kappa \frac{A_{1, o c t}^{\mathrm{nf}}\left(m_{\psi}^{2}\right)}{A_{1}\left(m_{\psi}^{2}\right)} \\
\xi_{2}=1+\frac{A_{2}^{\mathrm{nf}}\left(m_{\psi}^{2}\right)}{A_{2}\left(m_{\psi}^{2}\right)}+\kappa \frac{A_{2, o c t}^{\mathrm{nf}}\left(m_{\psi}^{2}\right)}{A_{2}\left(m_{\psi}^{2}\right)} \\
\xi_{V}=1+\frac{V^{\mathrm{nf}}\left(m_{\psi}^{2}\right)}{V\left(m_{\psi}^{2}\right)}+\kappa \frac{V_{o c t}^{\mathrm{nf}}\left(m_{\psi}^{2}\right)}{V\left(m_{\psi}^{2}\right)} .
\end{gathered}
$$

$\kappa$ est défini après l'équation (2.9). Avec ces définitions, le taux de désintégration de $B_{q} \rightarrow \psi K^{*}(892)$ est donné par :

$$
\Gamma\left(B_{q} \rightarrow \psi K^{*}\right)=\frac{|\vec{p}|}{8 \pi m_{B}^{2}}\left(\left|H_{0}\right|^{2}+\left|H_{+1}\right|^{2}+\left|H_{-1}\right|^{2}\right),
$$

et les polarisations longitudinale et transverse sont définies par :

$$
P_{L}=\frac{\Gamma_{L}}{\Gamma}=\frac{\left|H_{0}\right|^{2}}{\left|H_{0}\right|^{2}+\left|H_{+1}\right|^{2}+\left|H_{-1}\right|^{2}}=\frac{\left(a \xi_{1}-b x \xi_{2}\right)^{2}}{\left(a \xi_{1}-b x \xi_{2}\right)^{2}+2\left(\xi_{1}^{2}+c^{2} y^{2} \xi_{V}^{2}\right)}
$$

\footnotetext{
${ }^{11}$ Les conventions utilisées sont $g^{\mu \nu}=\operatorname{diag}(1,-1,-1,-1)$ et $\epsilon^{0123}=-\epsilon_{0123}=1$.
} 


$$
P_{T}=1-P_{L}=\frac{\Gamma_{T}}{\Gamma}=\frac{\left|H_{+1}\right|^{2}+\left|H_{-1}\right|^{2}}{\left|H_{0}\right|^{2}+\left|H_{+1}\right|^{2}+\left|H_{-1}\right|^{2}}=\frac{2\left(\xi_{1}^{2}+c^{2} y^{2} \xi_{V}^{2}\right)}{\left(a \xi_{1}-b x \xi_{2}\right)^{2}+2\left(\xi_{1}^{2}+c^{2} y^{2} \xi_{V}^{2}\right)}
$$

avec

$$
\Gamma_{L}=\frac{|\vec{p}|}{8 \pi m_{B}^{2}}\left|H_{0}\right|^{2}, \quad \Gamma_{T}=\frac{|\vec{p}|}{8 \pi m_{B}^{2}}\left(\left|H_{+1}\right|^{2}+\left|H_{-1}\right|^{2}\right) .
$$

À noter que la factorisation naïve donne $\xi_{1}=\xi_{2}=\xi_{V}=1$. Dans la factorisation généralisée, en supposant l'universalité des termes non-factorisables, on aboutit à $\xi_{1}=\xi_{2}=\xi_{V}=\xi$. Il est alors possible de définir un paramètre $a_{2}^{\text {eff }}$ par $a_{2} \rightarrow a_{2}^{\text {eff }}=a_{2} \xi$. Autrement dit, si les termes nonfactorisables ne sont pas universels, il n'est plus possible de définir le paramètre $a_{2}^{\text {eff }}$ [95]. De plus, les rapports $R_{\psi}, \Gamma_{L} / \Gamma$ et $\Gamma_{T} / \Gamma$ sont indépendants du paramètres $a_{2}$ et donc du paramètre $a_{2}^{\text {eff. La }}$ factorisation généralisée modifie le paramètre $a_{2} \rightarrow a_{2}^{\text {eff }}$ mais donne les mêmes résultats pour les précédents rapports que la factorisation naïve.

Les différentes mesures expérimentales obtenues pour $B_{q} \rightarrow J / \psi K^{*}(892)$ [119] et $B_{q} \rightarrow \psi(2 S)$ $K^{*}(892)$ [120] ne s'accordent pas avec les prédictions de la factorisation $[81,87,95,97]$ (naïve ou généralisée). En général, le rapport $R_{\psi}$ est trop important alors que la polarisation longitudinale $P_{L}$ est trop faible. L'approche développée récemment par Beneke-Buchalla-Neubert-Sachrajda [103], appelée factorisation QCD améliorée, permet de calculer les corrections non-factorisables dans la limite des quarks lourds $\left(m_{b} \rightarrow \infty\right)$, au lieu que celles-ci soient ajustées sur les données et considérées universelles. Des calculs utilisant la factorisation QCD améliorée [80, 104] montrent que les corrections non-factorisables dépendent de l'hélicité des mésons vecteurs. L'approche de la factorisation QCD améliorée vient très récemment d'être critiquée pour les désintégrations exclusives d'un méson $B$ en charmonium [121].

\subsection{Distributions angulaires}

Les différentes observables des désintégrations $B_{q} \rightarrow \psi K^{*}(892)$ sont accessibles par une analyse angulaire et/ou temporelle des produits de désintégration des mésons vecteurs. Différentes bases permettent d'exprimer ces distributions : la base en ondes partielles $(S, P, D)$, la base en hélicité $\left(H_{0}, H_{+1}, H_{-1}\right)$ et la base en transversité $\left(A_{0}, A_{\|}, A_{\perp}\right)$. De plus, le fait que le méson $B$ évolue dans le temps implique que les amplitudes dépendent du temps. Dans la dérivation des distributions angulaires, il est nécessaire et important d'apporter un soin tout particulier aux définitions des repères et des angles puisque les désintégrations traitées proviennent de processus faibles qui violent la parité, à la différence de l'utilisation de ces distributions dans l'étude du spin-parité des résonances qui implique des processus forts conservant la parité. Nous verrons en effet que des conclusions physiques peuvent être erronées si ces repères sont mal définis (cf. §6.9).

Dans une première partie, je dérive les distributions angulaires pour la désintégration $B_{q} \rightarrow$ $\psi K^{*}(892)(\psi=J / \psi$ ou $\psi(2 S))$ sans considérer l'évolution temporelle du méson $B$. Cette évolution temporelle, dépendante du type de désintégration du $K^{*}(892)$, est ensuite prise en compte. Enfin, je répète l'exercice avec la désintégration $B_{q} \rightarrow \chi_{c 1} K^{*}(892)$. Seuls les canaux $B \rightarrow J / \psi K^{*}(892)$ sont étudiés dans les chapitres 5 (mesure des amplitudes de transversité) et 6 (mesure de $\sin 2 \beta$ et $\cos 2 \beta)$. Cette section a aussi pour but la préparation des analyses futures des canaux charmonium$K^{*}(892)$, avec, par exemple, la distribution angulaire de la désintégration $B_{q} \rightarrow \chi_{c 1} K^{*}(892)$ qui est 
inédite.

\subsubsection{Généralités}

\section{Amplitudes d'hélicité}

L'obtention de distributions angulaires en utilisant le formalisme d'hélicité est détaillée dans l'appendice A, en insistant sur les conventions des opérateurs de rotation finie et sur la phase de la « seconde particule ». La distribution angulaire pour la désintégration d'une particule $\alpha$ en deux particules $1+2,(J, M) \rightarrow\left(s_{1}, \lambda_{1}\right)+\left(s_{2}, \lambda_{2}\right)$, est donnée par :

$$
\frac{\mathrm{d} \Gamma}{\mathrm{d} \Omega}=\frac{p_{f}}{32 \pi m_{\alpha}^{2}}\left|\mathcal{M}_{\lambda_{1}, \lambda_{2}}^{J, M}(\theta, \varphi)\right|^{2}
$$

où $m_{\alpha}$ est la masse de la particule $\alpha, p_{f}$ l'impulsion des particules 1 et 2 dans le repère au repos de $\alpha$ et

$$
\mathcal{M}_{\lambda_{1}, \lambda_{2}}^{J, M}(\theta, \varphi)=\sqrt{\frac{2 J+1}{4 \pi}} D_{M, \delta}^{J *}(\varphi, \theta, 0) H_{\lambda_{1}, \lambda_{2}}^{J} .
$$

Dans la formule ci-dessus, $\delta \equiv \lambda_{1}-\lambda_{2}$, et $H_{\lambda_{1}, \lambda_{2}}^{J} \equiv\left\langle J M \lambda_{1} \lambda_{2}\left|\mathcal{H}_{\text {eff }}\right| J M\right\rangle$ est l'amplitude d'hélicité (helicity couplings), qui est une fonction de $J$, des hélicités $\lambda_{1}$ et $\lambda_{2}$, et des masses des particules, mais est indépendante des angles $\theta$ et $\varphi$ (l'amplitude d'hélicité est invariante par rotation). Les fonctions $D_{m, m^{\prime}}^{J}$ sont les opérateurs représentant les rotations finies (cf. appendice A).

Le méson $B$ étant de spin nul $(J=0)$ implique que les hélicités des mésons vecteurs doivent être égales, i.e. $\lambda_{1}=\lambda_{2}=\lambda$ avec $\lambda=0, \pm 1$ (par conservation du moment angulaire total). Ainsi, $H_{\lambda} \equiv H_{\lambda, \lambda}^{J=0}$. L'amplitude d'hélicité la plus générale décrivant la désintégration d'un méson $B$ en paire de mésons vecteurs prend la forme $[122,123]$

$$
H_{\lambda}=\epsilon_{1}^{* \mu}(\lambda) \epsilon_{2}^{* \nu}(\lambda)\left[g_{\mu \nu} A+\frac{p_{B \mu} p_{B \nu}}{m_{1} m_{2}} B+i \epsilon_{\mu \nu \alpha \beta} \frac{p_{1}^{\alpha} p_{2}^{\beta}}{m_{1} m_{2}} C\right]
$$

où $\epsilon_{1}, \epsilon_{2}, m_{1}, m_{2}$ et $p_{1}, p_{2}$ sont les polarisations (circulaires), les masses et les quadrivecteurs énergieimpulsion des mésons vecteurs $V_{1}$ et $V_{2}$, respectivement. $p_{B}$ le quadrivecteur énergie-impulsion du méson $B$. $A, B, C$ sont des amplitudes invariantes. D'après les équations (2.25) et (2.26), les expressions des invariants $A, B, C$ sont

$$
\begin{aligned}
A & =-\frac{G_{F}}{\sqrt{2}} V_{c s} V_{c b}^{*} a_{2} f_{\psi} m_{\psi}\left(m_{B}+m_{K^{*}}\right) A_{1}\left(q^{2}\right) \xi_{1}, \\
B & =2 \frac{G_{F}}{\sqrt{2}} V_{c s} V_{c b}^{*} a_{2} \frac{f_{\psi} m_{\psi}^{2} m_{K^{*}}}{m_{B}+m_{K^{*}}} A_{2}\left(q^{2}\right) \xi_{2}, \\
C & =2 \frac{G_{F}}{\sqrt{2}} V_{c s} V_{c b}^{*} a_{2} \frac{f_{\psi} m_{\psi}^{2} m_{K^{*}}}{m_{B}+m_{K^{*}}} V\left(q^{2}\right) \xi_{V} .
\end{aligned}
$$

On a (pour les expressions des polarisations, voir les équations (2.21) et (2.22) avec $1 \equiv \psi$ et $2 \equiv K^{*}$ ):

$$
H_{0}=-\left(a A+B\left(a^{2}-1\right)\right), \quad H_{ \pm 1}=A \pm C \sqrt{a^{2}-1},
$$


où $a$ est défini par (cf. eq. (2.27)) :

$$
a=\frac{p_{1} \cdot p_{2}}{m_{1} m_{2}}=\frac{m_{B}^{2}-m_{1}^{2}-m_{2}^{2}}{2 m_{1} m_{2}}, \quad a^{2}-1=\frac{m_{B}^{2} p_{B}^{2}}{m_{1}^{2} m_{2}^{2}} .
$$

Les amplitudes d'hélicité sont reliées aux amplitudes en ondes partielles par [125] :

$$
H_{0}=-\sqrt{\frac{1}{3}} S+\sqrt{\frac{2}{3}} D, \quad H_{ \pm 1}=\sqrt{\frac{1}{3}} S \pm \sqrt{\frac{1}{2}} P+\sqrt{\frac{1}{6}} D
$$

où $(S, P, D)$ correspondent aux ondes partielles de moment orbital relatif $L$ entre $V_{1}$ et $V_{2}$, égal à $L=0,1,2$. L'expression des amplitudes $S, P, D$ en termes des invariants $A, B, C$ est

$$
S=\frac{1}{\sqrt{3}}\left[A(2+a)+B\left(a^{2}-1\right)\right], \quad P=C \sqrt{2\left(a^{2}-1\right)}, \quad D=\sqrt{\frac{2}{3}}\left[A(1-a)-B\left(a^{2}-1\right)\right],
$$

ce qui montre que $A$ et $B$ impliquent les ondes $S$ et $D$ alors que $C$ n'implique que l'onde $P$.

\section{Amplitudes d'hélicité et parité $C P$}

L'hélicité étant une quantité pseudo-scalaire, elle change de signe sous l'action de l'opérateur parité :

$$
\mathcal{P}\left|J, M, \lambda_{1}, \lambda_{2}\right\rangle=\eta_{1} \eta_{2}(-1)^{J-s_{1}-s_{2}}\left|J, M,-\lambda_{1},-\lambda_{2}\right\rangle .
$$

Cela montre que les états $\left|J, M, \lambda_{1}, \lambda_{2}\right\rangle$ ne sont pas, en général, des états propres de l'opérateur parité. Cependant, les combinaisons linéaires suivantes sont états propres de l'opérateur parité :

$$
\mathcal{P} \frac{1}{\sqrt{2}}\left(\left|J, M, \lambda_{1}, \lambda_{2}\right\rangle \pm\left|J, M,-\lambda_{1},-\lambda_{2}\right\rangle\right)= \pm \eta_{1} \eta_{2}(-1)^{J-s_{1}-s_{2}} \frac{1}{\sqrt{2}}\left(\left|J, M, \lambda_{1}, \lambda_{2}\right\rangle \pm\left|J, M,-\lambda_{1},-\lambda_{2}\right\rangle\right) .
$$

On définit alors les amplitudes, dites de transversité, par :

$$
A_{\|} \equiv \frac{1}{\sqrt{2}}\left(H_{+1}+H_{-1}\right), \quad A_{0} \equiv H_{0}, \quad A_{\perp} \equiv \frac{1}{\sqrt{2}}\left(H_{+1}-H_{-1}\right) .
$$

Le méson $B$ n'étant pas un état propre de $C P$, on a en toute rigueur la même chose pour les amplitudes (cf. §1.2). Ainsi, par abus de langage, on dit, dans le cas d'un état final propre de $C P$ de parité $C P$ intrinsèque $\eta_{C P}(f)$, que les amplitudes d'hélicité $H_{ \pm 1}$ ne sont pas états propres de $C P$, alors que les amplitudes de transversité $\left(A_{0}, A_{\|}, A_{\perp}\right)$ sont des états propres de $C P$ de parité $C P, \eta_{C P}(f)$, $\eta_{C P}(f)$ et $-\eta_{C P}(f)$, respectivement.

\section{Amplitudes de transversité}

L'hélicité est la quantification du spin d'une particule le long de la direction de son impuslsion. Pour des mésons vecteurs massifs, cela correspond à des polarisations circulaires droite et gauche $(\lambda= \pm 1)$ et une polarisation longitudinale $(\lambda=0)$. La transversité est la quantification du spin d'une particule perpendiculairement à la direction de son impulsion [63, 131, 132], ce qui, dans le cas de mésons vecteurs, correspond à une polarisation linéaire[125, 127]. $A_{0}$ correspond à une 
polarisation longitudinale des deux mésons vecteurs, alors que $A_{\|}$et $A_{\perp}$ correspondent à des polarisations transverse parallèle et transverse perpendiculaire, respectivement [133]. En termes des invariants $A, B, C$ (cf. eq. (2.38)), on obtient

$$
A_{0}=-\left(a A+B\left(a^{2}-1\right)\right), \quad A_{\|}=\sqrt{2} A, \quad A_{\perp}=C \sqrt{2\left(a^{2}-1\right)} .
$$

Les amplitudes de transversité sont reliées aux amplitudes en ondes partielles par :

$$
A_{0}=-\sqrt{\frac{1}{3}} S+\sqrt{\frac{2}{3}} D, \quad A_{\|}=\sqrt{\frac{2}{3}} S+\sqrt{\frac{1}{3}} D, \quad A_{\perp}=P .
$$

\subsubsection{Les canaux $J / \psi K^{*}(892)$ et $\psi(2 S) K^{*}(892)$}

\section{Distribution angulaire en base d'hélicité}

On considère la désintégration d'un méson $B$ en $J / \psi K^{*}(892)$ ou $\psi(2 S) K^{*}(892)$. Le méson charmonium $\psi=J / \psi$ ou $\psi(2 S)$ se désintègre en paire de leptons $\ell^{+} \ell^{-}$avec $\ell=e$ ou $\mu$ et le $K^{*}(892)$ en $K \pi$. Cette désintégration étant une chaîne de désintégrations séquentielles indépendantes, 1 'amplitude totale est le produit des amplitudes de chaque désintégration :

$$
\begin{aligned}
A\left(\lambda_{\ell^{+}}, \lambda_{\ell^{-}}\right)=\sum_{\lambda_{\psi}, \lambda_{K^{*}}}\left\langle\theta_{K^{*}}, \varphi_{K^{*}}, \lambda_{K}, \lambda_{\pi}\left|\mathcal{T}_{K^{*}}\right| 1, m_{K^{*}}=-\lambda_{K^{*}}\right\rangle \\
\quad \otimes\left\langle\theta_{\psi}, \varphi_{\psi}, \lambda_{\ell^{+}}, \lambda_{\ell^{-}}\left|\mathcal{T}_{\psi}\right| 1, m_{\psi}=\lambda_{\psi}\right\rangle \otimes\left\langle\theta_{B}, \varphi_{B}, \lambda_{\psi}, \lambda_{K^{*}}\left|\mathcal{T}_{B}\right| 0,0\right\rangle .
\end{aligned}
$$

Les kets sont des états canoniques $|j, m\rangle$ et les bras sont des états d'hélicité $\left\langle\theta, \varphi, \lambda_{1}, \lambda_{2}\right|$ (voir appendice $\mathrm{A})$. Cela signifie que chaque désintégration est évaluée dans le repère de la particule mère au repos, impliquant que les angles $\theta$ et $\varphi$ décrivant la désintégration à deux corps $\left\langle\theta, \varphi, \lambda_{1}, \lambda_{2}|\mathcal{T}| j, m\right\rangle$ sont évalués dans le repère au repos de leur mère. En général, les quadrivecteurs des particules sont donnés dans le référentiel du laboratoire, il convient donc de «booster » d'abord ces particules dans le repère du méson $B$, puis dans le repère de leur mère respective. À noter que cette approche est non covariante [134].

Analysons chaque désintégration séquentielle (on utilise la convention de Jackson pour les opérateurs de rotations finies $U\left[\mathcal{R}_{a}(\varphi, \theta, 0)\right]$ et on définit l'état d'hélicité $\left|-\vec{p}, \lambda_{2}\right\rangle$ de la deuxième fille dans le repère au repos de sa mère par une rotation autour de l'axe $x$ (voir appendice $\mathrm{A})$ ) :

1. $B \rightarrow \psi K^{*}(892):$

$$
\left\langle\theta_{B}, \varphi_{B}, \lambda_{\psi}, \lambda_{K^{*}}\left|\mathcal{T}_{B}\right| 0,0\right\rangle=\sqrt{\frac{1}{4 \pi}} D_{0, \lambda_{\psi}-\lambda_{K^{*}}}^{0 *}\left(\varphi_{B}, \theta_{B}, 0\right) H_{\lambda_{\psi}, \lambda_{K^{*}}}^{B}=\sqrt{\frac{1}{4 \pi}} D_{0,0}^{0 *}\left(\varphi_{B}, \theta_{B}, 0\right) H_{\lambda},
$$

où $D_{0,0}^{0 *}\left(\varphi_{B}, \theta_{B}, 0\right)=1$ pour tout $\left(\theta_{B}, \varphi_{B}\right)$, traduisant l'isotropie de la désintégration du méson $B$ et $\lambda_{\psi}=\lambda_{K^{*}}=\lambda$. L'axe $z$ du repère associé au méson $B$ au repos est un axe arbitraire de quantification du spin du méson $B$. L'axe $z_{\psi}$ du repère associé au méson $\psi$ au repos n'est pas arbitraire : c'est la direction de l'impulsion du $\psi$ dans le repère du méson $B$ au repos, impliquant que la projection du spin du $\psi$ sur l'axe $z_{\psi}$ vérifie $m_{\psi}=\lambda$ (voir Fig. 2.4). De plus, puisque $\vec{p}_{K^{*}}=-\vec{p}_{\psi}$ dans le repère du méson $B$ au repos, on a pour la projection $\mathrm{du}$ spin $\mathrm{du}$ 


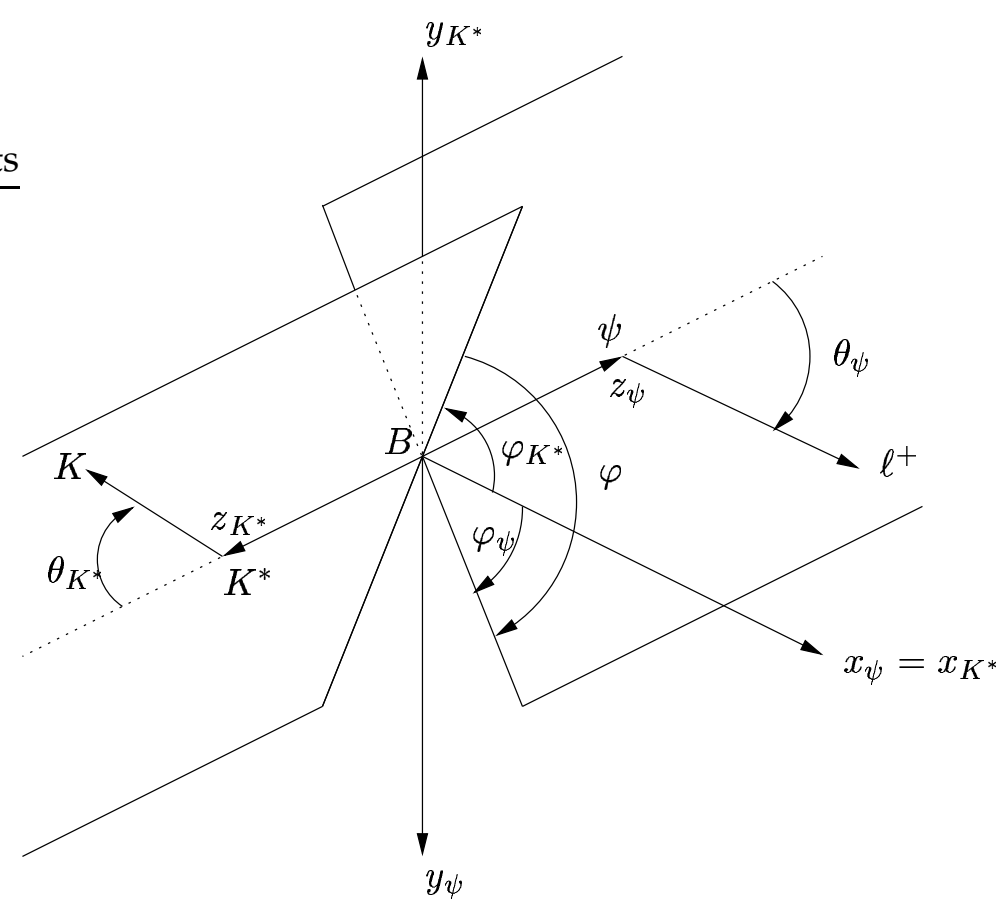

FIG. 2.4: Définition des plans de désintégration et des différents repères pour la dérivation de la distribution angulaire $B \rightarrow \psi\left(\rightarrow \ell^{+} \ell^{-}\right) K^{*}(\rightarrow K \pi)$. La convention choisie pour définir l'état d'hélicité $\left|\vec{p}_{K^{*}}, \lambda_{K^{*}}\right\rangle$ est une rotation autour de l'axe $x$ (voir appendice A), impliquant $x_{\psi}=x_{K^{*}}$. À noter que $\varphi=\varphi_{\psi}-\varphi_{K^{*}}$. Les angles $\theta_{\psi}$ et $\theta_{K^{*}}$ sont évalués dans les repères au repos des mésons $\psi$ et $K^{*}$, respectivement.

$K^{*}$ sur l'axe $z_{\psi}, m_{K^{*}}=-\lambda$.

2. $\psi \rightarrow \ell^{+} \ell^{-}$:

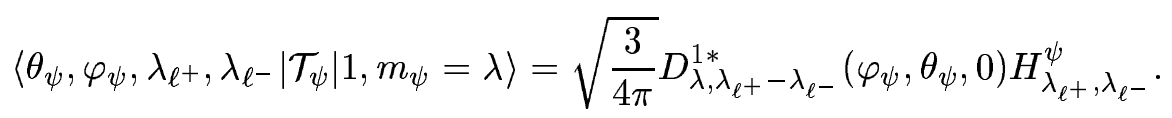

On se place dans la limite des leptons de masse nulle, soit $\alpha \equiv \lambda_{\ell^{+}}-\lambda_{\ell^{-}}= \pm 1$. Les angles $\left(\theta_{\psi}, \varphi_{\psi}\right)$ sont définis par la direction du lepton positif $\ell^{+}$dans le repère du $\psi$ au repos. La désintégration du méson $\psi$ en deux leptons étant un processus électromagnétique, la parité est conservée ce qui implique $H_{\frac{1}{2},-\frac{1}{2}}^{\psi}=H_{-\frac{1}{2}, \frac{1}{2}}^{\psi}$.

3. $K^{*} \rightarrow K \pi$ :

$$
\left\langle\theta_{K^{*}}, \varphi_{K^{*}}, \lambda_{K}, \lambda_{\pi}\left|\mathcal{T}_{K^{*}}\right| 1, m_{K^{*}}=-\lambda\right\rangle=\sqrt{\frac{3}{4 \pi}} D_{-\lambda, \lambda_{K}-\lambda_{\pi}}^{1 *}\left(\varphi_{K^{*}}, \theta_{K^{*}}, 0\right) H_{\lambda_{K}, \lambda_{\pi}}^{K^{*}}
$$

avec $\lambda_{K}-\lambda_{\pi}=0$. Les angles $\left(\theta_{K^{*}}, \varphi_{K^{*}}\right)$ sont définis par la direction du kaon dans le repère du $K^{*}$ au repos.

La distribution angulaire dépend des angles polaires $\theta_{\psi}, \theta_{K^{*}}$ et de l'angle azimutal $\varphi=\varphi_{\psi}-\varphi_{K^{*}}$ défini ${ }^{12}$ comme l'angle entre les deux plans des produits de désintégration du $\psi$ et du $K^{*}$, en allant

\footnotetext{
${ }^{12} \varphi=\varphi_{\psi}-\varphi_{K^{*}}$ provient du fait que l'on a choisi l'axe $z$ positif pour décrire la désintégration des filles du méson $B$ dans son repère au repos par la direction du $\psi$, de sorte que $\vec{p}_{\psi}=|\vec{p}| \hat{z}$ et $\vec{p}_{K^{*}}=-|\vec{p}| \hat{z}$. Cela a pour conséquence $m_{\psi}=+\lambda$ et $m_{K^{*}}=-\lambda$. Si on avait choisi le contraire, à savoir $\vec{p}_{\psi}=-|\vec{p}| \hat{z}$ et $\vec{p}_{K^{*}}=|\vec{p}| \hat{z}$, de sorte que $m_{\psi}=-\lambda$ et $m_{K^{*}}=\lambda$, l'angle azimutal $\varphi$ serait alors égal à $\varphi_{K^{*}}-\varphi_{\psi}$, défini comme l'angle entre les deux plans des produits de désintégration du $\psi$ et du $K^{*}$, en allant du lepton positif vers le kaon avec une rotation positive autour de l'axe $z_{K^{*}}$.
} 
du kaon vers le lepton positif avec une rotation positive autour de l'axe ${ }^{13} z_{\psi}$ (voir Fig. 2.4) :

$$
\sin (\varphi)=\left(\hat{p}_{\psi} \times \hat{c}\right) \cdot \hat{d}, \quad \cos (\varphi)=\hat{c} . \hat{d}
$$

où les vecteurs unités $\hat{c}$ et $\hat{d}$ sont définis par :

$$
\hat{c}=\left(\hat{p}_{K^{*}} \times \hat{p}_{K}\right) \times \hat{p}_{K^{*}}, \quad \hat{d}=\left(\hat{p}_{\psi} \times \hat{p}_{\ell^{+}}\right) \times \hat{p}_{\psi} .
$$

Les impulsions de chaque particule sont mesurées dans le repère au repos de leur mère respective. À noter que dans le cas particulier où $\varphi_{K^{*}}=0\left(\varphi_{\psi}=0\right)$, l'angle $\varphi$ devient simplement l'angle azimutal du lepton positif $\ell^{+}$, i.e. $\varphi_{\psi}$ (l'opposé de l'angle azimutal du kaon, i.e. $\left.-\varphi_{K^{*}}\right)$.

En résumé, l'amplitude $A\left(B \rightarrow \psi\left(\rightarrow \ell^{+} \ell^{-}\right) K^{*}(\rightarrow K \pi)\right)$ se réduit à

$$
\begin{aligned}
A(\alpha) & =\sum_{\lambda=0, \pm 1}\left\langle\theta_{K^{*}}, \varphi_{K^{*}}, \lambda_{K}, \lambda_{\pi}\left|\mathcal{T}_{K^{*}}\right| 1,-\lambda\right\rangle\left\langle\theta_{\psi}, \varphi_{\psi}, \lambda_{\ell^{+}}, \lambda_{\ell^{-}}\left|\mathcal{T}_{\psi}\right| 1, \lambda\right\rangle H_{\lambda} \\
& \propto \sum_{\lambda=0, \pm 1} D_{-\lambda, 0}^{1 *}\left(\varphi_{K^{*}}, \theta_{K^{*}}, 0\right) D_{\lambda, \alpha}^{1 *}\left(\varphi_{\psi}, \theta_{\psi}, 0\right) H_{\lambda}
\end{aligned}
$$

où on somme sur toutes les hélicités autorisées des particules intermédiaires $\psi$ et $K^{*}$ puisqu'elles ne peuvent être mesurées. La distribution angulaire non-polarisée est définie par :

$$
\mathrm{d} \Gamma=\frac{(2 \pi)^{4}}{2 m_{B}} \sum_{\alpha}|A(\alpha)|^{2} \mathrm{~d} \phi_{4}\left(p_{B} ; p_{\ell^{+}}, p_{\ell^{-}}, p_{K}, p_{\pi}\right),
$$

où $\mathrm{d} \phi_{4}\left(p_{B} ; p_{\ell^{+}}, p_{\ell^{-}}, p_{K}, p_{\pi}\right)$ est l'espace des phases à quatre corps. Il est donné en fonction des espaces des phases à deux corps de chaque désintégration par :

$$
\mathrm{d} \phi_{4}\left(p_{B} ; p_{\ell^{+}}, p_{\ell^{-}}, p_{K}, p_{\pi}\right)=(2 \pi)^{6} \mathrm{~d} \phi_{2}\left(p_{B} ; p_{\psi}, p_{K^{*}}\right) \mathrm{d} \phi_{2}\left(p_{\psi} ; p_{\ell^{+}}, p_{\ell^{-}}\right) \mathrm{d} \phi_{2}\left(p_{K^{*}} ; p_{K}, p_{\pi}\right) \mathrm{d} p_{\psi}^{2} \mathrm{~d} p_{K^{*}}^{2} .
$$

On obtient $[123]^{14}$

$$
\frac{1}{\Gamma} \frac{\left.\mathrm{d}^{3} \Gamma \stackrel{(-)}{B} \rightarrow \psi\left(\rightarrow \ell^{+} \ell^{-}\right) \stackrel{(-)}{K^{*}}(\rightarrow K \pi)\right)}{\mathrm{d} \cos \theta_{K^{*}} \mathrm{~d} \cos \theta_{\psi} \mathrm{d} \varphi}=\frac{1}{\left|\stackrel{(-)}{H_{+1}}\right|^{2}+\left|\stackrel{(-)}{H_{0}}\right|^{2}+\left|\stackrel{(-)}{H_{-1}}\right|^{2}} \sum_{i=1}^{7} \stackrel{(-)}{X_{i}} h_{i}\left(\theta_{K^{*}}, \theta_{\psi}, \varphi\right),
$$

où les expressions de $\stackrel{(-)}{X_{i}}$ et $h_{i}\left(\theta_{K^{*}}, \theta_{\psi}, \varphi\right)$ sont données dans la table 2.1. La distribution angulaire est normalisée par construction. À noter que l'invariance sous la symétrie $C P T$ implique que $\left|H_{1}\right|^{2}+$ $\left|H_{0}\right|^{2}+\left|H_{-1}\right|^{2}=\left|\bar{H}_{1}\right|^{2}+\left|\bar{H}_{0}\right|^{2}+\left|\bar{H}_{-1}\right|^{2}$ uniquement en l'absence de FSI.

\section{Distribution angulaire en base de transversité}

Considérons la désintégration d'un méson $B$ en deux particules instables $A$ et $C$, de spins $s_{a}$, $s_{c}$, d'hélicités $\lambda_{a}, \lambda_{c}$ et de parités intrinsèques $\pi_{a}$ et $\pi_{c}$ ( $A$ et $C$ sont instables pour que l'on puisse

\footnotetext{
${ }^{13}$ On lit souvent dans la littérature que $\varphi$ est l'angle entre les deux plans de désintégration. Cette définition est ambiguë et conduit à quatre angles possibles : $\varphi, \pi-\varphi,-\varphi$ et $\varphi-\pi$. Le formalisme d'hélicité définit cet angle de manière unique.

${ }^{14}$ Attention aux erreurs de signe dans cette référence.
} 
ТАВ. 2.1: Observables et fonctions angulaires de la distribution angulaire $\stackrel{(-)}{B} \rightarrow \psi\left(\rightarrow \ell^{+} \ell^{-}\right) \stackrel{(-)}{K^{*}}(\rightarrow K \pi)$ dans la base d'hélicité pour un méson $B$ initialement $\stackrel{(-)}{B}$.

\begin{tabular}{|c|c|c|}
\hline$i$ & $\begin{array}{l}(-) \\
X_{i}\end{array}$ & $h_{i}\left(\theta_{K^{*}}, \theta_{\psi}, \varphi\right)$ \\
\hline 1 & $\left|\stackrel{(-)}{H_{+1}}\right|^{2}$ & $\frac{9}{16 \pi} \frac{1}{4} \sin ^{2}\left(\theta_{K^{*}}\right)\left(1+\cos ^{2}\left(\theta_{\psi}\right)\right)$ \\
\hline 2 & $\left|\stackrel{(-)}{H_{-1}}\right|^{2}$ & $\frac{9}{16 \pi} \frac{1}{4} \sin ^{2}\left(\theta_{K^{*}}\right)\left(1+\cos ^{2}\left(\theta_{\psi}\right)\right)$ \\
\hline 3 & $\left|\stackrel{(-)}{H_{0}}\right|^{2}$ & $\frac{9}{16 \pi} \cos ^{2}\left(\theta_{K^{*}}\right) \sin ^{2}\left(\theta_{\psi}\right)$ \\
\hline 4 & $\begin{array}{c}(-)(-) \\
\Re e\left(H_{+1} H_{-1}^{*}\right)\end{array}$ & $-\frac{9}{16 \pi} \frac{1}{2} \sin ^{2}\left(\theta_{K^{*}}\right) \sin ^{2}\left(\theta_{\psi}\right) \cos (2 \varphi)$ \\
\hline 5 & $\Im m\left(\stackrel{(-)}{\left(H_{+1}\right.} H_{-1}^{*}\right)$ & $\frac{9}{16 \pi} \frac{1}{2} \sin ^{2}\left(\theta_{K^{*}}\right) \sin ^{2}\left(\theta_{\psi}\right) \sin (2 \varphi)$ \\
\hline 6 & $\Re e\left(\left(\stackrel{(-)}{H_{+1}}+\stackrel{(-)}{H_{-1}}\right) \stackrel{(-)}{H_{0}^{*}}\right)$ & $\frac{9}{16 \pi} \frac{1}{4} \sin \left(2 \theta_{K^{*}}\right) \sin \left(2 \theta_{\psi}\right) \cos (\varphi)$ \\
\hline 7 & $\Im m\left(\left(\stackrel{(-)}{H_{+1}}-\stackrel{(-)}{H_{-1}}\right) \stackrel{(-)}{H_{0}^{*}}\right)$ & $-\frac{9}{16 \pi} \frac{1}{4} \sin \left(2 \theta_{K^{*}}\right) \sin \left(2 \theta_{\psi}\right) \sin (\varphi)$ \\
\hline
\end{tabular}

obtenir des informations sur leur spin depuis leurs produits de désintégration). La particule $C$ se désintègre en $C_{1} C_{2}$ de spins $s_{1}$ et $s_{2}$. On définit l'axe transverse comme la normale au plan contenant les trois particules $A, C_{1}, C_{2}$. Les états propres de $C P$ du mode $A C_{1} C_{2}$ peuvent être classés selon la projection du spin des particules sur cet axe transverse (transversité). L'état de transversité $\tau_{i}$ de chaque particule $i$ est défini comme une combinaison linéaire d'états d'hélicité ce qui représente une composante du spin le long de l'axe transverse dans le repère où la particule $i$ est au repos. La transversité $\tau$ est définie comme la somme des transversités $\tau_{i}$ de chaque particule, $\tau=\tau(A)+\tau\left(C_{1}\right)+\tau\left(C_{2}\right)$. La parité $C P$ du système peut être écrite comme une fonction de $\tau$, en considérant $A, C_{1}$ et $C_{2}$ états propres de $C P[63,135]$ :

$$
\mathcal{C P}\left|A C_{1} C_{2}\right\rangle=\eta_{C P}(A) \eta_{C P}\left(C_{1}\right) \eta_{C P}\left(C_{2}\right)(-1)^{\tau}\left|A C_{1} C_{2}\right\rangle=\eta_{C P}\left(A C_{1} C_{2}\right)\left|A C_{1} C_{2}\right\rangle,
$$

où $\eta_{C P}$ est la parité $C P$ intrinsèque de la particule.

Dans le cas de la désintégration $B_{d}^{0} \rightarrow \psi\left(K_{S}^{0} \pi^{0}\right)^{* 0}, A=\psi, C_{1}=K_{S}^{0}$ et $C_{2}=\pi^{0}$. Comme $C_{1}$ et $C_{2}$ sont de spin 0, la transversité de $A C_{1} C_{2}$ devient la transversité de $A$. Étant donné que $\eta_{C P}(A)$, $\eta_{C P}\left(C_{1}\right)$ et $\eta_{C P}\left(C_{2}\right)$ valent $+1,+1$ et -1 respectivement, on a

$$
\eta_{C P}\left(A C_{1} C_{2}\right)=(-1)^{\tau(A)+1}
$$

La parité $C P$ de $A C_{1} C_{2}$ est entièrement déterminée par la transversité de $A$.

Pour obtenir la distribution angulaire en transversité à partir de celle obtenue en hélicité, il est nécessaire d'effectuer les étapes suivantes : 


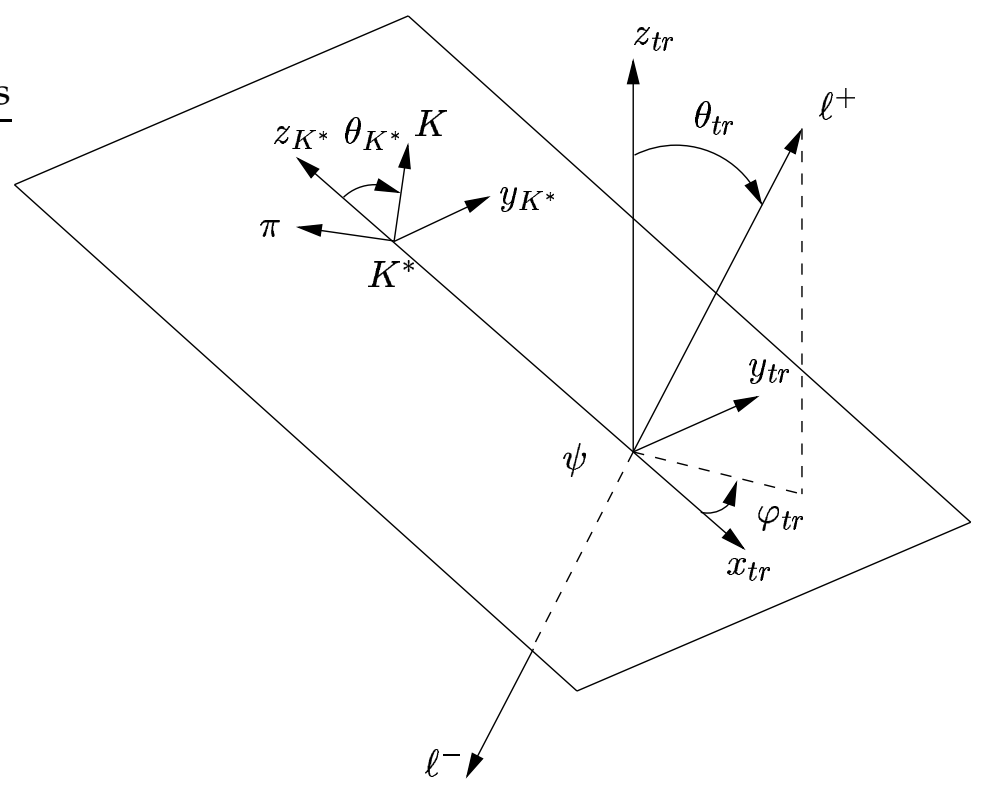

FIG. 2.5: Définition du plan et des angles de transversité. Le repère de transversité est défini dans le repère du $\psi$ au repos. La direction opposée à l'impulsion du $K^{*}$ définit l'axe $x_{t r}$. Le plan de désintégration du $K^{*}$ en $K \pi$ définit le plan de transversité $\left(x_{t r}, y_{t r}\right)$, où l'axe $y_{t r}$ est orienté de sorte que $p_{y}(K)>0$. L'axe $z_{t r}$ est perpendiculaire au plan de transversité afin de former un trièdre direct. Les angles $\left(\theta_{t r}, \varphi_{t r}\right)$ décrivent la direction du lepton positif provenant de la désintégration du $\psi$ dans le repère de transversité. $\theta_{K^{*}}$ est l'angle entre la direction du kaon dans le repère du $K^{*}$ au repos et la direction opposée de l'axe $x_{t r}$.

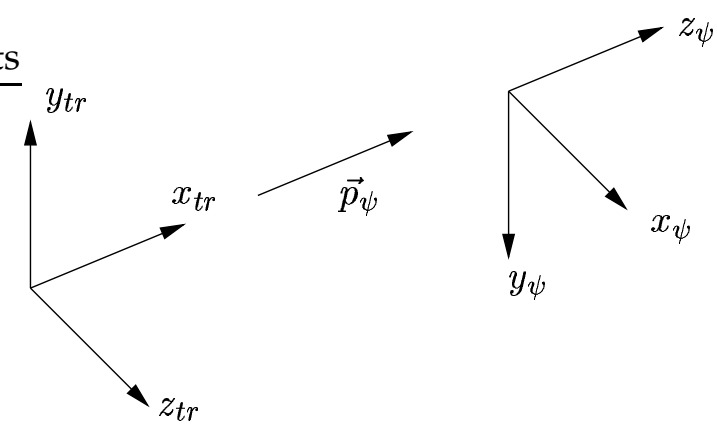

FIG. 2.6: Passage du repère d'hélicité au repère de transversité pour le méson $\psi$ : le repère de transversité se déduit du repère d'hélicité par une rotation passive $R_{p}(0, \pi / 2, \pi)$.

- changer les amplitudes d'hélicité en amplitudes de transversité ${ }^{15}$, du fait du nouvel axe de quantification du $\psi$ :

$$
A_{\|}=\frac{1}{\sqrt{2}}\left(H_{+1}+H_{-1}\right), \quad A_{0}=H_{0}, \quad A_{\perp}=\frac{1}{\sqrt{2}}\left(H_{+1}-H_{-1}\right)
$$

- changer les repères (cf. Fig. 2.5) : en comparant aux repères définis pour la distribution en hélicité, celui du $K^{*}$ reste inchangé, sauf que ces produits de désintégration sont contraints dans le plan $\left(y_{K^{*}}, z_{K^{*}}\right)$, avec $p_{y}(K)>0$ sur l'axe $y_{K^{*}}$. On a donc pour chaque événement $\varphi_{K^{*}}=\pi / 2$. L'axe $x_{t r}$ est défini par l'impulsion du $\psi$ dans le repère du méson $B$ au repos. L'axe $y_{t r}$ est défini par l'axe $y_{K^{*}}$. Enfin, l'axe $z_{t r}$ est perpendiculaire au plan de désintégration

\footnotetext{
${ }^{15}$ La définition utilisée dans CLEO CONF 96-24 [124] pour l'angle entre les deux plans de désintégration n'est pas celle donnée par le formalisme d'hélicité (voir la note en bas de page 12) mais $\chi=\pi-\varphi$. Pour obtenir la distribution en transversité donnée dans la référence [125] $A_{0}$ doit être défini égal à $-H_{0}$ [126], alors que la référence [125] définit $A_{0}=H_{0}$. Ainsi, on peut lire dans la littérature « we follow the (sign) convention of [125]» [97].
} 
du $K^{*}$ en $K \pi$, pour donner un repère direct. Les angles $\left(\theta_{t r}, \varphi_{t r}\right)$ décrivent la direction du lepton positif $\ell^{+}$dans le repère de transversité du $\psi$ au repos. Ainsi, le repère de transversité $\mathrm{du} \psi$ est obtenu à partir de celui en hélicité par, tout d'abord, une rotation passive de $\pi$ autour de l'axe $z_{\psi}$ suivie d'une rotation passive de $\pi / 2$ autour de l'axe $y_{\psi}$, soit $R_{p}(0, \pi / 2, \pi)$ (voir Fig. 2.6). On obtient

$$
x_{\psi}=z_{t r}, \quad y_{\psi}=-y_{t r}, \quad z_{\psi}=x_{t r} .
$$

La direction du lepton positif $\ell^{+}$dans le repère d'hélicité du $\psi$ au repos est :

$$
\left(\hat{p}_{x_{\psi}}, \hat{p}_{y_{\psi}}, \hat{p}_{z_{\psi}}\right)=\left(\sin \left(\theta_{\psi}\right) \cos \left(\varphi_{\psi}\right), \sin \left(\theta_{\psi}\right) \sin \left(\varphi_{\psi}\right), \cos \left(\theta_{\psi}\right)\right)
$$

et dans le repère de transversité du $\psi$ au repos est :

$$
\left(\hat{p}_{x_{t r}}, \hat{p}_{y_{t r}}, \hat{p}_{z_{t r}}\right)=\left(\sin \left(\theta_{t r}\right) \cos \left(\varphi_{t r}\right), \sin \left(\theta_{t r}\right) \sin \left(\varphi_{t r}\right), \cos \left(\theta_{t r}\right)\right)
$$

Ainsi, la transformation entre les deux repères est :

$$
\begin{aligned}
\sin \left(\theta_{\psi}\right) \cos \left(\varphi_{\psi}\right) & =\cos \left(\theta_{t r}\right) \\
\sin \left(\theta_{\psi}\right) \sin \left(\varphi_{\psi}\right) & =-\sin \left(\theta_{t r}\right) \sin \left(\varphi_{t r}\right), \\
\cos \left(\theta_{\psi}\right) & =\sin \left(\theta_{t r}\right) \cos \left(\varphi_{t r}\right) .
\end{aligned}
$$

En termes de l'angle $\varphi=\varphi_{\psi}-\varphi_{K^{*}}=\varphi_{\psi}-\pi / 2$, on obtient:

$$
\begin{aligned}
\sin \left(\theta_{\psi}\right) \sin (\varphi) & =-\cos \left(\theta_{t r}\right) \\
\sin \left(\theta_{\psi}\right) \cos (\varphi) & =-\sin \left(\theta_{t r}\right) \sin \left(\varphi_{t r}\right) \\
\cos \left(\theta_{\psi}\right) & =\sin \left(\theta_{t r}\right) \cos \left(\varphi_{t r}\right)
\end{aligned}
$$

À noter que l'on peut adopter une autre approche pour obtenir les transformations ci-dessus. En effet, l'état de transversité du $\psi$ est relié à son état d'hélicité par ${ }^{16}$ :

$$
\left|s_{\psi}=1, \lambda\right\rangle=\sum_{\tau} D_{\tau, \lambda}^{1}\left(-\pi,-\frac{\pi}{2}, 0\right)\left|s_{\psi}=1, \tau\right\rangle=\sum_{\tau}(-1)^{\tau} d_{\tau, \lambda}^{1}\left(-\frac{\pi}{2}\right)\left|s_{\psi}=1, \tau\right\rangle
$$

L'amplitude totale en transversité s'écrit alors

$$
\begin{aligned}
A(\alpha) & =\sum_{\lambda, \tau}\left\langle\theta_{K^{*}}, \pi / 2, \lambda_{K}, \lambda_{\pi}\left|\mathcal{T}_{K^{*}}\right| 1,-\lambda\right\rangle\left\langle\theta_{t r}, \varphi_{t r}, \lambda_{\ell^{+}}, \lambda_{\ell^{-}}\left|\mathcal{T}_{\psi}\right| 1, \tau\right\rangle\langle 1, \tau \mid 1, \lambda\rangle H_{\lambda} \\
& =\sum_{\tau=0, \pm 1} D_{\tau, \alpha}^{1 *}\left(\varphi_{t r}, \theta_{t r}, 0\right)\left[\sum_{\lambda=0, \pm 1} D_{-\lambda, 0}^{1 *}\left(\frac{\pi}{2}, \theta_{K^{*}}, 0\right) D_{\tau, \lambda}^{1}\left(-\pi,-\frac{\pi}{2}, 0\right) H_{\lambda}\right] .
\end{aligned}
$$

\footnotetext{
${ }^{16}$ Une remarque importante : la rotation qui fait passer le repère d'hélicité au repère de transversité est une rotation passive (description du même système physique dans deux repères différents, l'un étant déduit de l'autre par une rotation) $R_{p}(0, \pi / 2, \pi)$, illustrée par la figure 2.6. Les fonctions $D$ données dans le PDG [28] sont obtenues à partir de rotation active, la relation entre les deux types de rotation étant $R_{p}(\alpha, \beta, \gamma)=R_{a}^{-1}(\alpha, \beta, \gamma)=R_{a}(-\gamma,-\beta,-\alpha)$ (voir appendice A). On a donc $R_{p}(0, \pi / 2, \pi)=R_{a}(-\pi,-\pi / 2,0)$.
} 
TAB. 2.2: Observables et fonctions angulaires de la distribution angulaire $\stackrel{(-)}{B} \rightarrow \psi\left(\rightarrow \ell^{+} \ell^{-}\right) \stackrel{(-)}{K^{*}}(\rightarrow K \pi)$ dans la base de transversité pour un méson $B$ initialement $\stackrel{(-)}{B}$.

\begin{tabular}{|c||c|c|}
\hline$i$ & $\stackrel{(-)}{K_{i}}$ & $f_{i}\left(\theta_{K^{*}}, \theta_{t r}, \varphi_{t r}\right)$ \\
\hline \hline 1 & $\left|\stackrel{(-)}{A_{0}}\right|^{2}$ & $\frac{9}{32 \pi} 2 \cos ^{2}\left(\theta_{K^{*}}\right)\left[1-\sin ^{2}\left(\theta_{t r}\right) \cos ^{2}\left(\varphi_{t r}\right)\right]$ \\
\hline 2 & $\left|\stackrel{(-)}{A_{\|}}\right|^{2}$ & $\frac{9}{32 \pi} \sin ^{2}\left(\theta_{K^{*}}\right)\left[1-\sin ^{2}\left(\theta_{t r}\right) \sin ^{2}\left(\varphi_{t r}\right)\right]$ \\
\hline 3 & $\left|\stackrel{(-)}{A_{\perp}}\right|^{2}$ & $\frac{9}{32 \pi} \sin ^{2}\left(\theta_{K^{*}}\right) \sin ^{2}\left(\theta_{t r}\right)$ \\
\hline 4 & $\Im m\left(A_{\|}^{*} A_{\perp}\right)$ & $\frac{9}{32 \pi} \sin ^{2}\left(\theta_{K^{*}}\right) \sin \left(2 \theta_{t r}\right) \sin \left(\varphi_{t r}\right)$ \\
\hline 5 & $\Re e\left(\stackrel{(-)(-)}{\left.A_{\|} A_{0}^{*}\right)}\right.$ & $-\frac{9}{32 \pi} \frac{1}{\sqrt{2}} \sin \left(2 \theta_{K^{*}}\right) \sin { }^{2}\left(\theta_{t r}\right) \sin \left(2 \varphi_{t r}\right)$ \\
\hline 6 & $\Im m\left(A_{\perp} A_{0}^{*}\right)$ & $\frac{9}{32 \pi} \frac{1}{\sqrt{2}} \sin \left(2 \theta_{K^{*}}\right) \sin \left(2 \theta_{t r}\right) \cos \left(\varphi_{t r}\right)$ \\
\hline \hline
\end{tabular}

Les éléments de matrice $D_{\tau, \lambda}^{1}(-\pi,-\pi / 2,0)$ prennent les valeurs :

\begin{tabular}{|l|c|ccc|}
\hline & $\lambda$ & +1 & 0 & -1 \\
\hline+1 & & & & \\
\hline 0 & & $-1 / 2$ & $-1 / \sqrt{2}$ & $-1 / 2$ \\
-1 & & $-1 / \sqrt{2}$ & 0 & $1 / \sqrt{2}$ \\
& & $-1 / 2$ & $1 / \sqrt{2}$ & $-1 / 2$ \\
\hline
\end{tabular}

où l'on voit apparaître les combinaisons d'amplitudes d'hélicité qui donnent les amplitudes de transversité.

On obtient alors pour la distribution angulaire en transversité [125, 127, 128] :

$$
\frac{1}{\Gamma} \frac{\left.\mathrm{d}^{3} \Gamma \stackrel{(-)}{B} \rightarrow \psi\left(\rightarrow \ell^{+} \ell^{-}\right) \stackrel{(-)}{K^{*}}(\rightarrow K \pi)\right)}{\mathrm{d} \cos \theta_{K^{*}} \mathrm{~d} \cos \theta_{t r} \mathrm{~d} \varphi_{t r}}=\frac{1}{\left|\stackrel{(-)}{A_{\|}}\right|^{2}+\left|\stackrel{(-)}{A_{\perp}}\right|^{2}+\left|\stackrel{(-)}{A_{0}}\right|^{2}} \sum_{i=1}^{6} \stackrel{(-)}{K_{i}} f_{i}\left(\theta_{K^{*}}, \theta_{t r}, \varphi_{t r}\right),
$$

où les expressions de $\stackrel{(-)}{K_{i}}$ et $f_{i}\left(\theta_{K^{*}}, \theta_{t r}, \varphi_{t r}\right)$ sont données dans la table 2.2. La distribution angulaire est normalisée par construction.

Remarquons qu'en général il y a neuf observables dans la désintégration d'un méson $B$ en deux mésons vecteurs. Seules six de ces observables peuvent être mesurées via les distributions angulaires des produits de désintégration de ces mésons vecteurs du fait de la conservation de la parité pour ces désintégrations. Les trois observables restantes nécessitent de mesurer la polarisation d'un des produits de désintégration [127], ce qui n'est pas envisageable expérimentalement à présent. Les phases fortes $\left(\delta_{0}, \delta_{\|}, \delta_{\perp}\right)$ étant mesurées avec les termes d'interférences $\Im m\left(A_{\|}^{*} A_{\perp}\right)$, $\Re e\left(A_{\|} A_{0}^{*}\right)$ et $\Im m\left(A_{\perp} A_{0}^{*}\right)$, il existe l'ambiguïté, en prenant $\delta_{0}=0,\left(\delta_{\|}, \delta_{\perp}\right) \rightarrow\left(-\delta_{\|}, \pi-\delta_{\perp}\right)$. 


\section{Distributions angulaires réduites}

En intégrant sur les différentes paires d'angles, on obtient les distributions angulaires réduites :

$$
\begin{aligned}
\frac{1}{\Gamma} \frac{\mathrm{d} \Gamma}{\mathrm{d} \cos \theta_{t r}} & =\frac{3}{8}\left[\left(1+\cos ^{2}\left(\theta_{t r}\right)\right)+R_{\perp}\left(1-3 \cos ^{2}\left(\theta_{t r}\right)\right)\right] \\
\frac{1}{\Gamma} \frac{\mathrm{d} \Gamma}{\mathrm{d} \cos \theta_{K^{*}}} & =\frac{3}{4}\left[\left(1-\cos ^{2}\left(\theta_{K^{*}}\right)\right)+R_{0}\left(3 \cos ^{2}\left(\theta_{K^{*}}\right)-1\right)\right], \\
\frac{1}{\Gamma} \frac{\mathrm{d} \Gamma}{\mathrm{d} \varphi_{t r}} & =\frac{1}{4 \pi}\left[2+\left(R_{\|}-R_{0}\right) \cos \left(2 \varphi_{t r}\right)\right],
\end{aligned}
$$

où les paramètres $R_{\perp}, R_{0}$ et $R_{\|}$représentent les fractions de polarisation définies par :

$$
R_{(\perp, 0, \|)}=\frac{\left|A_{(\perp, 0,||)}\right|^{2}}{\left|A_{\|}\right|^{2}+\left|A_{\perp}\right|^{2}+\left|A_{0}\right|^{2}}, \quad R_{\perp}+R_{0}+R_{\|}=1 .
$$

\section{Dépendance temporelle}

Le fait que le méson $B$ évolue dans le temps implique une dépendance temporelle des amplitudes ${ }^{17} X_{i} \rightarrow X_{i}(t)$ (eq. (2.61)) et $K_{i} \rightarrow K_{i}(t)$ (eq. (2.73)). On a donc factorisation entre la dépendance temporelle et la dépendance angulaire. Cette dépendance temporelle diffère suivant l'état final, c'est-à-dire suivant les désintégrations du $K^{*}[64,129]$ :

- désintégration dans un état propre de $C P: K^{* 0} \rightarrow K_{S}^{0} \pi^{0}$;

- désintégration dans un état spécifique de saveur : $K^{* 0} \rightarrow K^{+} \pi^{-}$;

- désintégration chargée : $K^{*+}$.

Le fait que la partie angulaire et la partie temporelle se factorisent implique que les désintégrations $B_{q} \rightarrow \psi K^{*}(892)$ ont la même distribution angulaire avec des dépendances temporelles différentes (cf. eq. (2.73)).

Commençons par analyser le mode propre de $C P$. Afin d'exprimer cette dépendance, il est pratique d'utiliser la base de transversité, puisque chaque amplitude contribue à un seul état propre de $C P$. En désignant par $\left|f_{\sigma}\right\rangle$ l'état $\left|\psi\left(K_{S}^{0} \pi^{0}\right)^{* 0}\right\rangle_{\sigma}$, on a $\mathcal{C P}\left|f_{\sigma}\right\rangle=\eta_{C P}\left(f_{\sigma}\right)\left|f_{\sigma}\right\rangle(\sigma=0, \|, \perp)$. Les valeurs propres de $C P, \eta_{C P}\left(f_{\sigma}\right)$, valent $\eta_{C P}\left(f_{0}\right)=\eta_{C P}\left(f_{\|}\right)=1$ et $\eta_{C P}\left(f_{\perp}\right)=-1$ puisque la parité $C P$ de l'état final $\left|\psi\left(K_{S}^{0} \pi^{0}\right)^{* 0}\right\rangle$ vaut $\eta_{C P}(f)(-1)^{L}$, où $L$ est le moment angulaire relatif entre $\psi$ et $K^{*}$ et $\eta_{C P}(f)$ est la parité $C P$ intrinsèque de l'état final $\left(\eta_{C P}(f)=+1\right.$ pour $\psi=J / \psi$ ou $\left.\psi(2 S)\right)$. En utilisant les expressions d'évolution temporelle des mésons $B_{d}^{0}$ (cf. eqs. (1.24) et (1.25)) et en se restreignant au cas $\Delta \Gamma \ll \Gamma_{0}$, de sorte que

$$
\begin{aligned}
& g_{+}(t)=e^{-\frac{1}{2} \Gamma_{0} t} e^{-i M_{0} t} \cos \left(\frac{\Delta M}{2} t\right) \\
& g_{-}(t)=e^{-\frac{1}{2} \Gamma_{0} t} e^{-i M_{0} t} i \sin \left(\frac{\Delta M}{2} t\right)
\end{aligned}
$$

\footnotetext{
${ }^{17}$ Seules les distributions angulaires et temporelles (ou intégrées en temps) sont à considérer. Les distributions « indépendantes du temps ", comme on le lit trop souvent dans la littérature, n'existent pas lorsque l'on traite des données. Cela a son importance, notamment dans le cas des états propres de $C P$.
} 
ТАВ. 2.3: Évolution temporelle des observables présentes dans la distribution angulaire en transversité de la désintégration $\stackrel{(-)}{B_{d}^{0}} \rightarrow \psi\left(\rightarrow \ell^{+} \ell^{-}\right) \stackrel{(-)}{K^{* 0}}\left(\rightarrow K_{S}^{0} \pi^{0}\right)$ pour un méson $B$ initialement $(i . e . t=0) \stackrel{(-)}{B_{d}^{0}}$ dans l'hypothèse de conservation de la symétrie $C P$ dans la désintégration. Les phases fortes $\delta_{1}$ et $\delta_{2}$ sont définies par $\delta_{1} \equiv \delta_{\perp}-\delta_{\|}$et $\delta_{2} \equiv \delta_{\perp}-\delta_{0}$. $\left|A_{\sigma}\right| \equiv\left|A_{\sigma}(0)\right|(\sigma=0, \|, \perp)$.

\begin{tabular}{|c|c|c|}
\hline $\mathrm{i}$ & $\stackrel{(-)}{K_{i}(t)}$ & évolution temporelle \\
\hline 1 & $\mid \stackrel{(-)}{\left.A_{0}(t)\right|^{2}}$ & $\left|A_{0}\right|^{2} e^{-\Gamma_{0} t}[1 \stackrel{(-)}{+} \sin (2 \beta) \sin (\Delta M t)]$ \\
\hline 2 & $\left|\stackrel{(-)}{A_{\|}}(t)\right|^{2}$ & $\left|A_{\|}\right|^{2} e^{-\Gamma_{0} t}[1 \stackrel{(-)}{+} \sin (2 \beta) \sin (\Delta M t)]$ \\
\hline 3 & $\left|\stackrel{(-)}{A_{\perp}}(t)\right|^{2}$ & 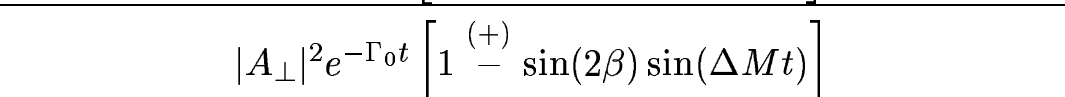 \\
\hline 4 & $\Im m\left({ }^{(-)}(t) \stackrel{(-)}{A} A_{\perp}(t)\right)$ & $\stackrel{(-)}{+}\left|A_{\|} \| A_{\perp}\right| e^{-\Gamma_{0} t}\left[\sin \left(\delta_{1}\right) \cos (\Delta M t)-\cos \left(\delta_{1}\right) \cos (2 \beta) \sin (\Delta M t)\right]$ \\
\hline 5 & $\Re e\left(\stackrel{(-)}{A_{\|}}(t) \stackrel{(-)}{A_{0}^{*}}(t)\right)$ & $\left|A_{\|}\right|\left|A_{0}\right| \cos \left(\delta_{2}-\delta_{1}\right) e^{-\Gamma_{0} t}[1 \stackrel{(-)}{+} \sin (2 \beta) \sin (\Delta M t)]$ \\
\hline 6 & $\Im m\left(\stackrel{(-)}{A_{\perp}}(t) \stackrel{(-)}{A_{0}^{*}}(t)\right)$ & $\stackrel{(-)}{+}\left|A_{\perp} \| A_{0}\right| e^{-\Gamma_{B} t}\left[\sin \left(\delta_{2}\right) \cos (\Delta M t)-\cos \left(\delta_{2}\right) \cos (2 \beta) \sin (\Delta M t)\right]$ \\
\hline
\end{tabular}

on obtient, pour un méson $B$ initialement $B_{d}^{0}$ et $\bar{B}_{d}^{0}$, respectivement :

$$
\begin{aligned}
& A_{\sigma}(t)=\left\langle f_{\sigma}\left|\mathcal{H}_{\mathrm{eff}}\right| B_{d}^{0}(t)\right\rangle=A_{\sigma}(0) e^{-\frac{1}{2} \Gamma_{0} t} e^{-i M_{0} t}\left[\cos \left(\frac{\Delta M}{2} t\right)+i \lambda_{\sigma} \sin \left(\frac{\Delta M}{2} t\right)\right], \\
& \bar{A}_{\sigma}(t)=\left\langle f_{\sigma}\left|\mathcal{H}_{\mathrm{eff}}\right| \bar{B}_{d}^{0}(t)\right\rangle=\bar{A}_{\sigma}(0) e^{-\frac{1}{2} \Gamma_{0} t} e^{-i M_{0} t}\left[\cos \left(\frac{\Delta M}{2} t\right)+i \lambda_{\sigma}^{-1} \sin \left(\frac{\Delta M}{2} t\right)\right],
\end{aligned}
$$

avec $\lambda_{\sigma}=(q / p)\left(\bar{A}_{\sigma}(0) / A_{\sigma}(0)\right)$ (voir eq. (1.30) avec $\left.A_{\sigma} \equiv A_{\sigma}(0)\right)$. Pour le moment, aucune hypothèse n'est effectuée sur les amplitudes $A_{\sigma}(0)$. Deux situations sont à considérer :

- soit il y a violation de $C P$ dans la désintégration (p. ex. par la présence de nouvelle physique) : $\left|A_{\sigma}(0)\right| \neq\left|\bar{A}_{\sigma}(0)\right|$. Dans ce cas, $\lambda_{\sigma}$ est différent pour chaque amplitude de transversité, i.e. $\lambda_{0} \neq \lambda_{\|} \neq \lambda_{\perp}$

- soit il n'y a pas de violation de $C P$ dans la désintégration : $\left|A_{\sigma}(0)\right|=\left|\bar{A}_{\sigma}(0)\right|$. Il existe alors une relation ${ }^{18}$ entre $\bar{A}_{\sigma}(0)$ et $A_{\sigma}(0)$ (cf. eq. (1.43)) : $\bar{A}_{\sigma}(0)=\eta_{C P}\left(f_{\sigma}\right) e^{-i \alpha_{B}} A_{\sigma}(0)$ et $\lambda_{\sigma}=\eta_{C P}\left(f_{\sigma}\right) e^{-2 i \beta}$. Les expressions des $K_{i}(t)$ intervenant dans la distribution angulaire (voir eq. (2.73) avec $\left.\stackrel{(-)}{K_{i}} \stackrel{(-)}{K_{i}}(0)\right)$ et temporelle sont données dans la table 2.3. À noter la présence de $\cos 2 \beta$ dans les termes d'interférence entre amplitudes de parité $C P$ opposée.

Passons aux désintégrations spécifiques de saveur. Un état spécifique de saveur est une désintégration qui permet de signer sans ambiguïté la saveur du méson $B$ au moment de sa désintégration.

\footnotetext{
${ }^{18}$ Avec la convention $\mathcal{C P}\left|B_{d}^{0}\right\rangle=\left|\bar{B}_{d}^{0}\right\rangle\left(\alpha_{B}=0\right)$, on a $\bar{A}_{0}=A_{0}, \bar{A}_{\|}=A_{\|}$et $\bar{A}_{\perp}=-A_{\perp}$ [125], ce qui donne pour les amplitudes d'hélicité $\bar{H}_{0}=H_{0}$ et $\bar{H}_{ \pm 1}=H_{\mp 1}$.
} 
Pour la désintégration $B_{d}^{0} \rightarrow \psi\left(K^{+} \pi^{-}\right)^{* 0}$, on a :

$$
\begin{array}{ll}
B_{d}^{0} \rightarrow \psi\left(\rightarrow \ell^{+} \ell^{-}\right) K^{* 0}\left(\rightarrow K^{+} \pi^{-}\right) \equiv f_{\sigma}, & \bar{B}_{d}^{0} \rightarrow \psi\left(\rightarrow \ell^{+} \ell^{-}\right) \bar{K}^{* 0}\left(\rightarrow K^{-} \pi^{+}\right) \equiv \bar{f}_{\sigma}, \\
\bar{B}_{d}^{0} \not \rightarrow \psi\left(\rightarrow \ell^{+} \ell^{-}\right) K^{* 0}\left(\rightarrow K^{+} \pi^{-}\right) \equiv f_{\sigma}, & B_{d}^{0} \nrightarrow \rightarrow \psi\left(\rightarrow \ell^{+} \ell^{-}\right) \bar{K}^{* 0}\left(\rightarrow K^{-} \pi^{+}\right) \equiv \bar{f}_{\sigma},
\end{array}
$$

c'est-à-dire que les amplitudes $\left\langle f_{\sigma}\left|\mathcal{H}_{\text {eff }}\right| \bar{B}_{d}^{0}\right\rangle$ et $\left\langle\bar{f}_{\sigma}\left|\mathcal{H}_{\text {eff }}\right| B_{d}^{0}\right\rangle$ sont nulles. Du fait des oscillations des mésons $B$ neutres, l'évolution temporelle des amplitudes de transversité dépend de la différence de saveur du méson $B$ entre l'instant initial $(t=0)$ et l'instant de désintégration $t$. Si les saveurs sont identiques, on a :

$$
\begin{aligned}
& A_{f_{\sigma}}(t)=\left\langle f_{\sigma}|\mathcal{H}| B_{d}^{0}(t)\right\rangle=g_{+}(t) A_{\sigma}(0)=e^{-\frac{1}{2} \Gamma_{0} t} e^{-i M_{0} t} \cos \left(\frac{\Delta M}{2} t\right) A_{\sigma}(0) \\
& \bar{A}_{\bar{f}_{\sigma}}(t)=\left\langle\bar{f}_{\sigma}|\mathcal{H}| \bar{B}_{d}^{0}(t)\right\rangle=g_{+}(t) \bar{A}_{\sigma}(0)=e^{-\frac{1}{2} \Gamma_{0} t} e^{-i M_{0} t} \cos \left(\frac{\Delta M}{2} t\right) \bar{A}_{\sigma}(0)
\end{aligned}
$$

pour un méson $B$ initialement $B_{d}^{0}$ et $\bar{B}_{d}^{0}$, respectivement et $\stackrel{(-)}{A_{\sigma}}(0) \equiv\left\langle\stackrel{(-)}{f_{\sigma}}\left|\mathcal{H}_{\text {eff }}\right| \stackrel{(-)}{B_{d}^{0}}\right\rangle$. Si les saveurs sont opposées alors

$$
\begin{aligned}
& A_{\bar{f}_{\sigma}}(t)=\left\langle\bar{f}_{\sigma}|\mathcal{H}| B_{d}^{0}(t)\right\rangle=\frac{q}{p} g_{-}(t) A_{\sigma}(0)=i \frac{q}{p} e^{-\frac{1}{2} \Gamma_{0} t} e^{-i M_{0} t} \sin \left(\frac{\Delta M}{2} t\right) A_{\sigma}(0), \\
& \bar{A}_{f_{\sigma}}(t)=\left\langle f_{\sigma}|\mathcal{H}| \bar{B}_{d}^{0}(t)\right\rangle=\frac{p}{q} g_{-}(t) \bar{A}_{\sigma}(0)=i \frac{p}{q} e^{-\frac{1}{2} \Gamma_{0} t} e^{-i M_{0} t} \sin \left(\frac{\Delta M}{2} t\right) \bar{A}_{\sigma}(0),
\end{aligned}
$$

pour un méson $B$ initialement $B_{d}^{0}$ et $\bar{B}_{d^{\prime}}^{0}$ respectivement. Les amplitudes $A_{\bar{f}_{\sigma}}(t)$ et $\bar{A}_{f_{\sigma}}(t)$ s'annulent à $t=0$ mais deviennent différentes de zéro pour $t \neq 0 \mathrm{du}$ fait du mélange des mésons $B$ neutres. Dans le cas où l'on considère l'absence de violation de $C P$ dans la désintégration $\left(\left|A_{\sigma}(0)\right|=\left|\bar{A}_{\sigma}(0)\right|\right)$, la relation qui lie $A_{\sigma}(0)$ à $\bar{A}_{\sigma}(0)$ est $\bar{A}_{\sigma}(0)=e^{-i\left(\alpha_{B}-\alpha_{f_{\sigma}}\right)} A_{\sigma}(0)$ (cf. eq. (1.43)), $\operatorname{avec}^{19} e^{i \alpha_{f_{0}}}=e^{i \alpha_{f_{\|}}}=-e^{i \alpha_{f_{\perp}}}\left(\equiv e^{i \alpha_{f}}\right)$.

Puisque la transition faible $b \rightarrow c \bar{c} s$ est un transition $\Delta I=0$, cela implique l'égalité des amplitudes $A\left(\bar{B}_{d}^{0} \rightarrow(c \bar{c}) \bar{K}^{* 0}\right)=A\left(B_{u}^{-} \rightarrow(c \bar{c}) \bar{K}^{*-}\right)$ [130]. L'évolution temporelle des canaux chargés est donnée en prenant $\Delta M=0$ dans les équations (2.82) et (2.83) avec le canal relié d'isospin. Remarquons que la symétrie d'isospin est un ingrédient important dans la détermination des amplitudes car elle implique que tous les modes $B \rightarrow \psi K^{*}(892)$, neutres et chargés, sont décrits par les mêmes amplitudes. On peut donc utiliser toute la statistique des désintégrations $K^{*}(892) \rightarrow K \pi$ pour déterminer les amplitudes de transversité $A_{\sigma}(0)(\sigma=0, \|, \perp)$.

Dans la production cohérente de paires de mésons $B$ (cf. le chapitre 3), le temps $t$ s'étend de $-\infty$ à $+\infty$. Les distributions angulaires intégrées en temps s'obtiennent à partir des distributions dépendantes du temps en "substituant » $X_{i}(t)$ ou $K_{i}(t)$ par $X_{i}(0)$ ou $K_{i}(0)$ sauf pour le mode propre de $C P$. En effet, l'intégration des termes $K_{4}(t)$ et $K_{6}(t)$ (cf. la table 2.3) conduit à $K_{4}(0) /(1+$ $x_{d}^{2}$ ) et $K_{6}(0) /\left(1+x_{d}^{2}\right)$, où $x_{d}=\Delta M / \Gamma_{0}$ (voir la remarque en bas de page 17).

\footnotetext{
${ }^{19}$ Dans le cas $K^{* 0} \rightarrow K^{+} \pi^{-}$, on a $\mathcal{C P}\left|\psi K^{* 0}\left(\rightarrow K^{+} \pi^{-}\right)\right\rangle=\eta_{C P}(\psi) e^{i \alpha_{f}}(-1)^{L}\left|\psi \bar{K}^{* 0}\left(\rightarrow K^{-} \pi^{+}\right)\right\rangle$, avec $\mathcal{C P} \mid K^{* 0}(\rightarrow$ $\left.\left.K^{+} \pi^{-}\right)\right\rangle=e^{i \alpha_{f}}\left|\bar{K}^{* 0}\left(\rightarrow K^{-} \pi^{+}\right)\right\rangle$.
} 


\subsubsection{Le canal $\chi_{c 1} K^{*}(892)$}

\section{Distribution angulaire en base d'hélicité}

Jusqu'ici, nous avons traité le cas des désintégrations $B \rightarrow \psi K^{*}(892)$ avec $\psi=J / \psi$ ou $\psi(2 S)$. On considère maintenant la désintégration d'un méson $B$ en $\chi_{c 1} K^{*}(892)$, où le $\chi_{c 1}$ est reconstruit dans sa désintégration radiative $J / \psi \gamma$ (le $J / \psi$ est reconstruit dans sa désintégration leptonique $\ell^{+} \ell^{-}(\ell=e$ ou $\mu)$ ). L'amplitude totale s'écrit ${ }^{20}$ (une version complète de la distribution en hélicité incluant la désintégration du $J / \psi$ est donnée dans l'appendice $C$ ) :

$$
\begin{aligned}
A\left(\lambda_{\gamma}, \lambda_{\psi}\right)=\sum_{\lambda_{\chi}, \lambda_{K^{*}}}\left\langle\theta_{K^{*}}, \varphi_{K^{*}}, \lambda_{K}, \lambda_{\pi}\left|\mathcal{T}_{K^{*}}\right| 1, m_{K^{*}}=-\lambda_{K^{*}}\right\rangle \\
\left\langle\theta_{\chi}, \varphi_{\chi}, \lambda_{\psi}, \lambda_{\gamma}\left|\mathcal{T}_{\chi}\right| 1, m_{\chi}=\lambda_{\chi}\right\rangle\left\langle\theta_{B}, \varphi_{B}, \lambda_{\chi}, \lambda_{K^{*}}\left|\mathcal{T}_{B}\right| 0,0\right\rangle,
\end{aligned}
$$

soit, à une constante globale près,

$$
A\left(\lambda_{\gamma}, \nu\left(\lambda_{\gamma}\right)\right)=\sum_{\lambda=0, \pm 1} D_{\lambda, \nu}^{1 *}\left(\varphi_{\chi}, \theta_{\chi}, 0\right) D_{-\lambda, 0}^{1 *}\left(\varphi_{K^{*}}, \theta_{K^{*}}, 0\right) H_{\lambda} H_{\lambda_{\psi}, \lambda_{\gamma}}^{\chi}
$$

où $\nu=\lambda_{\psi}-\lambda_{\gamma}$ avec

$$
\nu=-1,0 \text { pour } \lambda_{\gamma}=+1, \quad \nu=0,+1 \text { pour } \lambda_{\gamma}=-1,
$$

l'hélicité du photon ne pouvant prendre que les valeurs +1 et -1 et $|\nu| \leq J_{\chi}=1$. La conservation de la parité dans le processus électromagnétique $\chi_{c 1} \rightarrow J / \psi \gamma$ implique

$$
H_{\lambda_{\psi}, \lambda_{\gamma}}^{\chi}=\eta_{P}(J / \psi) \eta_{P}(\gamma) \eta_{P}\left(\chi_{c 1}\right)(-1)^{s_{\chi_{c 1}}-s_{J / \psi}-s_{\gamma}} H_{-\lambda_{\psi},-\lambda_{\gamma}}^{\chi}=-H_{-\lambda_{\psi},-\lambda_{\gamma}}^{\chi} .
$$

Les études théoriques basées sur QCD [136] ainsi que les résultats expérimentaux [137, 138] confirment la large domination d'une transition $E 1$ entre le $\chi_{c 1}$ et le $J / \psi$. Dans cette hypothèse, les amplitudes valent

$$
\frac{\left|H_{1,1}^{\chi}\right|^{2}}{\left|H_{1,1}^{\chi}\right|^{2}+\left|H_{0,1}^{\chi}\right|^{2}}=\frac{\left|H_{0,1}^{\chi}\right|^{2}}{\left|H_{1,1}^{\chi}\right|^{2}+\left|H_{0,1}^{\chi}\right|^{2}}=\frac{1}{2} .
$$

Les différents angles intervenant dans cette distribution angulaire sont définis comme suit : l'angle $\theta_{K^{*}}\left(\theta_{\chi}\right)$ est l'angle polaire de l'impulsion du kaon $(J / \psi)$ dans le repère au repos du $K^{*}$ $\left(\chi_{c 1}\right)$ par rapport à l'impulsion du $K^{*}\left(\chi_{c 1}\right)$ dans le repère du méson $B$ au repos. L'angle azimutal $\varphi=\varphi_{\chi}-\varphi_{K^{*}}$ est l'angle entre les deux plans des produits de désintégration du $\chi_{c 1}$ et du $K^{*}$, en allant du kaon vers le $J / \psi$ avec une rotation positive autour de l'axe $z_{\chi}$ (voir Fig. 2.7) :

$$
\sin (\varphi)=\left(\hat{p}_{\chi} \times \hat{c}\right) \cdot \hat{d}, \quad \cos (\varphi)=\hat{c} . \hat{d}
$$

où les vecteurs unités $\hat{c}$ et $\hat{d}$ sont définis par :

$$
\hat{c}=\left(\hat{p}_{K^{*}} \times \hat{p}_{K}\right) \times \hat{p}_{K^{*}}, \quad \hat{d}=\left(\hat{p}_{\chi} \times \hat{p}_{\psi}\right) \times \hat{p}_{\chi} .
$$

\footnotetext{
${ }^{20}$ Les conventions utilisées sont les mêmes que celles utilisées pour $\psi K^{*}(892)$ : convention de Jackson pour les opérateurs de rotation finie et rotation autour de l'axe $x$ pour définir l'état $\left|-\vec{p}, \lambda_{2}\right\rangle$ (voir appendice A).
} 


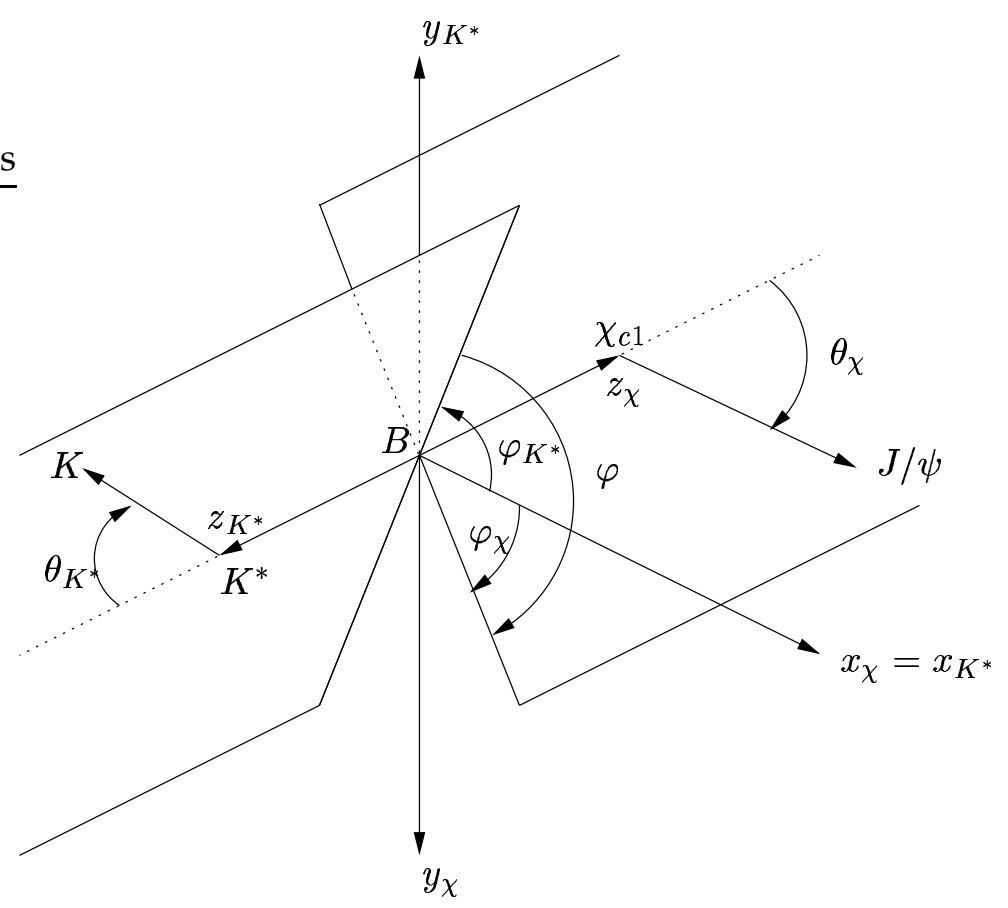

FIG. 2.7: Définition des plans de désintégration et des différents repères pour la dérivation de la distribution angulaire $B \rightarrow \chi_{c 1}(\rightarrow J / \psi \gamma) K^{*}(\rightarrow K \pi)$. La convention choisie pour définir l'état d'hélicité $\left|\vec{p}_{K^{*}}, \lambda_{K^{*}}\right\rangle$ est une rotation autour de l'axe $x$ (voir appendice A), impliquant $x_{\chi}=x_{K^{*}}$. À noter que $\varphi=\varphi_{\chi}-\varphi_{K^{*}}$. Les angles $\theta_{\chi}$ et $\theta_{K^{*}}$ sont évalués dans les repères au repos des mésons $\chi_{c 1}$ et $K^{*}$, respectivement.

Les impulsions de chaque particule sont mesurées dans le repère au repos de leur mère respective.

La distribution angulaire en base d'hélicité est donnée, après sommation sur les états d’hélicité du photon, par :

$$
\begin{aligned}
\frac{1}{\Gamma} & \frac{\mathrm{d}^{3} \Gamma \stackrel{(-)}{B} \rightarrow \chi_{c 1}(\rightarrow J / \psi \gamma) \stackrel{(-)}{K}}{\mathrm{~d} \cos \theta_{K^{*}} \mathrm{~d} \cos \theta_{\chi} \mathrm{d} \varphi} \\
& \frac{1}{\left|\stackrel{(-)}{H}_{+1}\right|^{2}+\left|\stackrel{(-)}{H_{0}}\right|^{2}+\left|\stackrel{(-)}{H_{-1}}\right|^{2}} \frac{1}{\left|H_{1,1}^{\chi}\right|^{2}+\left|H_{0,1}^{\chi}\right|^{2}} \sum_{i=1}^{7} \stackrel{(-)}{X_{i}} h_{i}\left(\theta_{K^{*}}, \theta_{\chi}, \varphi\right),
\end{aligned}
$$

où les expressions de $\stackrel{(-)}{X_{i}}$ et $h_{i}\left(\theta_{K^{*}}, \theta_{\chi}, \varphi\right)$ sont données dans la table 2.4. La distribution angulaire est normalisée par construction.

Dans le cas particulier $\left|H_{1,1}^{\chi}\right|^{2}=0$, soit $\left|H_{0,1}^{\chi}\right|^{2}=1$, on retrouve la distribution angulaire en hélicité de la désintégration $B \rightarrow J / \psi\left(\rightarrow \ell^{+} \ell^{-}\right) K^{*}(\rightarrow K \pi)$ en faisant la substitution $\theta_{\chi} \rightarrow \theta_{\psi}$. Cela provient du fait que dans ce cas, seule $\lambda_{\psi}=0$ contribue, équivalent à $\nu= \pm 1$, qui devient l'analogue du $\alpha= \pm 1$ dans l'amplitude $A(\alpha)$ (cf. eq. (2.58)). Les distributions angulaires reflètent la dynamique en spin sans se soucier du contenu en particules, qui lui intervient dans les amplitudes d'hélicité $H_{\lambda_{1}, \lambda_{2}}^{J}$.

\section{Distribution angulaire en base de transversité}

La distribution angulaire en transversité s'obtient en effectuant, soit les transformations d'amplitudes et d'angles directement sur la distribution angulaire en hélicité d'après les équations (2.64) 
TAB. 2.4: Observables et fonctions angulaires de la distribution angulaire $\stackrel{(-)}{B} \rightarrow \chi_{c 1}(\rightarrow J / \psi \gamma) \stackrel{(-)}{K^{*}}(\rightarrow K \pi)$ dans la base d'hélicité pour un méson $B$ initialement $\stackrel{(-)}{B}$.

\begin{tabular}{|c||c|c|}
\hline$i$ & $\stackrel{(-)}{X}_{i}$ & $h_{i}\left(\theta_{K^{*}}, \theta_{\chi}, \varphi\right)$ \\
\hline \hline 1 & $\left|H_{+1}\right|^{2}$ & $\frac{9}{16 \pi} \frac{1}{4} \sin ^{2}\left(\theta_{K^{*}}\right)\left[2\left|H_{1,1}^{\chi}\right|^{2} \sin ^{2}\left(\theta_{\chi}\right)+\left|H_{0,1}^{\chi}\right|^{2}\left(1+\cos ^{2}\left(\theta_{\chi}\right)\right)\right]$ \\
\hline 2 & $\left|H_{-1}\right|^{2}$ & $\frac{9}{16 \pi} \frac{1}{4} \sin ^{2}\left(\theta_{K^{*}}\right)\left[2\left|H_{1,1}^{\chi}\right|^{2} \sin ^{2}\left(\theta_{\chi}\right)+\left|H_{0,1}^{\chi}\right|^{2}\left(1+\cos ^{2}\left(\theta_{\chi}\right)\right)\right]$ \\
\hline 3 & $\left|H_{0}\right|^{2}$ & $\frac{9}{16 \pi} \cos ^{2}\left(\theta_{K^{*}}\right)\left[2\left|H_{1,1}^{\chi}\right|^{2} \cos ^{2}\left(\theta_{\chi}\right)+\left|H_{0,1}^{\chi}\right|^{2} \sin ^{2}\left(\theta_{\chi}\right)\right]$ \\
\hline 4 & $\Re e\left(H_{+1} H_{-1}^{*}\right)$ & $\frac{9}{16 \pi} \frac{1}{2} \sin ^{2}\left(\theta_{K^{*}}\right) \sin ^{2}\left(\theta_{\chi}\right) \cos (2 \varphi)\left[2\left|H_{1,1}^{\chi}\right|^{2}-\left|H_{0,1}^{\chi}\right|^{2}\right]$ \\
\hline 5 & $\Im m\left(H_{+1} H_{-1}^{*}\right)$ & $-\frac{9}{16 \pi} \frac{1}{2} \sin ^{2}\left(\theta_{K^{*}}\right) \sin ^{2}\left(\theta_{\chi}\right) \sin (2 \varphi)\left[2\left|H_{1,1}^{\chi}\right|^{2}-\left|H_{0,1}^{\chi}\right|^{2}\right]$ \\
\hline 6 & $\Re e\left(\left(H_{+1}+H_{-1}\right) H_{0}^{*}\right)$ & $-\frac{9}{16 \pi} \frac{1}{4} \sin \left(2 \theta_{K^{*}}\right) \sin \left(2 \theta_{\chi}\right) \cos (\varphi)\left[2\left|H_{1,1}^{\chi}\right|^{2}-\left|H_{0,1}^{\chi}\right|^{2}\right]$ \\
\hline 7 & $\Im m\left(\left(H_{+1}-H_{-1}\right) H_{0}^{*}\right)$ & $\frac{9}{16 \pi} \frac{1}{4} \sin \left(2 \theta_{K^{*}}\right) \sin \left(2 \theta_{\chi}\right) \sin (\varphi)\left[2\left|H_{1,1}^{\chi}\right|^{2}-\left|H_{0,1}^{\chi}\right|^{2}\right]$ \\
\hline \hline
\end{tabular}

et (2.69), soit à partir de l'amplitude d'hélicité :

$$
\begin{aligned}
& A\left(\lambda_{\gamma}, \nu\left(\lambda_{\gamma}\right)\right)= \\
& \quad \sum_{\lambda, \tau}\left\langle\theta_{K^{*}}, \pi / 2, \lambda_{K}, \lambda_{\pi}\left|\mathcal{T}_{K^{*}}\right| 1,-\lambda\right\rangle\left\langle\theta_{t r}, \varphi_{t r}, \lambda_{\psi}, \lambda_{\gamma}\left|\mathcal{T}_{\chi}\right| 1, \tau\right\rangle\langle 1, \tau \mid 1, \lambda\rangle H_{\lambda}= \\
& \quad H_{\lambda_{\psi}, \lambda_{\gamma}}^{\chi} \sum_{\tau=0, \pm 1} D_{\tau, \nu}^{1 *}\left(\varphi_{t r}, \theta_{t r}, 0\right)\left[\sum_{\lambda=0, \pm 1} D_{-\lambda, 0}^{1 *}\left(\frac{\pi}{2}, \theta_{K^{*}}, 0\right) D_{\tau, \lambda}^{1}\left(-\pi,-\frac{\pi}{2}, 0\right) H_{\lambda}\right],
\end{aligned}
$$

où les angles sont définis sur la figure 2.8. On obtient alors pour la distribution angulaire en transversité :

$$
\begin{aligned}
& \frac{1}{\Gamma} \frac{\left.\mathrm{d}^{3} \Gamma \stackrel{(-)}{B} \rightarrow \chi_{c 1}(\rightarrow J / \psi \gamma) \stackrel{(-)}{K^{*}}(\rightarrow K \pi)\right)}{\mathrm{d} \cos \theta_{K^{*}} \mathrm{~d} \cos \theta_{t r} \mathrm{~d} \varphi_{t r}}= \\
& \quad \frac{1}{\left|\stackrel{(-)}{A_{\|}}\right|^{2}+\left|\stackrel{(-)}{A_{\perp}}\right|^{2}+\left|\stackrel{(-)}{A_{0}}\right|^{2}} \frac{1}{\left|H_{1,1}^{\chi}\right|^{2}+\left|H_{0,1}^{\chi}\right|^{2}} \sum_{i=1}^{6} \stackrel{(-)}{K_{i}} f_{i}\left(\theta_{K^{*}}, \theta_{t r}, \varphi_{t r}\right),
\end{aligned}
$$

où les expressions de $\stackrel{(-)}{K_{i}}$ et $f_{i}\left(\theta_{K^{*}}, \theta_{t r}, \varphi_{t r}\right)$ sont données dans la table 2.5. La distribution angulaire est normalisée par construction. La remarque sur le retour à la distribution angulaire du $B \rightarrow$ $J / \psi\left(\rightarrow \ell^{+} \ell^{-}\right) K^{*}(\rightarrow K \pi)$ quand $\left|H_{1,1}^{\chi}\right|^{2}=0$ reste valable en transversité. L'évolution temporelle des amplitudes, donnée dans le paragraphe 2.2.2, s'applique encore aux amplitudes $B \rightarrow \chi_{c 1} K^{*}(892)$ sans oublier de prendre en compte l'évolution temporelle du type temps de vie des amplitudes $H^{\chi}$. À noter que la parité $C P$ intrinsèque du $\chi_{c 1}$ est identique à celle du $J / \psi$ et $\psi(2 S)$. 


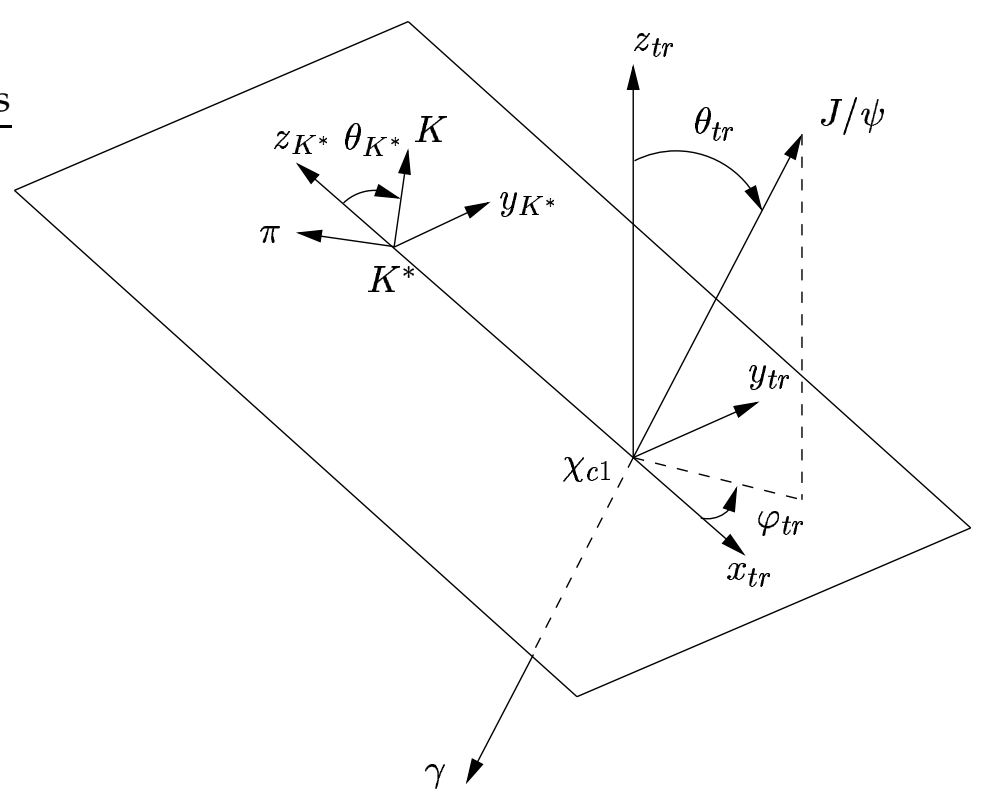

FIG. 2.8: Définition du plan et des angles de transversité. Le repère de transversité est défini dans le repère du $\chi_{c 1}$ au repos. La direction opposée à l'impulsion du $K^{*}$ définit l'axe $x_{t r}$. Le plan de désintégration du $K^{*}$ en $K \pi$ définit le plan de transversité $\left(x_{t r}, y_{t r}\right)$, où l'axe $y_{t r}$ est orienté de sorte que $p_{y}(K)>0$. L'axe $z_{t r}$ est perpendiculaire au plan de transversité afin de former un trièdre direct. Les angles $\left(\theta_{t r}, \varphi_{t r}\right)$ décrivent la direction du $J / \psi$ provenant de la désintégration du $\chi_{c 1}$ dans le repère de transversité. $\theta_{K^{*}}$ est l'angle entre la direction du kaon dans le repère du $K^{*}$ au repos et la direction opposée de l'axe $x_{t r}$.

TAB. 2.5: Observables et fonctions angulaires de la distribution angulaire $\stackrel{(-)}{B} \rightarrow \chi_{c 1}(\rightarrow J / \psi \gamma) \stackrel{(-)}{K^{*}}(\rightarrow K \pi)$ dans la base de transversité pour un méson $B$ initialement $\stackrel{(-)}{B}$.

\begin{tabular}{|c|c|c|}
\hline$i$ & $\stackrel{(-)}{K_{i}}$ & $f_{i}\left(\theta_{K^{*}}, \theta_{t r}, \varphi_{t r}\right)$ \\
\hline 1 & $\mid(-))$ & $\frac{9}{32 \pi} 2 \cos ^{2}\left(\theta_{K^{*}}\right)\left[2\left|H_{1,1}^{\chi}\right|^{2} \sin ^{2}\left(\theta_{t r}\right) \cos ^{2}\left(\varphi_{t r}\right)+\left|H_{0,1}^{\chi}\right|^{2}\left(1-\sin ^{2}\left(\theta_{t r}\right) \cos ^{2}\left(\varphi_{t r}\right)\right)\right]$ \\
\hline 2 & $\mid(-))$ & $\frac{9}{32 \pi} \sin ^{2}\left(\theta_{K^{*}}\right)\left[2\left|H_{1,1}^{\chi}\right|^{2} \sin ^{2}\left(\theta_{t r}\right) \sin ^{2}\left(\varphi_{t r}\right)+\left|H_{0,1}^{\chi}\right|^{2}\left(1-\sin ^{2}\left(\theta_{t r}\right) \sin ^{2}\left(\varphi_{t r}\right)\right)\right]$ \\
\hline 3 & $\left|\stackrel{(-)}{A_{\perp}}\right|^{2}$ & $\frac{9}{32 \pi} \sin ^{2}\left(\theta_{K^{*}}\right)\left[2\left|H_{1,1}^{\chi}\right|^{2} \cos ^{2}\left(\theta_{t r}\right)+\left|H_{0,1}^{\chi}\right|^{2} \sin ^{2}\left(\theta_{t r}\right)\right]$ \\
\hline 4 & $\Im m\left(A_{\|}^{*} A_{\perp}\right)$ & $-\frac{9}{32 \pi} \sin ^{2}\left(\theta_{K^{*}}\right) \sin \left(2 \theta_{t r}\right) \sin \left(\varphi_{t r}\right)\left[2\left|H_{1,1}^{\chi}\right|^{2}-\left|H_{0,1}^{\chi}\right|^{2}\right]$ \\
\hline 5 & $\Re e((-))(-))$ & $\frac{9}{32 \pi} \frac{1}{\sqrt{2}} \sin \left(2 \theta_{K^{*}}\right) \sin ^{2}\left(\theta_{t r}\right) \sin \left(2 \varphi_{t r}\right)\left[2\left|H_{1,1}^{\chi}\right|^{2}-\left|H_{0,1}^{\chi}\right|^{2}\right]$ \\
\hline 6 & $\Im m\left(\stackrel{(-)(-)}{\left.A_{\perp} A_{0}^{*}\right)}\right.$ & $-\frac{9}{32 \pi} \frac{1}{\sqrt{2}} \sin \left(2 \theta_{K^{*}}\right) \sin \left(2 \theta_{t r}\right) \cos \left(\varphi_{t r}\right)\left[2\left|H_{1,1}^{\chi}\right|^{2}-\left|H_{0,1}^{\chi}\right|^{2}\right]$ \\
\hline
\end{tabular}




\section{Distributions angulaires réduites}

En intégrant successivement sur les différents angles, on obtient les distributions angulaires réduites :

$$
\begin{aligned}
\frac{1}{\Gamma} \frac{\mathrm{d} \Gamma}{\mathrm{d} \cos \theta_{K^{*}}}= & \frac{3}{8}\left[\left(1+\cos ^{2}\left(\theta_{t r}\right)\right)+R_{\perp}\left(1-3 \cos ^{2}\left(\theta_{t r}\right)\right)\right] \\
\frac{1}{\Gamma} \frac{\mathrm{d} \Gamma}{\mathrm{d} \cos \theta_{t r}}= & \frac{3}{8}\left[\left\{\left(1+\cos ^{2}\left(\theta_{t r}\right)\right)+R_{1,1}^{\chi}\left(1-3 \cos ^{2}\left(\theta_{t r}\right)\right)\right\}+\right. \\
& \left.R_{\perp}\left(3 R_{1,1}^{\chi}-1\right)\left(3 \cos ^{2}\left(\theta_{t r}\right)-1\right)\right] \\
\frac{1}{\Gamma} \frac{\mathrm{d} \Gamma}{\mathrm{d} \varphi_{t r}}= & \frac{1}{4 \pi}\left[2+\left(1-3 R_{1,1}^{\chi}\right)\left(R_{\|}-R_{0}\right) \cos \left(2 \varphi_{t r}\right)\right]
\end{aligned}
$$

où les paramètres $R_{\perp}, R_{0}$ et $R_{\|}$sont définis par l'équation (2.77) et où $R_{1,1}^{\chi}$ est donné par:

$$
R_{1,1}^{\chi}=\frac{\left|H_{1,1}^{\chi}\right|^{2}}{\left|H_{1,1}^{\chi}\right|^{2}+\left|H_{0,1}^{\chi}\right|^{2}}=1-\frac{\left|H_{0,1}^{\chi}\right|^{2}}{\left|H_{1,1}^{\chi}\right|^{2}+\left|H_{0,1}^{\chi}\right|^{2}} .
$$

\subsection{Résonances : spectre $K \pi$}

Loin d'être uniquement les produits de désintégration du $K^{*}(892)$, le spectre $K \pi$ est un mélange complexe de résonances, de leurs interférences et d'une production non résonante. Dans le but de mesurer les amplitudes de la désintégration $B \rightarrow \psi K^{*}(892)$, il convient de prendre en compte les contributions de ces différentes sources.

La dépendance en masse des amplitudes (hélicité, transversité, etc.) est en général complexe. Cependant, dans le cas où ces amplitudes sont complètement dominées par une résonance à l'énergie du centre de masse $E$ on peut alors les décomposer en une dépendance en énergie généralement paramétrée par une Breit-Wigner relativiste, $B_{B W}(m)$, et une dépendance en spin, $F_{\lambda_{1}, \lambda_{2}}^{J}$ :

$$
H_{\lambda_{1}, \lambda_{2}}^{J}=B_{B W}(m) F_{\lambda_{1}, \lambda_{2}}^{J}
$$

Les sections suivantes décrivent la forme retenue pour la Breit-Wigner, puis analysent le spectre $K \pi$ en ondes $S, P, D$ à partir de moments et se terminent par la distribution angulaire de la désintégration $B \rightarrow J / \psi(K \pi)_{S+P}$.

\subsubsection{Breit-Wigner relativiste avec facteur de barrière centrifuge}

Pour une introduction à la théorie de la diffusion, on pourra consulter les ouvrages de la référence [139]. Une synthèse appliquée à l'étude des résonances est donnée dans la référence [140]. On considère la désintégration $B \rightarrow J / \psi X, X \rightarrow K \pi$. Soient $p$ l'impulsion de la résonance $X$ (système $K \pi$ ) dans le repère du méson $B$ au repos et $q$ l'impulsion du kaon dans le repère de la résonance au repos : $p$ et $q$ dépendent tous les deux de $m$, la masse du système $K \pi$. La fonction $B_{B W}(m)$ prend la forme

$$
B_{B W}(m)=D_{L}(p) \cdot B W(m) \cdot D_{J_{X}}(q), \quad B W(m)=\frac{m_{0} \Gamma_{0}}{m_{0}^{2}-m^{2}-i m_{0} \Gamma(m)}
$$


avec

$$
\Gamma(m)=\Gamma_{0}\left(\frac{m_{0}}{m}\right)\left(\frac{q}{q_{0}}\right)\left[\frac{D_{J_{X}}(q)}{D_{J_{X}}\left(q_{0}\right)}\right]^{2},
$$

où $m_{0}$ et $\Gamma_{0}$ sont la masse nominale et la largeur totale nominale de la résonance et $q_{0}=q$ pour $m=m_{0}$ de sorte que $\Gamma\left(m_{0}\right)=\Gamma_{0}$. D'après la forme de la fonction $D_{J_{X}}(q)$ donnée plus bas, la dépendance en énergie de la largeur est

$$
\Gamma(m) \propto\left(\frac{q}{q_{0}}\right)^{2 J_{X}+1}\left(\frac{m_{0}}{m}\right) .
$$

Dans la désintégration $B \rightarrow J / \psi K^{*}(892)$, le moment angulaire entre la résonance $K^{*}(892)$ et le méson $J / \psi$ peut prendre les valeurs $L=0,1,2$ du fait du spin 0 du méson $B$. L'amplitude résultante contient alors une barrière centrifuge, $D_{L}(p) \propto p^{L}$. Puisque $p$ dépend de la masse $K \pi$, la forme de la résonance est légèrement différente selon le moment angulaire $L$. Il en va de même pour les autres résonances $K \pi$.

La dépendance en masse de l'amplitude de diffusion pour une onde partielle $j$ est donnée par $[141,142]$ :

$$
T_{j}=e^{i \delta_{j}(m)} \sin \delta_{j}(m)=\frac{1}{\cot \delta_{j}(m)-i} .
$$

Une résonance de spin $j$ s'écrit de la manière conventionnelle

$$
\cot \delta_{j}(m)=\frac{m_{0}^{2}-m^{2}}{m_{0} \Gamma(m)}
$$

de sorte que

$$
T_{j}=\frac{m_{0} \Gamma(m)}{m_{0}^{2}-m^{2}-i m_{0} \Gamma(m)}=\frac{\Gamma(m)}{\Gamma_{0}} B W(m) .
$$

À noter que $T_{j}$ contient le facteur d'espace des phases à deux corps de la désintégration $X \rightarrow K \pi$. À la masse nominale de la résonance, l'amplitude $T_{j}$ vaut $i$ de sorte que $\delta_{j}\left(m_{0}\right)=90^{\circ}$.

Les fonctions $D_{L}(p)$ et $D_{J_{X}}(q)$ sont les facteurs de barrière centrifuge de Blatt-Weisskopf [143] tels que les ont donné von Hippel et Quigg [144] :

$$
\begin{aligned}
& D_{0}(p)=1 \\
& D_{1}(p)=\sqrt{\frac{2 z}{z+1}}, \\
& D_{2}(p)=\sqrt{\frac{13 z^{2}}{(z-3)^{2}+9 z}}, \\
& D_{3}(p)=\sqrt{\frac{277 z^{3}}{z(z-15)^{2}+9(2 z-5)^{2}}}, \\
& D_{4}(p)=\sqrt{\frac{12746 z^{4}}{\left(z^{2}-45 z+105\right)^{2}+25 z(2 z-21)^{2}}}
\end{aligned}
$$

où $z=\left(p / p_{R}\right)^{2}$ et $p_{R}=0.1973 \mathrm{GeV} / c$ correspondant à une longueur de $1 \mathrm{fm}$, taille typique de l'interaction. En général, les fonctions $D_{L}$ sont définies de sorte que $D_{L}(p \rightarrow \infty)=1$, mais ici elles sont 
renormalisées pour avoir $D_{L}\left(p_{R}\right)=1$ afin que les paramètres ajustés pour l'onde $L$ reflètent sa taille " réelle » à $p=p_{R}$. Les effets de la barrière centrifuge ne peuvent être isolés des propriétés internes de la résonance quand les particules sont à l'intérieur de la région d'interaction. Le problème de diffusion est un problème complexe puisqu'en général le potentiel (en se restreignant à la physique quantique non relativiste) modélisant l'interaction est inconnu, rendant la résolution de l'équation de Schrödinger impossible. Les barrières centrifuges données par Blatt-Weisskopf sont obtenues en prenant un puits de potentiel. À noter que la dépendance au seuil de l'amplitude $B_{B W}(m)$ est celle attendue [141], à savoir $B_{B W} \propto p^{L}$ pour $p \approx 0$ et $B_{B W} \propto q^{J_{X}}$ pour $q \approx 0$.

La décomposition de l'amplitude $T_{j}$ en fonction de ses parties réelle et imaginaire donne

$$
\Re e\left(T_{j}\right)=\frac{\left(m_{0}^{2}-m^{2}\right) m_{0} \Gamma(m)}{\left(m_{0}^{2}-m^{2}\right)^{2}+m_{0}^{2} \Gamma^{2}(m)}, \quad \Im m\left(T_{j}\right)=\frac{m_{0}^{2} \Gamma^{2}(m)}{\left(m_{0}^{2}-m^{2}\right)^{2}+m_{0}^{2} \Gamma^{2}(m)},
$$

représentées dans le plan complexe $\left(\Re e\left(T_{j}\right), \Im m\left(T_{j}\right)\right)$ par un diagramme, appelé diagramme d'Argand (cf. Fig. 2.9).

\subsubsection{Ondes $S, P, D$ du système $K \pi$}

En se basant sur la distribution décrivant la désintégration $B \rightarrow J / \psi X, X \rightarrow K \pi$ avec $X$ de spin $0,1,2$, donnée dans l'appendice $\mathrm{D}$, on dérive les moments intervenant dans la description du spectre $K \pi$. On se limite aux résonances de spin $0,1,2$ (ondes $S, P, D$ ), limitation motivée par la présence des résonances dans cette région qui se couplent fortement à $K \pi$ d'après [28, 146]. L'amplitude décrivant l'interférence des ondes $S, P, D$ a pour expression

$$
|\mathcal{M}|^{2}=\left|\sum_{J_{X}=0}^{2} \sum_{M_{X}\left(J_{X}\right)} \sqrt{\frac{2 J_{X}+1}{4 \pi}} D_{M_{X}, 0}^{J_{X} *}\left(\varphi_{X}=0, \theta_{X}, 0\right) H_{M_{X}}^{J_{X}}\right|^{2} .
$$

L'expression $M_{X}\left(J_{X}\right)$ signifie $M_{X}\left(J_{X}=0\right)=0, M_{X}\left(J_{X} \geq 1\right)=0, \pm 1$. L'absence de $M_{X}= \pm 2$ pour $J_{X}=2$ provient du fait que la résonance $X$ est produite par la désintégration $B \rightarrow J / \psi X$. La désintégration $B \rightarrow J / \psi X$ est décrite par trois angles, $\theta_{\psi}, \theta_{X}$ et $\varphi=\varphi_{\psi}-\varphi_{X}$. On choisit de prendre $\varphi=\varphi_{\psi}$ de sorte que $\varphi_{X}=0$ sans perte de généralité. $\theta_{X}$ est l'équivalent de $\theta_{K^{*}}$ pour $J_{X}=1$.

En développant le module au carré, on obtient

$$
|\mathcal{M}|^{2}=\sum_{J_{X}, J_{X}^{\prime}} \sum_{M_{X}, M_{X}^{\prime}} \sqrt{\frac{2 J_{X}+1}{4 \pi}} \sqrt{\frac{2 J_{X}^{\prime}+1}{4 \pi}} D_{M_{X}, 0}^{J_{X}{ }^{*}}\left(0, \theta_{X}, 0\right) D_{M_{X}^{\prime}, 0}^{J_{X}^{\prime}}\left(0, \theta_{X}, 0\right) H_{M_{X}}^{J_{X}} H_{M_{X}^{\prime}}^{J_{X}^{\prime}}
$$

En utilisant les équations (B.3), (B.4) et (B.6), on aboutit à

$$
\begin{aligned}
|\mathcal{M}|^{2}= & \sum_{J_{X}, J_{X}^{\prime}} \sum_{M_{X}, M_{X}^{\prime}}(-1)^{M_{X}^{\prime}} H_{M_{X}}^{J_{X}} H_{M_{X}^{\prime}}^{J_{X}^{\prime}} \sqrt{\frac{2 J_{X}+1}{2 J+1}} \sqrt{\frac{2 J_{X}^{\prime}+1}{4 \pi}} \\
& \sum_{\left|J_{X}-J_{X}^{\prime}\right| \leq J \leq J_{X}+J_{X}^{\prime}}\left\langle J_{X},-M_{X} ; J_{X}^{\prime}, M_{X}^{\prime} \mid J, M_{X}^{\prime}-M_{X}\right\rangle\left\langle J_{X}, 0 ; J_{X}^{\prime}, 0 \mid J, 0\right\rangle Y_{J, M}\left(\theta_{X}, 0\right)
\end{aligned}
$$




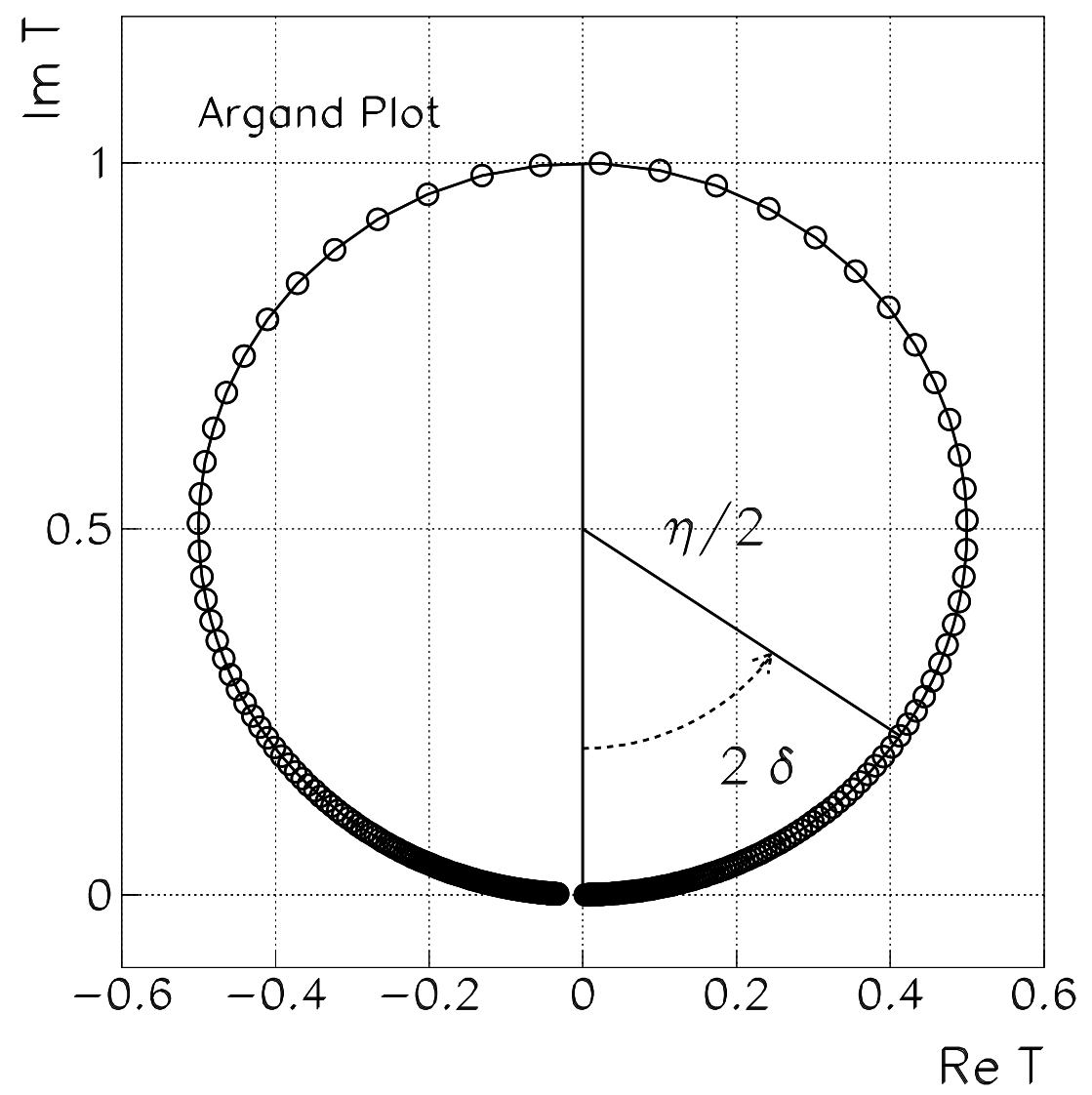

FIG. 2.9: Diagramme d'Argand correspondant à l'amplitude $T_{j}$ (diffusion élastique sans absorption $(\eta=1$ )). La causalité implique que lorsque la masse $m$ augmente, le vecteur $T_{j}$ tourne dans le sens trigonométrique (sens inverse des aiguilles d'une montre), appelé condition de Wigner [145]. Les points sont équidistants en masse, montrant la montée rapide de la phase $\delta_{j}$ à l'approche du pic de la résonance. D'après [147].

ТАВ. 2.6: Table de correspondance entre les valeurs prises par $J$ et celles prises par $J_{X}$ et $J_{X}^{\prime}$.

\begin{tabular}{|c|c|c||c|c|c||c|c|c|}
\hline$J$ & $J_{X}$ & $J_{X}^{\prime}$ & $J$ & $J_{X}$ & $J_{X}^{\prime}$ & $J$ & $J_{X}$ & $J_{X}^{\prime}$ \\
\hline \hline 0 & 0 & 0 & 1 & 0 & 1 & 2 & 0 & 2 \\
& 1 & 1 & & 1 & 0 & & 2 & 0 \\
& 2 & 2 & & 1 & 1 & & 1 & 1 \\
\hline 3 & 1 & 2 & & 1 & 2 & & 1 & 2 \\
& 2 & 1 & & 2 & 1 & & 2 & 1 \\
& 2 & 2 & & 2 & 2 & & 2 & 2 \\
\hline 4 & 2 & 2 & & & & & & \\
\hline \hline
\end{tabular}


avec $M=M_{X}-M_{X}^{\prime}$. La conservation du moment angulaire total implique que l'amplitude décrivant la distribution angulaire des produits de la réaction contient des harmoniques sphériques d'ordre $L$ ne dépassant pas le moment angulaire maximal de l'état initial, i.e. $J_{X}$. La distribution angulaire étant proportionnelle au module au carré de l'amplitude, elle contient donc des harmoniques sphériques jusqu'à l'ordre $2 J_{X}$. Les moments $T_{J M}$ sont définis par :

$$
T_{J M}=2 \pi \int|\mathcal{M}|^{2} Y_{J, M}^{*}\left(\theta_{X}, 0\right) \mathrm{d} \cos \theta_{X}
$$

et valent

$$
\begin{array}{r}
T_{J M}=\sum_{J_{X}, J_{X}^{\prime}} \sum_{M_{X}, M_{X}^{\prime}}(-1)^{M_{X}^{\prime}} H_{M_{X}}^{J_{X}} H_{M_{X}^{\prime}}^{J_{X}^{\prime}} \sqrt{\frac{2 J_{X}+1}{2 J+1}} \sqrt{\frac{2 J_{X}^{\prime}+1}{4 \pi}} \\
\left\langle J_{X},-M_{X} ; J_{X}^{\prime}, M_{X}^{\prime} \mid J, M_{X}^{\prime}-M_{X}\right\rangle\left\langle J_{X}, 0 ; J_{X}^{\prime}, 0 \mid J, 0\right\rangle,
\end{array}
$$

où les valeurs prises par $J$ en fonction de $J_{X}$ et $J_{X}^{\prime}$ sont données dans la table 2.6.

Les expressions explicites des moments en fonction des amplitudes sont, en se limitant aux moments $T_{J 0}$ :

$$
\begin{aligned}
\sqrt{4 \pi} T_{00} & =\left|H_{0}^{0}\right|^{2}+\left|H_{0}^{1}\right|^{2}+\left|H_{1}^{1}\right|^{2}+\left|H_{-1}^{1}\right|^{2}+\left|H_{0}^{2}\right|^{2}+\left|H_{1}^{2}\right|^{2}+\left|H_{-1}^{2}\right|^{2}, \\
\sqrt{4 \pi} T_{10} & =2 \Re e\left(H_{0}^{0 *} H_{0}^{1}\right)+\frac{4}{\sqrt{5}} \Re e\left(H_{0}^{1} H_{0}^{2 *}\right)+2 \sqrt{\frac{3}{5}}\left(\Re e\left(H_{1}^{1} H_{1}^{2 *}\right)+\Re e\left(H_{-1}^{1} H_{-1}^{2 *}\right)\right) \\
\sqrt{4 \pi} T_{20} & =2 \Re e\left(H_{0}^{0 *} H_{0}^{2}\right)+\frac{1}{\sqrt{5}}\left(2\left|H_{0}^{1}\right|^{2}-\left|H_{1}^{1}\right|^{2}-\left|H_{-1}^{1}\right|^{2}\right)+\frac{\sqrt{5}}{7}\left(2\left|H_{0}^{2}\right|^{2}+\left|H_{1}^{2}\right|^{2}+\left|H_{-1}^{2}\right|^{2}\right), \\
\sqrt{4 \pi} T_{30} & =2 \sqrt{\frac{27}{35}} \Re e\left(H_{0}^{1} H_{0}^{2 *}\right)-6 \sqrt{\frac{1}{35}}\left(\Re e\left(H_{1}^{1} H_{1}^{2 *}\right)+\Re e\left(H_{-1}^{1} H_{-1}^{2 *}\right)\right) \\
\sqrt{4 \pi} T_{40} & =\frac{2}{7}\left(3\left|H_{0}^{2}\right|^{2}-2\left(\left|H_{1}^{2}\right|^{2}+\left|H_{-1}^{2}\right|^{2}\right)\right) .
\end{aligned}
$$

L'analyse des résonances présentes dans la région $0.6-2.2 \mathrm{GeV} / c^{2}$ (cf. la table 4.5 ) se désintégrant en $K \pi$ permettent d'associer les ondes $S, P, D$ aux mésons $K_{0}^{*}(1430), K^{*}(892)$ et $K_{2}^{*}(1430)$ dont les Breit-Wigner sont représentées sur la figure 2.10. À noter la forme particulière de l'onde $S$ qui, en partant du seuil, croit d'abord lentement, puis plus rapidement jusqu'à atteindre le pic de la résonance. Elle occupe une grande portion de l'espace des phases et donc chevauche de nombreuses résonances, rendant l'extraction de ces différentes résonances difficiles. Il faut bien distinguer l'onde $S$ provenant de la diffusion $K \pi$ qui est décrite dans la référence [146] de l'onde $S$ provenant de la production $K \pi[142,147]$ qui intervient dans la désintégration $B \rightarrow J / \psi X, X \rightarrow K \pi$ et qui est présentée sur la figure 2.11. Un autre point qu'il convient de souligner est que l'onde $S$ est un système $K \pi$ résonant [146], bien que la partie qui croit lentement est souvent interprétée comme étant du $K \pi$ non résonant. Nous limitons l'appellation non résonant à une production de trois corps provenant de la désintégration du méson $B$, c'est-à-dire $B \rightarrow J / \psi K \pi$. En désignant par $l$ le moment angulaire du système $K \pi$ dans son repère au repos et $L$ le moment angulaire orbital du système $J / \psi(K \pi)$, on obtient par conservation du moment angulaire total les valeurs suivantes 
pour le « spin » $l$ du système $K \pi$ provenant de la désintégration non résonante $B \rightarrow J / \psi K \pi$ :

$$
l=0, L=1 ; \quad l=1, L=0,1,2 ; \quad l=2, L=1,2,3 ; \quad l=3, L=2,3,4 ; \quad \text { etc. }
$$

À noter que la désintégration $B \rightarrow J / \psi K \pi$ étant une désintégration faible, aucune contrainte ne peut être obtenue de la parité ou de la conjugaison de charge. Ainsi, le système $K \pi$ peut être dans un état d'onde $S, P, D, \ldots$ Il n'est pas possible de distinguer l'onde $S(P, D, \ldots)$ résonante de celle non résonante par une analyse angulaire seulement.

\subsubsection{Distribution angulaire avec ondes $S, P$ pour le système $K \pi$}

Afin de quantifier la présence de l'onde $S$ sous le pic du $K^{*}(892)$ ainsi que dans la région plateau $\left(\left|m_{K \pi}-1.2\right|<0.1 \mathrm{GeV} / c^{2}\right)$, on étend la distribution angulaire de la désintégration $B \rightarrow J / \psi K^{*}(892)$ (cf. eq. (2.73)) en ajoutant un terme correspondant à l'onde $S$. La distribution angulaire en base de transversité de la désintégration $B \rightarrow J / \psi X, X \rightarrow(K \pi)_{S+P}$ est donnée par :

$$
\begin{aligned}
& \frac{\left.\mathrm{d}^{4} \sigma \stackrel{(-)}{B} \rightarrow J / \psi \stackrel{(-)}{X}, \stackrel{(-)}{X} \rightarrow(K \pi)_{S+P}\right)}{\mathrm{d} m_{K \pi} \mathrm{d} \cos \theta_{X} \mathrm{~d} \cos \theta_{t r} \mathrm{~d} \varphi_{t r}} \propto p q\left[3 | B ^ { 1 } | ^ { 2 } \left[f_{1}\left|\stackrel{(-)}{A_{0}^{1}}\right|^{2}+f_{2}\left|\stackrel{(-)}{A_{\|}^{1}}\right|^{2}\right.\right. \\
& \left.+f_{3}\left|\stackrel{(-)}{A_{\perp}^{1}}\right|^{2}+f_{4} \Im m\left(\stackrel{(-)}{A_{\|}^{1 *}} A_{\perp}^{1}\right)+f_{5} \Re e\left(\stackrel{(-)}{A_{\|}^{1}} \stackrel{(-)}{A_{0}^{1 *}}\right)+f_{6} \Im m\left(\stackrel{(-)}{A_{\perp}^{1}} \stackrel{(-)}{A_{0}^{1 *}}\right)\right]+f_{7}\left|B^{0}\right|^{2}\left|\stackrel{(-)}{A_{0}^{0}}\right|^{2} \\
& \left.+f_{8} \Re e\left(B^{1} B^{0 *} \stackrel{(-)}{A_{\|}^{1}} \stackrel{(-)}{A_{0}^{0 *}}\right)+f_{9} \Im m\left(B^{1} B^{0 *} \stackrel{(-)}{A_{\perp}^{1}} \stackrel{(-)}{A_{0}^{0 *}}\right)+f_{10} \Re e\left(B^{1} B^{0 *} \stackrel{(-)}{A_{0}^{1}} \stackrel{(-)}{A_{0}^{0 *}}\right)\right],
\end{aligned}
$$

où les fonctions $f_{i}\left(\theta_{X}, \theta_{t r}, \varphi_{t r}\right)(i=1, \ldots, 10)$ sont définies par :

$$
\begin{aligned}
f_{1} & =\frac{3}{32 \pi} 2 \cos ^{2}\left(\theta_{X}\right)\left[1-\sin ^{2}\left(\theta_{t r}\right) \cos ^{2}\left(\varphi_{t r}\right)\right] \\
f_{2} & =\frac{3}{32 \pi} \sin ^{2}\left(\theta_{X}\right)\left[1-\sin ^{2}\left(\theta_{t r}\right) \sin ^{2}\left(\varphi_{t r}\right)\right] \\
f_{3} & =\frac{3}{32 \pi} \sin ^{2}\left(\theta_{X}\right) \sin ^{2}\left(\theta_{t r}\right) \\
f_{4} & =\frac{3}{32 \pi} \sin ^{2}\left(\theta_{X}\right) \sin \left(2 \theta_{t r}\right) \sin \left(\varphi_{t r}\right) \\
f_{5} & =-\frac{3}{32 \pi} \frac{1}{\sqrt{2}} \sin \left(2 \theta_{X}\right) \sin ^{2}\left(\theta_{t r}\right) \sin \left(2 \varphi_{t r}\right) \\
f_{6} & =\frac{3}{32 \pi} \frac{1}{\sqrt{2}} \sin \left(2 \theta_{X}\right) \sin \left(2 \theta_{t r}\right) \cos \left(\varphi_{t r}\right) \\
f_{7} & =\frac{3}{32 \pi} 2\left[1-\sin { }^{2}\left(\theta_{t r}\right) \cos ^{2}\left(\varphi_{t r}\right)\right] \\
f_{8} & =-\frac{3}{32 \pi} \sqrt{6} \sin \left(\theta_{X}\right) \sin ^{2}\left(\theta_{t r}\right) \sin \left(2 \varphi_{t r}\right) \\
f_{9} & =\frac{3}{32 \pi} \sqrt{6} \sin \left(\theta_{X}\right) \sin \left(2 \theta_{t r}\right) \cos \left(\varphi_{t r}\right) \\
f_{10} & =\frac{3}{32 \pi} 4 \sqrt{3} \cos \left(\theta_{X}\right)\left[1-\sin ^{2}\left(\theta_{t r}\right) \cos ^{2}\left(\varphi_{t r}\right)\right]
\end{aligned}
$$

$A^{J_{X}}=\langle J / \psi(\lambda) X(\lambda)|\mathcal{T}| B\rangle$ et $B^{J_{X}}=\langle K \pi|\mathcal{T}| X\rangle=B_{B W}^{J_{X}}(m) F^{J_{X}}$ (cf. eq. (2.101)). $p$ et $q$ sont les impulsions respectivement du système $(K \pi)$ dans le repère du méson $B$ au repos et du kaon dans 

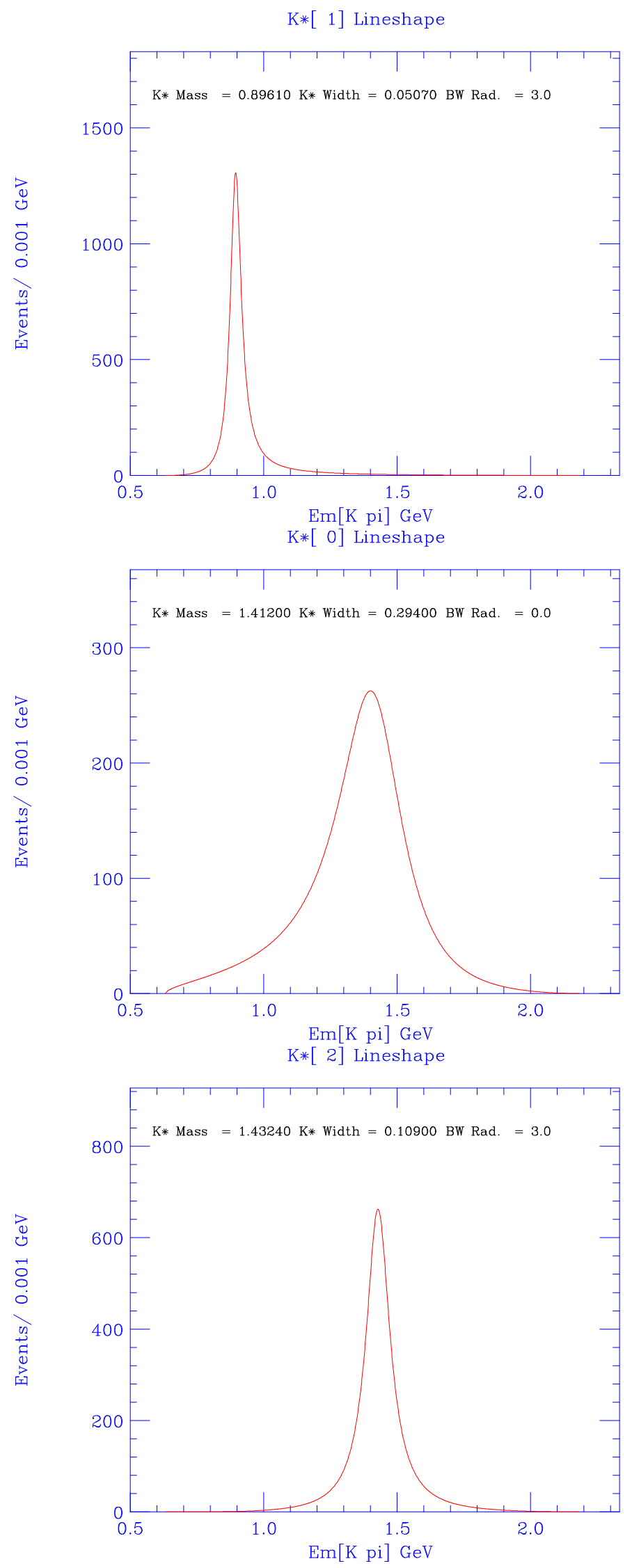

FIG. 2.10: Résonances $K^{*}(892), K_{0}^{*}(1430)$ et $K_{2}^{*}(1430)$ de spin $1,0,2$, respectivement. Les résonances sont normalisées de façon à avoir la même aire. 

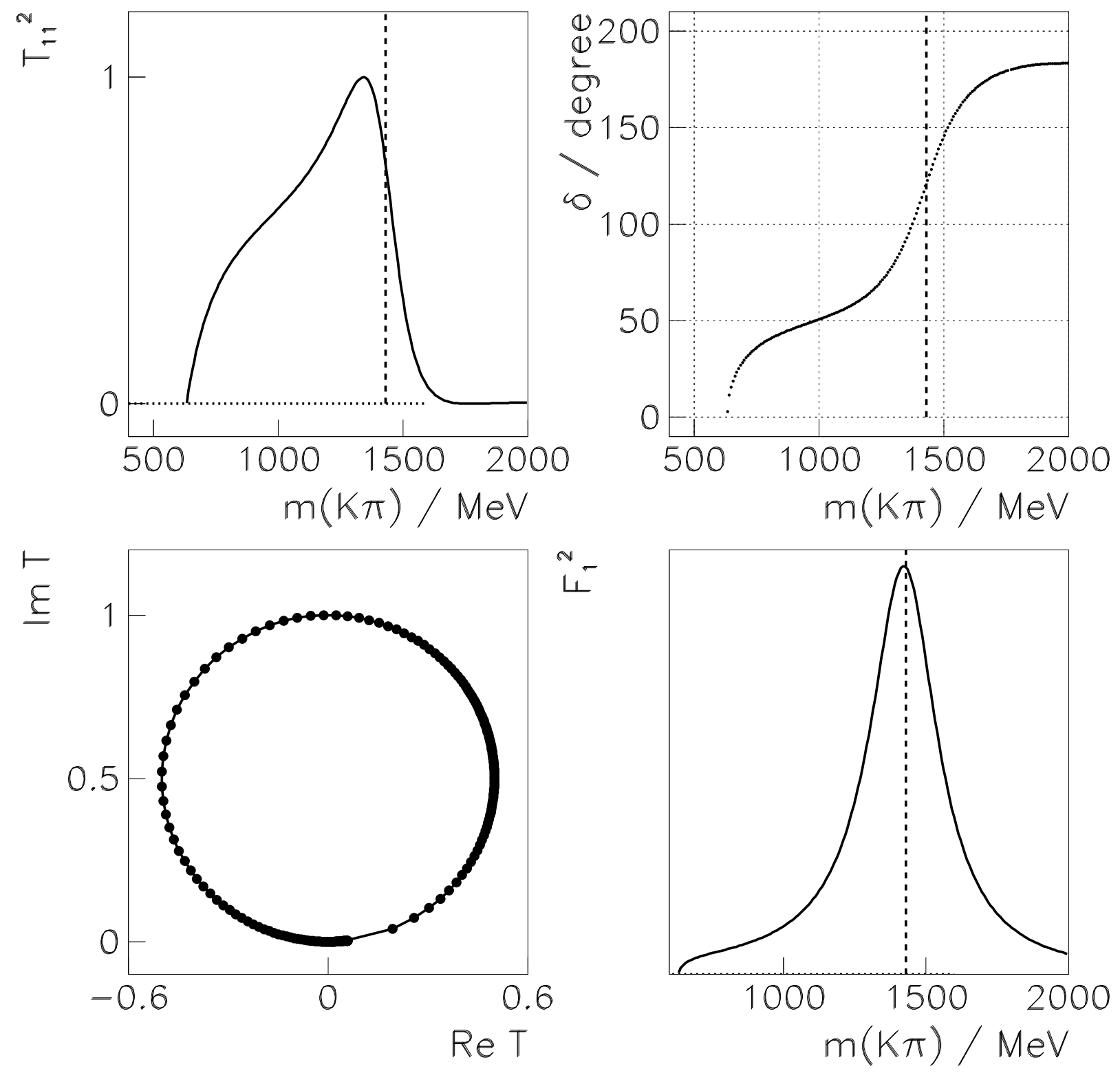

FIG. 2.11: Haut : module au carré de l'amplitude de diffusion $K \pi$ et la phase $\delta_{j}$ pour une onde $S$. La position du pôle de l'amplitude $T_{j}$ est représentée par une ligne en pointillé. Bas : diagramme d'Argand. Le mouvement rapide de la phase en fonction de la masse $K \pi$ est décalé de $(0, i)$. La figure de droite montre l'amplitude de production, à comparer avec la figure 2.10. D’après [147]. 
le repère du système $(K \pi)$ au repos. En l'absence de violation de $C P$ dans la désintégration, les amplitudes $\bar{A}^{J_{X}}$ et $A^{J_{X}}$ sont reliées par (cf. §2.2.2) :

$$
\bar{A}_{0}^{0}=A_{0}^{0}, \quad \bar{A}_{0}^{1}=A_{0}^{1}, \bar{A}_{\|}^{1}=A_{\|}^{1}, \bar{A}_{\perp}^{1}=-A_{\perp}^{1},
$$

en prenant les conventions $\mathcal{C P}|B\rangle=|\bar{B}\rangle$ et $\mathcal{C} \mathcal{P}|X\rangle=(-1)^{J_{X}+1}|\bar{X}\rangle$ (le choix $(-1)^{J_{X}+1}$ est motivé par la désintégration $X \rightarrow K_{S}^{0} \pi^{0}$, état propre de $C P$ de valeur propre $(-1)^{J_{X}+1}$. Les autres désintégrations $X \rightarrow K \pi$ n'étant pas états propres de $C P$, la transformation $C P$ reliant $X$ à $\bar{X}$ est définie à une phase arbitraire près que l'on peut choisir à sa convenance sans conséquence sur les résultats physiques. Ce choix permet aussi d'avoir les mêmes relations entre les amplitudes de transversité $A$ et $\bar{A}$, quel que soit le spin de la particule $X$ ). À noter que les amplitudes $B^{J_{X}}$ décrivent le processus fort $X \rightarrow K \pi$, la symétrie $C P$ est donc conservée. Une version complète incluant les ondes $S, P, D$ en bases d'hélicité et de transversité est donnée dans l'appendice D. 


\section{Chapitre 3}

\section{Dispositif expérimental}

Le but principal de l'expérience BABAR est la mesure de la violation de $C P$ dans le secteur des mésons $B$. Leurs taux d'embranchement sont relativement faibles $\left(\leq 10^{-3}\right)$, ce qui nécessite une production importante de mésons $B$, d'où le terme " usine à $B$ ". L'étude de la violation de $C P$ étant basée sur la comparaison de taux de désintégration entre mésons $B_{d}^{0}$ et $\bar{B}_{d}^{0}$, il nous faut connaître la saveur du méson $B$. Le méson $B$ reconstruit dans un état propre de $C P$ ne permet pas à lui seul de déterminer sa saveur. Dans les usines à $B$, les mésons $B$ sont produits par paires via la réaction $e^{+} e^{-} \rightarrow \Upsilon(4 S) \rightarrow B \bar{B}$, où la résonance $\Upsilon(4 S)$ (état lié $b \bar{b}$ ), se désintègre en $B_{u}^{+} B_{u}^{-}$ $(\sim 50 \%)$ et $B_{d}^{0} \bar{B}_{d}^{0}(\sim 50 \%)$. Le point essentiel est que la paire $B_{d}^{0} \bar{B}_{d}^{0}$ provenant de la désintégration de la résonance $\Upsilon(4 S)$, état $J^{P C}=1^{--}$, est générée dans un état cohérent $L=1$, antisymétrique sous l'échange des deux mésons. L'état initial s'écrit dans le repère de $1^{\prime} \Upsilon(4 S)$ au repos [22, 23] :

$$
|i\rangle=\frac{1}{\sqrt{2}}\left(\left|B_{d}^{0}(\vec{k})\right\rangle \otimes\left|\bar{B}_{d}^{0}(-\vec{k})\right\rangle-\left|\bar{B}_{d}^{0}(\vec{k})\right\rangle \otimes\left|B_{d}^{0}(-\vec{k})\right\rangle\right),
$$

où $\vec{k}$ et $-\vec{k}$ sont les impulsions de chaque méson $B$, respectivement. La conséquence de ceci est qu'à tout moment, et ce malgré leur oscillation, les deux mésons $B$ neutres restent de saveurs opposées jusqu'à ce qu'un méson $B$ se désintègre. Ainsi, au moment de la désintégration du premier méson $B$ neutre, l'autre méson $B$ neutre est de saveur opposée [148]. C'est un exemple du paradoxe EPR [149]. Pour déterminer la saveur du méson $B$ reconstruit dans un état propre de $C P, B_{C P}$, on mesure en pratique la saveur opposée de l'autre méson $B, B_{t a g}$, au moment de sa désintégration grâce à ses produits de désintégration en utilisant notamment le signe de leptons ou de kaons. Cette mesure s'appelle l'étiquetage. Cela nécessite l'emploi de dispositifs d'identification d'électrons, de muons et de kaons (pour plus de détails sur l'étiquetage, voir §6.2).

En plus de sa saveur, il nous faut connaître la durée de vie du méson $B_{C P}$, entrant dans l'équation (1.57). Rappelons que dans (1.57), $t=0$ définit l'instant de production du méson $B$, dans un état de saveur bien défini, $B_{d}^{0}$ ou $\bar{B}_{d}^{0}$. Plus généralement, nous introduisons la variable $\Delta t$, où $\Delta t=0$ définit un instant où le méson $B_{C P}$ est de saveur connue (pas nécessairement l'instant de production). Dans la production cohérente de paires de mésons $B$ neutres, $\Delta t=0$ est pris comme l'instant 


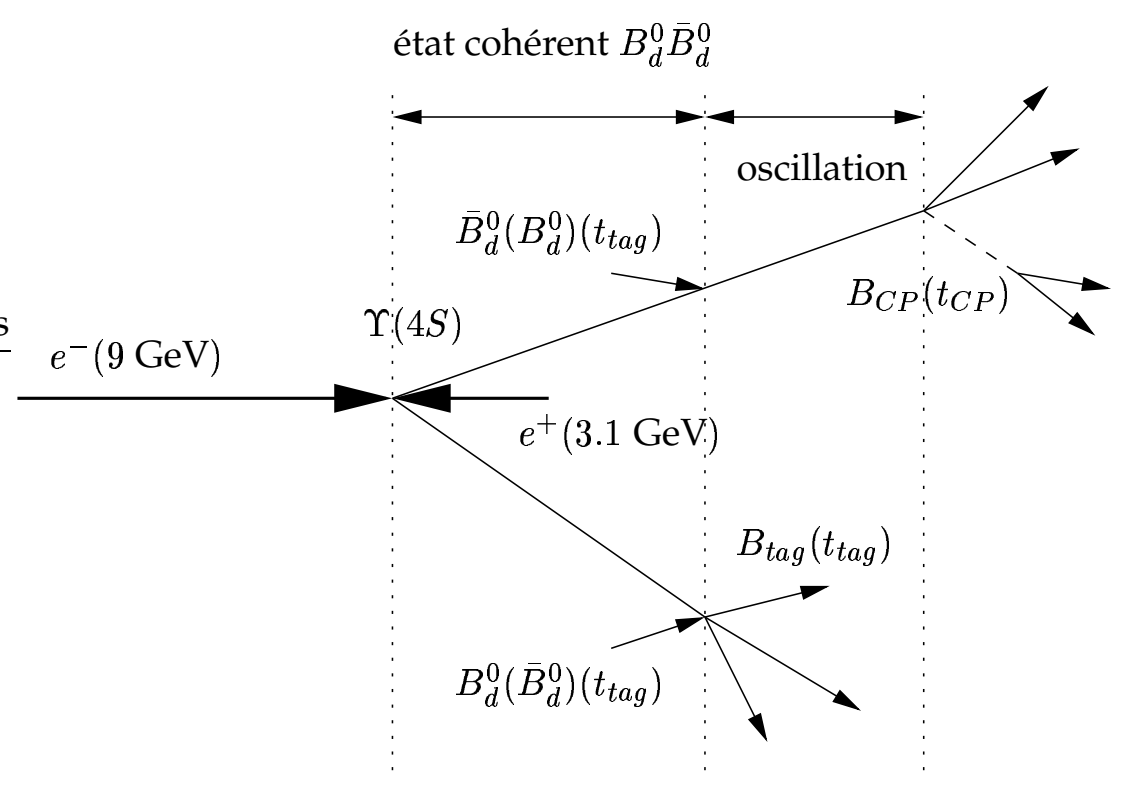

FIG. 3.1: Principe de la mesure de $\Delta t \equiv t_{C P}-t_{\text {tag }}$ basé sur la production cohérente d'une paire de mésons $B_{d}^{0} \bar{B}_{d}^{0}$, via la résonance $\Upsilon(4 S)$, dans un collisionneur asymétrique en énergie. Si le méson $B_{t a g}$ est dans un état $B_{d}^{0}\left(\bar{B}_{d}^{0}\right)$ alors le méson $B_{C P}$ est dans l'état $\bar{B}_{d}^{0}\left(B_{d}^{0}\right)$ à l'instant de désintégration, $t_{t a g}$, du méson $B_{\text {tag }}$.

de désintégration du méson $B_{t a g}$ (voir figure 3.1). L'asymétrie prend alors la forme [150] :

$$
\begin{aligned}
\mathcal{A}_{f_{C P}}(\Delta t) & =\frac{\Gamma\left(B_{t a g} \rightarrow \bar{B}_{d}^{0}\left(t_{t a g}\right), B_{C P} \rightarrow f_{C P}\left(t_{C P}\right)\right)-\Gamma\left(B_{t a g} \rightarrow B_{d}^{0}\left(t_{t a g}\right), B_{C P} \rightarrow f_{C P}\left(t_{C P}\right)\right)}{\Gamma\left(B_{t a g} \rightarrow \bar{B}_{d}^{0}\left(t_{t a g}\right), B_{C P} \rightarrow f_{C P}\left(t_{C P}\right)\right)+\Gamma\left(B_{t a g} \rightarrow B_{d}^{0}\left(t_{t a g}\right), B_{C P} \rightarrow f_{C P}\left(t_{C P}\right)\right)} \\
& =\frac{1-\left|\lambda_{f_{C P}}\right|^{2}}{1+\left|\lambda_{f_{C P}}\right|^{2}} \cdot \cos (\Delta M \Delta t)-\frac{2 I m \lambda_{f_{C P}}}{1+\left|\lambda_{f_{C P}}\right|^{2}} \cdot \sin (\Delta M \Delta t)
\end{aligned}
$$

où $\Delta t$ est la différence de temps propres entre la désintégration du méson $B_{C P}$ et du méson $B_{t a g}$, $\Delta t \equiv t_{C P}-t_{\text {tag }} . \Gamma\left(B_{\text {tag }} \rightarrow \bar{B}_{d}^{0}\left(B_{d}^{0}\right)\left(t_{t a g}\right), B_{C P} \rightarrow f_{C P}\left(t_{C P}\right)\right)$ est le taux de désintégration du méson $B_{t a g}$ en un état final spécifique de saveur permettant de signer cette saveur à l'instant de sa désintégration, $t_{t a g}$, et du méson $B_{C P}$ dans l'état propre de $C P$ au temps $t_{C P}$. L'asymétrie (3.2) dépend de la différence de temps, $\Delta t$, du fait de l'antisymétrie ${ }^{1}$ de l'état défini à l'équation (3.1). Par rapport à l'asymétrie définie à l'équation (1.57), le temps $t \equiv t_{C P}$ est translaté de $t=0$ à $t=t_{\text {tag }}$. Le méson $B_{C P}$ pouvant se désintégrer avant ou après le méson $B_{t a g}, \Delta t$ s'étend de $-\infty$ à $+\infty$. Remarquons qu'un tel système ne permet pas la mesure d'une asymétrie intégrée en temps, qui est nulle. Il est donc nécessaire d'effectuer la mesure d'une asymétrie dépendante du temps.

La nécessité de mesurer cette différence de temps a déterminé le choix d'un collisionneur asymétrique en énergie. Dans un collisionneur asymétrique, les deux mésons $B$ voyagent dans la même direction, quasiment le long des faisceaux, de sorte que la différence temporelle des deux désintégrations, $t_{C P}-t_{t a g}$, est proportionnelle à la séparation spatiale des vertex de désintégration. Cela permet de s'affranchir de la connaissance du vertex de production. En revanche (cf. Fig. 3.2), dans un collisionneur symétrique, les deux mésons $B$ voyagent dans des directions opposées et la séparation spatiale des vertex de désintégration est proportionnelle à la somme des temps, $t_{C P}+t_{t a g}$. La mesure de la différence requiert également la mesure du vertex de production des mésons $B$ afin

\footnotetext{
${ }^{1}$ Dans le cas d'un état symétrique, (tel est le cas de la paire $B^{0} \bar{B}^{0}$ provenant de la désintégration $e^{+} e^{-} \rightarrow B^{0 *} \bar{B}^{0} \rightarrow$ $B^{0} \bar{B}^{0} \gamma$ ) l'asymétrie (3.2) dépendrait de la somme des temps propres [22].
} 

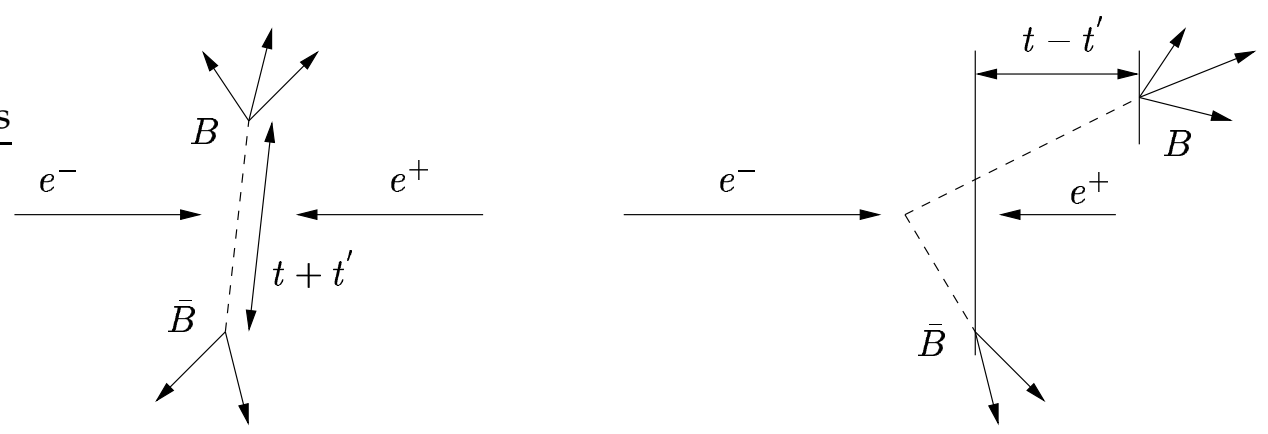

FIG. 3.2: Illustration de la désintégration $\Upsilon(4 S) \rightarrow B \bar{B}$ dans le cas d'un collisionneur symétrique et d'un collisionneur asymétrique en énergie. La distance entre les vertex de désintégration des mésons dans le cas d'un collisionneur symétrique est proportionnelle à la somme des temps de vie de chaque méson $B$. Dans un collisionneur asymétrique, la séparation spatiale entre les vertex de désintégration dans la direction du «boost » est proportionnelle à la différence des temps de vie de chaque méson $B[22,23,151]$.

de mesurer individuellement $t$ et $t^{\prime}$. Cependant, puisque la masse de la résonance $\Upsilon(4 S)$ est proche du seuil de création de la paire $B \bar{B}$, chaque méson $B$ ne parcourt en moyenne que $30 \mu \mathrm{m}$ (voir la note en bas de page 2), une distance qui, en pratique, est insuffisante pour la mesure de $t_{C P}-t_{t a g}$.

De ce qui précède, nous concluons que la mesure de la violation de $C P$ dans le système des mésons $B$ produit par la résonance $\Upsilon(4 S)$ requiert :

- une haute luminosité ;

- de bonnes performances d'identification de leptons et kaons ;

- une bonne mesure des vertex de désintégration des mésons $B$;

- un collisionneur asymétrique.

Ce sont ces considérations qui ont guidé la conception de l'usine à $B$, PEP-II [152], et du détecteur BABAR [153].

\subsection{Le collisionneur PEP-II}

Installé à la suite de l'accélérateur linéaire présent sur le site du Stanford Linear Accelerator Center (SLAC) en Californie (USA), PEP-II est un collisionneur composé de deux anneaux de stockage de $2.2 \mathrm{~km}$ de circonférence (cf. Fig. 3.3). L'anneau dit de « haute énergie » produit le faisceau d'électron de $9 \mathrm{GeV}$ (HER $\equiv$ High Energy Ring) ; tandis que l'anneau dit de «basse énergie » produit le faisceau de positons de $3.1 \mathrm{GeV}$ (LER $\equiv$ Low Energy Ring). Les faisceaux entrent en collision frontale et sont séparés du plan horizontal par une paire de dipôles magnétiques permanents située à $\pm 21 \mathrm{~cm}$ du point d'interaction. La présence d'éléments de PEP-II à l'intérieur du détecteur BABAR s'explique $\mathrm{du}$ fait que l'espacement entre les paquets est relativement faible (initialement prévu de $1.26 \mathrm{~m}$ mais qui est jusqu'à présent de $2.52 \mathrm{~m}$ (cf. la table 3.1)). Cela implique que le premier point de croisement parasite est situé à environ $1 \mathrm{~m}$ (63 cm avec les paramètres de conception). Afin d'éviter une collision en ce point, les faisceaux doivent être séparés aussitôt après le point de collision. À noter que l'axe de collision est légèrement décalé de $20 \mathrm{mrad}$ dans le plan horizontal par rapport à l'axe $z$ du détecteur BABAR afin d'atténuer la perturbation de l'orbite des faisceaux par le champ solénoïdal de BABAR $[153,154]$.

L'énergie dans le centre de masse est de $10.58 \mathrm{GeV}$, correspondant à la masse de la résonance 


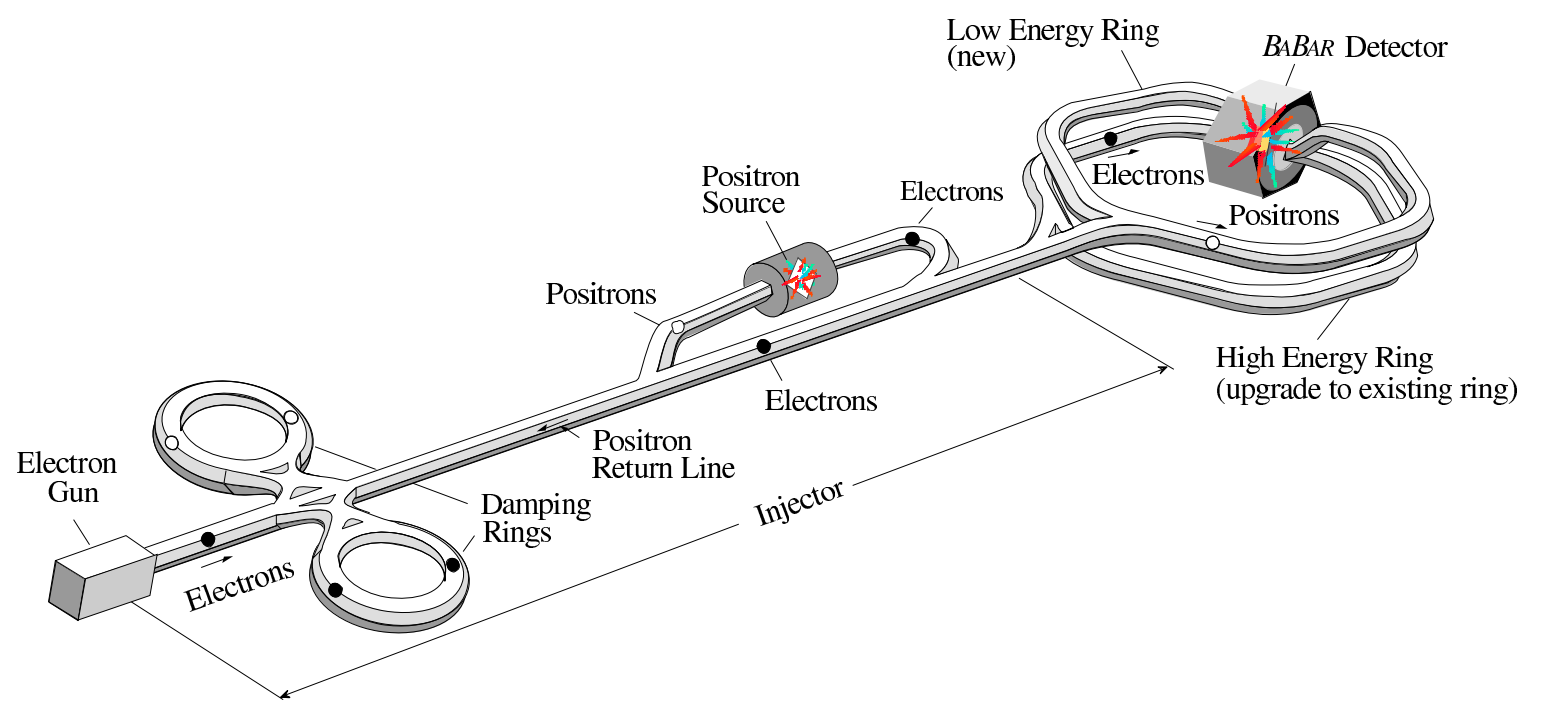

FIG. 3.3: Le collisionneur PEP-II et l'accélérateur linéaire de SLAC.

$\Upsilon(4 S)$, produite avec un $\langle\beta \gamma\rangle$ de 0.56 par rapport au laboratoire dans la direction du faisceau d'électrons (HER). L'impulsion des mésons $B$ dans le repère de $1^{\prime} \Upsilon(4 S)$ au repos est $p_{B}^{*}=\sqrt{s / 4-M_{B}^{2}} \approx$ $341 \mathrm{MeV} / c$. À noter que dans ce repère, $\gamma^{*}=E_{B}^{*} / M_{B}=1.002$ et $\beta^{*}=p_{B}^{*} / E_{B}^{*}=0.064$, les mésons $B$ sont non relativistes (dans le repère du laboratoire, $\beta_{B}=p_{B} / E_{B} \in[0.462,0.512]$ et $\left.\gamma_{B}=E_{B} / M_{B} \in[1.111,1.183]\right)$. La différence des temps propres de désintégration $\Delta t$ des deux mésons $B$ est, à une excellente approximation, proportionnelle à la séparation spatiale $\Delta z$ le long de l'axe $z$ de BABAR, $\Delta z \approx\left\langle\beta_{z} \gamma\right\rangle c \Delta t$. L'utilisation de $\beta_{z}$, projection de $\vec{\beta}$ sur l'axe $z$ de BABAR, au lieu de $\beta$, prend en compte le léger décalage de $20 \mathrm{mrad}$ dans le plan horizontal entre l'axe de collision des faisceaux et l'axe $z$ de BABAR, réduisant ainsi le «boost » initial d'environ $0.02 \%$. La séparation moyenne entre les deux vertex de désintégration des mésons $B$ est $\Delta z=\left\langle\beta_{z} \gamma\right\rangle c \tau_{B}=260 \mu \mathrm{m}$, mesurable par le détecteur de vertex ${ }^{2}$ de BABAR. Les paramètres de ces anneaux de stockage sont résumés dans la table 3.1.

TAB. 3.1: Paramètres des faisceaux de PEP-II.

\begin{tabular}{lcc}
\hline Paramètres & Conception & Typique \\
\hline Énergie HER/LER $(\mathrm{GeV})$ & $9.0 / 3.1$ & $9.0 / 3.1$ \\
Courant HER/LER $(\mathrm{A})$ & $0.75 / 2.15$ & $1.05 / 1.78$ \\
$\mathrm{Nb}$. de paquets & 1658 & 800 \\
Espacement entre paquet $(\mathrm{ns})$ & 4.2 & 8.4 \\
$\sigma_{L x}(\mu \mathrm{m})$ & 110 & 120 \\
$\sigma_{L y}(\mu \mathrm{m})$ & 3.3 & 5.6 \\
$\sigma_{L z}(\mathrm{~mm})$ & 9 & 9 \\
Luminosité $\left(10^{33} \mathrm{~cm}^{-2} \mathrm{~s}^{-1}\right)$ & 3 & 4 \\
Luminosité $\left(\mathrm{pb}^{-1} /\right.$ jour $)$ & 135 & 240 \\
\hline
\end{tabular}

\footnotetext{
${ }^{2}$ Sans «boost », i.e. dans un collisionneur symétrique, on peut montrer que la séparation moyenne entre les vertex de désintégration des mésons $B$ est $\Delta z=2\left\langle\beta^{*} \gamma^{*}\right\rangle c \tau_{B}=60 \mu \mathrm{m}$ [151], inférieure au pouvoir de séparation des détecteurs de vertex actuels.
} 
La production de $b \bar{b}$ n'est pas le seul processus se produisant au pic de la résonance $\Upsilon(4 S)$. La table 3.2 montre les sections efficaces de production des autres processus. Deux types de données sont fournis par PEP-II : les données au pic de la résonance (on-peak) et les données $40 \mathrm{MeV}$ environ en dessous du pic de la résonance pour l'étude des bruits de fond non résonants (off-peak). Ces données off-peak représentent près de $10 \%$ du volume total des données. D’octobre 1999 à Juin 2002, PEP-II a fourni pratiquement $100 \mathrm{fb}^{-1}$, illustré par la figure 3.4, soit un échantillon de près de 50 millions de paires $B_{d}^{0} \bar{B}_{d}^{0}$.

TAB. 3.2: Sections efficaces à $\sqrt{s}=10.58 \mathrm{GeV}$.

\begin{tabular}{|c|c|}
\hline$e^{+} e^{-} \rightarrow$ & section efficace (nb) \\
\hline \hline$b b$ & 1.10 \\
$c \bar{c}$ & 1.30 \\
$s \bar{s}$ & 0.35 \\
$u \bar{u}$ & 1.39 \\
$d \bar{d}$ & 0.35 \\
\hline$\tau^{+} \tau^{-}$ & 0.94 \\
$\mu^{+} \mu^{-}$ & 1.16 \\
$e^{+} e^{-}$ & $\sim 40$ \\
\hline
\end{tabular}

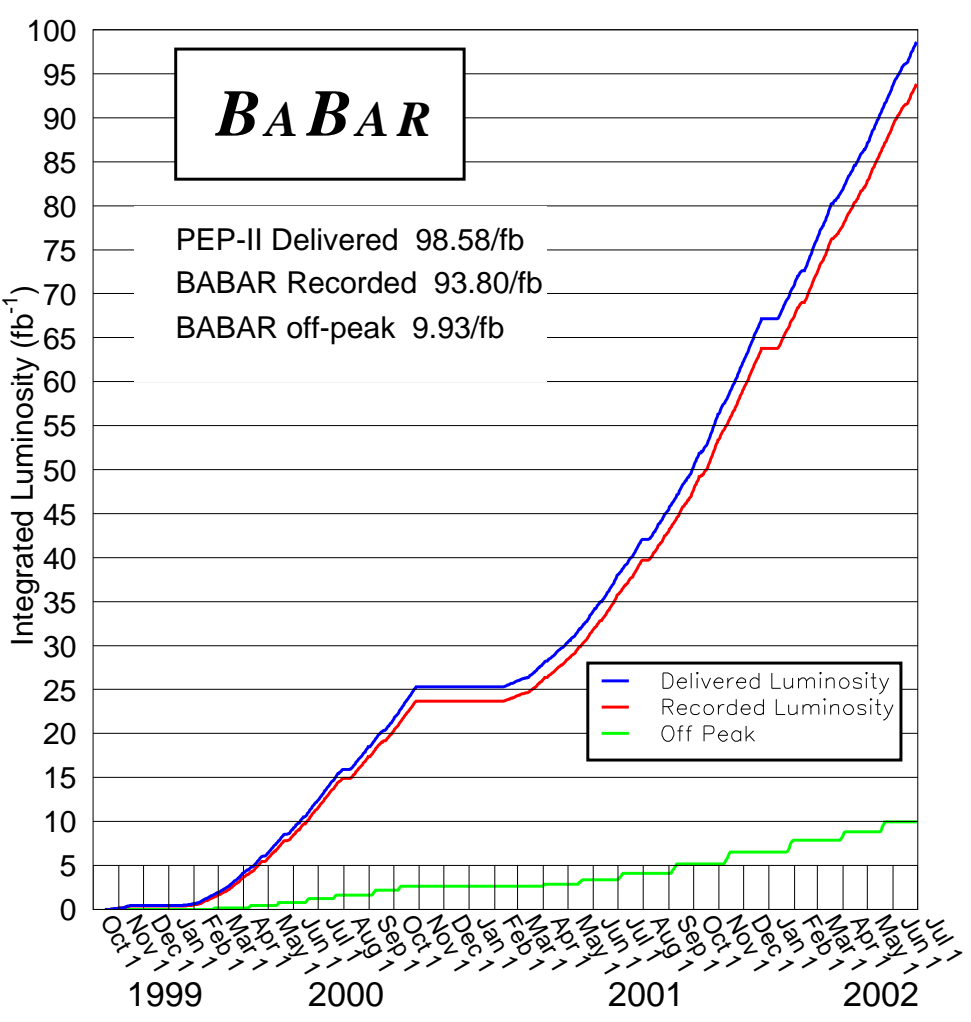

FIG. 3.4: Luminosité intégrée fournie par PEP-II et enregistrée par BABAR. 


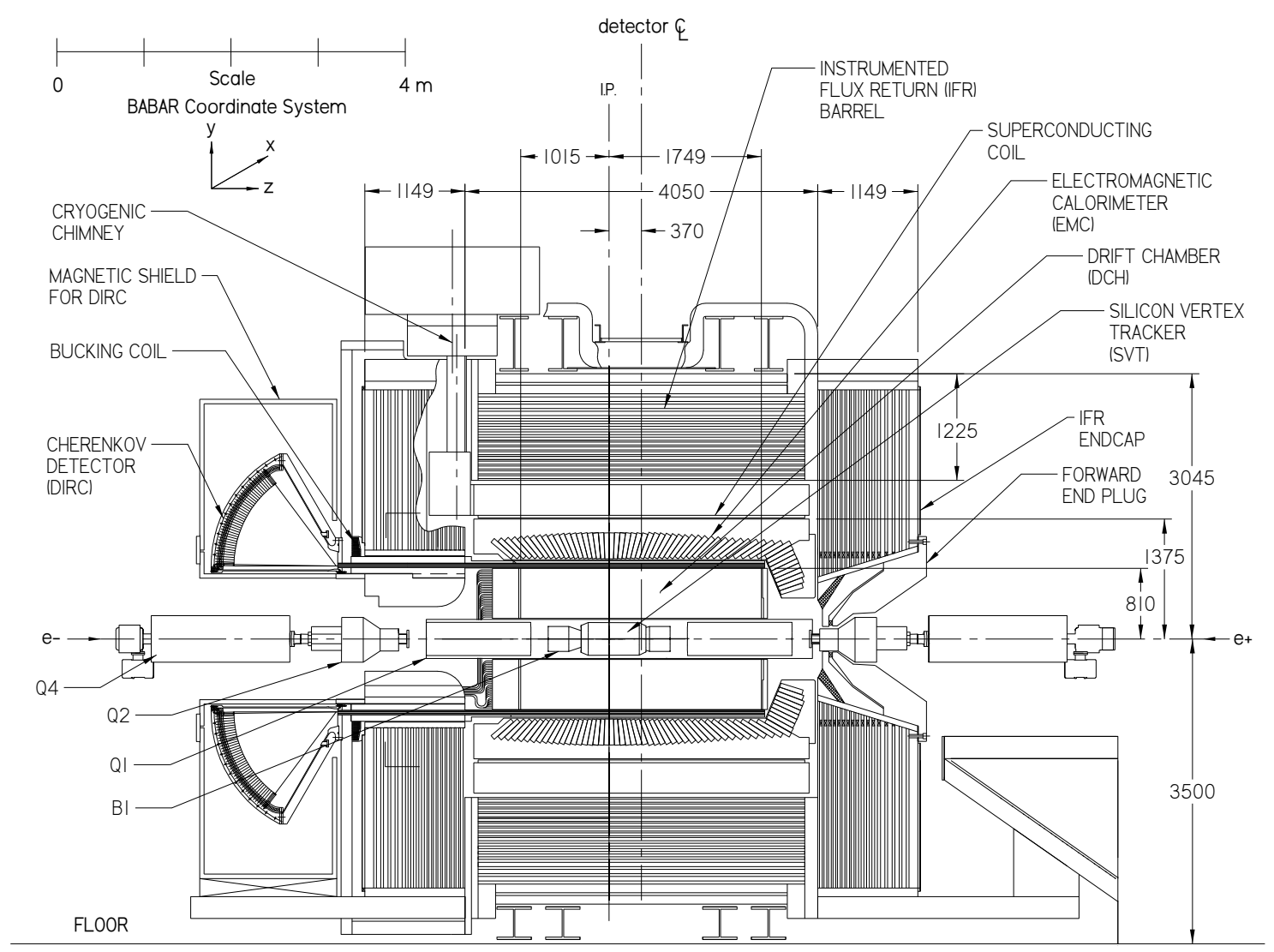

FIG. 3.5: Coupe longitudinale du détecteur BABAR.

\subsection{Le détecteur BAB AR}

Le détecteur installé sur le collisionneur PEP-II, du nom de BABAR, est de conception classique, cylindrique, asymétrique avec une acceptance géométrique proche de $4 \pi$. La figure 3.5 montre une section longitudinale du détecteur passant par son centre et la figure 3.6 montre la vue arrière du détecteur avec les dimensions principales. En allant du point d'interaction vers l'extérieur, on trouve :

- un détecteur de vertex (SVT) permettant la détermination des vertex des deux mésons $B$ ainsi que la mesure des impulsions des traces chargées de faible énergie $\left(p_{t} \leq 100 \mathrm{MeV} / c\right)$;

- la chambre à dérive $(\mathrm{DCH})$ mesurant les impulsions des traces chargées. Le SVT et la DCH fournissent des informations de $d E / d x$ pour l'identification des particules ;

- le DIRC, basé sur l'effet Cherenkov, est optimisé pour l'identification des $K, \pi$ et $p$;

- le calorimètre $(E M C)$ permettant la détection des photons et servant à l'identification des électrons ;

- un aimant supraconducteur fournissant un champ de $1.5 \mathrm{~T}$;

- le retour de flux instrumenté (IFR) pour la détection des muons et des $K_{L}^{0}$.

Cette section décrit l'ensemble des sous-détecteurs qui constituent le détecteur BABAR. 


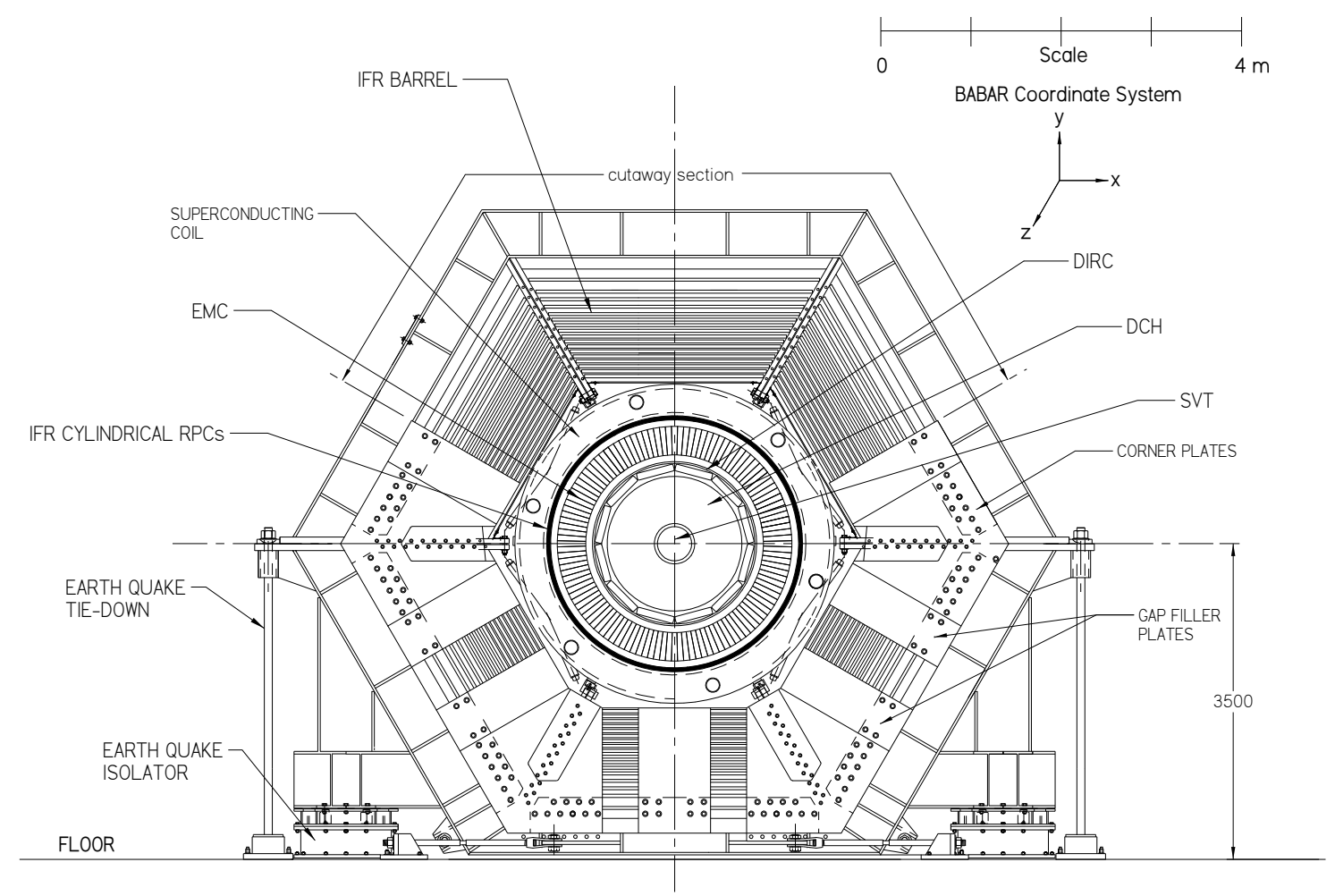

FIG. 3.6: Vue arrière du détecteur BABAR.

\subsubsection{Détecteur de vertex (SVT)}

Le SVT (Silicon Vertex Tracker) a été conçu principalement pour la reconstruction des vertex de désintégration des mésons $B$. Cependant, il permet aussi la reconstruction des trajectoires des particules chargées. Pour les particules d'impulsion transverse, $p_{t}$, inférieure à $100 \mathrm{MeV} / c$, seul le SVT permet la reconstruction de leur trajectoire. Pour les particules ayant une impulsion plus importante, les informations fournies par le SVT et par la chambre à dérive sont combinées. Enfin, il contribue à l'identification des particules par la mesure de leur perte d'énergie par ionisation $(d E / d x)$.

Le SVT est installé au plus proche du point d'interaction. Il est composé de cinq couches cylindriques concentriques de plaquettes de silicium (cf. Fig. 3.7) de $300 \mu \mathrm{m}$ d'épaisseur à lecture sur les deux faces. Les trois couches internes sont composées de six modules. Les deux couches externes

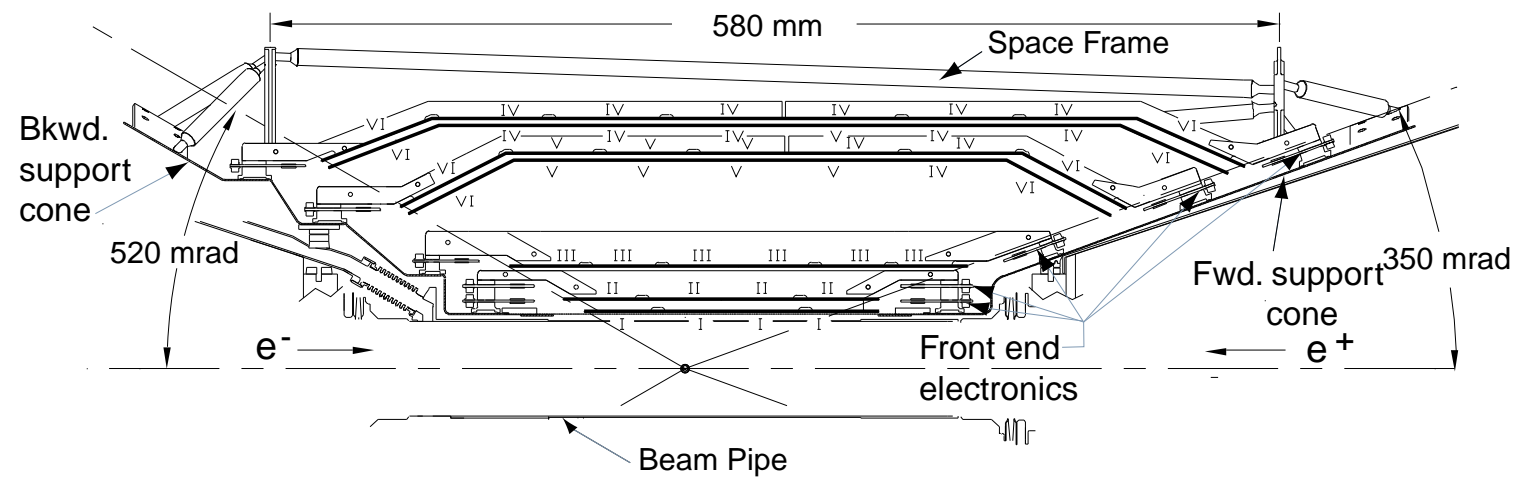

FIG. 3.7: Section longitudinale du SVT. 


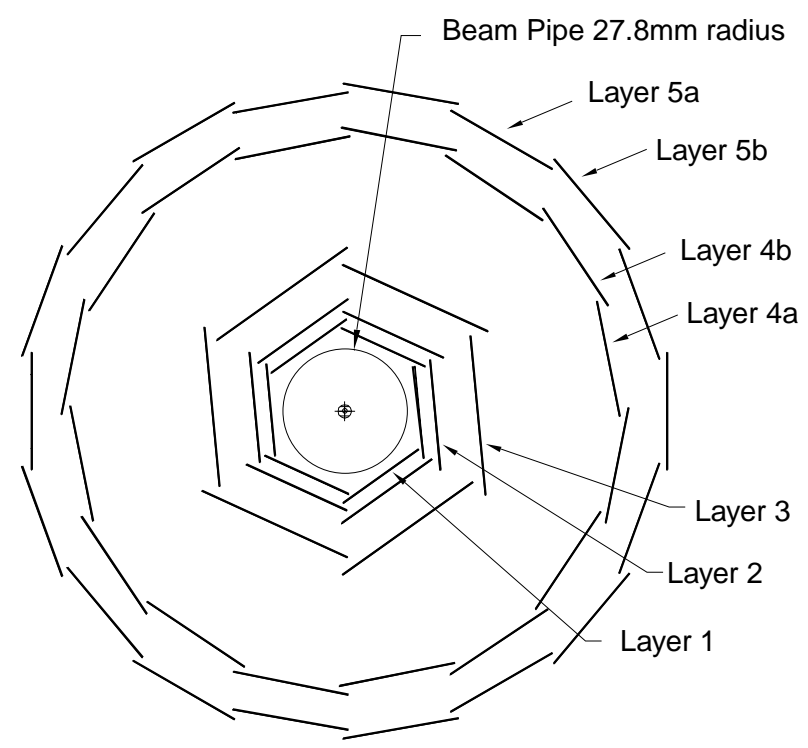

FIG. 3.8: Section transverse du SVT.

consistent en 16 et 18 modules, respectivement. Les faces internes ont leurs pistes orientées perpendiculairement à la direction des faisceaux pour la mesure de la coordonnée $z$, alors que les faces externes, avec leurs pistes parallèles à la direction des faisceaux, permettent la mesure de la coordonnée $\phi$. Afin d'éviter les zones mortes en $\phi$ les couches 4 et 5 sont divisées en deux sous-couches $(4 a, 4 b, 5 a, 5 b)$ et placées à des rayons légèrement différents (voir Fig. 3.8). Le SVT totalise 340 détecteurs au silicium couvrant une surface de $0.96 \mathrm{~m}^{2}$, avec un total de canaux de lecture de l'ordre de 150000 dont la répartition est détaillée dans la table 3.3. L'acceptance géométrique du SVT, limitée par les éléments de PEP-II situés à l'intérieur de BABAR (dipôles magnétiques), couvre l'angle polaire, dans le référentiel du laboratoire, de $20^{\circ}$ à $150^{\circ}$, soit $-0.87<\cos \theta<0.94$. Cela correspond à $90 \%$ de l'angle solide dans le centre de masse $\left(-0.95<\cos \theta^{*}<0.87\right)$. Enfin, le SVT doit pouvoir supporter 2 Mrad de radiation. La dose attendue est de $1 \mathrm{rad} /$ jour dans le plan horizontal, à cause de la séparation des faisceaux, immédiatemment après le tube à vide et $0.1 \mathrm{rad} /$ jour en moyenne ailleurs. La dose accumulée par le SVT depuis le début des prises de données est illustrée sur la figure 3.9.

La distance moyenne de séparation entre les deux mésons $B$ est de l'ordre de $260 \mu \mathrm{m}$. Afin d'éviter un impact trop important de la résolution sur la mesure d'asymétries $C P$, la résolution moyenne sur le vertex le long de l'axe $z$ pour un méson $B$ entièrement reconstruit doit être meilleure que $80 \mu \mathrm{m}$. Les diffusions multiples limitent la résolution, meilleure sur les couches internes que sur les couches externes. Les mesures effectuées montrent que la résolution spatiale, pour les traces perpendiculaires, est de $15 \mu \mathrm{m}$ pour les trois premières couches et de l'ordre de $40 \mu \mathrm{m}$ pour les deux dernières couches, en accord avec les résultats obtenus par simulation Monte Carlo.

\subsubsection{Chambre à dérive $(\mathrm{DCH})$}

La chambre à dérive (Drift CHamber) a pour but principal la détection des traces chargées et la mesure précise de leurs impulsions et angles. Elle fournit jusqu'à 40 points de mesure assurant une grande efficacité de reconstruction pour les traces d'impulsion transverse supérieure à $100 \mathrm{MeV} / c$. 
TAB. 3.3: Caractéristiques du SVT. Les résolutions sont calculées pour des traces incidentes à $90^{\circ}$, en considérant un rapport signal sur bruit, $S / N=20: 1$. Les chiffres entre parenthèses correspondent aux rayons des couches 4 a et $5 a$ dans la région centrale.

\begin{tabular}{|l|c|c|c|c|c|c|c|}
\hline & Couche & Couche & Couche & Couche & Couche & Couche & Couche \\
& 1 & 2 & 3 & $4 \mathrm{a}$ & $4 \mathrm{~b}$ & $5 \mathrm{a}$ & $5 \mathrm{~b}$ \\
\hline \hline Rayon $(\mathrm{mm})$ & 32 & 40 & 54 & $91(124)$ & 127 & $114(140)$ & 144 \\
Modules/couche & 6 & 6 & 6 & 8 & 8 & 9 & 9 \\
\hline Séparation des pistes : & & & & & & \\
$\quad$ en $\phi(\mu \mathrm{m})$ & $50-100$ & $55-110$ & 110 & 100 & 100 \\
en $z(\mu \mathrm{m})$ & 100 & 100 & 100 & 210 & 210 \\
\hline Canaux de lecture & 18432 & 24576 & 30720 & 32768 & 36864 \\
\hline$\sigma_{\phi}(\mu \mathrm{m})$ & 10 & 10 & 10 & $10-12$ & $10-12$ \\
$\sigma_{z}(\mu \mathrm{m})$ & 12 & 12 & 12 & 25 & 25 \\
\hline
\end{tabular}

SVTRAD : Accumulated Dose and Budget

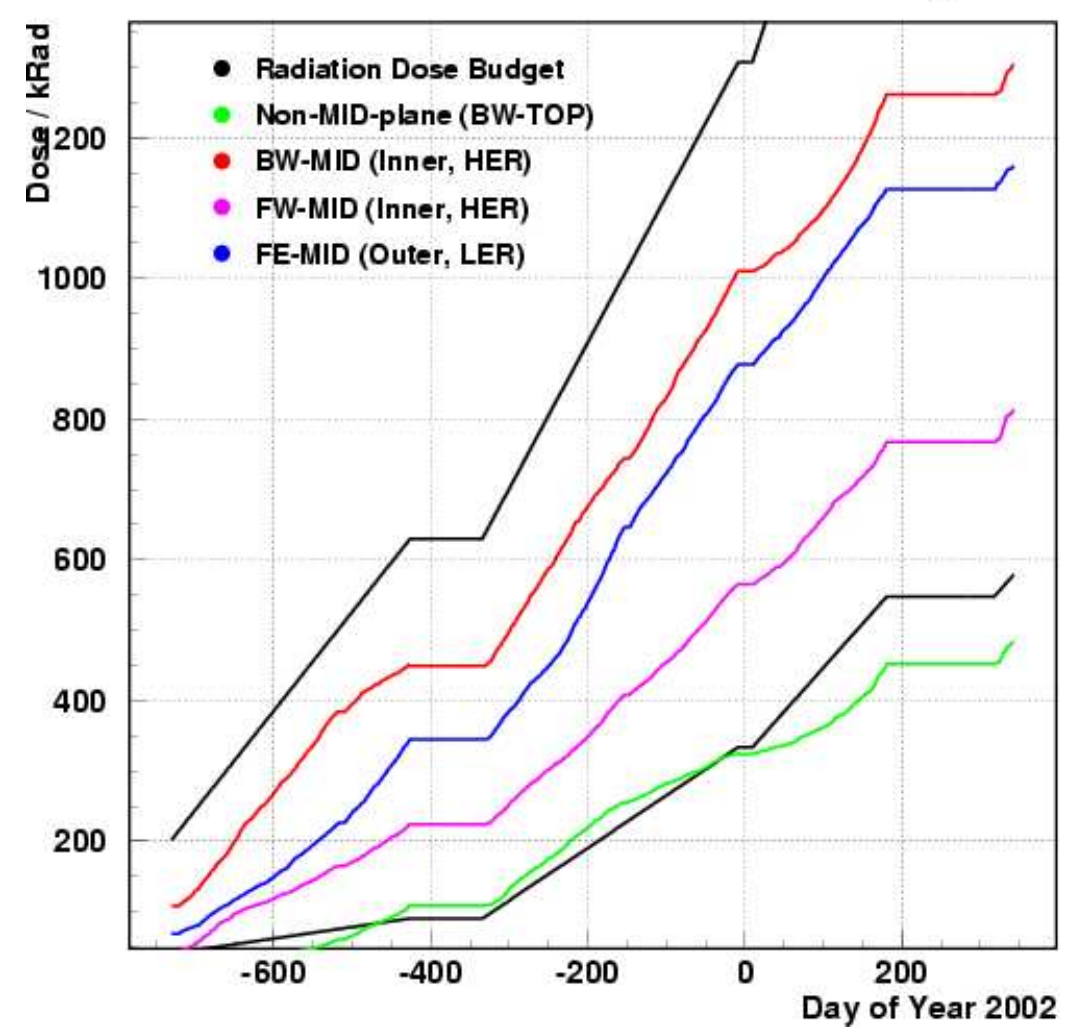

FIG. 3.9: Dose de radiation accumulée par le SVT depuis le début des prises de données. 


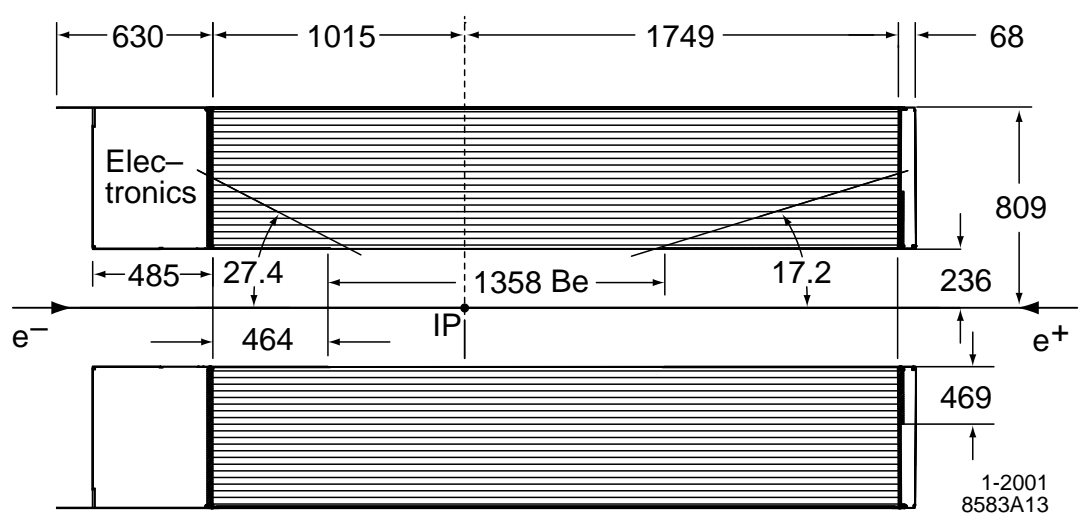

FIG. 3.10: Section longitudinale de la DCH avec ses dimensions principales. Le centre de la chambre est décalé de $370 \mathrm{~mm}$ du point d'interaction (IP).

Elle participe à l'identification des particules par la mesure de leur perte d'énergie par ionisation $(d E / d x)$, notamment dans la discrimination $K / \pi$ à basse impulsion. Enfin, la DCH contribue au système de déclenchement du détecteur BABAR.

La DCH, entourant le SVT, est un cylindre dont les dimensions sont données sur la figure 3.10. L'acceptance géométrique couvre l'angle polaire, dans le référentiel du laboratoire, de $17.2^{\circ}$ à $152.6^{\circ}$ $(-0.89<\cos \theta<0.96)$. La DCH consiste en 7104 cellules de dérive, rangées en 40 couches cylindriques. Les couches sont regroupées par quatre avec la même orientation des fils pour former dix super-couches. Pour obtenir une mesure spatiale en trois dimensions, l'angle stéréo des supercouches alterne entre une configuration axiale $(\mathrm{A})$ et stéréo $(\mathrm{U}, \mathrm{V})$ dans l'ordre AUVAUVAUVA. 24 des 40 couches font ainsi un faible angle avec l'axe $z$. Cela permet d'obtenir l'information sur la position longitudinale avec une résolution de l'ordre de $1 \mathrm{~mm}$. L'angle stéréo varie de \pm 45 à \pm 76 mrad. Les cellules de dérive sont hexagonales, de dimension typique $11.9 \times 19.0 \mathrm{~mm}^{2}$. Chaque cellule comprend un fil sensible entouré de six fils de champ (voir Fig. 3.11). Le fil sensible est fabriqué en tungstène-rhénium plaqué d'or de $20 \mu \mathrm{m}$ de diamètre. Les fils de champ sont fabriqués en aluminium plaqué d'or de $80 \mu \mathrm{m}$ et $120 \mu \mathrm{m}$ de diamètre. Au total, il y a 28768 fils. Les fils sensibles sont portés à une tension de $1930 \mathrm{~V}$ et les fils de champ qui séparent les super-couches (guard wires) à $340 \mathrm{~V}$. Les autres fils de champ sont mis à la terre. Le gaz choisi est un mélange hélium-isobutane en proportion $80: 20 \%$. Le gain d'avalanche est de $5 \times 10^{4}$.

La résolution en $d E / d x$ est de $7.5 \%$. La résolution sur l'impulsion transverse, mesurée avec un échantillon de muons cosmiques traversant la DCH et le SVT, est de

$$
\frac{\sigma_{p_{t}}}{p_{t}}=(0.13 \pm 0.01) \% \cdot p_{t}+(0.45 \pm 0.03) \%
$$

\subsubsection{DIRC}

Le DIRC (Detection of Internally Reflected Cherenkov light) est un détecteur de conception nouvelle pour l'identification de particules basée sur l'émission de lumière Cherenkov par une particule traversant une barre de quartz. Son rôle est la séparation $K / \pi$ sur une large plage en impulsion, essentielle dans l'étiquetage des mésons $B$ par les kaons et la séparation des modes tels que $B_{d}^{0} \rightarrow$ $K^{+} \pi^{-}$et $B_{d}^{0} \rightarrow \pi^{+} \pi^{-}$. La DCH permet une bonne séparation $K / \pi$ pour des impulsions allant 


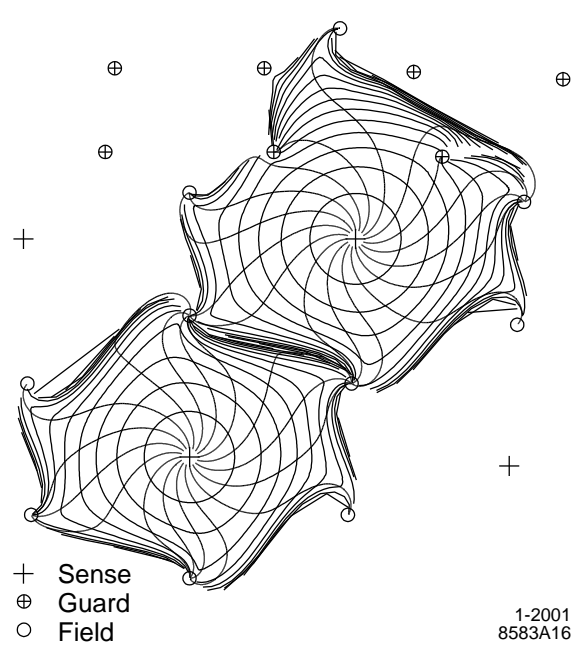

FIG. 3.11: Isochrones de cellules à dérive, c'est-à-dire les contours des temps de dérive égaux des ions. Les isochrones sont espacées de $100 \mathrm{~ns}$.

jusqu'à $0.7 \mathrm{GeV} / c$. Le DIRC doit pouvoir fournir une bonne séparation jusqu'à des impulsions de $4.2 \mathrm{GeV} / c$.

Une particule traversant un milieu d'indice $n$ avec une vitesse $\beta c$ produit une émission de photons (photons Cherenkov) si sa vitesse vérifie $\beta>1 / n$. Cette lumière est produite dans un cône d'ouverture $\theta_{C}$, angle Cherenkov, autour de la direction de la particule selon $\cos \theta_{C}=1 /(n \beta)$. Le matériau choisi pour le DIRC est une barre de quartz d'indice $n=1.473$. Une partie de la lumière Cherenkov est capturée par réflexion interne dans la barre (voir Fig. 3.12) et est transmise à une large matrice de 10752 photomultiplicateurs située à l'arrière du détecteur (à l'avant, les photons sont réfléchis par un miroir placé au bout de la barre de quartz (voir Fig. 3.13)). La haute qualité optique et géométrique des barres conserve l'angle d'émission des photons. La mesure de cet angle ainsi que la connaissance de l'impulsion de la trace provenant de la $\mathrm{DCH}$ permettent de déterminer la masse de la particule et donc sa nature. En effet, l'impulsion de la particule est reliée à sa masse par :

$$
p=\frac{m \beta c}{\sqrt{1-\beta^{2}}}
$$

conduisant à

$$
\cos \theta_{C}=\frac{1}{n} \sqrt{1+\left(\frac{m c}{p}\right)^{2}} .
$$

Les barres de quartz sont collées bout à bout par groupe de quatre pour former un radiateur de $4.9 \mathrm{~m}$ de long, $17 \mathrm{~mm}$ d'épaisseur et $35 \mathrm{~mm}$ de largeur. Les radiateurs sont rassemblés par douze pour former une boîte (Bar Box). Les boîtes sont réparties autour de la DCH en formant un polygone à douze côtés, couvrant $94 \%$ de l'angle azimutal et $83 \%$ du cosinus de l'angle polaire, dans le référentiel du centre de masse. Les photons circulent ensuite dans un volume d'expansion (Standoff Box) rempli de 60001 d'eau purifiée $(n \approx 1.346)$ avant d'atteindre les photomultiplicateurs. Afin de réduire la surface de détection du Standoff Box, un prisme en bout de barre, à l'arrière (cf. Fig. 3.13), renvoie les photons vers le centre (cf. Fig. 3.12). Le nombre moyen de photons en fonction de l'angle d'incidence de la trace traversant la barre de quartz (cf. Fig. 3.14) varie de 20 pour les petits angles polaires d'incidence, jusqu'à 65 pour les grands angles polaires d'incidence. La résolution 


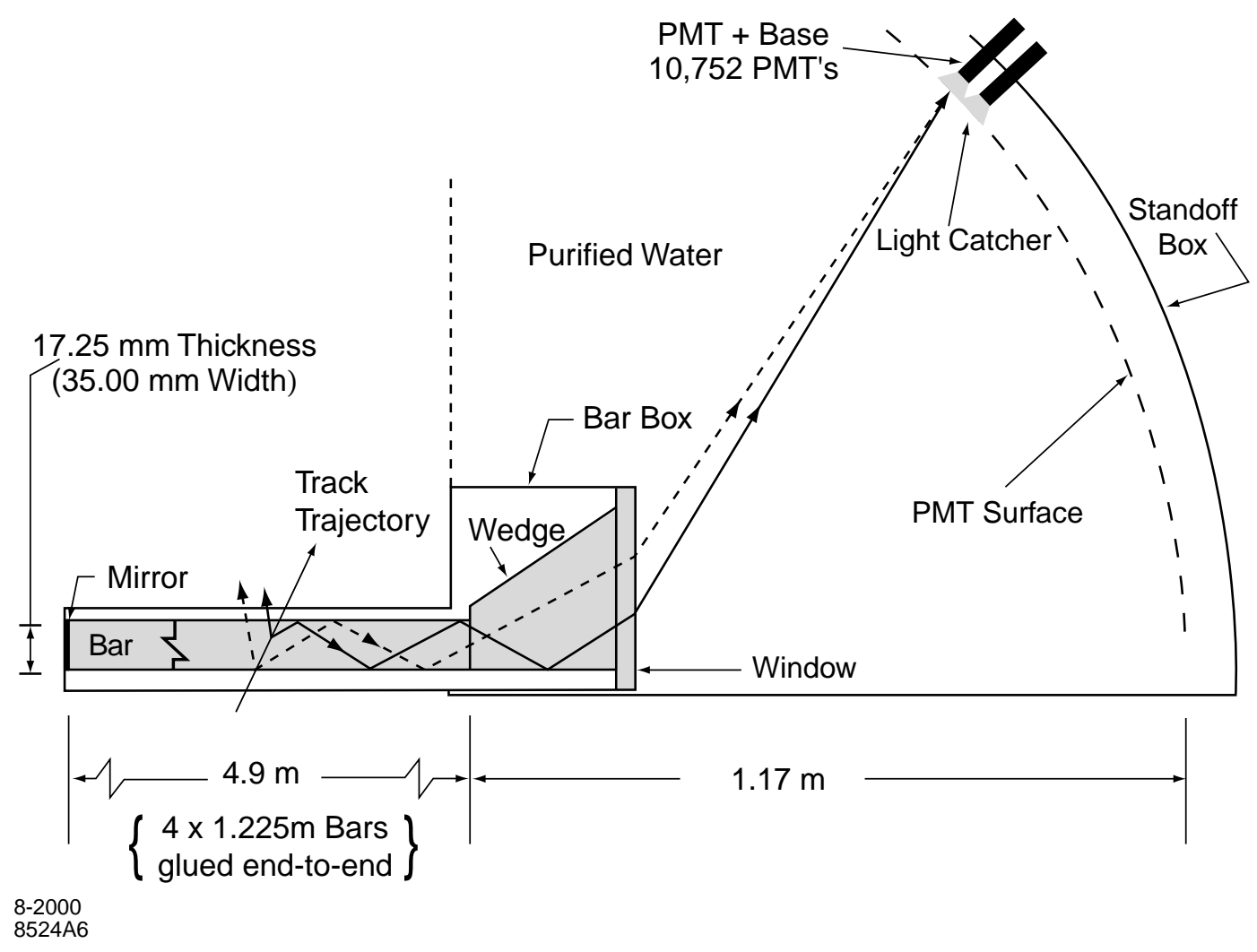

FIG. 3.12: Principe de fonctionnement du DIRC. La direction des $z$ positifs est de droite à gauche : le volume d'expansion (Standoff Box) se situe à l'arrière du détecteur BABAR.

du DIRC sur l'angle Cherenkov est de 2.5 mrad, illustrée sur la figure 3.15.

Les performances du DIRC sont illustrées sur la figure 3.16 : la séparation $K / \pi$ est de l'ordre de $8 \sigma$ pour une trace de $2 \mathrm{GeV} / c$ jusqu'à $2.5 \sigma$ pour une trace de $4 \mathrm{GeV} / c$ où $\sigma$ est la résolution moyenne sur $\theta_{C}$. Ces résultats sont obtenus à partir d'un échantillon de désintégrations $D^{*+} \rightarrow D^{0} \pi^{+}$et $D^{0} \rightarrow K^{-} \pi^{+}$provenant des données.

\subsubsection{Calorimètre (EMC)}

Le calorimètre électromagnétique (EMC) est conçu pour mesurer l'énergie et la résolution angulaire des gerbes électromagnétiques avec une excellente efficacité sur une large plage en énergie, allant de $20 \mathrm{MeV}$ à $9 \mathrm{GeV}$. Cette capacité est nécessaire à la détection des photons provenant de la désintégration des $\pi^{0}$ ou des $\eta$ ainsi que des processus électromagnétiques ou radiatifs. De plus, il participe à l'identification des électrons, utilisée dans l'étiquetage de la saveur des mésons $B$ neutres via leurs désintégrations semileptoniques et dans l'état final de nombreux canaux d'intérêt pour l'étude de la violation de $C P$. La limite supérieure en énergie est déterminée par la nécessité de mesurer des processus électromagnétiques tels que $e^{+} e^{-} \rightarrow e^{+} e^{-}(\gamma)$ et $e^{+} e^{-} \rightarrow \gamma \gamma$, pour la calibration et la détermination de la luminosité. La limite inférieure est déterminée par la nécessité d'une grande efficacité de reconstruction des désintégrations de mésons $B$ contenant des $\pi^{0}$ et des $\eta$. Enfin, le calorimètre participe au système de déclenchement.

Le calorimètre est composé de 6580 cristaux d'iodure de césium dopés au thallium (CsI(Tl)), projectifs en $\phi$ et quasi projectifs en $\theta$, couvrant un angle solide correspondant à $-0.775<\cos \theta<$ 


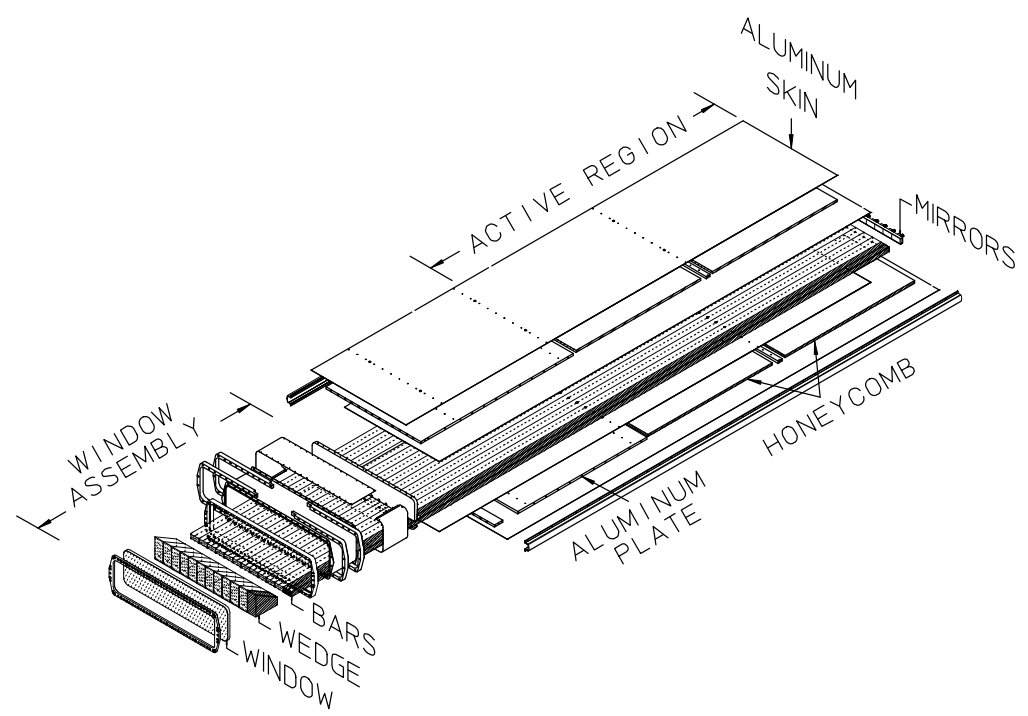

FIG. 3.13: Schéma d'une boîte du DIRC.

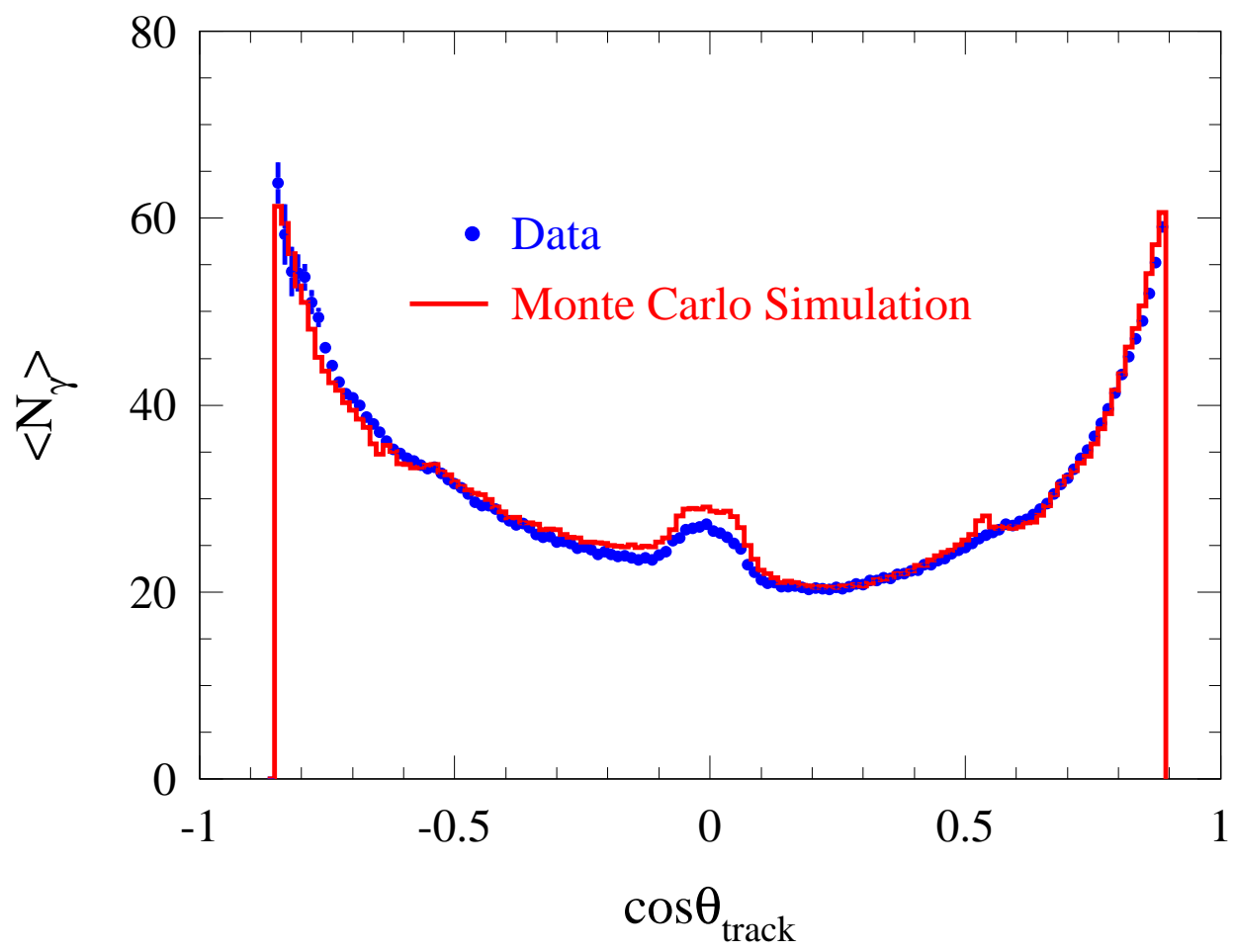

FIG. 3.14: Nombre de photons détectés en fonction de l'angle polaire de la trace chargée pour des traces reconstruites provenant d'événements dimuons. Est superposé le résultat d'une simulation Monte Carlo, où le nombre de photons est ajusté sur celui des données. 


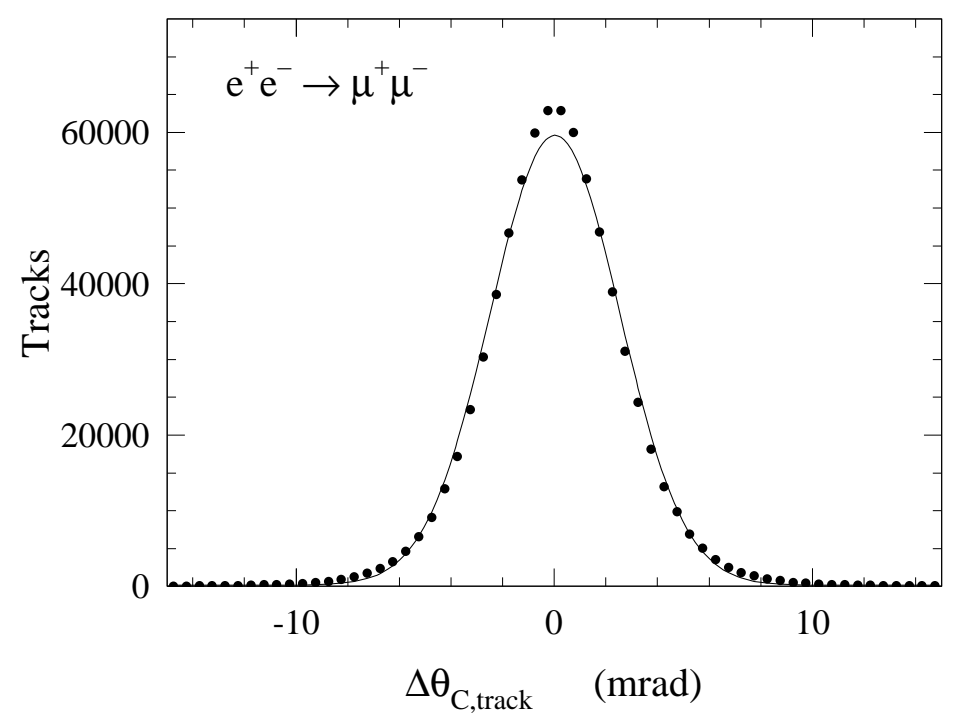

FIG. 3.15: Différence entre les angles Cherenkov mesuré et attendu, $\Delta \theta_{c, t r a c k}$, pour des muons provenant d'événements dimuons. La courbe représente un ajustement par une distribution gaussienne sur les données avec une largeur de 2.5 mrad.

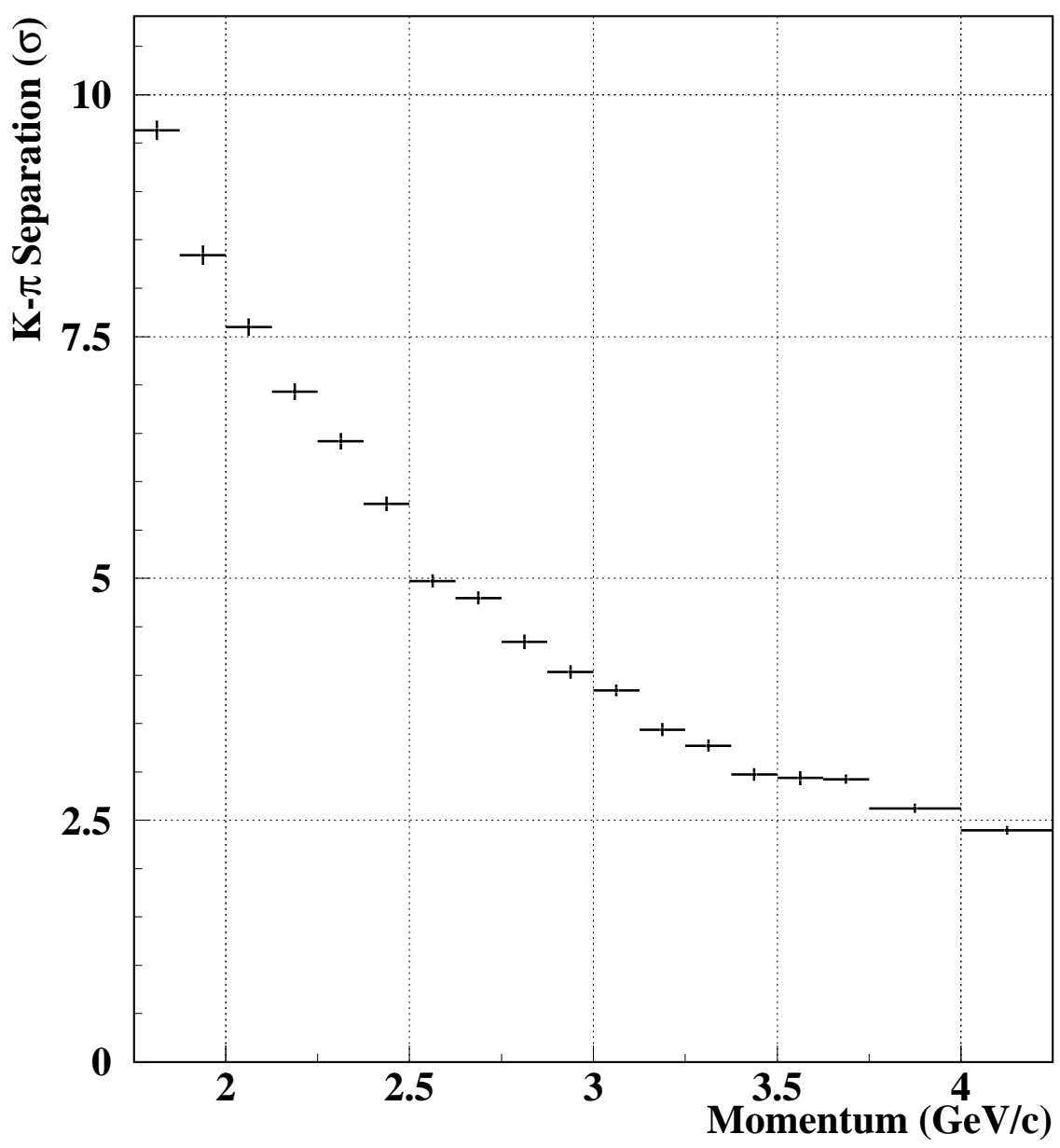

FIG. 3.16: Variation de la séparation des angles Cherenkov des kaons et des pions avec l'impulsion, obtenue à partir d'un échantillon de désintégrations $D^{*+} \rightarrow D^{0} \pi^{+}$et $D^{0} \rightarrow K^{-} \pi^{+}$provenant des données. 


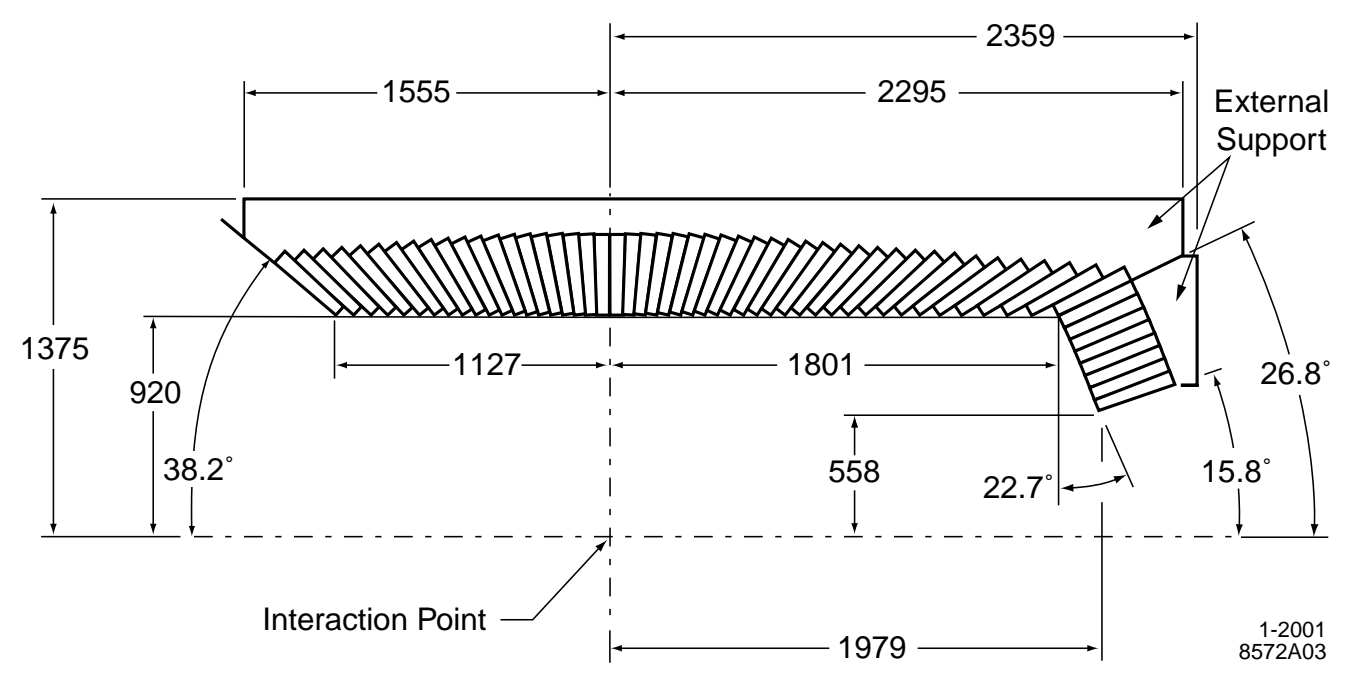

FIG. 3.17: Section longitudinale de l’EMC (partie supérieure uniquement).

0.962 dans le référentiel du laboratoire, soit $-0.916<\cos \theta^{*}<0.962$ dans le référentiel du centre de masse (cf. Fig. 3.17). La taille des cristaux augmente de $29.6 \mathrm{~cm}$ à $32.4 \mathrm{~cm}$ en allant de l'arrière vers l'avant afin de limiter les fuites. La lumière de scintillation est lue par deux photodiodes collées sur l'arrière de chaque cristal.

La résolution en énergie obtenue est de

$$
\frac{\sigma_{E}}{E}=\frac{(2.32 \pm 0.30) \%}{\sqrt[4]{E(\mathrm{GeV})}} \oplus(1.85 \pm 0.12) \%
$$

moins bonne que la résolution de conception mais en accord avec la simulation détaillée Monte Carlo. La résolution angulaire est de

$$
\sigma_{\theta, \phi}=\left(\frac{(3.87 \pm 0.07)}{\sqrt{E(\mathrm{GeV})}}+(0.00 \pm 0.04)\right) \operatorname{mrad}
$$

\subsubsection{Retour de flux instrumenté (IFR)}

Les IFR (Instrumented Flux Return) ont pour but l'identification des muons avec une grande efficacité et une bonne pureté, ainsi que la détection des hadrons neutres (principalement les $K_{L}^{0}$ ). Les muons interviennent dans l'étiquetage de la saveur des mésons $B$ neutres via ses désintégrations semileptoniques et dans l'état final de nombreux canaux d'intérêt pour l'étude de la violation de $C P$. Les $K_{L}^{0}$ permettent l'étude de désintégrations exclusives de mésons $B$, en particulier des états propres de $C P$.

Les IFR utilisent le retour de flux du champ magnétique en fer segmenté en l'instrumentant de détecteurs RPC (Resistive Plate Chamber). Les IFR sont composés d'un tonneau et de deux bouchons (cf. Fig. 3.18). L'acceptance géométrique couvre l'angle polaire de $17.2^{\circ}$ à $157.1^{\circ}(-0.92<\cos \theta<$ 0.96) dans le référentiel du laboratoire. Le fer est segmenté en 18 plaques, augmentant en épaisseur de $2 \mathrm{~cm}$ pour les 9 premières plaques, jusqu'à $10 \mathrm{~cm}$ pour les toutes dernières plaques. Il y a 21 couches de détecteurs RPC dans le tonneau : une RPC bicouche cylindrique entre le calorimètre et l'aimant, une entre l'aimant et le fer, 17 entre les plaques de fer et une à l'extérieur de la structure 


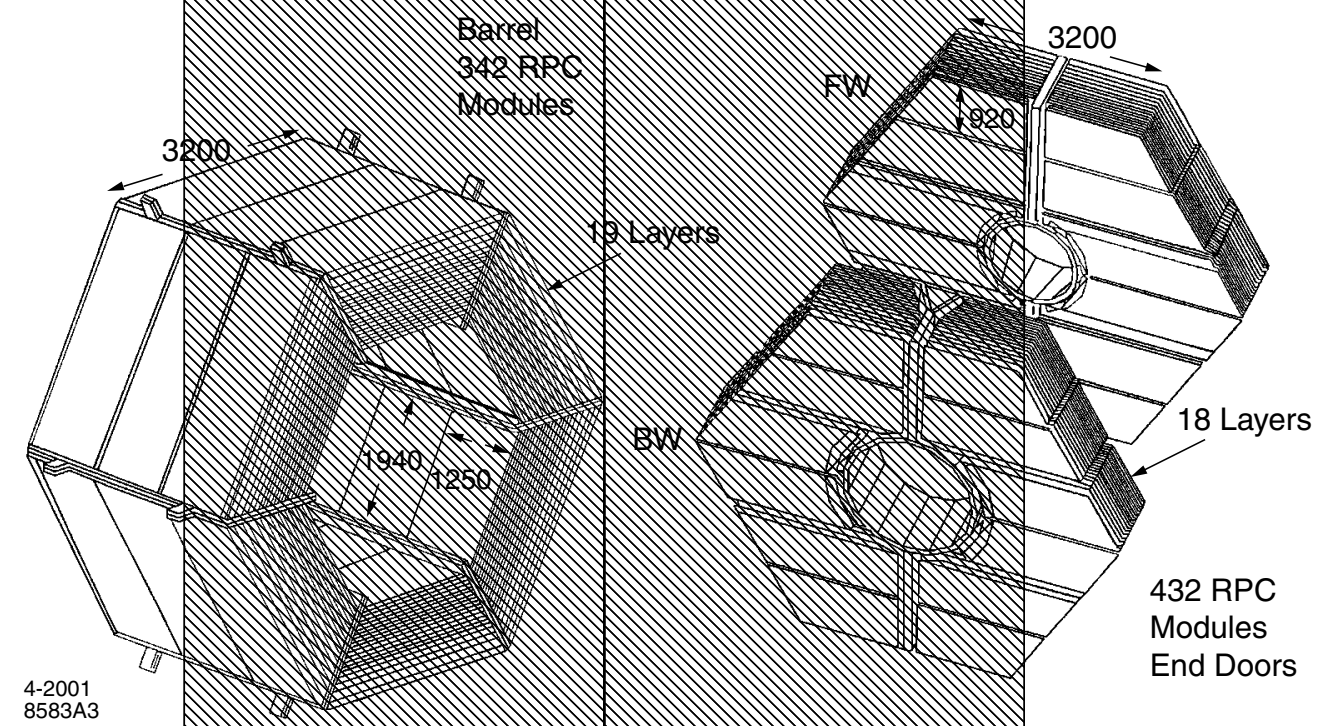

FIG. 3.18: Vue des IFR : le tonneau et les deux bouchons.

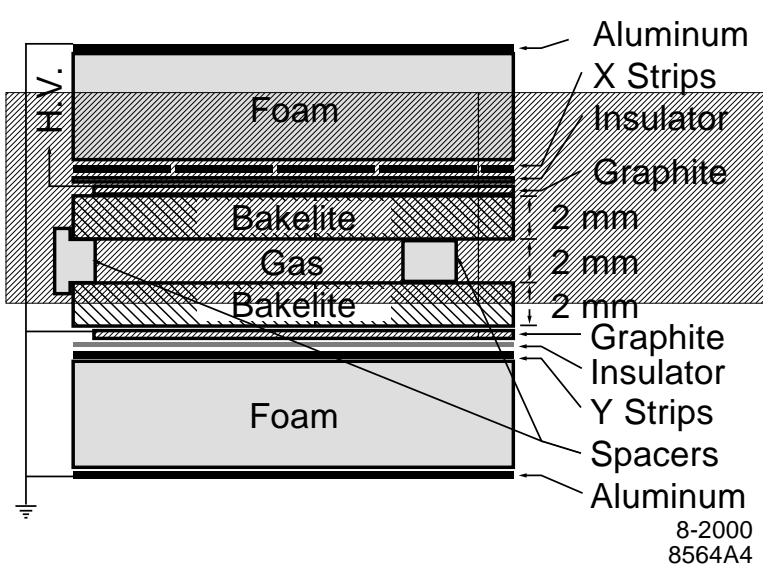

FIG. 3.19: Section d'un détecteur RPC.

de fer et 18 couches de détecteurs RPC dans les bouchons. Au total, il y a 806 détecteurs RPC. Les détecteurs RPC, de $3 \mathrm{~cm}$ d'épaisseur, sont constitués de deux plaques de bakélite de $2 \mathrm{~mm}$ d'épaisseur séparées de $2 \mathrm{~mm}$ (cf. Fig. 3.19). Cet espace est rempli de gaz ininflammable composé de $56.7 \%$ d'argon, $38.8 \%$ de fréon $\left(C_{2} H_{2} F_{4}\right)$ et $4.5 \%$ d'isobutane. Les deux plaques de bakélite sont recouvertes sur l'extérieur par du graphite, connecté à une haute tension $(\sim 8 \mathrm{kV})$ et à la terre. Les faces internes sont recouvertes d'huile de lin. Le passage d'une particule ionise le gaz, ce qui induit par effet capacitif un signal sur des pistes disposées en un maillage bidimensionnel $: z-\phi$ pour le tonneau et $x-y$ pour les bouchons.

L'efficacité des IFR, stable au début de fonctionnement, a commencé une lente dégradation continue pour aboutir, plus récemment, par une dégradation plus rapide. Les causes de cette perte d'efficacité, illustrée sur la figure 3.20, sont en cours d'étude mais il semble que de nombreux effets doivent être pris en compte et tous ne sont pas encore identifiés. 


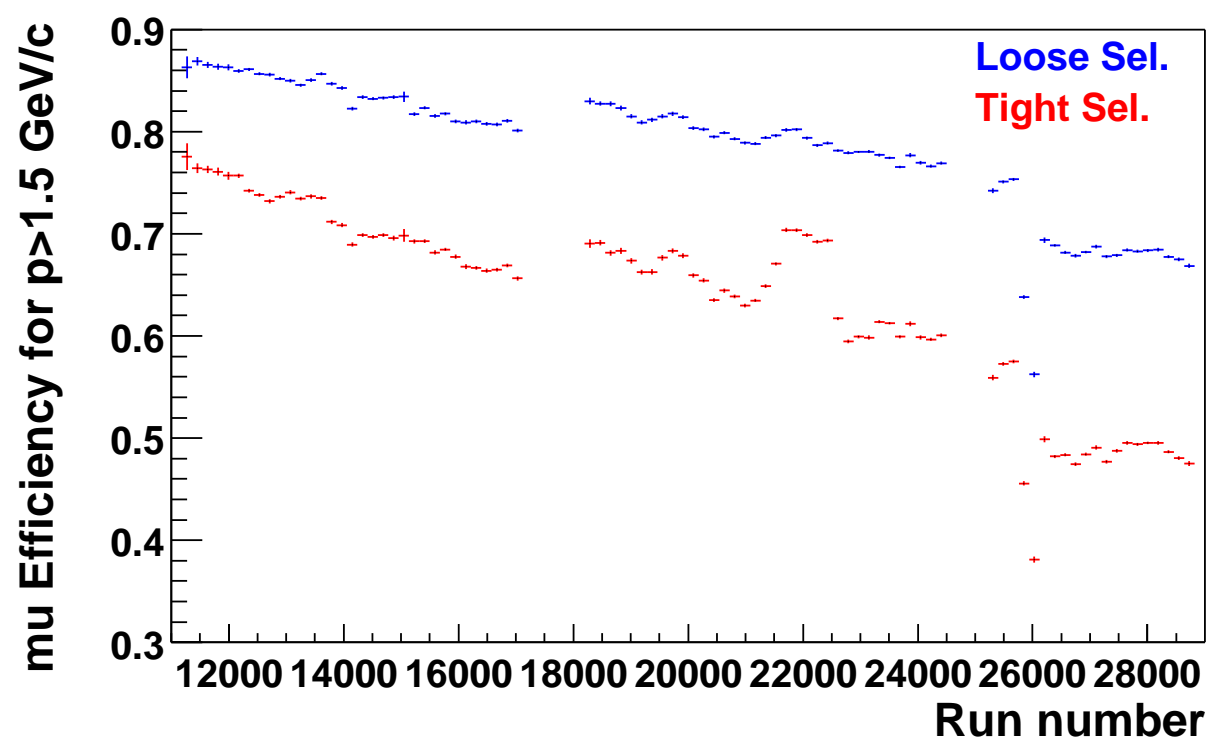

FIG. 3.20: Efficacité des IFR pour deux types de sélection de muons : Loose (courbe du haut) et Tight (courbe du bas). Les runs 11000 à 17000 correspondent aux données enregistrées en 2000, les runs 18000 à 25000 celles enregistrées en 2001. Les runs suivants correspondent aux données enregistrées en 2002.

\subsubsection{Système de déclenchement}

Le système de déclenchement a pour but la sélection des événements d'intérêt avec une efficacité élevée, stable et bien comprise, tout en rejetant les événements de bruit de fond. Le système de déclenchement de BABAR est composé de deux niveaux, le niveau 1 (level 1 trigger) et le niveau 3 (level 3 trigger) (il n'y a pas de niveau 2). Le niveau 1 intervient en temps réel au moment de la prise des données et est limité à un taux de $2.5 \mathrm{kHz}$. Le niveau 3, qui fait partie du système de reconstruction en ligne, opère une deuxième sélection à partir des événements acceptés par le niveau 1 et ne peut excéder un taux de $100 \mathrm{~Hz}$.

Le niveau 1 est basé sur un déclencheur global (GLT $\equiv$ Global Level Trigger) qui utilise les informations qui lui sont fournies par un déclencheur associé à la chambre à dérive (DCT $\equiv D C H$ Trigger) basé sur les traces chargées, un déclencheur basé sur les dépôts d'énergie dans le calorimètre (EMT $\equiv E M C$ Trigger) et un déclencheur associé aux IFR (IFT $\equiv$ IFR Trigger) basé sur les muons et les rayons cosmiques. Le niveau 1 peut sélectionner des événements en utilisant soit un seul des déclencheurs (DCT ou EMT), soit les deux. Dans ce cas, il tente d'associer à un objet neutre une trace chargée. L'efficacité du niveau 1 est supérieure à $99.9 \%$ pour les événements $B \bar{B}$ pour un taux typique de $1 \mathrm{kHz}$ pour une luminosité de $3-4 \times 10^{33} \mathrm{~cm}^{-2} s^{-1}$. L'efficacté du niveau 3 est aussi supérieure à $99.9 \%$ pour les événements $B \bar{B}$ passant le niveau 1 .

\subsubsection{Conclusion}

Le détecteur BABAR a fonctionné dès le début proche des caractéristiques de conception. En parallèle, PEP-II a dépassé ses paramètres de conception, à la fois en termes de luminosité instantanée et intégrée par jour et par mois. D'octobre 1999 à juin 2002, PEP-II a fourni $98.6 \mathrm{fb}^{-1}$ avec une efficacité d'enregistrement de BABAR supérieure à $95 \%$, illustrée sur la figure 3.4. Ainsi, le détecteur 
BABAR a enregistré $83.87 \mathrm{fb}^{-1}$ de paires de mésons $B$, soit un total de 184.5 millions de mésons $B$. Ce sont ces données de haute qualité qui sont utilisées pour l'étude de la violation de $C P$ et la mesure des amplitudes de transversité des canaux charmonium- $K^{*}(892)$.

\subsection{Sélection des particules finales}

La reconstruction des désintégrations exclusives de mésons $B$ commence par l'identification des candidats des produits finals de désintégration. Les particules chargées sont reconstruites en tant que traces dans le SVT et/ou la DCH. Les leptons et les kaons sont identifiés à partir des informations fournies par la DCH, l'EMC (pour les électrons), les IFR (pour les muons) et le DIRC (pour les kaons). Les photons sont identifiés à partir de leur dépôt d'énergie dans l'EMC.

\subsubsection{Sélection des particules chargées}

Les traces chargées sont sélectionnées en demandant au moins douze points de mesure dans la $\mathrm{DCH}$ afin que leur impulsion et leur $d E / d x$ soient bien mesurés. En plus, on demande qu'elles aient une impulsion transverse supérieure à $100 \mathrm{MeV} / c$ et que la distance de moindre approche soit inférieure à $1.5 \mathrm{~cm}$ en $x y$ et $10 \mathrm{~cm}$ en $z$ (le système de coordonnées attaché au détecteur BABAR est défini sur les figures 3.5 et 3.6). La seule exception faite concerne les deux pions provenant d'un $K_{S}^{0}$, où toutes les traces reconstruites dans la DCH et le SVT sont utilisées.

\subsubsection{Sélection des neutres}

L'énergie déposée dans des cristaux contigus de l'EMC est sommée dans un cluster. La distribution en énergie des cristaux dans le cluster est utilisée pour discriminer les clusters provenant de gerbes électromagnétiques de ceux provenant de gerbes hadroniques. Les variables utilisées pour décrire cette distribution en énergie sont la distribution latérale en énergie (LAT) [155] et les moments de Zernike $A_{m n}$ [156]. La distribution latérale en énergie est définie par :

$$
L A T=\frac{\sum_{i=3}^{N} E_{i} r_{i}^{2}}{\sum_{i=3}^{N} E_{i} r_{i}^{2}+E_{1} r_{0}^{2}+E_{2} r_{0}^{2}},
$$

où le cluster est composé de $N$ cristaux ordonnés en énergie de sorte que $E_{1}>E_{2}>\ldots>E_{N}$ et $r_{0}$ est la distance moyenne entre deux cristaux (typiquement $5 \mathrm{~cm}$ pour les cristaux de l'EMC). $r_{i}$ est la distance du centre du cristal $i$ au centre du cluster. Le LAT est une mesure du profil radial en énergie d'un cluster. Le moment de Zernike $A_{42}$ mesure l'asymétrie du cluster par rapport à son maximum. Les gerbes électromagnétiques ont un LAT piqué autour de 0.25 et un $A_{42}$ proche de zéro, alors que les gerbes hadroniques ont une distribution en LAT plus dispersée et un $A_{42}$ s'étendant sur de plus larges valeurs.

Les photons sont identifiés comme des clusters de l'EMC n'ayant pas de traces chargées associées, ayant une énergie minimale demandée de $30 \mathrm{MeV}$ et un LAT inférieur à 0.8 . Ceci permet de rejeter les clusters formés par les débris hadroniques et les photons provenant du bruit de fond machine. 
TAB. 3.4: Résumé des critères d'identification d'électrons. La différence entre le $d E / d x$ mesuré $(\langle d E / d x\rangle \sim 650)$ et la valeur attendue pour un électron doit se situer dans un intervalle donné en termes de la résolution mesurée $\sigma(\sigma \sim 50)$.

\begin{tabular}{lcccc}
\hline \hline & VeryLoose & Loose & Tight & VeryTight \\
\hline$d E / d x$ (mesuré-attendu) & {$[-3 \sigma,+7 \sigma]$} & {$[-3 \sigma,+7 \sigma]$} & {$[-3 \sigma,+7 \sigma]$} & {$[-2 \sigma,+4 \sigma]$} \\
$E / p$ & $0.5-5.0$ & $0.65-5.0$ & $0.75-1.3$ & $0.89-1.2$ \\
$N_{\text {crys }}$ & $>3$ & $>3$ & $>3$ & $>3$ \\
LAT & - & - & $0.0-0.6$ & $0.1-0.6$ \\
$A_{42}$ & - & - & - & $<0.11$ \\
$\theta_{C}$ (mesuré-attendu) & - & - & - & {$[-3 \sigma,+3 \sigma]$} \\
\hline \hline
\end{tabular}

\subsubsection{Identification des particules}

L'identification des électrons, muons et kaons est un ingrédient essentiel à la fois dans la reconstruction et dans l'étiquetage de mésons $B$. Les particules sont identifiées par leur perte d'énergie par ionisation $(d E / d x)$ dans le SVT et dans la DCH, le nombre de photons Cherenkov et l'angle Cherenkov dans le DIRC, les gerbes électromagnétiques dans l'EMC et la longueur de pénétration dans les IRF. Les critères de sélection sont basés sur ces quantités, sur des rapports de probabilités dérivés de ces dernières ou sur des réseaux de neurones combinant des probabilités dérivées de différents détecteurs. Les efficacités et les probabilités de mauvaise identification sont obtenues à partir d'échantillons de données de contrôle avec des caractéristiques similaires.

\section{Identification des électrons}

Les candidats électrons sont principalement identifiés par le rapport de l'énergie mesurée dans $l^{\prime}$ EMC à l'impulsion de la trace, $E / p$. De plus, ils doivent avoir un $d E / d x$ mesuré dans la DCH compatible avec l'hypothèse électron. Enfin, pour certaines applications, le LAT et le moment de Zernike $A_{42}$, ainsi que la compatibilité entre l'angle Cherenkov observé et attendu provenant du DIRC sont utilisés pour l'identification. Quatre catégories de candidats électrons (VeryLoose, Loose, Tight et VeryTight) sont définies avec les critères donnés dans la table 3.4. Les candidats ayant une trace chargée non associée à un cluster de l'EMC définissent la catégorie noCal si leur $d E / d x$ mesuré satisfait les mêmes critères que la catégorie VeryTight. L'efficacité d'identification des électrons dans un intervalle en impulsion $0.5<p<3.0 \mathrm{GeV} / c$ varie de $98 \%$ à $88 \%$ pour les catégories de VeryLoose à VeryTight, avec un taux de mauvaise identification de pions allant de $5 \%$ à $0.1 \%$ (cf. Fig. 3.21).

\section{Identification des muons}

Pour identifier les muons, la présence d'un dépôt d'énergie compatible avec une trace d'ionisation minimale, ainsi que les distributions des coups dans les IFR sont utilisées. En particulier, le nombre de longueurs d'interaction, $n_{\lambda}$, traversées dans les IFR doit être compatible avec l'hypothèse muon. La moyenne et la variance du nombre de coups par couche doivent être petites afin de rejeter les contaminations des gerbes hadroniques. L'ajustement des traces aux coups dans les détecteurs RPC doit avoir un faible $\chi^{2}$, à la fois à l'intérieur des IFR $\left(\chi_{\mathrm{IFR}}^{2}\right)$ et dans l'extrapolation de 
ТАВ. 3.5: Résumé des critères d'identification des muons. $\dagger:$ le critère donné n'est valable que pour un candidat muon d'impulsion $p>1.2 \mathrm{GeV} / c$. Pour $0.5<p<1.2 \mathrm{GeV} / c$, on a pour la sélection Tight (VeryTight), $\mid n_{\lambda}-n_{\lambda}($ attendu) $\mid<$ $(p-0.5) / 0.7(<0.8(p-0.5) / 0.7)$, et vaut 0 pour $p<0.5 \mathrm{GeV} / c$ pour les deux catégories.

\begin{tabular}{lcccc}
\hline \hline & VeryLoose & Loose & Tight & VeryTight \\
\hline$E_{\mathrm{EMC}} \mathrm{GeV}$ & $<0.5$ & $<0.5$ & $0.05-0.4$ & $0.05-0.4$ \\
$n_{\lambda}$ & $>2$ & $>2$ & $>2.2$ & $>2.2$ \\
$\mid n_{\lambda}-n_{\lambda}($ attendu $) \mid$ & $<2.5$ & $<2.0$ & $<1^{\dagger}$ & $<0.8^{\dagger}$ \\
$\left\langle N_{\text {coups }}\right\rangle$ & $<10$ & $<10$ & $<8$ & $<8$ \\
$\sigma_{N_{\text {coups }}}$ & $<6$ & $<6$ & $<4$ & $<4$ \\
$\chi_{\mathrm{IFR}}^{2} / N_{\text {couche }}$ & - & 4 & 3 & 3 \\
$\chi_{\text {match }}^{2} / N_{\text {couche }}$ & - & 7 & 5 & 5 \\
\hline \hline
\end{tabular}

la trace vers les IFR $\left(\chi_{\text {match }}^{2}\right)$. Quatre catégories de candidats muons (VeryLoose, Loose, Tight et VeryTight) sont définies avec les critères donnés dans la table 3.5. Pour les catégories Tight et VeryTight, un veto kaon est appliqué en demandant que le candidat muon ne satisfasse pas la sélection de kaon Tight. Dans le cas où aucune information provenant des IFR n'est disponible, seule l'information de l'EMC est utilisée, définissant la catégorie MIP (Minimum Ionizing Particle). L'efficacité $^{3} \mathrm{~d}^{\prime}$ identification des muons dans un intervalle en impulsion $1.2<p<3.0 \mathrm{GeV} / c$ varie de $92 \%$ à $60 \%$ pour les catégories de VeryLoose à VeryTight, avec un taux de mauvaise identification de pions allant de $15 \%$ à $3 \%$ (cf. Fig. 3.21).

En plus de ces critères de sélection, la sélection des leptons est restreinte à une région fiducielle à l'intérieur de laquelle l'efficacité est bien mesurée sur des échantillons de données de contrôle, et le matériau dans le détecteur est modélisé avec précision dans la simulation Monte Carlo. L'intervalle accepté en angle polaire $\theta$ est pour les électrons $0.410<\theta_{e}<2.409$ rad et pour les muons $0.30<$ $\theta_{\mu}<2.70 \mathrm{rad}$. Cela correspond à une couverture de $84 \%$ de l'angle solide dans le centre de masse pour les électrons, et à $92 \%$ pour les muons.

\section{Identification des kaons}

Les kaons sont distingués des pions et des protons par les pertes d'énergie par ionisation, $d E / d x$, dans le SVT et la DCH ainsi que le nombre de photons Cherenkov et l'angle Cherenkov dans le DIRC, compatible avec une hypothèse kaon. Les informations fournies par ces détecteurs permettent de définir des probabilités $\mathcal{L}_{K}, \mathcal{L}_{\pi}$ et $\mathcal{L}_{p}$. Une probabilité combinée utilise les probabilités du SVT et de la $\mathrm{DCH}$ pour les traces d'impulsion inférieure à $0.5 \mathrm{GeV} / c$, la probabilité de la $\mathrm{DCH}$ uniquement pour les traces d'impulsion comprise entre $0.5 \mathrm{GeV} / c$ et $0.6 \mathrm{GeV} / c$, la probabilité du DIRC uniquement pour les traces d'impulsion supérieure à $0.6 \mathrm{GeV} / c$. La reconstruction exclusive de la plupart des états finals de mésons $B$, ce qui est le cas des canaux $J / \psi K^{*}(892)$, ne nécessite pas d'identification explicite de kaons. La sélection des canaux $J / \psi K^{*}(892)$ contenant un kaon dans l'état final utilise la catégorie notAPion, pour laquelle un candidat kaon est rejeté si $r \mathcal{L}_{\pi}>\mathcal{L}_{K}$ et $r \mathcal{L}_{\pi}>\mathcal{L}_{p}$, avec $r=1$ pour $p \geq 0.5 \mathrm{GeV} / c$ et $r=0.1$ pour $p<0.5 \mathrm{GeV} / c$. Cette catégorie a une

\footnotetext{
${ }^{3}$ Les efficacités, ainsi que les taux de mauvaise identification de pions, sont obtenus grâce à des échantillons de données de contrôle de grande pureté. Les nombres donnés proviennent de ces échantillons enregistrés en 2000 et 2001. L'effet observé dans la perte d'efficacité des IFR plus rapide en 2002 (cf. Fig. 3.20) n'est pas inclus.
} 
efficacité de l'ordre de $97 \%$ avec un taux de réjection des pions de l'ordre de $80 \%$. Pour la même raison que pour les leptons, la région fiducielle pour les kaons est réduite à $0.45<\theta_{K}<2.50$ rad.
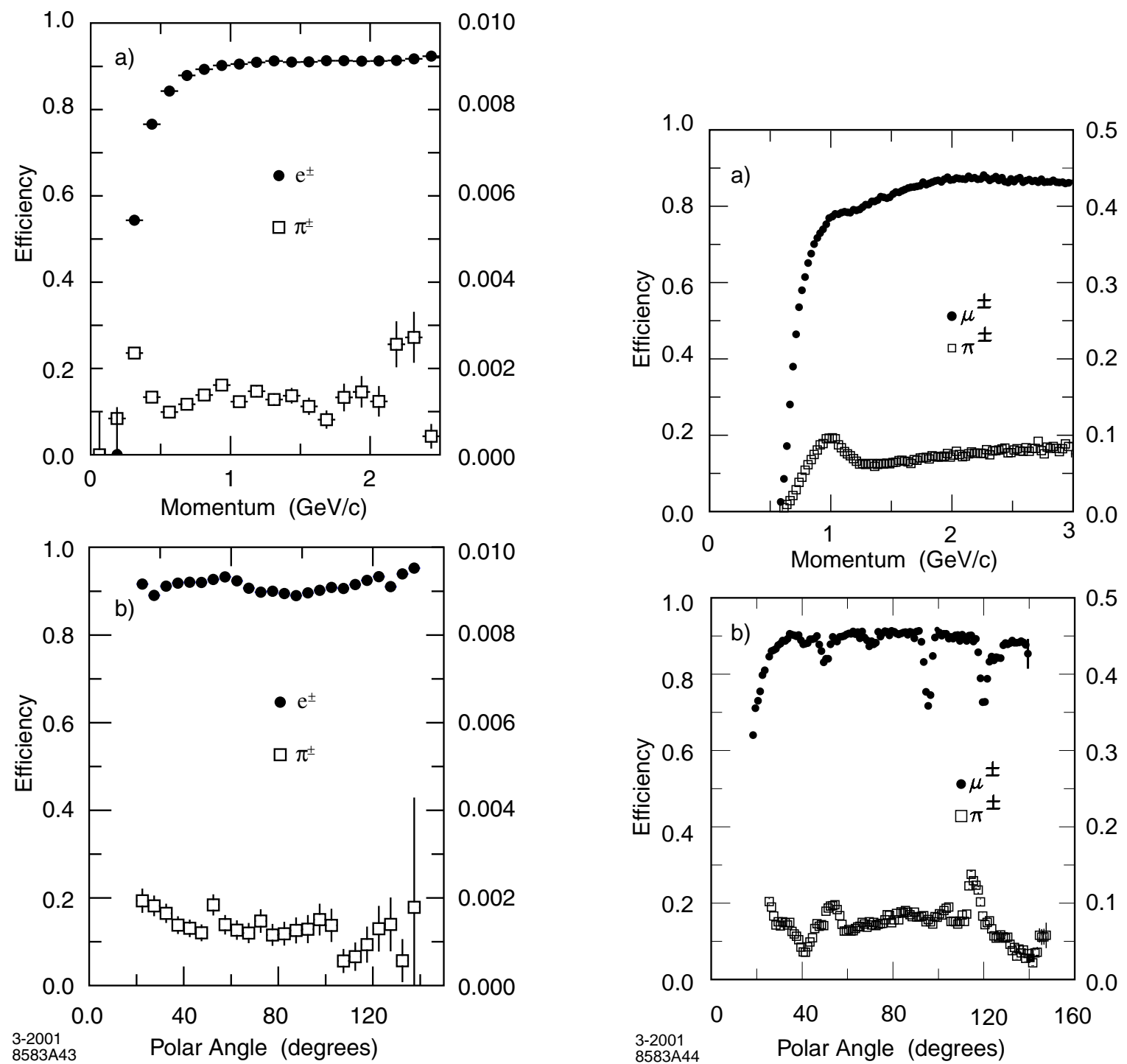

FIG. 3.21: Efficacité et taux de mauvaise identification de pions pour les critères de sélection VeryTight pour les électrons (figures de gauche) et Loose pour les muons (figures de droite) en fonction a) de l'impulsion, b) de l'angle polaire $(1.5<p<3 \mathrm{GeV} / \mathrm{c})$. Les échelles de gauches donnent les efficacités et celles de droite les taux de mauvaise identification de pions.

\section{Identification des particules dans la simulation Monte Carlo}

Afin de prendre en compte les éventuelles différences entre les données et les données simulées, des tables d'efficacité et de mauvaises identifications sont mesurées sur des échantillons de données de contrôle de grande pureté, tabulées en bins d'impulsion et d'angles polaire et azimutal. Ces tables sont alors utilisées lors de la reconstruction des événements Monte Carlo, en remplacement de l'identification basée sur les grandeurs simulées. Cette procédure permet de refléter l'efficacité d'identification des particules directement sur celle des données. 


\section{Chapitre 4}

\section{Sélection des canaux exclusifs}

\section{$B \rightarrow J / \psi K^{*}(892)$}

Ce chapitre détaille la sélection des canaux exclusifs $B \rightarrow J / \psi K^{*}(892)$, servant dans la mesure des amplitudes de transversité ainsi que dans la mesure de $\sin 2 \beta$ et $\cos 2 \beta$ par une analyse angulaire complète dépendante du temps. Parmi les canaux vecteur-vecteur, les désintégrations $B \rightarrow J / \psi K^{*}(892)$ ont les taux d'embranchement les plus élevés. Les différentes caractéristiques des particules impliquées sont résumées dans le tableau ci-dessous [28] :

\begin{tabular}{lccl}
\hline Méson & $\begin{array}{c}\text { Masse } \\
\left(\mathrm{MeV} / c^{2}\right)\end{array}$ & $\begin{array}{c}\text { Largeur ou temps de vie } \\
(\mathrm{MeV} \text { ou })\end{array}$ & $J^{P C}$ \\
\hline \hline$B_{d}^{0}$ & $5279.4 \pm 0.5$ & $(1.542 \pm 0.016) \times 10^{-12}$ & $0^{-}$ \\
$B_{u}^{+}$ & $5279.0 \pm 0.5$ & $(1.674 \pm 0.018) \times 10^{-12}$ & $0^{-}$ \\
$J / \psi$ & $3096.87 \pm 0.04$ & $0.087 \pm 0.005$ & $1^{--}$ \\
$K^{* 0}$ & $896.10 \pm 0.27$ & $50.7 \pm 0.6$ & $1^{-}$ \\
$K^{*+}$ & $891.66 \pm 0.26$ & $50.8 \pm 0.9$ & $1^{-}$ \\
$K_{S}^{0}$ & $497.672 \pm 0.031$ & $(0.8935 \pm 0.0008) \times 10^{-10}$ & $0^{-}$ \\
$K^{+}$ & $493.677 \pm 0.016$ & $(1.2384 \pm 0.0024) \times 10^{-8}$ & $0^{-}$ \\
$\pi^{0}$ & $134.9766 \pm 0.0006$ & $(8.4 \pm 0.6) \times 10^{-17}$ & $0^{-+}$ \\
$\pi^{+}$ & $139.57018 \pm 0.00035$ & $(2.6033 \pm 0.0005) \times 10^{-8}$ & $0^{-}$ \\
\hline \hline
\end{tabular}

Les désintégrations considérées sont les suivantes (les modes conjugués de charges sont implicites et $\ell=e$ ou $\mu$ ), et les rapports d'embranchement secondaires sont donnés dans la table 4.1 :

\begin{tabular}{l}
\hline \hline$B_{d}^{0} \rightarrow J / \psi K^{* 0}, J / \psi \rightarrow \ell^{+} \ell^{-}, K^{* 0} \rightarrow K_{S}^{0} \pi^{0}, K_{S}^{0} \rightarrow \pi^{+} \pi^{-}, \pi^{0} \rightarrow \gamma \gamma$ \\
$B_{d}^{0} \rightarrow J / \psi K^{* 0}, J / \psi \rightarrow \ell^{+} \ell^{-}, K^{* 0} \rightarrow K^{+} \pi^{-}$ \\
\hline$B_{u}^{+} \rightarrow J / \psi K^{*+}, J / \psi \rightarrow \ell^{+} \ell^{-}, K^{*+} \rightarrow K_{S}^{0} \pi^{+}, K_{S}^{0} \rightarrow \pi^{+} \pi^{-}$ \\
$B_{u}^{+} \rightarrow J / \psi K^{*+}, J / \psi \rightarrow \ell^{+} \ell^{-}, K^{*+} \rightarrow K^{+} \pi^{0}, \pi^{0} \rightarrow \gamma \gamma$ \\
\hline \hline
\end{tabular}


TАВ. 4.1: Rapports d'embranchement (BF) intervenant dans les désintégrations $B \rightarrow J / \psi K^{*}(892)$ [28] (ceux du $K^{*}$ sont obtenus par conservation de la symétrie d'isospin).

\begin{tabular}{lc}
\hline Désintégration & BF \\
\hline \hline$B_{d}^{0} \rightarrow J / \psi K^{* 0}$ & $(1.31 \pm 0.09) \times 10^{-3}$ \\
$B_{u}^{+} \rightarrow J / \psi K^{*+}$ & $(1.39 \pm 0.13) \times 10^{-3}$ \\
$J / \psi \rightarrow e^{+} e^{-}$ & $(5.93 \pm 0.10) \%$ \\
$J / \psi \rightarrow \mu^{+} \mu^{-}$ & $(5.88 \pm 0.10) \%$ \\
$K^{* 0} \rightarrow K^{0} \pi^{0}$ & $1 / 3$ \\
$K^{* 0} \rightarrow K^{+} \pi^{-}$ & $2 / 3$ \\
$K^{*+} \rightarrow K^{0} \pi^{+}$ & $2 / 3$ \\
$K^{*+} \rightarrow K^{+} \pi^{0}$ & $1 / 3$ \\
$K^{0} \rightarrow K_{S}^{0}$ & $1 / 2$ \\
$K_{S}^{0} \rightarrow \pi^{+} \pi^{-}$ & $(68.60 \pm 0.27) \%$ \\
$\pi^{0} \rightarrow \gamma \gamma$ & $(98.798 \pm 0.032) \%$ \\
\hline \hline
\end{tabular}

\subsection{Reconstruction des mésons $B$}

\subsubsection{Présélection des événements}

Les événements $B \bar{B}$ produits au pic de la résonance $\Upsilon(4 S)$ sont sélectionnés (après avoir passé le niveau 3 de déclenchement) en demandant :

- au moins trois traces chargées dans la région fiducielle $0.41<\theta<2.54$ rad (ces traces doivent satisfaire la sélection des traces chargées définie au paragraphe 3.3.1);

- une énergie totale des particules chargées et des neutres supérieure à $4.5 \mathrm{GeV}$ (les neutres sont définis comme des clusters de l'EMC d'énergie supérieure à $30 \mathrm{MeV}$ dans une région fiducielle $0.410<\theta<2.409$ rad non associés à une trace chargée) ;

- que le vertex primaire se situe au plus à $0.5 \mathrm{~cm}$ du centre de la tâche lumineuse (beamspot) dans le plan $x y$ et $\pm 6 \mathrm{~cm}$ en $z$ (ce vertex primaire est calculé événement par événement, par une procédure itérative qui commence par considérer toutes les traces chargées se trouvant dans le volume fiduciel, puis élimine toutes celles qui ont un $\chi^{2}$ important jusqu'à ce que l'ajustement soit stable ou qu'il ne reste plus que deux traces);

- que le moment de Fox-Wolfram [157] d'ordre deux normalisé, $R_{2}$, de l'événement soit inférieur à 0.5 (la variable $R_{2}$ fournit une bonne séparation entre les événements du continuum de type « jet » et les événements $B \bar{B}$ plus sphériques).

Estimée à partir d'une simulation Monte Carlo d'événements $B \bar{B}$, l'efficacité de ces critères est de $95.4 \pm 1.4 \%$.

Un deuxième niveau de filtrage est appliqué afin de rejeter les événements inintéressants. Il s'agit de sélectionner les événements qui contiennent au moins un candidat $J / \psi$ reconstruit dans sa désintégration leptonique $\ell^{+} \ell^{-}$. Le taux d'embranchement $B \rightarrow J / \psi X$ représente $(1.16 \pm 0.1) \%$ et celui du méson $J / \psi$ en paires d'électrons et de muons est de $(11.8 \pm 0.2) \%$ [28]. On applique une sélection lâche, dans laquelle toutes les traces chargées reconstruites sont considérées. L'hypothèse de masse électron ou muon est assignée à ces traces suivant la désintégration du $J / \psi$ considérée. Pour $e^{+} e^{-}$, on applique un algorithme de récupération des photons de freinage (bremsstrahlung). 
Une des deux traces doit être identifiée comme électron suivant les critères Loose ou NoCal définis au chapitre précédent. Aucune sélection n'est appliquée sur l'autre trace. La masse invariante du $J / \psi$, obtenue par addition des quadrivecteurs énergie-impulsion, doit se trouver dans l'intervalle de masse de $[2.5,3.3] \mathrm{GeV} / c^{2}$. Pour $\mu^{+} \mu^{-}$, l'une des traces doit satisfaire une sélection VeryLoose. Aucune sélection n'est appliquée sur l'autre trace. La masse invariante du $J / \psi$ doit se trouver dans l'intervalle $[2.8,3.3] \mathrm{GeV} / c^{2}$. L'échantillon final représente moins de $2 \%$ de l'ensemble des données enregistrées au pic de la résonance $\Upsilon(4 S)$.

\subsubsection{Sélection des mésons intermédiaires $\pi^{0}, K_{S}^{0}, K^{*}$ et $J / \psi$}

Les mésons intermédiaires sont reconstruits en combinant un ensemble de traces chargées et/ou de neutres. Les particules chargées et les neutres utilisés dans la reconstruction des mésons intermédiaires doivent avoir satisfait aux critères de sélection définis au chapitre précédent (volume fiduciel, énergie minimal, etc.). En reconstruisant les mésons intermédiaires, les traces qui les composent sont assignées à leur masse attendue $(e, \mu, \pi, K)$. On applique ensuite un ajustement du vertex avant de déterminer leur masse invariante afin d'améliorer les mesures des positions et des quadrivecteurs énergie-impulsion. Dans de rares cas, l'ajustement ne converge pas (moins de 1\% pour tous les candidats mésons) mais le candidat méson est conservé. La masse invariante est alors obtenue par addition des quadrivecteurs énergie-impulsion des traces/neutres composant le candidat méson après l'ajustement. Si un ou plusieurs produits de désintégration sont eux-mêmes des mésons intermédiaires, ils sont contraints à leur masse nominale, à l'exception du méson $K^{*}$ dont la largeur naturelle domine la résolution expérimentale.

\section{Sélection des $\pi^{0} \rightarrow \gamma \gamma$}

Les candidats pions neutres $\left(\pi^{0}\right)$ sont reconstruits à partir de paires de candidats photons, avec l'hypothèse qu'ils proviennent du point d'interaction. Le candidat $\pi^{0}$ doit avoir une masse invariante qui se situe dans l'intervalle $106<M_{\pi^{0}}<153 \mathrm{MeV} / c^{2}$ (la résolution est $\sim 6.8 \mathrm{MeV} / c^{2}$ ), et une énergie supérieure à $200 \mathrm{MeV}$. La borne inférieure sur la masse invariante est relâchée pour tenir compte des pertes d'énergie par fuite à l'arrière des cristaux du calorimètre (leakage).

\section{Sélection des $K_{S}^{0} \rightarrow \pi^{+} \pi^{-}$}

Les candidats $K_{S}^{0} \rightarrow \pi^{+} \pi^{-}$sont reconstruits à partir d'une combinaison de deux traces chargées de signes opposés. Leur masse invariante, calculée au vertex des deux traces, doit être comprise entre 489 et $507 \mathrm{MeV} / c^{2}$ (la résolution est $\sim 3.5 \mathrm{MeV} / c^{2}$ ). Afin de rejeter davantage de bruit de fond, on demande que l'angle dans le plan transverse (2DAngle) entre la ligne de vol du $K_{S}^{0}$ et la ligne joignant les vertex du $J / \psi$ et du $K_{S}^{0}$ soit inférieur à $200 \mathrm{mrad}$ et que la distance de vol au vertex du $J / \psi(f l t)$ soit plus grande que $1 \mathrm{~mm}$ (en trois dimensions). L'ajustement du vertex doit aussi converger $\left(p_{\chi^{2}}>0\right)$. 


\section{Sélection des $K^{*} \rightarrow K \pi$}

Les candidats $K^{*}$ sont reconstruits à partir de paires de candidats $(K, \pi)$, dont la masse invariante est à moins de $100 \mathrm{MeV} / c^{2}$ de la masse nominale du méson $K^{*}$ [28]. Pour les désintégrations avec $\pi^{0}$, un ajustement cinématique est effectué. De plus, on demande que le cosinus de l'angle $\theta_{K^{*}}$ (cf. Fig. 2.5) soit inférieur à 0.7 . Un veto des événements $B \rightarrow J / \psi\left(K \pi^{+}\right)^{*}$ dans le lot des événements reconstruits $B \rightarrow J / \psi\left(K \pi^{0}\right)^{*}$ est aussi appliqué (cf. §4.2.3). Pour les désintégrations avec $\pi^{+}$, le critère de sélection des particules chargées (cf. §3.3.1) est relâché en enlevant la coupure sur l'impulsion transverse du pion.

\section{Sélection des $J / \psi \rightarrow \ell^{+} \ell^{-}$}

La masse invariante des candidats $J / \psi$ doit se situer dans l'intervalle $2.95<M_{J / \psi}<3.14 \mathrm{GeV} / c^{2}$ et $3.06<M_{J / \psi}<3.14 \mathrm{GeV} / c^{2}$ pour les désintégrations $J / \psi \rightarrow e^{+} e^{-}$et $J / \psi \rightarrow \mu^{+} \mu^{-}$, respectivement. Les bornes représentent une coupure à environ $\pm 3 \sigma\left(\sigma \sim 13 \mathrm{MeV} / c^{2}\right)$ sauf pour la borne inférieure dans le cas $J / \psi \rightarrow e^{+} e^{-}$, qui est relâchée pour tenir compte des pertes par rayonnement de freinage (bremsstrahlung). Les critères de sélection retenus sont une sélection Tight ou NoCal pour chaque électron et Loose pour chaque muon. Pour augmenter l'efficacité de la sélection d'événements, les candidats électrons sont combinés avec un maximum de trois candidats photons pour récupérer une partie de l'énergie perdue par bremsstrahlung. En plus des critères de sélection des photons décrits au paragraphe 3.3.2, les photons utilisés pour la récupération du bremsstrahlung doivent avoir un moment de Zernike $A_{42}<0.25$. Ils doivent aussi former des angles maximaux de $35 \mathrm{mrad}$ en $\theta$ et de $50 \mathrm{mrad}$ en $\phi$ par rapport à la direction de la trace projetée sur les cristaux de l'EMC. Cette procédure permet d'augmenter l'efficacité de reconstruction du $J / \psi$ en $e^{+} e^{-}$de $30 \%$.

\subsubsection{Sélection des mésons $B$}

Les mésons $B$ sont reconstruits en combinant les candidats $J / \psi$ avec les candidats $K^{*}$. Deux variables cinématiques quasi non-corrélées sont utilisées pour sélectionner le signal. La première est la différence entre l'énergie reconstruite du candidat $B$ et l'énergie des faisceaux dans le centre de masse $\left(E_{\text {beam }}^{*}\right), \Delta E$. L'autre variable est la masse substituée définie par :

$$
m_{\mathrm{ES}}=\sqrt{E_{\exp }^{2}-\vec{p}_{B}^{2}}
$$

où, dans le repère du laboratoire, $E_{\text {exp }}=\left(s / 2-\vec{p}_{B} \cdot \vec{p}_{i}\right) / E_{i}$ est l'énergie attendue pour un candidat $B$ d'impulsion $\vec{p}_{B}$ et $\left(E_{i}, \vec{p}_{i}\right)$ le quadrivecteur énergie-impulsion de l'état initial $e^{+} e^{-} ; \sqrt{s}$ est l'énergie dans le centre de masse. Cette procédure permet de s'affranchir de «booster » dans le centre de masse et ainsi évite d'assigner des masses aux différentes traces chargées. Les légères variations de $E_{\text {beam }}^{*}$ au cours de la durée d'un run sont prises en compte dans le calcul de $m_{\mathrm{ES}}$. Les événements signal ont un $\Delta E$ proche de zéro et un $m_{\mathrm{ES}}$ piqué à la masse nominale du méson $B$. La résolution en $m_{\mathrm{ES}}$, de l'ordre de $3 \mathrm{MeV} / c^{2}$, est dominée par la résolution sur l'énergie des faisceaux. La résolution en $\Delta E$ est de l'ordre de $10 \mathrm{MeV}(20 \mathrm{MeV})$ pour les désintégrations $K^{*}$ contenant un pion chargé (neutre). 
On ne considère alors que les événements qui ont un $\left(m_{\mathrm{ES}}, \Delta E\right)$ compris dans la région définie par $5.2<m_{\mathrm{ES}}<5.3 \mathrm{GeV} / c^{2}$ et $|\Delta E|<0.120 \mathrm{GeV}$. Si, pour un même événement, plusieurs candidats $B$ sont définis dans cette région, seul le candidat ayant la plus petite valeur de $|\Delta E|$ est conservé. Cette procédure de sélection d'un unique candidat $B$ choisit dans $3.4 \%, 1.2 \%, 1.5 \%, 3.4 \%$ des cas, pour les désintégrations $K_{S}^{0} \pi^{0}, K^{+} \pi^{-}, K_{S}^{0} \pi^{+}$et $K^{+} \pi^{0}$ respectivement, un candidat $B$ différent de celui généré alors que ce dernier était présent parmi les candidats $B$ reconstruits. Différentes régions, définies dans le plan $\left(m_{\mathrm{ES}}, \Delta E\right)$, sont illustrées sur la figure 4.1 :

- la région signal correspond typiquement à moins de $3 \sigma$ des valeurs nominales pour un méson $B\left(m_{\mathrm{ES}}=m_{B}, \Delta E \simeq 0\right), \sigma$ est la résolution en $\left(m_{\mathrm{ES}}, \Delta E\right)$ déterminée à partir d'une simulation Monte Carlo;

- une région à bas $m_{\mathrm{ES}}$ définie par $5.2<m_{\mathrm{ES}}<5.27 \mathrm{GeV} / c^{2}$ et $|\Delta E|<3 \sigma(\Delta E)$.

Les divers critères de sélection pour les canaux $B \rightarrow J / \psi K^{*}(892)$ sont résumés dans la table 4.2.

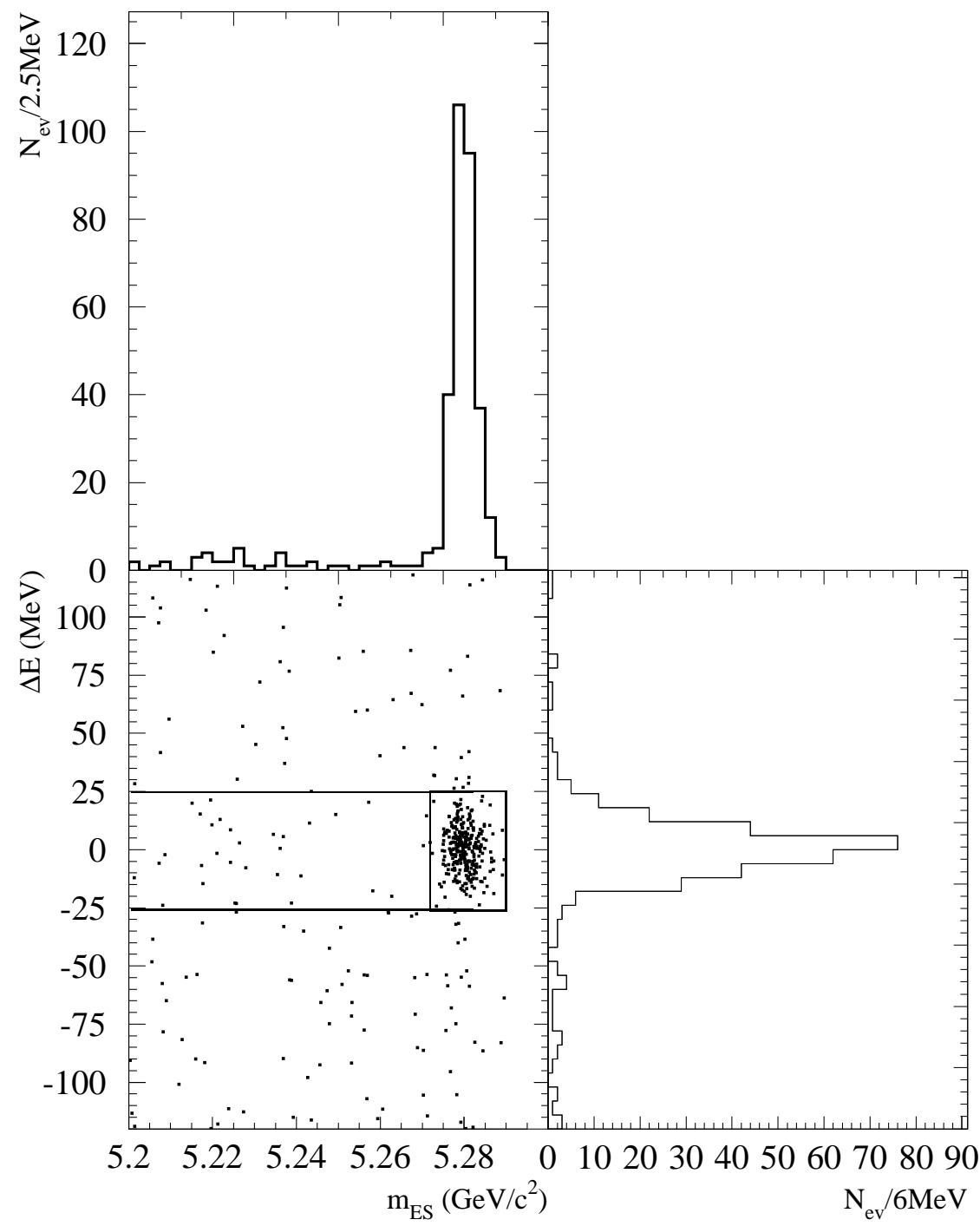

FIG. 4.1: Illustration du plan $\left(m_{\mathrm{ES}}, \Delta E\right)$ avec la région signal $\left(\left|m_{\mathrm{ES}}-m_{B_{d}^{0}}\right|<3 \sigma\left(m_{\mathrm{ES}}\right)\right.$ et $\left.|\Delta E|<3 \sigma(\Delta E)\right)$ et les projections en $m_{\mathrm{ES}}$ pour $|\Delta E|<3 \sigma(\Delta E)$ et $\Delta E$ pour $\left|m_{\mathrm{ES}}-m_{B_{d}^{0}}\right|<3 \sigma\left(m_{\mathrm{ES}}\right)$, obtenue à partir d'un échantillon $B_{d}^{0} \rightarrow J / \psi K_{S}^{0}\left(\pi^{+} \pi^{-}\right)$. 
TAB. 4.2: Résumé des critères de sélection pour les états finals $B \rightarrow J / \psi K^{*}(892)$.

\begin{tabular}{|c|c|c|c|c|}
\hline Critère & $J / \psi\left(K_{S}^{0} \pi^{0}\right)^{* 0}$ & $J / \psi\left(K^{+} \pi^{-}\right)^{* 0}$ & $J / \psi\left(K_{S}^{0} \pi^{+}\right)^{*+}$ & $J / \psi\left(K^{+} \pi^{0}\right)^{*+}$ \\
\hline$\overline{\mathrm{M}(e e)\left(\mathrm{GeV} / c^{2}\right)}$ & \multicolumn{4}{|c|}{$\overline{[2.95,3.14]}$} \\
\hline $\mathrm{M}(\mu \mu)\left(\mathrm{GeV} / c^{2}\right)$ & \multicolumn{4}{|c|}{$[3.06,3.14]$} \\
\hline$e$ PID & \multicolumn{4}{|c|}{ (Tight ou NoCal) et (Tight ou NoCal) } \\
\hline$\mu \mathrm{PID}$ & \multicolumn{4}{|c|}{ Loose et Loose } \\
\hline$\theta_{e}(\mathrm{rad})$ & \multicolumn{4}{|c|}{$[0.410,2.409]$} \\
\hline$\theta_{\mu}(\mathrm{rad})$ & \multicolumn{4}{|c|}{$[0.30,2.70]$} \\
\hline $\mathrm{M}\left(K_{S}^{0}\right)\left(\mathrm{GeV} / c^{2}\right)$ & {$[0.489,0.507]$} & & {$[0.489,0.507]$} & \\
\hline$K_{S}^{0} 2$ DAngle & $<200 \mathrm{mrad}$ & & $<200 \mathrm{mrad}$ & \\
\hline$K_{S}^{0}$ flt & $>1 \mathrm{~mm}$ & & $>1 \mathrm{~mm}$ & \\
\hline $\mathrm{M}\left(K^{*}\right)\left(\mathrm{GeV} / c^{2}\right)$ & \multicolumn{2}{|c|}{$[0.796,0.996]$} & \multicolumn{2}{|c|}{$[0.792,0.992]$} \\
\hline $\cos \left(\theta_{K^{*}}\right)$ & $<0.7+$ veto & & & $<0.7+$ veto \\
\hline $\mathrm{M}\left(\pi^{0}\right)\left(\mathrm{GeV} / c^{2}\right)$ & {$[0.106,0.153]$} & & & {$[0.106,0.153]$} \\
\hline$K^{+}$PID & & notAPion & & notAPion \\
\hline$\theta_{K}(\mathrm{rad})$ & & {$[0.45,2.50]$} & & {$[0.45,2.50]$} \\
\hline$E_{\gamma}(\mathrm{MeV})$ & $>30$ & & & $>30$ \\
\hline $\mathrm{LAT}_{\gamma}$ & $<0.8$ & & & $<0.8$ \\
\hline $\begin{array}{l}m_{\mathrm{ES}}\left(\mathrm{GeV} / c^{2}\right) \\
\text { région signal }\end{array}$ & \multicolumn{4}{|c|}{$[5.27,5.29]$} \\
\hline $\begin{array}{l}\Delta E(\mathrm{GeV}) \\
\text { région signal }\end{array}$ & {$[-0.07,0.05]$} & {$[-0.03,0.03]$} & {$[-0.03,0.03]$} & {$[-0.07,0.05]$} \\
\hline
\end{tabular}

\section{2 Étude des bruits de fond}

Les bruits de fond proviennent essentiellement de trois sources :

- les désintégrations de mésons $B$ contenant un $J / \psi \rightarrow \ell^{+} \ell^{-}$dans l'état final ;

- les désintégrations de mésons $B$ ne contenant pas de $J / \psi \rightarrow \ell^{+} \ell^{-}$dans l'état final ;

- les événements du continuum.

Ces trois sources peuvent être regroupées en deux catégories. La première, appelée combinatoire, provient de la combinaison aléatoire de traces chargées et de neutres provenant des deux mésons $B$ ou du continuum. Ce bruit de fond est distribué quasi uniformément en $m_{\mathrm{ES}}$ et ne pique pas à la masse du méson $B$. L'autre bruit de fond, de cinématique similaire à celle du signal (par exemple un pion mou provenant de la désintégration du $K^{*}$ est remplacé par un autre pion mou provenant de l'autre méson $B$ ), a tendance à s'accumuler dans la région signal. Le bruit de fond dominant des désintégrations $B \rightarrow J / \psi K^{*}(892)$ provient d'une mauvaise reconstruction du $K^{*}$, principalement due aux pions mous. Le bruit de fond combinatoire est estimé par une distribution empirique d'espace des phases, appelée distribution Argus [158], qui a pour forme

$$
A\left(m_{\mathrm{ES}} ; m_{0}, \xi\right)=A_{B} \cdot m_{\mathrm{ES}} \cdot \sqrt{1-x_{\mathrm{ES}}^{2}} \cdot e^{\xi\left(1-x_{\mathrm{ES}}^{2}\right)} .
$$

$x_{\mathrm{ES}} \equiv m_{\mathrm{ES}} / m_{0}<1, m_{0}$ représente la limite supérieure cinématique, fixée à l'énergie des faisceaux dans le centre de masse, soit $E_{\text {beam }}^{*}=5.291 \mathrm{GeV}$, et $A_{B}$ et $\xi$ sont des paramètres libres. 


\subsubsection{Bruits de fond sans charmonium}

Un échantillon représentant $9.6 \mathrm{fb}^{-1}$ de données prises en dessous du pic de la résonance $\Upsilon(4 S)$ (off-peak) est utilisé pour évaluer l'importance des bruits de fond du continuum $(q \bar{q}, q=u, d, s, c)$ et vérifier qu'aucune composante ne vienne s'accumuler dans la région signal. Les sections efficaces de production des divers processus produits à la résonance $\Upsilon(4 S)$ sont données dans la table 3.2 et peuvent donc potentiellement conduire à des bruits de fond importants. Pour corriger de la différence de $40 \mathrm{MeV}$ pour cet échantillon, $m_{\mathrm{ES}}$ est calculé avec des paramètres de faisceaux fixés aux valeurs typiques de ces derniers au pic de la résonance $\Upsilon(4 S)$. Les distributions en $m_{\mathrm{ES}}$ pour l'ensemble des données présentes dans plan $\left(m_{\mathrm{ES}}, \Delta E\right)$ sont représentées sur la figure 4.2. Un ajustement par une fonction Argus est intégrée sur l'intervalle $m_{\mathrm{ES}} \in[5.27,5.29] \mathrm{GeV} / c^{2}$. La normalisation de ces résultats à la luminosité des données prises au pic de la résonance (les sections efficaces en dessous du pic et sous le pic de la résonance $\Upsilon(4 S)$ sont quasiment identiques) et à la région en $|\Delta E|<3 \sigma(\Delta E)$ (cf. la table 4.2) permet d'obtenir une estimation de la quantité des bruits de fond provenant du continuum dans la région signal (cf. la table 4.3) pour les données prises au pic de la résonance $\left(\mathcal{L}=81.9 \mathrm{fb}^{-1}\right)$.

Un échantillon représentant $72.1 \mathrm{fb}^{-1}$ de données simulées de désintégrations $B \bar{B}$ est utilisé pour estimer les bruits de fond provenant des désintégrations de mésons $B$ où tout événement généré $B \rightarrow J / \psi\left(\rightarrow \ell^{+} \ell^{-}\right) X$ est éliminé. La procédure d'estimation du nombre d'événements dans la région signal est identique à celle effectuée pour les données off-peak. Les résultats sont résumés dans la table 4.4 et illustrés sur la figure 4.3.

\subsubsection{Bruits de fond $B \rightarrow J / \psi\left(\rightarrow \ell^{+} \ell^{-}\right) X$}

La simulation Monte Carlo des événements contenant un vrai $J / \psi$ provenant de la désintégration d'un méson $B$ contient les dernières mesures des rapports d'embranchement et prend en compte les désintégrations non résonantes, $B \rightarrow J / \psi K \pi$, et des résonances $K^{*}$ plus lourdes (voir la table 4.5). Un échantillon représentant $91 \mathrm{fb}^{-1}$ de données simulées, $B \rightarrow J / \psi\left(\rightarrow \ell^{+} \ell^{-}\right) X$, est utilisé en éliminant les événements contenant les désintégrations $B \rightarrow J / \psi K^{*}(892)$. Les résultats sont représentés sur la figure 4.4. L'essentiel des bruits de fond combinatoires est dominé par un vrai $J / \psi$ provenant de la désintégration d'un méson $B$ (plus de $98 \%$ des événements représentés sur la figure 4.4 contiennent un vrai $J / \psi$ ) et provient de la combinatoire $K \pi$ pour la reconstruction du $K^{*}$. L'accumulation d'événements sous le pic du méson $B$ dans le canal reconstruit $\left(K^{+} \pi^{-}\right)^{* 0}$ provient de la désintégration non résonante $B_{d}^{0} \rightarrow J / \psi K^{+} \pi^{-}$.

\subsubsection{Bruits de fond provenant du transfert entre les canaux $B \rightarrow J / \psi K^{*}(892)$}

Les échantillons d'événements reconstruits $B \rightarrow J / \psi K^{*}(892)$ contiennent des bruits de fond qui s'accumulent dans la région signal provenant du transfert entre les canaux $B \rightarrow J / \psi K^{*}(892)$. Ils peuvent être classés en deux catégories :

- les événements sélectionnés provenant du canal signal mais qui diffèrent du signal pur (SFA $\equiv$ Self Feed Across) (par exemple, le pion chargé dans le canal reconstruit $J / \psi\left(K_{S}^{0} \pi^{+}\right)^{*+}$ provient de la désintégration de l'autre méson $B$ ); 
ТАВ. 4.3: Estimation des bruits de fond provenant du continuum dans la région signal pour les quatre canaux $B \rightarrow$ $J / \psi K^{*}(892)$ à partir d'un échantillon représentant $9.6 \mathrm{fb}^{-1}$ de données off-peak, normalisée à la luminosité des données prises au pic de la résonance $\left(\mathcal{L}=81.9 \mathrm{fb}^{-1}\right)$.

\begin{tabular}{cccc}
\hline$J / \psi\left(K_{S}^{0} \pi^{0}\right)^{* 0}$ & $J / \psi\left(K^{+} \pi^{-}\right)^{* 0}$ & $J / \psi\left(K_{S}^{0} \pi^{+}\right)^{*+}$ & $J / \psi\left(K^{+} \pi^{0}\right)^{*+}$ \\
\hline \hline $0.2 \pm 0.1$ & $1.2 \pm 0.4$ & $0.1 \pm 0.1$ & $0.7 \pm 0.4$ \\
\hline \hline
\end{tabular}
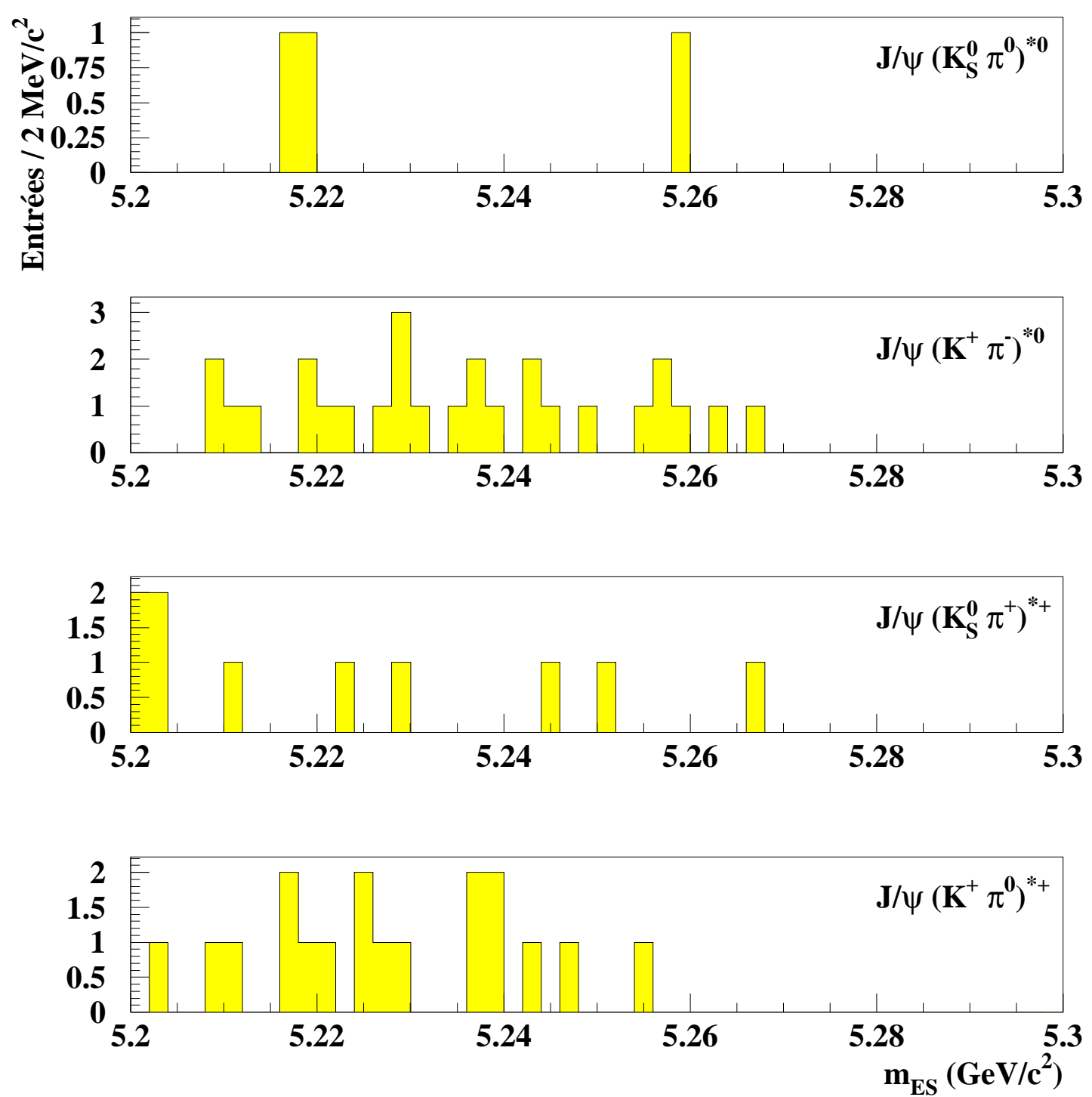

FIG. 4.2: Distributions en $m_{\mathrm{ES}}$ pour les quatre canaux $B \rightarrow J / \psi K^{*}(892)$ obtenues à partir de données off-peak représentant $9.6 \mathrm{fb}^{-1}$ sur l'ensemble de la région $\Delta E(|\Delta E|<0.12 \mathrm{GeV})$. 
TAB. 4.4: Estimation à partir d'un échantillon représentant $72.1 \mathrm{fb}^{-1}$ de données simulées des bruits de fond provenant de désintégrations $B \bar{B}$ ne contenant pas de désintégrations leptoniques de $J / \psi$ dans la région signal pour les quatre canaux $B \rightarrow J / \psi K^{*}(892)$, normalisée à la luminosité des données prises au pic de la résonance $\left(\mathcal{L}=81.9 \mathrm{fb}^{-1}\right)$.

\begin{tabular}{cccc}
\hline$J / \psi\left(K_{S}^{0} \pi^{0}\right)^{* 0}$ & $J / \psi\left(K^{+} \pi^{-}\right)^{* 0}$ & $J / \psi\left(K_{S}^{0} \pi^{+}\right)^{*+}$ & $J / \psi\left(K^{+} \pi^{0}\right)^{*+}$ \\
\hline \hline $0.2 \pm 0.1$ & $1.2 \pm 0.2$ & $0.3 \pm 0.1$ & $1.2 \pm 0.3$ \\
\hline \hline
\end{tabular}
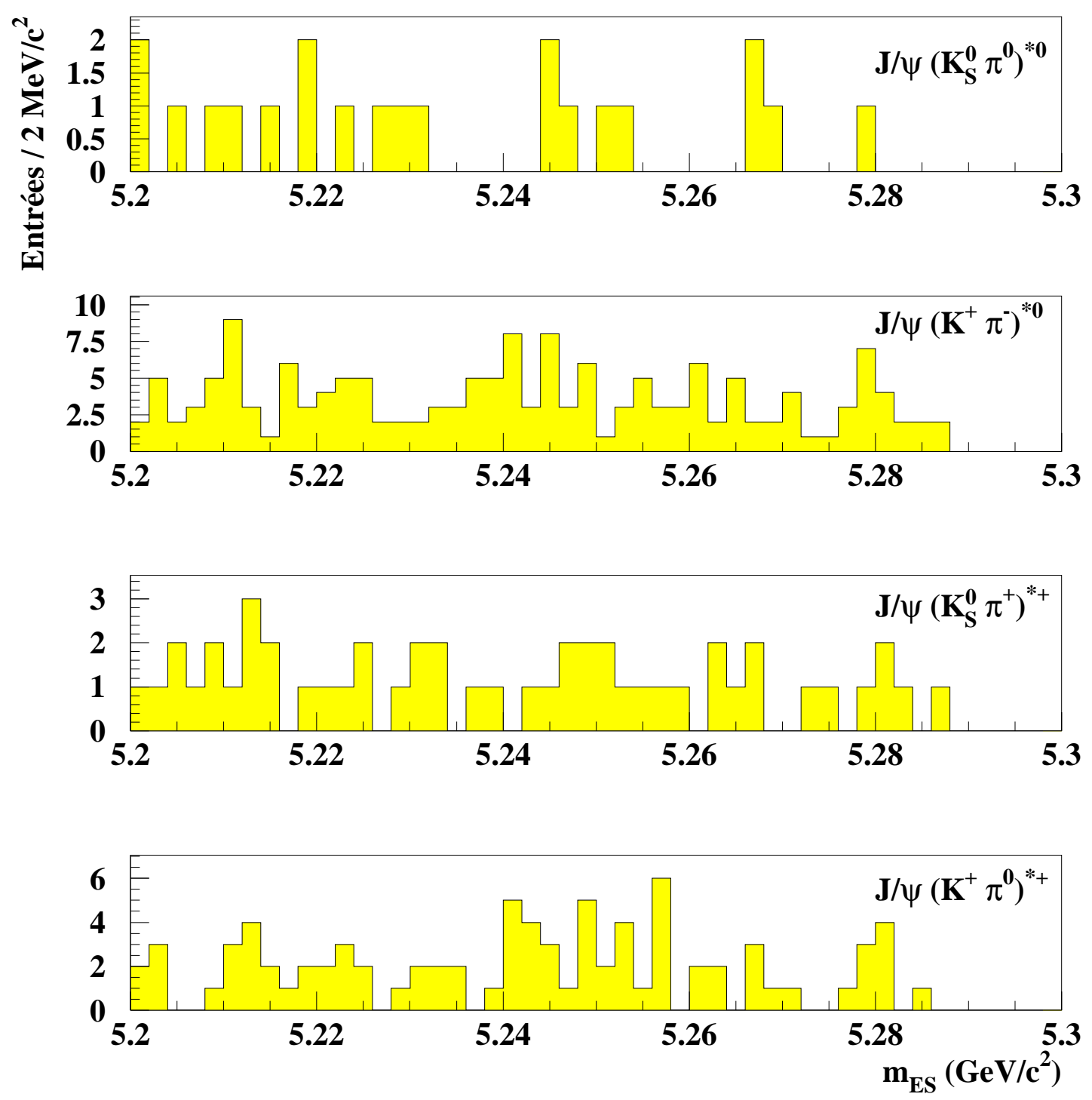

FIG. 4.3: Distributions en $m_{\mathrm{ES}}$ pour les quatre canaux $B \rightarrow J / \psi K^{*}(892)$ obtenues à partir de données simulées $B \bar{B}$ ne contenant pas de désintégrations leptoniques de $J / \psi$ sur l'ensemble de la région $\Delta E(|\Delta E|<0.12 \mathrm{GeV})$. 

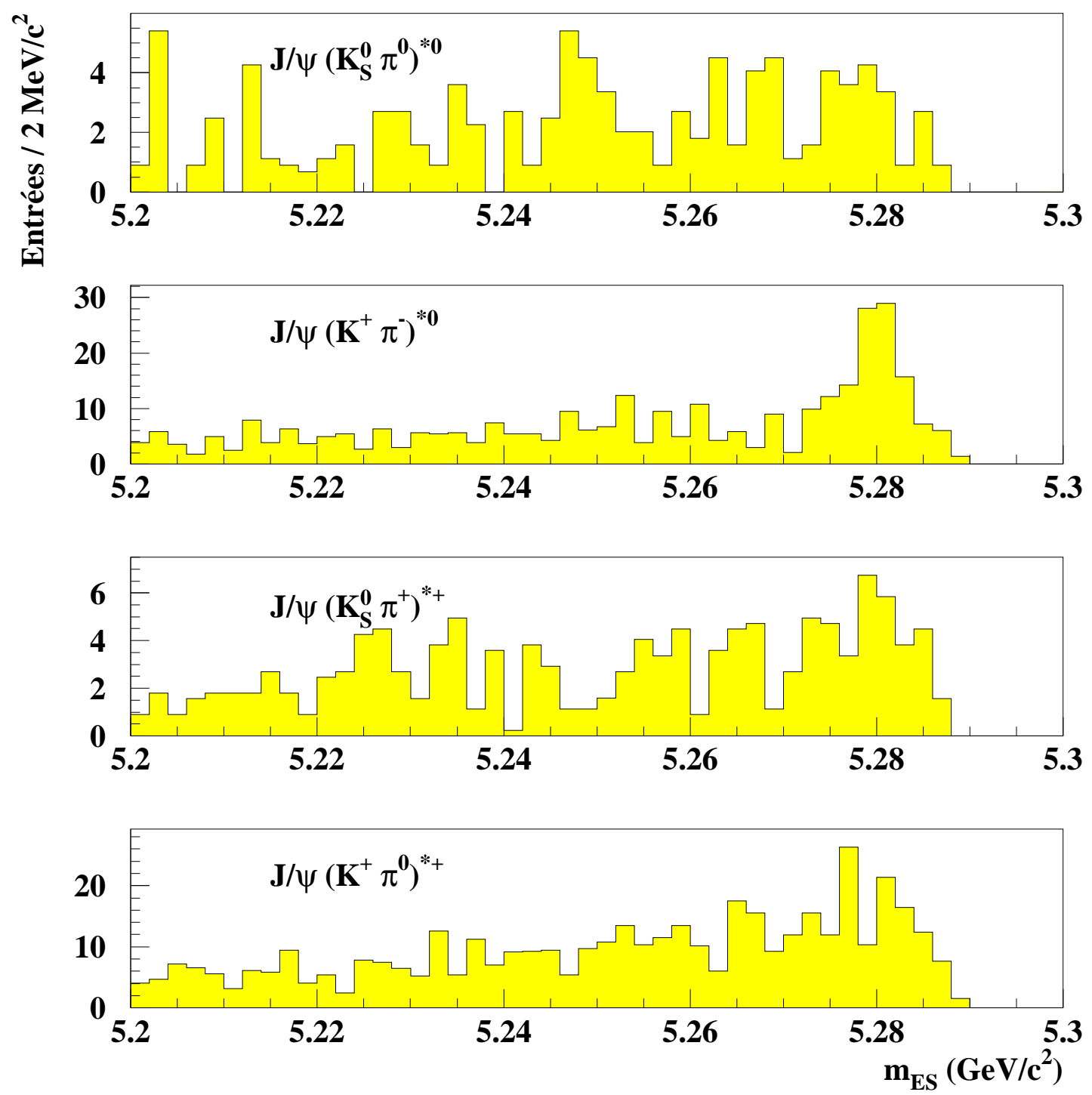

FIG. 4.4: Distributions en $m_{\mathrm{ES}}$ après coupure sur $\Delta E$, obtenues à partir de données simulées $B \rightarrow J / \psi X$ pour les quatre canaux $B \rightarrow J / \psi K^{*}(892)$, normalisées à la luminosité des données $\left(\mathcal{L}=81.9 \mathrm{fb}^{-1}\right)$. Seuls les événements ne contenant pas une désintégration $B \rightarrow J / \psi K^{*}(892)$ sont représentés. Le nombre d'événements pour $m_{E S} \in[5.27,5.29] \mathrm{GeV} / c^{2}$ est de $22,126,38$ et 135 pour les désintégrations $K_{S}^{0} \pi^{0}, K^{+} \pi^{-}, K_{S}^{0} \pi^{+}$et $K^{+} \pi^{0}$, respectivement. 
TAB. 4.5: Désintégrations de $K^{*}$ de masses supérieures au $K^{*}(892)$ [28].

\begin{tabular}{lcclll}
\hline Mode & $\begin{array}{c}\text { Masse } \\
\left(\mathrm{MeV} / c^{2}\right)\end{array}$ & $\begin{array}{c}\text { Largeur } \\
(\mathrm{MeV})\end{array}$ & $J^{P}$ & \multicolumn{2}{c}{ BF dominants } \\
& $1273 \pm 7$ & $90 \pm 20$ & $1^{+}$ & $K \rho$ & Fractions (\%) \\
\hline \hline$K_{1}(1270)$ & & & & $K_{0}^{*}(1430) \pi$ & $28 \pm 4$ \\
& & & & $K^{*}(892) \pi$ & $16 \pm 5$ \\
\hline$K_{1}(1400)$ & $1402 \pm 7$ & $174 \pm 13$ & $1^{+}$ & $K^{*}(892) \pi$ & $94 \pm 6$ \\
\hline$K^{*}(1410)$ & $1414 \pm 15$ & $232 \pm 21$ & $1^{-}$ & $K^{*}(892) \pi$ & $>40$ \\
& & & & $K \pi$ & $6.6 \pm 1.3$ \\
\hline$K_{0}^{*}(1430)$ & $1412 \pm 6$ & $294 \pm 23$ & $0^{+}$ & $K \pi$ & $93 \pm 10$ \\
\hline$K_{2}^{*}(1430)^{ \pm}$ & $1425.6 \pm 1.5$ & $98.5 \pm 2.7$ & $2^{+}$ & $K \pi$ & $49.9 \pm 1.2$ \\
$K_{2}^{*}(1430)^{0}$ & $1432.4 \pm 1.3$ & $109 \pm 5$ & & $K^{*}(892) \pi$ & $24.7 \pm 1.5$ \\
& & & & $K^{*}(892) \pi \pi$ & $13.4 \pm 2.2$ \\
\hline$K^{*}(1680)$ & $1717 \pm 27$ & $322 \pm 110$ & $1^{-}$ & $K \pi$ & $38.7 \pm 2.5$ \\
& & & & $K \rho$ & $31.4_{-2.1}^{+4.7}$ \\
& & & & $K^{*}(892) \pi$ & $29.9_{-4.7}^{+2.2}$ \\
\hline \hline
\end{tabular}

- les événements provenant des désintégrations $B \rightarrow J / \psi K^{*}(892)$ autres que les événements du canal signal (FA $\equiv$ Feed Across) (par exemple, le canal généré $J / \psi\left(K_{S}^{0} \pi^{+}\right)^{*+}$ est reconstruit en tant que canal $J / \psi\left(K_{S}^{0} \pi^{0}\right)^{* 0}$, le pion chargé ayant été remplacé par un pion neutre provenant de l'autre méson $B)$.

Ces bruits de fond sont beaucoup plus importants dans les désintégrations avec $\pi^{0}$ dans l'état final. Les différents niveaux de contamination sont donnés dans la table 4.6. Les éléments diagonaux de la table 4.6 sont la somme de deux contributions, à savoir l'efficacité de reconstruction et la contamination due au SFA (cf. la table 4.7). Cela correspond à un niveau de contamination du FA (SFA), après veto, pour les quatre canaux $B \rightarrow J / \psi K^{*}(892)$ de $4.8(14.3) \%, 1.8(3.0) \%, 4.5(4.0) \%$ et $3.3(14.8) \%$ par rapport au signal pur (cf. Figs. 4.5 et 4.6). La composition du FA est donnée dans la table 4.8. Le FA des canaux contenant un $\pi^{0}$ est majoritairement dû aux canaux contenant un $\pi^{+}$. Afin de limiter ce bruit de fond, un veto est appliqué : pour un même événement, si les désintégrations chargée et neutre sont sélectionnées dans la région signal, l'événement sélectionné dans sa désintégration neutre est rejeté. Les résultats de ce veto sont donnés dans la table 4.6. Le veto n'affecte quasiment pas l'efficacité de sélection du canal mais diminue le FA provenant du canal chargé de l'ordre de $75 \%$. La composition du SFA est majoritairement due à de faux $\pi^{0}$ (les photons ayant servi à reconstruire le $\pi^{0}$ sont différents de ceux générés) pour les canaux $K_{S}^{0} \pi^{0}(87 \%$ du SFA) et $K^{+} \pi^{0}\left(91 \%\right.$ du SFA). Pour le canal $K^{+} \pi^{-}\left(K_{S}^{0} \pi^{+}\right)$, il est dû pour $92 \%(97 \%)$ à un faux $K^{*}$ (le kaon $\left(K_{S}^{0}\right)$ et/ou le pion servant à la reconstruction du $K^{*}$ sont différents de ceux générés).

Les résolutions angulaires des trois angles de transversité et les distributions angulaires réduites après sélection sont données sur les figures 4.7 et 4.8. Chaque figure montre le signal pur, la contribution du SFA et du FA. Les résolutions angulaires sont de l'ordre de $10 \mathrm{mrad}$ pour le signal pur. Les distributions angulaires réduites permettent de déterminer les efficacités réduites (cf. Fig. 4.9) dont l'efficacité moyenne de reconstruction est obtenue en intégrant ces distributions. À noter que dans les distributions angulaires réduites, la partie $\cos \left(\theta_{K^{*}}\right)$ proche de $1(-1)$ correspond à la région 
TAB. 4.6: Probabilité (en \%) de reconstruction dans la région signal d'un canal $j$, généré $i$, pour les canaux $B \rightarrow$ $J / \psi K^{*}(892)$ (aucune pondération par les rapports d'embranchement n'est effectuée). Les chiffres donnés entre crochets sont obtenus avant d'avoir appliqué le veto. Les chiffres entre parenthèses correspondent aux événements qui sont étiquetés et qui ont passé les critères d'exigence sur les vertex des deux mésons $B$.

\begin{tabular}{|l||cccc|}
\hline \multicolumn{1}{|c||}{} & \multicolumn{4}{c|}{ Canal reconstruit $\downarrow$} \\
\hline \hline Canal généré $\downarrow$ & $J / \psi\left(K_{S}^{0} \pi^{0}\right)^{* 0}$ & $J / \psi\left(K^{+} \pi^{-}\right)^{* 0}$ & $J / \psi\left(K_{S}^{0} \pi^{+}\right)^{*+}$ & $J / \psi\left(K^{+} \pi^{0}\right)^{*+}$ \\
\hline$J / \psi\left(K_{S}^{0} \pi^{0}\right)^{* 0}$ & $10.97[11.14](7.13[7.24])$ & 0.09 & 1.28 & $0.34[0.34]$ \\
\hline$J / \psi\left(K^{+} \pi^{-}\right)^{* 0}$ & $0.01[0.01](0.01[0.01])$ & 25.23 & 0.07 & $0.12[0.52]$ \\
\hline$J / \psi\left(K_{S}^{0} \pi^{+}\right)^{*+}$ & $0.11[0.45](0.07[0.29])$ & 0.26 & 20.50 & $0.07[0.07]$ \\
\hline$J / \psi\left(K^{+} \pi^{0}\right)^{*+}$ & $0.07[0.07](0.06[0.06])$ & 0.69 & 0.02 & $14.35[14.49]$ \\
\hline \hline
\end{tabular}

TAB. 4.7: Décomposition de la probabilité de reconstruction dans la région signal d'un canal $i$ en efficacité de reconstruction et en SFA (en \%). Les chiffres donnés entre crochets sont obtenus avant d'avoir appliqué le veto.

\begin{tabular}{lcccc}
\hline & $J / \psi\left(K_{S}^{0} \pi^{0}\right)^{* 0}$ & $J / \psi\left(K^{+} \pi^{-}\right)^{* 0}$ & $J / \psi\left(K_{S}^{0} \pi^{+}\right)^{*+}$ & $J / \psi\left(K^{+} \pi^{0}\right)^{*+}$ \\
\hline \hline efficacité & $9.60[9.71]$ & 24.51 & 19.71 & $12.50[12.60]$ \\
SFA & $1.37[1.43]$ & 0.72 & 0.79 & $1.85[1.89]$ \\
\hline \hline
\end{tabular}

cinématique des pions (kaons) mous, d'après la définition de cet angle. Ainsi, la coupe à 0.7 permet de réduire les bruits de fond provenant de la combinatoire du $K^{*}$ contenant des pions mous.

\subsection{Estimation du nombre d'événements signal}

L'échantillon utilisé de données accumulées au pic de la résonance $\Upsilon(4 S)$ correspond à une période comprise entre octobre 1999 et juin 2002 (run 9931 - run 29435), soit un échantillon de 90 millions de paires de méson $B\left(81.9 \mathrm{fb}^{-1}\right)$. Dans le but d'illustrer les nombres d'événements mis en jeu, une estimation simplifiée est effectuée avec la distribution en $m_{\mathrm{ES}}$ (après coupure sur $\Delta E$ ). Cette estimation est obtenue par un ajustement avec une distribution gaussienne pour le signal et par une distribution Argus. Cette estimation surestime le nombre d'événements signal du fait des bruits de fond qui s'accumulent sous le pic du méson $B$, bien qu'une partie soit absorbée dans la distribution Argus. Les résultats sont présentés dans la table 4.9 et sur la figure 4.12.

Afin d'illustrer la perte d'efficacité des IFR pour la reconstruction des muons (cf. Fig. 3.20), les

TAB. 4.8: Composition du FA dans la région signal en $\%$, après pondération par les rapports d'embranchement [28]. Les chiffres donnés entre crochets sont obtenus avant d'avoir appliqué le veto.

\begin{tabular}{|l||cccc|}
\hline & \multicolumn{4}{c|}{ Canal reconstruit $\downarrow$} \\
\hline \hline Canal généré $\downarrow$ & $J / \psi\left(K_{S}^{0} \pi^{0}\right)^{* 0}$ & $J / \psi\left(K^{+} \pi^{-}\right)^{* 0}$ & $J / \psi\left(K_{S}^{0} \pi^{+}\right)^{*+}$ & $J / \psi\left(K^{+} \pi^{0}\right)^{*+}$ \\
\hline$J / \psi\left(K_{S}^{0} \pi^{0}\right)^{* 0}$ & $\times$ & 3.4 & 72.1 & $28.2[9.7]$ \\
\hline$J / \psi\left(K^{+} \pi^{-}\right)^{* 0}$ & $9.2[3.8]$ & $\times$ & 24.5 & $60.7[86.5]$ \\
\hline$J / \psi\left(K_{S}^{0} \pi^{+}\right)^{*+}$ & $47.5[78.4]$ & 19.5 & $\times$ & $11.1[3.8]$ \\
\hline$J / \psi\left(K^{+} \pi^{0}\right)^{*+}$ & $43.3[17.8]$ & 77.1 & 3.4 & $\times$ \\
\hline \hline
\end{tabular}


efficacités de reconstruction pour le canal $K^{+} \pi^{-}$pour les années 2000, 2001 et 2002 sont de 25.4\%, $24.1 \%$ et $18.7 \%$ pour la désintégration en muons alors qu'elles sont stables (voire en légère hausse) pour la désintégration en électrons $(26.1 \%, 26.2 \%$ et $26.5 \%)$.
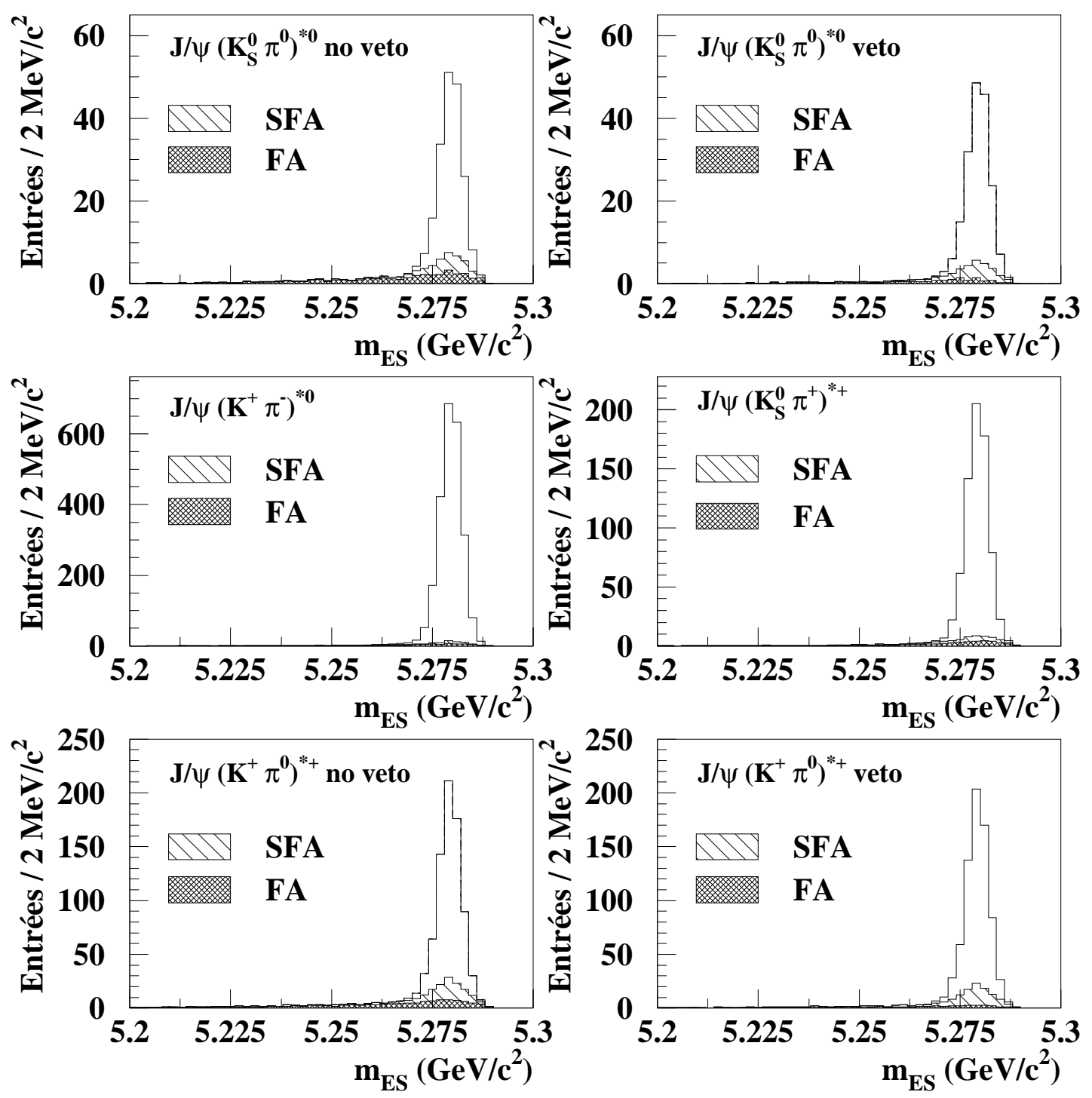

FIG. 4.5: Distributions en $m_{\mathrm{ES}}$ après coupure sur $\Delta E$, pour les quatre canaux $B \rightarrow J / \psi K^{*}(892)$, obtenues à partir de données simulées. Sur chaque distribution sont superposées les contributions du FA et du SFA, après repondération par les rapports d'embranchement [28]. Les distributions sont normalisées à la luminosité des données $\left(81.9 \mathrm{fb}^{-1}\right)$. Pour les canaux contenant un $\pi^{0}$, les distributions avant et après veto sont représentées. 

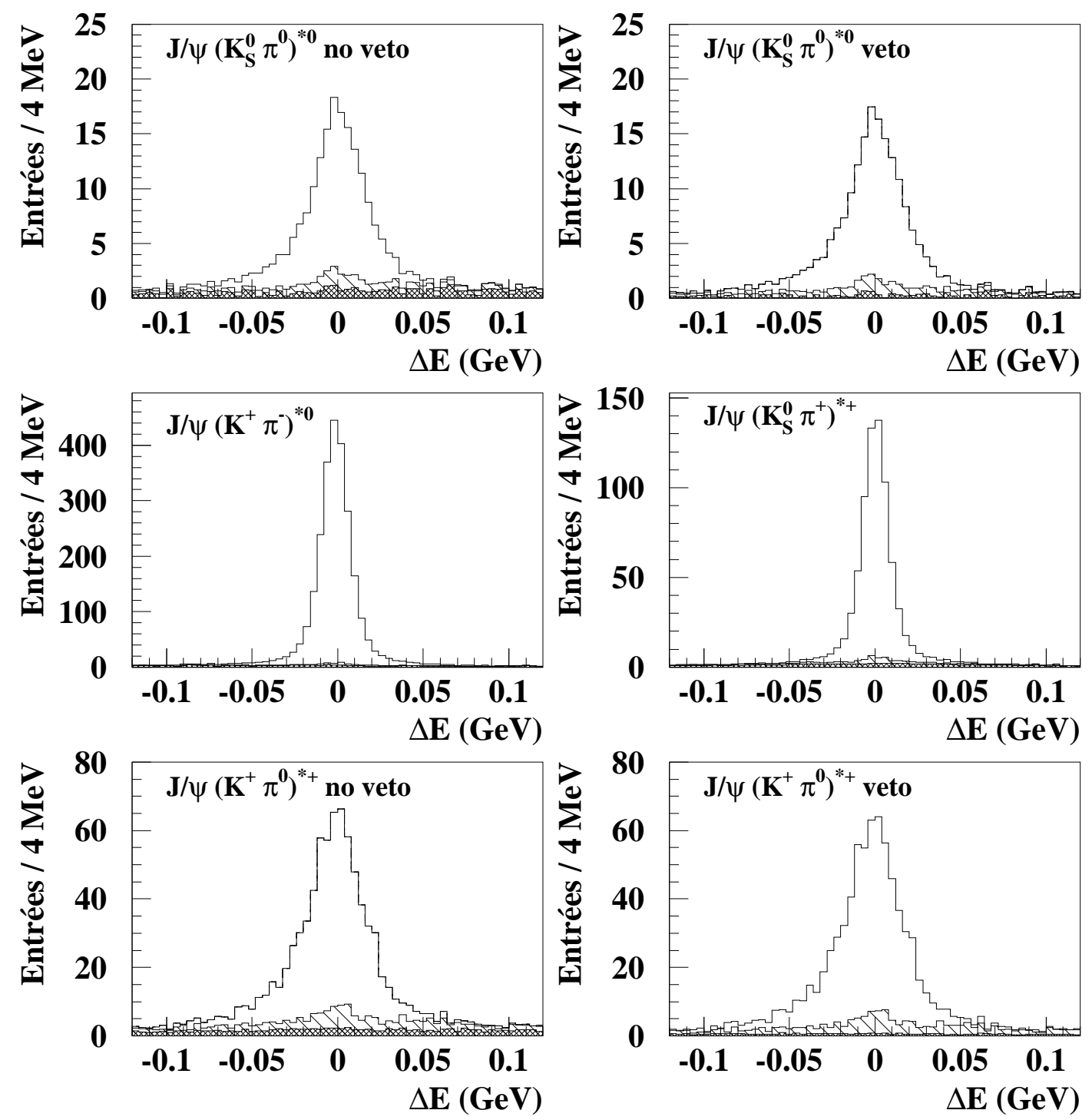

FIG. 4.6: Distributions en $\Delta E$ après coupure sur $m_{\mathrm{ES}}$, pour les quatre canaux $B \rightarrow J / \psi K^{*}(892)$, obtenues à partir de données simulées. Sur chaque distribution sont superposées les contributions du FA et du SFA, après repondération par les rapports d'embranchement [28]. Les distributions sont normalisées à la luminosité des données $\left(81.9 \mathrm{fb}^{-1}\right)$. Pour les canaux contenant un $\pi^{0}$, les distributions avant et après veto sont représentées. 

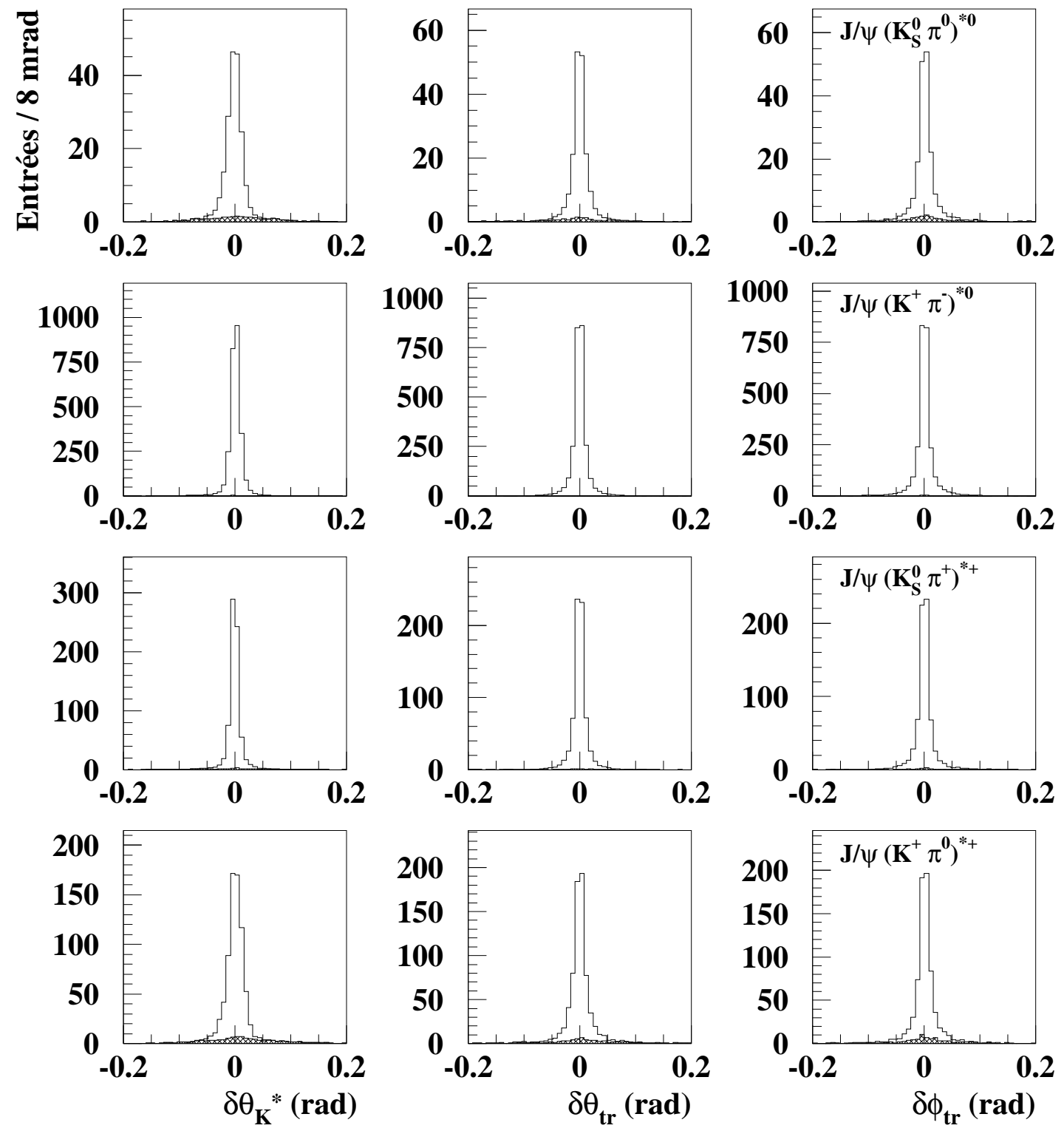

FIG. 4.7: Résolutions angulaires pour les quatre canaux $B \rightarrow J / \psi K^{*}(892)$ obtenues à partir de données simulées. Sur chaque distribution est superposée la contribution du FA et du SFA (partie hachurée), après repondération par les rapports d'embranchements [28]. Les distributions sont normalisées à la luminosité des données $\left(81.9 \mathrm{fb}^{-1}\right)$. 

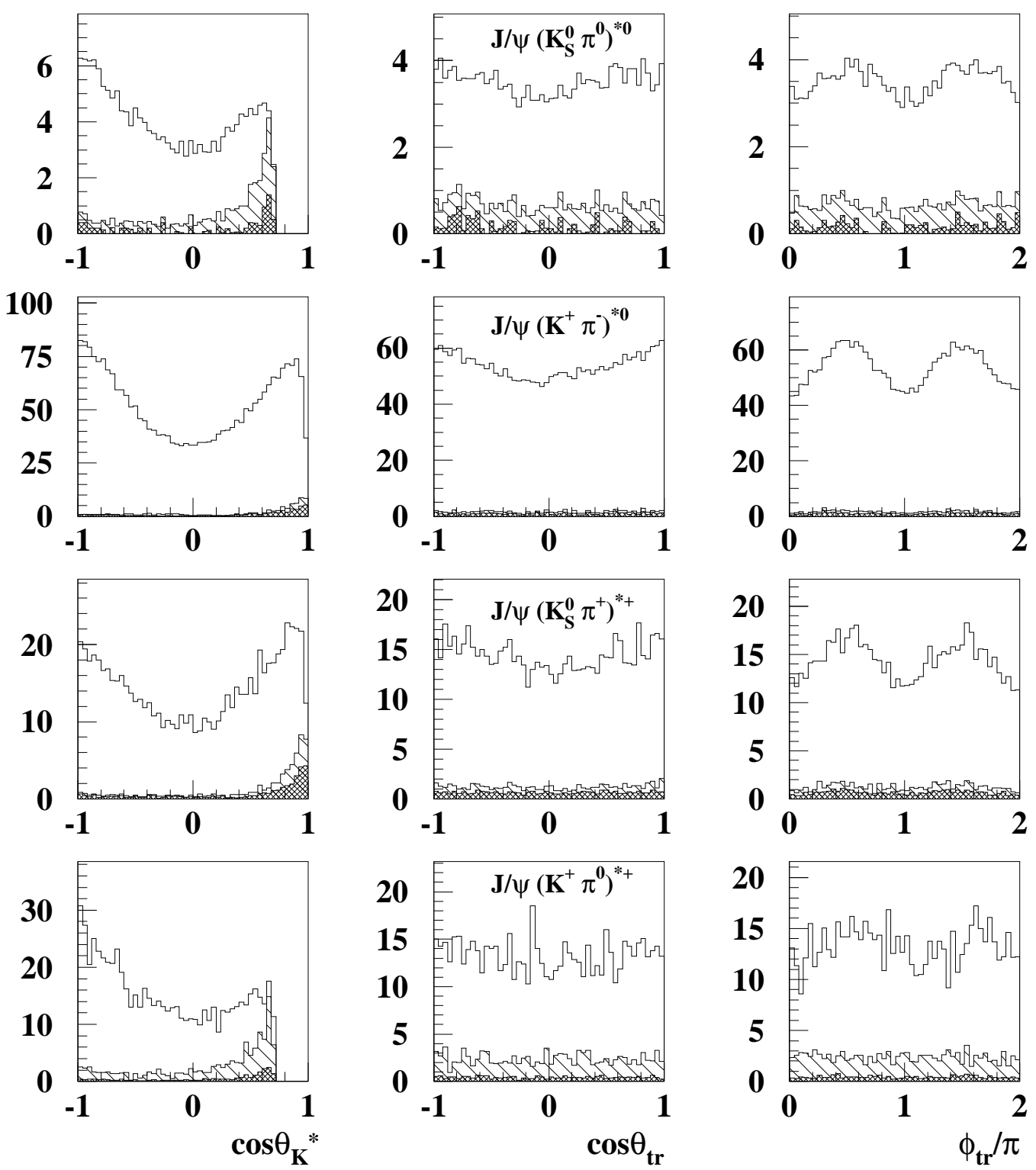

FIG. 4.8: Distributions angulaires réduites pour les quatre canaux $B \rightarrow J / \psi K^{*}(892)$ obtenues à partir de données simulées, après veto pour les canaux contenant un $\pi^{0}$. Sur chaque distribution sont superposées les contributions du FA et du SFA individuellement (partie hachurée, voir Fig. 4.5), après repondération par les rapports d'embranchements [28]. Les distributions sont normalisées à la luminosité des données $\left(81.9 \mathrm{fb}^{-1}\right)$. 

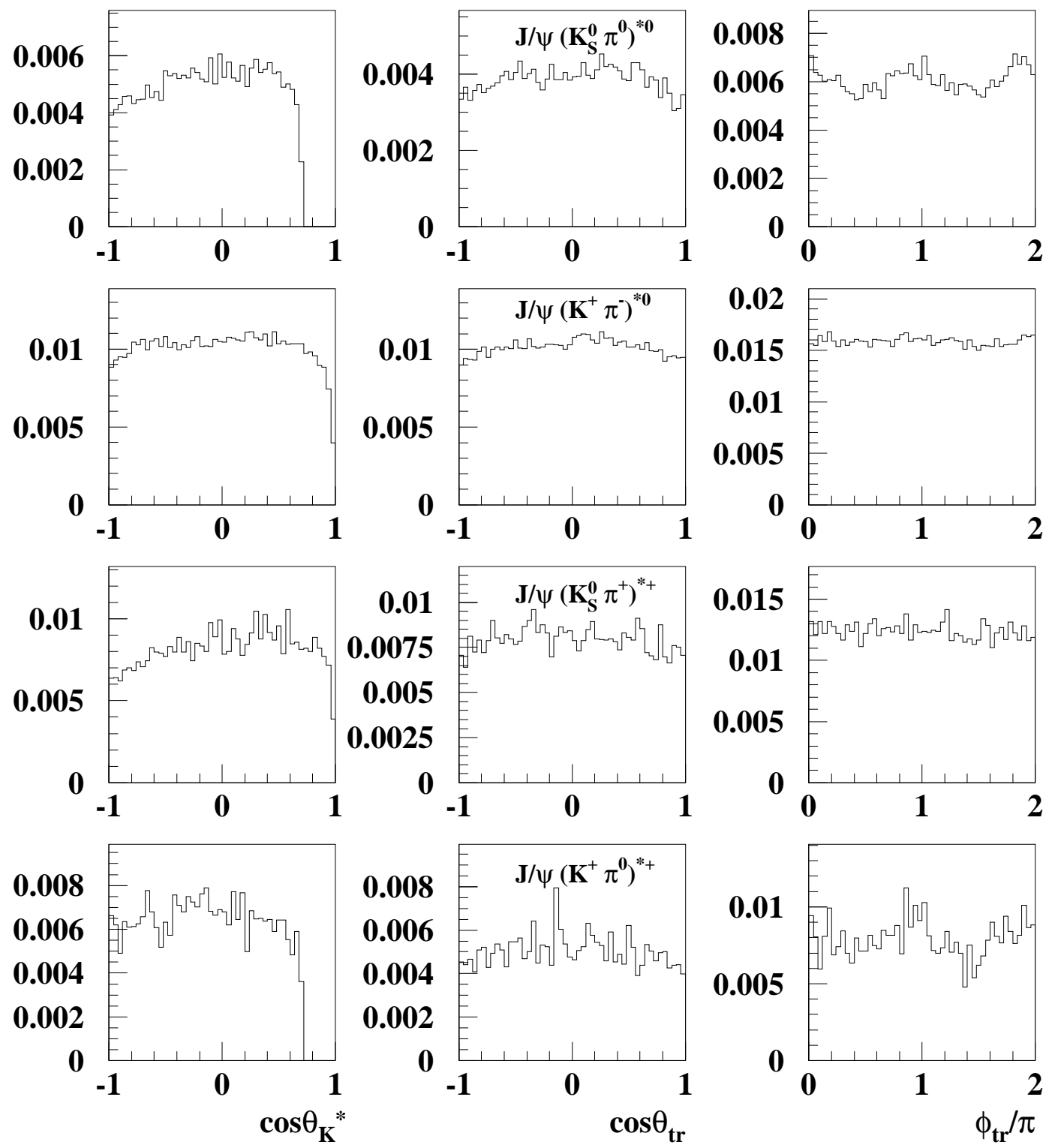

FIG. 4.9: Efficacités réduites obtenues après intégration sur deux des trois angles de transversité, après veto pour les canaux contenant un $\pi^{0}$. L'efficacité moyenne de reconstruction pour chaque canal est obtenue en intégrant ces distributions. 

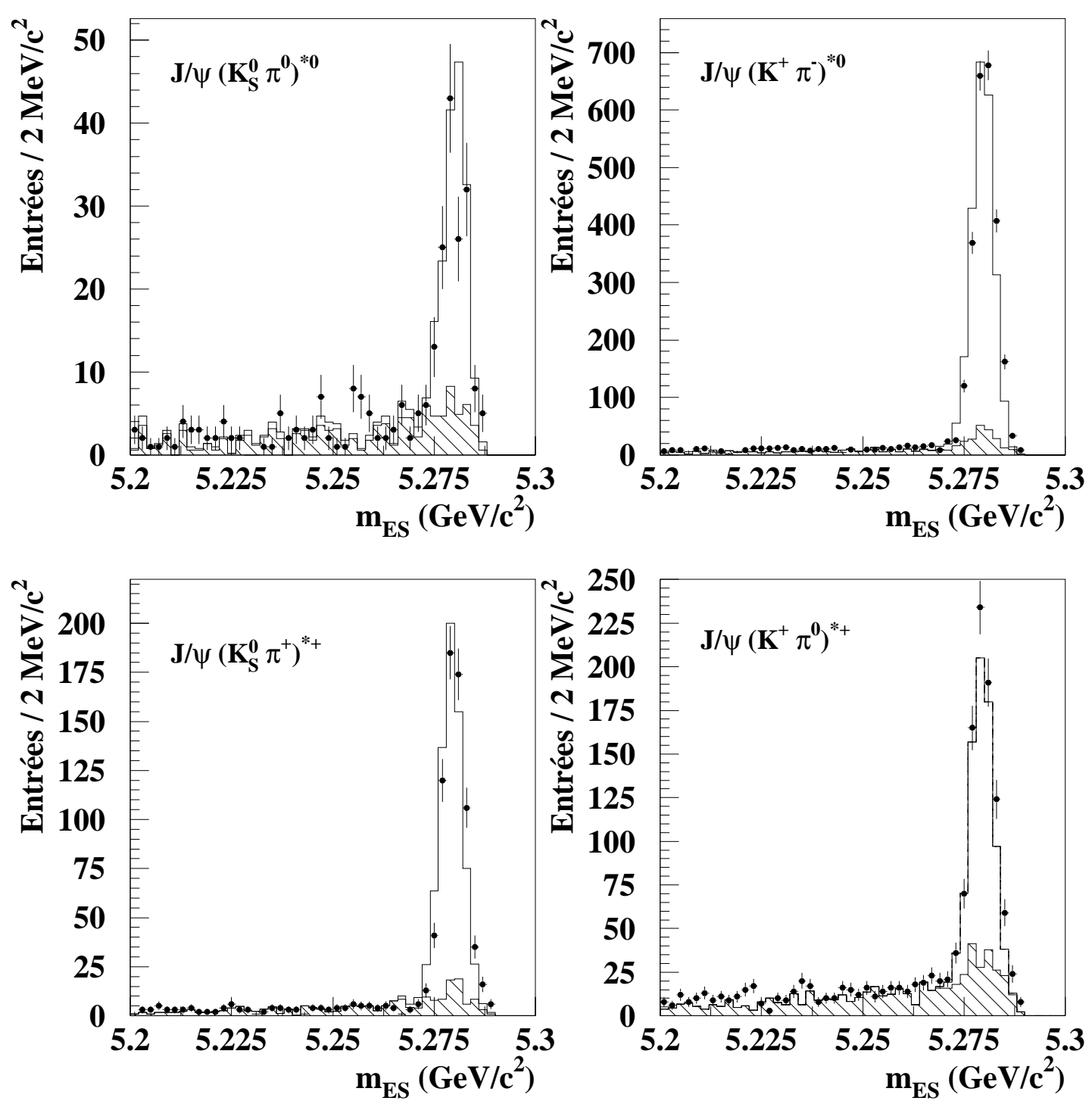

FIG. 4.10: Comparaison données et données simulées provenant de l'échantillon $B \rightarrow J / \psi\left(\rightarrow \ell^{+} \ell^{-}\right) X$ pour les distributions en $m_{\mathrm{ES}}$. La partie hachurée correspond aux bruits de fond contenant un $J / \psi\left(\rightarrow \ell^{+} \ell^{-}\right)$incluant le FA et le SFA. 

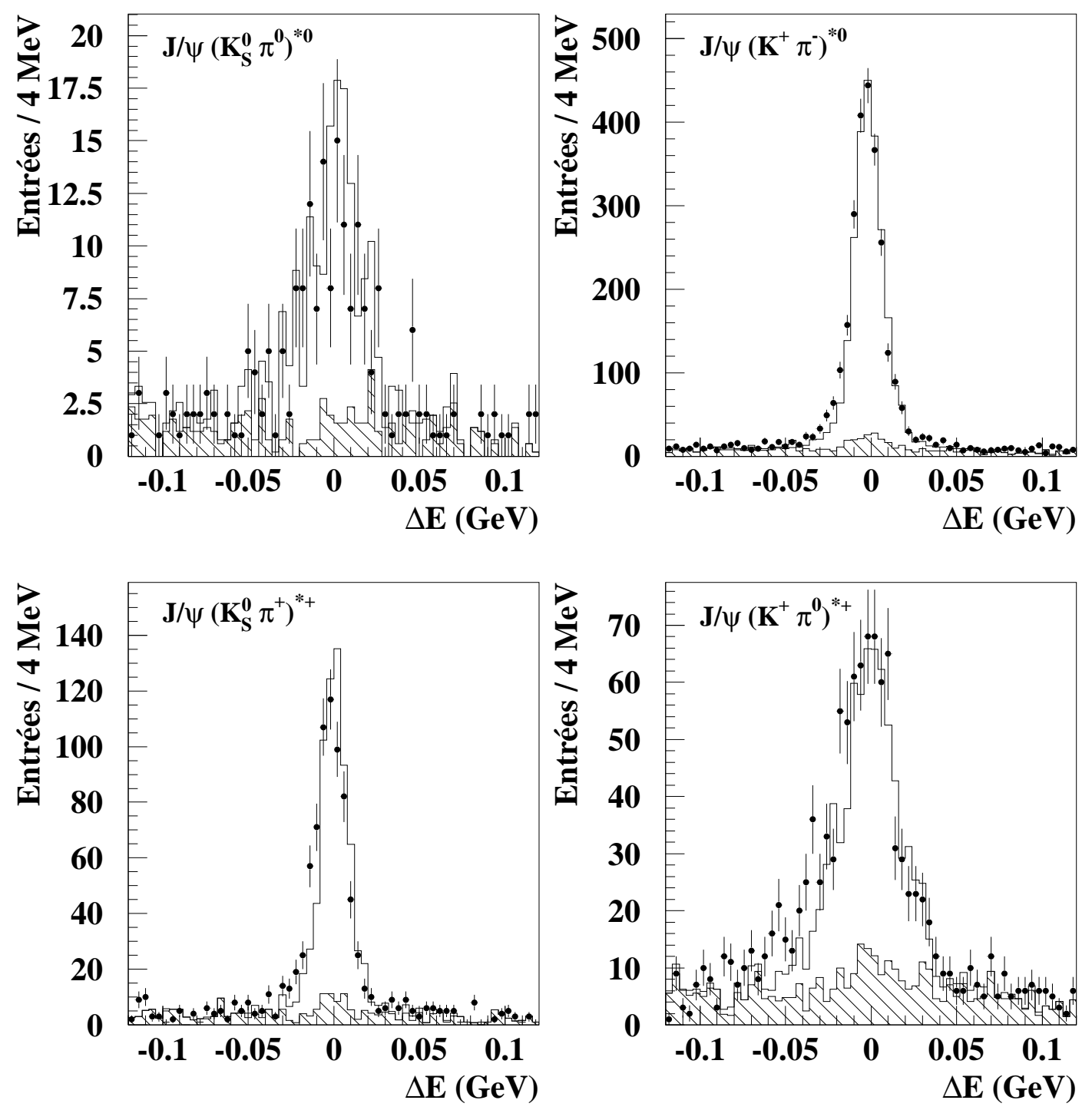

FIG. 4.11: Comparaison données et données simulées provenant de l'échantillon $B \rightarrow J / \psi\left(\rightarrow \ell^{+} \ell^{-}\right) X$ pour les distributions en $\Delta E$. On remarque un léger décalage entre les données et les données simulées. La partie hachurée correspond aux bruits de fond contenant un $J / \psi\left(\rightarrow \ell^{+} \ell^{-}\right)$incluant le FA et le SFA. 
TAB. 4.9: Résolution en $m_{\mathrm{ES}}$ et $\Delta E$ du signal, estimation du nombre d'événements signal (Yield) et pureté obtenues à partir d'un ajustement du spectre en $m_{\mathrm{ES}}$ (après coupure sur $\Delta E$ ) par une gaussienne et une fonction Argus sur les données pour chaque désintégration $B \rightarrow J / \psi K^{*}(892)$. La résolution en $\Delta E$ est obtenue à partir de la distribution en $\Delta E$ après coupure sur $m_{\mathrm{ES}}$ par un ajustement par une gaussienne et un polynôme du second degré. Les Yields correspondent aux nombres d'événements de la gaussienne pour $m_{\mathrm{ES}}>5.27 \mathrm{GeV} / c^{2}$. La pureté est déterminée comme étant le rapport entre le Yield et le nombre total d'événements pour $m_{\mathrm{ES}}>5.27 \mathrm{GeV} / c^{2}$.

\begin{tabular}{|l||c|c|c|c|c|}
\hline Canal & $\begin{array}{c}\sigma\left(m_{\mathrm{ES}}\right) \\
\left(\mathrm{MeV} / c^{2}\right)\end{array}$ & $\begin{array}{c}\sigma(\Delta E) \\
(\mathrm{MeV})\end{array}$ & $\begin{array}{c}\text { \# evts } \\
\text { région signal }\end{array}$ & Yield & Pureté $(\%)$ \\
\hline \hline$J / \psi\left(K_{S}^{0} \pi^{0}\right)^{* 0}$ & $2.96 \pm 0.26$ & $14.3 \pm 1.7$ & 163 & $131 \pm 13.9$ & 81.6 \\
\hline$J / \psi\left(K^{+} \pi^{-}\right)^{* 0}$ & $2.60 \pm 0.04$ & $9.04 \pm 0.23$ & 2486 & $2376.2 \pm 50.7$ & 95.8 \\
\hline$J / \psi\left(K_{S}^{0} \pi^{+}\right)^{*+}$ & $2.85 \pm 0.10$ & $9.27 \pm 0.39$ & 702 & $670.0 \pm 27.2$ & 95.7 \\
\hline$J / \psi\left(K^{+} \pi^{0}\right)^{*+}$ & $2.98 \pm 0.11$ & $17.5 \pm 1.3$ & 932 & $791.3 \pm 33.3$ & 85.0 \\
\hline \hline
\end{tabular}
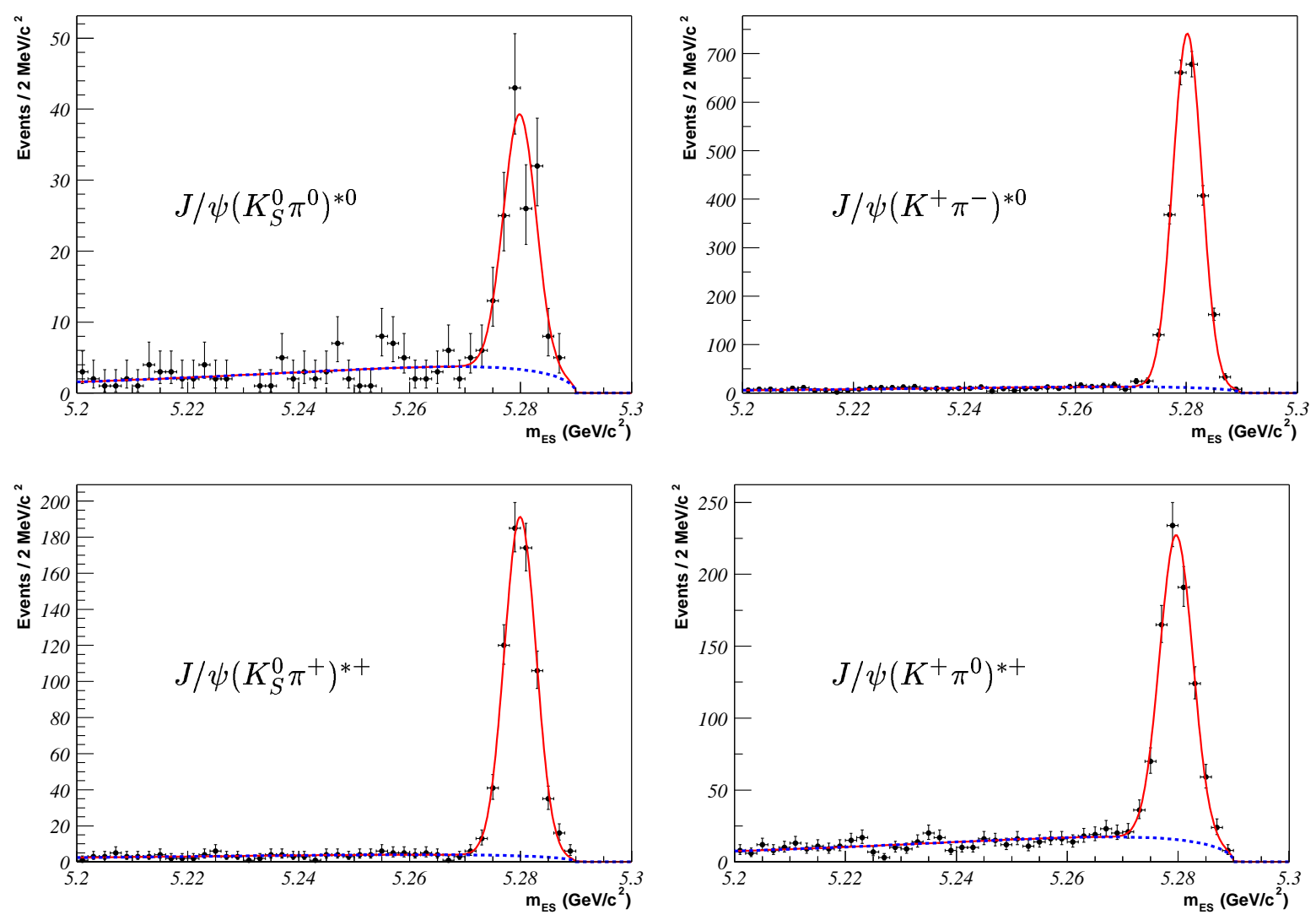

FIG. 4.12: Distributions en $m_{E S}$ après coupure sur $\Delta E$ des quatre désintégrations $B \rightarrow J / \psi K^{*}(892)$ obtenues à partir des données. Un ajustement par une gaussienne et une distribution Argus est superposée. 


\section{Chapitre 5}

\section{Mesure des amplitudes de transversité des canaux $B \rightarrow J / \psi K^{*}(892)$}

Ce chapitre décrit la méthode utilisée pour mesurer les amplitudes de transversité des canaux $B \rightarrow J / \psi K^{*}(892)$. Cette mesure est un préalable aux mesures de $\sin 2 \beta$ et $\cos 2 \beta$ avec le canal $B_{d}^{0} \rightarrow J / \psi\left(K_{S}^{0} \pi^{0}\right)^{* 0}$. L'analyse $C P$ est plus ambitieuse dans la compréhension des bruits de fond puisqu'il faut déterminer leur contenu $C P$ conditionnant leur évolution temporelle. Cela nécessite une description complète de la densité de probabilité normalisée utilisée dans un maximum de vraisemblance. La stratégie choisie dans la mesure des amplitudes est plus simple car basée sur l'utilisation d'un pseudo-maximum de vraisemblance. La densité de probabilité normalisée du signal est donnée par l'équation (2.73). Seules les distributions angulaires sont considérées, à savoir celles obtenues après intégration sur le temps. De plus, on se place dans l'hypothèse de l'absence de violation de $C P$ dans la désintégration (violation de $C P$ directe) de sorte que $\left|A_{\sigma}(0)\right|=\left|\bar{A}_{\sigma}(0)\right|$ $(\sigma=0, \|, \perp)$ (cf. $§ 2.2 .2)$.

Dans un premier temps, je détaille quelle serait la méthode pour la mesure des amplitudes avec un maximum de vraisemblance qui nécessite une description exhaustive du problème. Cela me permet de mettre en avant les difficultés liées à la nécessité de connaître la fonction d'efficacité de détection qui devient obligatoire en présence de bruits de fond, même dans le cas d'une bonne résolution angulaire. Dans un deuxième temps, j'expose la méthode utilisée pour la mesure des amplitudes de transversité, basée sur l'utilisation d'un pseudo-maximum de vraisemblance dont le grand avantage est de pouvoir s'affranchir de la connaissance de la fonction d'efficacité de détection, même en présence de bruits de fond, dans le cas d'une bonne résolution angulaire. Les bruits de fond sont estimés à partir des événements à bas $m_{\mathrm{ES}}\left(5.2<m_{\mathrm{ES}}<5.27 \mathrm{GeV} / c^{2},|\Delta E|<3 \sigma(\Delta E)\right)$ et entrent dans le pseudo-maximum de vraisemblance avec un poids négatif, permettant de soustraire la contribution de ces derniers dans la région signal.

\subsection{Ajustement par maximum de vraisemblance}

Cette section décrit comment on pourrait mesurer les amplitudes de transversité avec un maximum de vraisemblance. L'ajustement par un maximum de vraisemblance nécessite une description complète du problème, ce qui parfois n'est pas l'approche la plus attractive. Tel est le cas lorsque 
différentes composantes du problème sont difficilement descriptibles en termes d'une densité de probabilité normalisée telles que la connaissance de la fonction d'efficacité de détection qui implique des performances du détecteur ou la dépendance angulaire des bruits de fond. Ce n'est pas cette méthode qui est utilisée pour mesurer les amplitudes de transversité mais une méthode simplifiée basée sur un pseudo-maximum de vraisemblance décrite au paragraphe 5.2. Cette section a pour but d'introduire différents concepts, qui sont utilisés dans le pseudo-maximum de vraisemblance et dans le maximum de vraisemblance pour les mesures de $\sin 2 \beta$ et $\cos 2 \beta$ avec le canal $B_{d}^{0} \rightarrow J / \psi\left(K_{S}^{0} \pi^{0}\right)^{* 0}$ décrit au chapitre suivant.

Chaque canal reconstruit $B \rightarrow J / \psi K^{*}(892)$ reçoit les contributions du signal avec effets d'efficacité de détection, du transfert entre les canaux $B \rightarrow J / \psi K^{*}(892)$ (le FA et le SFA) et de divers bruits de fond. La densité de probabilité normalisée entrant dans un maximum de vraisemblance doit prendre en compte ces différentes contributions afin d'obtenir une estimation correcte des amplitudes. Dans un premier temps, je détaille la densité de probabilité normalisée observée dans le cas d'un unique canal reconstruit $B \rightarrow J / \psi K^{*}(892)$. Je généralise ensuite aux quatre canaux reconstruits $B \rightarrow J / \psi K^{*}(892)$ en prenant en compte le FA et le SFA.

\subsubsection{Fonction de transfert}

Afin d'alléger les notations, nous désignons par $g(\vec{\omega} ; \vec{A})$ la distribution signal, soit

$$
\stackrel{(-)}{g}(\vec{\omega} ; \vec{A}) \equiv \frac{\left.\mathrm{d}^{3} \Gamma \stackrel{(-)}{B} \rightarrow J / \psi\left(\rightarrow \ell^{+} \ell^{-}\right) \stackrel{(-)}{K^{*}}(\rightarrow K \pi)\right)}{\mathrm{d} \cos \theta_{K^{*}} \mathrm{~d} \cos \theta_{t r} \mathrm{~d} \varphi_{t r}}
$$

avec $\vec{\omega} \equiv\left(\cos \theta_{K^{*}}, \cos \theta_{t r}, \varphi_{t r}\right)$ et $\vec{A} \equiv\left(A_{0}, A_{\|}, A_{\perp}\right)$. Sous l'hypothèse d'absence de violation de $C P$ dans la désintégration, on a $\bar{g}(\vec{\omega} ; \vec{A})=g(\vec{\omega} ; \vec{A})$ avec $\vec{A} \equiv\left(A_{0}, A_{\|},-A_{\perp}\right)$. Les divers critères de sélection, l'acceptance géométrique du détecteur, la résolution sur les traces et les neutres, etc., font que la distribution observée est quelque peu différente de celle générée. La densité de probabilité normalisée observée doit tenir compte de ces effets. Elle prend la forme [159] :

$$
g_{o b s}\left(\vec{\omega}^{\prime} ; \vec{A}\right)=\frac{\int g(\vec{\omega} ; \vec{A}) \cdot T\left(\vec{\omega}, \vec{\omega}^{\prime}\right) \mathrm{d} \vec{\omega}}{\iint g(\vec{\omega} ; \vec{A}) \cdot T\left(\vec{\omega}, \vec{\omega}^{\prime}\right) \mathrm{d} \vec{\omega} \mathrm{d} \vec{\omega}^{\prime}},
$$

où $T\left(\vec{\omega}, \vec{\omega}^{\prime}\right)$ est la fonction de transfert [159] qui inclut les pertes par acceptance et les effets de résolution. $\vec{\omega}$ et $\vec{\omega}^{\prime}$ sont les angles générés et reconstruits. La fonction de transfert représente la densité de probabilité (non normalisée) qu'un événement produit avec les angles $\vec{\omega}$ soit reconstruit avec les angles $\vec{\omega}^{\prime}$. L'efficacité est donnée par :

$$
\varepsilon(\vec{\omega})=\int T\left(\vec{\omega}, \vec{\omega}^{\prime}\right) \mathrm{d} \vec{\omega}^{\prime}
$$

De manière générale, la fonction de résolution $R\left(\vec{\omega}, \vec{\omega}^{\prime}\right)$ (normalisée) peut être définie par :

$$
T\left(\vec{\omega}, \vec{\omega}^{\prime}\right)=\varepsilon(\vec{\omega}) \cdot R\left(\vec{\omega}, \vec{\omega}^{\prime}\right)
$$


Dans le cas d'une bonne résolution angulaire, que l'on idéalise par une distribution de Dirac :

$$
R\left(\vec{\omega}, \vec{\omega}^{\prime}\right)=\delta\left(\vec{\omega}-\vec{\omega}^{\prime}\right)
$$

on obtient

$$
g_{o b s}\left(\vec{\omega}^{\prime} ; \vec{A}\right)=g\left(\vec{\omega}^{\prime} ; \vec{A}\right) \cdot \frac{\varepsilon\left(\vec{\omega}^{\prime}\right)}{\langle\varepsilon\rangle(\vec{A})},
$$

où l'efficacité moyenne $\langle\varepsilon\rangle(\vec{A})$, qui dépend des amplitudes, est donnée par :

$$
\langle\varepsilon\rangle(\vec{A})=\int g\left(\vec{\omega}^{\prime} ; \vec{A}\right) \cdot \varepsilon\left(\vec{\omega}^{\prime}\right) \mathrm{d} \vec{\omega}^{\prime} .
$$

En l'absence de bruits de fond et sous l'hypothèse d'une bonne résolution angulaire, le maximum de vraisemblance prend la forme (cf. eq. (5.6)) :

$$
\ln \mathcal{L}=\sum_{i=1}^{N} \ln \left[g\left(\vec{\omega}^{\prime} ; \vec{A}\right) \cdot \frac{\varepsilon\left(\vec{\omega}^{\prime}\right)}{\langle\varepsilon\rangle(\vec{A})}\right]=\sum_{i=1}^{N} \ln \left[\frac{g\left(\vec{\omega}^{\prime} ; \vec{A}\right)}{\langle\varepsilon\rangle(\vec{A})}\right]+\sum_{i=1}^{N} \ln \varepsilon\left(\vec{\omega}^{\prime}\right) .
$$

On peut laisser tomber le dernier terme qui ne dépend pas des amplitudes et qui n'a donc aucune incidence sur le résultat de l'ajustement. Seule la connaissance de l'efficacité moyenne $\langle\varepsilon\rangle(\vec{A})$ est nécessaire. En utilisant la linéarité de la distribution angulaire (cf. eq. (2.73)) :

$$
g(\vec{\omega} ; \vec{A})=\sum_{l=1}^{6} K_{l} \cdot f_{l}(\vec{\omega})
$$

l'efficacité moyenne s'écrit

$$
\langle\varepsilon\rangle(\vec{A})=\sum_{l=1}^{6} K_{l} \cdot \Phi_{l},
$$

où les constantes $\Phi_{l}=\int f_{l}\left(\vec{\omega}^{\prime}\right) \cdot \varepsilon\left(\vec{\omega}^{\prime}\right) \mathrm{d} \vec{\omega}^{\prime}$ sont indépendantes des amplitudes de transversité et peuvent être évaluées en utilisant une méthode d'intégration Monte Carlo. Dans le cas présent, les données simulées utilisées pour la détermination des constantes $\Phi_{l}$ sont générées avec pour densité de probabilité la distribution $g\left(\vec{\omega} ; \vec{A}_{M C}\right)$ avec des amplitudes $\vec{A}_{M C}$ correspondants aux premières mesures faites par BABAR [160]:

$$
\Phi_{l} \simeq \frac{1}{N_{g e n}} \sum_{i=1}^{N_{a c c}} \frac{f_{l}\left(\vec{\omega}^{\prime}\right)}{g\left(\vec{\omega}^{\prime} ; \vec{A}_{M C}\right)} .
$$

$N_{a c c}$ est le nombre d'événements détectés parmi les $N_{\text {gen }}$ événements Monte Carlo générés. Dans le cas où l'état final $B \rightarrow J / \psi K \pi$ diffère entre un $B$ et un $\bar{B}$ (tel est le cas des canaux $K^{+} \pi^{-}, K_{S}^{0} \pi^{+}$ et $K^{+} \pi^{0}$ ), la réponse du détecteur est différente puisque les sections efficaces matière-matière et matière-antimatière diffèrent. Pour tenir compte de ces effets, on sépare les constantes $\Phi_{l}$ en deux séries suivant l'étiquetage du méson $B$, étiquetage obtenu grâce au signe du kaon chargé ou du pion chargé de l'état final. En conclusion, la connaissance de l'efficacité $\varepsilon\left(\vec{\omega}^{\prime}\right)$ n'est pas nécessaire, seule celle de l'efficacité moyenne suffit. En présence de bruits de fond, la situation se complique. 
La densité de probabilité normalisée observée devient

$$
g_{o b s}\left(\vec{\omega}^{\prime} ; \vec{A}\right)=x \cdot g\left(\vec{\omega}^{\prime} ; \vec{A}\right) \cdot \frac{\varepsilon\left(\vec{\omega}^{\prime}\right)}{\langle\varepsilon\rangle(\vec{A})}+(1-x) \cdot b\left(\vec{\omega}^{\prime}\right)
$$

où $b\left(\vec{\omega}^{\prime}\right)$ est la densité de probabilité normalisée des bruits de fond et $x$ la fraction de signal. La présence de bruit de fond brise la possibilité de factoriser l'efficacité de détection qu'il faut désormais connaître. L'approche simplifiée du pseudo-maximum de vraisemblance permet de s'affranchir de la connaissance de la fonction d'efficacité de détection même en présence de bruits de fond (voir $\S 5.2)$.

\subsubsection{Densité de probabilité normalisée du signal : canaux $B \rightarrow J / \psi K^{*}(892)$}

Soient $i$ les canaux à la génération et $j$ les canaux à la reconstruction, $(i, j=1,2,3,4$, correspondant aux modes $K_{S}^{0} \pi^{0}, K^{+} \pi^{-}, K_{S}^{0} \pi^{+}$et $K^{+} \pi^{0}$, respectivement). On considère que les quatre canaux $B \rightarrow J / \psi K^{*}(892)$ mesurent les mêmes amplitudes en se basant sur la symétrie d'isospin (cf. la fin du paragraphe 2.2.2). Cette hypothèse sera vérifiée en effectuant un ajustement sur chaque canal $B \rightarrow J / \psi K^{*}(892)$. En reconstruisant le canal $j$, celui-ci reçoit les contributions des canaux $i=1,2,3,4$ avec le signal pur $(j=i)$, le SFA $(j=i)$ et le FA $(j \neq i)$. La distribution observée du canal $j$ devient en prenant en compte ces contributions :

$$
\begin{aligned}
g_{o b s}^{j}\left(\vec{\omega}^{\prime} ; \vec{A}\right) & =\sum_{i=1}^{4} x_{o b s}^{i \rightarrow j} \cdot g_{o b s}^{i \rightarrow j}\left(\vec{\omega}^{\prime} ; \vec{A}\right) \\
& =\frac{\int g(\vec{\omega} ; \vec{A}) \times\left\{\sum_{i=1}^{4} F_{i} \cdot T^{i \rightarrow j}\left(\vec{\omega}, \vec{\omega}^{\prime}\right)\right\} \mathrm{d} \vec{\omega}}{\iint g(\vec{\omega} ; \vec{A}) \times\left\{\sum_{i=1}^{4} F_{i} \cdot T^{i \rightarrow j}\left(\vec{\omega}, \vec{\omega}^{\prime}\right)\right\} \mathrm{d} \vec{\omega} \mathrm{d} \vec{\omega}^{\prime}} .
\end{aligned}
$$

Dans l'expression ci-dessus, $x_{o b s}^{i \rightarrow j}$ est la fraction d'événements observés dans le canal $j$ provenant du canal généré $i$ et $g_{o b s}^{i \rightarrow j}$ est la densité de probabilité normalisée individuelle observée :

$$
g_{o b s}^{i \rightarrow j}\left(\vec{\omega}^{\prime} ; \vec{A}\right) \equiv \frac{\int g(\vec{\omega} ; \vec{A}) \cdot T^{i \rightarrow j}\left(\vec{\omega}, \vec{\omega}^{\prime}\right) \mathrm{d} \vec{\omega}}{\iint g(\vec{\omega} ; \vec{A}) \cdot T^{i \rightarrow j}\left(\vec{\omega}, \vec{\omega}^{\prime}\right) \mathrm{d} \vec{\omega} \mathrm{d} \vec{\omega}^{\prime}}
$$

$x_{o b s}^{i \rightarrow j} \equiv N_{o b s}^{i \rightarrow j} / N_{o b s}^{j}$, où $N_{o b s}^{j}$ est le nombre d'événements observés dans le canal $j$ :

$$
N_{o b s}^{j}=\iint g(\vec{\omega} ; \vec{A}) \times\left\{\sum_{i=1}^{4} N_{g e n}^{i} \cdot T^{i \rightarrow j}\left(\vec{\omega}, \vec{\omega}^{\prime}\right)\right\} \mathrm{d} \vec{\omega} \mathrm{d} \vec{\omega}^{\prime}=\sum_{i=1}^{4} N_{o b s}^{i \rightarrow j}
$$

avec $N_{\text {gen }}^{i}$ le nombre d'événements généré dans le canal $i$ et

$$
N_{o b s}^{i \rightarrow j}=N_{g e n}^{i} \times \iint g(\vec{\omega} ; \vec{A}) \cdot T^{i \rightarrow j}\left(\vec{\omega}, \vec{\omega}^{\prime}\right) \mathrm{d} \vec{\omega} \mathrm{d} \vec{\omega}^{\prime}
$$

$T^{i \rightarrow j}\left(\vec{\omega}, \vec{\omega}^{\prime}\right)$ est la fonction de transfert reliant un événement produit dans le canal $i$ avec les angles $\vec{\omega}$ et reconstruit dans le canal $j$ avec les angles $\vec{\omega}^{\prime}$. Enfin, $F_{i}(i=1,2,3,4)$ est la fraction relative des rapports d'embranchement prenant en compte les divers rapports d'embranchement secondaire 
pour chaque canal (cf. la table 4.1). On a par définition $\sum_{i=1}^{4} F_{i}=1$. Les transferts entre les canaux $B \rightarrow J / \psi K^{*}(892)$ contiennent de l'information sur les amplitudes puisqu'ils proviennent d'un canal généré $i$ décrit par une distribution $g(\vec{\omega} ; \vec{A})$ et sont reconstruits dans un canal $j \neq i$ décrit par la distribution $g_{o b s}^{i \rightarrow j \neq i}\left(\vec{\omega}^{\prime} ; \vec{A}\right) \propto \int g(\vec{\omega} ; \vec{A}) \cdot T^{i \rightarrow j \neq i}\left(\vec{\omega}, \vec{\omega}^{\prime}\right) \mathrm{d} \vec{\omega}$. Ils sont donc considérés comme du signal.

La distribution globale $g_{o b s}\left(\vec{\omega}^{\prime} ; \vec{A}\right)$, obtenue en sommant sur les contributions des quatre canaux reconstruits et en la normalisant proprement pour obtenir une densité de probabilité normalisée, est définie par :

$$
\begin{aligned}
g_{o b s}\left(\vec{\omega}^{\prime} ; \vec{A}\right) & =\sum_{j=1}^{4} x_{o b s}^{j} \cdot g_{o b s}^{j}\left(\vec{\omega}^{\prime} ; \vec{A}\right) \\
& =\frac{\int g(\vec{\omega} ; \vec{A}) \times\left\{\sum_{i, j=1}^{4} F_{i} \cdot T^{i \rightarrow j}\left(\vec{\omega}, \vec{\omega}^{\prime}\right)\right\} \mathrm{d} \vec{\omega}}{\iint g(\vec{\omega} ; \vec{A}) \times\left\{\sum_{i, j=1}^{4} F_{i} \cdot T^{i \rightarrow j}\left(\vec{\omega}, \vec{\omega}^{\prime}\right)\right\} \mathrm{d} \vec{\omega} \mathrm{d} \vec{\omega}^{\prime}},
\end{aligned}
$$

où $x_{o b s}^{j}=N_{o b s}^{j} / N_{o b s}$ et le nombre total d'événements observés est $N_{o b s}=\sum_{j=1}^{4} N_{o b s}^{j}$.

\subsubsection{Traitement des fonctions de transfert}

En utilisant la linéarité de la distribution angulaire (voir eq. (5.9)), la distribution angulaire des événements observés dans le canal $j$ provenant du canal généré $i$ s'écrit, d'après l'équation (5.15) :

$$
g_{o b s}^{i \rightarrow j}\left(\vec{\omega}^{\prime} ; \vec{A}\right)=\frac{\sum_{l=1}^{6} K_{l} \times\left\{\int f_{l}(\vec{\omega}) \cdot T^{i \rightarrow j}\left(\vec{\omega}, \vec{\omega}^{\prime}\right) \mathrm{d} \vec{\omega}\right\}}{\sum_{l=1}^{6} K_{l} \times\left\{\iint f_{l}(\vec{\omega}) \cdot T^{i \rightarrow j}\left(\vec{\omega}, \vec{\omega}^{\prime}\right) \mathrm{d} \vec{\omega} \mathrm{d} \vec{\omega}^{\prime}\right\}}
$$

Le traitement des fonctions de transfert revient à la détermination des intégrales suivantes :

$$
\varphi_{l}^{i \rightarrow j}\left(\vec{\omega}^{\prime}\right) \equiv \int f_{l}(\vec{\omega}) \cdot T^{i \rightarrow j}\left(\vec{\omega}, \vec{\omega}^{\prime}\right) \mathrm{d} \vec{\omega}
$$

et

$$
\Phi_{l}^{i \rightarrow j} \equiv \iint f_{l}(\vec{\omega}) \cdot T^{i \rightarrow j}\left(\vec{\omega}, \vec{\omega}^{\prime}\right) \mathrm{d} \vec{\omega} \mathrm{d} \vec{\omega}^{\prime}=\int \varphi_{l}^{i \rightarrow j}\left(\vec{\omega}^{\prime}\right) \mathrm{d} \vec{\omega}^{\prime}
$$

Commençons par l'estimation des constantes $\Phi_{l}^{i \rightarrow j}$ qui s'obtient par intégration Monte Carlo (cf. eq. (5.11)). D'après la relation (cf. eq. (5.3))

$$
\varepsilon^{i \rightarrow j}(\vec{\omega})=\int T^{i \rightarrow j}\left(\vec{\omega}, \vec{\omega}^{\prime}\right) \mathrm{d} \vec{\omega}^{\prime},
$$

où $\varepsilon^{i \rightarrow j}(\vec{\omega})$ représente la densité de probabilité (non normalisée) qu'un événement produit dans le canal $i$ avec les angles $\vec{\omega}$ soit accepté dans le canal $j$, quels que soient les angles reconstruits $\vec{\omega}^{\prime}$, on a

$$
\Phi_{l}^{i \rightarrow j}=\int f_{l}(\vec{\omega}) \cdot\left\{\int T^{i \rightarrow j}\left(\vec{\omega}, \vec{\omega}^{\prime}\right) \mathrm{d} \vec{\omega}^{\prime}\right\} \mathrm{d} \vec{\omega}=\int f_{l}(\vec{\omega}) \cdot \varepsilon^{i \rightarrow j}(\vec{\omega}) \mathrm{d} \vec{\omega}
$$


En désignant par le symbole $\asymp$ « a pour estimateur », on a :

$$
\begin{aligned}
\Phi_{l}^{i \rightarrow j} & =\int f_{l}(\vec{\omega}) \cdot \varepsilon^{i \rightarrow j}(\vec{\omega}) \cdot \frac{1}{h(\vec{\omega})} \times h(\vec{\omega}) \mathrm{d} \vec{\omega}\left(=E\left[f_{l} \cdot \varepsilon^{i \rightarrow j} \cdot \frac{1}{h}\right]\right) \\
& \asymp \frac{1}{N_{g e n}^{i}} \cdot \sum_{\alpha=1}^{N_{g e n}^{i}} \varepsilon_{\alpha}^{i \rightarrow j} \cdot f_{l}\left(\vec{\omega}_{\alpha}\right) \cdot \frac{1}{h\left(\vec{\omega}_{\alpha}\right)}=\frac{1}{N_{g e n}^{i}} \cdot \sum_{\beta=1}^{N_{a c c}^{i \rightarrow j}} f_{l}\left(\vec{\omega}_{\beta}\right) \cdot \frac{1}{h\left(\vec{\omega}_{\beta}\right)}
\end{aligned}
$$

où $E$ est l'espérance (expectation value) et $\varepsilon_{\alpha}^{i \rightarrow j}$ vaut 1 si l'événement $\alpha$ est accepté et 0 autrement. $N_{a c c}^{i \rightarrow j}$ est le nombre d'événements acceptés avec les angles reconstruits $\vec{\omega}_{\beta}^{\prime}\left(\beta=1, \ldots, N_{a c c}^{i \rightarrow j}\right)$ dans le canal $j$ parmi les $N_{g e n}^{i}$ événements générés avec les angles $\vec{\omega}_{\alpha}\left(\alpha=1, \ldots, N_{g e n}^{i}\right)$ dans le canal $i$. Les angles $\vec{\omega}_{\alpha}$ sont distribués selon une densité de probabilité $h(\vec{w})$. À noter qu'il est nécessaire de repondérer chaque événement par $1 / h\left(\vec{\omega}_{\beta}\right)$ et que les angles sont les angles générés. Aucune hypothèse sur la résolution n'est effectuée, les constantes $\Phi_{l}^{i \rightarrow j}$ étant identiques quelle que soit la résolution, parfaite ou non.

Passons maintenant aux fonctions $\varphi_{l}^{i \rightarrow j}\left(\vec{\omega}^{\prime}\right)$. La difficulté dans l'évaluation des ces fonctions est qu'elles dépendent des angles reconstruits $\vec{\omega}^{\prime}$. En supposant une résolution angulaire parfaite ${ }^{1}$ pour les événements signal (ce qui inclut les événements SFA et FA), motivée par les résultats obtenues aux chapitres 4 (cf. Fig. 4.7), on a :

$$
\varphi_{l}^{i \rightarrow j}\left(\vec{\omega}^{\prime}\right)=f_{l}\left(\vec{\omega}^{\prime}\right) \cdot \varepsilon^{i \rightarrow j}\left(\vec{\omega}^{\prime}\right)
$$

L'efficacité $\varepsilon^{i \rightarrow j}\left(\vec{\omega}^{\prime}\right)$ est développée sur la base orthonormée des harmoniques sphériques (c'est une méthode de décomposition en moments, dont celle pour le canal $B \rightarrow J / \psi K^{*}(892)$ est donnée dans l'appendice B) :

$$
\varepsilon^{i \rightarrow j}\left(\vec{\omega}^{\prime}\right)=\sum_{L R M} T_{L R M}^{\varepsilon^{i \rightarrow j}} \cdot \mathcal{Y}_{L R M}\left(\vec{\omega}^{\prime}\right)
$$

Les fonctions $\mathcal{Y}_{L R M}\left(\vec{\omega}^{\prime}\right)$ sont définies par :

$$
\mathcal{Y}_{L R M}\left(\vec{\omega}^{\prime}\right)=\sqrt{2 \pi} \cdot Y_{L, M}\left(\theta_{\psi}, \varphi_{\psi}\right) \cdot Y_{R,-M}\left(\theta_{K^{*}}, 0\right)
$$

où $Y_{l m}$ sont les harmoniques sphériques, $\left(\theta_{\psi}, \varphi_{\psi}, \theta_{K^{*}}\right)$ sont les angles d'hélicité correspondant aux angles de transversité $\vec{\omega}^{\prime}$. La connaissance de la fonction $\varepsilon^{i \rightarrow j}\left(\vec{\omega}^{\prime}\right)$ revient à la connaissance des composantes $T_{L R M}^{\varepsilon^{i \rightarrow j}}$, qui sont obtenues avec des données simulées (cf. eqs (5.25)-(5.26) en remplaçant $\left.f_{l}(\vec{\omega}) \operatorname{par} \mathcal{Y}_{L R M}^{*}\left(\vec{\omega}^{\prime}\right)\right)$ :

$$
T_{L R M}^{\varepsilon^{i \rightarrow j}}=\int \varepsilon^{i \rightarrow j}\left(\vec{\omega}^{\prime}\right) \cdot \mathcal{Y}_{L R M}^{*}\left(\vec{\omega}^{\prime}\right) \mathrm{d} \vec{\omega}^{\prime} \asymp \frac{1}{N_{g e n}^{i}} \sum_{\beta=1}^{N_{a c c}^{i \rightarrow j}} \mathcal{Y}_{L R M}^{*}\left(\vec{\omega}_{\beta}^{\prime}\right) \cdot \frac{1}{h\left(\vec{\omega}_{\beta}^{\prime}\right)} .
$$

En principe, la somme sur le triplet $(L, R, M)$ s'étend de $(0,0,0)$ jusqu'à $(\infty, \infty, \infty)$ du fait de la forme complexe de $\varepsilon^{i \rightarrow j}\left(\vec{\omega}^{\prime}\right)$ (cf. les efficacités réduites figure 4.9).

\footnotetext{
${ }^{1}$ Un traitement complet, i.e. sans hypothèse concernant la résolution, des fonctions $\varphi_{l}^{i \rightarrow j}\left(\vec{\omega}^{\prime}\right)$ est donné dans l'appendice $\mathrm{E}$.
} 
La densité de probabilité normalisée observée pour le canal reconstruit $j$ devient :

$$
g_{o b s}^{j}\left(\vec{\omega}^{\prime} ; \vec{A}\right)=\frac{\left\{\sum_{l=1}^{6} K_{l} \cdot f_{l}\left(\vec{\omega}^{\prime}\right)\right\} \times\left\{\sum_{i=1}^{4} F_{i} \cdot \varepsilon^{i \rightarrow j}\left(\vec{\omega}^{\prime}\right)\right\}}{\sum_{l=1}^{6} K_{l} \cdot \sum_{i=1}^{4} F_{i} \cdot \Phi_{l}^{i \rightarrow j}} .
$$

Le terme $\left\{\sum_{i=1}^{4} F_{i} \cdot \varepsilon^{i \rightarrow j}\left(\vec{\omega}^{\prime}\right)\right\}$ représente une « efficacité moyenne ».

Dans le cas où on considère un échantillon unique composé des événements des quatre canaux, la densité de probabilité normalisée observée est, sous l'hypothèse d'une résolution angulaire parfaite :

$$
g_{\text {obs }}\left(\vec{\omega}^{\prime} ; \vec{A}\right)=\frac{\left\{\sum_{l=1}^{6} K_{l} \cdot f_{l}\left(\vec{\omega}^{\prime}\right)\right\} \times\left\{\sum_{i=1}^{4} F_{i} \cdot \sum_{j=1}^{4} \varepsilon^{i \rightarrow j}\left(\vec{\omega}^{\prime}\right)\right\}}{\sum_{l=1}^{6} K_{l} \cdot \sum_{i=1}^{4} F_{i} \cdot \sum_{j=1}^{4} \Phi_{l}^{i \rightarrow j}} .
$$

Le terme $\sum_{j=1}^{4} \varepsilon^{i \rightarrow j}\left(\vec{\omega}^{\prime}\right)$ représente la probabilité qu'un événement généré dans le canal $i$ soit reconstruit sans tenir compte du canal de reconstruction. Le terme $\left\{\sum_{i=1}^{4} F_{i} \cdot \sum_{j=1}^{4} \varepsilon^{i \rightarrow j}\left(\vec{\omega}^{\prime}\right)\right\}$ représente l'efficacité totale des événements $J / \psi K^{*}(892)$, sans tenir compte des canaux de génération et de reconstruction.

\subsubsection{Ajustement en présence de bruits de fond}

En présence de bruits de fond, la densité de probabilité normalisée décrivant la distribution angulaire observée pour le canal reconstruit $j$ devient :

$$
x_{j} \cdot g_{o b s}^{j}\left(\vec{\omega}^{\prime} ; \vec{A}\right)+\left(1-x_{j}\right) \cdot b^{j}\left(\vec{\omega}^{\prime}\right)
$$

avec $x_{j}$ la fraction de signal du canal $j$ estimée au préalable à partir d'un ajustement de la distribution en $m_{\mathrm{ES}}$ (après coupure sur $\Delta E$ ) du canal $j$ par une distribution gaussienne et une distribution Argus. De même que pour l'efficacité, la densité de probabilité normalisée des bruits de fond du canal $j, b^{j}\left(\vec{\omega}^{\prime}\right)$, est estimée par sa décomposition en moments :

$$
b^{j}\left(\vec{\omega}^{\prime}\right)=\sum_{L R M} b_{L R M}^{j} \cdot \mathcal{Y}_{L R M}\left(\vec{\omega}^{\prime}\right)
$$

avec

$$
b_{L R M}^{j}=\int \mathcal{Y}_{L R M}^{*}\left(\vec{\omega}^{\prime}\right) \cdot b^{j}\left(\vec{\omega}^{\prime}\right) \mathrm{d} \vec{\omega}^{\prime} \asymp \frac{1}{N_{a c c}^{j}} \cdot \sum_{\beta=1}^{N_{a c c}^{j}} \mathcal{Y}_{L R M}^{*}\left(\vec{\omega}_{\beta}^{\prime}\right),
$$

où les angles $\vec{\omega}_{\beta}^{\prime}$ sont distribués selon la densité de probabilité normalisée $b^{j}\left(\vec{\omega}^{\prime}\right)$. Les moments $b_{L R M}^{j}$ sont estimés sur les données, avant d'effectuer l'ajustement pour la mesure des amplitudes, à partir des événements ayant une masse $m_{\mathrm{ES}}<5.27 \mathrm{GeV} / c^{2}$ après coupure sur $\Delta E$.

Deux types d'ajustement seraient alors effectués :

- un ajustement pour chaque canal reconstruit $j$, afin de tester la compatibilité des résultats 
obtenus :

$$
\ln \mathcal{L}=\left[\sum_{n=1}^{N_{j}^{d a t a, B^{0} / B^{+}}} \ln \mathcal{L}_{j}+\sum_{n=1}^{N_{j}^{d a t a, \bar{B}^{0} / B^{-}}} \ln \overline{\mathcal{L}}_{j}\right]
$$

où $\stackrel{(-)}{\mathcal{L}_{j}}=x_{j} \cdot \stackrel{(-)}{g_{\text {obs }}^{j}}\left(\vec{\omega}^{\prime} ; \vec{A}\right)+\left(1-x_{j}\right) \cdot b^{j}\left(\vec{\omega}^{\prime}\right) ;$

- un ajustement sur l'ensemble des quatre canaux :

$$
\begin{array}{r}
\ln \mathcal{L}=\sum_{n=1}^{N_{\text {data }, B^{0} / B^{+}}} \ln \left[x \cdot g_{\text {obs }}\left(\vec{\omega}^{\prime} ; \vec{A}\right)+(1-x) \cdot b\left(\vec{\omega}^{\prime}\right)\right]+ \\
\sum_{n=1}^{N_{\text {data }, \bar{B}^{0} / B^{-}}} \ln \left[x \cdot \bar{g}_{\text {obs }}\left(\vec{\omega}^{\prime} ; \vec{A}\right)+(1-x) \cdot b\left(\vec{\omega}^{\prime}\right)\right]
\end{array}
$$

où l'expression de $g_{\text {obs }}\left(\vec{\omega}^{\prime} ; \vec{A}\right)$ est donnée par l'équation (5.18), $b\left(\vec{\omega}^{\prime}\right)$ est la densité de probabilité normalisée des bruits de fond et $x$ est la fraction globale de signal.

La mise en œuvre de ce formalisme est délicate notamment par la nécessité de développer en moments des densités de probabilité inconnues décrivant la dépendance angulaire des bruits de fond et la fonction d'efficacité de détection pour le signal. La conséquence de ceci est qu'il n'existe pas de critères permettant de connaître l'ordre du développement et il est possible que des moments $\mathrm{d}$ 'ordre très élevé contribuent. La présence de bruits de fond rend obligatoire la connaissance de la fonction d'efficacité de détection (voir eq. (5.32)). Le fait d'avoir moins de contraintes dans la connaissance des bruits de fond pour l'analyse angulaire par rapport à l'analyse $C P$ m'a conduit à utiliser une approche simplifiée du problème permettant de s'affranchir de la connaissance de l'efficacité de détection (en supposant le cas d'une bonne résolution angulaire) ainsi que les densités de probabilité normalisées décrivant la dépendance angulaire des bruits de fond.

\subsection{Ajustement par pseudo-maximum de vraisemblance}

\subsubsection{Principe}

La méthode du pseudo-maximum de vraisemblance, inspirée d'une discussion avec Bill Dunwoodie et mise en forme par Bob Cahn [161], est assez intuitive. Supposons que nous ayons un échantillon $\mathcal{S B}$ correspondant à l'échantillon des événements dans la région signal. Cet échantillon est un mélange d'événements signal $(S)$ et de bruits de fond $(B)$ que l'on ne peut séparer. En écrivant un maximum de vraisemblance pour les événements de l'échantillon $\mathcal{S B}$, en considérant tous les événements en tant que signal, on a :

$$
\ln \mathcal{L}=\sum_{S+B} \ln g_{o b s}\left(\vec{\omega}^{\prime} ; \vec{A}\right)=\sum_{S} \ln g_{o b s}\left(\vec{\omega}^{\prime} ; \vec{A}\right)+\sum_{B} \ln g_{o b s}\left(\vec{\omega}^{\prime} ; \vec{A}\right)
$$

où $g_{o b s}\left(\vec{\omega}^{\prime} ; \vec{A}\right)$ est donné par l'équation (5.18). Supposons que l'on a un échantillon de purs bruits de fond, $B_{2}$, qui possèdent les mêmes caractéristiques angulaires que les événements de bruits de fond dans la région signal. En notant par $n_{B_{2}}$ le nombre d'événements de bruits de fond dans la 
région $B_{2}$ et $n_{B}$ le nombre d'événements de bruits de fond dans la région signal, on peut estimer $\sum_{B} \ln g_{o b s}\left(\vec{\omega}^{\prime} ; \vec{A}\right)$ par :

$$
\sum_{B} \ln g_{o b s}\left(\vec{\omega}^{\prime} ; \vec{A}\right) \approx \frac{n_{B}}{n_{B_{2}}} \sum_{B_{2}} \ln g_{o b s}\left(\vec{\omega}^{\prime} ; \vec{A}\right)
$$

On peut donc soustraire la contribution des bruits de fond :

$$
\ln \mathcal{L}=\sum_{S+B} \ln g_{\text {obs }}\left(\vec{\omega}^{\prime} ; \vec{A}\right)-\frac{n_{B}}{n_{B_{2}}} \sum_{B_{2}} \ln g_{o b s}\left(\vec{\omega}^{\prime} ; \vec{A}\right)
$$

aboutissant à

$$
\begin{aligned}
\ln \mathcal{L} & =\sum_{S+B} \ln g_{o b s}\left(\vec{\omega}^{\prime} ; \vec{A}\right)-\frac{n_{B}}{n_{B_{2}}} \sum_{B_{2}} \ln g_{o b s}\left(\vec{\omega}^{\prime} ; \vec{A}\right) \\
& =\sum_{S} \ln g_{o b s}\left(\vec{\omega}^{\prime} ; \vec{A}\right)+\sum_{B} \ln g_{o b s}\left(\vec{\omega}^{\prime} ; \vec{A}\right)-\frac{n_{B}}{n_{B_{2}}} \sum_{B_{2}} \ln g_{o b s}\left(\vec{\omega}^{\prime} ; \vec{A}\right) \\
& \approx \sum_{S} \ln g_{o b s}\left(\vec{\omega}^{\prime} ; \vec{A}\right) .
\end{aligned}
$$

Cette méthode a l'avantage de pouvoir s'affranchir de la description de la dépendance angulaire des bruits de fond, seule la connaissance de la distribution angulaire observée du signal est nécessaire. De plus, dans le cas d'une bonne résolution angulaire, on a (cf. eqs. (5.6) et (5.32)) :

$$
g_{o b s}\left(\vec{\omega}^{\prime} ; \vec{A}\right)=g\left(\vec{\omega}^{\prime} ; \vec{A}\right) \cdot \frac{\varepsilon\left(\vec{\omega}^{\prime}\right)}{\langle\varepsilon\rangle(\vec{A})}
$$

ce qui permet de s'affranchir de la connaissance de la fonction d'efficacité de détection (voir eq. (5.8)).

\subsubsection{Le problème des incertitudes}

L'utilisation d'un pseudo-maximum de vraisemblance dans un programme de maximum de vraisemblance, tel que Minuit, peut conduire à une sous-estimation des erreurs. Pour illustrer ce problème nous effectuons une simulation « toy Monte Carlo » dans laquelle nous générons un ensemble d'échantillons signal, selon une acceptance parfaite, auxquels nous ajoutons une fraction connue de bruit de fond, ce bruit de fond étant de dépendance angulaire connue également. Pour le signal nous considérons une paramétrisation sphérique des amplitudes, à savoir :

$$
\begin{aligned}
& A_{0}=\cos \theta_{A} \cdot e^{i \delta_{0}} \\
& A_{\|}=\sin \theta_{A} \cos \phi_{A} \cdot e^{i \delta_{\|}} \\
& A_{\perp}=\sin \theta_{A} \sin \phi_{A} \cdot e^{i \delta_{\perp}}
\end{aligned}
$$

et nous choisissons $\theta_{A}=\phi_{A}=\delta_{\|}=\delta_{\perp}=1, \delta_{0}=0$. Pour le bruit de fond, nous choisissons la distribution angulaire suivante :

$$
b(\vec{\omega})=\frac{1}{\sqrt{8 \pi}} \cdot \mathcal{Y}_{000}(\vec{\omega})+\frac{1}{15} \cdot \mathcal{Y}_{030}(\vec{\omega})=\frac{1}{8 \pi}+\frac{1}{15} \cdot \mathcal{Y}_{030}(\vec{\omega})
$$




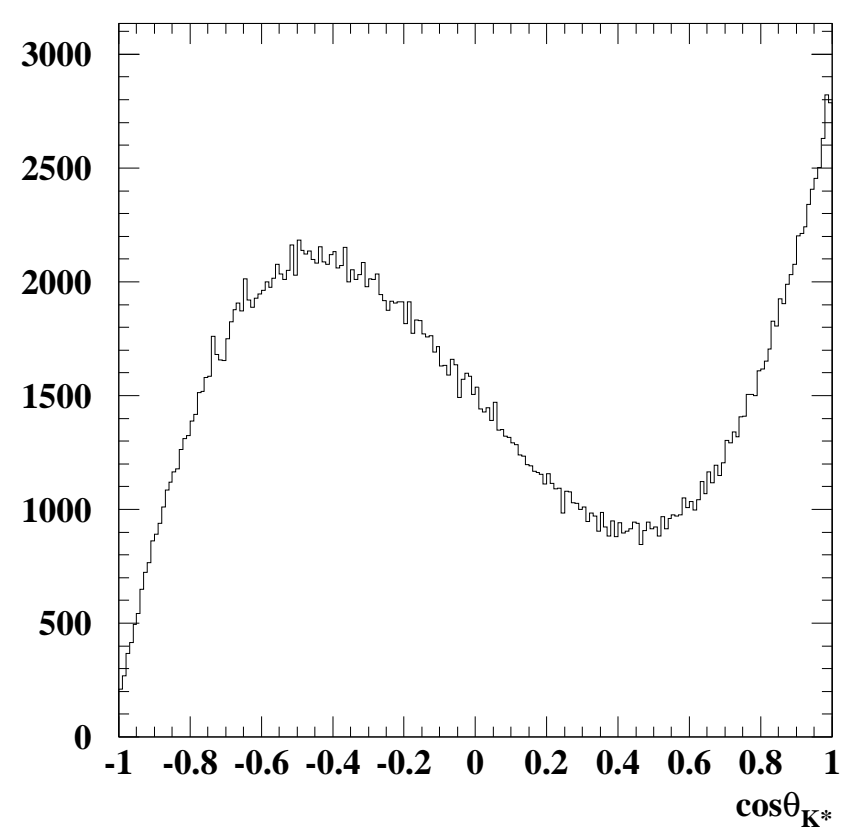

FIG. 5.1: Distribution en $\cos \theta_{K^{*}}$ du bruit de fond.

c'est-à-dire un bruit de fond n'ayant qu'une dépendance en $\cos \theta_{K^{*}}$. Le terme constant $1 / 8 \pi$ assure une normalisation égale à 1 et le facteur $1 / 15$ est suffisamment petit pour avoir $b(\vec{\omega}) \geq 0, \forall \vec{\omega}$. La forme de $b(\vec{\omega})$ est représentée sur la figure 5.1 .

Nous associons également une dépendance en masse $\left(m_{\mathrm{ES}}\right)$ au signal et au bruit de fond, en générant les événements signal suivant une gaussienne $(G)$ centrée sur la masse du méson $B$ et de largeur $3 \mathrm{MeV} / c^{2}$, et le bruit de fond suivant une fonction Argus $(A)$. Nous considérons 3 niveaux de pureté, $x$, définis sur la plage $5.2<m_{\mathrm{ES}}<5.3 \mathrm{GeV} / c^{2}$, à savoir $75 \%, 50 \%$ et $25 \%$. Pour chaque pureté nous générons 400 expériences de 4000 événements signal, ce qui correspond environ au nombre d'événements signal attendu sur les données. Pour chaque expérience nous effectuons trois ajustements :

- un premier ajustement sur le spectre en masse pour extraire les paramètres de la gaussienne, de la fonction Argus et la fraction de signal sur la plage $5.2<m_{\mathrm{ES}}<5.3 \mathrm{GeV} / c^{2}$;

- puis deux ajustements angulaires :

- l'un avec la technique du pseudo-maximum de vraisemblance, en utilisant les paramètres en masse précédemment ajustés pour estimer la fraction de bruit sous le pic signal ;

- l'autre avec un ajustement par maximum de vraisemblance en utilisant la densité de probabilité normalisée :

$$
\mathrm{pdf}=x \cdot G\left(m_{\mathrm{ES}}\right) \cdot g(\vec{\omega})+(1-x) \cdot A\left(m_{\mathrm{ES}}\right) \cdot b(\vec{\omega})
$$

Nous formons alors pour chaque ajustement les «pulls », définis, pour le paramètre ajusté $p$, par :

$$
\operatorname{pull}(p)=\left(p_{\text {ajusté }}-p_{\text {généré }}\right) / \operatorname{Erreur}(p),
$$

où $\operatorname{Erreur}(p)$ est l'incertitude donnée par Minuit sur $p$. Chaque distribution de pull est ensuite 
ajustée avec une gaussienne. La valeur moyenne de cette gaussienne mesure le biais moyen obtenu sur l'ajustement du paramètre et la déviation standard de la gaussienne le rapport moyen entre la dispersion vraie $\left(p_{\text {ajusté }}-p_{\text {généré}}\right)$ et Erreur $(p)$, la dispersion estimée. Ce rapport est idéalement de 1.

Les figures 5.2 et 5.3 montrent la variation de ce biais moyen et de ce rapport moyen de dispersion avec la fraction de bruit. Nous voyons donc que l'ajustement par pseudo-vraisemblance permet une estimation non-biaisée des paramètres, mais que l'erreur donnée par Minuit est sousestimée, et ceux d'autant plus que le bruit de fond est élevé. L'ajustement par maximum de vraisemblance fournit également une estimation non-biaisée des paramètres ainsi qu'une estimation correcte des erreurs. Le prix à payer pour la plus grande simplicité dans la mise en oeuvre de la méthode de pseudo-vraisemblance est donc une estimation des erreurs plus délicate.

\subsubsection{Ajustement sur les données}

L'ajustement sur les données par un pseudo-maximum de vraisemblance (cf. eq.( 5.40)) est effectué :

- pour chaque canal reconstruit $j(j=2,3,4)$ en utilisant la distribution angulaire observée donnée à l'équation (5.31). On ne considère pas le canal reconstruit $K_{S}^{0} \pi^{0}(j=1)$ qui est utilisé pour les mesures de $\sin 2 \beta$ et $\cos 2 \beta$ puisqu'il a un faible impact en termes de statistique. De plus, il est nécessaire pour ce canal de faire intervenir l'étiquetage afin de distinguer les événements décrits par une distribution $g$ ou $\bar{g}$ alors que les trois autres canaux $B \rightarrow J / \psi K^{*}(892)$ sont étiquetés par le kaon chargé ou le pion chargé de l'état final ;

- sur l'ensemble des événements obtenus en sommant sur la contribution des trois canaux $j=$ 2, 3, 4 en utilisant la distribution angulaire observée donnée à l'équation (5.32) (en limitant la sommation sur $j$ à $j=2,3,4)$.

La fraction $n_{B} / n_{B_{2}}$ est obtenue à partir d'un ajustement par une gaussienne et une distribution Argus sur le spectre en $m_{\mathrm{ES}}$ après coupure sur $\Delta E$ en considérant la région signal $\left(m_{\mathrm{ES}}>5.27 \mathrm{GeV} / c^{2}\right)$ et la région à bas $m_{\mathrm{ES}}\left(m_{\mathrm{ES}}<5.27 \mathrm{GeV} / c^{2}\right)$. Pour chaque canal reconstruit $j$, les constantes $\Phi_{l}^{i \rightarrow j}$ sont déterminées à partir de données simulées des canaux exclusifs $B \rightarrow J / \psi K^{*}(892)$ en les séparant en deux séries suivant l'étiquetage du méson $B$.

\subsection{Validations}

Dans la suite nous considérons une paramétrisation sphérique des amplitudes (cf. eq. (5.45)). Par abus de langage, lorsque nous parlerons d'ajustement des amplitudes, nous sous-entendrons en réalité l'ajustement de $\theta_{A}$ et $\phi_{A}$. Les erreurs sur les modules des amplitudes sont obtenues par propagation des erreurs, au premier ordre.

\subsubsection{Validation sur simulation complète}

Dans un premier temps, nous utilisons les échantillons de simulation complète des canaux exclusifs pour déterminer les facteurs de correction d'acceptance $\Phi_{l}^{i \rightarrow j}$. Nous déterminons ces facteurs 

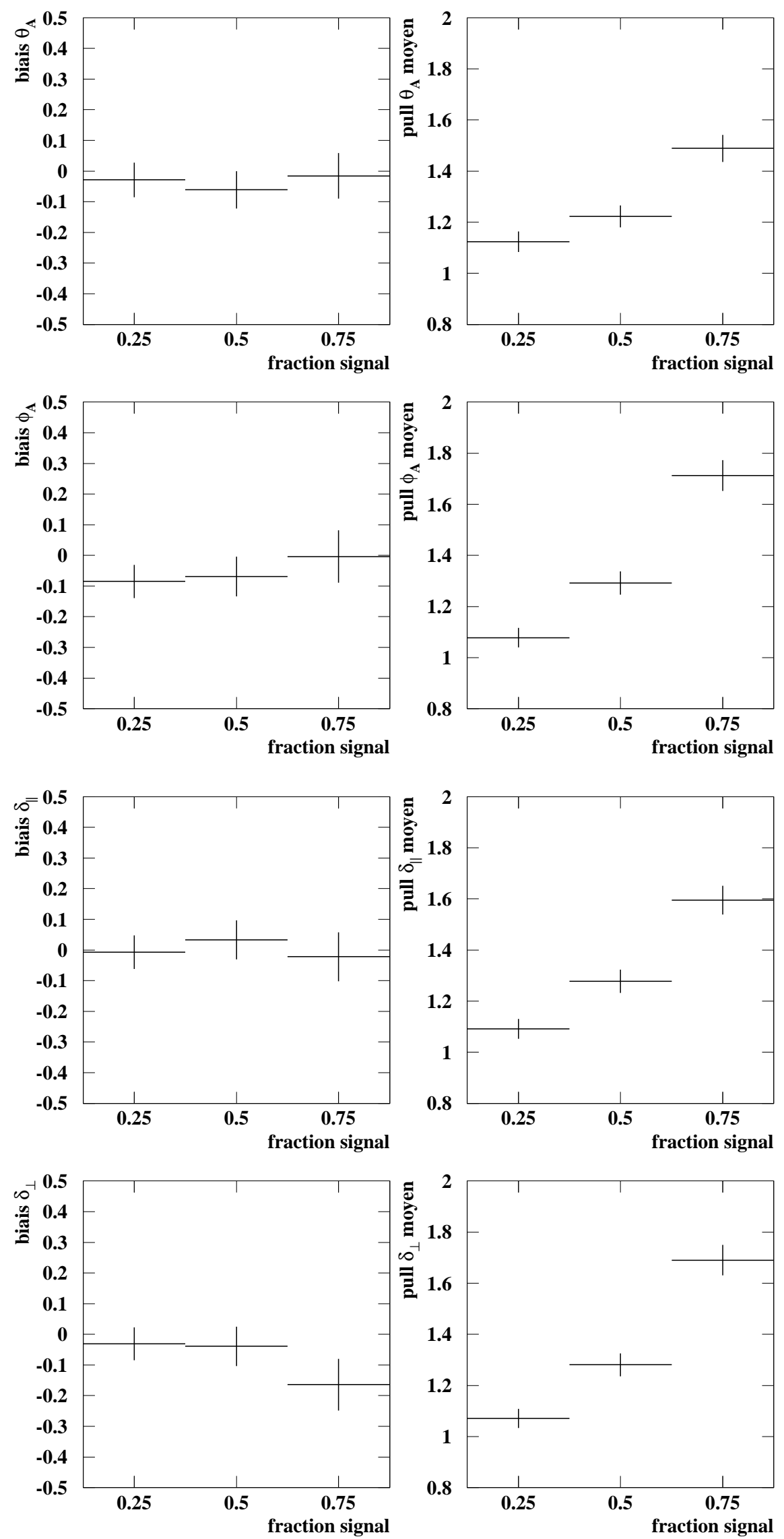

FIG. 5.2: Biais moyen et pulls moyens en fonction de la fraction de signal pour l'ajustement par pseudo-vraisemblance. 

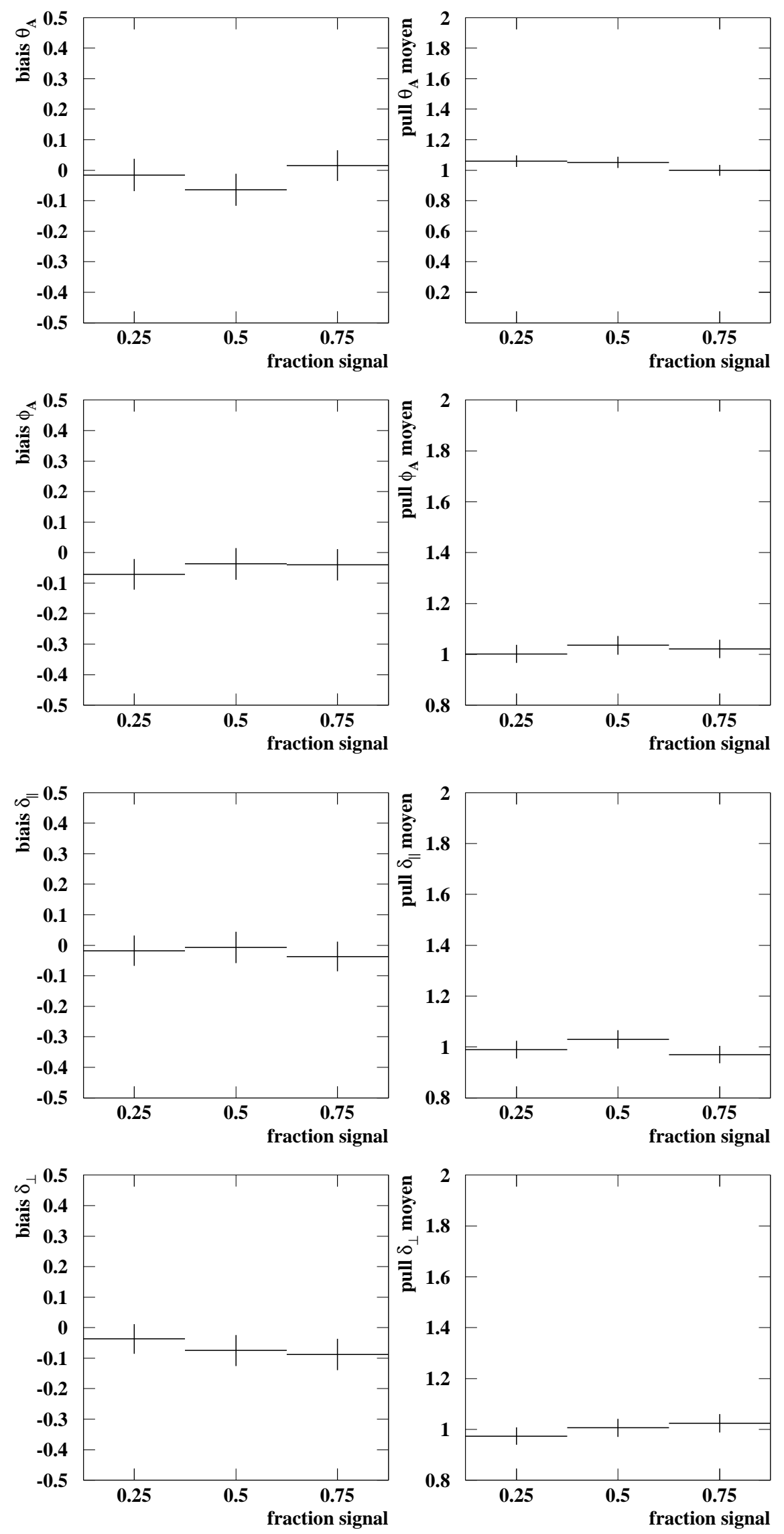

FIG. 5.3: Biais moyens et pulls moyens en fonction de la fraction de signal pour l'ajustement par maximum de vraisemblance. 
pour chaque année de prise de données ${ }^{2}$ en séparant, pour un même canal $B^{+}$de $B^{-}$ou $B^{0}$ de $\bar{B}^{0}$. Dans le cas d'un signal pur ( $B$ reconstruit sans FA ni SFA), nous obtenons les valeurs de la table 5.1. On constate qu'en sommant les termes $l=1,2,3$ pour $B$ et $\bar{B}$ on trouve une valeur voisine de l'efficacité dans le canal. On constate également que ces coefficients sont légèrement, mais significativement, différents pour $B$ et $\bar{B}$, ce qui n'est pas surprenant compte tenu que la détection des particules de matière peut différer de celle des particules d'antimatière. En prenant en compte le SFA et le FA, les termes diagonaux $\Phi_{l}^{i \rightarrow i}$ sont légèrement modifiés par le SFA et les termes nondiagonaux $\Phi_{l}^{i \rightarrow j \neq i}$ apparaissent, comme montré sur la table 5.2. Enfin nous rappelons que pour un même mode reconstruit $j$ les $\Phi_{l}^{i \rightarrow j}$ sont sommés en étant repondérés par le rapport de branchement du canal $i$ avant d'être utilisés dans l'ajustement (cf. eq. (5.31)).

TAB. 5.1: Coefficients $\Phi_{l}^{i \rightarrow i}$ pour l'année 2000, en l'absence de FA et SFA.

\begin{tabular}{|l|ccc|}
\hline Mode & $\Phi_{1}^{i \rightarrow i}$ & $\Phi_{2}^{i \rightarrow i}$ & $\Phi_{3}^{i \rightarrow i}$ \\
\hline$K^{+} \pi^{-}$ & $0.1240 \pm 0.0003$ & $0.1377 \pm 0.0005$ & $0.1406 \pm 0.0006$ \\
$K^{-} \pi^{+}$ & $0.1212 \pm 0.0003$ & $0.1338 \pm 0.0005$ & $0.1358 \pm 0.0006$ \\
\hline$K_{S}^{0} \pi^{+}$ & $0.0974 \pm 0.0005$ & $0.1121 \pm 0.0009$ & $0.1138 \pm 0.0011$ \\
$K_{S}^{0} \pi^{-}$ & $0.0978 \pm 0.0005$ & $0.1104 \pm 0.0009$ & $0.1104 \pm 0.0010$ \\
\hline$K^{+} \pi^{0}$ & $0.0560 \pm 0.0004$ & $0.0817 \pm 0.0010$ & $0.0832 \pm 0.0012$ \\
$K^{-} \pi^{0}$ & $0.0526 \pm 0.0004$ & $0.0796 \pm 0.0010$ & $0.0830 \pm 0.0012$ \\
\hline \hline Mode & $\Phi_{4}^{i \rightarrow i}$ & $\Phi_{5}^{i \rightarrow i}$ & $\Phi_{6}^{i \rightarrow i}$ \\
\hline$K^{+} \pi^{-}$ & $-0.0008 \pm 0.0003$ & $0.0006 \pm 0.0001$ & $-0.0010 \pm 0.0001$ \\
$K^{-} \pi^{+}$ & $0.0004 \pm 0.0003$ & $0.0027 \pm 0.0001$ & $0.0001 \pm 0.0001$ \\
\hline$K_{S}^{0} \pi^{+}$ & $-0.0019 \pm 0.0006$ & $0.0009 \pm 0.0002$ & $0.0008 \pm 0.0002$ \\
$K_{S}^{0} \pi^{-}$ & $0.0006 \pm 0.0006$ & $0.0016 \pm 0.0002$ & $-0.0006 \pm 0.0002$ \\
\hline$K^{+} \pi^{0}$ & $0.0024 \pm 0.0006$ & $0.0039 \pm 0.0002$ & $-0.0007 \pm 0.0002$ \\
$K^{-} \pi^{0}$ & $0.0009 \pm 0.0006$ & $0.0010 \pm 0.0002$ & $0.0006 \pm 0.0002$ \\
\hline
\end{tabular}

\section{Validations sur un échantillon de $J / \psi$ inclusif}

Cette validation est basée sur un large échantillon de $J / \psi$ inclusif, c'est-à-dire des événements $\Upsilon(4 S) \rightarrow B \bar{B}$ dans lesquels un $J / \psi$ est produit, sans tenir compte de sa source. Cet échantillon de $705 \mathrm{~K}$ d'événements produits comporte un seuil à $1.3 \mathrm{GeV} / c$ sur l'impulsion du $J / \psi$ dans le centre de masse de $l^{\prime} \Upsilon(4 S)$. Ce seuil n'affecte pas la région signal, ce qui nous permet de continuer à utiliser les $\Phi_{l}^{i \rightarrow j}$ précédemment calculés. Compte tenu du seuil sur l'impulsion du $J / \psi$, cet échantillon est équivalent à une luminosité intégrée de $\sim 591 \mathrm{fb}^{-1}$. Ce seuil a par contre un effet modéré sur la forme du spectre dans la région $m_{\mathrm{ES}}<5.27 \mathrm{GeV} / c^{2}$ (cf. Fig. 5.4), mais la haute statistique de cet échantillon fait qu'il demeure intéressant pour cette validation.

Nous effectuons un premier ensemble d'ajustements dans lesquels nous ne considérons que les contributions provenant de $B \rightarrow J / \psi K^{*}(892)$ (voir figures 5.5 à 5.8). Nous vérifions tout d'abord la polarisation du Monte Carlo en effectuant un ajustement sur tous les événements $B \rightarrow J / \psi K^{*}(892)$

\footnotetext{
${ }^{2}$ Dans la suite, nous n'illustrerons les validations que pour des échantillons Monte Carlo produits avec les tables d'efficacités de l'année 2000, les ajustements effectués en utilisant les efficacités des années 2001 et 2002 amenant à des résultats similaires, en terme de validation.
} 
TAB. 5.2: Coefficients $\Phi_{l}^{i \rightarrow j}$ pour l'année 2000. Les valeurs $1,2,3,4$ désignent les modes $K_{S}^{0} \pi^{0}, K^{+} \pi^{-}, K_{S}^{0} \pi^{+}, K^{+} \pi^{0}$, respectivement. Le premier bloc de 4 lignes montrent les coefficients pour le mode $j$ reconstruit en $B^{0}\left(B^{+}\right)$, le second bloc pour ce même mode reconstruit en $\bar{B}^{0}\left(B^{-}\right)$.

\begin{tabular}{|c|c|c|c|c|c|c|}
\hline$\left[\theta^{\circ}\right.$ & 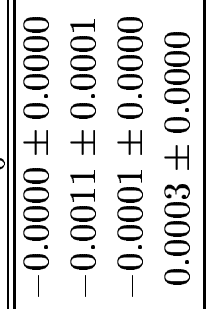 & 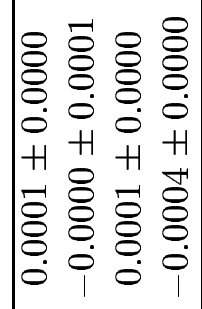 & 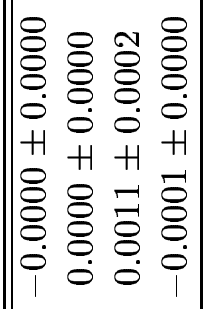 & 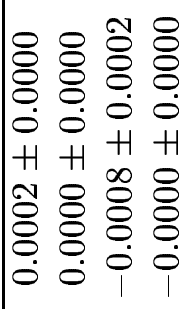 & 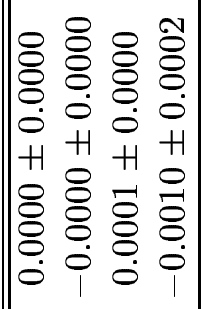 & 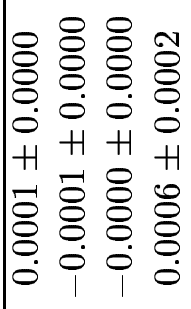 \\
\hline ini & 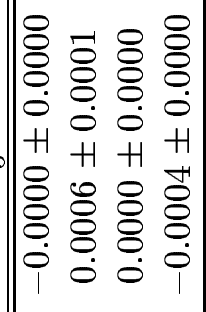 & 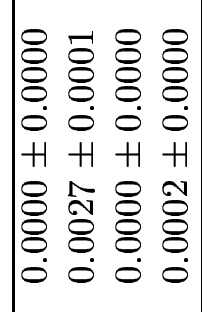 & 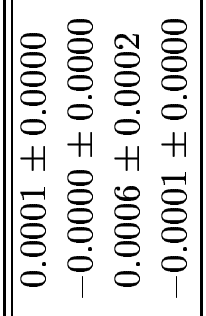 & 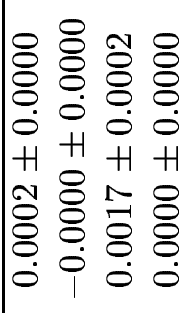 & 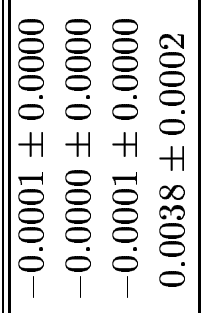 & 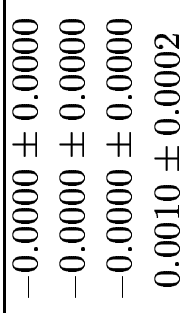 \\
\hline$[\theta 1$ & 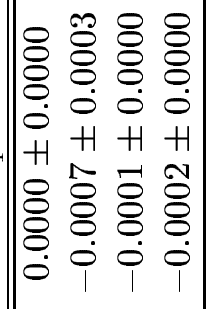 & 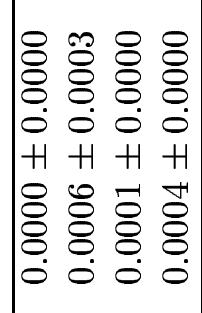 & 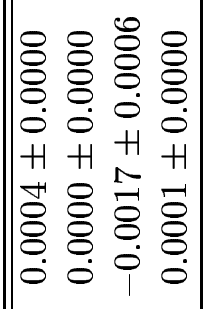 & 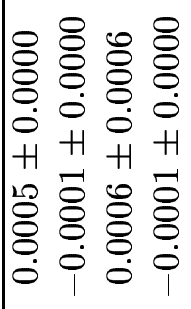 & 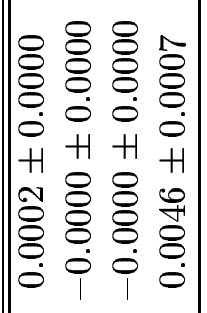 & 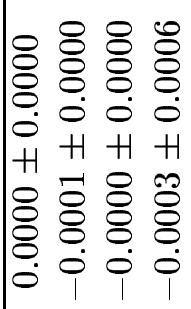 \\
\hline$[\theta i$ & 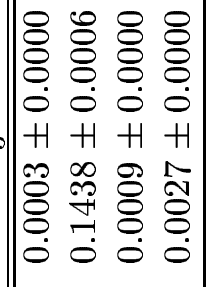 & 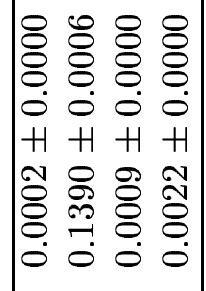 & 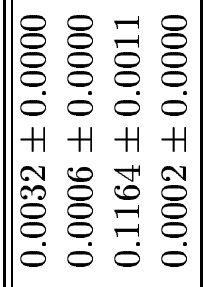 & 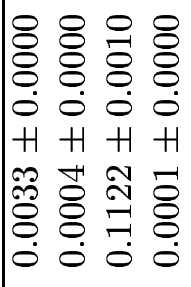 & $\left|\begin{array}{||cccc|}\delta & 8 & 8 & \overrightarrow{0} \\
0 & 0 & 0 & 0 \\
0 & 0 & 0 & 0 \\
0 & 0 & 0 \\
H & H & H & H \\
0 & 0 & 0 & 0 \\
\hdashline & 0 & 0 & 0 \\
8 & 0 & 0 & 0 \\
0 & 0 & 0 & 0\end{array}\right|$ & 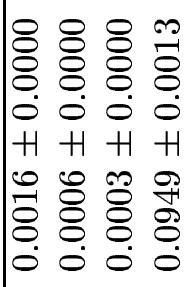 \\
\hline & 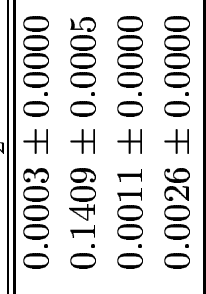 & 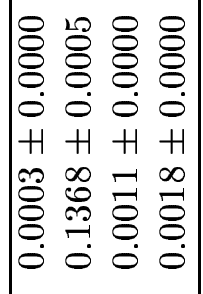 & 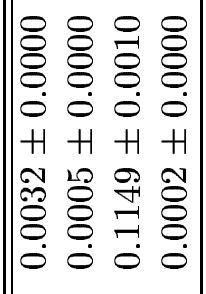 & 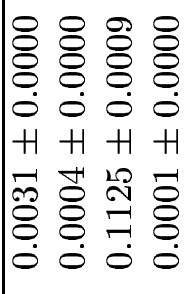 & 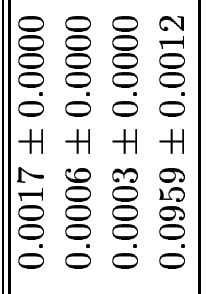 & 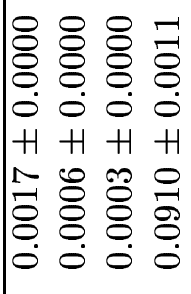 \\
\hline$|\theta|$ & 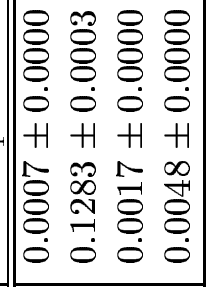 & 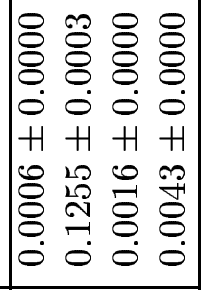 & 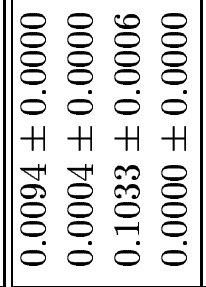 & 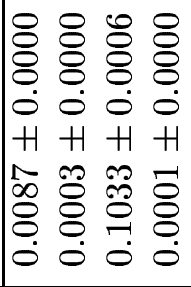 & 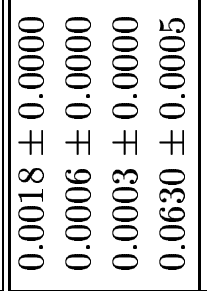 & 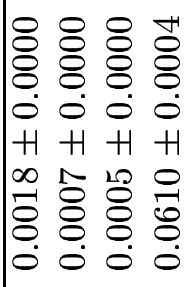 \\
\hline & $\begin{array}{llll}\sim & \sim & N & N \\
\uparrow & \uparrow & \uparrow & \uparrow\end{array}$ & $\mid \begin{array}{ll}N & N \\
1 & 1\end{array}$ & $\mid \begin{array}{cccc}m & n & n & \infty \\
\uparrow & \uparrow & \uparrow & \uparrow\end{array}$ & $\begin{array}{l}0 \\
1\end{array}$ & $\left|\begin{array}{llll}H & + & + & + \\
\uparrow & \uparrow & \uparrow & \uparrow\end{array}\right|$ & $\begin{array}{ll}+1 & + \\
\uparrow & \uparrow\end{array}$ \\
\hline
\end{tabular}



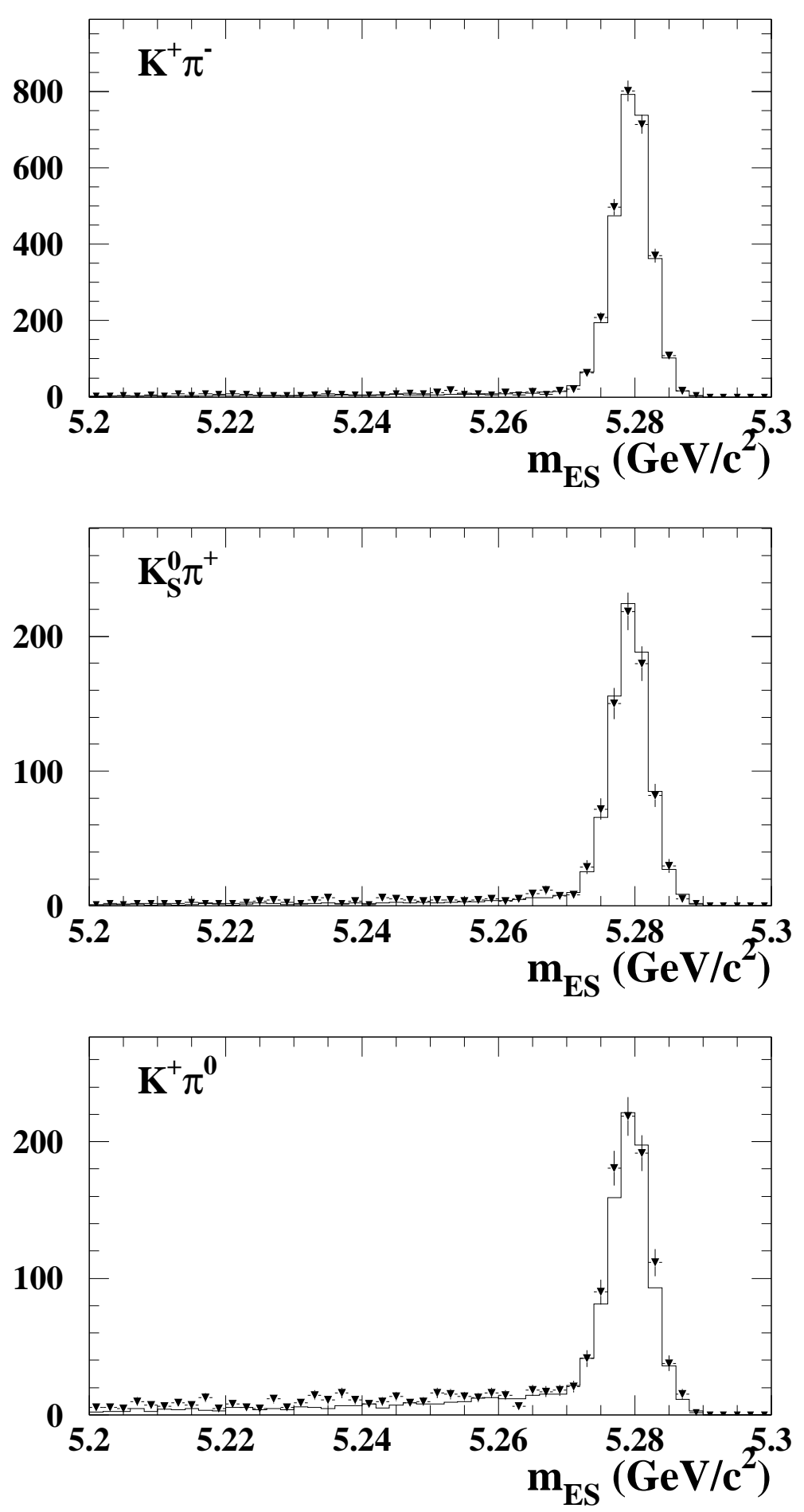

FIG. 5.4: Effet sur les spectres en $m_{\mathrm{ES}}$ du seuil à $1.3 \mathrm{GeV} / c$ sur l'impulsion du $J / \psi$ dans le centre de masse de $l^{\prime} \Upsilon(4 S)$. Les histogrammes en trait plein représentent les spectres obtenus pour l'échantillon de 705K événements avec seuil, équivalent à une luminosité intégrée de $\sim 591 \mathrm{fb}^{-1}$. Les histogrammes avec triangles représentent les spectres obtenus sur un échantillon plus restreint de $264 \mathrm{~K}$ événements, sans seuil, équivalent à une luminosité intégrée de $\sim 91 \mathrm{fb}^{-1}$. Les histogrammes sont normalisés à la luminosité des données $\left(\mathcal{L}=81.9 \mathrm{fb}^{-1}\right)$. 
générés de l'échantillon (valeurs (a) des figures 5.5 à 5.8). Puis nous considérons les candidats ayant passé la sélection, c'est-à-dire l'échantillon après effet d'acceptance (valeurs (b)). Nous utilisons alors les $\Phi_{l}^{i \rightarrow j}$ de la table 5.1. Il faut noter que si l'on ne fait pas de correction d'acceptance, les valeurs ajustées sont très loin des valeurs générées $\left(\left|A_{0}\right|^{2}=0.60,\left|A_{\|}\right|^{2}=0.24,\left|A_{\perp}\right|^{2}=0.16, \delta_{\|}=\right.$ $\left.2.5, \delta_{\perp}=-0.17\right)$, comme montré dans la table ci-dessous :

\begin{tabular}{|l|ccccc|}
\hline Canal & $\left|A_{0}\right|^{2}$ & $\left|A_{\|}\right|^{2}$ & $\left|A_{\perp}\right|^{2}$ & $\delta_{\|}$ & $\delta_{\perp}$ \\
\hline \hline$K^{+} \pi^{-}$ & $0.549 \pm 0.005$ & $0.255 \pm 0.007$ & $0.195 \pm 0.007$ & $2.61 \pm 0.04$ & $-0.14 \pm 0.03$ \\
\hline$K_{S}^{0} \pi^{+}$ & $0.529 \pm 0.010$ & $0.259 \pm 0.015$ & $0.212 \pm 0.015$ & $2.56 \pm 0.08$ & $-0.21 \pm 0.06$ \\
\hline$K^{+} \pi^{0}$ & $0.386 \pm 0.011$ & $0.332 \pm 0.015$ & $0.282 \pm 0.015$ & $2.73 \pm 0.08$ & $-0.10 \pm 0.06$ \\
\hline 3 canaux & $0.520 \pm 0.004$ & $0.268 \pm 0.006$ & $0.213 \pm 0.006$ & $2.62 \pm 0.03$ & $-0.14 \pm 0.03$ \\
\hline \hline
\end{tabular}

En effet, la correction d'acceptance est la correction la plus importante. Nous ajoutons ensuite les événements de (S)FA, utilisant les $\Phi_{l}^{i \rightarrow j}$ modifiés de la table 5.2 pour tenir compte de cette contribution (valeurs (c)). Jusqu'alors, nous n'avions utilisé que les angles générés. Pour estimer l'effet de la résolution sur les angles et notamment l'effet de la mauvaise résolution sur les angles de (S)FA, nous recommençons l'ajustement avec cette fois les angles reconstruits (valeurs (d)). Nous voyons que l'effet est minime. Ceci justifie notre approximation de ne pas introduire de fonction de résolution pour les événements de (S)FA. Nous ajoutons enfin les bruits de fond. Le premier ajustement (valeurs (e)) est fait sans corriger de l'effet des bruits de fond. Nous voyons que l'ajout des bruits de fond n'a en général pas un effet trop important, du fait de la bonne pureté des canaux reconstruits. La correction des bruits de fond améliore globalement les résultats, notamment les modules des amplitudes. Les phases fortes semblent peu affectées par cette correction.

\subsubsection{Facteurs de correction d'erreur}

\section{Qualité de la simulation rapide}

Afin de mesurer le facteur de correction d'erreur à appliquer, nous avons besoin de générer, comme dans la section 5.2.2, un nombre important d'expériences, chacune d'elle simulant l'échantillon de données dont nous disposons. L'idéal serait de disposer d'une grande statistique de simulation détaillée afin de pouvoir former ces $N$ expériences. Comme ce n'est malheureusement pas le cas, nous avons généré, utilisant la simulation rapide de BABAR, Pravda [162], les échantillons suivants :

- 1 million d'événements dans chacun des quatre modes $B \rightarrow J / \psi K^{*}(892)$, ce qui nous permet de calculer les facteurs $\Phi_{l}^{i \rightarrow j}$ de correction d'acceptance ;

- 48 millions d'événements de $J / \psi$ inclusif, ce qui nous permet de former 168 expériences de même statistique que les données. Cette production est réalisée sans seuil sur l'impulsion du $J / \psi$.

Bien que rapide, cette simulation est néanmoins de bonne qualité. Elle utilise en effet les tables d'efficacité d'identification de particules mesurée sur les données ce qui nous permet d'obtenir des effets d'acceptance réalistes. Ceci se traduit sur les coefficients $\Phi_{l}^{i \rightarrow j}$. Si nous formons les rapports $\operatorname{des} \Phi_{l}^{i \rightarrow i}$ entre simulations rapide et complète pour les termes $l=1,2,3$, nous obtenons les valeurs 


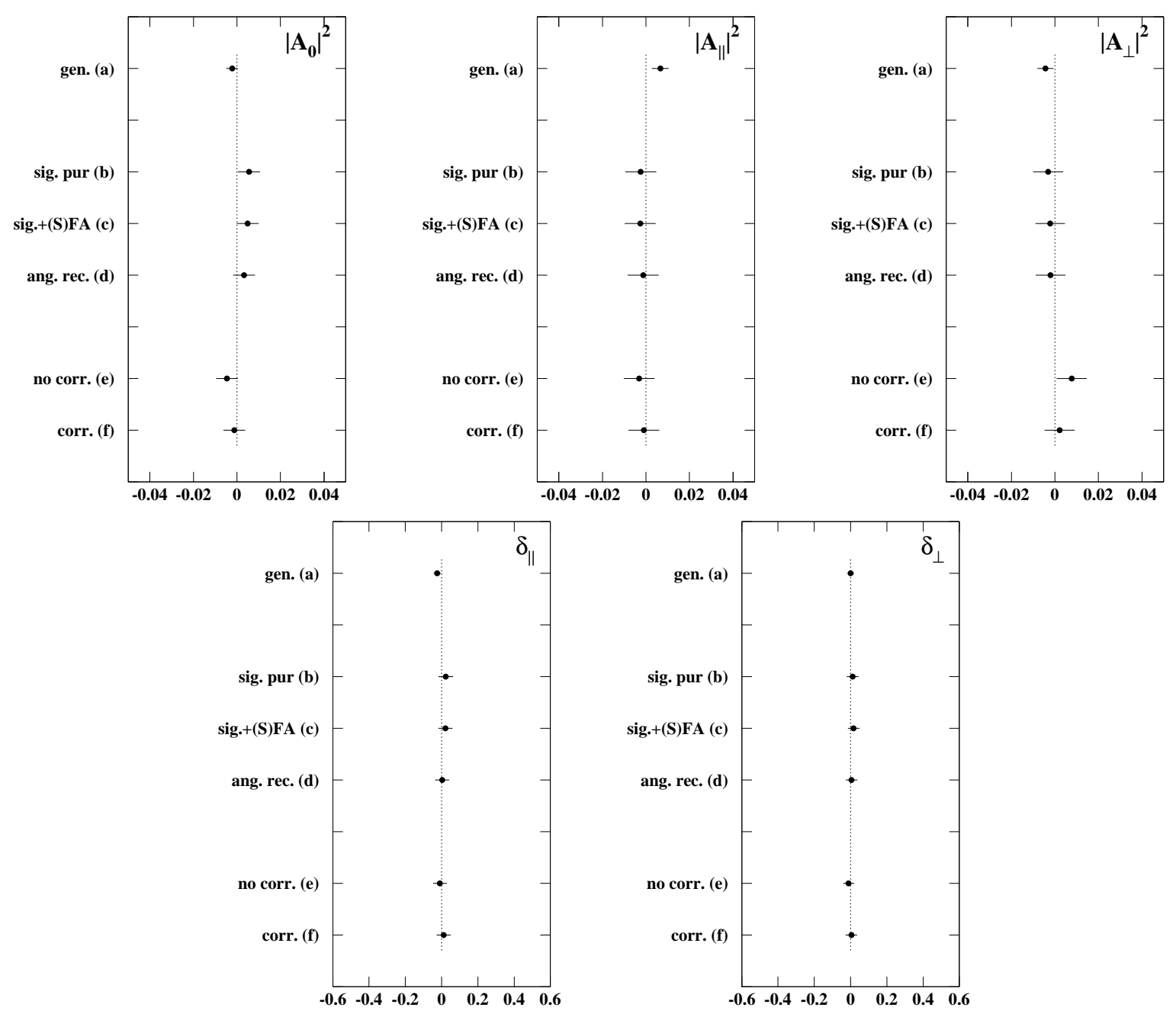

FIG. 5.5: Différences entre les amplitudes et les phases ajustées et les valeurs vraies de ces amplitudes et phases pour le canal $K^{+} \pi^{-}$: (a) échantillon à la génération, (b) échantillon pur après sélection et angles générés, (c) échantillon avec FA + SFA et angles générés, (d) idem (c) en utilisant les angles reconstruits, (e) ajout des événements de bruits de fond, sans correction, (f) idem avec correction des bruits de fond. 


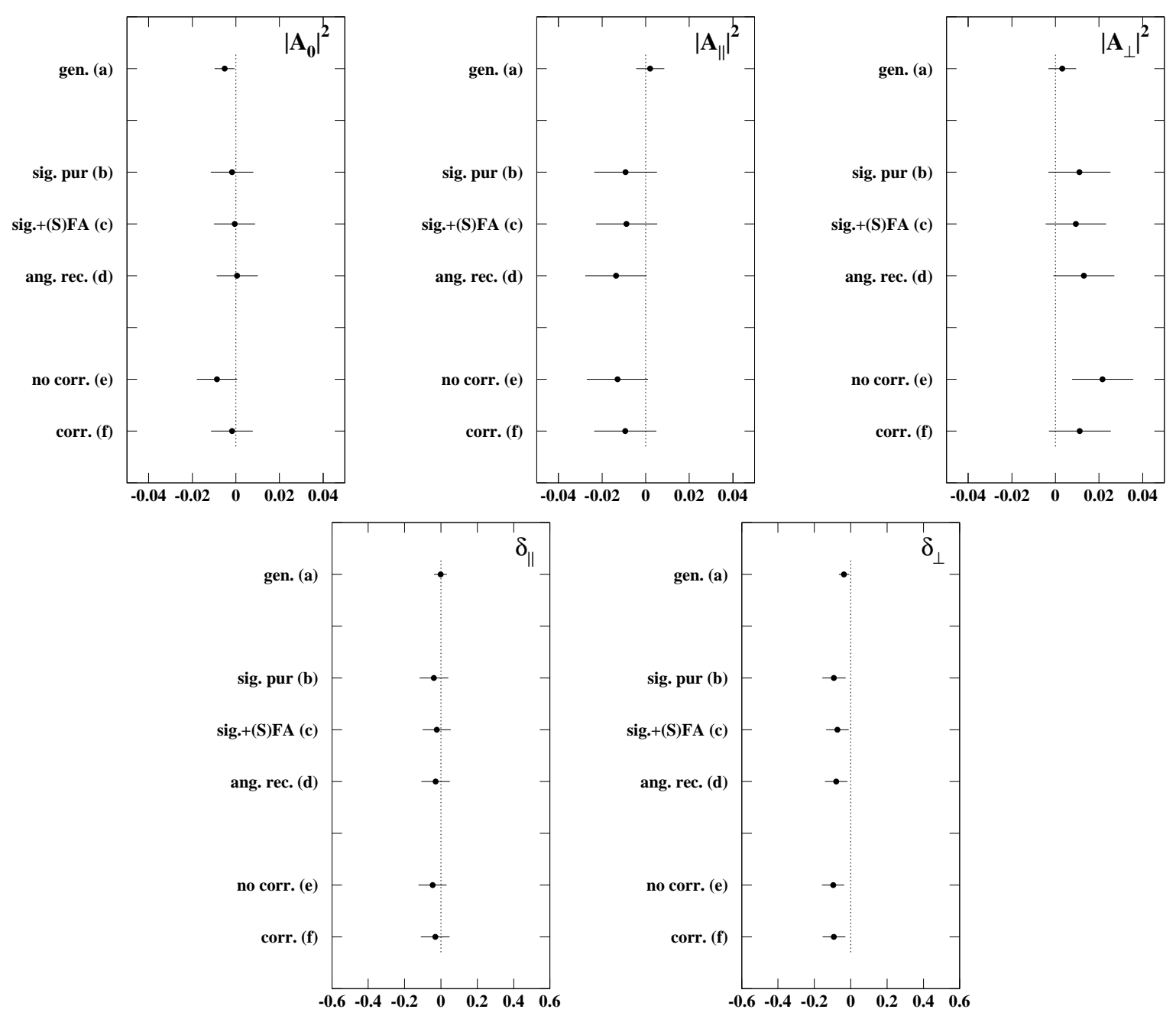

FIG. 5.6: Différences entre les amplitudes et les phases ajustées et les valeurs vraies de ces amplitudes et phases pour le canal $K_{S}^{0} \pi^{+}$: (a) échantillon à la génération, (b) échantillon pur après sélection et angles générés, (c) échantillon avec FA + SFA et angles générés, (d) idem (c) en utilisant les angles reconstruits, (e) ajout des événements de bruits de fond, sans correction, (f) idem avec correction des bruits de fond. 

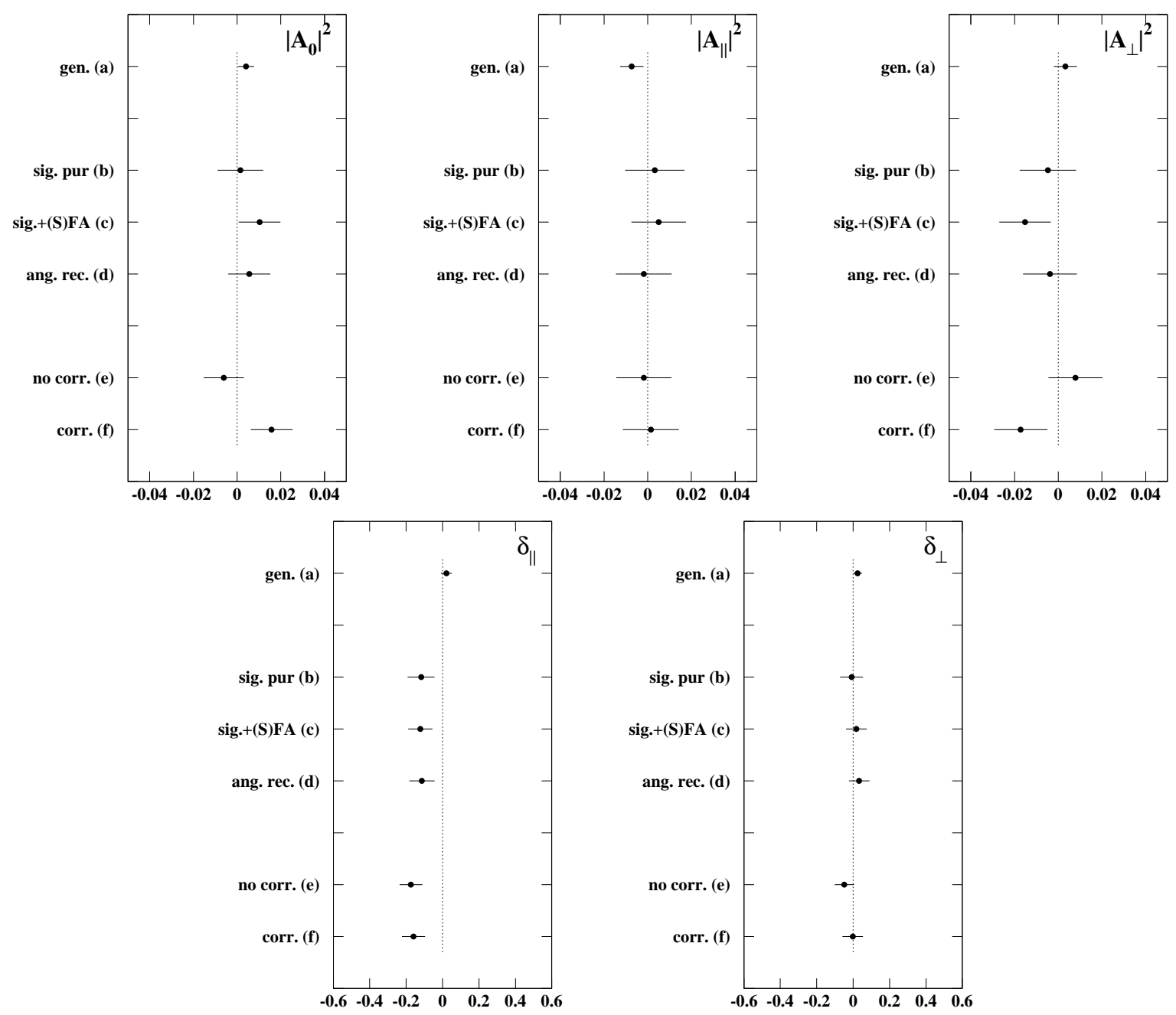

FIG. 5.7: Différences entre les amplitudes et les phases ajustées et les valeurs vraies de ces amplitudes et phases pour le canal $K^{+} \pi^{0}$ : (a) échantillon à la génération, (b) échantillon pur après sélection et angles générés, (c) échantillon avec FA + SFA et angles générés, (d) idem (c) en utilisant les angles reconstruits, (e) ajout des événements de bruits de fond, sans correction, (f) idem avec correction des bruits de fond. 

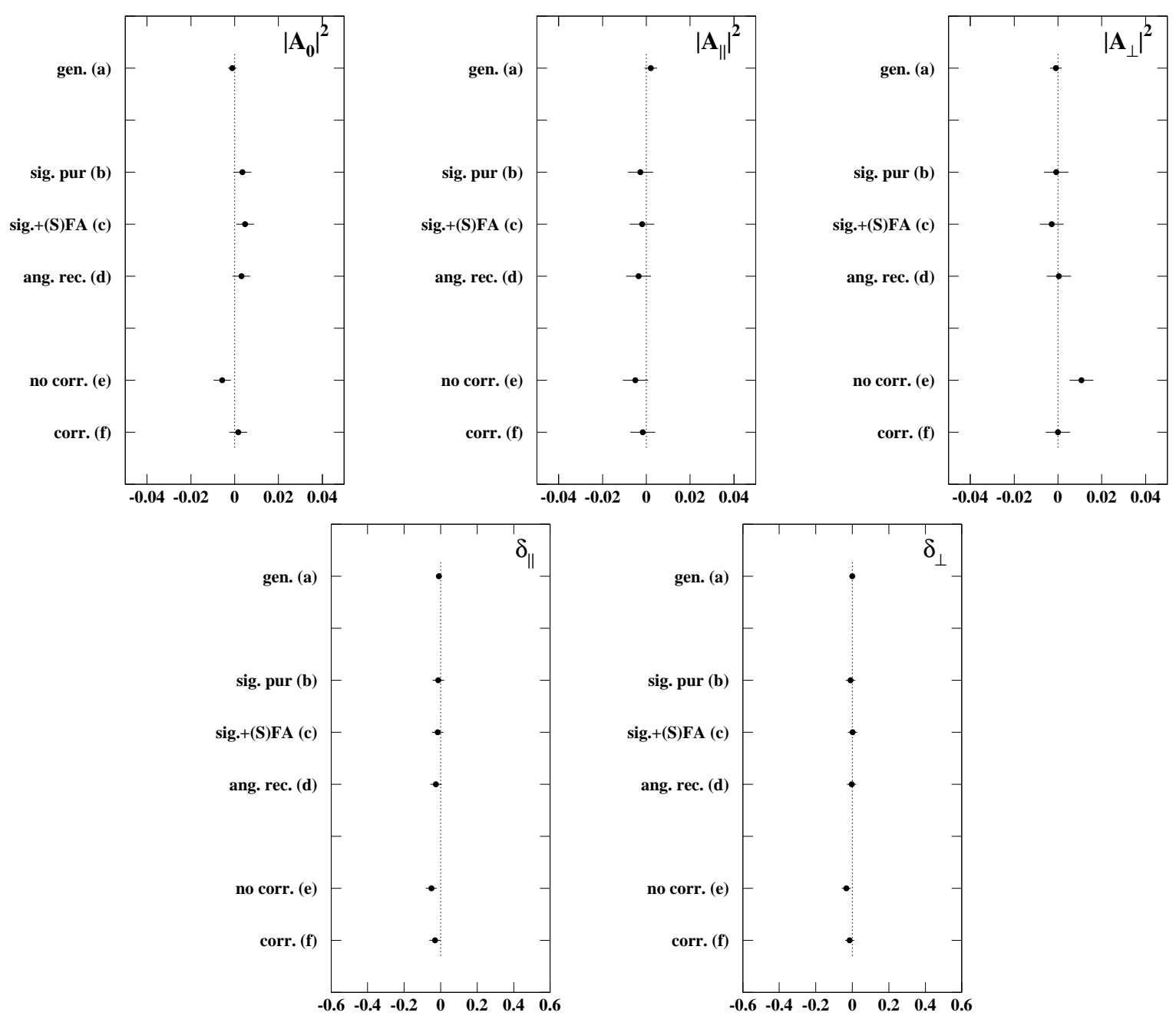

FIG. 5.8: Différences entre les amplitudes et les phases ajustées et les valeurs vraies de ces amplitudes et phases pour sur la somme des canaux $K^{+} \pi^{-}, K_{S}^{0} \pi^{+}$et $K^{+} \pi^{0}$ : (a) échantillon à la génération, (b) échantillon pur après sélection et angles générés, (c) échantillon avec FA + SFA et angles générés, (d) idem (c) en utilisant les angles reconstruits, (e) ajout des événements de bruits de fond, sans correction, (f) idem avec correction des bruits de fond. 
de la table ci-dessous :

\begin{tabular}{|l|ccc|}
\hline \hline mode & $\Phi_{1}^{i \rightarrow i, \text { rap. }} / \Phi_{1}^{i \rightarrow i, c o m p .}$ & $\Phi_{2}^{i \rightarrow i, \text { rap. }} / \Phi_{2}^{i \rightarrow i, \text { comp. }}$ & $\Phi_{3}^{i \rightarrow i, \text { rap. }} / \Phi_{3}^{i \rightarrow i, \text { comp } . ~}$ \\
\hline \hline$K^{+} \pi^{-}$ & 0.980 & 0.989 & 0.987 \\
$K^{-} \pi^{+}$ & 0.995 & 1.011 & 1.018 \\
\hline$K_{S}^{0} \pi^{+}$ & 1.022 & 1.039 & 1.042 \\
$K_{S}^{0} \pi^{-}$ & 1.027 & 1.058 & 1.080 \\
\hline$K^{+} \pi^{0}$ & 1.111 & 1.102 & 1.108 \\
$K^{-} \pi^{0}$ & 1.139 & 1.156 & 1.138 \\
\hline \hline
\end{tabular}

Nous voyons que l'accord est très bon pour les modes n'ayant que des particules chargées dans l'état final. En revanche, la simulation rapide surestime de 10 à $15 \%$ l'efficacité du canal $K^{ \pm} \pi^{0}$, traduisant une simulation rapide des photons de moins bonne qualité que celle des chargés. Nous pouvons cependant voir, aussi bien pour $K^{+} \pi^{0}$ que $K^{-} \pi^{0}$, que cette différence se traduit essentiellement par un facteur constant, autrement dit un facteur global sur l'acceptance, sans déformation de celle-ci. Les termes $l=4,5,6$ peuvent quant à eux différer largement entre les deux simulations, mais étant donnée la petitesse de ces termes, il s'agit là d'un effet négligeable. Dans le même genre d'idée, si nous comparons les distributions angulaires obtenues à partir des simulations rapide et complète dans la région signal pour pour les candidats $B \rightarrow J / \psi K^{*}(892)$ reconstruits, nous obtenons un très bon accord (figure 5.9) pour les canaux $K^{+} \pi^{-}$et $K_{S}^{0} \pi^{+}$, la différence d'efficacité observée sur le canal $K^{+} \pi^{0}$ se traduisant par un excès d'événements signal pour la simulation rapide. On note également quelques différences à grand $\cos \theta_{K^{*}}$ pour ce canal.

Passons maintenant à la comparaison des simulations rapide et complète dans la région $m_{\mathrm{ES}}<$ $5.27 \mathrm{GeV} / c^{2}$. Nous pourrions pour cela utiliser un échantillon de $J / \psi$ inclusif de $264 \mathrm{~K}$, sans seuil sur l'impulsion du $J / \psi$ (équivalent à $\sim 91 \mathrm{fb}^{-1}$ ), à notre disposition ${ }^{3}$, mais la statistique de cet échantillon est trop pauvre pour amener à des comparaisons intéressantes. Pour palier cela, nous introduisons pour les figures qui suivent, une coupure additionnelle sur l'impulsion du $J / \psi$ dans le centre de masse de $1^{\prime} \Upsilon(4 S)$ à $1.3 \mathrm{GeV} / c$ pour les événements de la simulation rapide. La figure 5.10 montre la comparaison des spectres de masse obtenus dans ces conditions. On observe un bon accord, à l'excès près des événements $K^{+} \pi^{0}$ dans la région signal. Enfin, la figure 5.11 montre la comparaison des distributions angulaires dans la région $m_{\mathrm{ES}}<5.27 \mathrm{GeV} / c^{2}$, réalisée dans les mêmes conditions que précédemment. L'accord n'est pas parfait, mais reste raisonnable.

En conclusion, la simulation rapide de BABAR est globalement de bonne qualité, et nous l'utilisons donc pour extraire les corrections d'erreurs.

${ }^{3} C^{\prime}$ est l'échantillon utilisé dans la figure 5.4 

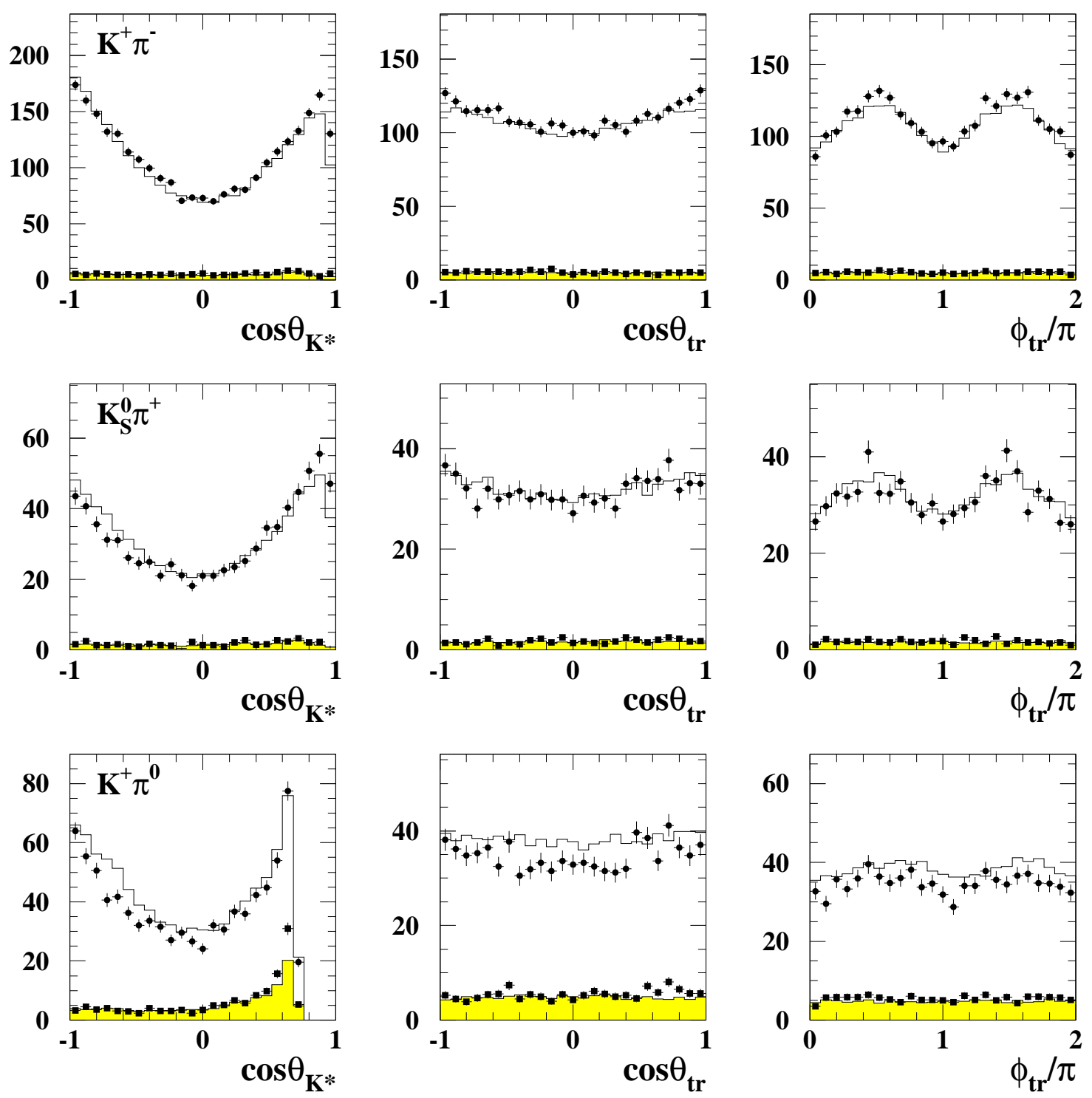

FIG. 5.9: Comparaison des distributions angulaires dans la région signal obtenues avec les simulations rapide et détaillée. Les histogrammes en trait plein sont ceux de la simulation rapide, la partie blanche représente le total des événements, la partie grisée la contribution des bruits de fond non $B \rightarrow J / \psi K^{*}(892)$. La simulation détaillée est représentée par les marqueurs ronds pour le total et carrés pour les bruits de fond. Les histogrammes sont normalisés à la luminosité des données $\left(\mathcal{L}=81.9 \mathrm{fb}^{-1}\right)$. 

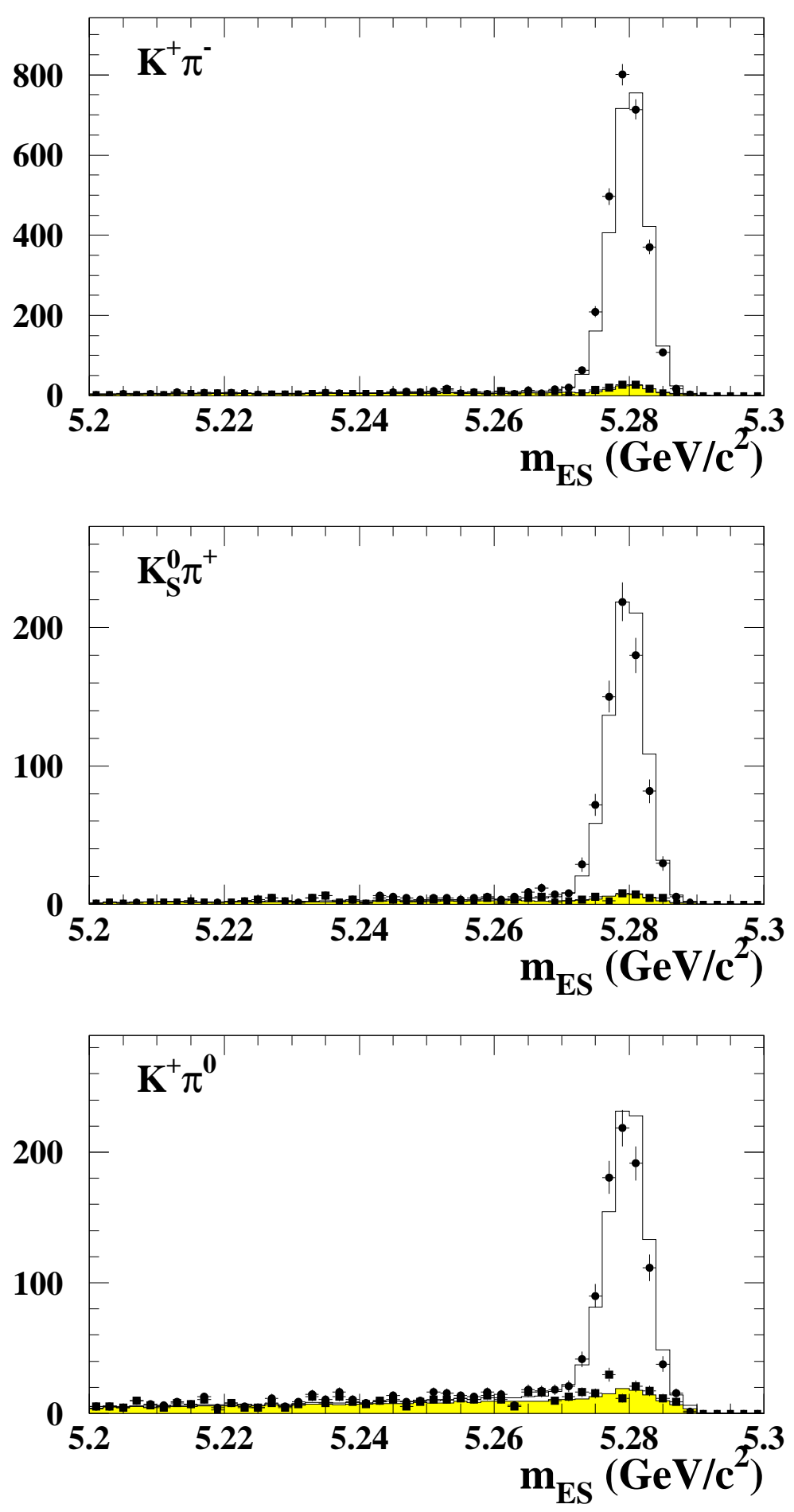

FIG. 5.10: Comparaison des distributions $m_{\mathrm{ES}}$ (après coupure sur $\Delta E$ ) pour les simulations rapide et détaillée. Les histogrammes en trait plein sont ceux de la simulation rapide, la partie blanche représente le total des événements, la partie grisée la contribution des bruits de fond non $B \rightarrow J / \psi K^{*}(892)$. La simulation détaillée est représentée par les marqueurs ronds pour le total et carrés pour les bruits de fond. Un seuil additionnel sur l'impulsion du $J / \psi$ dans le centre de masse de $l^{\prime} \Upsilon(4 S)$ est utilisé pour la simulation rapide, comme expliqué dans le texte. Les histogrammes sont normalisés à la luminosité des données $\left(\mathcal{L}=81.9 \mathrm{fb}^{-1}\right)$. 

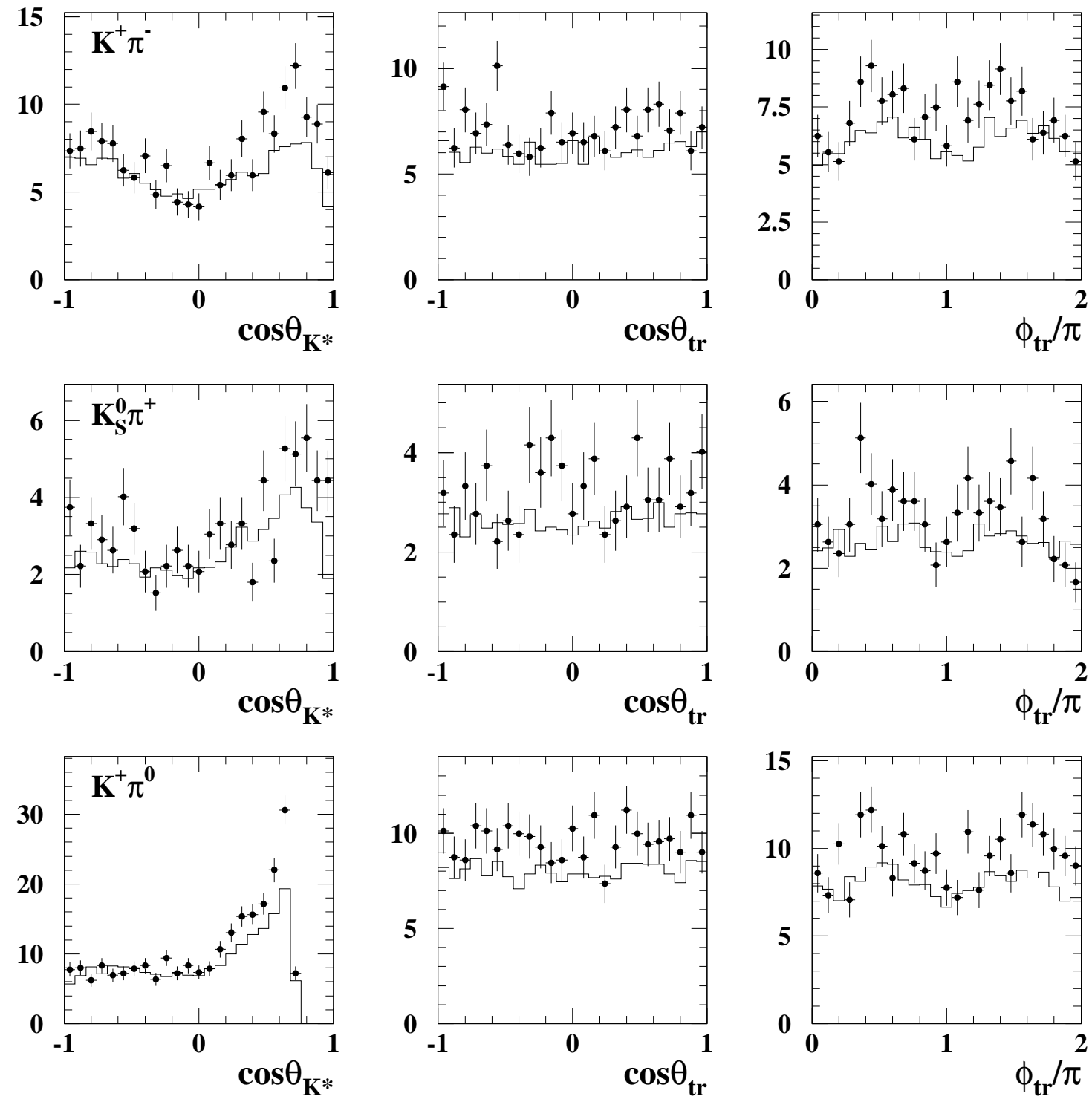

FIG. 5.11: Comparaison des distributions angulaires dans la région $m_{\mathrm{ES}}<5.27 \mathrm{GeV} / c^{2}$ (après coupure sur $\Delta E$ ) obtenues avec les simulations rapide et détaillée. Les histogrammes en trait plein sont ceux de la simulation rapide, la simulation détaillée est représentée par les marqueurs ronds. Un seuil additionnel sur l'impulsion du $J / \psi$ dans le centre de masse de $l^{\prime} \Upsilon(4 S)$ est utilisé pour la simulation rapide, comme expliqué dans le texte. Les histogrammes sont normalisés à la luminosité des données $\left(\mathcal{L}=81.9 \mathrm{fb}^{-1}\right)$. 


\section{Facteurs de correction d'erreur}

Nous formons donc 168 expériences, en respectant la répartition des événements observés dans les données, année par année et canal par canal, à savoir :

\begin{tabular}{|c|ccc|c|}
\hline \hline Canal & 2000 & 2001 & 2002 & Total \\
\hline \hline$K^{+} \pi^{-}$ & 724 & 1305 & 788 & 2817 \\
$K_{S}^{0} \pi^{+}$ & 213 & 368 & 220 & 819 \\
$K^{+} \pi^{0}$ & 323 & 640 & 414 & 1380 \\
\hline \hline
\end{tabular}

où ces nombres d'événements correspondent aux événements sélectionnés dans la bande en $m_{\mathrm{ES}}$ de 5.2 à $5.3 \mathrm{GeV} / c^{2}$ (après coupure sur $\Delta E$ ). Nous ajustons les amplitudes et phases pour chaque expérience, pour les 3 canaux séparément et ensembles. Les figures 5.12 à 5.15 montrent, pour les canaux $K^{+} \pi^{-}, K_{S}^{0} \pi^{+}, K^{+} \pi^{0}$ et l'ensemble des canaux, respectivement, les distributions des paramètres ajustés $\theta_{A}, \phi_{A}, \delta_{\|}$et $\delta_{\perp}$ et de leurs pulls, ainsi que les distributions des modules des amplitudes et de leurs pulls.

La table 5.3 résume la liste des facteurs de correction d'erreur à appliquer aux erreurs fournies par Minuit obtenus à partir des pulls précédents. Nous voyons que pour le canal $K^{+} \pi^{0}$, qui est celui comportant le plus de bruits, les erreurs fournies par Minuit peuvent être sous-estimées de près de $30 \%$. Nous observons également que $\phi_{A}$ est le paramètre nécessitant la plus grande correction d'erreur.

TAB. 5.3: Facteurs de correction d'erreur.

\begin{tabular}{|l|cccc|}
\hline \hline Mode & $\theta_{A}$ & $\phi_{A}$ & $\delta_{\|}$ & $\delta_{\perp}$ \\
\hline \hline$K^{+} \pi^{-}$ & $0.993 \pm 0.054$ & $1.175 \pm 0.064$ & $1.073 \pm 0.059$ & $1.041 \pm 0.057$ \\
$K_{S}^{0} \pi^{+}$ & $1.122 \pm 0.061$ & $1.160 \pm 0.063$ & $1.033 \pm 0.056$ & $0.989 \pm 0.054$ \\
$K^{+} \pi^{0}$ & $1.192 \pm 0.065$ & $1.287 \pm 0.071$ & $1.296 \pm 0.071$ & $1.055 \pm 0.058$ \\
3 canaux & $1.005 \pm 0.055$ & $1.131 \pm 0.062$ & $1.115 \pm 0.061$ & $1.034 \pm 0.056$ \\
\hline \hline
\end{tabular}

Ces facteurs de correction se répercutent sur les erreurs des modules des amplitudes, comme montré dans la table 5.4.

TAB. 5.4: Facteurs de correction d'erreur pour les modules des amplitudes.

\begin{tabular}{|l|ccc|}
\hline \hline Mode & $\left|A_{0}\right|^{2}$ & $\left|A_{\|}\right|^{2}$ & $\left|A_{\perp}\right|^{2}$ \\
\hline \hline$K^{+} \pi^{-}$ & $0.991 \pm 0.054$ & $1.166 \pm 0.064$ & $1.168 \pm 0.064$ \\
$K_{S}^{0} \pi^{+}$ & $1.127 \pm 0.062$ & $1.191 \pm 0.065$ & $1.217 \pm 0.067$ \\
$K^{+} \pi^{0}$ & $1.194 \pm 0.065$ & $1.256 \pm 0.069$ & $1.296 \pm 0.072$ \\
3 canaux & $1.002 \pm 0.055$ & $1.128 \pm 0.062$ & $1.112 \pm 0.061$ \\
\hline \hline
\end{tabular}

Lors de l'ajustement sur les données, nous appliquerons ces corrections aux erreurs données par Minuit. En ce qui concerne l'obtention de la matrice de covariance nous ferons l'hypothèse que la technique du pseudo-maximum de vraisemblance permet une obtention correcte de la matrice de 

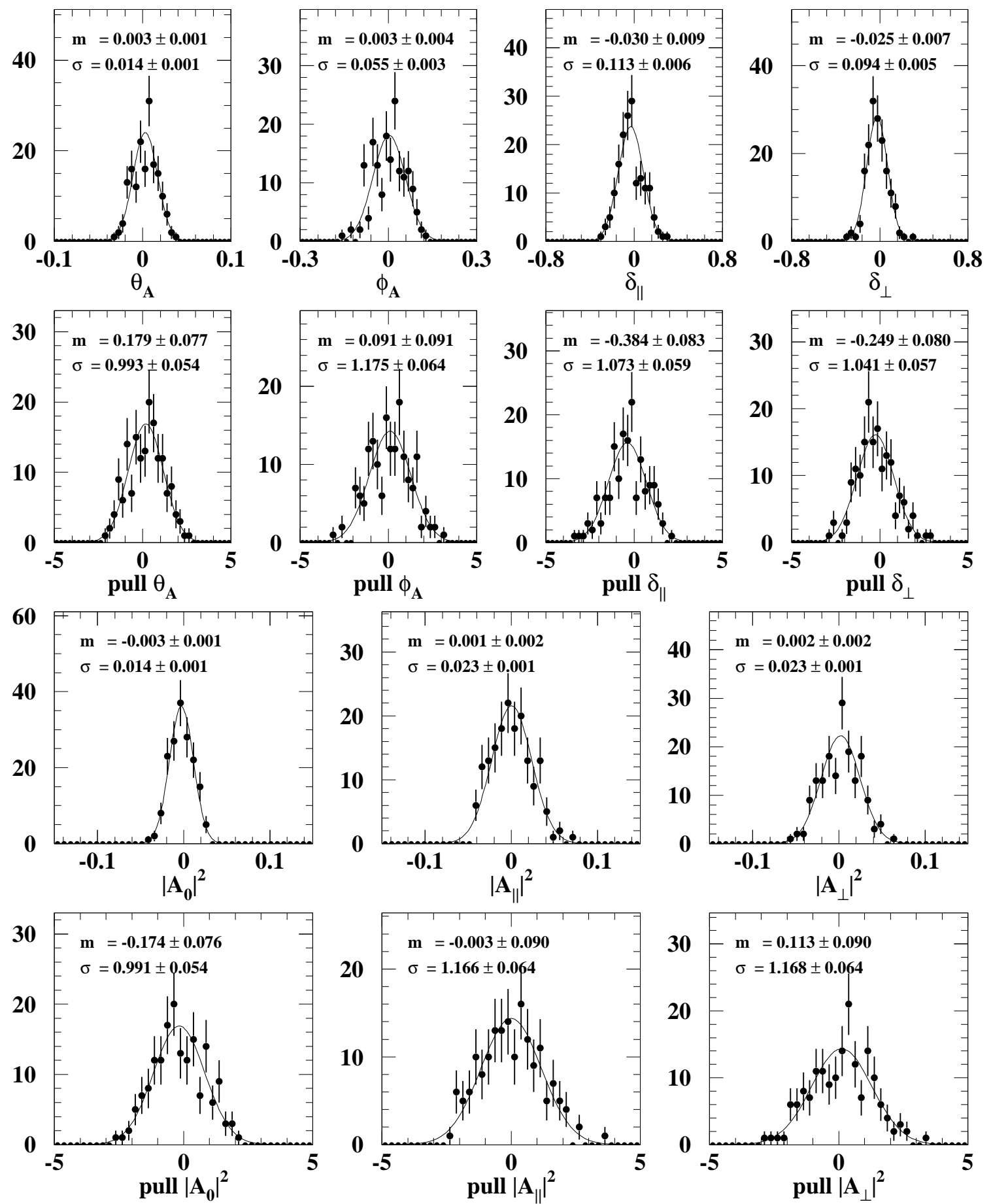

FIG. 5.12: Distribution des paramètres ajustés $\theta_{A}, \phi_{A}, \delta_{\|}$et $\delta_{\perp}$, de leurs pulls et distribution des modules des amplitudes, dérivés de $\theta_{A}$ et $\phi_{A}$ pour le canal $K^{+} \pi^{-}$. Chaque ajustement correspond à une expérience de même statistique que les données. Sur chaque histogramme sont indiquées la valeur moyenne et la déviation standard de la distribution obtenus par un ajustement gaussien. 

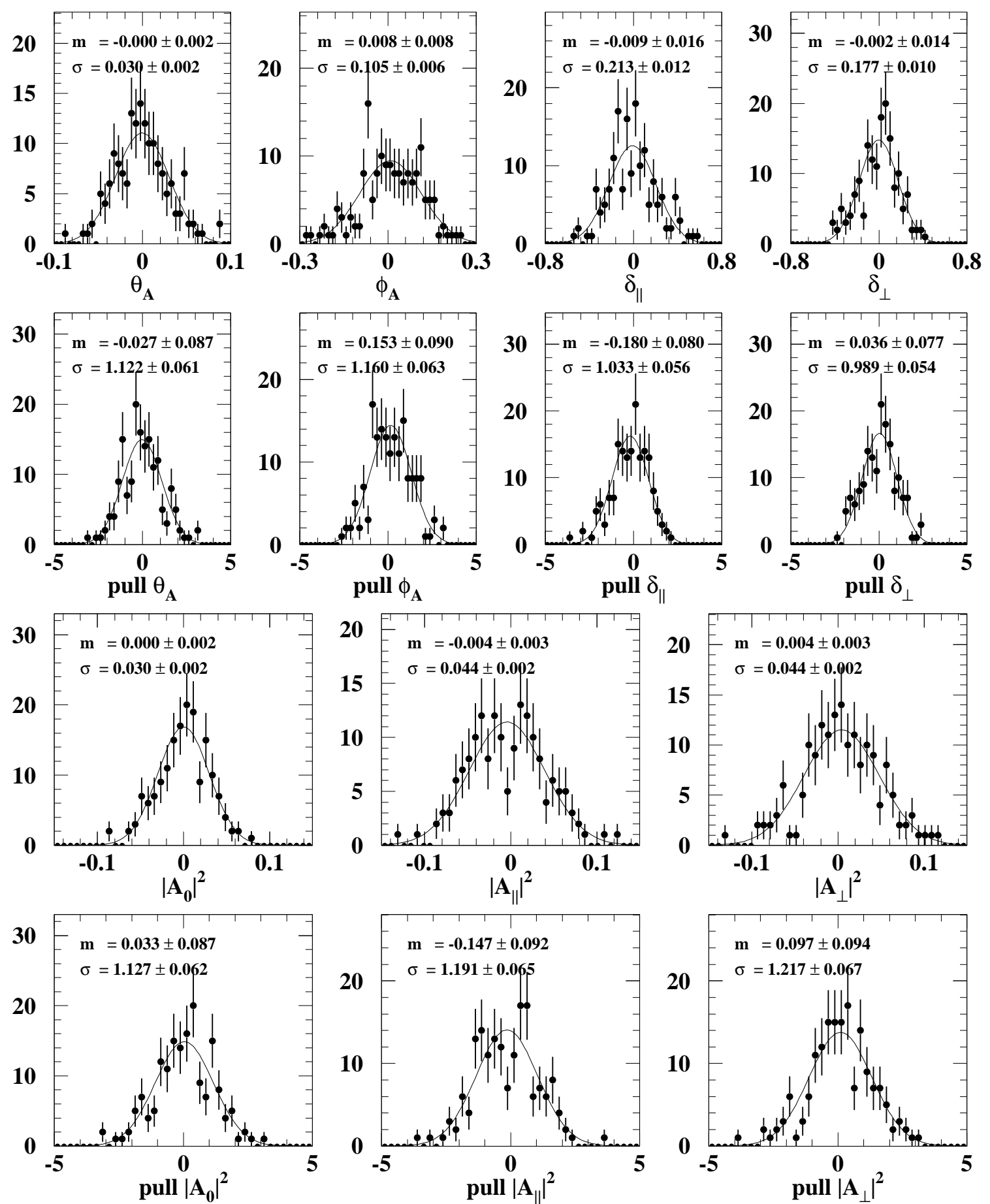

FIG. 5.13: Distribution des paramètres ajustés $\theta_{A}, \phi_{A}, \delta_{\|}$et $\delta_{\perp}$, de leurs pulls et distribution des modules des amplitudes, dérivés de $\theta_{A}$ et $\phi_{A}$ pour le canal $K_{S}^{0} \pi^{+}$. Chaque ajustement correspond à une expérience de même statistique que les données. Sur chaque histogramme sont indiquées la valeur moyenne et la déviation standard de la distribution obtenus par un ajustement gaussien. 

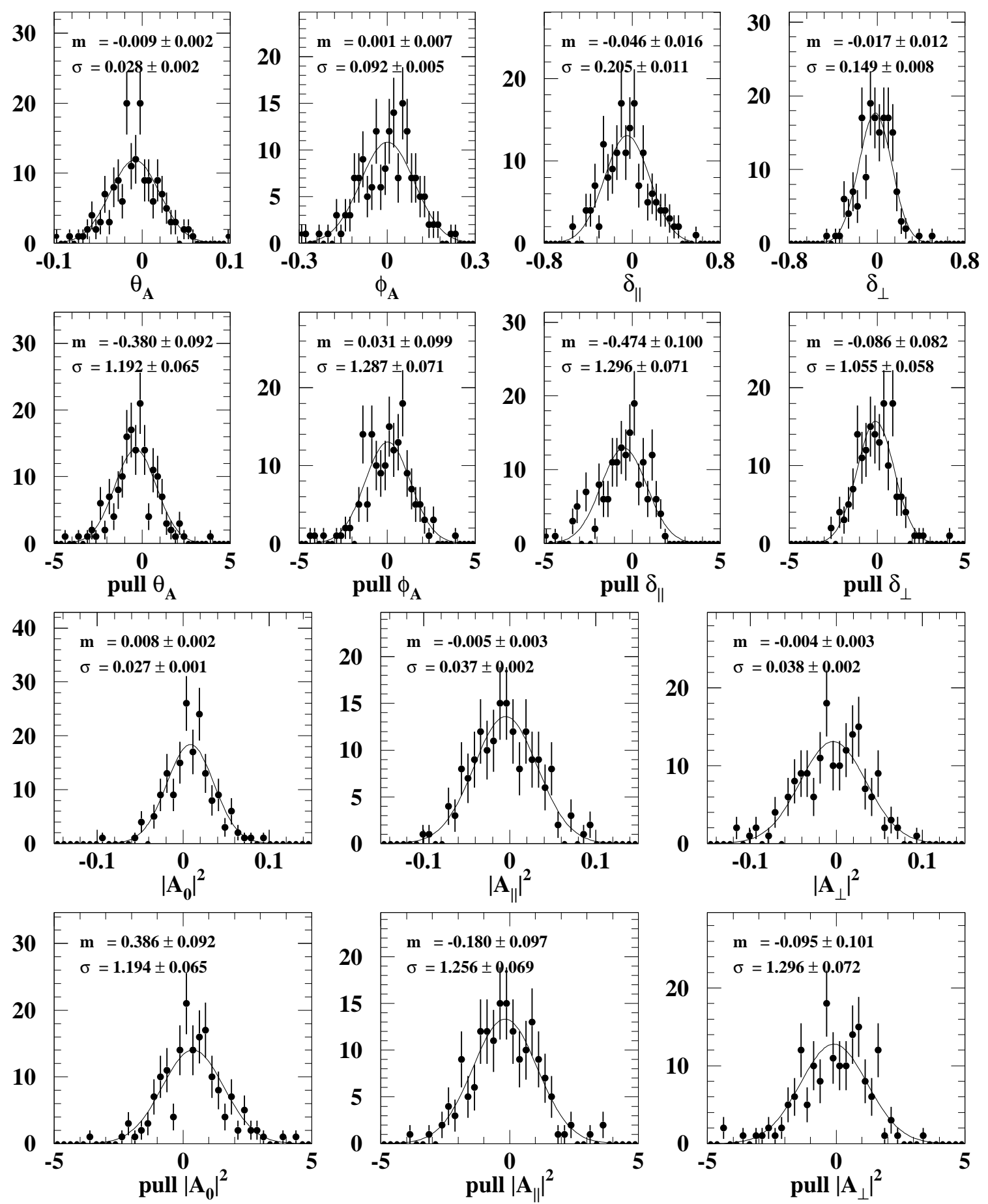

FIG. 5.14: Distribution des paramètres ajustés $\theta_{A}, \phi_{A}, \delta_{\|}$et $\delta_{\perp}$, de leurs pulls et distribution des modules des amplitudes, dérivés de $\theta_{A}$ et $\phi_{A}$ pour le canal $K^{+} \pi^{0}$. Chaque ajustement correspond à une expérience de même statistique que les données. Sur chaque histogramme sont indiquées la valeur moyenne et la déviation standard de la distribution obtenus par un ajustement gaussien. 

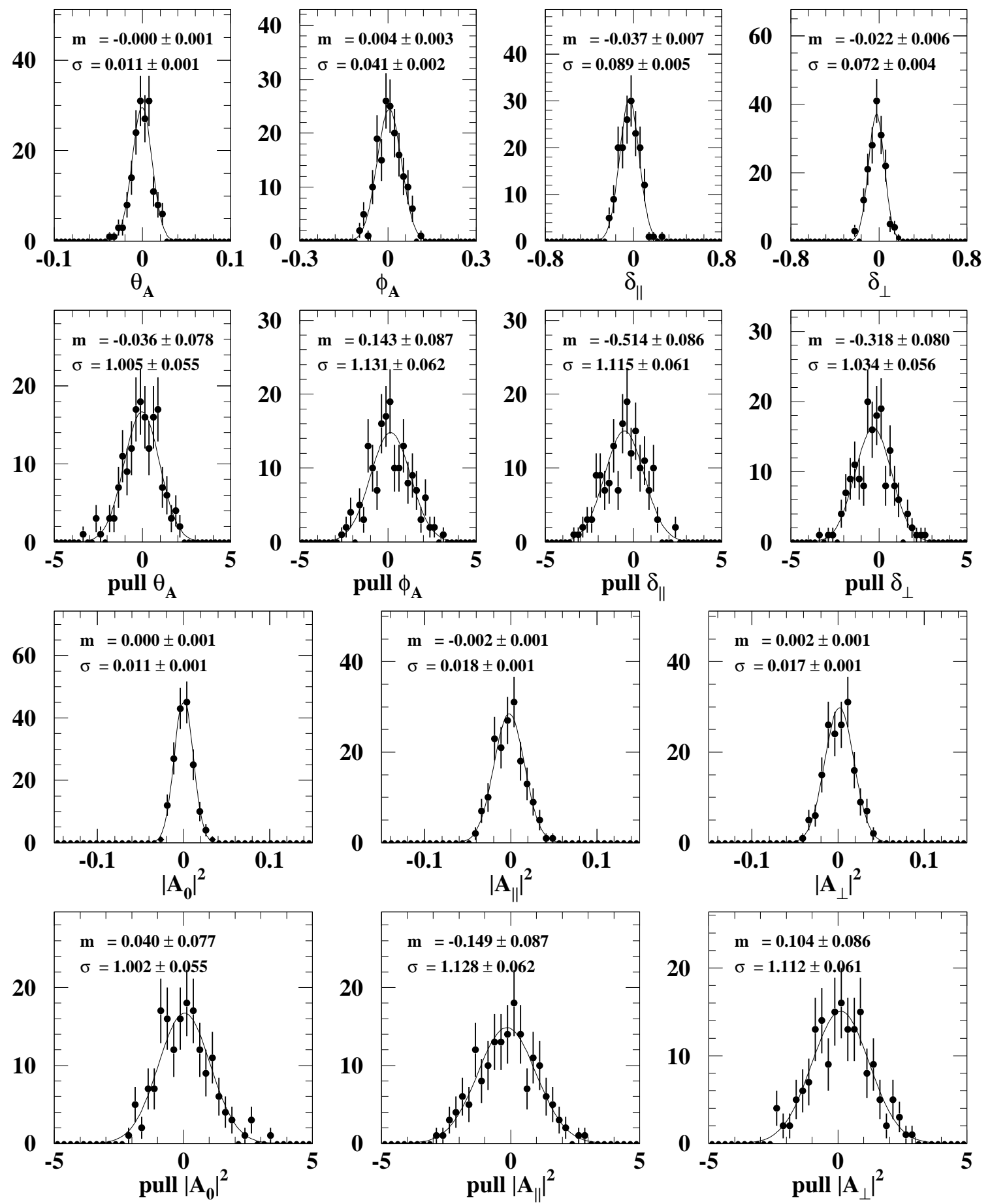

FIG. 5.15: Distribution des paramètres ajustés $\theta_{A}, \phi_{A}, \delta_{\|}$et $\delta_{\perp}$, de leurs pulls et distribution des modules des amplitudes, dérivés de $\theta_{A}$ et $\phi_{A}$ pour les trois canaux ensembles. Chaque ajustement correspond à une expérience de même statistique que les données. Sur chaque histogramme sont indiquées la valeur moyenne et la déviation standard de la distribution obtenus par un ajustement gaussien. 
corrélation. Nous nous contenterons alors de redimensionner la covariance $\operatorname{Cov}\left(p_{i}, p_{j}\right)$ entre les paramètres $p_{i}$ et $p_{j}$ comme :

$$
\operatorname{Cov}\left(p_{i}, p_{j}\right)=\rho_{i j} \cdot \sigma_{i} \cdot \sigma_{j}
$$

où $\rho_{i j}$ est la corrélation entre $p_{i}$ et $p_{j}$, et $\sigma_{i}, \sigma_{j}$ leurs incertitudes corrigées.

\section{Corrections de biais}

Les figures 5.12 à 5.15 montrent également que l'on observe un certain nombre de biais dans l'estimation des paramètres. Ces biais sont résumés dans les tables 5.5 et 5.6.

TAB. 5.5: Biais observés sur $\theta_{A}, \phi_{A}, \delta_{\|}$et $\delta_{\perp}$.

\begin{tabular}{|l|cccc|}
\hline \hline Mode & $\theta_{A}$ & $\phi_{A}$ & $\delta_{\|}$ & $\delta_{\perp}$ \\
\hline \hline$K^{+} \pi^{-}$ & $0.003 \pm 0.001$ & $0.003 \pm 0.004$ & $-0.030 \pm 0.009$ & $-0.025 \pm 0.007$ \\
$K_{S}^{0} \pi^{+}$ & $-0.000 \pm 0.002$ & $0.008 \pm 0.008$ & $-0.009 \pm 0.016$ & $-0.002 \pm 0.014$ \\
$K^{+} \pi^{0}$ & $-0.009 \pm 0.002$ & $0.001 \pm 0.007$ & $-0.046 \pm 0.016$ & $-0.017 \pm 0.012$ \\
3 canaux & $-0.000 \pm 0.001$ & $0.004 \pm 0.003$ & $-0.037 \pm 0.007$ & $-0.022 \pm 0.006$ \\
\hline \hline
\end{tabular}

TAB. 5.6: Biais observés sur $\left|A_{0}\right|^{2},\left|A_{\|}\right|^{2}$ et $\left|A_{\perp}\right|^{2}$.

\begin{tabular}{|l|ccc|}
\hline \hline Mode & $\left|A_{0}\right|^{2}$ & $\left|A_{\|}\right|^{2}$ & $\left|A_{\perp}\right|^{2}$ \\
\hline \hline$K^{+} \pi^{-}$ & $-0.003 \pm 0.001$ & $0.001 \pm 0.002$ & $0.002 \pm 0.002$ \\
$K_{S}^{0} \pi^{+}$ & $0.000 \pm 0.002$ & $-0.004 \pm 0.003$ & $0.004 \pm 0.003$ \\
$K^{+} \pi^{0}$ & $0.008 \pm 0.002$ & $-0.005 \pm 0.003$ & $-0.004 \pm 0.003$ \\
3 canaux & $0.000 \pm 0.001$ & $-0.002 \pm 0.001$ & $0.002 \pm 0.001$ \\
\hline \hline
\end{tabular}

Ces biais se retrouvent si l'on effectue un unique ajustement sur l'ensemble des événements de simulation rapide :

\begin{tabular}{|l|cccc|}
\hline \hline Mode & $\theta_{A}$ & $\phi_{A}$ & $\delta_{\|}$ & $\delta_{\perp}$ \\
\hline \hline$K^{+} \pi^{-}$ & $0.003 \pm 0.001$ & $0.003 \pm 0.003$ & $-0.035 \pm 0.008$ & $-0.029 \pm 0.006$ \\
$K_{S}^{0} \pi^{+}$ & $-0.001 \pm 0.002$ & $0.013 \pm 0.006$ & $-0.019 \pm 0.014$ & $-0.008 \pm 0.011$ \\
$K^{+} \pi^{0}$ & $-0.009 \pm 0.002$ & $0.006 \pm 0.006$ & $-0.054 \pm 0.012$ & $-0.029 \pm 0.010$ \\
3 canaux & $-0.000 \pm 0.001$ & $0.005 \pm 0.003$ & $-0.036 \pm 0.006$ & $-0.025 \pm 0.005$ \\
\hline \hline
\end{tabular}

En d'autre termes, le biais moyen est la moyenne des biais. Ces chiffres sont intéressants car ils montrent les limites de notre méthode d'ajustement. Cet effet systématique est notamment dû aux différences de distribution angulaire des bruits de fond entre les régions signal et à bas $m_{\mathrm{ES}}$. Dans l'ajustement sur les données, nous utiliserons les valeurs des tables 5.5 et 5.6 comme estimations des corrections à apporter, et nous attribuerons comme erreur systématique, la moitié de la valeur de ce biais. 
TAB. 5.7: Résultat de l'ajustement des amplitudes et des phases, corrigées des biais mentionnés dans la section 5.3.2, par année, par canal et pour l'échantillon total. Les incertitudes sont statistiques (et corrigées). Rappelons que $\left(\delta_{\|}, \delta_{\perp}\right) \rightarrow$ $\left(-\delta_{\|}, \pi-\delta_{\perp}\right)$ est aussi une solution.

\begin{tabular}{|l|cc|}
\hline \hline Échantillon & $\theta_{A}$ & $\phi_{A}$ \\
\hline \hline$K^{+} \pi^{-}$ & $0.723 \pm 0.014$ & $0.810 \pm 0.046$ \\
$K_{S}^{0} \pi^{+}$ & $0.726 \pm 0.030$ & $0.750 \pm 0.080$ \\
$K^{+} \pi^{0}$ & $0.701 \pm 0.030$ & $0.882 \pm 0.083$ \\
\hline \hline 2000 & $0.720 \pm 0.023$ & $0.692 \pm 0.073$ \\
2001 & $0.725 \pm 0.017$ & $0.834 \pm 0.048$ \\
2002 & $0.710 \pm 0.022$ & $0.855 \pm 0.069$ \\
\hline \hline Total & $0.720 \pm 0.012$ & $0.811 \pm 0.034$ \\
\hline
\end{tabular}

\begin{tabular}{|l|ccc|cc|}
\hline Échantillon & $\left|A_{0}\right|^{2}$ & $\left|A_{\|}\right|^{2}$ & $\left|A_{\perp}\right|^{2}$ & $\delta_{\|}$ & $\delta_{\perp}$ \\
\hline \hline$K^{+} \pi^{-}$ & $0.562 \pm 0.014$ & $0.208 \pm 0.021$ & $0.230 \pm 0.022$ & $2.703 \pm 0.125$ & $0.184 \pm 0.084$ \\
$K_{S}^{0} \pi^{+}$ & $0.559 \pm 0.030$ & $0.237 \pm 0.040$ & $0.204 \pm 0.040$ & $2.756 \pm 0.221$ & $0.126 \pm 0.165$ \\
$K^{+} \pi^{0}$ & $0.584 \pm 0.030$ & $0.169 \pm 0.036$ & $0.247 \pm 0.038$ & $2.950 \pm 0.315$ & $0.346 \pm 0.158$ \\
\hline \hline 2000 & $0.565 \pm 0.022$ & $0.258 \pm 0.034$ & $0.177 \pm 0.033$ & $2.587 \pm 0.191$ & $-0.113 \pm 0.142$ \\
2001 & $0.560 \pm 0.017$ & $0.198 \pm 0.023$ & $0.241 \pm 0.023$ & $2.863 \pm 0.149$ & $0.307 \pm 0.099$ \\
2002 & $0.575 \pm 0.021$ & $0.183 \pm 0.031$ & $0.242 \pm 0.032$ & $2.721 \pm 0.231$ & $0.325 \pm 0.128$ \\
\hline \hline Total & $0.565 \pm 0.011$ & $0.206 \pm 0.016$ & $0.228 \pm 0.016$ & $2.766 \pm 0.105$ & $0.207 \pm 0.067$ \\
\hline \hline
\end{tabular}

\subsection{Ajustement sur les données}

Utilisant l'échantillon de données dont la sélection est décrite au chapitre 4, nous effectuons les ajustements par canal et par année. Le résultat des ajustements est montré sur la figure 5.16. Nous voyons que les mesures sont compatibles de canal à canal et d'année à année. Les mesures sont rassemblées dans le tableau 5.7. La corrélation entre les paramètres en sortie d'ajustement est donnée dans la table 5.8. Nous voyons que ces corrélations ne dépassent pas $67 \%$.

TAB. 5.8: Matrice de corrélation.

\begin{tabular}{|l|c|cccc|}
\hline \hline Paramètre & global & $\theta_{A}$ & $\phi_{A}$ & $\delta_{\|}$ & $\delta_{\perp}$ \\
\hline \hline$\theta_{A}$ & 0.08197 & 1.000 & -0.008 & 0.039 & -0.027 \\
$\phi_{A}$ & 0.24344 & -0.008 & 1.000 & 0.233 & 0.100 \\
$\delta_{\|}$ & 0.66906 & 0.039 & 0.233 & 1.000 & 0.645 \\
$\delta_{\perp}$ & 0.64890 & -0.027 & 0.100 & 0.645 & 1.000 \\
\hline \hline
\end{tabular}

\section{5 Étude des incertitudes systématiques}

Les effets systématiques sont rassemblés dans les tables 5.9 à 5.12. Nous considérons les sources d'effet systématique suivantes : 


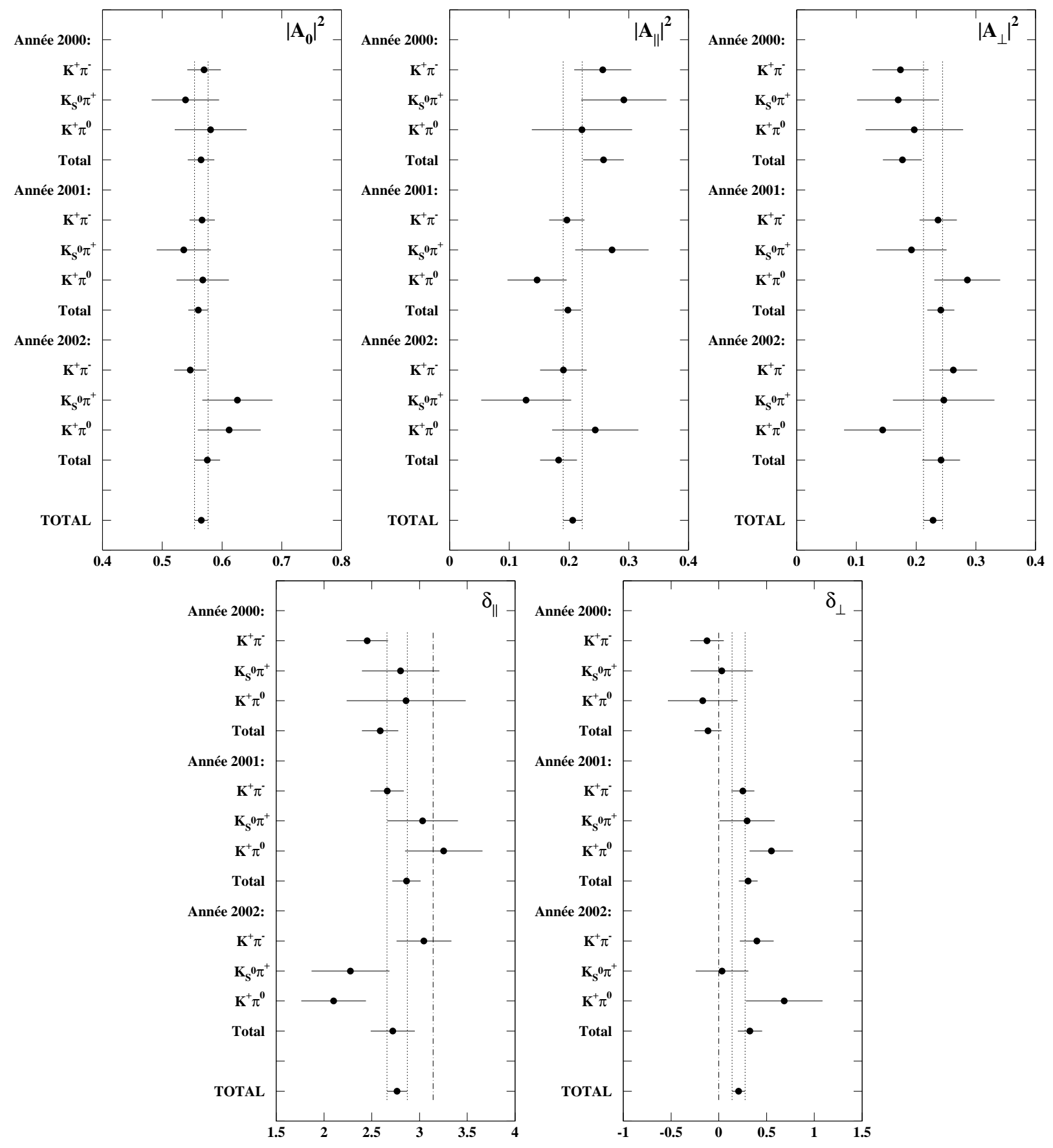

FIG. 5.16: Ajustement des amplitudes et des phases sur les données. 
- « énergie CM »: l'incertitude sur l'énergie dans le centre de masse est prise en compte en variant le paramètre $m_{0}$ de la fonction Argus (cf. eq. (4.2)). Partant de la valeur nominale de ce paramètre $\left(5.291 \mathrm{GeV} / c^{2}\right)$, nous effectuons des ajustements en changeant cette valeur de $\pm 2 \mathrm{MeV} / c^{2}$. La plus grande variation est retenue comme incertitude;

- « forme bruit» : le rapport des aires entre les régions $m_{\mathrm{ES}}<5.27 \mathrm{GeV} / c^{2}$ et $m_{\mathrm{ES}}>5.27 \mathrm{GeV} / c^{2}$ pour les bruits de fond, utilisé pour soustraire la contribution des bruits de fond dans la région $m_{\mathrm{ES}}>5.27 \mathrm{GeV} / c^{2}$, dépend de la forme de ces bruits de fond. Dans l'ajustement sur le spectre en $m_{\mathrm{ES}}$, cette forme est contrôlée par le paramètre $\xi$ de la fonction Argus. Nous faisons varier ce paramètre de \pm 1 déviation standard et gardons la plus grande déviation comme incertitude systématique;

- « BR » : le FA opère entre canaux chargé et neutre, par remplacement d'un pion chargé en un pion neutre. Une variation des rapports de branchement entre $B^{0} \rightarrow J / \psi K^{* 0}$ et $B^{+} \rightarrow$ $J / \psi K^{*+}$ peut affecter la valeur de $g_{o b s}\left(\vec{\omega}^{\prime} ; \vec{A}\right)$ à travers les fractions $F_{i}$. Cet effet est estimé en faisant varier indépendamment de \pm 1 déviation standard les rapports de branchement de $B^{0} \rightarrow J / \psi K^{* 0}$ et $B^{+} \rightarrow J / \psi K^{*+}$ [28]. La plus grande déviation est retenue comme incertitude systématique.

- «stat. MC » : la statistique finie du Monte Carlo induit un effet systématique à travers le calcul des coefficients $\Phi_{l}^{i \rightarrow j}$. Cet effet est estimé en variant individuellement de \pm 1 déviation standard ces coefficients $\Phi_{l}^{i \rightarrow j}$. La plus grande déviation est retenue comme incertitude systématique;

- « corr. biais » : comme expliqué dans la section 5.3.2, nous observons un certain nombre de biais, notamment dû au fait que la composition angulaire des bruits de fond présente des différences entre les régions $m_{\mathrm{ES}}<5.27 \mathrm{GeV} / c^{2}$ et $m_{\mathrm{ES}}>5.27 \mathrm{GeV} / c^{2}$. Nous utilisons les biais mesurés comme corrections. Comme la mesure de ces biais provient d'une simulation rapide, nous assignons la moitié de la valeur du biais comme incertitude systématique;

- «PID» : l'acceptance angulaire dépend de l'identification de particules. Rappelons que les efficacités de détection sont mesurées sur les données et utilisées par le Monte Carlo pour simuler de façon réaliste le PID. Les incertitudes sur les efficacités de détection sont connues et une méthode naturelle consisterait à varier ces efficacités pour chaque type de particules dans le Monte Carlo. Ceci serait néanmoins une procédure lourde. Pour estimer, de façon très conservative, l'effet sur nos mesures, nous avons choisi d'utiliser les tables d'efficacité des années 2000, 2001 et 2002, successivement, pour l'ensemble de l'échantillon de données. Les différences d'efficacité entre ces tables sont bien supérieures aux incertitudes sur ces efficacités. L'effet sur nos mesures est faible, essentiellement car les variations d'efficacité mettent en jeu un facteur global, sans déformation de l'acceptance ;

- « onde $S$ » : il s'agit là de la systématique la plus intéressante. Nous attribuons la même incertitude systématique à tous les modes. La façon dont est déterminée cette systématique est expliquée dans la section suivante. 
TAB. 5.9: Incertitudes systématiques pour le mode $K^{+} \pi^{-}$.

\begin{tabular}{|l|cccc|ccc|}
\hline Source & $\theta_{A}$ & $\phi_{A}$ & $\delta_{\|}$ & $\delta_{\perp}$ & $\left|A_{0}\right|^{2}$ & $\left|A_{\|}\right|^{2}$ & $\left|A_{\perp}\right|^{2}$ \\
\hline énergie CM & 0.0007 & 0.0007 & 0.0010 & 0.0009 & 0.0010 & 0.0007 & 0.0001 \\
forme bruit & 0.0014 & 0.0014 & 0.0018 & 0.0010 & 0.0027 & 0.0014 & 0.0001 \\
BR & 0.0011 & 0.0011 & 0.0007 & 0.0014 & 0.0013 & 0.0011 & 0.0006 \\
stat. MC & 0.0013 & 0.0013 & 0.0075 & 0.0058 & 0.0019 & 0.0013 & 0.0033 \\
corr. biais & 0.0014 & 0.0013 & 0.0149 & 0.0124 & 0.0013 & 0.0003 & 0.0010 \\
PID & 0.0021 & 0.0040 & 0.0109 & 0.0088 & 0.0021 & 0.0023 & 0.0025 \\
onde S & 0.0030 & 0.0025 & 0.0335 & 0.0371 & 0.0029 & 0.0025 & 0.0005 \\
\hline \hline total & 0.0046 & 0.0054 & 0.0390 & 0.0406 & 0.0053 & 0.0041 & 0.0043 \\
\hline \hline
\end{tabular}

TAB. 5.10: Incertitudes systématiques pour le mode $K_{S}^{0} \pi^{+}$.

\begin{tabular}{|l|cccc|ccc|}
\hline Source & $\theta_{A}$ & $\phi_{A}$ & $\delta_{\|}$ & $\delta_{\perp}$ & $\left|A_{0}\right|^{2}$ & $\left|A_{\|}\right|^{2}$ & $\left|A_{\perp}\right|^{2}$ \\
\hline énergie CM & 0.0005 & 0.0003 & 0.0014 & 0.0018 & 0.0005 & 0.0001 & 0.0003 \\
forme bruit & 0.0020 & 0.0017 & 0.0059 & 0.0082 & 0.0020 & 0.0003 & 0.0017 \\
BR & 0.0021 & 0.0003 & 0.0003 & 0.0003 & 0.0021 & 0.0012 & 0.0009 \\
stat. MC & 0.0026 & 0.0138 & 0.0103 & 0.0039 & 0.0026 & 0.0065 & 0.0071 \\
corr. biais & 0.0002 & 0.0042 & 0.0046 & 0.0011 & 0.0001 & 0.0022 & 0.0021 \\
PID & 0.0045 & 0.0083 & 0.0196 & 0.0186 & 0.0045 & 0.0060 & 0.0033 \\
onde S & 0.0030 & 0.0025 & 0.0335 & 0.0371 & 0.0029 & 0.0025 & 0.0005 \\
\hline \hline total & 0.0067 & 0.0169 & 0.0409 & 0.0425 & 0.0066 & 0.0095 & 0.0084 \\
\hline \hline
\end{tabular}

TAB. 5.11: Incertitudes systématiques pour le mode $K^{+} \pi^{0}$.

\begin{tabular}{|l|cccc|ccc|}
\hline Source & $\theta_{A}$ & $\phi_{A}$ & $\delta_{\|}$ & $\delta_{\perp}$ & $\left|A_{0}\right|^{2}$ & $\left|A_{\|}\right|^{2}$ & $\left|A_{\perp}\right|^{2}$ \\
\hline énergie CM & 0.0014 & 0.0005 & 0.0014 & 0.0027 & 0.0014 & 0.0004 & 0.0010 \\
forme bruit & 0.0050 & 0.0014 & 0.0136 & 0.0143 & 0.0049 & 0.0014 & 0.0035 \\
BR & 0.0010 & 0.0003 & 0.0024 & 0.0008 & 0.0010 & 0.0004 & 0.0007 \\
stat. MC & 0.0040 & 0.0229 & 0.0142 & 0.0043 & 0.0039 & 0.0096 & 0.0115 \\
corr. biais & 0.0043 & 0.0003 & 0.0232 & 0.0084 & 0.0043 & 0.0024 & 0.0018 \\
PID & 0.0058 & 0.0106 & 0.0480 & 0.0293 & 0.0057 & 0.0063 & 0.0069 \\
onde S & 0.0030 & 0.0025 & 0.0335 & 0.0371 & 0.0029 & 0.0025 & 0.0005 \\
\hline \hline total & 0.0103 & 0.0254 & 0.0660 & 0.0504 & 0.0101 & 0.0121 & 0.0140 \\
\hline \hline
\end{tabular}


TAB. 5.12: Incertitudes systématiques pour l'ensemble des trois modes.

\begin{tabular}{|l|cccc|ccc|}
\hline \hline Source & $\theta_{A}$ & $\phi_{A}$ & $\delta_{\|}$ & $\delta_{\perp}$ & $\left|A_{0}\right|^{2}$ & $\left|A_{\|}\right|^{2}$ & $\left|A_{\perp}\right|^{2}$ \\
\hline \hline énergie CM & 0.0008 & 0.0009 & 0.0010 & 0.0014 & 0.0008 & 0.0000 & 0.0008 \\
forme bruit & 0.0013 & 0.0011 & 0.0018 & 0.0033 & 0.0013 & 0.0001 & 0.0011 \\
BR & 0.0002 & 0.0005 & 0.0005 & 0.0009 & 0.0002 & 0.0002 & 0.0003 \\
stat. MC & 0.0020 & 0.0124 & 0.0100 & 0.0029 & 0.0020 & 0.0055 & 0.0064 \\
corr. biais & 0.0003 & 0.0021 & 0.0186 & 0.0112 & 0.0002 & 0.0008 & 0.0008 \\
PID & 0.0018 & 0.0041 & 0.0070 & 0.0068 & 0.0018 & 0.0024 & 0.0027 \\
onde S & 0.0030 & 0.0025 & 0.0335 & 0.0371 & 0.0029 & 0.0025 & 0.0005 \\
\hline \hline total & 0.0043 & 0.0135 & 0.0403 & 0.0396 & 0.0042 & 0.0066 & 0.0071 \\
\hline \hline
\end{tabular}

\subsection{Analyse du spectre $K \pi$}

La désintégration $B \rightarrow J / \psi K \pi$ est dominée par la contribution du $K^{*}(892)$, mais reçoit d'autres contributions $K \pi$. La plage cinématique disponible pour le système $K \pi$ s'étend en effet de ( $m_{K}+$ $m_{\pi}$ ) jusqu'à la limite cinématique fixée par la différence $m_{B}-m_{J / \psi}$, autorisant la production d'états plus lourds que le $K^{*}(892)$. Les différents spectres $K \pi$ sont représentés sur la figure 5.17 , et sont obtenus après soustraction des bruits de fond. Un ajustement prenant en compte les facteurs de barrière centrifuge est effectué sur le spectre ayant la plus grande statistique, à savoir $K^{+} \pi^{-}$avec les paramètres des résonances $K^{*}(892)$ et $K_{2}^{*}$ (1430) fixés aux valeurs du PDG [28], étant connus plus précisément (cf. Fig. 5.18). Les spectres en $m_{\mathrm{ES}}$ (après coupure sur $\Delta E$ ) pour différentes régions du spectre $K \pi$ sont représentés sur les figures 5.19 et 5.20 .

La présence de l'onde $S$ peut être mise en évidence de manière simple en étudiant l'asymétrie avant-arrière en $\cos \theta_{K^{*}}$. En effet, en considérant la distribution angulaire $S+P$ (cf. eq. (2.121)) on a :

$$
\begin{aligned}
N_{F} & \equiv \int_{0}^{1}\left\{\iint g_{S+P}\left(\vec{\omega}, m_{K \pi}\right) \mathrm{d} \cos \theta_{t r} \mathrm{~d} \cos \varphi_{t r}\right\} \mathrm{d} \cos \theta_{K^{*}} \\
& =\frac{1}{2} \times\left[\left|B^{1}\right|^{2}\left[\left|A_{0}^{1}\right|^{2}+\left|A_{\|}^{1}\right|^{2}+\left|A_{\perp}^{1}\right|^{2}\right]+\left.\left|B^{0}\right|^{2}|| A_{0}^{0}\right|^{2}+\sqrt{3} \Re e\left(B^{1} B^{0 *} A_{0}^{1} A_{0}^{0 *}\right)\right], \\
N_{B} & \equiv \int_{-1}^{0}\left\{\iint g_{S+P}\left(\vec{\omega}, m_{K \pi}\right) \mathrm{d} \cos \theta_{t r} \mathrm{~d} \cos \varphi_{t r}\right\} \mathrm{d} \cos \theta_{K^{*}} \\
& =\frac{1}{2} \times\left[\left|B^{1}\right|^{2}\left[\left|A_{0}^{1}\right|^{2}+\left|A_{\|}^{1}\right|^{2}+\left|A_{\perp}^{1}\right|^{2}\right]+\left.\left|B^{0}\right|^{2}|| A_{0}^{0}\right|^{2}-\sqrt{3} \Re e\left(B^{1} B^{0 *} A_{0}^{1} A_{0}^{0 *}\right)\right],
\end{aligned}
$$

d'où l'asymétrie avant-arrière, $A_{F B}$ :

$$
A_{F B} \equiv \frac{N_{F}-N_{B}}{N_{F}+N_{B}}=\frac{\sqrt{3} \Re e\left(B^{1} B^{0 *} A_{0}^{1} A_{0}^{0 *}\right)}{\left|B^{1}\right|^{2}\left[\left|A_{0}^{1}\right|^{2}+\left|A_{\|}^{1}\right|^{2}+\left|A_{\perp}^{1}\right|^{2}\right]+\left.\left|B^{0}\right|^{2}|| A_{0}^{0}\right|^{2}} .
$$

Rappelons que $B^{1}$ et $B^{0}$ contiennent la dépendance en masse $K \pi$. L'asymétrie avant-arrière ne conserve donc que l'interférence entre l'onde $P$ et l'onde $S$. S'il y a une onde $S$, l'asymétrie avantarrière doit montrer l'évolution de cette interférence avec la masse.

Pour calculer $A_{F B}$, il faut au préalable corriger d'acceptance puisque la région avant est affectée 

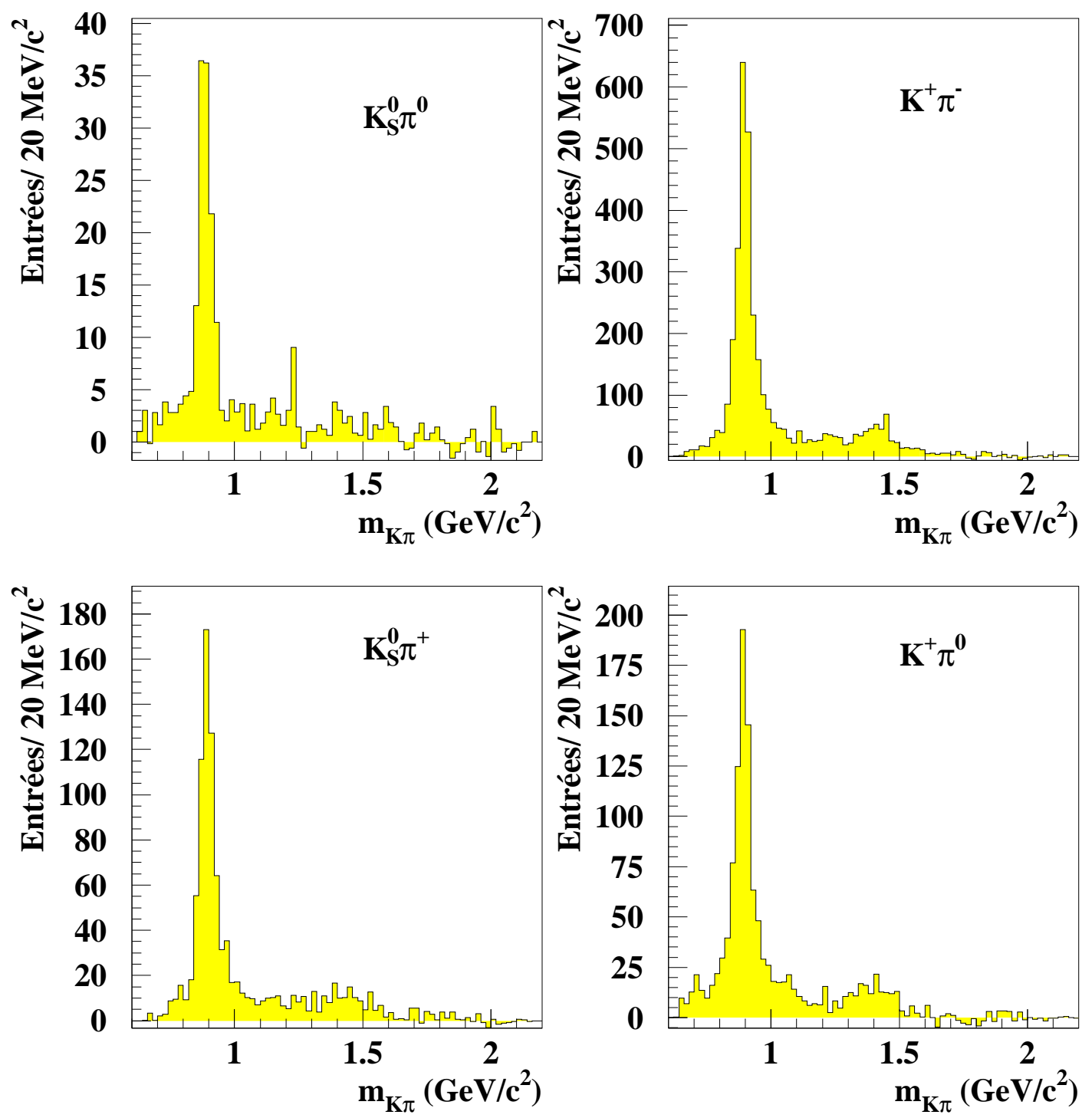

FIG. 5.17: Distributions en masse $K \pi$ pour les événements provenant de la désintégration $B \rightarrow J / \psi K \pi$ dans la région signal après soustraction du bruit de fond obtenue à partir d'un ajustement par une gaussienne et une distribution Argus sur la distribution en $m_{\mathrm{ES}}$ après coupure sur $\Delta E$. 


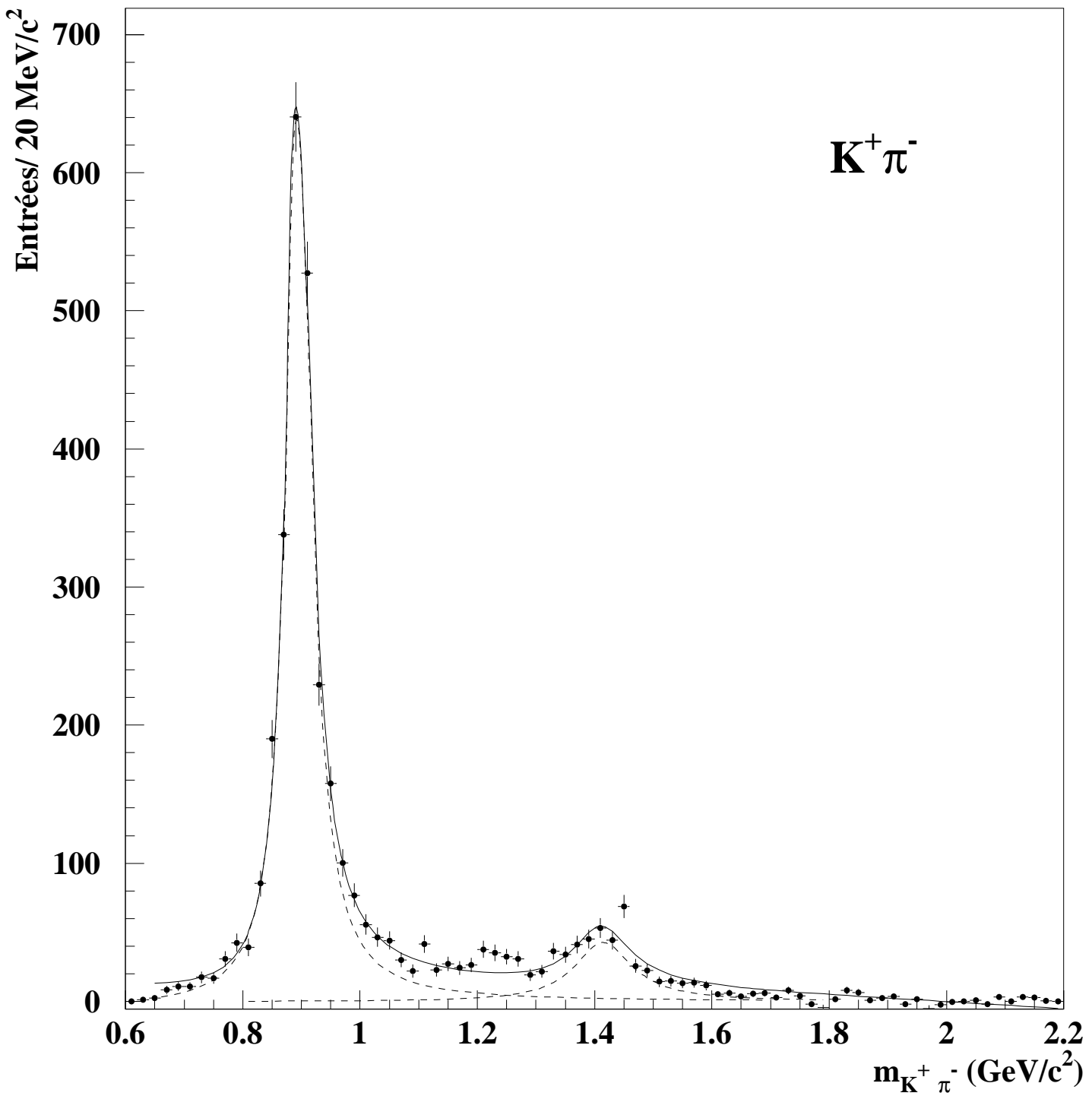

FIG. 5.18: Distribution en masse $K \pi$ pour les événements provenant de la désintégration $B^{0} \rightarrow J / \psi K^{+} \pi^{-}$dans la région signal après soustraction du bruit de fond obtenue à partir d'un ajustement par une gaussienne et une distribution Argus sur la distribution en $m_{\mathrm{ES}}$ après coupure sur $\Delta E$. Un ajustement par deux fonctions Breit-Wigner relativistes avec barrière centrifuge est superposé. 

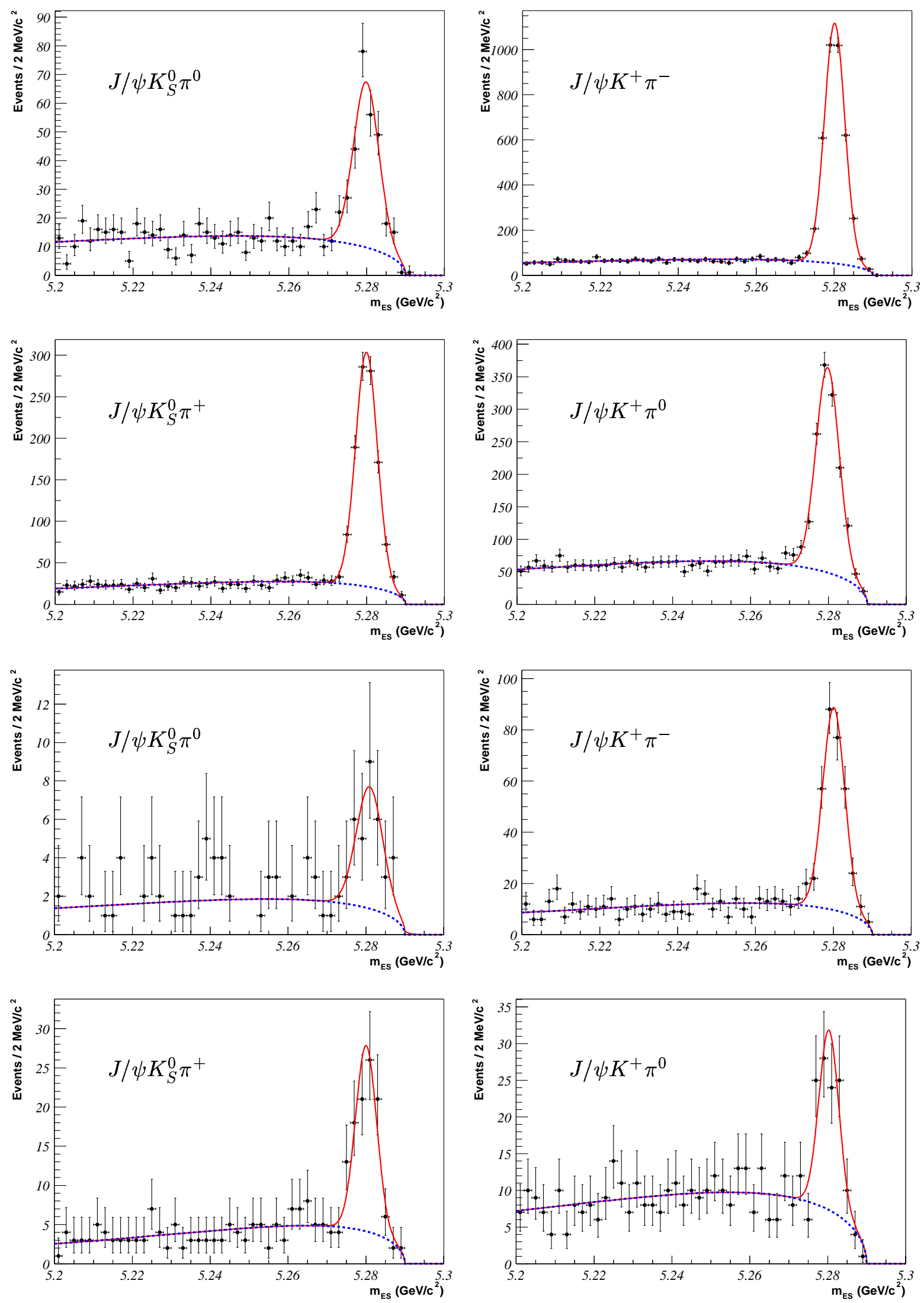

FIG. 5.19: Distributions en $m_{\mathrm{ES}}$ après coupure sur $\Delta E$ pour les événements ayant une masse $K \pi$ comprise à $\pm 100 \mathrm{MeV} / c^{2}$ par rapport à la masse nominale de la masse de la résonance $K^{*}(892)$ (quatre figures du haut) et une masse $K \pi$ comprise entre 1.1 et $1.3 \mathrm{GeV} / c^{2}$ (quatre figures du bas) pour les quatre désintégrations $K \pi$. 

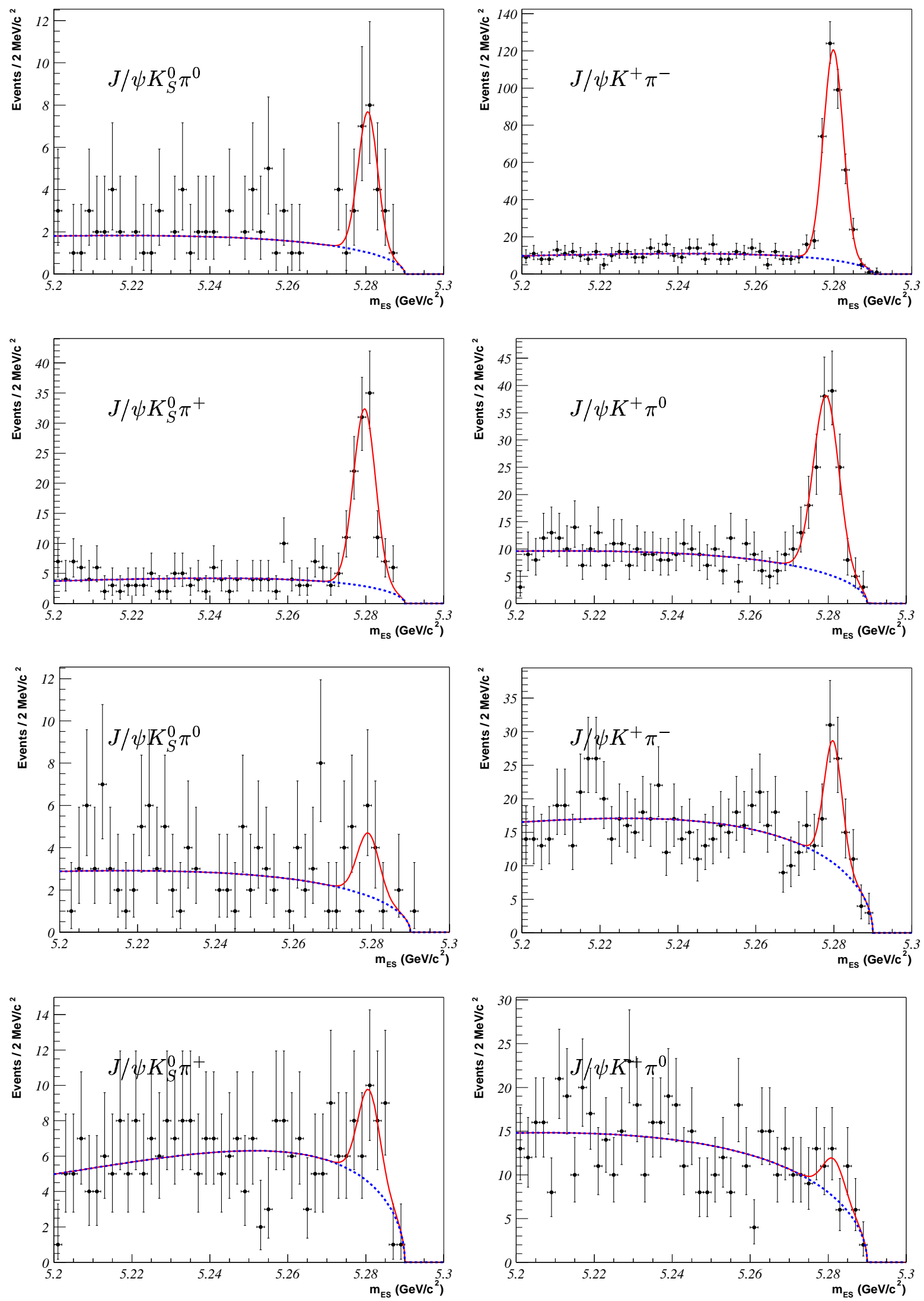

FIG. 5.20: Distributions en $m_{\mathrm{ES}}$ après coupure sur $\Delta E$ pour les événements ayant une masse $K \pi$ comprise entre 1.35 et $1.55 \mathrm{GeV} / c^{2}$ (quatre figures du haut) et une masse $K \pi$ supérieure à $1.7 \mathrm{GeV} / c^{2}$ (quatre figures du bas) pour les quatre désintégrations $K \pi$. 


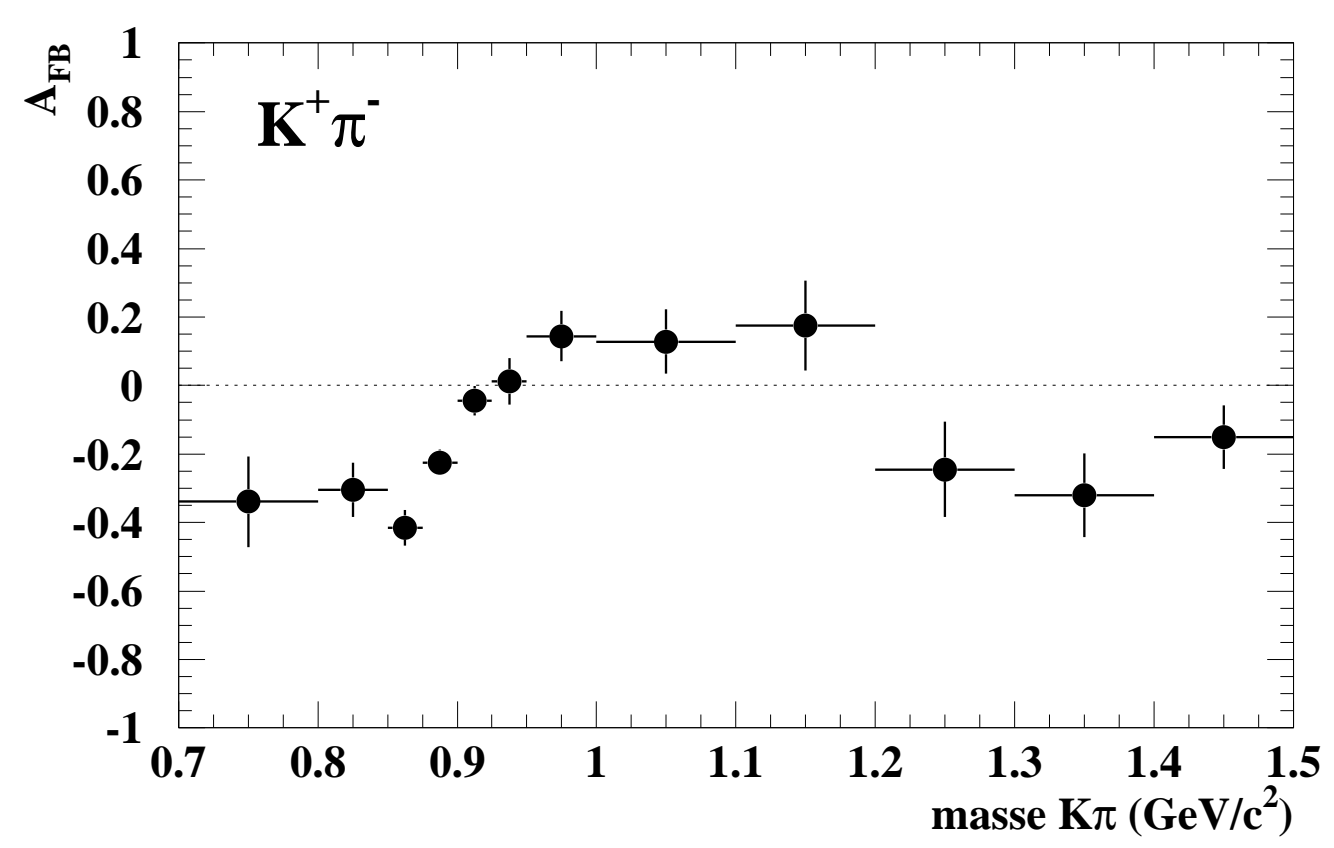

FIG. 5.21: Asymétrie avant-arrière en $\cos \theta_{K^{*}}$ en fonction de la masse $K \pi$ pour le canal $K^{+} \pi^{-}$obtenue sur les données.

par la perte d'événements, à cause du régime mou des pions dans ce domaine. Ce problème d'acceptance est plus simple que celui de la mesure des amplitudes puisqu'il s'agit d'une correction 1D. Nous repondérons donc les événements, en utilisant l'acceptance $1 \mathrm{D}$ en $\cos \theta_{K^{*}}{ }^{4}$. Pour chaque tranche de masse nous effectuons une soustraction des bruits de fond, ayant préalablement réalisé un ajustement sur le spectre en $m_{\mathrm{ES}}$ pour déterminer la fraction de signal. Dans ce dernier ajustement, seule la fraction de signal est laissée libre, les autres paramètres étant fixés à des valeurs raisonnables.

L'asymétrie avant-arrière ainsi obtenue est montrée sur la figure 5.21, pour le canal $K^{+} \pi^{-}$. On voit clairement l'évolution de cette asymétrie avec la masse. Cette évolution étant dirigée par la variation de la différence de phase entre l'onde $P$ et l'onde $S$. On voit nettement la variation rapide de la phase de l'onde $P$ au fur et à mesure que l'on traverse la région de $K^{*}(892)$. La phase de l'onde $S$ ne commence à varier qu'à partir de $1.1 \mathrm{GeV} / c^{2}$. Il s'agit là d'un comportement présentant de grandes similitudes avec les expériences de diffusion élastique [146, 163]. C'est la première mise en évidence de l'onde $S$ dans le système $K \pi$ provenant de la désintégration de $B$. La figure 5.22 montre cette même évolution pour les canaux ${ }^{5} K_{S}^{0} \pi^{+}$et $K^{+} \pi^{0}$. La même tendance se dégage, mais la signifiance statistique est bien moindre qu'avec le canal $K^{+} \pi^{-}$. La figure 5.23 montre l'asymétrie avant-arrière, pour un échantillon de simulation complète, ne comportant donc pas d'onde $S$. Nous nous assurons ainsi que notre procédure ne «fabrique » pas d'asymétrie.

En toute rigueur, il faudrait considérer un ajustement prenant en compte la présence de l'onde

\footnotetext{
${ }^{4}$ Nous tenons également en compte de la différence de polarisation entre les données et le Monte Carlo, en incluant aussi la pondération $g(\vec{\omega} ; \vec{A}) / g\left(\vec{\omega} ; \vec{A}_{M C}\right)$ dans ce calcul.

${ }^{5}$ Pour le canal $K^{+} \pi^{0}$ nous sommes obligés de couper aussi les événements à $\cos \theta_{K^{*}}<0.7$ afin de symétriser la distribution observée, avant de corriger d'acceptance. Il n'est en effet pas possible de corriger d'acceptance les régions coupées.
} 

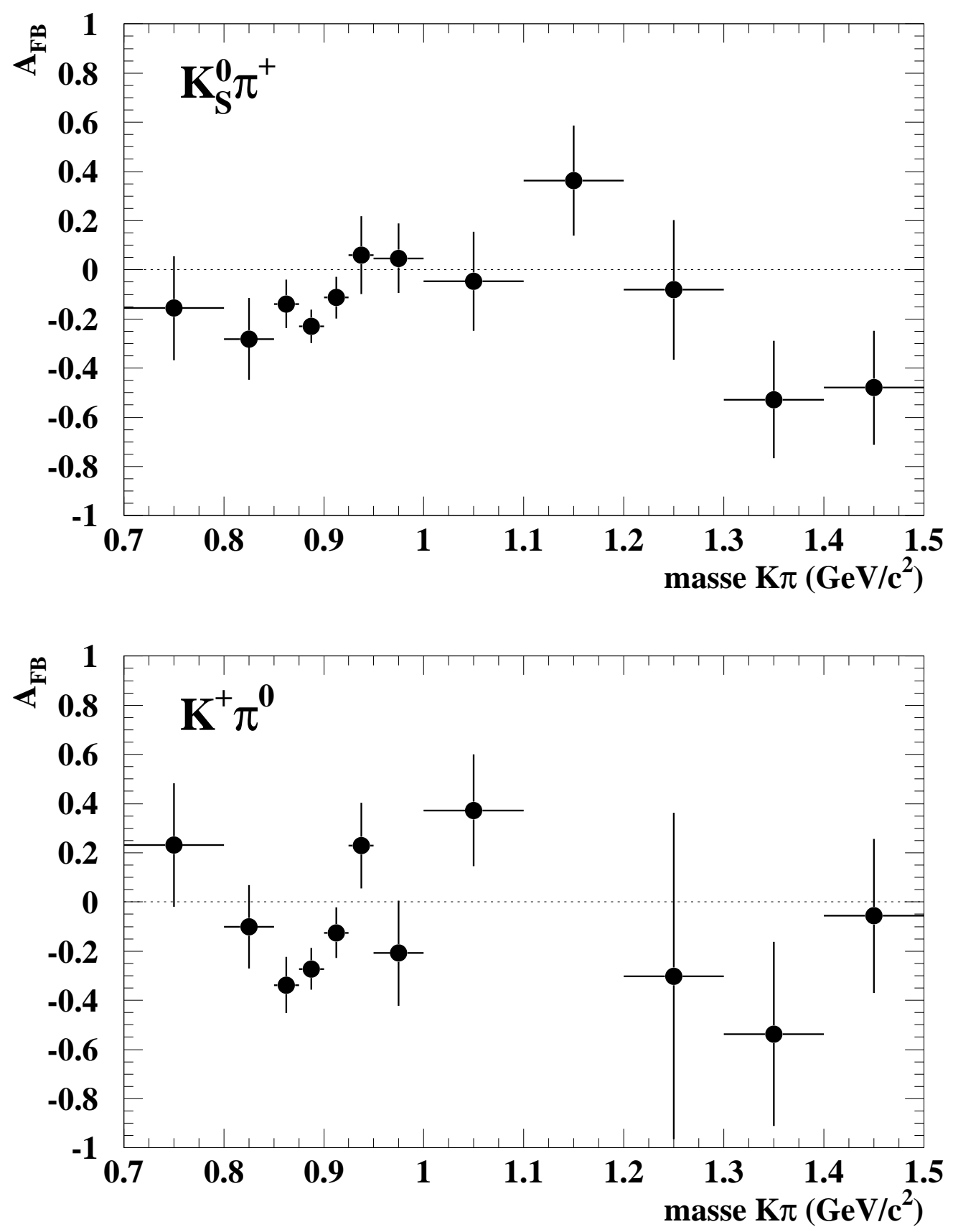

FIG. 5.22: Asymétrie avant-arrière en $\cos \theta_{K^{*}}$ en fonction de la masse $K \pi$ pour les canaux $K_{S}^{0} \pi^{+}$et $K^{+} \pi^{0}$ obtenue sur les données. 


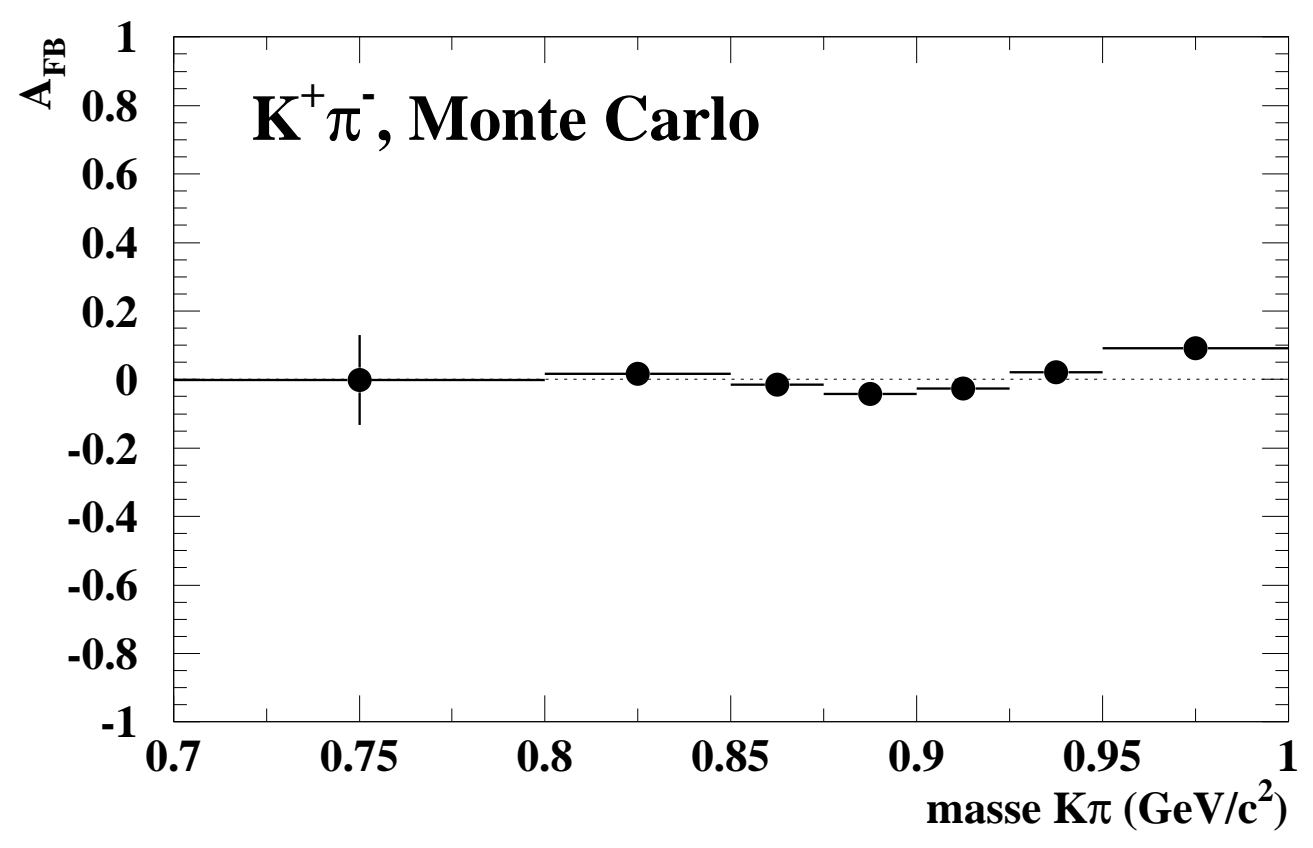

FIG. 5.23: Asymétrie avant-arrière en $\cos \theta_{K^{*}}$ en fonction de la masse $K \pi$ pour le canal $K^{+} \pi^{-}$, pour un échantillon de simulation complète, sans onde $S$.

$S$. Mais un tel ajustement implique une sophistication accrue avec par exemple l'introduction de dépendance en masse $K \pi$ dans l'ajustement. De plus, d'un point de vue pratique, nous ne disposons pas d'échantillon Monte Carlo de simulation détaillée avec interférence d'onde $S$ et $P$ pour effectuer une validation réaliste. Nous traitons donc la présence de l'onde $S$ comme une source d'incertitude systématique dont l'effet va être mesuré sur l'échantillon $K^{+} \pi^{-}$. Pour cela nous considérons un ajustement avec la distribution angulaire $S+P$. La correction d'acceptance procède de la même façon qu'auparavant, en étendant le nombre de coefficients $\Phi_{l}^{i \rightarrow j}$ de $l=1, \ldots, 6$ à $l=1, \ldots, 10$. Ne disposant pas de simulation complète pour ce type d'événements, nous effectuons une validation « toy Monte Carlo ». Pour cela nous effectuons une première génération d'événements, selon une distribution $g$ pour une onde $P$ uniquement et en introduisant une acceptance imparfaite. Ceci nous permet de calculer les $\Phi_{l}^{i \rightarrow j}, l=1, \ldots, 10$. Puis nous générons un échantillon d'événements selon une distribution angulaire $S+P$, faisant passer ces événements à travers la même acceptance. L'ajustement qui s'en suit ne pose pas de problème, et les valeurs générées sont bien retrouvées.

Nous assignons comme incertitude systématique la différence observée sur les amplitudes et les phases entre les ajustements $S+P$ et $P$ seul. Nous assignons la même erreur systématique à tous les modes, étant donné que seul le canal $K^{+} \pi^{-}$nous permet de mesurer cet effet.

\subsection{Conclusion}

Les résultats obtenus par la méthode de pseudo-maximum de vraisemblance sur $81.9 \mathrm{fb}^{-1}$ de données collectées par le détecteur BABAR au pic de la résonance $\Upsilon(4 S)$ sont

$$
\left|A_{0}\right|^{2}=0.565 \pm 0.011 \pm 0.004,\left|A_{\|}\right|^{2}=0.206 \pm 0.016 \pm 0.007,\left|A_{\perp}\right|^{2}=0.228 \pm 0.016 \pm 0.007
$$




$$
\delta_{\|}=-2.766 \pm 0.105 \pm 0.040, \delta_{\perp}=2.935 \pm 0.067 \pm 0.040 .
$$

À noter que $\left(\delta_{\|}, \delta_{\perp}\right) \rightarrow\left(-\delta_{\|}, \pi-\delta_{\perp}\right)$ est aussi une solution. Les phases fortes $\delta_{\|}$et $\delta_{\perp}$ sont à $3 \sigma$ de $\pm \pi$, signant ainsi la présence d'interactions dans l'état final et la rupture de l'approximation de factorisation pour les canaux $B \rightarrow J / \psi K^{*}(892)$. Aucune forme de la factorisation (naïve, généralisée et QCD améliorée) n'arrive à expliquer les modules des amplitudes (ainsi que les rapports d'embranchement). La table ci-dessous donne les prédictions de la factorisation QCD améliorée calculées avec différents modèles de facteurs de forme [80] (cf. §2.1) :

\begin{tabular}{lccc}
\hline Modèle & $\left|A_{0}\right|^{2}$ & $\left|A_{\|}\right|^{2}$ & $\left|A_{\perp}\right|^{2}$ \\
\hline \hline BSW & 0.43 & 0.24 & 0.33 \\
BSWII & 0.38 & 0.26 & 0.36 \\
LF & 0.41 & 0.25 & 0.34 \\
NS & 0.40 & 0.25 & 0.34 \\
Yang & 0.38 & 0.25 & 0.36 \\
BB & 0.41 & 0.25 & 0.34 \\
MS & 0.40 & 0.25 & 0.35 \\
YYK & 0.44 & 0.23 & 0.32 \\
\hline \hline
\end{tabular}

On remarque que l'amplitude de polarisation longitudinale, $\left|A_{0}\right|^{2}$, est sous-estimée alors que l'amplitude $\left|A_{\perp}\right|^{2}$ est surestimée. La statistique accumulée a permis de signer la présence d'une onde $S$ cohérente dans le spectre $K \pi$ venant interférer avec le $K^{*}(892)$. C'est la première démonstration de la présence de cette onde dans le système $K \pi$ provenant de la désintégration d'un méson $B$. L'analyse future des amplitudes devra donc prendre en compte cette onde. 


\section{Chapitre 6}

\section{Analyse angulaire complète dépendante du temps du canal $B_{d}^{0} \rightarrow J / \psi\left(K_{S}^{0} \pi^{0}\right)^{* 0}$}

Le principe de la mesure de $\sin 2 \beta$ est donné en détail dans les références [164, 165] avec une mise à jour dans la référence [166]. Pour cette mesure, différents échantillons de mésons $B$ entièrement reconstruits $\left(B_{r e c}\right)$ sont utilisés, soit dans un état spécifique de saveur $\left(B_{\text {rec }}=B_{\text {sav }}\right)$, soit dans un mode $C P\left(B_{r e c}=B_{C P}\right)$. Une fois le méson $B_{\text {rec }}$ reconstruit, il est nécessaire de déterminer sa saveur grâce à l'autre méson $B$, dénommé $B_{t a g}$, provenant de la désintégration $\Upsilon(4 S) \rightarrow B_{d}^{0} \bar{B}_{d}^{0}$ (voir le chapitre 3), ainsi que la différence des temps propres, $\Delta t \equiv t_{\text {rec }}-t_{t a g}$, entre les deux mésons $B$. La particularité du canal $B_{d}^{0} \rightarrow J / \psi\left(K_{S}^{0} \pi^{0}\right)^{* 0}$ est d'avoir une distribution qui dépend de la différence des temps propres, $\Delta t$, des deux mésons $B$, mais aussi des angles de transversité $\vec{\omega}$, permettant de mesurer $\sin 2 \beta$ et $\cos 2 \beta$.

L'échantillon $B_{s a v}$, qui ne viole pas $C P$, est utilisé pour estimer des paramètres de l'ajustement à partir des données. Il est composé des désintégrations spécifiques de saveur suivantes : $B_{d}^{0} \rightarrow$ $D^{(*)-} \pi^{+} / \rho^{+} / a_{1}^{+}$et $B_{d}^{0} \rightarrow J / \psi K^{* 0}\left(K^{* 0} \rightarrow K^{+} \pi^{-}\right)$, où les produits de désintégration permettent de signer la saveur au moment de la désintégration, $t_{s a v}$. L'autre méson $B, B_{t a g}$, sert, quant à lui, à l'étiquetage par ses propres produits de désintégration permettant de connaître la saveur de $B_{\text {sav }}$ à l'instant $t_{t a g}$. La probabilité pour le mélange $B_{d}^{0}-\bar{B}_{d}^{0}$ est une fonction de $\Delta M$ et de la différence des temps propres des deux mésons $B$ (cf. §1.1.3) :

$$
\operatorname{Prob}\left(B_{d}^{0}\left(t_{t a g}\right) \bar{B}_{d}^{0}\left(t_{t a g}\right) \rightarrow\left\{\begin{array}{l}
B_{d}^{0}\left(t_{\text {tag }}\right) \bar{B}_{d}^{0}\left(t_{\text {sav }}\right) \\
B_{d}^{0}\left(t_{\text {sav }}\right) \bar{B}_{d}^{0}\left(t_{\text {tag }}\right)
\end{array}\right)=\frac{\Gamma_{0}}{2} e^{-\Gamma_{0}|\Delta t|}(1+\cos (\Delta M \Delta t)),\right.
$$

dans le cas où les saveurs initiales et finales sont identiques (Unmixed) et

$$
\operatorname{Prob}\left(B_{d}^{0}\left(t_{t a g}\right) \bar{B}_{d}^{0}\left(t_{t a g}\right) \rightarrow\left\{\begin{array}{c}
B_{d}^{0}\left(t_{\text {tag }}\right) B_{d}^{0}\left(t_{\text {sav }}\right) \\
\bar{B}_{d}^{0}\left(t_{\text {sav }}\right) \bar{B}_{d}^{0}\left(t_{\text {tag }}\right)
\end{array}\right)=\frac{\Gamma_{0}}{2} e^{-\Gamma_{0}|\Delta t|}(1-\cos (\Delta M \Delta t)),\right.
$$

pour des saveurs initiales et finales opposées (Mixed). Ces probabilités ne sont valables que pour un étiquetage parfait. Dans le cas où l'algorithme d'étiquetage identifie incorrectement la saveur, avec une probabilité $w$, l'amplitude des oscillations est réduite par un facteur de dilution $D=(1-2 w)$. Si plusieurs catégories d'étiquetage existent, chacune apporte sa propre probabilité de mauvais 


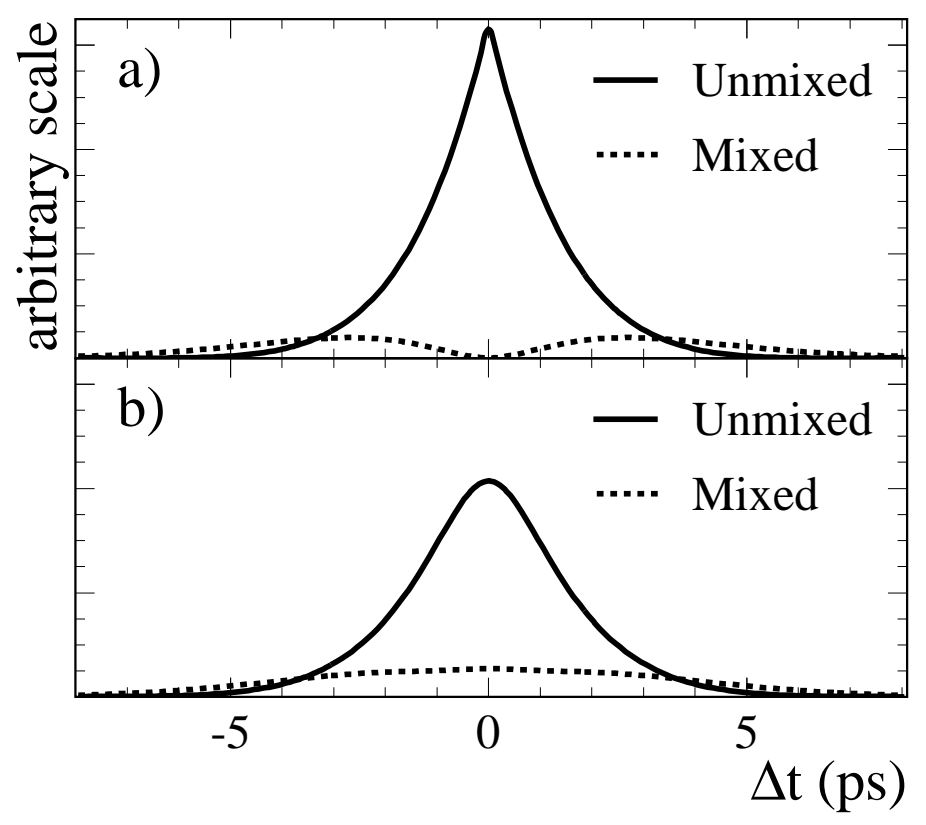

FIG. 6.1: Distribution temporelle des événements spécifiques de saveur ayant la même saveur (Mixed) ou la saveur opposée (Unmixed) du méson $B_{\text {tag }}$ dans le cas, a) d'une résolution temporelle et d'un étiquetage parfaits, b) d'une résolution finie et d'une probabilité de mauvais étiquetage.

étiquetage $w_{c}$. On obtient alors comme densité de probabilité normalisée :

$$
h_{\substack{U \text { nixixed } \\ \text { Mixed }}}\left(\Delta t ; \Gamma_{0}, \Delta M, w\right)=\frac{\Gamma_{0}}{2} e^{-\Gamma_{0}|\Delta t|}(1 \pm D \cos (\Delta M \Delta t))
$$

Il convient aussi de prendre en compte la fonction de résolution en $\Delta t, \mathcal{R}\left(\delta(\Delta t) \equiv \Delta t-\Delta t_{\text {vrai }} ; \hat{a}\right)$, conduisant à

$$
\mathcal{H}_{\text {Mixed }}^{\text {nimixed }}\left(\Delta t ; \Gamma_{0}, \Delta M, w, \hat{a}\right)=h_{\text {Mixed }}^{\text {Mnixed }}\left(\Delta t_{\text {vrai }} ; \Gamma_{0}, \Delta M, w\right) \otimes \mathcal{R}(\delta(\Delta t) ; \hat{a})
$$

où $\delta(\Delta t)$ est la différence entre le $\Delta t$ mesuré $(\Delta t)$ et le $\Delta t$ généré $\left(\Delta t_{v r a i}\right)$ et $\hat{a}$ sont les paramètres de la fonction de résolution. L'impact de la résolution finie en $\Delta t$, ainsi que de la probabilité de mauvais étiquetage sur les distributions dépendantes du temps, est illustré sur la figure 6.1.

Cet échantillon permet de déterminer avec un maximum de vraisemblance non-binné les différentes probabilités de mauvais étiquetage, les paramètres de la fonction de résolution en $\Delta t$ ainsi que $\Delta M[167]$ :

$$
\ln \mathcal{L}_{\text {sav }}=\sum_{c}\left[\sum_{i=1}^{N_{U n \text { mixed }}} \ln \mathcal{H}_{\text {Unmixed }}\left(\Delta t ; \Gamma_{0}, \Delta M, w_{c}, \hat{a}_{c}\right)+\sum_{i=1}^{N_{\text {Mixed }}} \ln \mathcal{H}_{\text {Mixed }}\left(\Delta t ; \Gamma_{0}, \Delta M, w_{c}, \hat{a}_{c}\right)\right] .
$$

Pour la mesure de $\sin 2 \beta$, on se base sur un échantillon de modes propres de $C P, B_{C P}$, composé des désintégrations $B_{d}^{0} \rightarrow J / \psi K_{S}^{0}, \psi(2 S) K_{S}^{0}, \chi_{c 1} K_{S}^{0}, \eta_{c} K_{S}^{0}, J / \psi K_{L}^{0}$. La désintégration $B_{d}^{0} \rightarrow$ $J / \psi\left(K_{S}^{0} \pi^{0}\right)^{* 0}$ a la particularité de ne pas être un état propre de $C P$ mais un mélange d'états propres 


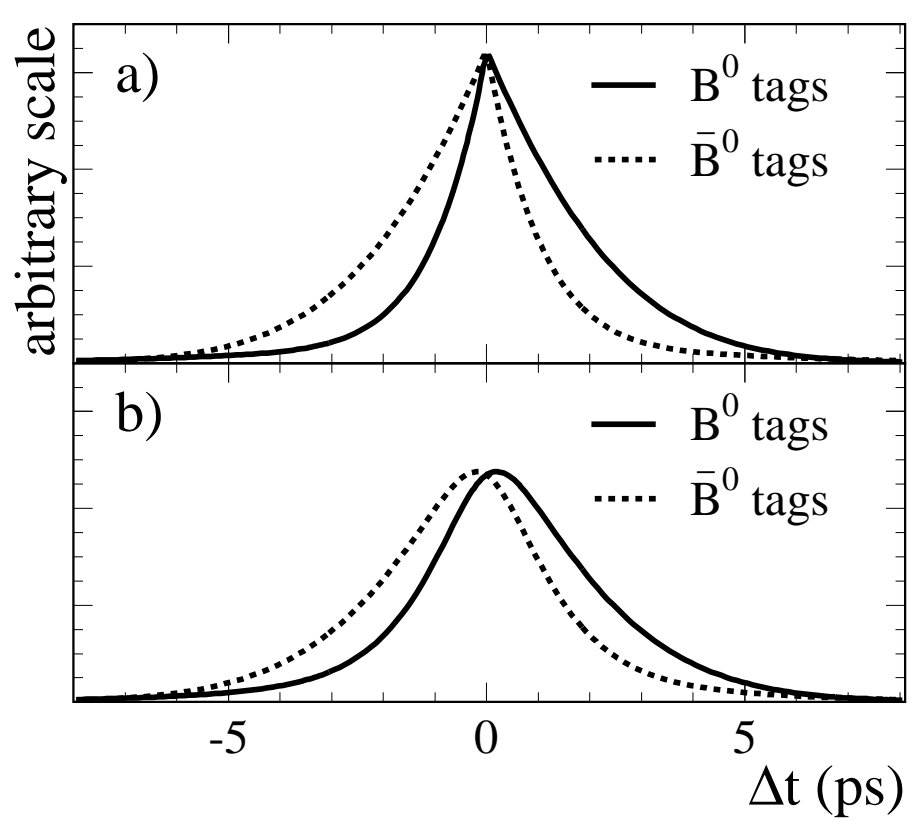

FIG. 6.2: Distribution temporelle des événements propres de $C P$ où le méson $B_{\text {tag }}$ est de saveur $B^{0}$ ou $\bar{B}^{0}$ dans le cas, a) d'une résolution temporelle et d'un étiquetage parfaits, b) d'une résolution finie et d'une probabilité de mauvais étiquetage.

de $C P$. Les distributions temporelles sont données par (cf. eq. (1.59), en prenant $\left|\lambda_{f_{C P}}\right|=1$ ):

$$
f_{ \pm}\left(\Delta t ; \Gamma_{0}, \Delta M, \sin 2 \beta, w\right)=\frac{\Gamma_{0}}{2} e^{-\Gamma_{0}|\Delta t|}\left(1 \pm D \eta_{C P}(f) \sin 2 \beta \sin (\Delta M \Delta t)\right)
$$

où les signes + et - en indice de $f$ indique la saveur du méson $B_{t a g}, B_{d}^{0}$ ou $\bar{B}_{d}^{0}$, respectivement. Pour tenir compte de la résolution finie du détecteur, il convient de convoluer les distributions $f_{ \pm}$ avec la fonction de résolution en $\Delta t$ :

$$
\mathcal{F}_{ \pm}\left(\Delta t ; \Gamma_{0}, \Delta M, \sin 2 \beta, w, \hat{a}\right)=f_{ \pm}\left(\Delta t_{\text {vrai }} ; \Gamma_{0}, \Delta M, \sin 2 \beta, w\right) \otimes \mathcal{R}(\delta(\Delta t) ; \hat{a})
$$

En pratique, les événements sont séparés en quatre catégories d'étiquetage, chacune ayant sa propre probabilité de mauvais étiquetage. L'impact de la résolution finie en $\Delta t$ ainsi que de la probabilité de mauvais étiquetage sur les distributions $f_{ \pm}$est illustré sur la figure 6.2 (cf. Fig. 1.7).

La valeur de $\sin 2 \beta$ est obtenue avec un maximum de vraisemblance :

$$
\ln \mathcal{L}_{C P}=\sum_{c}\left[\sum_{B_{t a g}=B_{d}^{0}} \ln \mathcal{F}_{+}\left(\Delta t ; \Gamma_{0}, \Delta M, \sin 2 \beta, w_{c}, \hat{a}_{c}\right)+\sum_{B_{t a g}=\bar{B}_{d}^{0}} \ln \mathcal{F}_{-}\left(\Delta t ; \Gamma_{0}, \Delta M, \sin 2 \beta, w_{c}, \hat{a}_{c}\right)\right] .
$$

On remarque sur l'expression de la distribution (6.6) que l'échantillon $B_{C P}$ ne permet d'obtenir que $D \sin 2 \beta$ et non $\sin 2 \beta$.

Les sections suivantes sont organisées comme suit : on commence par décrire les critères de sélection de l'échantillon $B_{\text {sav }}$ (ceux de l'échantillon $B_{C P}$ que l'on restreint dans la suite uniquement au canal $B_{d}^{0} \rightarrow J / \psi\left(K_{S}^{0} \pi^{0}\right)^{* 0}$ sont donnés dans le chapitre 4$)$. Vient ensuite la description de l'algorithme d'étiquetage ainsi que la mesure de la séparation spatiale entre les vertex des deux mésons 
$B, \Delta z$, et de la conversion en $\Delta t$. Ensuite, la méthode d'ajustement pour extraire $\sin 2 \beta$ et $\cos 2 \beta$ est présentée, suivie de validations et de la détermination des incertitudes systématiques. Enfin, le chapitre se termine par une discussion sur les résultats obtenus. À noter qu'à chaque fois que cela est possible, les données sont utilisées plutôt qu'une simulation Monte Carlo. Ainsi, par exemple, les paramètres de la fonction de résolution en $\Delta t$ ainsi que les probabilités de mauvais étiquetage sont déterminés à partir des données. La technique basée sur des distributions temporelles par conversion de $\Delta z$ en $\Delta t$, ainsi que la convolution avec la fonction de résolution en $\Delta t$, a été utilisée dans les mesures de précision des temps de vie des mésons $B$ neutres et chargés [168]. Cette technique, avec la prise en compte des dilutions dues à l'étiquetage, a été mise en œuvre dans la mesure de précision de $\Delta M$ [167].

\section{1 Échantillons $B_{\text {sav }}, B_{C P}$ et $B^{+}$}

L'échantillon $B_{s a v}$ se compose des désintégrations $B_{d}^{0} \rightarrow D^{(*)-} \pi^{+}, B_{d}^{0} \rightarrow D^{(*)-} \rho^{+}, B_{d}^{0} \rightarrow$ $D^{(*)-} a_{1}^{+}$et $B_{d}^{0} \rightarrow J / \psi K^{* 0}\left(K^{* 0} \rightarrow K^{+} \pi^{-}\right)$. Les candidats $\rho^{+}$sont reconstruits dans leurs désintégrations $\pi^{+} \pi^{0}$, où la masse invariante de la combinaison $\pi^{+} \pi^{0}$ doit se trouver à $\pm 150 \mathrm{MeV} / c^{2}$ de la masse nominale du $\rho^{+}$[28]. Le candidat $\pi^{0}$, provenant de la désintégration du $\rho^{+}$, doit avoir une masse invariante comprise entre $\pm 20 \mathrm{MeV} / c^{2}$ par rapport à la masse nominale du $\pi^{0}$ et une énergie supérieure à $300 \mathrm{MeV}$. Les candidats $a_{1}$ sont reconstruits dans leurs désintégrations en trois pions chargés avec une masse invariante comprise entre 1.0 et $1.6 \mathrm{GeV} / c^{2}$. Les candidats $\bar{D}^{0}$ sont reconstruits dans leurs désintégrations $K^{+} \pi^{-}, K^{+} \pi^{-} \pi^{0}, K^{+} \pi^{-} \pi^{-} \pi^{+}$et $K_{S}^{0} \pi^{+} \pi^{-}$et les candidats $D^{-}$en $K^{+} \pi^{-} \pi^{-}$et $K_{S}^{0} \pi^{-}$. Les kaons et pions, chargés et neutres, doivent avoir une impulsion supérieure à $200 \mathrm{MeV} / c$, à l'exception des pions provenant de la désintégration $B_{d}^{0} \rightarrow D^{(*)-} a_{1}^{+}$pour lesquels la limite est abaissée à $150 \mathrm{MeV} / c$. Les candidats $\bar{D}^{0}$ et $D^{-}$sont sélectionnés par une coupure à $\pm 3 \sigma$ par rapport à leur masse nominale respective. Les candidats $D^{*-}$ sont reconstruits en combinant un candidat $\bar{D}^{0}$ et un pion chargé $\pi^{-}$d'impulsion supérieure à $70 \mathrm{MeV} / c$, puis sélectionnés en demandant que la masse invariante du candidat $\bar{D}^{0} \pi^{-}$se situe à $\pm 0.8 \mathrm{MeV} / c^{2}$ de la masse nominale du méson $D^{*-}$ [28] (élargie à $\pm 1.1 \mathrm{MeV} / c^{2}$ pour la désintégration $\bar{D}^{0} \rightarrow K^{+} \pi^{-} \pi^{0}$ ). La sélection de la désintégration $B_{d}^{0} \rightarrow J / \psi K^{* 0}\left(K^{+} \pi^{-}\right)$est donnée au chapitre 4, en retirant les coupures sur le volume fiduciel. Les distributions en $m_{\mathrm{ES}}$ (après coupure sur $\Delta E$ ) sont représentées sur la figure 6.3.

L'échantillon $B_{C P}$ se compose uniquement de la désintégration $B_{d}^{0} \rightarrow J / \psi\left(K_{S}^{0} \pi^{0}\right)^{* 0}$ dont la sélection est donnée dans le chapitre 4 . La distribution en $m_{\mathrm{ES}}$ après coupure sur $\Delta E$ est représentée sur la figure 4.12 .

L'échantillon $B^{+}$est composé des désintégrations $\bar{D}^{(*) 0} \pi^{+}, J / \psi K^{(*)+}, \psi(2 S) K^{+}$et $\chi_{c 1} K^{+}$. Les différentes désintégrations considérées du méson $\bar{D}^{0}$ sont identiques à celles prises en compte dans l'échantillon $B_{s a v}$. Les candidats $\bar{D}^{* 0}$ sont reconstruits en combinant un $\bar{D}^{0}$ avec un $\pi^{0}$, puis sélectionnés en demandant que la masse invariante $m\left(\bar{D}^{0} \pi^{0}\right)$ soit à $\pm 4 \mathrm{MeV} / c^{2}$ de la masse nominale du méson $\bar{D}^{* 0}$, après avoir contraint le méson $\bar{D}^{0}$ à sa masse nominale [28]. Les candidats $\psi(2 S)$ sont reconstruits dans leurs désintégrations $\ell^{+} \ell^{-}(\ell=e$ ou $\mu)$ et $J / \psi \pi^{+} \pi^{-}$. Les critères de sélection retenus sont $\mathrm{Tight}$ et VeryLoose pour les électrons et Loose et VeryLoose pour les muons (cf. §3.3.3). La masse invariante du candidat $\psi(2 S)$ doit se situer dans l'intervalle $3.436<M_{\psi(2 S)}<3.736 \mathrm{GeV} / c^{2}$ 

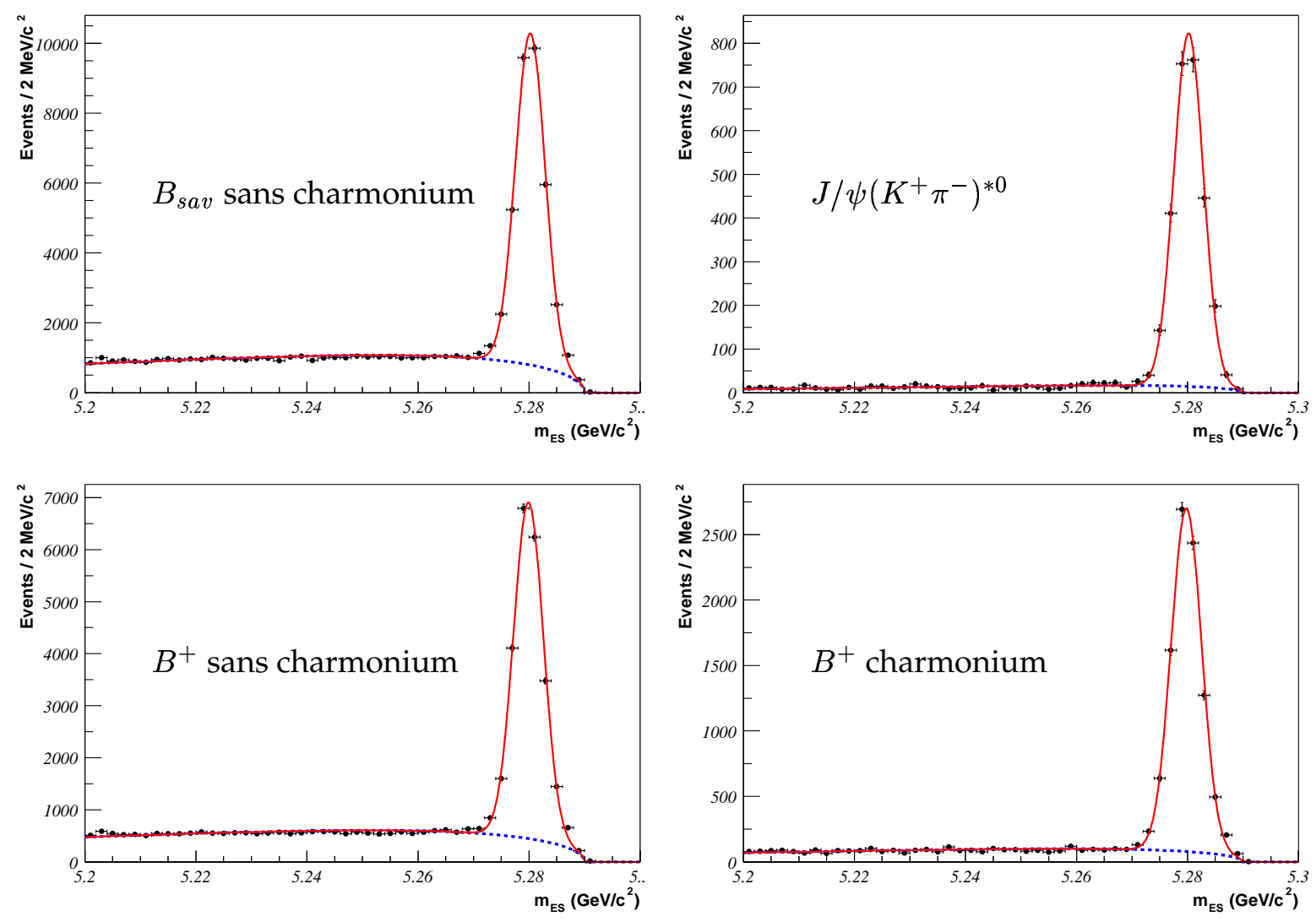

FIG. 6.3: Distributions en $m_{\mathrm{ES}}$ des échantillons $B_{\text {sav }}$ et $B^{+}$, séparés en désintégrations non charmonium (figures de gauche) et charmonium (figures de droite) avant l'application des critères de qualité sur les vertex des mésons $B$ et l'étiquetage. Un ajustement avec une gaussienne et une distribution Argus est superposé.

et $3.636<M_{\psi(2 S)}<3.736 \mathrm{GeV} / c^{2}$ pour les désintégrations $\psi(2 S) \rightarrow e^{+} e^{-}$et $\psi(2 S) \rightarrow \mu^{+} \mu^{-}$, respectivement. Pour la désintégration $\psi(2 S) \rightarrow J / \psi \pi^{+} \pi^{-}$, le méson $J / \psi$ est contraint à sa masse nominale, puis combiné avec une paire de traces de charges opposées supposées être des pions, de masse invariante comprise entre 400 et $600 \mathrm{MeV} / c^{2}$. Le candidat $\psi(2 S)$ est sélectionné en demandant que $0.574<M\left(J / \psi \pi^{+} \pi^{-}\right)-M(J / \psi)<0.604 \mathrm{MeV} / c^{2}$. Enfin, les candidats $\chi_{c 1}$ sont reconstruits en $J / \psi \gamma$. Le photon est sélectionné dans le volume fiduciel $0.41<\theta_{\gamma}<2.41 \mathrm{rad}$ et doit avoir une énergie supérieure à $150 \mathrm{MeV}$. De plus, si la combinaison du photon provenant du candidat $\chi_{c 1}$ avec tout autre photon de l'événement d'énergie supérieure à $70 \mathrm{MeV}$ a une masse invariante comprise entre 120 et $150 \mathrm{MeV} / c^{2}$, et est donc susceptible d'être un $\pi^{0}$, le candidat $\chi_{c 1}$ est rejeté. La masses invariante du candidat $\chi_{c 1}$ doit se situer dans l'intervalle $3.476<M_{\chi_{c 1}}<3.546 \mathrm{GeV} / c^{2}$. Les distributions en $m_{\mathrm{ES}}$ après coupure sur $\Delta E$ sont représentées sur la figure 6.3.

La table 6.1 résume les estimations des nombres d'événements (Yield) et la pureté obtenues à partir d'un ajustement avec une gaussienne et une distribution Argus avant d'appliquer les critères de qualité sur les vertex des mésons $B$ et l'étiquetage. 
TAB. 6.1: Estimations des nombres d'événements des différentes désintégrations considérées pour les échantillons utilisés dans cette analyse, avant d'appliquer l'étiquetage et les critères de qualité sur les vertex des mésons $B$. Ces estimations sont uniquement basées sur un ajustement par une gaussienne et une fonction Argus des distributions en $m_{\mathrm{ES}}$ après coupure sur $\Delta E$. La pureté est calculée à partir de l'ajustement pour les événements de $m_{\mathrm{ES}}>5.27 \mathrm{GeV} / c^{2}$.

\begin{tabular}{|l|c|c|c|}
\hline Échantillon & Désintégration & Yield & Pureté $(\%)$ \\
\hline \hline \multirow{3}{*}{$B_{\text {sav }}$} & $D^{(*)-} \pi^{+} / \rho^{+} / a_{1}^{+}$ & $31708 \pm 208$ & 81.1 \\
& $J / \psi K^{* 0}\left(K^{+} \pi^{-}\right)$ & $2689.1 \pm 54.3$ & 95.3 \\
\cline { 2 - 4 } & Total & $34405 \pm 215$ & 82.1 \\
\hline \hline \multirow{3}{*}{$B^{+}$} & $\bar{D}^{(*) 0} \pi^{+}$ & $21795 \pm 169$ & 84.2 \\
& $J / \psi K^{(*)+}, \psi(2 S) K^{+}, \chi_{c 1} K^{+}$ & $9026 \pm 102$ & 92.7 \\
\cline { 2 - 4 } & Total & $30799 \pm 198$ & 86.5 \\
\hline \hline
\end{tabular}

\section{2 Étiquetage du méson $B$}

Le but de l'étiquetage est d'identifier la saveur du méson $B$ de recul, $B_{t a g}$, afin de pouvoir mesurer l'asymétrie dépendante de la différence des temps propres, $t_{r e c}-t_{t a g}$ (cf. le chapitre 3 ). Cette saveur est directement reliée à la saveur du quark $b$ du méson $B$. On utilise la corrélation existante entre le signe de la charge du quark $b$ et ses produits de désintégration. Par exemple, le signe de la charge du lepton provenant de la désintégration semileptonique d'un méson $B$ est de même signe que la charge du quark $b$, à savoir un lepton positif (négatif) signe la présence d'un quark $\bar{b}(b)$ (cf. Fig. 6.4 a)). Cependant, le lepton peut provenir d'une désintégration en cascade, dans ce cas le signe de sa charge peut être opposé à celui du quark b (cf. Fig 6.4 b)) ou identique. Bien qu'il soit impossible de distinguer entre les deux types de désintégration en cascade, il est possible de reconnaître ceux provenant d'une désintégration directe $(b \rightarrow c)$ d'une désintégration en cascade, en utilisant le spectre en impulsion du lepton dans le repère de $1^{\prime} \Upsilon(4 S)$ au repos. En effet, on s'attend à ce que les leptons primaires (production directe) soient plus énergétiques que les leptons secondaires (production en cascade). Plus généralement, les événements peuvent se ranger en deux classes : celle avec le signe direct où le signe attribué aux produits de désintégration, est celui du quark $b$ et celle avec le signe opposé. Le but de l'étiquetage est d'effectuer la discrimination la plus efficace entre ces deux classes et de fournir ainsi la meilleure identification de la saveur du méson $B_{t a g}$.

a)

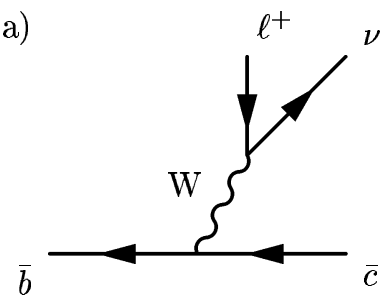

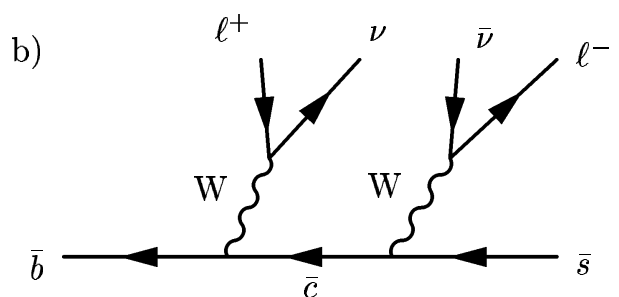

FIG. 6.4: a) Production directe d'un lepton. b) Production en cascade d'un lepton avec le signe opposé. 


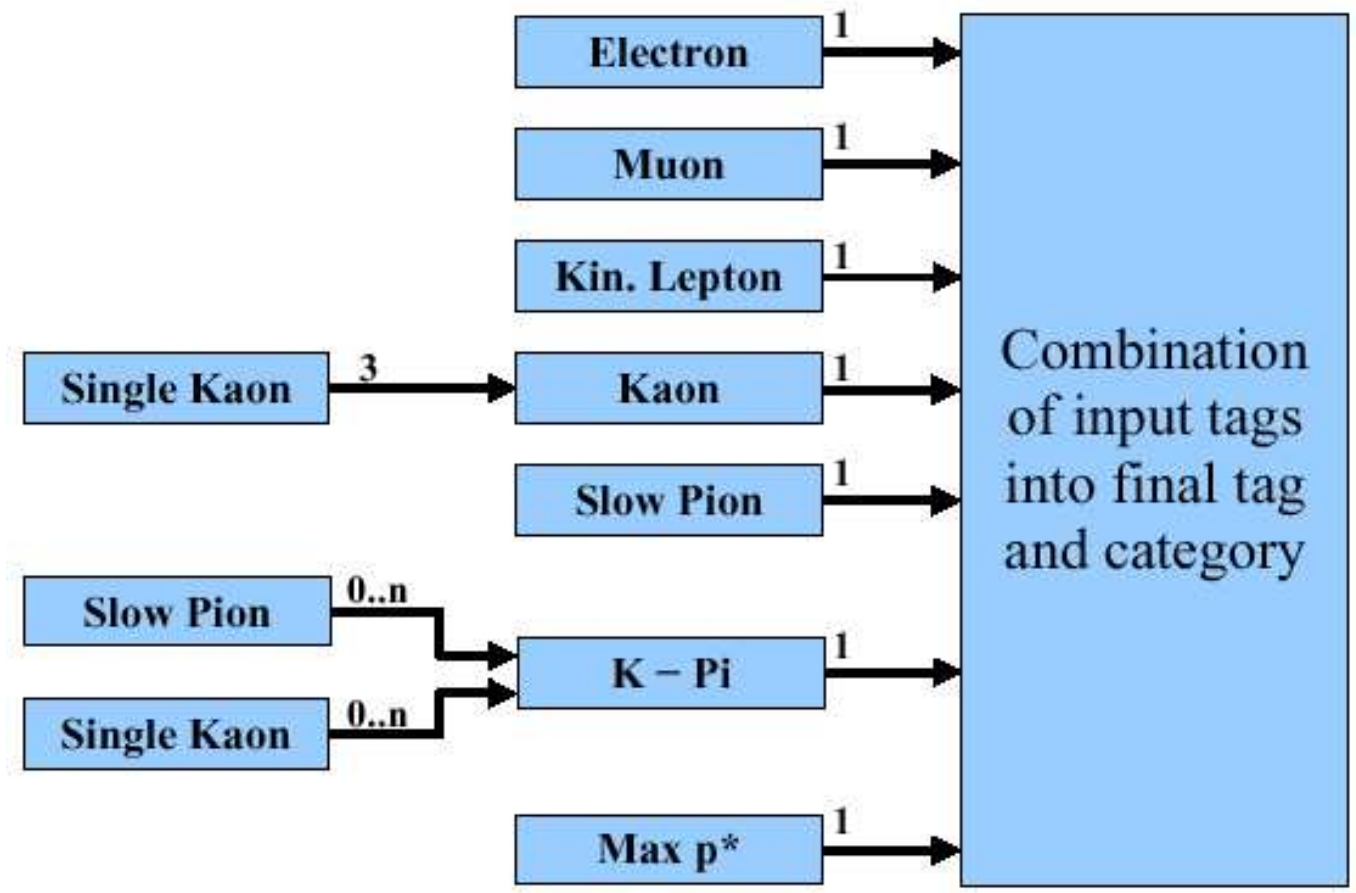

FIG. 6.5: Structure de l'algorithme Moriond Tagger et ses sept sources d'information de saveur. D'après [169].

\subsubsection{Algorithme d'étiquetage}

L'algorithme d'étiquetage adopté par la collaboration BABAR depuis la conférence de Moriond 2002 (d'où le nom de Moriond Tagger) est un algorithme multivariable avec des catégories basées sur la physique (i.e. sur le contenu en particules) de la désintégration du méson $B_{t a g}$. Les traces chargées n'appartenant pas au méson $B$ entièrement reconstruit $\left(B_{r e c}\right)$ sont analysées afin de déterminer la saveur du méson $B_{t a g}, B^{0}$ ou $\bar{B}^{0}$. Seules les traces ayant une distance de moindre approche inférieure à $0.4 \mathrm{~cm}$ en $x y$ et $4 \mathrm{~cm}$ en $z$ sont retenues. Sept sources d'information de saveur sont utilisées, illustrées sur la figure 6.5 :

- Electron

- Muon

- Kin. Lepton (« lepton identifié cinématiquement»)

- Kaon

- Slow Pion

- K-Pi (corrélation kaon pion mou)

- Maximum $p^{*}$.

Chaque source d'information de saveur est un réseau de neurones qui peut servir à lui seul comme algorithme d'étiquetage, ou être combiné avec d'autres sources en un algorithme d'étiquetage plus performant. On distingue deux types d'algorithme d'étiquetage : les algorithmes basés sur une trace (track based) et les algorithmes basés sur l'ensemble des traces (event based). Les algorithmes basés sur une trace fournissent les informations de saveur à partir d'une unique trace, avec la possibilité d'utiliser aussi des informations cinématiques. Les algorithmes basés sur l'ensemble des traces utilisent toutes les traces pour fournir une information de saveur. 
Les réseaux Electron et Muon utilisent uniquement les traces passant l'identification d'électrons VeryTight et de muons Tight, respectivement (cf. §3.3.3), ainsi que trois variables cinématiques qui permettent de séparer les leptons primaires des leptons de cascade et autres traces: l'impulsion de chacune de ces traces dans le centre de masse, $p^{*}$, et une paire de variables cinématiques, $E_{W}^{90}$ et $\cos \theta_{\ell \nu}$. La variable d'isolation, $E_{W}^{90}$, est donnée par la somme des énergies de toutes les traces se trouvant dans un cône d'ouverture de $90^{\circ}$ par rapport à la direction du $W$. L'impulsion du $W$ est déduite de la somme des impulsions des traces et de l'impulsion du neutrino, obtenue comme étant l'impulsion manquante dans le centre de masse en utilisant toutes les traces provenant du méson $B_{t a g}$. Cette variable est efficace car, dans les désintégrations semileptoniques, les hadrons de recul par rapport au $W$ sont généralement dans la direction opposée. L'autre variable cinématique utilisée, $\cos \theta_{\ell_{\nu}}$, est le cosinus de l'angle entre l'impulsion du candidat électron ou muon et la direction du neutrino. Le réseau Kin. Lepton sélectionne les leptons primaires en se basant uniquement sur les variables cinématiques $p^{*}, E_{W}^{90}$ et $\cos \theta_{\ell \nu}$, dont les distributions sont données sur la figure $6.6 \mathrm{a}$ ) et $\mathrm{b}$ ). Seules les traces n'ayant pas passé les identificateurs électron et muon sont considérées. La sortie de ce réseau contient une part significative de pions et kaons.

La charge d'un kaon provenant de la chaîne de désintégration $b \rightarrow c \rightarrow s$ fournit aussi une signature de la saveur du quark $b$ (un $K^{+}$signe un méson $B_{d}^{0}$ ). La majorité des kaons chargés provenant de la désintégration d'un méson $B$ ont le signe en adéquation avec la saveur du méson $B$ : il y a 0.8 kaons chargés en moyenne par désintégration de méson $B$, dont environ $15 \%$ ont un signe opposé par rapport à la saveur du méson $B$. Cependant, une fraction significative d'événements ont plus d'un kaon chargé (cf. Fig. 6.7). Aucune variable cinématique ne permettant de distinguer des kaons ayant un signe en adéquation ou non avec la saveur du méson $B$, le réseau Kaon utilise uniquement les traces passant l'identification de kaon VeryLoose ainsi qu'un réseau de neurone combinant les informations provenant du SVT, de la DCH et du DIRC. Les critères sur la distance de moindre approche sont relâchés : $1.5 \mathrm{~cm}$ en $x y$ et $10 \mathrm{~cm}$ en $z$. Les trois meilleurs candidats kaons sont retenus, ordonnés selon la sortie du réseau de neurone de l'identificateur de kaon. Bien que la charge du meilleur candidat kaon fournisse une performance d'étiquetage significative par elle-même, c'est la corrélation entre les trois meilleurs candidats kaons qui aide à augmenter les performances de l'étiquetage par les kaons.

Une autre source apportant des informations pour l'étiquetage sont les pions mous provenant de la désintégration $D^{*+} \rightarrow D^{0} \pi^{+}$, qui, contrairement aux leptons et aux kaons, ont le signe de leur charge opposé à celui du quark $b$. Le bruit de fond substantiel provenant des traces de faible impulsion peut être réduit par la corrélation existante entre les pions mous et les autres traces provenant du méson $B_{t a g}$. En effet, puisque le pion mou et le $D^{0}$ sont émis quasiment au repos, dans le repère du $D^{*}$ au repos, on s'attend à ce que la direction du pion mou dans le repère du méson $B_{t a g}$ au repos soit le long de la direction à la fois des produits de désintégration du $D^{0}$ et des traces restantes provenant du méson $B_{t a g}$. Cette direction est approchée en calculant l'axe de poussée (thrust) des produits de désintégration du méson $B_{t a g}$. Le réseau Slow Pion utilise les traces ayant une impulsion dans le centre de masse inférieure à $0.25 \mathrm{GeV} / c$ et les variables discriminantes suivantes : l'impulsion du pion mou dans le centre de masse, le cosinus de l'angle entre la direction de l'impulsion du pion mou et l'axe de poussée dans le centre de masse, $\cos \theta_{\text {thrust }}$ (voir Fig. 6.6 c), et des informations d'identification de kaons aidant à rejeter la contribution des 

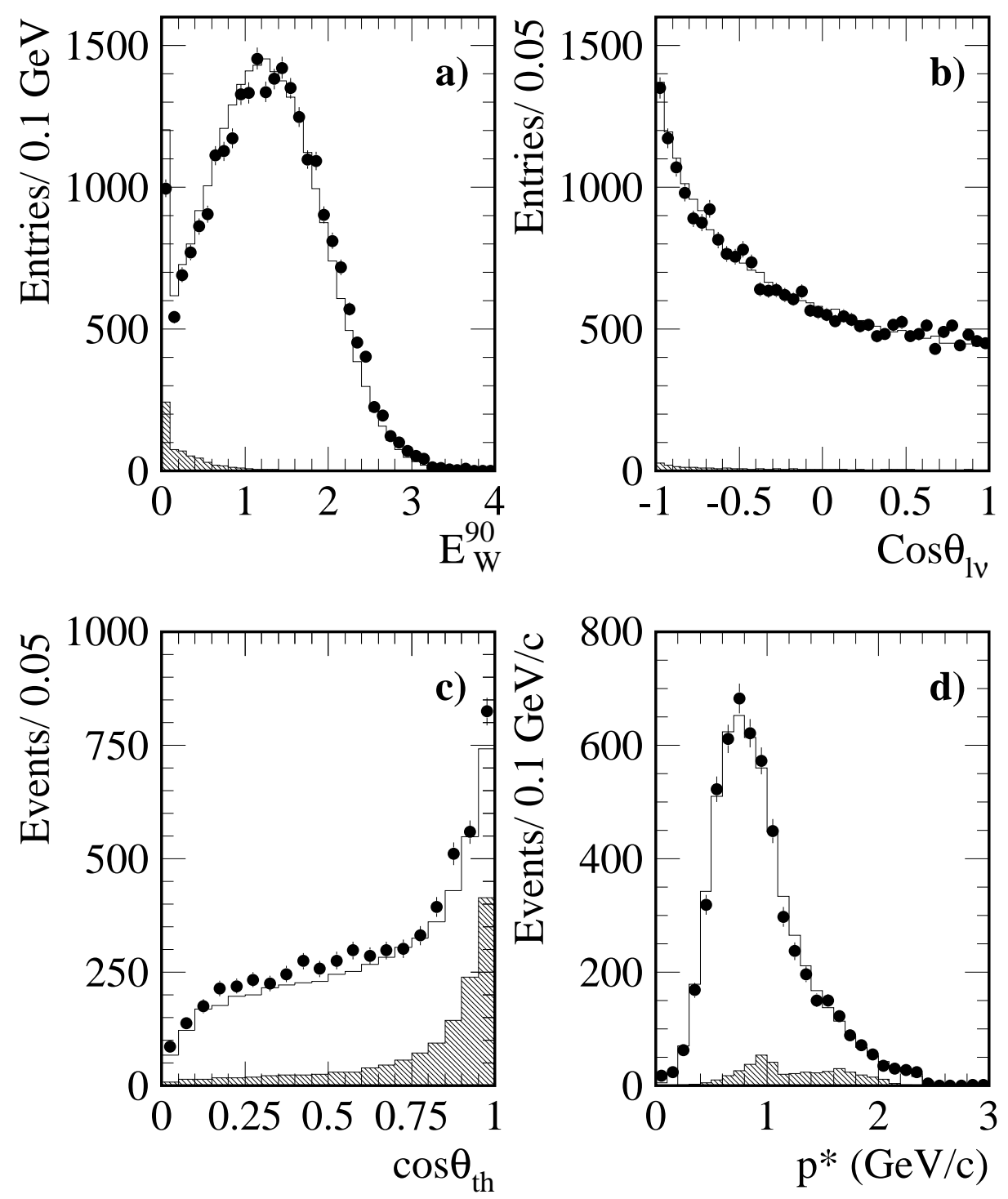

FIG. 6.6: Variables cinématiques utilisées par les source d'information de saveur après élimination des événements ayant un électron $\left(p^{*}>1.0 \mathrm{GeV} / c\right)$ ou un muon $\left(p^{*}>1.1 \mathrm{GeV} / c\right)$ identifié : a) $E_{W}^{90}$ b) $\left.\cos \theta_{\ell \nu} \mathrm{c}\right) \cos \theta_{t h(\text { rust })}$ (pour $p^{*}<$ $0.18 \mathrm{GeV} / c)$ d) $p^{*}$ de toutes les traces. Les points sont les données provenant de l'échantillon $B_{\text {sav }}$ après soustraction des bruits de fond avec la région à bas $m_{\mathrm{ES}}\left(m_{\mathrm{ES}}<5.27 \mathrm{GeV} / c^{2}\right)$, les histogrammes proviennent de données simulées normalisés au nombre total de mésons $B$ après soustraction des bruits de fond. Pour $\cos \theta_{t h(r u s t)}, l^{\prime}$ histogramme hachuré correspond à la contribution des pions mous provenant de la désintégration de mésons $D^{*}$, et pour tous les autres histogrammes il correspond à la contribution de leptons primaires. 


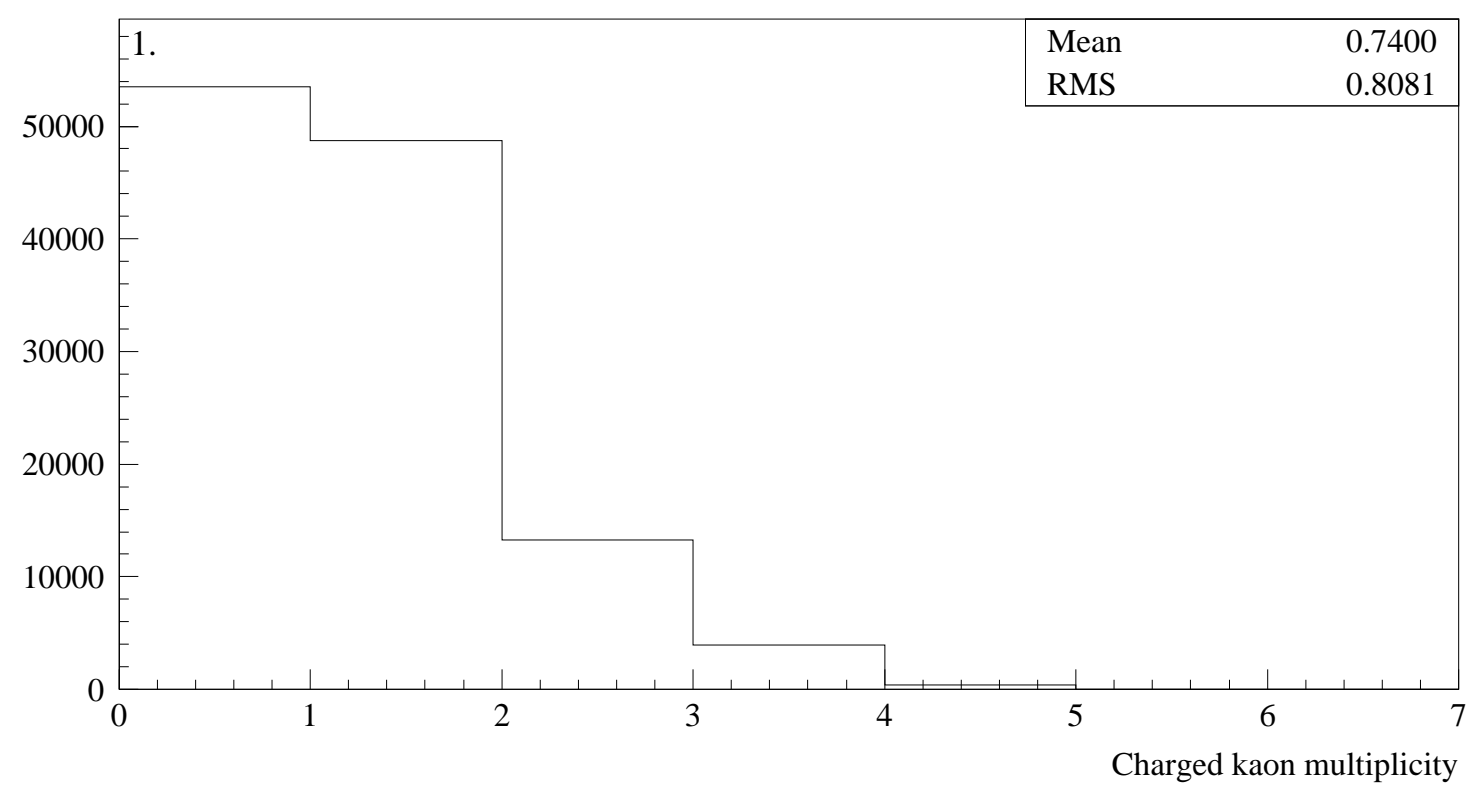

FIG. 6.7: Multiplicité de kaons chargés à la génération obtenue à partir de données simulées.

kaons de faible impulsion dans le calcul de l'axe de poussée. L'axe de poussée est déterminé en utilisant les traces chargées et les neutres.

Pour les événements où sont présents à la fois un kaon chargé et un pion mou, l'utilisation de la corrélation entre les deux peut améliorer les résultats sur l'étiquetage de la saveur. Par exemple, si un kaon et un pion mou proviennent de la désintégration d'un même $D^{*}$, ils auront quasiment la même direction de vol dans le centre de masse. Le réseau $\mathrm{K}-\mathrm{Pi}$ utilise des kaons et des pions de signes opposés ayant passé les mêmes critères que ceux des réseaux Kaon (sans se limiter aux trois meilleurs candidats) et Slow Pion, ainsi que le cosinus de l'angle entre les directions des impulsions du kaon et du pion mou, $\cos \theta_{K, \pi}$, calculé dans le centre de masse de $1^{\prime} \Upsilon(4 S)$.

La dernière source d'information de saveur, constituée par le réseau Maximum $p^{*}$, sélectionne la trace chargée de plus grande impulsion dans le centre de masse (voir Fig. $6.6 \mathrm{~d}$ ) et utilise sa charge pour étiqueter la saveur du méson $B_{t a g}$. Le but de cette source est d'identifier les particules rapides (par exemple les pions rapides de $B \rightarrow D^{*} \pi$ ) et de récupérer les leptons de haute impulsion qui n'ont pas été sélectionnés par les autres sources d'information de saveur. De telles particules étant rapides, la distance de moindre approche est réduite à être inférieure à $1 \mathrm{~mm}$ en $x y$.

Un réseau de neurones final combine les différentes sorties de ces algorithmes basés sur la physique du méson $B_{t a g}$. Il prend en compte les corrélations entre ces différentes sources d'étiquetage et fournit une estimation des probabilités de mauvais étiquetage pour chaque événement (voir Fig. 6.5). En se basant sur la sortie de ce réseau de neurones final, chaque événement est assigné à neuf catégories hiérarchisées, mutuellement exclusives d'étiquetage, i.e. chaque événement est assigné à une unique catégorie, définies dans la table 6.2. L'importance de définir des catégories d'étiquetage selon le contenu en particules du méson $B_{t a g}$ est motivée pour l'étude et la compréhension des bruits de fond et des effets systématiques. Dans le cas d'un algorithme purement probabiliste, i.e. la saveur est obtenue de façon continue de -1 à +1 , définie par $p\left(B^{0}\right)-p\left(\bar{B}^{0}\right)$, divers effets, tels que les corrélations entre l'étiquetage et la fonction de résolution en $\Delta t$ (cf. §6.3.3), les diffé- 
ТАв. 6.2: Définition des neuf catégories hiérarchisées, mutuellement exclusives d'étiquetage de l'algorithme Moriond Tagger.

\begin{tabular}{|c|c|c|}
\hline $\begin{array}{l}\text { Coupure sur la sortie des sources } \\
\text { d'information de saveur }\end{array}$ & $\begin{array}{c}\text { Coupure sur la sortie } \\
\text { du réseau final }\end{array}$ & Catégorie \\
\hline $\begin{array}{l}\text { ElectronTag } \mid>0.7 \text { et } \mid \text { KaonTag } \mid>0.2 \\
\mid \text { MuonTag } \mid>0.7 \text { et } \mid \text { KaonTag } \mid>0.2\end{array}$ & $\begin{array}{l}|\operatorname{tag}|>0.8 \\
|\operatorname{tag}|>0.8\end{array}$ & $\begin{array}{l}\text { Electron-kaon } \\
\text { Muon-kaon }\end{array}$ \\
\hline $\begin{array}{l}\mid \text { ElectronTag } \mid>0.7 \\
\mid \text { MuonTag } \mid>0.7\end{array}$ & $\begin{aligned}|\operatorname{tag}| & >0.8 \\
|\operatorname{tag}| & >0.8\end{aligned}$ & $\begin{array}{l}\text { Electron } \\
\text { Muon }\end{array}$ \\
\hline $\mid$ KaonSlowPionTag $\mid>0.1$ & $|\operatorname{tag}|>0.8$ & Kaon-slow pion \\
\hline $\mid \overline{\mid \text { KaonTag } \mid>0.2}$ & $|\overline{\mid t a g}|>0.7$ & Kaon 1 \\
\hline $\mid$ SlowPionTag $\mid>0.1$ & $|\operatorname{tag}|>0.5$ & Slow pion \\
\hline $\mid$ KaonTag $\mid>0.2$ & $|\operatorname{tag}|>0.4$ & Kaon 2 \\
\hline Aucune & $\overline{|l \operatorname{tag}|>0.2}$ & Autre \\
\hline
\end{tabular}

rents niveaux de bruits de fond, sont dilués et difficilement interprétables. Ainsi, chaque catégorie est appropriée à l'étude des bruits de fond, des erreurs systématiques et aux validations avec des échantillons de contrôle, permettant d'obtenir une estimation de la probabilité de mauvais étiquetage. Finalement, ces catégories sont fusionnées en quatre catégories hiérarchisées, mutuellement exclusives d'étiquetage, définies dans la table 6.3. La catégorie Lepton contient les événements avec un lepton identifié et une étiquette kaon en confirmation si elle est disponible. Les événements avec un candidat kaon et un pion mou, de charges opposées et des directions de vol similaires, sont assignés à la catégorie Kaon I. Les événements avec uniquement une étiquette kaon sont assignés à la catégorie Kaon I ou Kaon II selon leur estimation de probabilité de mauvais étiquetage. La catégorie Kaon I I contient aussi les événements restants avec un pion mou. Tous les autres événements sont soit assignés à la catégorie Inclusive, soit rejetés selon leur estimation de probabilité de mauvais étiquetage.

ТАВ. 6.3: Définition des quatre catégories hiérarchisées, mutuellement exclusives d'étiquetage en fonction des neuf catégories définies par l'algorithme Moriond Tagger.

\begin{tabular}{|l|c|}
\hline Catégories sources & Catégories finales \\
\hline \hline Electron-Kaon, Muon-Kaon, Electron, Muon & Lepton \\
\hline Kaon-Slow Pion, Kaon 1 & Kaon I \\
\hline Slow Pion, Kaon 2 & Kaon I I \\
\hline Autre & Inclus ive \\
\hline
\end{tabular}

\subsubsection{Performances}

La figure de mérite de l'étiquetage est donnée par l'efficacité d'étiquetage effective $Q \equiv \sum_{c} \epsilon_{c}(1-$ $\left.2 w_{c}\right)^{2}$, où la somme porte sur les catégories $(c)$ d'étiquetage. L'efficacité d'étiquetage $\epsilon$ est la fraction d'événements dont on sait donner une saveur. Cette efficacité est calculée à partir de l'échantillon de mésons $B$ entièrement reconstruits, $B_{r e c}$, après avoir appliqué l'ensemble des coupures et des 
TAB. 6.4: Performance de l'algorithme Moriond tagger sur des données simulées avec les quatre catégories finales d'étiquetage.

\begin{tabular}{|l|cc|cc|cc|}
\hline Catégorie & $\epsilon(\%)$ & $\Delta \epsilon(\%)$ & $w(\%)$ & $\Delta w(\%)$ & $Q(\%)$ & $\Delta Q(\%)$ \\
\hline \hline Lepton & $10.0 \pm 0.1$ & $0.5 \pm 0.2$ & $2.8 \pm 0.1$ & $-0.7 \pm 0.3$ & $9.0 \pm 0.1$ & $0.7 \pm 0.2$ \\
Kaon I & $17.6 \pm 0.1$ & $-0.4 \pm 0.2$ & $9.2 \pm 0.2$ & $-0.9 \pm 0.4$ & $11.7 \pm 0.1$ & $0.2 \pm 0.3$ \\
Kaon II & $19.9 \pm 0.1$ & $0.5 \pm 0.2$ & $21.2 \pm 0.3$ & $-3.0 \pm 0.5$ & $6.6 \pm 0.1$ & $1.5 \pm 0.3$ \\
Inclusive & $20.1 \pm 0.1$ & $-0.2 \pm 0.2$ & $30.9 \pm 0.3$ & $-2.3 \pm 0.6$ & $2.9 \pm 0.1$ & $0.7 \pm 0.2$ \\
\hline \hline Total & $67.7 \pm 0.2$ & $0.5 \pm 0.5$ & & & $30.2 \pm 0.2$ & $3.2 \pm 0.5$ \\
\hline
\end{tabular}

critères de qualité concernant les vertex des deux mésons $B, B_{\text {rec }}$ et $B_{t a g}$ (voir §6.3.1). La fraction de mauvais étiquetage, $w$, est définie comme étant la fraction où le méson $B$ est étiqueté de la saveur opposée (p. ex. un méson $B_{d}^{0}$ est étiqueté $\bar{B}_{d}^{0}$ ). Le but de l'algorithme d'étiquetage est de maximiser $Q$, puisqu'on peut montrer que l'erreur statistique sur $\sin 2 \beta$ varie en fonction de $Q$ comme $1 / \sqrt{Q}$ [170]. Les performances de l'algorithme Moriond Tagger sont résumées dans la table 6.4 avec $\Delta \epsilon=\epsilon_{B^{0}}-\epsilon_{\bar{B}^{0}}$ et $\Delta w=w_{B^{0}}-w_{\bar{B}^{0}}$. L'algorithme d'étiquetage est optimisé avec des données simulées ainsi que l'entraînement des réseaux de neurones. Les différences entre les données et les données simulées peuvent rendre l'algorithme quelque peu sous-optimal mais n'introduisent aucun biais du fait que les fractions de mauvais étiquetage $w_{c}$ sont obtenues directement avec les données. Les performances obtenues sur les données sont résumées dans la table 6.5.

TAB. 6.5: Performance de l'algorithme Moriond Tagger sur l'ensemble des données $\left(B_{C P}+B_{s a v}, B_{C P}\right.$ contenant l'ensemble des modes $C P$ ).

\begin{tabular}{|l|c|cc|c|}
\hline Catégorie & $\epsilon(\%)$ & $w(\%)$ & $\Delta w(\%)$ & $Q(\%)$ \\
\hline \hline Lepton & $9.1 \pm 0.2$ & $3.3 \pm 0.6$ & $-1.5 \pm 1.1$ & $7.9 \pm 0.3$ \\
Kaon I & $16.7 \pm 0.2$ & $10.0 \pm 0.7$ & $-1.3 \pm 1.1$ & $10.7 \pm 0.4$ \\
Kaon I I & $19.8 \pm 0.3$ & $20.9 \pm 0.8$ & $-4.4 \pm 1.2$ & $6.7 \pm 0.4$ \\
Inclusive & $20.0 \pm 0.3$ & $31.5 \pm 0.9$ & $-2.4 \pm 1.3$ & $2.7 \pm 0.3$ \\
\hline \hline Total & $65.6 \pm 0.5$ & & & $28.1 \pm 0.7$ \\
\hline
\end{tabular}

Les figures 6.8 à 6.10 montrent les sorties des sept algorithmes d'information de saveur ainsi que la sortie de l'algorithme Moriond Tagger. Les points avec leurs barres d'erreur sont les données (échantillon $B_{\text {sav }}$ ) après soustraction des bruits de fond basée sur la région à bas $m_{\mathrm{ES}}$ et les histogrammes proviennent de simulations Monte Carlo.

L'analyse de $\sin 2 \beta$ se base sur l'hypothèse que les fractions de mauvais étiquetage sont indépendantes du mode de désintégration du méson $B_{r e c}$. Cette hypothèse peut être vérifiée sur les données en comparant les performances d'étiquetage sur les désintégrations chargées (échantillon $B^{+}$) d'un échantillon non charmonium avec celles d'un échantillon charmonium. Ces performances sont déterminées en comparant la saveur obtenue avec l'algorithme d'étiquetage et la charge du mode entièrement reconstruit. Les résultats sont représentés sur la figure 6.11. Aucune différence significative n'est observée. 
Moriond Tagger: Track--Based Inputs
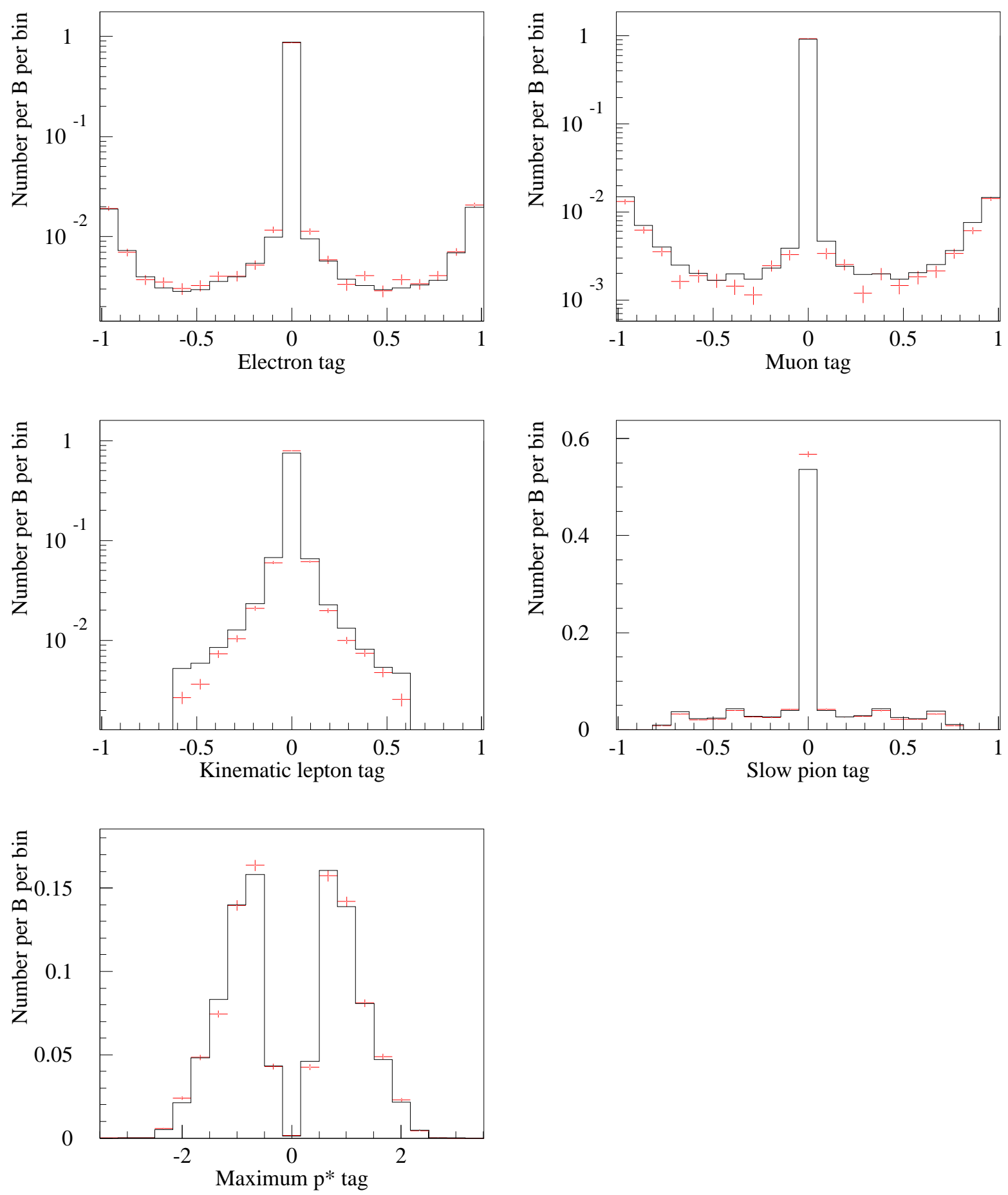

FIG. 6.8: Sorties des cinq (sur sept) algorithmes d'information de saveur basés sur une trace (track based) qui servent d'entrée à l'algorithme Moriond Tagger. Les points avec leurs barres d'erreur sont les données (échantillon $\boldsymbol{B}_{\text {sav }}$ ) après soustraction des bruits de fond basée sur la région à bas $m_{\mathrm{ES}}$ et les histogrammes proviennent de simulations Monte Carlo. D'après [169]. 
Moriond Tagger: Event--Based Inputs
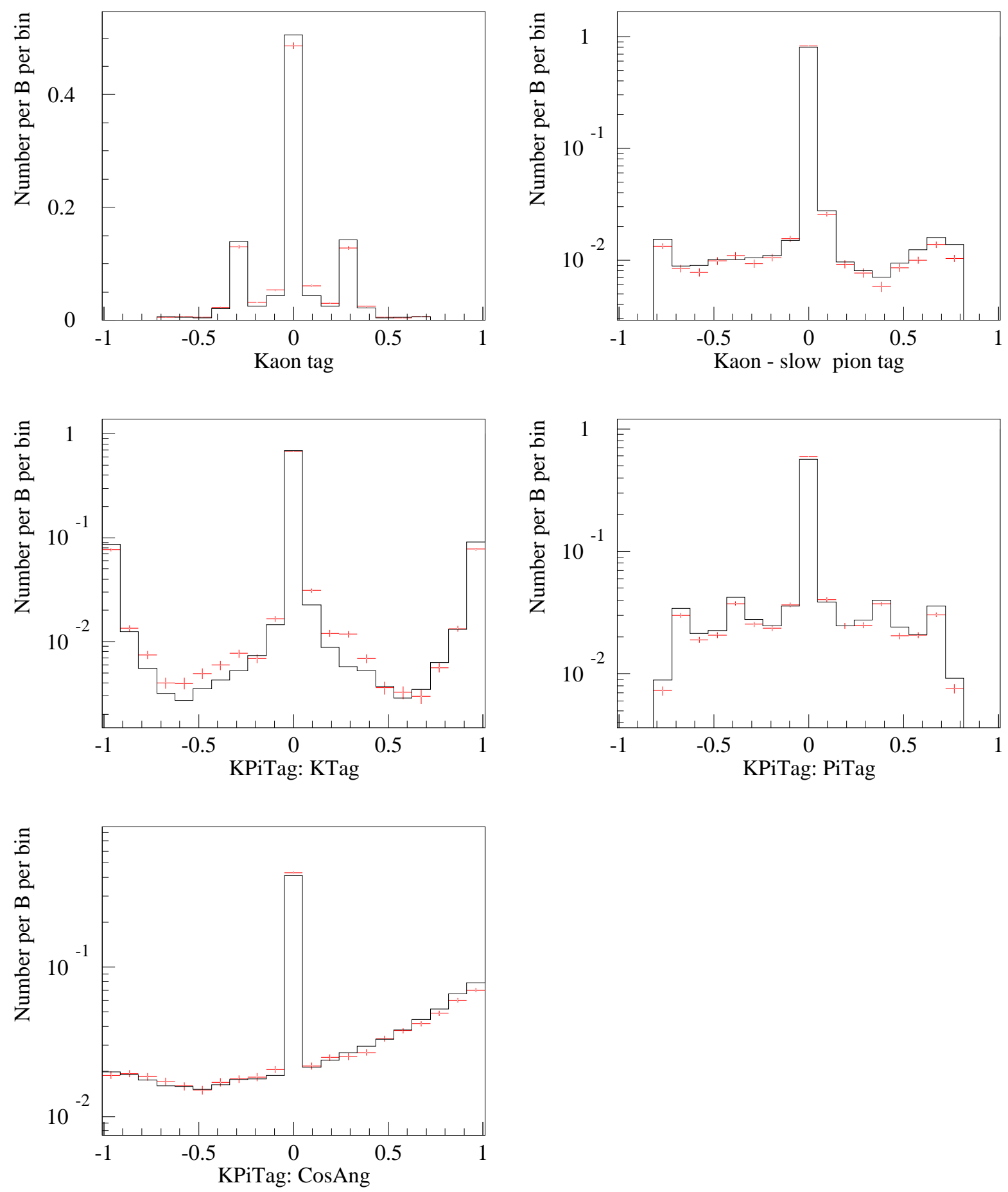

FIG. 6.9: Sorties des deux (sur sept) algorithmes d'information de saveur basés sur l'ensemble des traces (event based) qui servent d'entrée à l'algorithme Moriond Tagger (les deux figures du haut). Sont montrées en plus les entrées de l'algorithme $\mathrm{K}-\mathrm{Pi}$ (cf. Fig. 6.5). Les points avec leurs barres d'erreur sont les données (échantillon $\boldsymbol{B}_{\text {sav }}$ ) après soustraction des bruits de fond basée sur la région à bas $m_{\mathrm{ES}}$ et les histogrammes proviennent de simulations Monte Carlo. D'après [169]. 


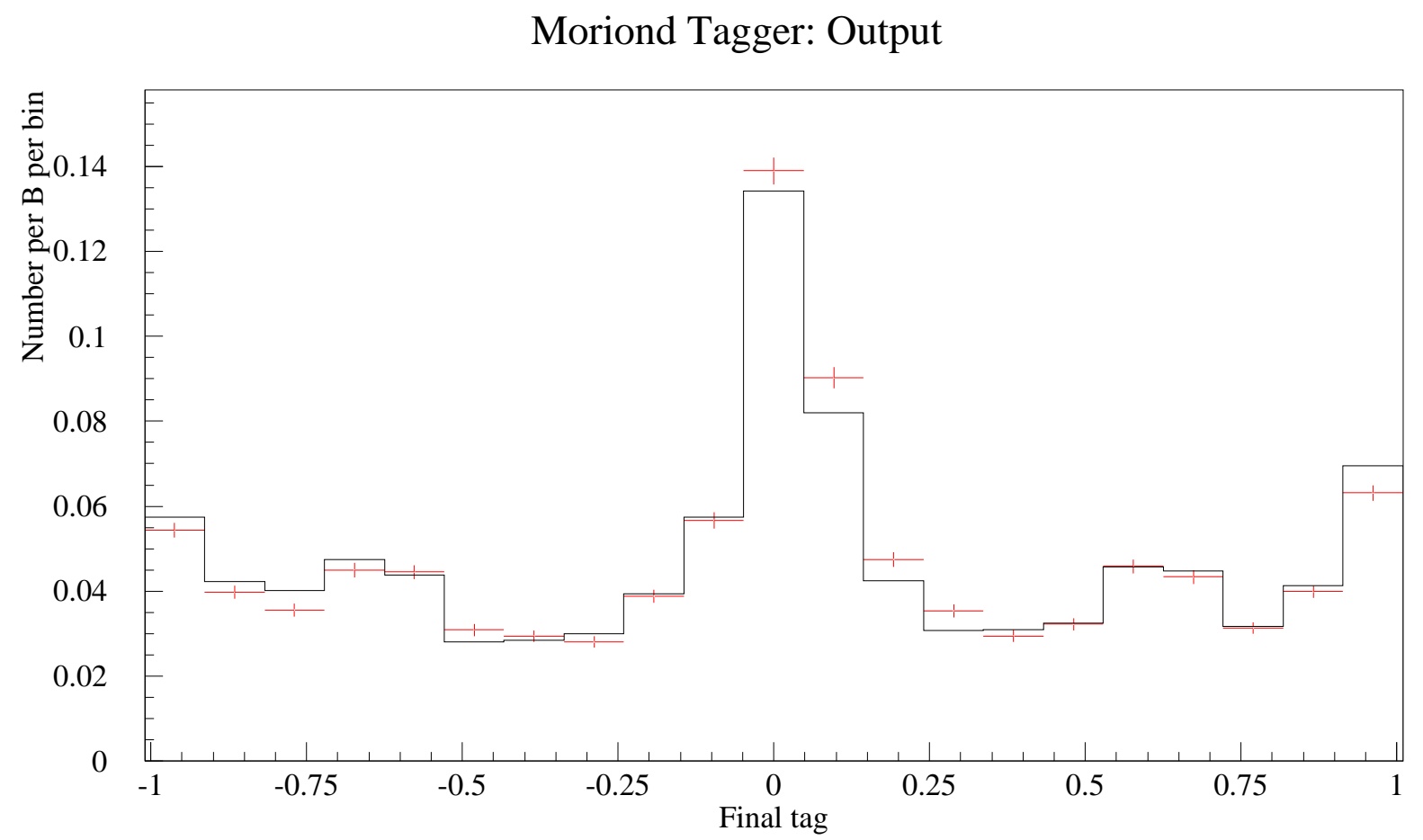

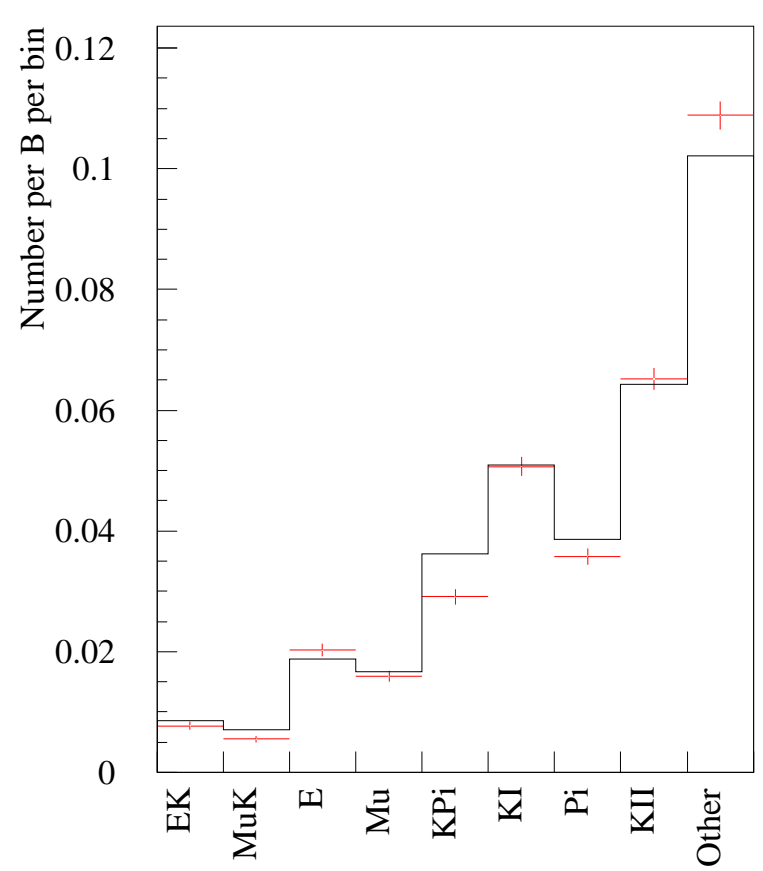

B0 tags: category number

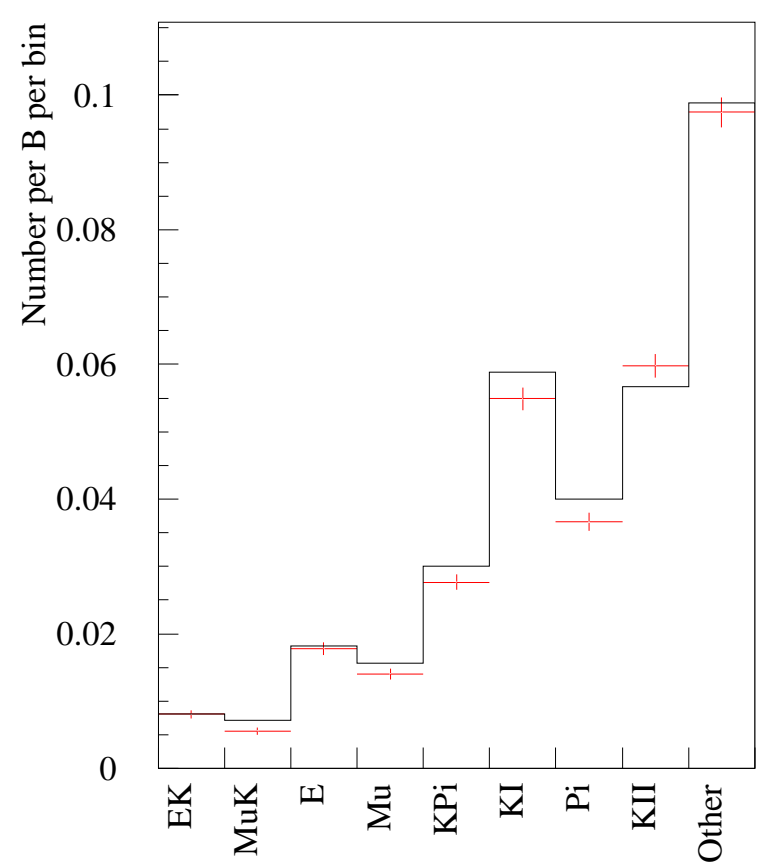

B0bar tags: category number

FIG. 6.10: Sortie de l'algorithme Moriond Tagger ainsi que la séparation de l'efficacité d'étiquetage des neuf catégories selon la saveur $B^{0}$ ou $\bar{B}^{0}$. Les points avec leurs barres d'erreur sont les données (échantillon $B_{\text {sav }}$ ) après soustraction des bruits de fond basée sur la région à bas $m_{\mathrm{ES}}$ et les histogrammes proviennent de simulations Monte Carlo. D'après [169]. 


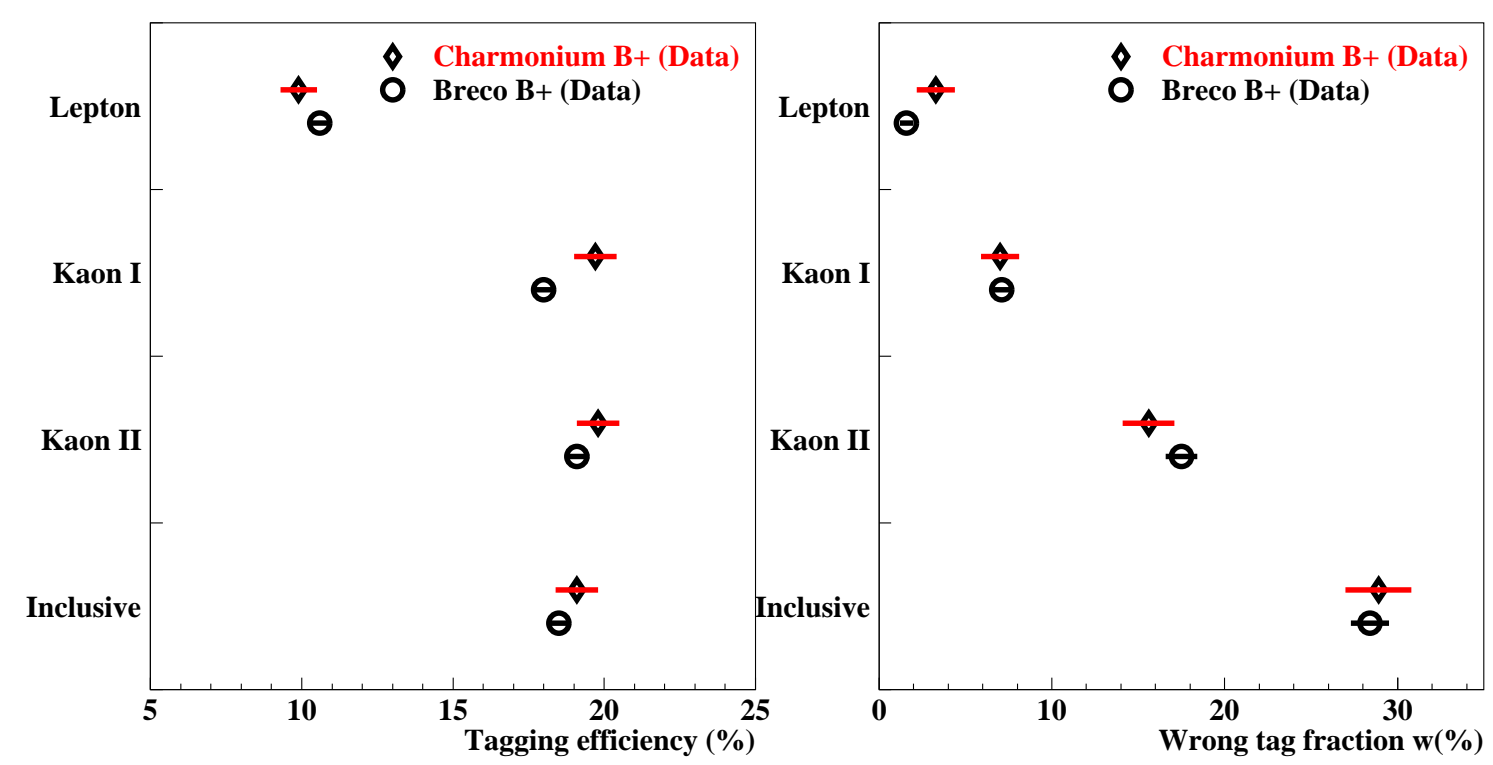

FIG. 6.11: Comparaison des performances de l'algorithme Moriond Tagger entre un échantillon charmonium (Charmonium $B^{+}$) et non charmonium (Breco $B^{+}$) de désintégrations chargées provenant des données. D'après [169].

\subsection{Mesure de la différence de temps propres}

La différence des temps propres des mésons $B, \Delta t=t_{\text {rec }}-t_{t a g}$, est déterminée par la mesure de la séparation spatiale le long de l'axe $z, \Delta z$, entre les vertex du méson $B$ entièrement reconstruit, $B_{r e c}$, et du méson de recul servant à l'étiquetage, $B_{t a g}$. La résolution sur $\Delta z$ est dominée par la résolution sur la position sur l'axe $z$ du vertex du méson $B_{t a g}$.

\subsubsection{Reconstruction des vertex}

Le vertex du méson $B_{r e c}$ est déterminé en ajustant à un vertex commun les traces chargées provenant de ses filles, à l'exception des candidats $K_{S}^{0}$ ou $D$, qui sont d'abord reconstruits avec un vertex séparé. Leurs impulsions et leurs positions sont ensuite utilisées dans l'ajustement du vertex du méson $B_{r e c}$. Les candidats $D$ sont contraints à leurs masses nominales, ce qui inclut leurs filles neutres, contrairement aux candidats $D^{*+}$ et $J / \psi$. Contraindre à la masse nominale améliore la résolution en $\Delta E$, ingrédient essentiel dans la sélection des candidats $B$ (cf. §4.1.2), mais pas la résolution sur le vertex. Cela permet, par exemple, de s'affranchir du rayonnement de freinage dans la désintégration $J / \psi \rightarrow e^{+} e^{-}$. La résolution (RMS) en $z$ pour le méson $B_{r e c}$ obtenue à partir d'une simulation Monte Carlo varie de $60 \mu \mathrm{m}$, pour les modes avec une faible multiplicité de traces et des impulsions élevées, à $80 \mu \mathrm{m}$ pour les modes les moins bien mesurés (haute multiplicité de traces, présence de neutres). Par exemple, la figure 6.12 illustre le résidu $z-z_{\text {vrai }}$ (différence entre le $z$ mesuré et le $z$ généré) pour des événements simulés $B^{0} \rightarrow J / \psi K_{S}^{0}$. Un ajustement fait avec une somme de deux gaussiennes donnent une fraction de $77 \%$ avec une résolution de $41 \mu \mathrm{m}$ pour la gaussienne centrale. Le RMS global est de $68 \mu \mathrm{m}$.

Le vertex du méson $B_{t a g}$ est déterminé en ajustant à un vertex commun les traces chargées qui n'appartiennent pas au méson $B_{r e c}$. Les candidats $K_{S}^{0}$ et $\Lambda^{0}$ reconstruits sont utilisés dans l'ajustement à la place de leurs filles. Les traces provenant de la conversion d'un photon $\left(\gamma \rightarrow e^{+} e^{-}\right)$sont 


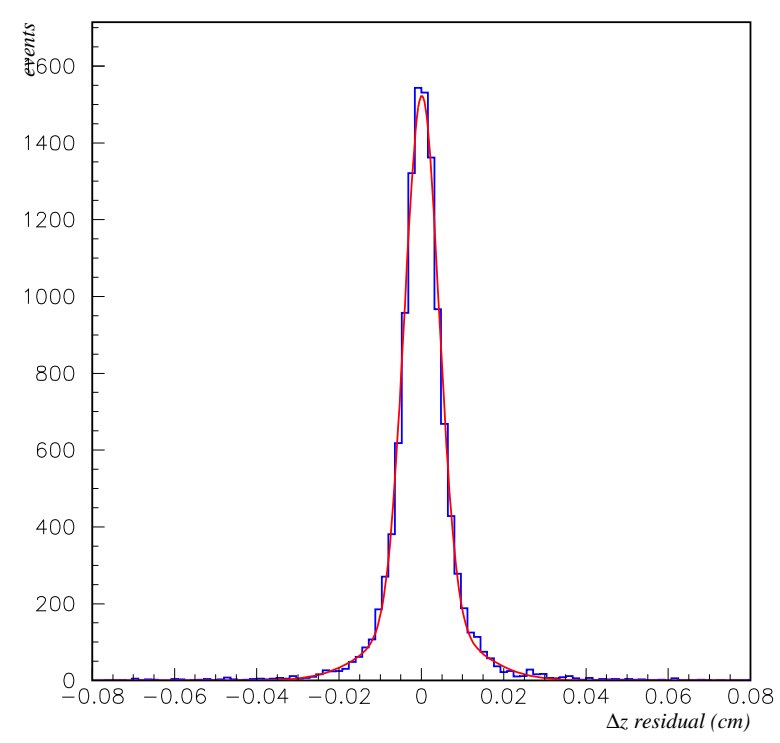

FIG. 6.12: Résidu $z-z_{v r a i}$ pour des événements simulés $B^{0} \rightarrow J / \psi K_{S}^{0}$. Un ajustement fait avec une somme de deux gaussiennes est superposé. D'après [171].

exclues de l'ajustement. Afin de réduire la contribution des désintégrations des mésons charmés, qui pourraient biaiser l'estimation du vertex, la trace avec le $\chi^{2}$ le plus grand et supérieur à 6 est retirée et l'ajustement du vertex est refait jusqu'à ce qu'aucune trace ne contribue à plus de 6 au $\chi^{2}$. Une contrainte supplémentaire est utilisée dans l'ajustement du vertex du méson $B_{t a g}$ : en utilisant l'impulsion du méson $B_{r e c}$, son vertex de désintégration avec sa matrice d'erreur associée, la connaissance de la position moyenne du point d'interaction et le «boost » moyen de la résonance $\Upsilon(4 S)$ (voir Fig. 6.13) permettent d'obtenir une estimation du quadrivecteur énergie-impulsion du méson $B_{t a g}$. Une pseudo-trajectoire peut être ainsi définie en partant de la région lumineuse (beam spot $\equiv$ région dans laquelle se produit les collisions $e^{+} e^{-}$). La condition de compatibilité des traces composant le vertex du méson $B_{t a g}$ avec cette pseudo-trajectoire aide à éliminer les traces qui ne proviennent pas directement de la désintégration du méson $B_{t a g}$ et réduit ainsi le biais dû aux désintégrations des mésons charmés.

Un événement est accepté si l'ajustement en $\Delta z \equiv z_{\text {rec }}-z_{\text {tag }}$ converge avec une erreur inférieure à $400 \mu \mathrm{m}$ et un $\Delta z$ mesuré compris dans l'intervalle $\pm 3 \mathrm{~mm}$. La figure 6.14 montre la distribution du résidu $\Delta z-\Delta z_{v r a i}$ (différence entre le $\Delta z$ reconstruit $(\Delta z)$ et le $\Delta z$ généré $\left(\Delta z_{v r a i}\right)$ ) et du pull $=$ $\left(\Delta z-\Delta z_{v r a i}\right) / \sigma_{\Delta z}$ obtenue à partir de données simulées $B^{0} \rightarrow J / \psi K_{S}^{0}$. Un ajustement effectué avec une somme de trois gaussiennes est superposé (une gaussienne centrale (core), une gaussienne plus élargie (tail) et une gaussienne quasi plate de valeur centrale $\mu=0$ et de largeur $\sigma=1.33 \mathrm{~mm}$ (outliers)). La troisième gaussienne permet de prendre en compte les cas où le résidu est beaucoup plus grand que l'incertitude calculée par l'ajustement. La gaussienne centrale contient $65 \%$ des événements et a pour résolution $92 \mu \mathrm{m}$ (voir la table 6.6). La fraction des outliers est de $2 \%$. La distribution est biaisée du fait de la présence de traces provenant de la désintégration de mésons charmés dans le vertex du méson $B_{t a g}$. L'algorithme de vertex du méson $B_{t a g}$ essaie d'éliminer ces traces mais la faible longueur de désintégration des mésons $D$ (p. ex. le $c \tau$ du méson $D^{0}$ est de 


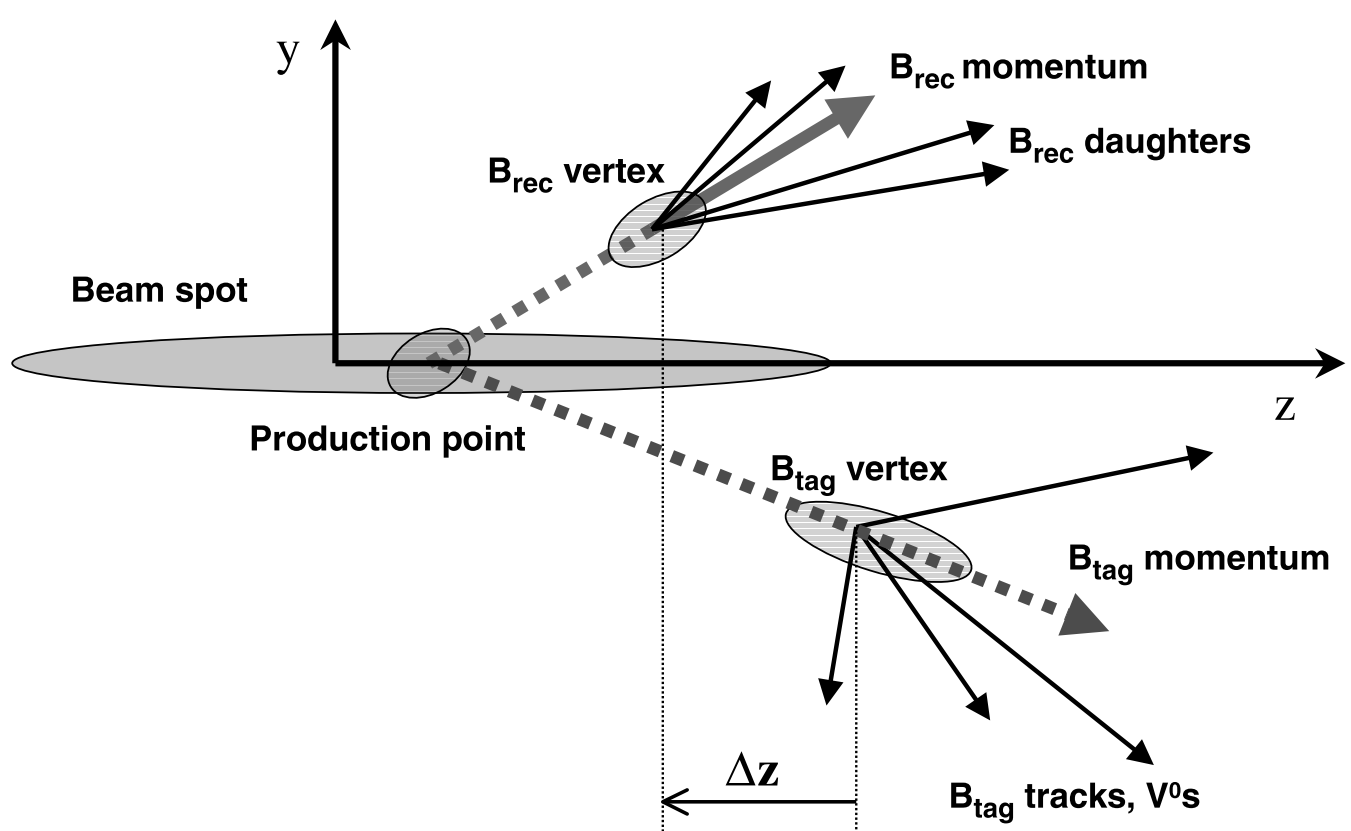

FIG. 6.13: Vue schématique dans le plan $y z$ de la désintégration $\Upsilon(4 S) \rightarrow B \bar{B}$. Pour les modes entièrement reconstruits, la ligne de vol du méson $B_{t a g}$ peut être estimée à partir de l'impulsion opposée et de la position du méson $B_{r e c}$, de la position de la région lumineuse (beamspot) et du «boost » moyen de la résonance $\Upsilon(4 S)$. À noter que l'échelle en $y$ est considérablement agrandie par rapport à l'échelle en $z$.

$124.3 \mu \mathrm{m}$ [28]) par rapport à la résolution ne permet pas une réjection efficace. En conséquence, les traces acceptées « tirent » le vertex vers les valeurs positives en $z$ (du fait du «boost»), ce qui induit un biais négatif sur $\Delta z$. Le biais est d'environ $-20 \mu \mathrm{m}$ pour le résidu. La figure 6.15 montre la distribution de l'erreur et de la probabilité de $\chi^{2}, p \chi^{2}$, où $4 \%$ des événements ont un $p \chi^{2}<1 \%$.

TAB. 6.6: Résultats de l'ajustement à trois gaussiennes (core, tail, outliers) sur les distributions du résidu et du pull de $\Delta z$ (cf. Fig. 6.14) obtenus avec des données simulées $B^{0} \rightarrow J / \psi K_{S}^{0}$. Le RMS inclut uniquement les résolutions des gaussiennes core et tail. D'après [171].

\begin{tabular}{lcc}
\hline & Résidu & pull \\
\hline \hline$f_{\text {core }}$ & $0.654 \pm 0.024$ & $0.842 \pm 0.028$ \\
$f_{\text {out }}$ & $0.020 \pm 0.002$ & $0.014 \pm 0.003$ \\
$\mu_{\text {core }}$ & $-19.4 \pm 1.4$ & $-0.193 \pm 0.017$ \\
$\sigma_{\text {core }}$ & $91.9 \pm 2.4$ & $1.054 \pm 0.020$ \\
$\mu_{\text {tail }}$ & $-60 \pm 2$ & $-1.09 \pm 0.15$ \\
$\sigma_{\text {tail }}$ & $224 \pm 8$ & $1.97 \pm 0.10$ \\
RMS & 151 & 1.25 \\
\hline \hline
\end{tabular}

\subsubsection{Mesure de $\Delta t$}

Pour chaque événement, $\Delta t$, différence des temps propres des deux mésons $B$, est estimé par :

$$
\Delta t=t_{r e c}-t_{t a g}=m_{B}\left[\frac{z_{r e c}}{p_{z}^{r e c}}-\frac{z_{t a g}}{p_{z}^{t a g}}\right] .
$$



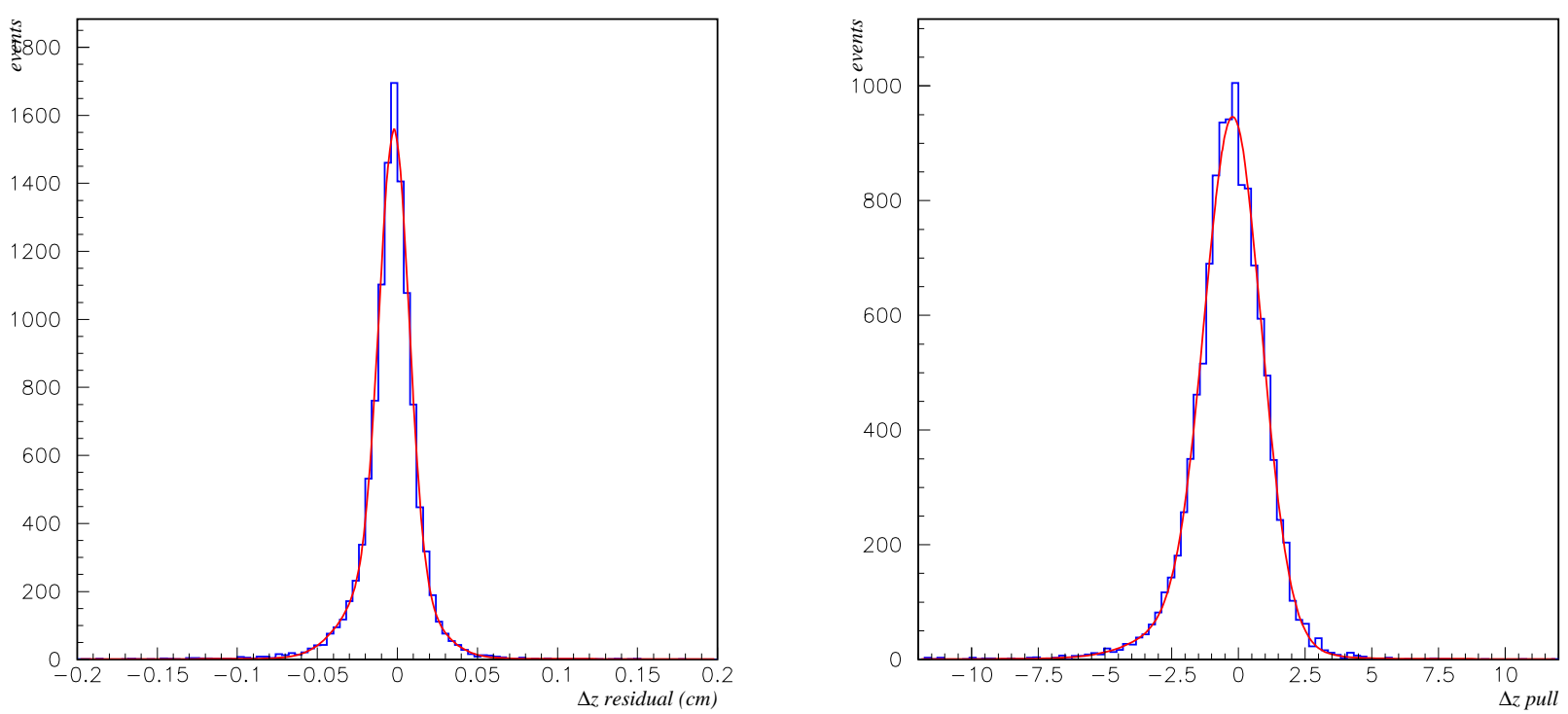

Fig. 6.14: Résidu (figure de gauche) et pull (figure de droite) de $\Delta z$ pour des données simulées $J / \psi K_{S}^{0}$. Un ajustement effectué avec une somme de trois gaussiennes est superposé. D'après [171].
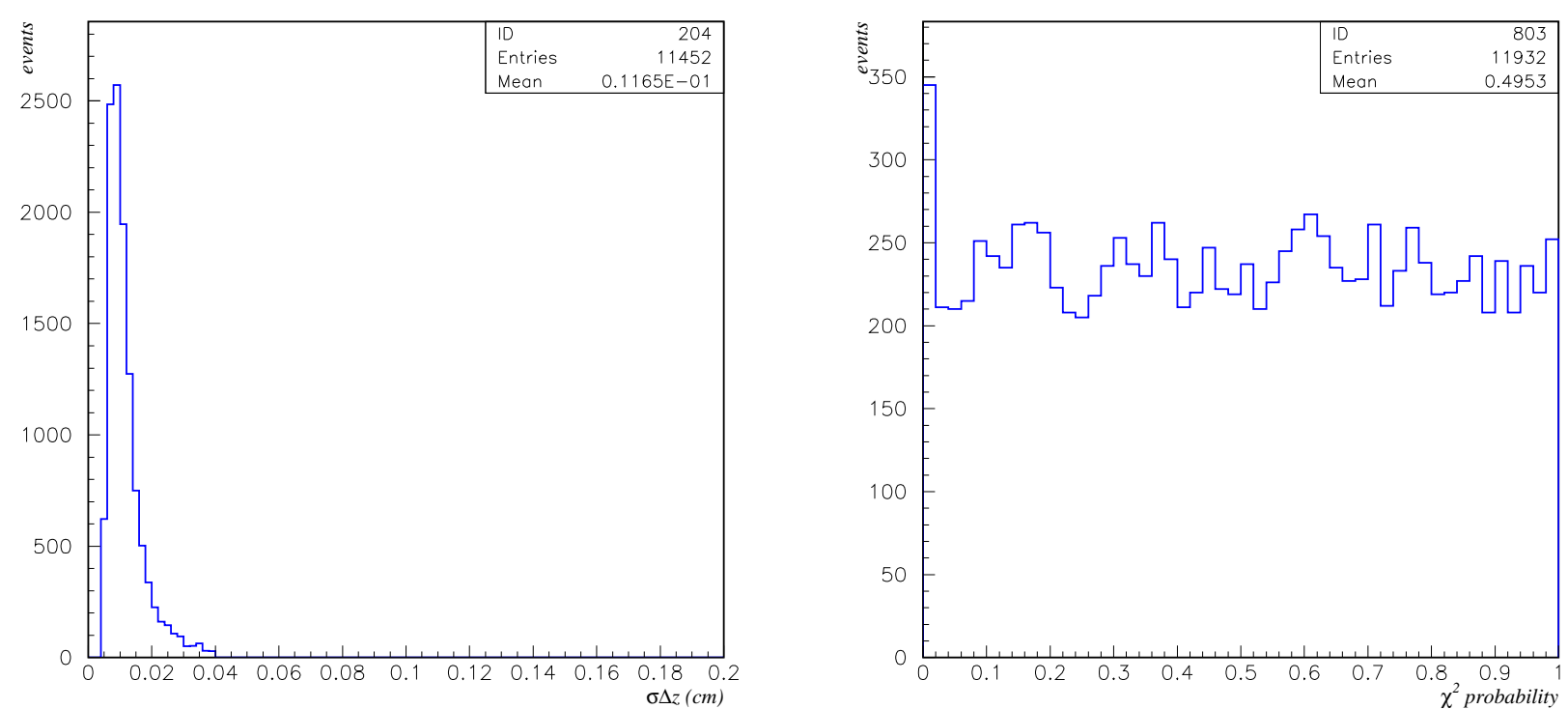

FIG. 6.15: Distributions de l'erreur sur $\Delta z$ (figure de gauche) et de la probabilité de $\chi^{2}$ (figure de droite) pour des données simulées $J / \psi K_{S}^{0}$. La coupure à $400 \mu \mathrm{m}$ sur la distribution de l'erreur de $\Delta z$ est visible. D'après [171]. 
Cependant, chaque temps propre est défini dans des repères différents, ce qui rend la transformation entre $\Delta z$ et $\Delta t$ non-triviale. L'approche prise dans BABAR est de négliger la faible impulsion des mésons $B$ dans le repère de l’ $(4 S)$ au repos $\left(p_{z}^{*} \approx 341 \mathrm{MeV} / c\right.$, cf. §3.1). Avec ces hypothèses,

$$
\Delta t=\frac{\Delta z}{\beta_{z} \gamma c}
$$

où $\gamma$ est le facteur de Lorentz de $1^{\prime} \Upsilon(4 S)$ dans le repère du laboratoire et $\beta_{z}$ est la projection de $\vec{\beta}$ sur l'axe $z$ de BABAR. Le «boost » moyen est calculé directement à partir de l'énergie des faisceaux toutes les cinq secondes avec une incertitude de $0.1 \%$.

Par rapport à la résolution expérimentale sur $\Delta z$, l'effet sur $\Delta t$ de cette approximation est faible mais non négligeable. Par exemple, une étude analytique montre que le biais introduit par cette approximation sur la mesure des temps de vie des mésons $B$ est de l'ordre de $0.4 \%$, ce qui n'est pas négligeable étant données les précisions atteintes avec la statistique accumulée par BABAR. Dans le cas où un des mésons $B$ est entièrement reconstruit (ce qui est le cas de l'échantillon $B_{\text {rec }}$ ), l'impulsion de ce dernier est déterminée avec précision et permet de corriger l'approximation précédente. La relation générale reliant $\Delta z$ à $\Delta t$ est

$$
\Delta z=\beta_{z} \gamma \gamma_{r e c}^{*} c\left(t_{r e c}-t_{t a g}\right)+\gamma \beta_{r e c}^{*} \gamma_{r e c}^{*} \cos \theta_{r e c}^{*} c\left(t_{r e c}+t_{t a g}\right)
$$

où $\beta_{r e c}^{*} c, \gamma_{r e c}^{*}$ et $\theta_{r e c}^{*}$ sont la vitesse, le facteur de Lorentz et l'angle polaire du méson $B_{r e c}$ dans le repère de l' $\Upsilon(4 S)$ au repos. La différence entre $\Delta t$ calculé avec l'équation (6.10) et l'équation (6.11) est faible puisque $\gamma_{r e c}^{*}=1.002$ et $\beta_{r e c}^{*}=0.064$. Cependant, la correction dépend de la somme des temps propres, $t_{r e c}+t_{t a g}$, qui ne peut être déterminée qu'avec une très médiocre résolution. Le problème est donc de pouvoir estimer $t_{r e c}+t_{t a g}$. Une possibilité est de prendre $\left\langle t_{r e c}+t_{t a g}\right\rangle=$ $2 \tau_{B}$, mais cette estimation ne prend pas en compte les variations de $t_{r e c}+t_{t a g}$ avec $\Delta t$. Une autre possibilité est de faire la moyenne sur l'intervalle en $\Delta t$ :

$$
\left.\left\langle t_{\text {rec }}+t_{t a g}\right\rangle\right|_{\Delta t}=\left(\int_{|\Delta t|}^{\infty} x e^{-x / \tau_{B}} \mathrm{~d} x\right) /\left(\int_{|\Delta t|}^{\infty} e^{-x / \tau_{B}} \mathrm{~d} x\right)=\tau_{B}+|\Delta t|,
$$

où la valeur de $\Delta t$ est fixée dans le calcul de la moyenne. On aboutit alors à l'expression

$$
\Delta z \simeq \beta_{z} \gamma \gamma_{r e c}^{*} c\left(t_{r e c}-t_{t a g}\right)+\gamma \beta_{r e c}^{*} \gamma_{r e c}^{*} \cos \theta_{r e c}^{*} c\left(\tau_{B}+|\Delta t|\right)
$$

Pour obtenir $\Delta t$, on suppose tout d'abord qu'il est de même signe que $\Delta z$, puis on vérifie si cette hypothèse est compatible avec l'équation (6.13). Dans le cas contraire, $\Delta t$ et $\Delta z$ sont supposés de signes opposés.

La différence de $\Delta t$ calculé par les deux méthodes (cf. eqs. (6.13) et (6.10)), événement par événement, a un RMS de 0.20 ps. La prise en compte de ce terme améliore la résolution sur $\Delta t$ d'environ $5 \%$. De plus, cette méthode (cf. eq. (6.13)) de calcul de $\Delta t$ permet de minimiser la corrélation entre la résolution sur $\Delta t$ et la valeur vraie de $\Delta t$, corrélation provenant du fait que le deuxième terme de l'équation (6.13) introduit une dépendance en $\left(t_{r e c}+t_{t a g}\right)^{2}$, qui dépend de $\Delta t$. L'estimation de l'erreur statistique, $\sigma_{\Delta t}$, est prise en considérant uniquement la contribution du premier terme de 


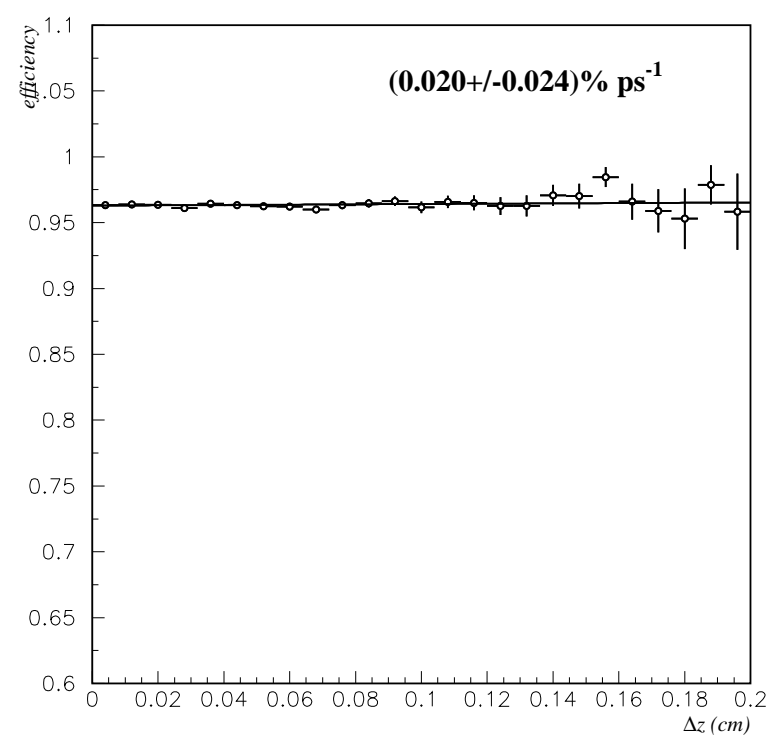

FIG. 6.16: Dépendance de l'efficacité de reconstruction de $\Delta z$ en fonction du $\Delta z$ généré pour des données simulées.

$\Delta z$, i.e.

$$
\sigma_{\Delta t}=\frac{\sigma_{\Delta z}}{\beta_{z} \gamma \gamma_{r e c}^{*} c}
$$

Un certain nombre d'exigences sont faites afin de s'assurer d'une bonne détermination de $\Delta z$. Les ajustements des vertex des mésons $B_{\text {rec }}$ et $B_{t a g}$ doivent converger. De plus, l'erreur sur $\Delta t$ $\left(\sigma_{\Delta t}\right)$ déterminée à partir des ajustements des vertex doit être inférieure à 2.5 ps et $|\Delta t|$ doit être inférieur à 20 ps. L'efficacité, après passage de ces critères, est d'environ $97 \%$ pour tous les modes $B_{r e c}$. L'efficacité de reconstruction de $\Delta z$ en fonction du $\Delta z$ généré est représentée sur la figure 6.16. Aucune dépendance n'est observée.

\subsubsection{Fonction de résolution}

Plusieurs paramétrisations de la fonction de résolution en $\Delta t$ sont possibles. Celle retenue est, comme pour $\Delta z$, la somme de trois gaussiennes (core, tail, outliers) avec des valeurs centrales et des largeurs différentes :

$$
\begin{aligned}
\mathcal{R}(\delta(\Delta t) ; \hat{a})= & \frac{f_{\text {core }}}{\sigma_{\text {core }} \sqrt{2 \pi}} \exp \left(-\frac{\left(\delta(\Delta t)-\mu_{\text {core }}\right)^{2}}{2 \sigma_{\text {core }}^{2}}\right)+\frac{f_{\text {tail }}}{\sigma_{\text {tail }} \sqrt{2 \pi}} \exp \left(-\frac{\left(\delta(\Delta t)-\mu_{\text {tail }}\right)^{2}}{2 \sigma_{\text {tail }}^{2}}\right) \\
& +\frac{f_{\text {out }}}{\sigma_{\text {out }} \sqrt{2 \pi}} \exp \left(-\frac{\delta(\Delta t)^{2}}{2 \sigma_{\text {out }}^{2}}\right)
\end{aligned}
$$

où $\delta(\Delta t)$ dénote le résidu $\Delta t-\Delta t_{\text {vrai }}$ et $f_{\text {core }}+f_{\text {tail }}+f_{\text {out }}=1$. L'estimation événement par événement de l'incertitude $\sigma_{\Delta t}$ contient de l'information quant à la qualité de reconstruction. Ainsi, pour les deux gaussiennes principales (core et tail), leurs largeurs sont proportionnelles à $\sigma_{\Delta t}$, i.e. $\sigma_{\text {core }}=$ $S_{\text {core }} \sigma_{\Delta t}$ et $\sigma_{t a i l}=S_{t a i l} \sigma_{\Delta t}$. Les facteurs d'échelle, $S_{\text {core }}$ et $S_{t a i l}$, permettent d'accommoder une sousestimation $(S<1)$ ou surestimation $(S>1)$ globale des erreurs pour tous les événements. La figure 
6.17 a) montre la corrélation existante entre le RMS de $\delta(\Delta t)$ et $\sigma_{\Delta t}$ obtenue à partir de données simulées. Leurs valeurs centrales sont autorisées à être différentes de zéro pour prendre en compte le biais dû aux désintégrations de mésons charmés dans la détermination du vertex du méson $B_{t a g}$. Dans la fonction de résolution, ces valeurs centrales sont proportionnelles à $\sigma_{\Delta t}$ ( $\mu_{\text {core }}=b_{\text {core }} \sigma_{\Delta t}$ et $\mu_{\text {tail }}=b_{t a i l} \sigma_{\Delta t}$ ) pour tenir compte de la corrélation observée [172] entre la valeur centrale et $\sigma_{\Delta t}$, illustrée sur la figure 6.17 b) à partir de données simulées.
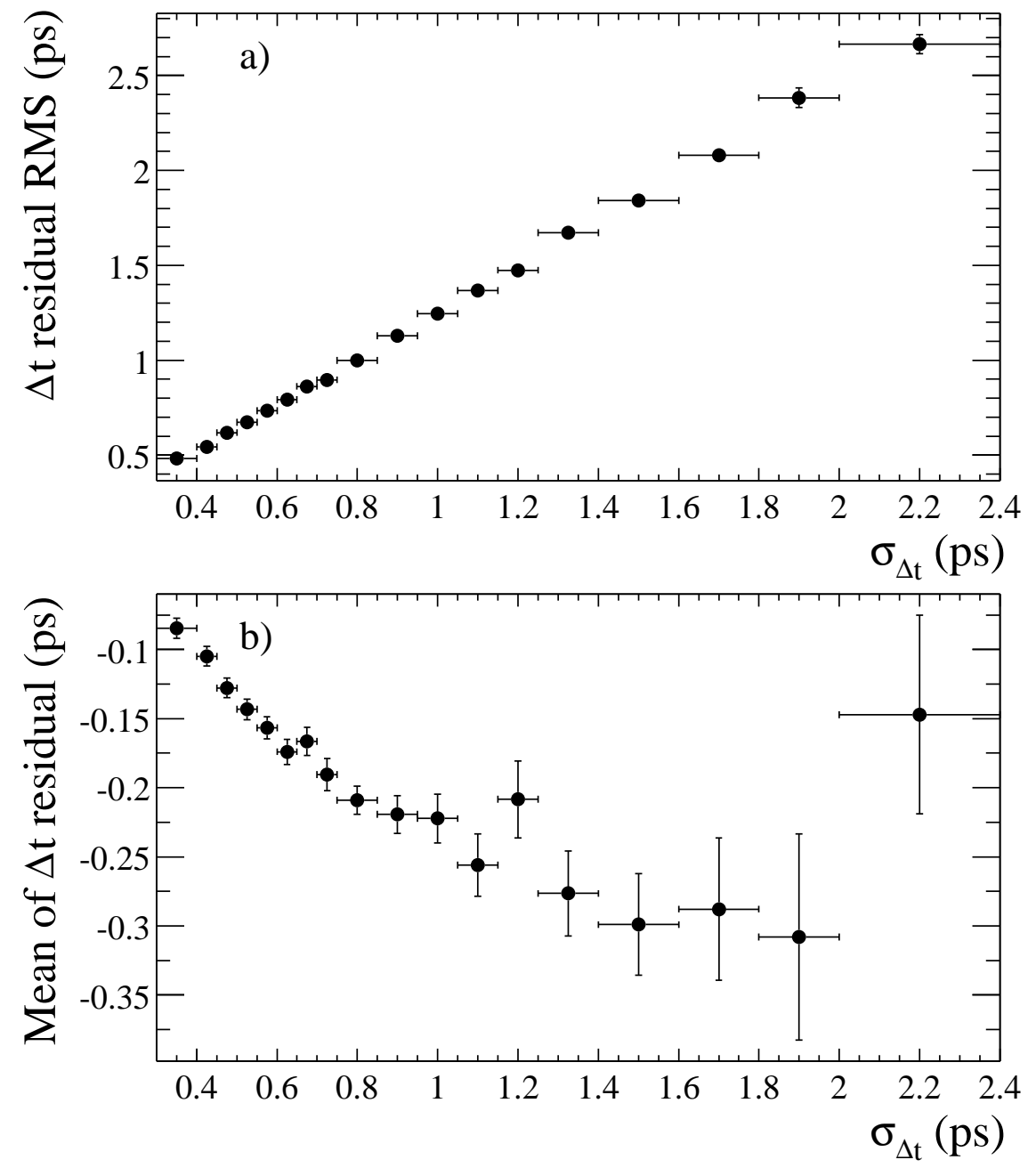

FIG. 6.17: Corrélations obtenues à partir de données simulées entre l'erreur sur $\Delta t$ déterminée événement par événement et a) le RMS de $\delta(\Delta t)$, b) l'écart à la valeur moyenne de $\delta(\Delta t)$.

La fonction de résolution dépend des catégories d'étiquetage simplement par le fait que cette dernière est moins biaisée pour la catégorie basée sur un lepton primaire que pour la catégorie basée sur les kaons. Ainsi, $b_{\text {core }}$ est autorisé à être différent selon la catégorie d'étiquetage alors que $b_{\text {tail }}$ est pris indépendant des catégories d'étiquetage. Enfin, la troisième gaussienne (outliers) a une largeur fixée à 8 ps et une valeur centrale nulle afin de prendre en compte la faible fraction d'événements qui ont un $\Delta z$, et par conséquent un $\Delta t$, mal reconstruit. Les paramètres de la fonction de résolution extraits de l'ajustement par vraisemblance sur les données sont résumés dans la table 6.7.

Bien que la résolution en $z$ du méson $B_{r e c}$ dépende de son mode de désintégration (cf. §6.3.1), le fait que la résolution en $\Delta z$ (et donc en $\Delta t$ ) soit dominée par la résolution du vertex du méson $B_{t a g}$ 
TAB. 6.7: Paramètres de la fonction de résolution signal obtenus sur l'échantillon $B_{\text {sav }}$.

\begin{tabular}{|l|c|}
\hline \multicolumn{2}{|c|}{ Fonction de résolution Signal } \\
\hline Paramètre & Résultat \\
\hline$S_{\text {core }}$ & $1.092 \pm 0.048$ \\
$S_{\text {tail }}$ & 3.0 (fixé) \\
$b_{\text {core }}$ Lepton & $0.0132 \pm 0.063$ \\
$b_{\text {core Kaon I }}$ & $-0.230 \pm 0.052$ \\
$b_{\text {core Kaon I I }}$ & $-0.246 \pm 0.046$ \\
$b_{\text {core }}$ Inclusive & $-0.207 \pm 0.047$ \\
$b_{\text {tail }}$ & $-1.003 \pm 0.289$ \\
$f_{\text {tail }}$ & $0.109 \pm 0.020$ \\
$f_{\text {out }}$ & $0.002 \pm 0.001$ \\
\hline
\end{tabular}

implique que l'on ne s'attende pas à une différence importante, pour la fonction de résolution, entre l'échantillon spécifique de saveur $B_{\text {sav }}$ et les modes $C P$. Ainsi, la même fonction de résolution est utilisée pour tous les modes $B_{r e c}$. Afin de vérifier cette hypothèse, une comparaison de la probabilité de $\chi^{2}$, de l'erreur sur $\Delta z$ événement par événement, ainsi que du nombre de traces intervenant dans le vertex du méson $B_{t a g}$ est donnée sur la figure 6.18. Aucune différence significative n'est observée, confirmant que la résolution de la mesure de $\Delta z$ est dominée par le méson $B_{t a g}$ et donc quasi indépendante des différents modes entièrement reconstruits. Cependant, la figure présentant les variation de l'erreur sur $\Delta z$, événement par événement, montre que cette erreur est légèrement meilleure pour les modes $C P$ que pour les modes spécifiques de saveur. Cela est dû au fait que le vertex du méson $B_{C P}$ est mieux déterminé à cause d'une multiplicité plus faible de traces et des impulsions plus élevées. Cet effet est pris en compte en grande partie en prenant la valeur de $\sigma_{\Delta t}$ obtenue événement par événement.

\subsection{Ajustement par maximum de vraisemblance}

Les valeurs de $\sin 2 \beta$ et de $\cos 2 \beta$ sont obtenues à partir d'un ajustement par maximum de vraisemblance, $\mathcal{L}_{C P}$, basé sur la densité de probabilité normalisée dépendante du temps et des angles de transversité du canal $B_{d}^{0} \rightarrow J / \psi\left(K_{S}^{0} \pi^{0}\right)^{* 0}$. Cependant, les dilutions $D_{c}$ ainsi que les paramètres de la fonction de résolution en $\Delta t$ sont nécessaires pour pouvoir extraire $\sin 2 \beta$ et $\cos 2 \beta$. Ces divers paramètres sont obtenus grâce à l'échantillon spécifique de saveur, ayant une statistique bien plus importante, en faisant l'hypothèse qu'ils sont indépendants du mode de désintégration du méson $B_{r e c}$. Afin de prendre en compte les corrélations entre $\sin 2 \beta, \cos 2 \beta$ et ces paramètres, un ajustement global est effectué en maximisant simultanément $\mathcal{L}_{C P}+\mathcal{L}_{\text {sav }}$ sur les événements combinés $B_{C P}+B_{\text {sav }}$.

On s'attend à ce que les probabilités de mauvais étiquetage $w$ pour un $B^{0}$ et un $\bar{B}^{0}$ soient proches mais par exactement égales. La réponse du détecteur à des pions et kaons positifs diffère de celle à des pions et kaons négatifs. Pour prendre en compte ces différences, différentes probabilités de 

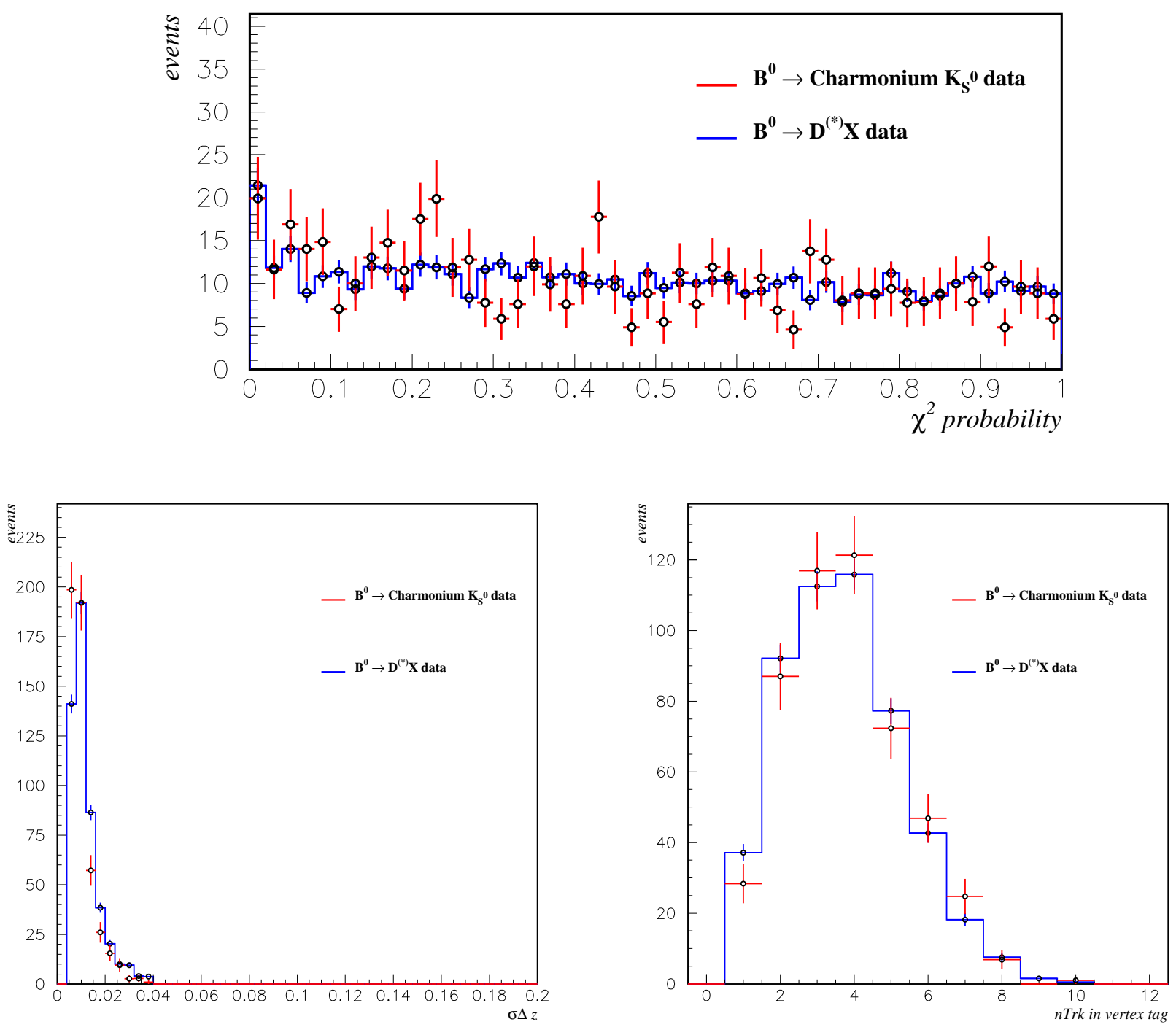

FIG. 6.18: Comparaison des distributions de la probabilité de $\chi^{2}$ (figure du haut), de l'erreur sur $\Delta z$ événement par événement (figure de gauche) et du nombre de traces intervenant dans le vertex du méson $B_{\text {ta } g}$ (figure de droite) à partir des données entre l'échantillon $B_{s a v}$ et l'échantillon $B_{C P}$ (comprenant tous les modes $C P$ ). 
mauvais étiquetage sont utilisées, w pour un $B^{0}$ et $\bar{w}$ pour un $\bar{B}^{0}$. On définit alors les dilutions

$$
D=1-2 w, \quad \bar{D}=1-2 \bar{w}
$$

conduisant à une dilution moyenne et une différence de dilution

$$
\langle D\rangle=\frac{D+\bar{D}}{2}, \quad \Delta D=D-\bar{D}
$$

Les probabilités de mauvais étiquetage s'expriment en fonction de $\langle D\rangle$ et $\Delta D$ :

$$
w=\frac{1-\langle D\rangle-\Delta D / 2}{2}, \quad \bar{w}=\frac{1-\langle D\rangle+\Delta D / 2}{2} .
$$

De manière générale, les distributions observées en fonction des distributions vraies étiquetées $B^{0}$ et $\bar{B}^{0}$ sont reliées par:

$$
\begin{aligned}
f_{B_{t a g}=B^{0}, o b s} & =(1-w) f_{B_{t a g}=B^{0}, v r a i}+\bar{w} f_{B_{t a g}=\bar{B}^{0}, v r a i} \\
f_{B_{t a g}=\bar{B}^{0}, o b s} & =(1-\bar{w}) f_{B_{t a g}=\bar{B}^{0}, v r a i}+w f_{B_{t a g}=B^{0}, v r a i} .
\end{aligned}
$$

Les sections suivantes détaillent les distributions utilisées pour les mesures de $\sin 2 \beta$ et $\cos 2 \beta$ en tenant compte des bruits de fond, des probabilités de mauvais étiquetage et de la résolution finie du détecteur.

\subsubsection{Ajustement pour l'échantillon $B_{s a v}$}

Afin de prendre en compte les possibles différences entre les probabilités de mauvais étiquetage, les distributions (6.3) sont modifiées :

$$
\begin{aligned}
& h_{\text {Mixed }}^{h_{\text {Mixed }}, B_{t a g}=B^{0}}(\Delta t) \propto\left[\left(1+\frac{\Delta D}{2}\right) \pm\langle D\rangle \cos (\Delta M \Delta t)\right], \\
& h_{\substack{\text { Mixmed } \\
h_{\text {nixed }}, B_{\text {tag }}=\bar{B}^{0}}}(\Delta t) \propto\left[\left(1-\frac{\Delta D}{2}\right) \pm\langle D\rangle \cos (\Delta M \Delta t)\right] .
\end{aligned}
$$

En présence de bruits de fond, les distributions $\mathcal{H}_{\begin{array}{l}\text { Unixixed } \\ \text { Mixed }\end{array}}$ (cf. eq. (6.4)) doivent être étendues pour décrire la dépendance temporelle de chaque source de bruits. Les paramétrisations des bruits de fond sont laissées libres de varier selon les quatre catégories d'étiquetage (voir Fig. 6.19). Chaque événement est définie par sa catégorie d'étiquetage, $c$, à laquelle il appartient (il ne peut appartenir qu'à une catégorie), par la différence entre les saveurs initiale et finale (Unmixed ou Mixed) et par la saveur du méson $B_{t a g}$. La distribution observée est donnée par :

$$
\begin{aligned}
& \mathcal{H}_{\text {Mixed }}^{\mathcal{U n}_{\text {nmixed }}, B_{\text {tag }}={ }_{\bar{B}^{0}}^{B^{0}}, c}=f_{c, s i g}^{\text {sav }} \mathcal{H}_{\text {Mixed }^{\text {nnmixed }}, B_{t a g}={ }_{\bar{B}^{0}}}\left(\Delta t ; \Gamma_{0}, \Delta M,\langle D\rangle_{c}, \Delta D_{c}, \hat{a}_{c}\right)+ \\
& f_{c, \text { peak }}^{\text {sav }} \mathcal{B}_{\text {Mixed }}^{\text {Mixixed }}, B_{\text {tag }}=\frac{B_{B^{0}}^{0}}{B^{0}}\left(\Delta t ; \mathcal{K}_{\text {peak }, c}^{\text {sav }}, \hat{a}_{c}\right)+ \\
& \sum_{\alpha=b k g} f_{c, \alpha}^{\text {sav }} \mathcal{B}_{\text {Mixed }}^{\text {Mixed }}, B_{t a g}={ }_{\bar{B}^{0}, \alpha}^{B^{0}}\left(\Delta t ; \mathcal{K}_{\alpha, c}^{\text {sav }}, \hat{b}_{c}\right) .
\end{aligned}
$$



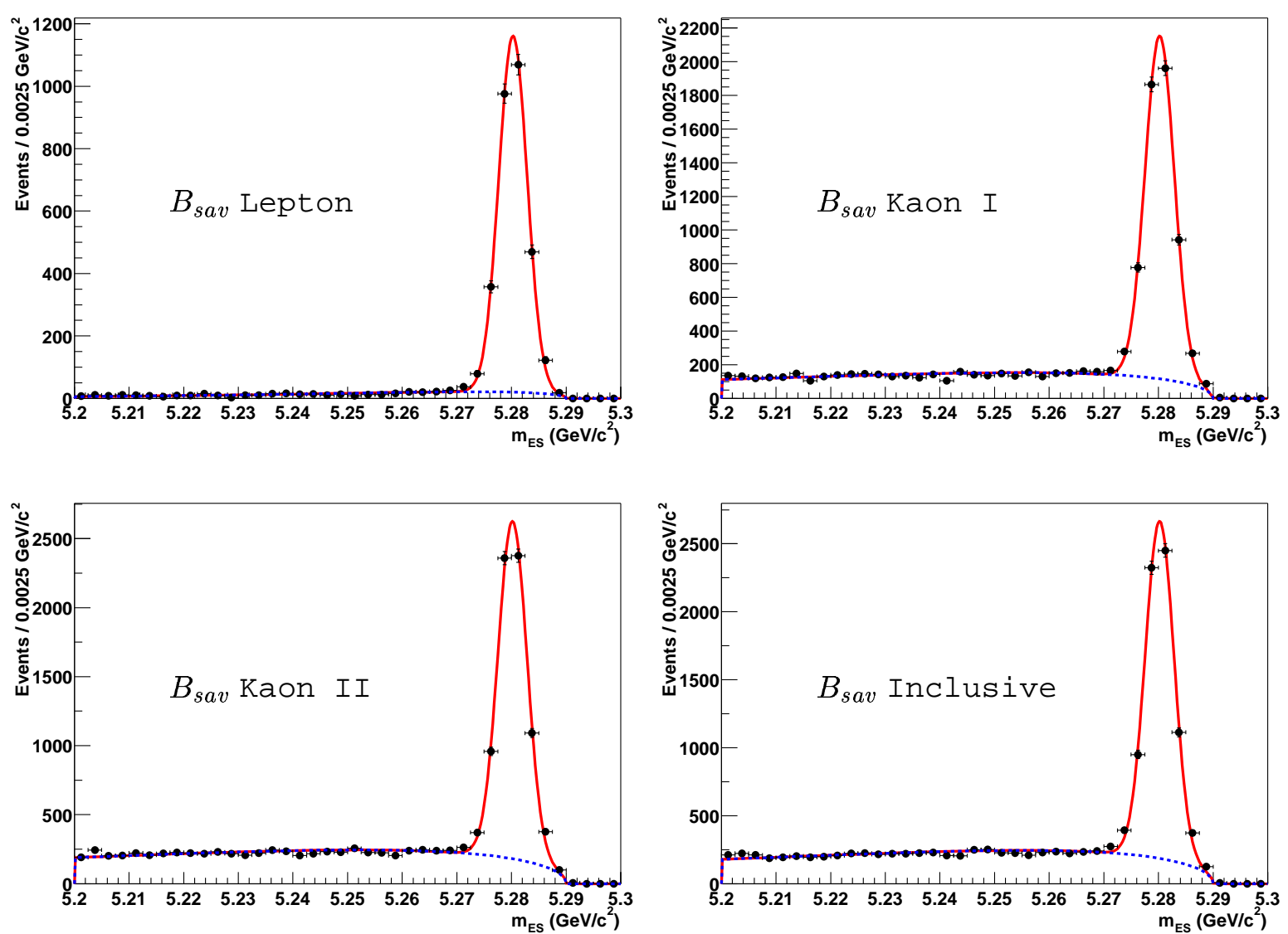

FIG. 6.19: Échantillon $B_{s a v}$, après étiquetage et application des critères de qualité sur les vertex, séparé selon les quatre catégories d'étiquetage: Lept on (haut gauche), Kaon I (haut droite), Kaon I I (bas gauche), et Inclusive (bas droite). Un ajustement par une gaussienne et une fonction Argus est superposé.

TAB. 6.8: Résultats des ajustements en $m_{\mathrm{ES}}$ pour l'échantillon $B_{\text {sav }}$ après étiquetage et application des critères de qualité sur les vertex. Le nombre d'événements de bruits de fond correspond à l'aire de la distribution Argus. La pureté est calculée pour les événements avec $m_{\mathrm{ES}}>5.27 \mathrm{GeV} / c^{2}$.

\begin{tabular}{|l|c|c|c|c|}
\hline$B_{\text {sav }}$ & Lepton & Kaon I & Kaon I I & Inclusive \\
\hline \hline$N_{\text {evt }}$ signal & $2979 \pm 57$ & $5450 \pm 83$ & $6489 \pm 92$ & $6535 \pm 94$ \\
\hline Moyenne $\left(\mathrm{MeV} / c^{2}\right)$ & $5280.30 \pm 0.05$ & $5280.20 \pm 0.04$ & $5280.20 \pm 0.04$ & $5280.20 \pm 0.04$ \\
\hline$\sigma(\mathrm{MeV})$ & $2.60 \pm 0.04$ & $2.64 \pm 0.04$ & $2.62 \pm 0.03$ & $2.60 \pm 0.04$ \\
\hline$N_{\text {evt }}$ bkg & $491 \pm 28$ & $4745 \pm 78$ & $7677 \pm 99$ & $7599 \pm 99$ \\
\hline Pureté & $0.956 \pm 0.005$ & $0.862 \pm 0.005$ & $0.826 \pm 0.005$ & $0.823 \pm 0.005$ \\
\hline \hline
\end{tabular}


La fraction signal $\left(f_{c, s i g}^{s a v}\right)$ et celle des bruits de fond $\left(f_{c, \alpha}^{s a v}\right)$ sont déterminées à partir des distributions en $m_{\mathrm{ES}}$ après étiquetage et application des critères de qualité sur les vertex des deux mésons $B$ en effectuant un ajustement par une gaussienne, $G_{c}\left(m_{\mathrm{ES}}\right)$, et une fonction Argus, $A_{c}\left(m_{\mathrm{ES}}\right)$, pour chaque catégorie d'étiquetage et avant d'effectuer l'ajustement global (cf. Fig. 6.19 et la table 6.8). À partir de ces ajustements sont extraites les probabilités signal et bruits de fond événement par événement par :

$$
\begin{aligned}
f_{c, s i g}^{s a v} & =\frac{\left(1-\delta_{\text {peak }}\right) G_{c}\left(m_{\mathrm{ES}}\right)}{G_{c}\left(m_{\mathrm{ES}}\right)+A_{c}\left(m_{\mathrm{ES}}\right)} \\
f_{c, p e a k}^{s a v} & =\frac{\delta_{p e a k} G_{c}\left(m_{\mathrm{ES}}\right)}{G_{c}\left(m_{\mathrm{ES}}\right)+A_{c}\left(m_{\mathrm{ES}}\right)}, \\
\sum_{\alpha=b k g} f_{c, \alpha}^{s a v} & =\frac{A_{c}\left(m_{\mathrm{ES}}\right)}{G_{c}\left(m_{\mathrm{ES}}\right)+A_{c}\left(m_{\mathrm{ES}}\right)} .
\end{aligned}
$$

La fraction de bruits de fond qui s'accumule sous le pic du signal, $\delta_{\text {peak }}$, est obtenue à partir de données simulées.

Étant donné que les bruits de fond peuvent avoir de nombreuses origines, une description empirique est utilisée dans l'ajustement plutôt qu'une identification de chaque source. Sont considérés les bruits de fond du type $\tau>0$ (temps de vie), $\tau=0$ et mélange, chacun avec son propre facteur de dilution effectif, $D_{\alpha, c}^{s a v}$, et convolués avec la même fonction de résolution en $\Delta t, \mathcal{R}\left(\delta(\Delta t) ; \hat{b}_{c}\right)$ :

$$
\begin{aligned}
& \mathcal{B}_{\text {Uixned }}^{\text {Mixixed }, B_{\text {tag }}={ }_{B^{0}}^{B^{0}}, \alpha=1}\left(\Delta t ; \mathcal{K}_{1, c}^{\text {sav }}, \hat{b}_{c}\right)=\left(1 \pm D_{1, c}^{\text {sav }}\right) \delta\left(\Delta_{\text {vrai }}\right) \otimes \mathcal{R}\left(\delta(\Delta t) ; \hat{b}_{c}\right), \\
& \mathcal{B}_{\text {Unixed }}{\text { Mixed }, B_{\text {tag }}={ }_{\bar{B}^{0}}^{B^{0}, \alpha=2}}\left(\Delta t ; \mathcal{K}_{2, c}^{\text {sav }}, \hat{b}_{c}\right)=\frac{\Gamma_{2, c}^{s a v}}{2} e^{-\Gamma_{2, c}^{s a v}\left|\Delta_{v r a i}\right|}\left(1 \pm D_{2, c}^{s a v}\right), \otimes \mathcal{R}\left(\delta(\Delta t) ; \hat{b}_{c}\right),
\end{aligned}
$$

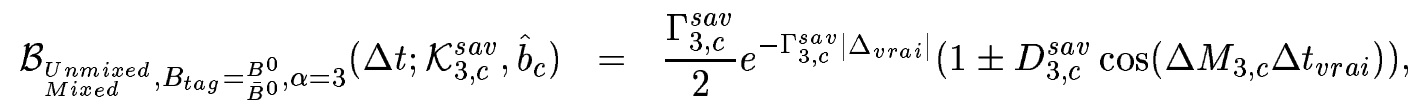

$$
\begin{aligned}
& \otimes \mathcal{R}\left(\delta(\Delta t) ; \hat{b}_{c}\right) \\
& \mathcal{B}_{\text {Mixixed }^{\text {nixed }}, B_{\text {tag }}={ }_{\bar{B}}^{B^{0}}, \text { peak }}\left(\Delta t ; \mathcal{K}_{\text {peak }, c}^{\text {sav }}, \hat{a}_{c}\right)=\frac{\Gamma_{\text {peak }, c}^{\text {sav }}}{2} e^{-\Gamma_{\text {peak }, c}^{\text {sav }}\left|\Delta_{\text {vrai }}\right|}\left(1 \pm D_{\text {peak }, c}^{\text {sav }} \cos \left(\Delta M_{\text {peak }, c} \Delta t_{\text {vrai }}\right)\right), \\
& \otimes \mathcal{R}\left(\delta(\Delta t) ; \hat{a}_{c}\right)
\end{aligned}
$$

où $\hat{b}_{c}$ représente les paramètres de la fonction de résolution des bruits de fond, pour une catégorie d'étiquetage $c$, et $\mathcal{K}_{1, c}^{s a v}=D_{1, c}^{\text {sav }} ; \mathcal{K}_{2, c}^{\text {sav }}=\Gamma_{2, c}^{\text {sav }}, D_{2, c}^{\text {sav }} ; \mathcal{K}_{3, c}^{\text {sav }}=\Gamma_{3, c}^{\text {sav }}, D_{3, c}^{\text {sav }}, \Delta M_{3, c}$ et $\mathcal{K}_{\text {peak }, c}^{\text {sav }}=$ $\Gamma_{\text {peak,c }}^{\text {sav }}, D_{\text {peak,c }}^{\text {sav }}, \Delta M_{\text {peak }, c}$.

\subsubsection{Ajustement pour le canal $B_{d}^{0} \rightarrow J / \psi\left(K_{S}^{0} \pi^{0}\right)^{* 0}$}

La distribution angulaire complète dépendante du temps du canal $B_{d}^{0} \rightarrow J / \psi\left(K_{S}^{0} \pi^{0}\right)^{* 0}$ est fonction de $\Delta t$ et $\vec{\omega} \equiv\left(\cos \theta_{K^{*}}, \cos \theta_{t r}, \varphi_{t r}\right)$, donnée par (cf. eq. (2.73)) :

$$
f_{ \pm}\left(\Delta t, \vec{\omega} ; \Gamma_{0}, \Delta M, \sin 2 \beta, \cos 2 \beta, \vec{A}\right)=\frac{\Gamma_{0}}{2} e^{-\Gamma_{0}|\Delta t|} \cdot \mathcal{A}(\vec{\omega} ; \vec{A}) \cdot[1 \mp a(\Delta t, \vec{\omega} ; \Delta M, \sin 2 \beta, \cos 2 \beta, \vec{A})]
$$


où l'asymétrie $a(\Delta t, \vec{\omega} ; \Delta M, \sin 2 \beta, \cos 2 \beta, \vec{A})$ est définie par :

$$
\begin{aligned}
a(\Delta t, \vec{\omega} ; \Delta M, \sin 2 \beta, \cos 2 \beta, \vec{A})=\left\{\frac{\mathcal{P}(\vec{\omega} ; \vec{A})}{\mathcal{A}(\vec{\omega} ; \vec{A})} \cdot \cos (\Delta M \Delta t)+\right. \\
\left.\sin (\Delta M \Delta t) \cdot\left[\frac{\mathcal{S}(\vec{\omega} ; \vec{A})}{\mathcal{A}(\vec{\omega} ; \vec{A})} \cdot \sin (2 \beta)+\frac{\mathcal{C}(\vec{\omega} ; \vec{A})}{\mathcal{A}(\vec{\omega} ; \vec{A})} \cdot \cos (2 \beta)\right]\right\} .
\end{aligned}
$$

Les signes + et - en indice de $f$ indique la saveur du méson $B_{t a g}, B_{d}^{0}$ ou $\bar{B}_{d}^{0}$, respectivement. Les termes $\mathcal{A}, \mathcal{P}, \mathcal{S}$ et $\mathcal{C}$ contiennent la dépendance angulaire et sont fonctions des trois amplitudes de transversité $\vec{A} \equiv\left(A_{0}, A_{\|}, A_{\perp}\right)$ normalisées :

$$
\begin{aligned}
& \mathcal{A}(\vec{\omega} ; \vec{A})=f_{1}(\vec{\omega})\left|A_{0}\right|^{2}+f_{2}(\vec{\omega})\left|A_{\|}\right|^{2}+f_{3}(\vec{\omega})\left|A_{\perp}\right|^{2}+f_{5}(\vec{\omega})\left|A_{\|}\right|\left|A_{0}\right| \cos \left(\delta_{\|}-\delta_{0}\right), \\
& \mathcal{P}(\vec{\omega} ; \vec{A})=f_{4}(\vec{\omega})\left|A_{\|}\right|\left|A_{\perp}\right| \sin \left(\delta_{\perp}-\delta_{\|}\right)+f_{6}(\vec{\omega})\left|A_{\perp}\right|\left|A_{0}\right| \sin \left(\delta_{\perp}-\delta_{0}\right), \\
& \mathcal{S}(\vec{\omega} ; \vec{A})=f_{1}(\vec{\omega})\left|A_{0}\right|^{2}+f_{2}(\vec{\omega})\left|A_{\|}\right|^{2}-f_{3}(\vec{\omega})\left|A_{\perp}\right|^{2}+f_{5}(\vec{\omega})\left|A_{\|}\right|\left|A_{0}\right| \cos \left(\delta_{\|}-\delta_{0}\right), \\
& \mathcal{C}(\vec{\omega} ; \vec{A})=-f_{4}(\vec{w})\left|A_{\perp}\right|\left|A_{\|}\right| \cos \left(\delta_{\perp}-\delta_{\|}\right)-f_{6}(\vec{\omega})\left|A_{\perp}\right|\left|A_{0}\right| \cos \left(\delta_{\perp}-\delta_{0}\right) .
\end{aligned}
$$

Les fonctions $f_{i}(\vec{\omega})(i=1, \ldots, 6)$ dépendent de la définition des repères de transversité dans lesquels les angles de transversité sont calculés. Elles sont données dans la table 2.2. Les phases fortes $\delta_{\|}$et $\delta_{\perp}$ sont définies par rapport à la phase de l'amplitude $A_{0}\left(\delta_{0}=0\right): \delta_{\|}=\arg \left(A_{\|} A_{0}^{*}\right)$ et $\delta_{\perp}=\arg \left(A_{\perp} A_{0}^{*}\right)$. En l'absence de ces phases fortes, le terme $\mathcal{P}$ vaut zéro. Les distributions de $\mathcal{A}, \mathcal{P} / \mathcal{A}, \mathcal{S} / \mathcal{A}$ et $\mathcal{C} / \mathcal{A}$ avec les amplitudes fixées aux premières valeurs mesurées par BABAR [160] sont représentées sur la figure 6.20, à la génération et à la reconstruction. L'efficacité en fonction de $\mathcal{A}, \mathcal{P} / \mathcal{A}, \mathcal{S} / \mathcal{A}$ et $\mathcal{C} / \mathcal{A}$ est représentée sur la figure 6.21. Les figures 6.21 et 6.22 montrent les fonctions angulaires $\mathcal{A}, \mathcal{P} / \mathcal{A}, \mathcal{S} / \mathcal{A}$ et $\mathcal{C} / \mathcal{A}$ en fonction de chaque angle de transversité. On remarque que le maximum de sensibilité pour $\sin 2 \beta$ correspond à l'absence de sensibilité pour $\cos 2 \beta(\mathcal{C} / \mathcal{A}$ pique à zéro). De plus, $\cos 2 \beta$ est moins sensible aux effets d'acceptance puisque $\mathcal{C} / \mathcal{A}$ pique à zéro pour $\cos \theta_{K^{*}}$ proche de 1 (cf. Fig. 4.9). À noter que les fonctions $\mathcal{S}$ et $\mathcal{C}$ sont orthogonales, ce qui implique que, dans le cas d'une acceptance parfaite, $\sin 2 \beta$ et $\cos 2 \beta$ ne sont pas corrélés (en traitant $\sin 2 \beta$ et $\cos 2 \beta$ comme deux paramètres dans l'ajustement).

D'après l'expression de l'asymétrie (6.28), on remarque que la dépendance temporelle en facteur de $\sin 2 \beta$ et $\cos 2 \beta$, pondérés par les fonctions angulaires $\mathcal{S} / \mathcal{A}$ et $\mathcal{C} / \mathcal{A}$ respectivement, est $\sin (\Delta M \Delta t)$. Ainsi, $\sin 2 \beta$ et $\cos 2 \beta$ sont distingués uniquement par la dépendance angulaire. Le terme $\mathcal{S} / \mathcal{A}$ pondérant $\sin 2 \beta$ représente une sorte de " parité $C P$ » continue $^{1}$ allant de -1 à +1 (cf. Fig. 6.20 c). L'expression du terme $\mathcal{C}$ pondérant $\cos 2 \beta$ dans l'équation (6.28) montre l'interférence de l'amplitude de parité $C P$ impaire, $A_{\perp}$, avec les amplitudes de parité $C P$ paires, $A_{\|}$et $A_{0}$. Du fait de la forme des fonctions angulaires $f_{4}(\vec{w})$ et $f_{6}(\vec{\omega}), \mathcal{C}$ pique à zéro (cf. Fig. $6.20 \mathrm{~d}$ ), conduisant à une suppression de sensibilité sur $\cos 2 \beta$.

L'utilisation de la distribution angulaire complète apporte une meilleure sensibilité sur $\sin 2 \beta$, illustrée sur la figure 6.23. En intégrant sur les angles de transversité, chaque événement serait

\footnotetext{
${ }^{1} \hat{A}$ noter que dans les configurations particulières d'amplitudes conduisant à un état final propre de $C P$, i.e. $\left\{\left|A_{\perp}\right|=1 ; A_{0}=0, A_{\|}=0\right\}$ et $\left.\left\{A_{\perp}=0 ;\left(A_{0}, A_{\|}\right) \neq(0,0)\right\}\right)$, l'asymétrie (6.28) se réduit à $\left[1 \mp \sin \left(\Delta m_{d} \Delta t\right) \sin (2 \beta)\right]$ et $\left[1 \pm \sin \left(\Delta m_{d} \Delta t\right) \sin (2 \beta)\right]$ respectivement, comme on s'y attend. Dans ces deux cas, $\cos 2 \beta$ disparaît.
} 

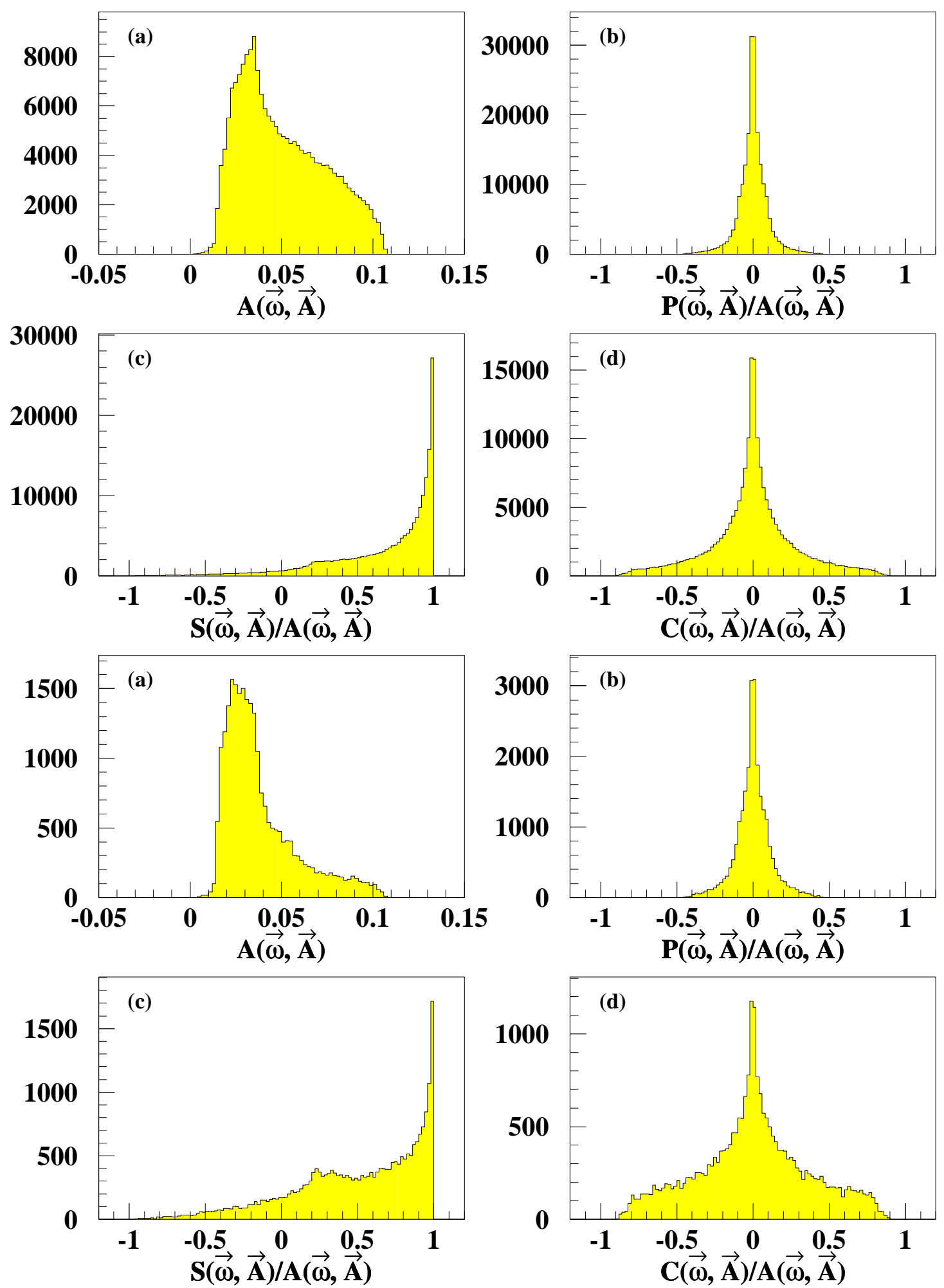

FIG. 6.20: Distributions de $\mathcal{A}, \mathcal{P} / \mathcal{A}, \mathcal{S} / \mathcal{A}$ et $\mathcal{C} / \mathcal{A}$ entrant dans l'équation (6.27) à la génération (les quatre figures du haut) et à la reconstruction (les quatre figures du bas). Les amplitudes de transversité sont fixées aux premières valeurs mesurées par BABAR [160], à savoir $\left|A_{0}\right|^{2}=0.597,\left|A_{\|}\right|^{2}=0.243,\left|A_{\perp}\right|=0.160, \delta_{\|}=2.5$ et $\delta_{\perp}=-0.17$ (ToyMC, 200K). 

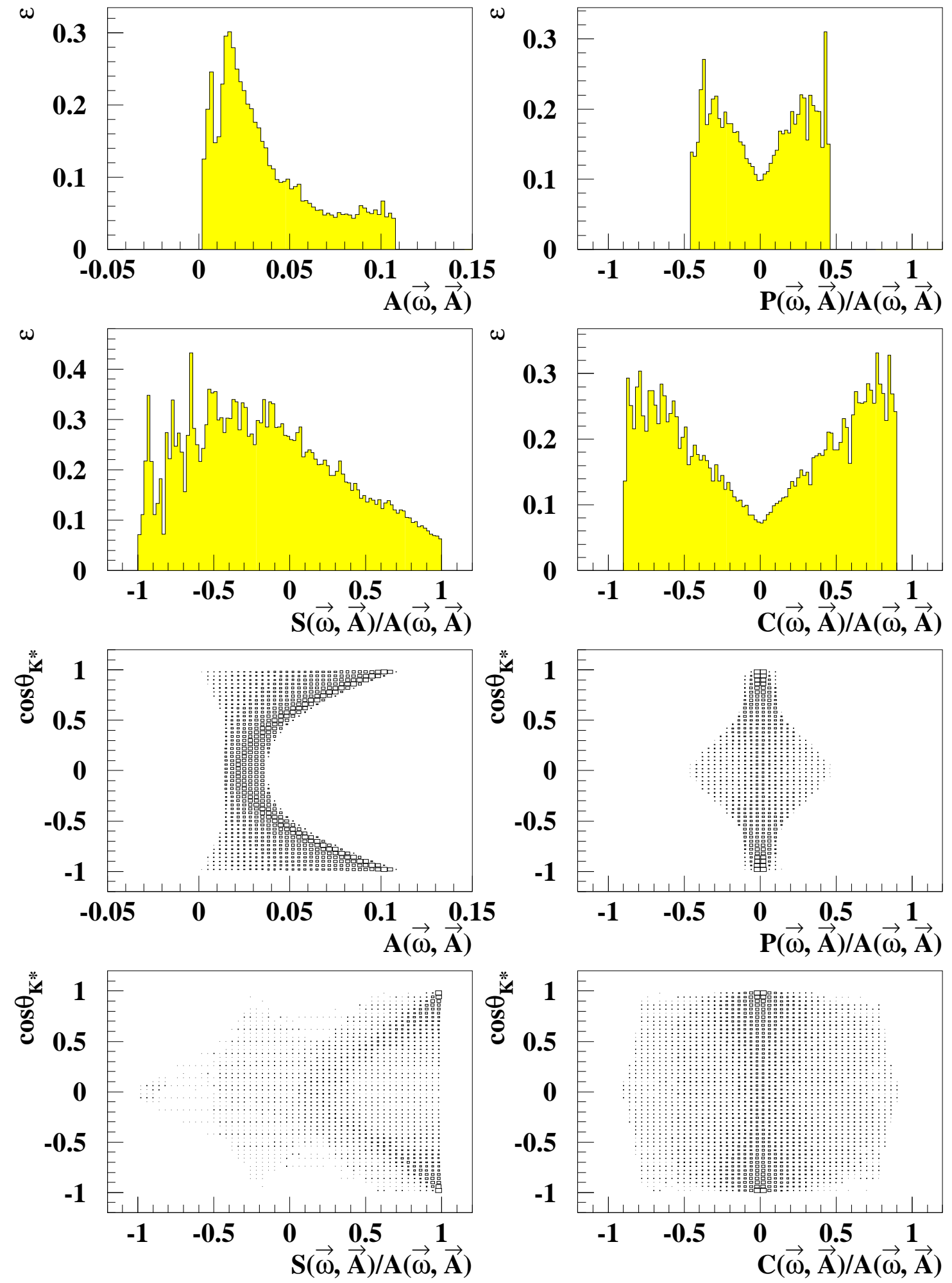

FIG. 6.21: Distributions de l'efficacité de reconstruction en fonction de $\mathcal{A}, \mathcal{P} / \mathcal{A}, \mathcal{S} / \mathcal{A}$ et $\mathcal{C} / \mathcal{A}$ (les quatre figures du haut). Les quatre figures du bas représentent $\mathcal{A}, \mathcal{P} / \mathcal{A}, \mathcal{S} / \mathcal{A}$ et $\mathcal{C} / \mathcal{A}$, entrant dans l'équation (6.27) en fonction de l'angle de transversité $\theta_{K^{*}}$ (ToyMC sans effet d'acceptance, 200K). 

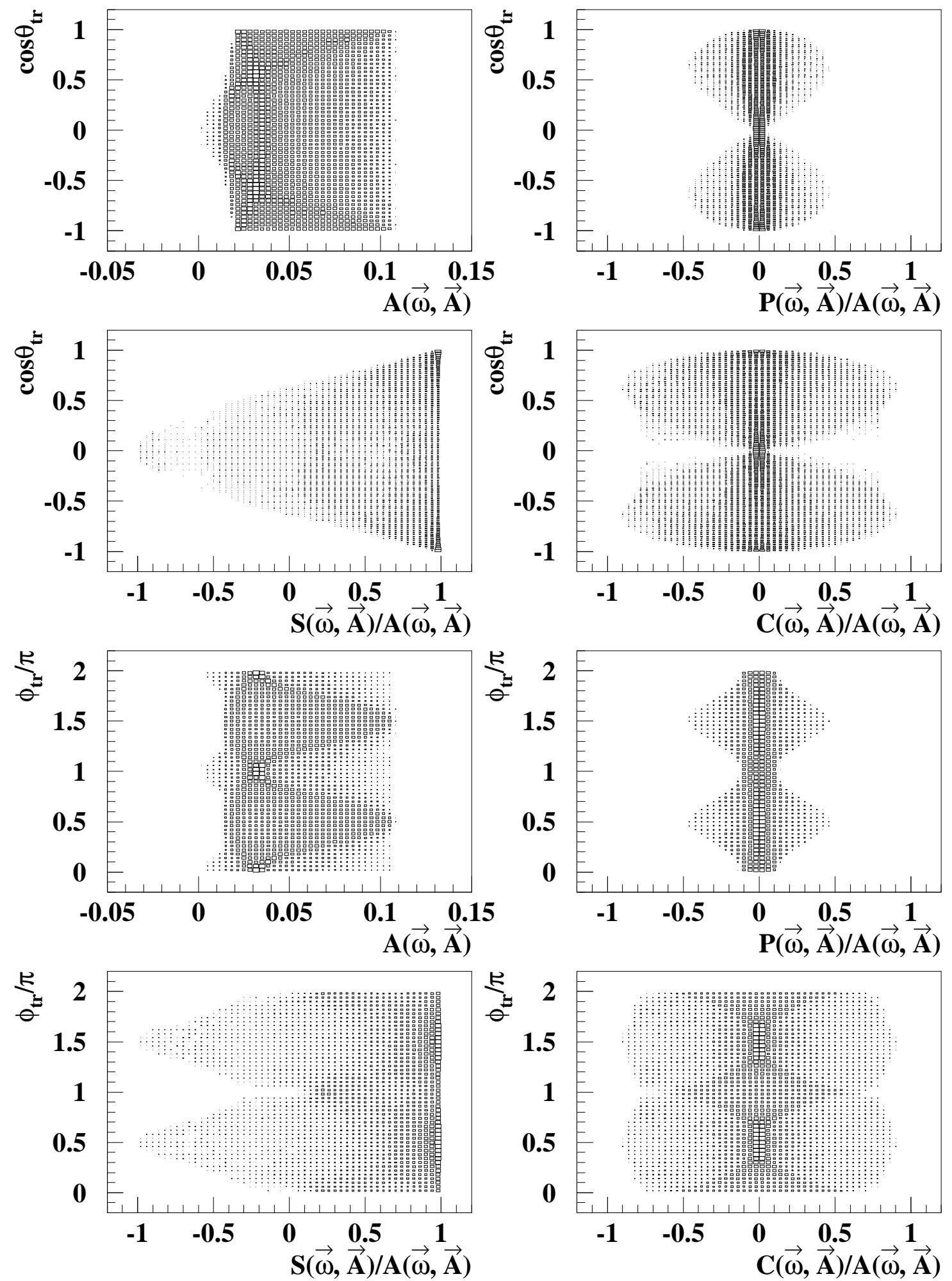

FIG. 6.22: Distributions de $\mathcal{A}, \mathcal{P} / \mathcal{A}, \mathcal{S} / \mathcal{A}$ et $\mathcal{C} / \mathcal{A}$, entrant dans l'équation (6.27) en fonction des angles de transversité $\theta_{t r}$ et $\varphi_{t r}$ (ToyMC sans effet d'acceptance, 200K). 


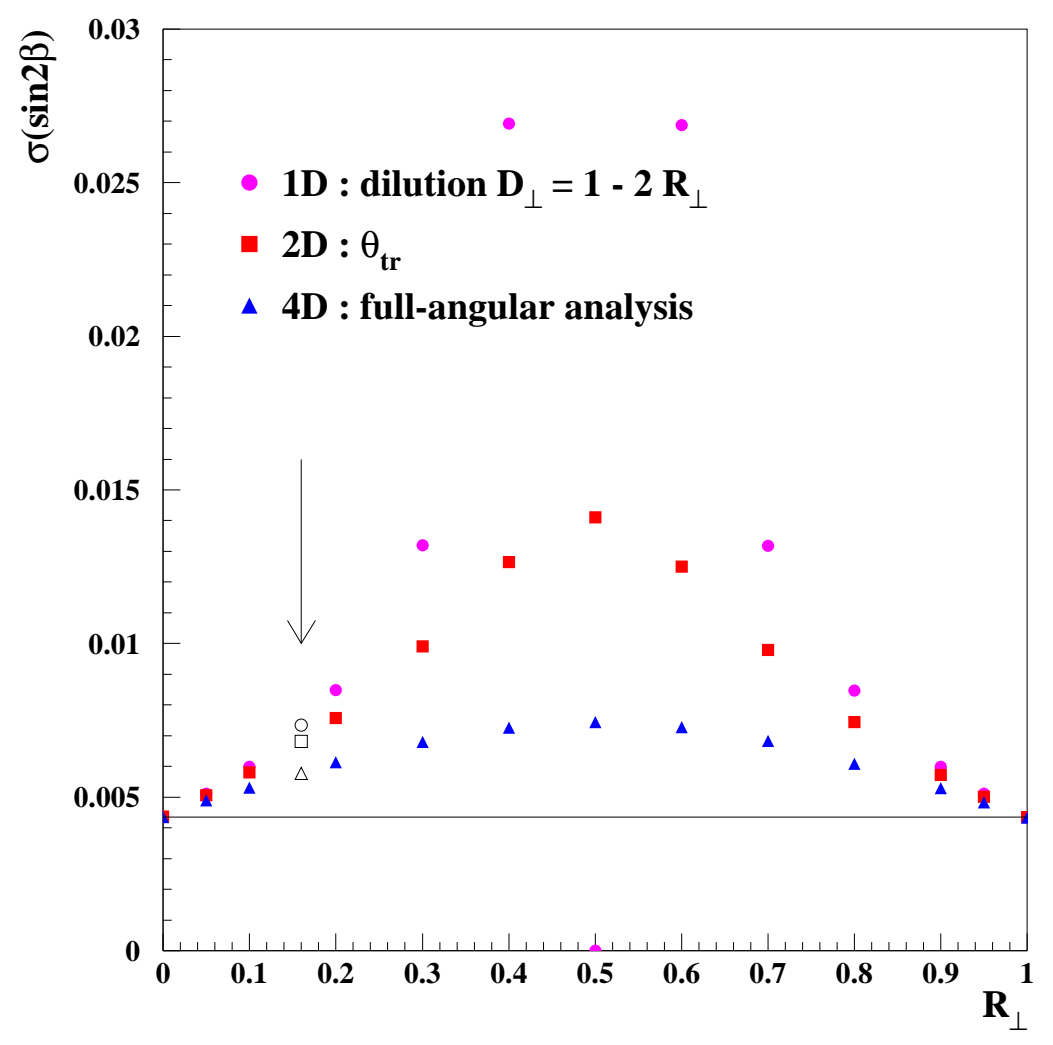

FIG. 6.23: Sensibilité de $\sin 2 \beta$ en fonction de $R_{\perp}$ généré (ToyMC sans effet d'acceptance, $100 \mathrm{~K}$ par point). $A_{0}=A_{\|}$et les phases fortes sont nulles. Les points creux sont obtenus avec les premières valeurs mesurées par BABAR [160]. " 1D » se réfère à l'ajustement dépendant du temps, « 2D » à l'ajustement dépendant du temps et de l'angle de transversité $\theta_{t r}$ et « $4 \mathrm{D}$ » à l'ajustement fait avec la distribution angulaire complète dépendante du temps.

pondéré par la même dilution $D_{\perp}=1-2 R_{\perp}$ :

$$
\int f_{ \pm}\left(\Delta t, \vec{\omega} ; \Gamma_{0}, \Delta M, \sin 2 \beta, \cos 2 \beta, \vec{A}\right) \mathrm{d} \vec{\omega}=\frac{\Gamma_{0}}{2} e^{-\Gamma_{0}|\Delta t|} \cdot\left[1 \mp D_{\perp} \cdot \sin 2 \beta \cdot \sin (\Delta M \Delta t)\right]
$$

alors que l'utilisation de la distribution angulaire complète permet d'obtenir une probabilité d'être de parité $C P$ impaire événement par événement.

La distribution observée est quelque peu différente du fait des effets d'acceptance et de résolution. La distribution observée se déduit de la distribution vraie par l'intermédiaire de la fonction de transfert [159] reliant un événement généré avec les angles $\vec{\omega}$ et reconstruit avec les angles $\vec{\omega}^{\prime}$ :

$$
\begin{aligned}
& f_{ \pm, o b s}\left(\Delta t, \vec{\omega}^{\prime} ; \Gamma_{0}, \Delta M, \sin 2 \beta, \cos 2 \beta, \vec{A}\right)= \\
& \quad \frac{\int f_{ \pm}\left(\Delta t, \vec{\omega} ; \Gamma_{0}, \Delta M, \sin 2 \beta, \cos 2 \beta, \vec{A}\right) \cdot T\left(\vec{\omega}^{\prime}, \vec{\omega}\right) \mathrm{d} \vec{\omega}}{\iiint f_{ \pm}\left(\Delta t, \vec{\omega} ; \Gamma_{0}, \Delta M, \sin 2 \beta, \cos 2 \beta, \vec{A}\right) \cdot T\left(\vec{\omega}^{\prime}, \vec{\omega}\right) \mathrm{d} \vec{\omega} \mathrm{d} \vec{\omega}^{\prime} \mathrm{d} \Delta t}
\end{aligned}
$$

où l'hypothèse d'indépendance de $T\left(\vec{\omega}^{\prime}, \vec{\omega}\right)$ avec $\Delta t$ est supposée (cf. Fig. 6.16), ainsi que celle de l'indépendance à l'étiquetage, à savoir $T_{B_{t a g}=B^{0}} \simeq T_{B_{t a g}=\bar{B}^{0}} \simeq T$. L'analyse angulaire montre que la résolution sur les angles a un effet négligeable, ce qui nous permet de réécrire la fonction de 
transfert comme $T\left(\vec{\omega}^{\prime}, \vec{\omega}\right) \simeq \delta\left(\vec{\omega}^{\prime}-\vec{\omega}\right) \varepsilon(\vec{\omega})$, $\varepsilon$ étant l'efficacité de reconstruction. Ces hypothèses permettent de réécrire $f_{ \pm, \text {obs }}$ sous une forme plus simple

$$
f_{ \pm, o b s}\left(\Delta t, \vec{\omega}^{\prime} ; \Gamma_{0}, \Delta M, \sin 2 \beta, \cos 2 \beta, \vec{A}\right)=f_{ \pm}\left(\Delta t, \vec{\omega}^{\prime} ; \Gamma_{0}, \Delta M, \sin 2 \beta, \cos 2 \beta, \vec{A}\right) \cdot \frac{\varepsilon\left(\vec{\omega}^{\prime}\right)}{\langle\varepsilon\rangle(\vec{A})}
$$

où $\langle\varepsilon\rangle(\vec{A})$ est l'efficacité moyenne définie par :

$$
\langle\varepsilon\rangle(\vec{A})=\iint f_{ \pm}\left(\Delta t, \vec{\omega}^{\prime} ; \Gamma_{0}, \Delta M, \sin 2 \beta, \cos 2 \beta, \vec{A}\right) \cdot \varepsilon\left(\vec{\omega}^{\prime}\right) \mathrm{d} \vec{\omega}^{\prime} \mathrm{d} \Delta t
$$

Enfin, en prenant en compte les probabilités de mauvais étiquetage, on aboutit à

$$
\begin{aligned}
& f_{ \pm, \text {obs }}\left(\Delta t, \vec{\omega}^{\prime} ; \Gamma_{0}, \Delta M, \sin 2 \beta, \cos 2 \beta, \vec{A}\right)= \\
& \quad \frac{\Gamma_{0}}{2} e^{-\Gamma_{0}|\Delta t|} \cdot \frac{\varepsilon\left(\vec{\omega}^{\prime}\right)}{\langle\varepsilon\rangle(\vec{A})} \cdot \mathcal{A}(\vec{\omega} ; \vec{A}) \cdot\left[\left(1 \pm \frac{\Delta D}{2}\right) \mp\langle D\rangle a(\Delta t, \vec{\omega} ; \Delta M, \sin 2 \beta, \cos 2 \beta, \vec{A})\right] .
\end{aligned}
$$

Une caractéristique particulière de l'analyse angulaire complète dépendante du temps, par rapport à une analyse dépendante du temps avec intégration sur un ou plusieurs angle(s) de transversité, est de pouvoir obtenir $(\sin 2 \beta, \cos 2 \beta)$ sans la connaissance de la fonction d'efficacité $\varepsilon\left(\vec{\omega}^{\prime}\right)$ dans un ajustement par maximum de vraisemblance en l'absence de bruits de fond. En effet, le logarithme de la fonction de vraisemblance sur un échantillon de données est

$$
\ln \mathcal{L}=\sum_{i=1}^{n} \ln f_{\text {obs }}\left(\Delta t_{i}, \vec{\omega}_{i} ; \theta\right)
$$

où $\theta$ est un paramètre à estimer (p. ex. $\cos 2 \beta$ ). Asymptotiquement, i.e. lorsque $n$ devient très grand, on a

$$
\ln \mathcal{L} \rightarrow n \int f_{\text {obs }}(\Delta t, \vec{\omega} ; \bar{\theta}) \ln f_{o b s}(\Delta t, \vec{\omega} ; \theta) \mathrm{d} t \mathrm{~d} \vec{\omega},
$$

où les événements sont distribués selon la densité de probabilité normalisée $f_{\text {obs }}(\Delta t, \vec{\omega} ; \bar{\theta})$. Montrons qu'en ajustant les événements distribués selon $f_{\text {obs }}(\Delta t, \vec{\omega} ; \bar{\theta})$ par $f(\Delta t, \vec{\omega} ; \bar{\theta})$ définie par :

$$
f_{o b s}(\Delta t, \vec{\omega} ; \bar{\theta})=f(\Delta t, \vec{\omega} ; \bar{\theta}) \cdot \frac{\varepsilon(\vec{\omega})}{\langle\varepsilon\rangle(\vec{A})}
$$

c'est-à-dire par la densité de probabilité normalisée à la génération, on obtient correctement $\theta$, ainsi que l'erreur $\operatorname{sur} \theta$. On a, asymptotiquement,

$$
\frac{\partial \ln \mathcal{L}}{\partial \theta} \rightarrow n \int f_{o b s}(\Delta t, \vec{\omega} ; \bar{\theta}) \frac{1}{f(\Delta t, \vec{\omega} ; \theta)} \frac{\partial f(\Delta t, \vec{\omega} ; \theta)}{\partial \theta} \mathrm{d} t \mathrm{~d} \vec{\omega} .
$$

En évaluant cette expression à $\theta=\bar{\theta}$, elle s'annule en conséquence de

$$
\left.\frac{\partial}{\partial \theta} \int f_{o b s}(\Delta t, \vec{\omega} ; \bar{\theta}) \mathrm{d} t \mathrm{~d} \vec{\omega}\right|_{\theta=\bar{\theta}}=0 .
$$


ТАВ. 6.9: Composition des événements reconstruits en tant que $B^{0} \rightarrow J / \psi K^{* 0}\left(K_{S}^{0} \pi^{0}\right)$ dans la région signal estimée à partir de données simulées $B \rightarrow J / \psi X$ avec une coupe $p^{*}>1.3 \mathrm{GeV} / c$. Les événements présents dans la région signal ne sont pas affectés par cette coupe. La première erreur est l'incertitude statistique, la seconde est l'incertitude systématique.

\begin{tabular}{|l||c|c|}
\hline & Fraction(\%) & $C P$ effectif \\
\hline \hline Signal & $83.0 \pm 1.2 \pm 5.7$ & \\
FA & $3.2 \pm 0.5 \pm 0.3$ & 0 \\
$B^{0} \rightarrow \chi_{c 1} K_{S}^{0}$ & $4.0 \pm 0.6 \pm 1.1$ & -1 \\
Hautes résonances $K^{*}$ & $3.5 \pm 0.6 \pm 1.8$ & $0 \pm 1$ \\
Non résonant $B^{0} \rightarrow J / \psi K_{S}^{0} \pi^{0}$ & $2.8 \pm 0.5 \pm 1.4$ & $0 \pm 1$ \\
Autres $B \rightarrow J / \psi X$ & $3.5 \pm 0.6 \pm 1.8$ & $0 \pm 1$ \\
\hline
\end{tabular}

En dérivant une nouvelle fois, on aboutit à

$$
\frac{\partial^{2} \ln \mathcal{L}}{\partial \theta^{2}} \rightarrow-n \int f_{o b s}(\Delta t, \vec{\omega} ; \bar{\theta}) \frac{1}{f^{2}(\Delta t, \vec{\omega} ; \theta)}\left(\frac{\partial f(\Delta t, \vec{\omega} ; \theta)}{\partial \theta}\right)^{2} \mathrm{~d} t \mathrm{~d} \vec{\omega}=-\sigma^{-2}
$$

en $\theta=\bar{\theta}$, soit

$$
\sigma^{-2}=\left.n \int \frac{1}{f} \frac{\varepsilon}{\langle\varepsilon\rangle(\vec{A})}\left(\frac{\partial f}{\partial \theta}\right)^{2} \mathrm{~d} t \mathrm{~d} \vec{\omega}\right|_{\theta=\bar{\theta}} .
$$

En conclusion, on aboutit aux mêmes résultats en ajustant les données distribuées selon $f_{\text {obs }}(\Delta t, \vec{\omega} ; \bar{\theta})$, que l'on prenne $f$ ou $f_{o b s}$ pour l'ajustement. La présence de bruits de fond rend la situation plus complexe puisque l'on ne peut plus s'affranchir de la connaissance de la fonction d'efficacité $\varepsilon(\vec{\omega})$.

À la différence de l'échantillon $B_{s a v}$, où tous les événements en $m_{\mathrm{ES}}$ après coupure sur $\Delta E$ sont utilisés permettant d'estimer les fractions signal et bruits de fond, on se restreint aux événements ayant un $m_{\mathrm{ES}}$ compris dans l'intervalle $5.27<m_{\mathrm{ES}}<5.29 \mathrm{GeV} / c^{2}$ du fait de la présence de bruits de fond qui s'accumulent dans la région signal tels que le FA ou celui provenant de la désintégration $B_{d}^{0} \rightarrow \chi_{c 1} K_{S}^{0}$ et qui ne permettent pas d'obtenir une estimation correcte des fractions signal et bruits de fond par un ajustement par une gaussienne et une fonction Argus (cf. §4.3). Les fractions sont alors estimées avec des données simulées et sont données dans la table 6.9 avec la parité $C P$ effective correspondante. La première erreur est l'erreur statistique, la seconde est l'erreur systématique prenant en compte les dernières mesures des rapports d'embranchement. Pour les rapports d'embranchement non mesurés, on suppose $50 \%$ d'erreur. De même, une erreur est associée aux parités $C P$ inconnues. Ces erreurs représentent les variations effectuées pour l'estimation des incertitudes systematiques sur $\sin 2 \beta$ et $\cos 2 \beta$ dues à la composition en bruits de fond. Ces bruits de fond provenant de canaux contenant un vrai $J / \psi$, on fait l'hypothèse qu'ils ont la même fonction de résolution et les mêmes performances d'étiquetage que le signal. Les distributions utilisées pour les bruits de fond sont

$$
\begin{aligned}
& b_{ \pm}\left(\Delta t, \vec{\omega}^{\prime} ; \Gamma_{0}, \Delta M, \sin 2 \beta, \Delta D,\langle D\rangle, \eta_{C P}\right)= \\
& \quad \frac{\Gamma_{0}}{2} e^{-\Gamma_{0}|\Delta t|}\left[\left(1 \pm \frac{\Delta D}{2}\right) \mp\langle D\rangle \eta_{C P} \sin 2 \beta \sin (\Delta M \Delta t)\right] b\left(\vec{\omega}^{\prime}\right),
\end{aligned}
$$


TАВ. 6.10: Séparation du nombre d'événements dans la région signal des échantillons $B_{C P}$ et $B_{s a v}$ selon les catégories d'étiquetage après application des critères de qualité sur les vertex des mésons $B$.

\begin{tabular}{|c|ccc|ccc|}
\hline & \multicolumn{3}{|c|}{$B_{C P}=J / \psi\left(K_{S}^{0} \pi^{0}\right)^{* 0}$} & \multicolumn{3}{c|}{$B_{\text {sav }}$} \\
\hline Tag & $B^{0}$ & $\bar{B}^{0}$ & Tot & $B^{0}$ & $\bar{B}^{0}$ & Tot \\
\hline Lepton & 6 & 5 & 11 & 1604 & 1523 & 3127 \\
Kaon I & 10 & 15 & 25 & 3170 & 3174 & 6344 \\
Kaon II & 19 & 13 & 32 & 4101 & 3797 & 7898 \\
Inclusive & 22 & 14 & 36 & 4118 & 3888 & 8006 \\
\hline No tag & \multicolumn{3}{|c|}{51} & \multicolumn{3}{c|}{14551} \\
\hline Total & \multicolumn{3}{|c|}{155} & \multicolumn{3}{c|}{39926} \\
\hline
\end{tabular}

où $b(\vec{\omega})$ est la densité de probabilité normalisée décrivant la partie angulaire des bruits de fond. Pour le bruit de fond provenant du transfert entre les canaux $B \rightarrow J / \psi K^{*}(892)$, dominé par les canaux $B^{+} \rightarrow J / \psi K^{*+}$ de parité $C P$ effective nulle, la distribution utilisée est

$$
b_{f a, \pm}\left(\Delta t, \vec{\omega}^{\prime} ; \Gamma_{+}, \Delta D,\langle D\rangle\right)=\frac{\Gamma_{+}}{2} e^{-\Gamma_{+}|\Delta t|}\left(1 \pm \frac{\Delta D}{2}\right) b_{f a}\left(\vec{\omega}^{\prime}\right)
$$

où $b_{f a}(\vec{\omega})$ la densité de probabilité normalisée décrivant la partie angulaire.

La partie la plus délicate est donc la détermination des fonctions angulaires $\varepsilon\left(\vec{\omega}^{\prime}\right), b\left(\vec{\omega}^{\prime}\right)$ et $b_{f a}\left(\vec{\omega}^{\prime}\right)$. La stratégie adoptée est de développer ces fonctions en moments (cf. §5.1.3) sur la base orthonormée des harmoniques sphériques jusqu'à un ordre suffisant pour avoir une bonne représentation de la forme des bruits de fond et de la fonction d'efficacité. La difficulté est de pouvoir quantifier la qualité de la représentation de ces fonctions angulaires en fonction de l'ordre de développement en moments. On détermine les moments de ces expressions sur les données simulées en développant l'acceptance jusqu'à un ordre correspondant à un spin 4 alors que le FA et le $\chi_{c 1} K_{S}^{0}$ sont développés à un ordre correspondant à un spin 6. Les autres bruits de fond (hautes résonances $K^{*}$, non résonant et autres $\left.B \rightarrow J / \psi X\right)$ sont considérés plats en $\vec{\omega}^{\prime}$.

La distribution finale pour l'échantillon $B_{C P}$, pour une catégorie d'étiquetage $c$ et après avoir convolué avec la fonction de résolution du signal, est donnée par :

$$
\begin{aligned}
\mathcal{F}_{ \pm, c} & = \\
& f_{c, s i g}^{C P} \mathcal{F}_{ \pm, c, o b s}\left(\Delta t, \vec{\omega}^{\prime} ; \Gamma_{0}, \Delta M, \sin 2 \beta, \cos 2 \beta, \vec{A}, \Delta D_{c},\langle D\rangle_{c}, \hat{a}_{c}\right)+ \\
& f_{c, f a}^{C P} \mathcal{B}_{f a, \pm}\left(\Delta t, \vec{\omega}^{\prime} ; \Gamma_{+}, \Delta D_{c},\langle D\rangle_{c}, \hat{a}_{c}\right)+ \\
& \sum_{\alpha=b k g \neq f a} f_{c, \alpha}^{C P} \mathcal{B}_{ \pm, \alpha}\left(\Delta t, \vec{\omega}^{\prime} ; \Gamma_{0}, \Delta M, \sin 2 \beta, \Delta D_{c},\langle D\rangle_{c}, \eta_{C P}, \hat{a}_{c}\right) .
\end{aligned}
$$

Les fractions $f_{c}^{C P}$ sont données dans la table 6.9.

Le nombre d'événements obtenu après étiquetage et application des critères de qualité sur les vertex des mésons $B$ est donné dans la table 6.10. 


\subsubsection{Ajustement global}

Les valeurs de $\sin 2 \beta$ et de $\cos 2 \beta$ sont obtenues par un ajustement global combinant l'échantillon $B_{C P}$ et $B_{\text {sav }}$ :

$$
\begin{aligned}
& \ln \mathcal{L}_{C P}+\ln \mathcal{L}_{\text {sav }}=\sum_{c=1}^{4}\left[\sum_{i=1}^{N_{C P, B_{\text {tag }}=B^{0}}} \ln \mathcal{F}_{+, c}+\sum_{i=1}^{N_{C P, B_{\text {tag }}=\bar{B}^{0}}} \ln \mathcal{F}_{-, c}+\right. \\
& \sum_{i=1}^{N_{\text {Unmixed }, B_{\text {tag }}=B^{0}}} \ln \mathcal{H}_{\text {Unmixed }, B_{\text {tag }}=B^{0}, c}+\sum_{i=1}^{N_{\text {Unmixed }, B_{\text {tag }}=\bar{B}^{0}}} \ln \mathcal{H}_{\text {Unmixed }, B_{\text {tag }}=\bar{B}^{0}, c} \\
&\left.\sum_{i=1}^{N_{\text {Mixed }, B_{\text {tag }}=B^{0}}} \ln \mathcal{H}_{\text {Mixed, }, B_{\text {tag }}=B^{0}, c}+\sum_{i=1}^{N_{\text {Mixed }, B_{\text {tag }}=\bar{B}^{0}}} \ln \mathcal{H}_{\text {Mixed, }, B_{\text {tag }}=\bar{B}^{0}, c}\right],
\end{aligned}
$$

où les densités de probabilités $\mathcal{F}$ et $\mathcal{H}$ sont définies par les équations (6.22) et (6.47). Cet ajustement par vraisemblance non-binné a un total de 34 paramètres (les valeurs de $\Delta M$ et $\Gamma_{0}$ étant fixées à celles données dans [28] puisque connues plus précisément) qui sont :

- les valeurs de $\sin 2 \beta$ et de $\cos 2 \beta$ (2);

- les paramètres de la fonction de résolution pour le signal $\hat{a}(8)$;

- les paramètres de dilution du signal : chaque catégorie d'étiquetage apporte deux paramètres, $D_{c}$ et $\langle D\rangle_{c}$, soit un total de huit paramètres (il y a quatre catégories d'étiquetage);

- les paramètres de la fonction de résolution pour les bruits de fond de l'échantillon $B_{\text {sav }}$ : $\hat{b}$ (3). La fonction de résolution des bruits de fond est réduite à une gaussienne avec pour paramètres le facteur d'échelle $S_{\text {core }}$, un biais $b_{\text {core }}$ commun aux quatre catégories d'étiquetage et la fraction $f_{\text {out }}$, la gaussienne outlier ayant un biais nul et une largeur fixée à 8 ps ;

- les paramètres de composition des bruits de fond de l'échantillon $B_{s a v}$ (13). Différentes hypothèses sont effectuées afin de simplifier la paramétrisation montrée à l'équation (6.26). On considère qu'il n'y a pas de bruits de fond oscillant en imposant $f_{c, 3}^{s a v}=0$ et en assignant une incertitude systématique en conséquence. La fraction de bruits de fond qui s'accumule dans la région signal est estimée à partir de données simulées, $\delta_{\text {peak }}=(1.5 \pm 0.5) \%$ pour chaque catégorie d'étiquetage. Cette contribution est dominée par des événements $B^{+}$, de sorte que $\Delta M_{\text {peak,c }}=0, \Gamma_{\text {peak,c }}^{s a v}=\Gamma_{+}$et les dilutions $D_{\text {peak }, c}^{s a v}$ sont fixées aux valeurs obtenues sur l'échantillon $B^{+}$(cf. la table 6.11). Les dilutions $D_{1, c}^{s a v}$ et $D_{2, c}^{s a v}$ sont laissées libres dans l'ajustement, soit huit paramètres. Les fractions relatives entre les bruits de fond $\tau=0$ et $\tau>0$ introduisent quatre paramètres. Enfin, $\Gamma_{2, c}^{s a v}$ est supposé être le même pour les quatre catégories d'étiquetage, soit un paramètre.

\subsection{Résultats de l'ajustement}

Les résultats obtenus avec l'ajustement global sont résumés dans la table 6.12. $\sin 2 \beta$ et $\cos 2 \beta$ sont peu corrélés avec les autres paramètres libres de l'ajustement, c'est-à-dire avec les paramètres liés à l'échantillon $B_{s a v}$. L'asymétrie observée est représentée sur la figure 6.24. Les résultats de $\sin 2 \beta$ et $\cos 2 \beta$ obtenus sur les échantillons de contrôle $B^{+} \rightarrow J / \psi K^{*+}$ sont donnés dans la table 
TAB. 6.11: Performances d'étiquetage sur l'échantillon $B^{ \pm}$. Le nombre d'événements signal provient d'un ajustement du spectre en $m_{\mathrm{ES}}$ par une gaussienne et une fonction Argus, après étiquetage et application des critères de qualité sur les vertex.

\begin{tabular}{|c|c|c|c|c|}
\hline Catégorie & $N_{\text {signal }}$ & $\varepsilon(\%)$ & $w(\%)$ & $Q(\%)$ \\
\hline Lepton & $2060 \pm 48$ & $9.9 \pm 0.2$ & $1.1 \pm 0.3$ & $9.5 \pm 0.2$ \\
Kaon I & $3710 \pm 68$ & $17.9 \pm 0.3$ & $7.7 \pm 0.5$ & $12.8 \pm 0.4$ \\
Kaon II & $4083 \pm 73$ & $19.7 \pm 0.3$ & $17.4 \pm 0.6$ & $8.3 \pm 0.4$ \\
Inclusive & $3870 \pm 70$ & $18.6 \pm 0.3$ & $27.7 \pm 0.8$ & $3.7 \pm 0.3$ \\
\hline Total & $20771 \pm 164$ & $66.1 \pm 0.6$ & & $34.3 \pm 0.6$ \\
\hline
\end{tabular}

6.13. Ils sont tous compatibles avec zéro.

\subsection{Validations}

Afin de déterminer la qualité de l'ajustement ainsi que l'erreur statistique attendue, deux mille expériences correspondant à la statistique du nombre d'événements de données reconstruits dans la région signal sont générées en utilisant des données simulées provenant d'une simulation rapide du détecteur BABAR (Pravda [162]). Les dilutions et les paramètres de la fonction de résolution sont fixés aux valeurs obtenues sur les données (cf. la table 6.12). Les amplitudes sont fixées aux valeurs obtenues au chapitre précédent. Les résultats sont représentés sur la figure 6.25. La fraction d'expériences qui donne un maximum de vraisemblance moins probable que celui obtenu sur les données est de $21.9 \%$.

Le deuxième type de validations est basé sur des données simulées, d'abord de signal pur, puis de désintégrations contenant un $J / \psi$. Chaque ajustement est effectué avec un échantillon Monte Carlo correspondant à l'échantillon $B_{\text {sav }}$ mais de plus grande statistique et en variant l'échantillon $B_{C P}$. Les résultats, compatibles avec les valeurs générées de $\sin 2 \beta$ et $\cos 2 \beta$, sont donnés dans la table 6.14.

La dépendance angulaire des bruits de fond ainsi que la fonction d'efficacité sont évaluées à partir de leur développement en moments jusqu'à un certain ordre correspondant à des spins donnés. La table 6.15 présente les résultats obtenus sur 104000 événements de données simulées provenant d'une simulation rapide du détecteur en faisant varier les differents ordres de développement de l'efficacité et des fonctions angulaires des bruits de fond. Le faible niveau de bruits de fond permet d'obtenir des résultats compatibles avec les valeurs générées dès l'ordre le plus bas en développement (i.e. une acceptance et une dépendance angulaire plates $($ spin $=0)$ ).

\subsection{Moment de $\mathcal{C}$}

L'asymétrie dépendante du temps ne permet d'obtenir qu'une visualisation de $\sin 2 \beta$ car, en intégrant sur les angles de transversité, on aboutit à l'équation (6.33) qui ne contient plus $\cos 2 \beta$. Afin d'obtenir une représentation graphique sensible à $\cos 2 \beta$, on utilise le moment de $\mathcal{C}$ qui utilise l'orthogonalité des fonctions angulaires $\mathcal{C}$ et $\mathcal{D}$ (cf. eqs. (6.31) et (6.32)). D'après l'expression de la 
ТАВ. 6.12: Résultat de l'ajustement global sur les échantillons de données $B_{\text {sav }}$ et $J / \psi K^{* 0}\left(K_{S}^{0} \pi^{0}\right)$. Les amplitudes de transversité sont fixées aux valeurs correspondant à l'autre solution pour les phases fortes (cf. la table 5.7).

\begin{tabular}{|c|c|c|c|}
\hline Paramètre & Résultat & Corr. avec $\sin 2 \beta$ & Corr. avec $\cos 2 \beta$ \\
\hline $\sin 2 \beta$ & $-0.10 \pm 0.57$ & 1.000 & -0.368 \\
\hline $\cos 2 \beta$ & $3.32_{-0.96}^{+0.76}$ & -0.368 & 1.000 \\
\hline \multicolumn{4}{|c|}{ Fonction de résolution signal } \\
\hline$S_{\text {core }}$ & $1.093 \pm 0.048$ & -0.020 & 0.028 \\
\hline$S_{t a i l}$ & 3.0 (fixé) & & \\
\hline$b_{\text {core }}$ Lepton & $0.012 \pm 0.063$ & 0.017 & -0.010 \\
\hline$b_{\text {core }}$ Kaon I & $-0.226 \pm 0.052$ & 0.008 & -0.050 \\
\hline$b_{\text {core }}$ Kaon II & $-0.248 \pm 0.046$ & 0.013 & -0.023 \\
\hline$b_{\text {core }}$ Inclusive & $-0.212 \pm 0.047$ & 0.020 & -0.020 \\
\hline$b_{t a i l}$ & $-1.01 \pm 0.286$ & -0.021 & 0.029 \\
\hline$f_{\text {tail }}$ & $0.109 \pm 0.020$ & 0.022 & -0.030 \\
\hline$f_{\text {out }}$ & $0.002 \pm 0.001$ & -0.004 & 0.006 \\
\hline \multicolumn{4}{|c|}{ Dilutions signal } \\
\hline$\langle D\rangle$, Lepton & $0.933 \pm 0.013$ & -0.002 & -0.004 \\
\hline$\langle D\rangle$, Kaon I & $0.799 \pm 0.014$ & -0.009 & 0.051 \\
\hline$\langle D\rangle$, Kaon II & $0.582 \pm 0.016$ & -0.001 & -0.006 \\
\hline$\langle D\rangle$, Inclusive & $0.368 \pm 0.017$ & 0.009 & 0.024 \\
\hline$\Delta D$, Lepton & $0.031 \pm 0.022$ & -0.003 & -0.001 \\
\hline$\Delta D$, Kaon I & $0.023 \pm 0.022$ & -0.010 & 0.039 \\
\hline$\Delta D$, Kaon II & $0.090 \pm 0.024$ & -0.008 & 0.002 \\
\hline$\Delta D$, Inclusive & $0.050 \pm 0.026$ & 0.002 & 0.004 \\
\hline \multicolumn{4}{|c|}{ Propriétés bruits de fond $\left(B_{\text {sav }}\right)$} \\
\hline$\tau$ [ps] & $1.335 \pm 0.064$ & 0.001 & 0.000 \\
\hline$f(\tau=0)$ Lepton & $0.293 \pm 0.174$ & 0.000 & -0.001 \\
\hline$f(\tau=0)$ Kaon I & $0.631 \pm 0.027$ & 0.000 & -0.001 \\
\hline$f(\tau=0)$ Kaon II & $0.659 \pm 0.024$ & 0.000 & -0.001 \\
\hline$f(\tau=0)$ Inclusive & $0.684 \pm 0.023$ & 0.000 & 0.000 \\
\hline \multicolumn{4}{|c|}{ Fonction de résolution bruits de fond } \\
\hline$S_{\text {core }}$ & $1.398 \pm 0.019$ & 0.001 & -0.002 \\
\hline$b_{\text {core }}$ & $-0.043 \pm 0.013$ & -0.001 & 0.002 \\
\hline$f_{\text {out }}$ & $0.015 \pm 0.002$ & 0.000 & 0.001 \\
\hline \multicolumn{4}{|c|}{ Dilutions bruits de fond } \\
\hline$\langle D\rangle$, Lepton,$\tau=0$ & $1.360 \pm 0.693$ & 0.000 & 0.001 \\
\hline$\langle D\rangle$, Kaon $I, \tau=0$ & $0.648 \pm 0.030$ & 0.001 & -0.004 \\
\hline$\langle D\rangle$, Kaon II , $\tau=0$ & $0.393 \pm 0.023$ & 0.000 & 0.000 \\
\hline$\langle D\rangle$, Inclusive,$\tau=0$ & $0.158 \pm 0.024$ & -0.001 & -0.002 \\
\hline$\langle D\rangle$, Lepton,$\tau>0$ & $0.168 \pm 0.108$ & 0.000 & 0.000 \\
\hline$\langle D\rangle$, Kaon $I, \tau>0$ & $0.251 \pm 0.048$ & 0.000 & 0.000 \\
\hline$\langle D\rangle$, Kaon II,$\tau>0$ & $0.278 \pm 0.042$ & 0.000 & 0.000 \\
\hline$\langle D\rangle$, Inclusive, $\tau>0$ & $0.031 \pm 0.046$ & 0.000 & 0.000 \\
\hline
\end{tabular}


TAB. 6.13: Résultat de l'ajustement global obtenu avec les échantillons de contrôle provenant des canaux $B^{+} \rightarrow J / \psi K^{*+}$.

\begin{tabular}{|l|c|c|}
\hline Échantillon & $\sin 2 \beta$ & $\cos 2 \beta$ \\
\hline \hline$B^{+} \rightarrow J / \psi\left(K_{S}^{0} \pi^{+}\right)^{*+}$ & $0.21 \pm 0.20$ & $-0.21 \pm 0.47$ \\
$B^{+} \rightarrow J / \psi\left(K^{+} \pi^{0}\right)^{*+}$ & $0.20 \pm 0.20$ & $-0.26 \pm 0.46$ \\
\hline
\end{tabular}

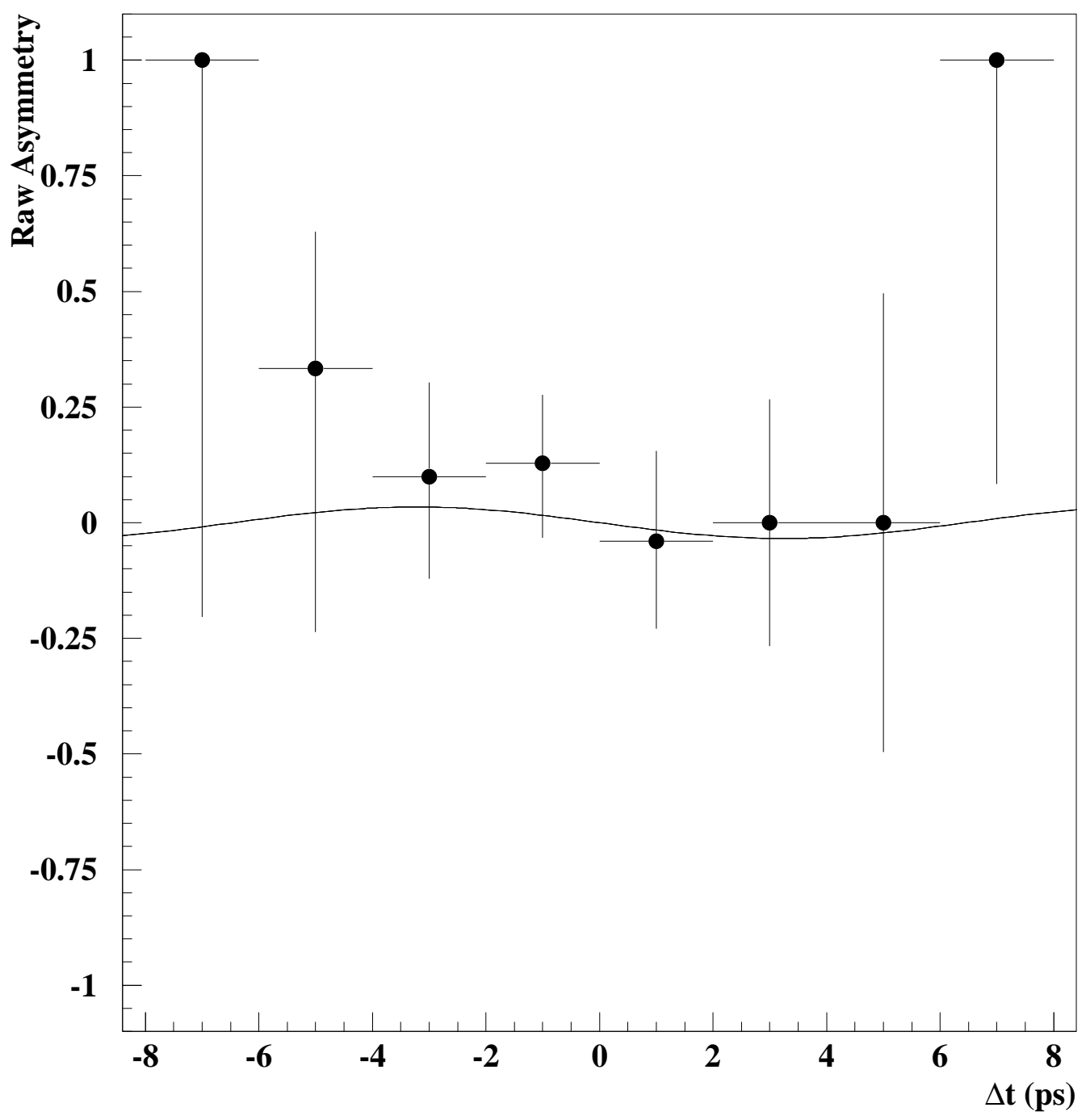

FIG. 6.24: Asymétrie temporelle observée pour le mode $J / \psi K^{* 0}\left(K_{S}^{0} \pi^{0}\right)$ avec le résultat du maximum de vraisemblance superposé. 

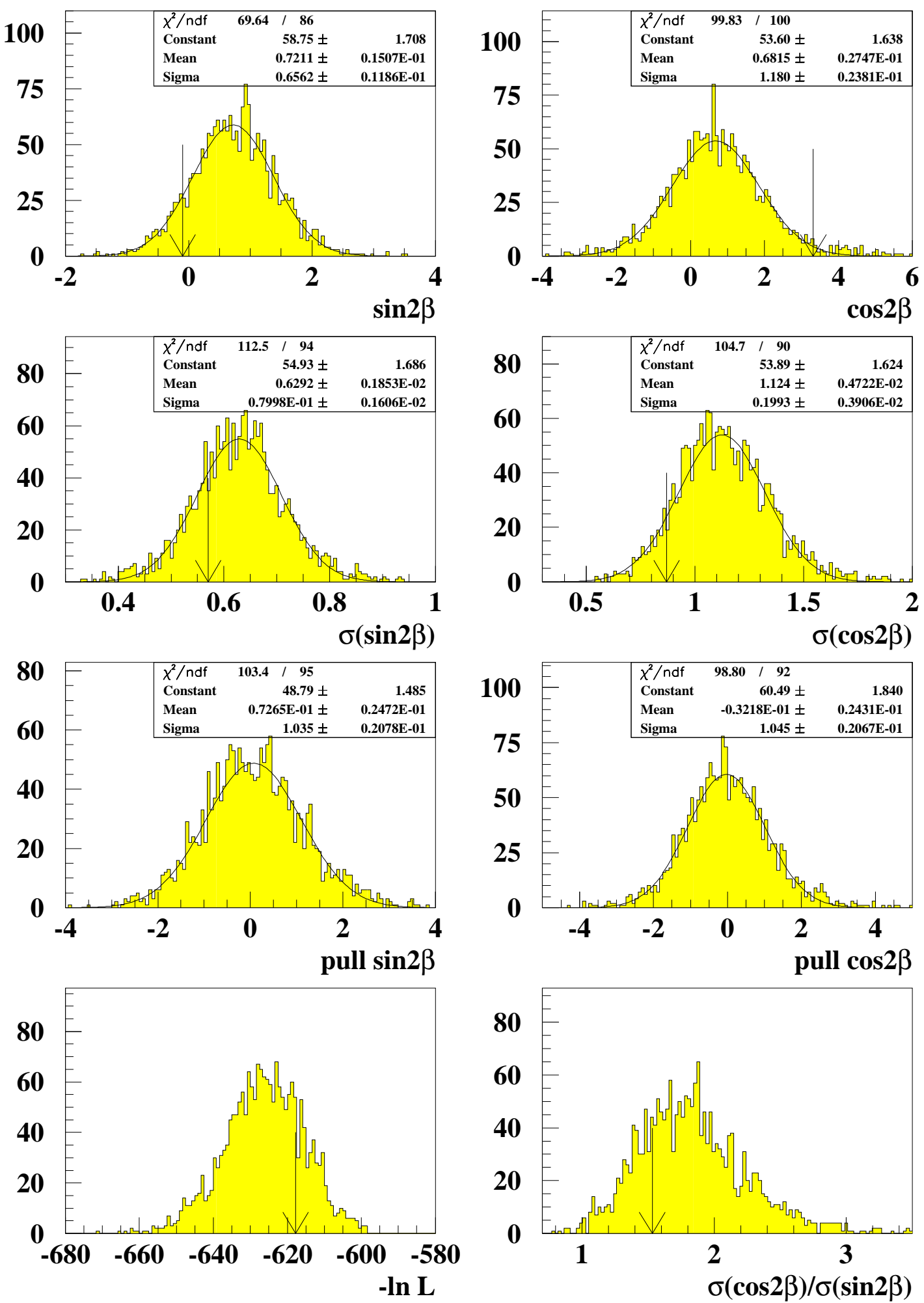

FIG. 6.25: Distributions de $\sin 2 \beta, \cos 2 \beta, \sigma(\sin 2 \beta), \sigma(\cos 2 \beta)$, les pulls correspondant, $-\ln \mathcal{L}$ ainsi que $\sigma(\cos 2 \beta) / \sigma(\sin 2 \beta)$ obtenues à partir de 2000 expériences de 104 événements de données simulées provenant d'une simulation rapide du détecteur BABAR. La flèche représente les valeurs obtenues avec les données (échantillon $J / \psi\left(K_{S}^{0} \pi^{0}\right)^{* 0}$ uniquement) à partir d'un ajustement où seuls $\sin 2 \beta$ et $\cos 2 \beta$ sont libres. 
TAB. 6.14: Résultats des ajustements sur les données simulées composées d'un échantillon correspondant à l'échantillon $B_{s a v}$ et d'un échantillon $B_{C P}$. Les valeurs générées de $\sin 2 \beta$ et de $\cos 2 \beta$ sont 0.7 et 0.714 , respectivement. La contribution du SFA a une effet négligeable sur les mesures de $\sin 2 \beta$ et $\cos 2 \beta$, malgré sa résolution angulaire dégradée. L'échantillon de $J / \psi$ inclusif est généré avec une coupe $p^{*}>1.3 \mathrm{GeV} / c$. Cette coupe n'affecte pas les événements présents dans la région signal.

\begin{tabular}{|l|l|c|c|}
\hline Mode $C P$ & luminosité $\left(\mathrm{fb}^{-1}\right)$ & $\sin 2 \beta$ & $\cos 2 \beta$ \\
\hline \hline$J / \psi\left(K_{S}^{0} \pi^{0}\right)^{* 0}$ signal pur & 6737 & $0.677 \pm 0.041$ & $0.744 \pm 0.090$ \\
$J / \psi\left(K_{S}^{0} \pi^{0}\right)^{* 0}+$ SFA & 6737 & $0.688 \pm 0.038$ & $0.762 \pm 0.084$ \\
$B \rightarrow J / \psi X$ avec $p^{*}>1.3 \mathrm{GeV} / c$ & 591 & $0.61 \pm 0.16$ & $0.20 \pm 0.32$ \\
\hline \hline
\end{tabular}

ТАВ. 6.15: Variation des résultats sur $\sin 2 \beta$ et $\cos 2 \beta$, en fonction de l'ordre de développement de l'efficacité et de la dépendance angulaire des bruits de fond, obtenue à partir d'une simulation rapide du détecteur BABAR. Les valeurs à la génération de $\sin 2 \beta$ et $\cos 2 \beta$ sont 0.7 et 0.714 , respectivement.

\begin{tabular}{|l|c|c|c|c|}
\hline Échantillon & rang $\varepsilon$ & rang bruits de fond & $\sin 2 \beta$ & $\cos 2 \beta$ \\
\hline \hline Signal pur & & & $0.699 \pm 0.014$ & $0.721 \pm 0.0312$ \\
Signal + bruits de fond & spin 0 & spin 0 & $0.678 \pm 0.016$ & $0.747 \pm 0.039$ \\
Signal + bruits de fond & spin 4 & spin 6 & $0.709 \pm 0.017$ & $0.705 \pm 0.036$ \\
Signal + bruits de fond & spin 8 & spin 10 & $0.710 \pm 0.017$ & $0.706 \pm 0.036$ \\
\hline \hline
\end{tabular}

densité de probabilité normalisée pour le mode $C P$, on a

$$
\begin{aligned}
& \frac{\int \mathcal{C}(\vec{\omega} ; \vec{A}) \cdot f_{ \pm}(\Delta t, \vec{\omega}) \mathrm{d} \vec{\omega}}{\frac{\Gamma_{0}}{2} e^{-\Gamma_{0}|\Delta t|}}= \\
& \quad \pm\left[\left\{\int \mathcal{C} \mathcal{P} \mathrm{d} \vec{\omega}\right\} \cos (\Delta M \Delta t)+\left\{\int \mathcal{C}^{2} \mathrm{~d} \vec{\omega}\right\} \cos (2 \beta) \sin (\Delta M \Delta t)\right]
\end{aligned}
$$

Á noter que dans le cas de phases fortes nulles, la fonction angulaire $\mathcal{P}(\vec{\omega} ; \vec{A})$ est nulle. En prenant en compte les effets d'acceptance ainsi que des probabilités de mauvais étiquetage, l'équation (6.49) devient

$$
\begin{array}{r}
\frac{\int \mathcal{C}(\vec{\omega} ; \vec{A}) \cdot f_{ \pm, \text {obs }}(\Delta t, \vec{\omega}) \mathrm{d} \vec{\omega}}{\frac{\Gamma_{0}}{2} e^{-\Gamma_{0}|\Delta t|}}=\left(1 \pm \frac{\Delta D}{2}\right)\left\{\int \mathcal{C} \mathcal{A} \varepsilon \mathrm{d} \vec{\omega}\right\} \mp\langle D\rangle\left[\left\{\int \mathcal{C} \mathcal{P} \varepsilon \mathrm{d} \vec{\omega}\right\} \cos (\Delta M \Delta t)\right. \\
\left.+\sin (\Delta M \Delta t)\left[\left\{\int \mathcal{C} \mathcal{S} \varepsilon \mathrm{d} \vec{\omega}\right\} \sin 2 \beta+\left\{\int \mathcal{C}^{2} \varepsilon \mathrm{d} \vec{\omega}\right\} \cos 2 \beta\right]\right],
\end{array}
$$

où les intégrales angulaires sont évaluées sur des données simulées en étendant la définition des $\Phi_{l}^{i \rightarrow j=i}$ (cf. eq. (5.24)) par

$$
\Phi_{l m}^{i \rightarrow j=i} \equiv \int f_{l}(\vec{\omega}) \cdot f_{m}(\vec{\omega}) \cdot \epsilon^{i \rightarrow j=i}(\vec{\omega}) \mathrm{d} \vec{\omega}
$$

Les valeurs de ces intégrales sont données dans la table 6.16. Les effets d'acceptance ont réintroduit $\sin 2 \beta$ qui est atténué par le coefficient $\int \mathcal{C} \mathcal{S} \varepsilon \mathrm{d} \vec{\omega}$, environ cent fois moins important que le coefficient devant $\cos 2 \beta$. 
TАВ. 6.16: Évaluation des intégrales sur des données simulées (9548 événements sélectionnés étiquetés obtenus à partir de 131000 événements générés).

\begin{tabular}{|l||c|}
\hline $\int \mathcal{C} \mathcal{A} \varepsilon \mathrm{d} \vec{\omega}$ & $-3.71 \times 10^{-5}$ \\
$\int \mathcal{C P} \varepsilon \mathrm{d} \vec{\omega}$ & $-3.72 \times 10^{-4}$ \\
$\int \mathcal{C} \mathcal{S} \varepsilon \mathrm{d} \vec{\omega}$ & $-2.83 \times 10^{-5}$ \\
$\int \mathcal{C}^{2} \varepsilon \mathrm{d} \vec{\omega}$ & $2.59 \times 10^{-3}$ \\
\hline
\end{tabular}

Chaque bin de temps reçoit la somme des poids $w_{i, \pm}$ en fonction de la saveur du méson $B_{\text {tag }}$, où $w_{i, \pm}$ est défini à l'équation (6.50) et l'erreur est donnée par $\sqrt{\sum_{i} w_{i, \pm}^{2}}$. La figure 6.26 représente le moment de $\mathcal{C}$ obtenu avec des données simulées (cf. la table 6.16) du canal $B_{d}^{0} \rightarrow J / \psi\left(K_{S}^{0} \pi^{0}\right)^{* 0}$ et avec les données. Une valeur de $\cos 2 \beta=\sqrt{1-\sin 2 \beta^{2}}$ avec $\sin 2 \beta$ pris à la dernière valeur mesurée par BABAR [166], à savoir $\sin 2 \beta=0.741 \pm 0.067$ est difficilement compatible avec les données du canal $B_{d}^{0} \rightarrow J / \psi\left(K_{S}^{0} \pi^{0}\right)^{* 0}$. À noter que dans la densité de probabilité normalisée du canal $B_{d}^{0} \rightarrow J / \psi\left(K_{S}^{0} \pi^{0}\right)^{* 0}$, les bruits de fond ayant une dépendance angulaire plate ne contribuent pas au moment de $\mathcal{C}$ puisque $\int \mathcal{C} \mathrm{d} \vec{\omega}=0$. Ceux qui pique à $\cos \theta_{K^{*}}$ proche de 1 sont atténués puisque la fonction $\mathcal{C}$ vaut zéro.

\section{8 Étude des incertitudes systématiques}

L'étude des incertitudes systématiques est regroupée en différentes sources : propriétés du signal, propriétés des bruits de fond, paramètres extérieurs fixes et limitation statistique des simulations Monte Carlo. Ces différentes sources sont résumées dans la table 6.20 et sont décrites dans les sections qui suivent, où chaque contribution individuelle est référencée par une lettre entre parenthèses.

\subsubsection{Propriétés du signal}

Les paramètres de la fonction de résolution en $\Delta t$, les dilutions moyennes $\langle D\rangle$ et les différences de dilution $\Delta D$ sont déterminés sur les données par l'ajustement global. Ainsi, ils ne contribuent pas aux incertitudes systématiques, étant incorporés dans l'erreur statistique. Cependant, il se peut que la paramétrisation utilisée ne prenne pas en compte tous les effets. De plus, il convient de quantifier l'impact de l'hypothèse que la fonction de résolution ainsi que les dilutions sont identiques entre l'échantillon $B_{\text {sav }}$ et $B_{C P}$.

Les paramètres de la fonction de résolution en $\Delta t$ ainsi que les dilutions sont obtenus à partir de données simulées à la génération des échantillons équivalents à $B_{s a v}$ et $B_{C P}$. La différence sur $\sin 2 \beta(\cos 2 \beta)$ obtenue avec les deux ensembles de paramètres de la fonction de résolution est assigné en tant qu'incertitude systématique (a). De même, la différence entre les dilutions des deux échantillons sert à assigner une incertitude systématique (b).

La contribution de la gaussienne outlier à l'incertitude systématique totale est obtenue par variation de ces paramètres. La largeur est changée de 4 à 12 ps et le biais de -2 à +2 ps par rapport aux valeurs nominales de 8 ps pour la largeur et 0 pour le biais (c). La même méthode est utilisée pour évaluer l'incertitude systématique associée à la fraction de la gaussienne tail. Le facteur d'échelle $S_{\text {tail }}$ est fixé aux valeurs 2 et 5 (la valeur nominale est de 3) (d). 

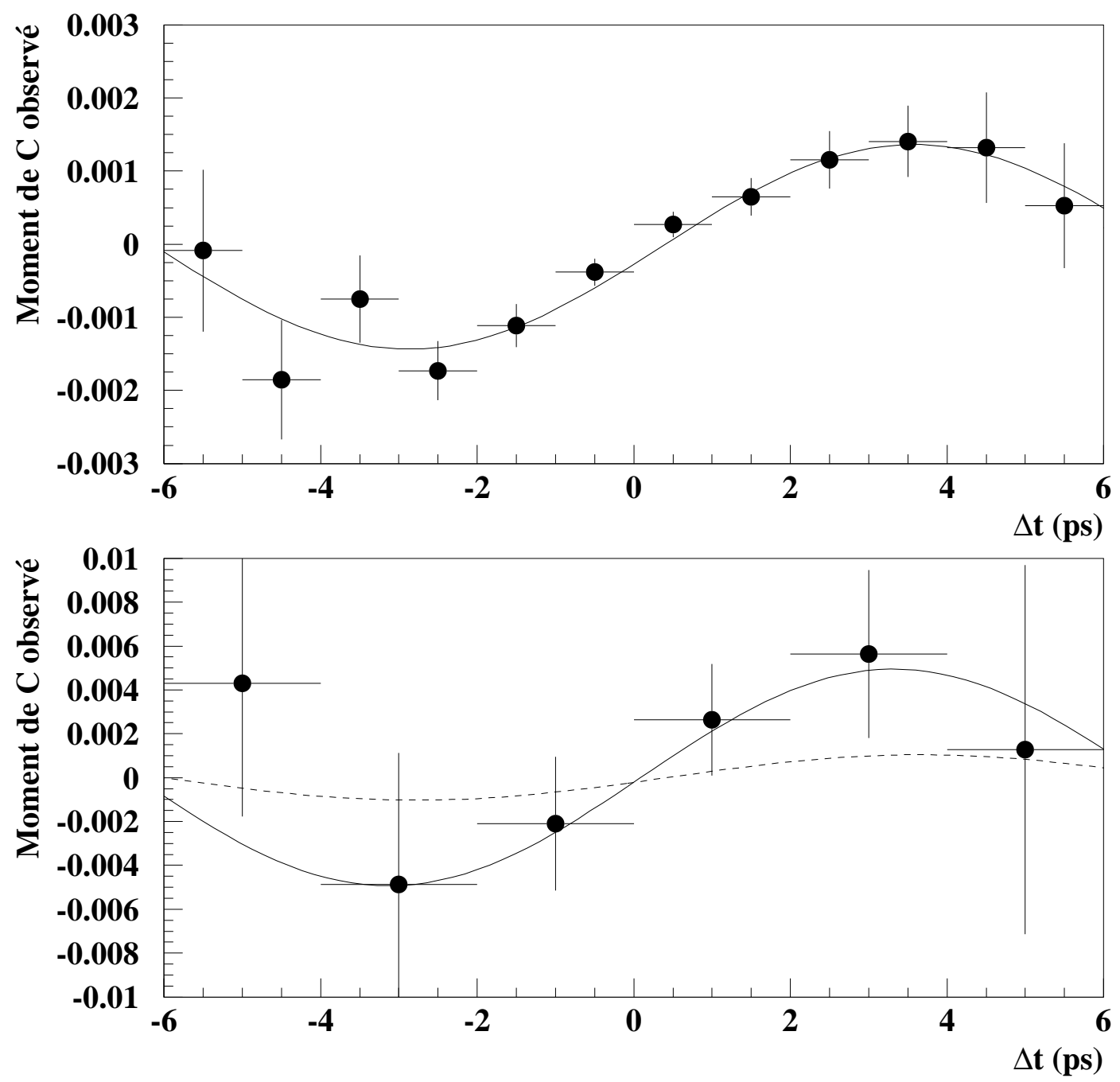

FIG. 6.26: (haut) Moment de $\mathcal{C}$ obtenu à partir de données simulées du canal $B_{d}^{0} \rightarrow J / \psi\left(K_{S}^{0} \pi^{0}\right)^{* 0}$. La courbe superposée correspond à la densité de probabilité normalisée prise aux valeurs centrales des paramètres obtenues avec l'ajustement par maximum de vraisemblance ; (bas) idem sur les données. Les courbes superposées correspondent à deux valeurs de $\cos 2 \beta: 3.32$ pour la courbe en trait plein, 0.67 pour la courbe en traits pointillés. 


\begin{tabular}{|l|c|c|}
\hline Largeur/biais $(\mathrm{ps})$ & $\delta \sin 2 \beta$ & $\delta \cos 2 \beta$ \\
\hline \hline $8.0 / 0.0$ & - & - \\
\hline $4.0 / 0.0$ & -0.0014 & +0.0005 \\
$12.0 / 0.0$ & -0.0001 & -0.0001 \\
\hline $8.0 /-2.0$ & -0.0001 & -0.0001 \\
$8.0 /+2.0$ & +0.0000 & -0.0000 \\
\hline Total & \pm 0.0014 & \pm 0.0005 \\
\hline
\end{tabular}

ТАВ. 6.17: Incertitude systématique sur $\sin 2 \beta$ et $\cos 2 \beta$ due aux valeurs fixes de la gaussienne outlier dans la fonction de résolution en $\Delta t$ estimée en variant la largeur et le biais de cette gaussienne. $\delta \sin 2 \beta$ est la différence de $\sin 2 \beta$ entre l'ajustement effectué avec les nouvelles valeurs et l'ajustement nominal de largeur 8 ps et de biais nul.

Afin d'évaluer les effets d'une fonction de résolution différente entre les événements dont la saveur est correctement ou incorrectement déterminée, on sépare en deux échantillons l'échantillon équivalent à $B_{C P}$ suivant que l'étiquetage est correct ou non et on estime $\sin 2 \beta(\cos 2 \beta)$ ainsi que les paramètres de la fonction de résolution sur ces deux échantillons (les dilutions $D$ sont fixées à +1 et -1 , respectivement). On compare la moyenne pondérée des deux valeurs de $\sin 2 \beta(\cos 2 \beta)$ obtenues à celle obtenue avec l'échantillon initiale où les dilutions sont fixées à leurs valeurs vraies. L'écart obtenu est assigné comme incertitude systématique (e).

Enfin, différents modèles d'incertitudes d'alignement ont été simulés d'où sont extraits différents ensembles de paramètres de la fonction de résolution. La moyenne des décalages de la valeur de $\sin 2 \beta(\cos 2 \beta)$ par rapport à l'alignement initiale des données simulées est prise comme incertitude systématique (f).

\subsubsection{Propriétés des bruits de fond : $B_{\text {sav }}$}

Un ensemble de paramètres sont fixés pour les bruits de fond soit parce qu'ils sont extraits à partir d'un ajustement indépendant (ajustement par une gaussienne et une fonction Argus du spectre en $m_{\mathrm{ES}}$ après coupure sur $\Delta E$ ), soit ils sont évalués par une source extérieure, telle que des données simulées. On décrit dans cette section les variations appliquées pour estimer les incertitudes systématiques sur $\sin 2 \beta$ et $\cos 2 \beta$.

L'effet des incertitudes provenant de l'ajustement en $m_{\mathrm{ES}} \operatorname{sur} \sin 2 \beta(\cos 2 \beta)$ est estimé comme suit : une probabilité globale pour les événements ayant un $m_{\mathrm{ES}}>5.27 \mathrm{GeV} / c^{2}$ est mesurée, puis assignée à ces événements (les événements ayant un $m_{\mathrm{ES}}<5.27 \mathrm{GeV} / c^{2}$ ont une probabilité signal nulle). La différence entre la valeur de $\sin 2 \beta(\cos 2 \beta)$ obtenue avec cette configuration et celle obtenue en variant cette probabilité globale de $\pm 1 \sigma$ est assignée comme incertitude systématique (f). Les résultats sont présentés dans la table 6.18.

\begin{tabular}{|l|cc|}
\hline Variation & $\delta \sin 2 \beta$ & $\delta \cos 2 \beta$ \\
\hline \hline$\Delta$ (up) $p_{\text {Sig, Mix }}$ & -0.0005 & -0.0017 \\
$\Delta$ (down) $p_{\text {Sig, Mix }}$ & +0.0003 & +0.0007 \\
\hline
\end{tabular}

TAB. 6.18: Incertitude systématique due aux incertitudes statistiques dans les probabilités signal événement par événement.

La limite supérieure de la fonction Argus est fixée à l'énergie des faisceaux dans le centre de 
masse, i.e. $5.291 \mathrm{GeV} / c^{2}$. Afin de tenir compte de l'incertitude sur l'énergie des faisceaux, on varie cette limite de $\pm 0.002 \mathrm{GeV} / c^{2}$. La variation maximale de $\sin 2 \beta(\cos 2 \beta)$ est prise comme incertitude systématique $(\mathrm{g})$. Les résultats sont présentés dans la table 6.19

\begin{tabular}{|l|cc|}
\hline Variation & $\delta \sin 2 \beta$ & $\delta \cos 2 \beta$ \\
\hline \hline$E_{\text {beam }}=5.289$ & -0.0024 & -0.0110 \\
$E_{\text {beam }}=5.293$ & -0.0006 & -0.0018 \\
\hline
\end{tabular}

TAB. 6.19: Incertitudes systématiques dues à la valeur fixe de $m_{0}$ dans la distribution Argus.

Dans l'ajustement global, la fraction de bruits de fond oscillant est prise égale à zéro. La fraction de bruits de fond du type temps de vie $(\tau>0)$ est remplacée par un bruit de fond du type mélange et la variation de $\sin 2 \beta(\cos 2 \beta)$ est prise comme incertitude systématique (h). Enfin, la fraction $\delta_{\text {peak }}$ estimée à partir de données simulées est de $1.5 \pm 0.5 \%$. La variation de $\sin 2 \beta(\cos 2 \beta)$ due à l'incertitude sur cette fraction est assignée comme incertitude systématique (i).

\subsubsection{Propriétés des bruits de fond : $B_{C P}$}

Dans l'ajustement global, les fractions signal et bruits de fond sont fixées ainsi que les parités $C P$ effectives des bruits de fond. On varie chaque fraction par $\pm 1 \sigma$ ainsi que les parités $C P$ effectives des bruits de fond selon la table 6.9 et on ajoute en quadrature ces variations pour obtenir l'incertitude systématique associée (k). Les bruits de fond sont supposés avoir les mêmes dilutions que le signal. Pour estimer cette incertitude, on utilise les dilutions obtenues avec l'échantillon $B^{+}$ données dans la table 6.11 (m). Les erreurs sur les amplitudes sont prises en compte en les faisant varier de $\pm 1 \sigma$ en utilisant la matrice de covariance afin d'avoir la condition de normalisation $\left|A_{0}\right|^{2}+\left|A_{\|}\right|^{2}+\left|A_{\perp}\right|^{2}=1$ (cf. Fig. 6.28)(n). Afin de tenir compte de la statistique finie des échantillons utilisées pour la déterminations des moments, on divise chaque échantillon par 10. L'incertitude systématique associée est obtenue en prenant le RMS de ces 10 expériences divisé par la racine carrée du nombre d'expériences (o). Les événements à bas $m_{\mathrm{ES}}$ sont utilisés pour déterminer les moments des bruits fond pris initialement plats $(p)$.

\subsubsection{Paramètres externes}

Afin d'évaluer l'effet de l'incertitude sur le «boost » et l'échelle en $z$, les valeurs de $\Delta t$ sont redimensionnées à $\pm 0.6 \%$ sur des données simulées (q). L'algorithme de calcul du vertex du méson $B_{t a g}$ utilise la position de la région lumineuse comme contrainte. La contrainte la plus importante provient de la position en $y$. On varie la position en $y$ de +20 et $+40 \mu \mathrm{m}$ et l'erreur sur $y$ est élargie de 30 et $60 \mu \mathrm{m}$ séparément (r). Enfin, la durée de vie ainsi que la fréquence d'oscillation des mésons

$B_{d}^{0}$ sont fixées aux valeurs moyennes mondiales [28]. On estime l'incertitude sur $\sin 2 \beta(\cos 2 \beta)$ à partir des incertitudes sur ces quantités $(s, t)$.

\subsubsection{Statistique Monte Carlo}

La technique de mesure de $\sin 2 \beta$ et $\cos 2 \beta$ est validée sur un échantillon de données simulées. Cet échantillon est découpé en des échantillons de taille équivalente à la statistique des données. 

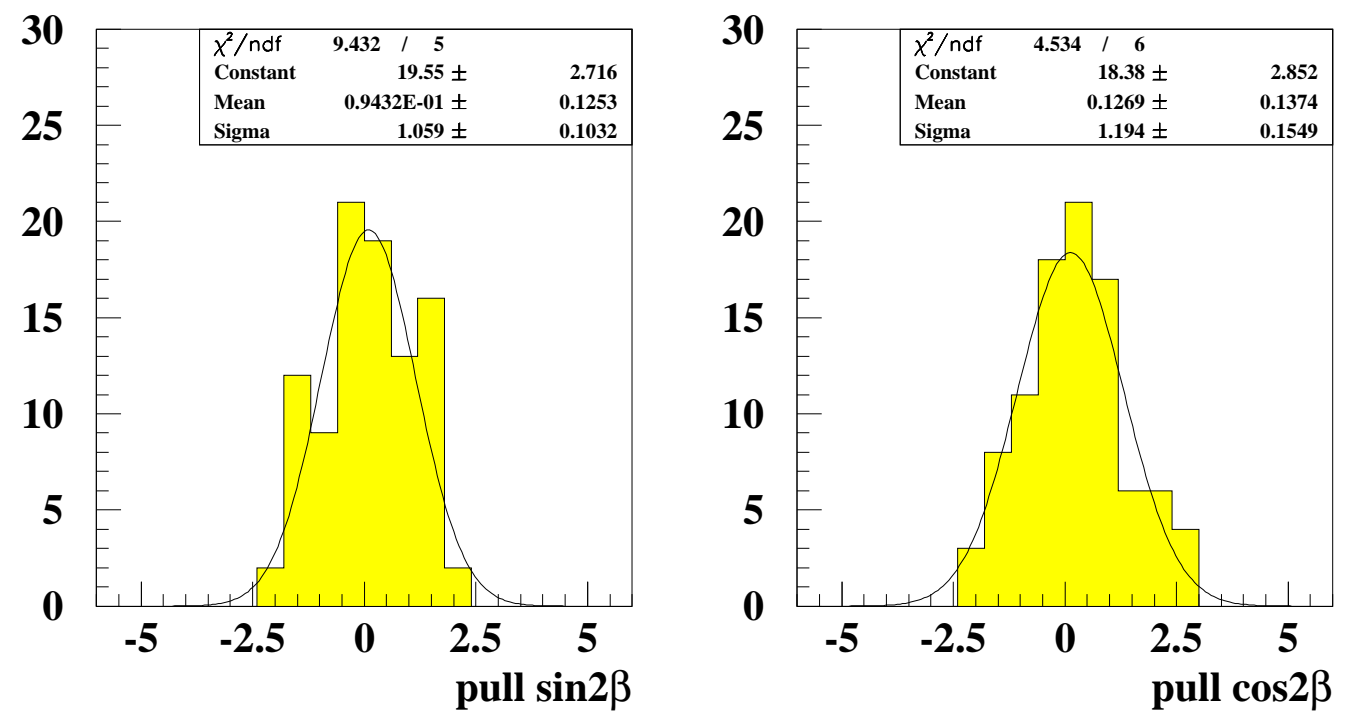

FIG. 6.27: Distributions des pulls de $\sin 2 \beta$ et $\cos 2 \beta$ obtenues à partir de 94 expériences de taille équivalente à la statistique des données obtenues avec des données simulées.
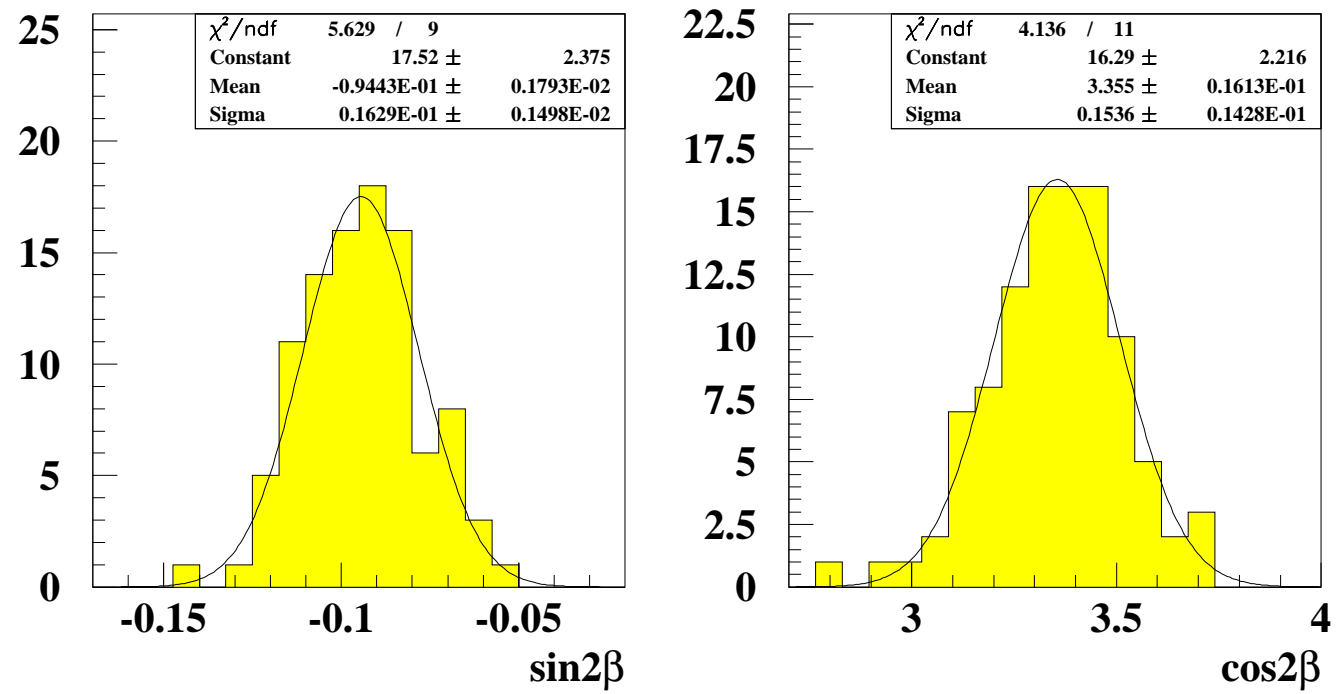

FIG. 6.28: Distributions de $\sin 2 \beta$ et $\cos 2 \beta$ obtenues en faisant varier les amplitudes de $\pm 1 \sigma$ tout en gardant la condition de normalisation.

La distribution des pulls de $\sin 2 \beta$ et $\cos 2 \beta$ est représenté sur la figure 6.27. La moyenne des pulls de $\sin 2 \beta$ et pour $\cos 2 \beta$ est compatible avec zéro. Aucune correction n'est appliquée sur les valeurs mesurées et une erreur systématique correspondant à l'erreur statistique sur ces moyennes est assignée $(u)$.

\subsection{Ambiguïté sur les phases fortes et signe de $\cos 2 \beta$}

La mesure de $\sin 2 \beta$ conduit à quatre ambiguïtés sur l'angle $\beta$ [173] :

$$
\beta, \frac{\pi}{2}-\beta, \pi+\beta, \frac{3 \pi}{2}-\beta
$$




\begin{tabular}{|l|l|l|}
\hline Source & $\sin 2 \beta$ & $\cos 2 \beta$ \\
\hline \hline \multicolumn{3}{|c|}{ Propriétés du signal } \\
\hline (a) fonction de résolution $\Delta t$ & \pm 0.002 & \pm 0.002 \\
\hline (b) dilutions signal $B_{C P}$ vs $B_{\text {sav }}$ & \pm 0.012 & \pm 0.013 \\
\hline (c) modélisation de la gaussienne outliers & \pm 0.001 & \pm 0.000 \\
\hline (d) paramètre $f_{\text {tail }}$ & \pm 0.002 & \pm 0.003 \\
\hline (e) corrélation résolution/étiquetage & \pm 0.001 & \pm 0.001 \\
\hline (f) alignement du SVT & \pm 0.010 & \pm 0.030 \\
\hline \multicolumn{3}{|c|}{ Propriétés des bruits de fond : $B_{\text {sav }}$} \\
\hline (g) probabilité signal & \pm 0.001 & \pm 0.001 \\
\hline (h) valeur de $m_{0}$ pour la fonction Argus & \pm 0.002 & \pm 0.010 \\
\hline (i) contribution oscillante & \pm 0.001 & \pm 0.022 \\
\hline (j) contribution de $\delta_{\text {peak }}$ & \pm 0.001 & \pm 0.003 \\
\hline \multicolumn{3}{|c|}{ Propriétés des bruits de fond : $B_{C P}$} \\
\hline (k) composition et parité $C P$ & \pm 0.032 & \pm 0.142 \\
\hline (m) paramétrisation & \pm 0.002 & \pm 0.006 \\
\hline (n) Incertitudes sur les amplitudes & \pm 0.016 & \pm 0.154 \\
\hline (o) Statistique moments & \pm 0.030 & \pm 0.030 \\
\hline (p) Distributions angulaires bruits de fond & \pm 0.024 & \pm 0.064 \\
\hline \multicolumn{3}{|c|}{ Paramètres externes } \\
\hline (q) échelle en $z$ et « boost » & \pm 0.001 & \pm 0.001 \\
\hline (r) région lumineuse & \pm 0.010 & \pm 0.040 \\
\hline (s) temps de vie $B^{0}$ & \pm 0.014 & \pm 0.040 \\
\hline (t) $\Delta M$ B $B^{0}$ & \pm 0.018 & \pm 0.032 \\
\hline \multicolumn{3}{|c|}{ (u) Monte Carlo } \\
\hline (u) statistique Monte Carlo & \pm 0.130 & \pm 0.140 \\
\hline \hline Total incertitude systématique & \pm 0.14 & \pm 0.27 \\
\hline Incertitude statistique & \pm 0.57 & ${ }_{-0.96}^{0.76}$ \\
\hline
\end{tabular}

ТАВ. 6.20: Résumé des diverses contributions aux incertitudes systématiques sur $\sin 2 \beta$ et $\cos 2 \beta$. Les lettres entre parenthèses renvoient à la description de l'évaluation de l'incertitude systématique dans le texte.

Afin de lever cette ambiguité, il est nécessaire de mesurer le signe de $\cos 2 \beta$ ainsi que le signe de $\sin \beta$. Une mesure du signe de $\cos 2 \beta$ permet d'éliminer deux des quatre ambiguïtés en ne gardant que les solutions :

$$
\beta, \pi+\beta \text { si } \cos 2 \beta>0, \quad \frac{\pi}{2}-\beta, \frac{3 \pi}{2}-\beta \text { si } \cos 2 \beta<0 .
$$

L'ambiguïté sur la détermination des phases fortes, $\left(\delta_{\|}, \delta_{\perp}\right) \rightarrow\left(-\delta_{\|}, \pi-\delta_{\perp}\right)$, ne permet pas de fixer le signe de la fonction $\mathcal{C}(\vec{\omega}, \vec{A})$ (les fonctions $\mathcal{A}(\vec{\omega} ; \vec{A}), \mathcal{P}(\vec{\omega} ; \vec{A})$ et $\mathcal{S}(\vec{\omega} ; \vec{A})$ restent invariantes sous le changement des phases fortes). Puisque $\cos 2 \beta$ est en produit avec la fonction $\mathcal{C}(\vec{\omega} ; \vec{A})$ (cf. eq. (6.28)), on ne peut donc distinguer la solution $\left(\delta_{\|}, \delta_{\perp}, \cos 2 \beta\right)$ de la solution $\left(-\delta_{\|}, \pi-\delta_{\perp},-\cos 2 \beta\right)$. Les deux solutions obtenues sur les données sont

$$
\begin{gathered}
\left(\delta_{\|}=2.77, \delta_{\perp}=0.21, \cos 2 \beta=-3.32\right)[\text { Solution I] } \\
\left(\delta_{\|}=-2.77, \delta_{\perp}=2.93, \cos 2 \beta=+3.32\right) \text { [Solution II] }
\end{gathered}
$$

Ces deux solutions impliquent des processus physiques assez différents [174]. Dans la désintégra- 


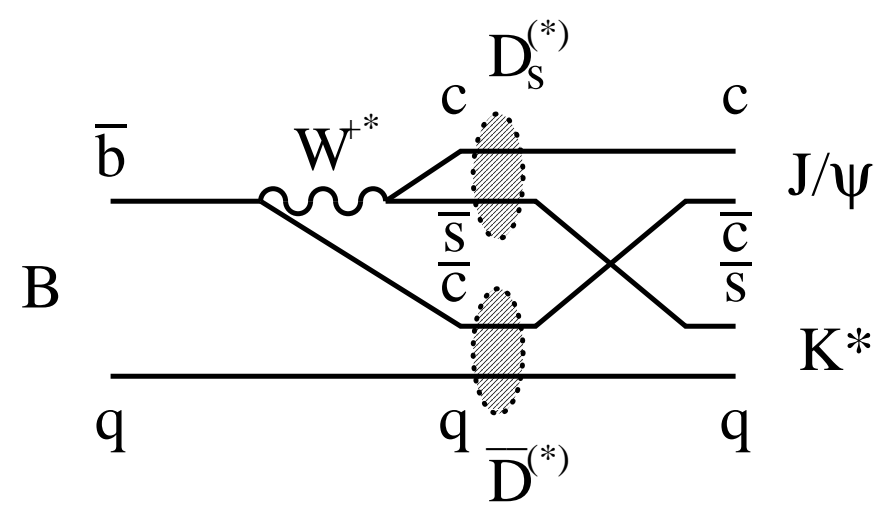

FIG. 6.29: Exemple de production de $J / \psi K^{*}$ via la rediffusion d'un état intermédiaire sur couche de masse autorisé de couleur, $B \rightarrow \bar{D}^{(*)} D_{s}^{(*)}$.

tion $B(q \bar{b}) \rightarrow J / \psi(c \bar{c}) K^{*}(q \bar{s})$, le quark $s$ est produit avec une hélicité $+1 / 2$ par l'interaction faible dans la limite $m_{s} \rightarrow 0$. L'interaction forte conserve cette hélicité si $m_{s}=0$. Ainsi, dans l'approximation du quark spectateur, le quark $\bar{s}_{L}$ s'associe avec le quark spectateur, $u$ ou $d$, afin de former le méson $K^{*}$. Si le quark $\bar{s}_{L}$ conserve son hélicité, l'hélicité du méson $K^{*}$ ne peut valoir -1 . En conséquence, on s'attend à ce que l'amplitude $H_{+1}$ domine sur l'amplitude $H_{-1}$, i.e $\left|H_{+1}\right| \gg\left|H_{-1}\right|$ [105]. Dans ce cas, $A_{\|} \simeq+A_{\perp}\left(H_{ \pm 1}=1 / \sqrt{2}\left(A_{\|} \pm A_{\perp}\right)\right)$, valable aussi bien en amplitude qu'en phase. Cette situation correspond à la solution II. L'autre situation, c'est-à-dire $\left|H_{-1}\right| \gg\left|H_{+1}\right|$ soit $A_{\|} \simeq-A_{\perp}$, correspond à la solution I. La solution I correspond à la situation où des FSI longue distance très importantes ont renversé l'hélicité du quark $\bar{s}$. Une telle situation, bien que peu probable, peut provenir d'un processus de rediffusion via un état intermédiaire sur couche de masse autorisé de couleur, tel que $B \rightarrow \bar{D}^{(*)} D_{s}^{(*)} \rightarrow J / \psi K^{*}$. Les FSI réarrangent les quarks pour produire au final un $J / \psi K^{*}$ (cf. Fig. 6.29). Un tel processus en deux étapes, passant par un état intermédiaire sur couche de masse autorisé de couleur, a plus de chance de dominer par rapport à la production directe de la transition supprimée de couleur.

En conclusion, la solution I, bien qu'elle ne puisse être exclue, a peu de chance de se produire étant donnée l'importance de la rediffusion nécessaire au retournement de l'hélicité du quark $\bar{s}$. La solution II, solution préférée des théoriciens, est celle qui conduit au signe positif pour $\cos 2 \beta$.

La figure 6.30 représente le plan $\bar{\rho}-\bar{\eta}$ où les quatre solutions pour $\beta$, obtenues à partir de la mesure de la moyenne mondiale de $\sin 2 \beta$, sont superposées. La solution préférée, avec le signe positif pour $\cos 2 \beta$, est compatible avec le Modèle Standard. À noter que cette conclusion physique est directement reliée aux phases fortes et donc aux distributions angulaires. On comprend l'importance du soin à apporter dans la dérivation de ces distributions qui conduisent à des définitions de repères et d'angles donnés. La forme de ces distributions dépend des conventions choisies, à travers l'orientation des repères et la définition des angles, qui ont un impact direct sur les résultats physiques. 


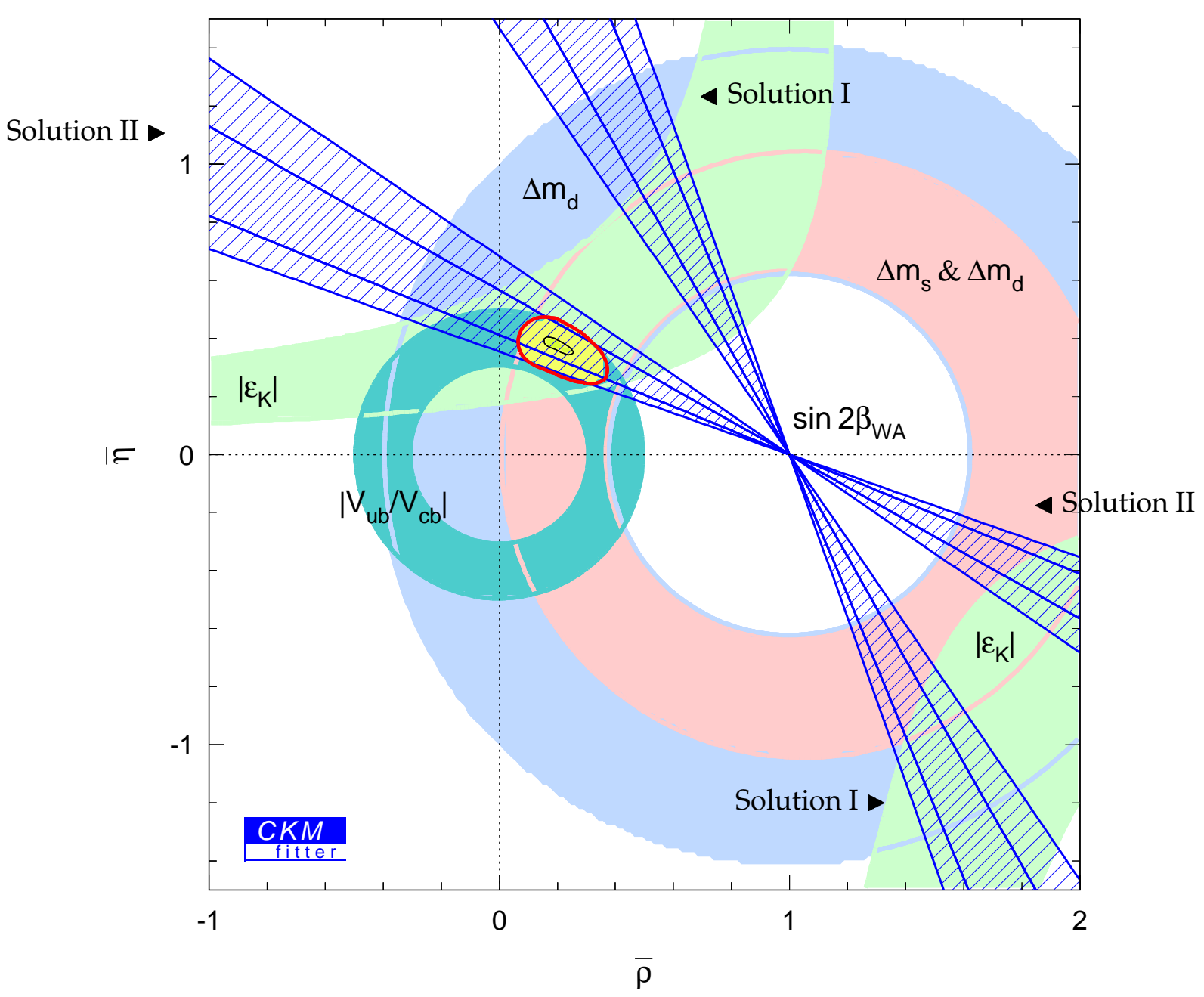

FIG. 6.30: Plan $\bar{\rho}-\bar{\eta}$ où les quatre solutions pour $\beta$, obtenues à partir de la mesure de la moyenne modiale de sin $2 \beta$, sont représentées par des bandes hachurées, correspondant aux niveaux de confiance de $68 \%$ et $95 \%$, respectivement. Les solutions I et II permettent d'exclure deux des quatre valeurs possibles pour $\beta$. 


\section{Conclusion}

Les travaux présentés dans cette thèse couvrent l'analyse des canaux vecteur-vecteur du type charmonium- $K^{*}(892)$. L'analyse des désintégrations $B \rightarrow J / \psi K^{*}(892)$ sur $81.9 \mathrm{fb}^{-1}$ de données collectées par le détecteur BABAR au pic de la résonance $\Upsilon(4 S)$ a conduit à des résultats de violation de $C P$, avec les mesures de $\sin 2 \beta$ et $\cos 2 \beta$, et de physique hadronique par la mesure des amplitudes de transversité. Les différentes distributions angulaires pour l'étude des autres désintégrations charmonium- $K^{*}(892)$, à savoir les désintégrations $\chi_{c 1} K^{*}(892)$ et $\psi(2 S) K^{*}(892)$ sont dérivées dans les bases d'hélicité et de transversité.

L'analyse du canal $B_{d}^{0} \rightarrow J / \psi\left(K_{S}^{0} \pi^{0}\right)^{* 0}$ par une analyse angulaire complète dépendante du temps a conduit à la mesure de $\sin 2 \beta$ et la première mesure de $\cos 2 \beta$ :

$$
\sin 2 \beta=-0.10 \pm 0.57 \pm 0.14, \quad \cos 2 \beta=3.32_{-0.96}^{+0.76} \pm 0.27
$$

Cette mesure nécessite au préalable la mesure des amplitudes de transversité, qui est effectuée avec les canaux symétriques d'isospin. Les résultats obtenus avec un ajustement par pseudo-maximum de vraisemblance sont

$$
\begin{gathered}
\left|A_{0}\right|^{2}=0.565 \pm 0.011 \pm 0.004,\left|A_{\|}\right|^{2}=0.206 \pm 0.016 \pm 0.007,\left|A_{\perp}\right|^{2}=0.228 \pm 0.016 \pm 0.007, \\
\delta_{\|}=-2.766 \pm 0.105 \pm 0.040, \delta_{\perp}=2.935 \pm 0.067 \pm 0.040 .
\end{gathered}
$$

À noter que $\left(\delta_{\|}, \delta_{\perp}\right) \rightarrow\left(-\delta_{\|}, \pi-\delta_{\perp}\right)$ est aussi une solution. Les phases fortes $\delta_{\|}$et $\delta_{\perp}$ sont à $\gtrsim 3 \sigma$ de $\pm \pi$, signant ainsi la présence d'interactions dans l'état final et la rupture de l'approximation de factorisation pour les canaux $B \rightarrow J / \psi K^{*}(892)$. De plus, les modules des amplitudes sont en désaccord avec les prédictions de la factorisation. L'analyse de l'asymétrie avant-arrière en fonction de la masse $K \pi$ a révélé la présence d'une onde $S$ cohérente qui interfère avec le $K^{*}(892)$. C'est la première démonstration de la présence de cette onde dans le système $K \pi$ provenant de la désintégration d'un méson $B$. L'analyse future des amplitudes devra prendre en compte cette onde.

Des considérations théoriques basées sur la conservation de l'hélicité du quark $s$ favorise le choix de phases fortes données ci-dessus conduisant au signe positif pour $\cos 2 \beta$. Ce signe de $\cos 2 \beta$ est celui compatible avec le Modèle Standard. À noter que cette conclusion physique est directement reliée aux phases fortes et donc aux distributions angulaires. On comprend l'importance du soin à apporter dans la dérivation de ces distributions qui conduisent à des définitions de repères et d'angles donnés. La forme de ces distributions dépend des conventions choisies, à travers l'orientation des repères et la définition des angles, qui ont un impact direct sur les résultats physiques. 


\section{Annexe A}

\section{Remarks on Conventions for the Derivation of Angular Distribution Using the Helicity Formalism}

\section{A.1 Introduction}

This appendix is not meant to be a complete guide to the use of the helicity formalism. There are several references that give a good introduction to the use of the helicity formalism for describing the dynamics of particle decays $[1,2,3,4,5]$. The reader is thus assumed to be familiar with this formalism. This appendix however emphasizes the conventions and their consequences in the derivation of angular distributions. These conventions come from two origins :

- the first one is the choice of the Euler angles. The choice of the Euler angles for a rotation fixes the orientation of the various coordinate systems involved in an angular distribution. The construction of these coordinate systems in which the decay angles are measured requires a particular care because the specific form of the angular distribution depends on it ;

- the second origin comes from the definition of the so-called "second" particle. In most books or articles on the helicity formalism, the examples consider only one unstable particle so that the orientation of the frame associated with the "second" particle does not matter. However, in $B$ decays, we often have to treat the case where the $B$ meson decays into two unstable mesons, whose decay products are analyzed. In this case, the orientation of the frame associated with the "second" particle which is linked with the definition of the "second" particle vector state matters. It has a direct consequence on the angle definitions.

We emphasize the construction of the various coordinate systems in which the decay angles are measured in two specific conventions used in the literature : the Jacob-Wick convention and the Jackson convention. We begin by a review on rotation by treating the active and passive views followed by the special case of inversion. We then turn to the definition of the one-particle plane-wave helicity state and explain where these conventions enter. We finally treat the two-particle planewave helicity state in the center-of-mass frame and finish by an example of the angular distribution of a general sequential two-by-two body decays. 


\section{A.2 Rotation in Euclidean Three-Dimensional Space}

\section{A.2.1 Definitions}

There is unfortunately a large variety of possibilities for writing down a rotation. Suppose we have a coordinate system $x, y, z$ (right-handed) in which we describe our physical system. Then, if we speak of the rotation $R$, it still remains open what shall be rotated : the coordinate frame or the physical system. A rotation is said to be ${ }^{1}$

- passive $\left[R_{p}\right]$ if the physical system remains fixed and the coordinate frame is rotated (see Fig. A.1 a)). [6] (i.e. the physical system is described with respect to a fixed Cartesian system of axes $x, y, z$ and another rotated $\left.x^{\prime}, y^{\prime}, z^{\prime}\right)$;

- active $\left[R_{a}\right]$ if the physical system is bodily rotated, whereas the coordinate frame remains fixed (see Fig. A.1 b)). It means that another coordinate system $x^{\prime}, y^{\prime}, z^{\prime}$ is attached to the physical system and rotated with respect to the fixed coordinate system $x, y, z[7]$.
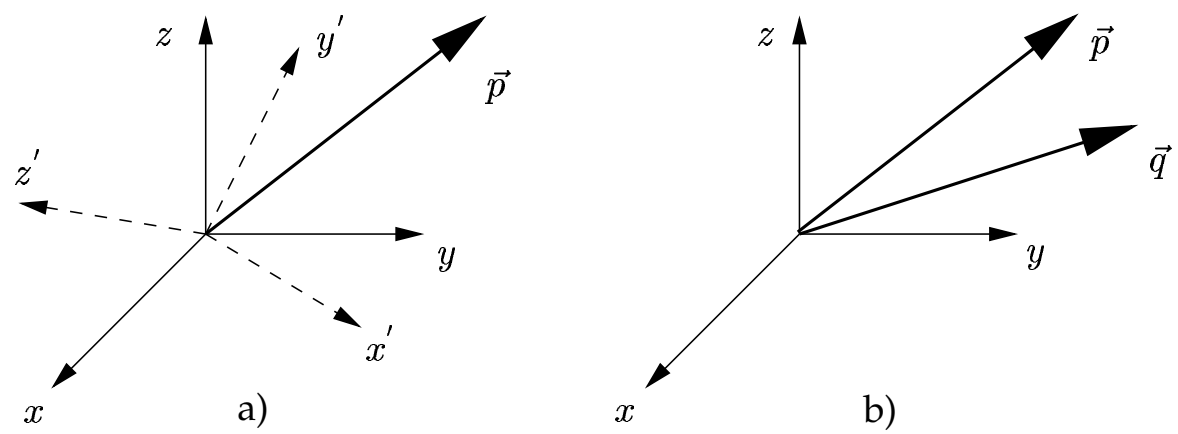

FIG. A.1: a) Passive interpretation of rotations. The same vector $\vec{p}$ is described with respect to a Cartesian system of axes $x, y, z$ and another rotated system $x^{\prime}, y^{\prime}, z^{\prime}$. b) Active interpretation of rotations. A rotation performed on the vector $\vec{p}$ transforms it into a new vector $\vec{q}$.

There are several possible parametrizations of a rotation $R$. A useful set of parameters is given by the Euler angles $\alpha, \beta, \gamma$. In fact, a rotation $R$ which brings a right-handed system of three orthogonal axes from an initial position coinciding with $x, y, z$ to some final position $x^{\prime}, y^{\prime}, z^{\prime}$ can be regarded as the result of the following three successive rotations :

1. rotation around the $z$-axis through $\gamma$;

2. rotation around the $y$-axis through $\beta$;

3. rotation around the $z$-axis through $\alpha$;

that we note $R(\alpha, \beta, \gamma)=R_{z}(\alpha) R_{y}(\beta) R_{z}(\gamma)$. The domain of $\alpha, \beta, \gamma$ is $0 \leq \alpha \leq 2 \pi, 0 \leq \beta \leq \pi$, $0 \leq \gamma \leq 2 \pi$. All the angles are defined counterclockwise. Let us note that the two views are linked by $[8]$

$$
R_{a}(\alpha, \beta, \gamma)=R_{p}^{-1}(\alpha, \beta, \gamma)=R_{p}(-\gamma,-\beta,-\alpha) .
$$

In the next sections, we only consider the ACTIVE VIEW.

\footnotetext{
${ }^{1}$ Without carefully keeping track of what is rotated against what, one gets almost immediately lost in confusion about + and - signs in matrices and exponential operators.
} 


\section{A.2.2 Representation}

A matrix representation of $R_{a}(\alpha, \beta, \gamma)=R_{a, z}(\alpha) R_{a, y}(\beta) R_{a, z}(\gamma)$ is

$$
\begin{aligned}
& \left(\begin{array}{ccc}
\cos \alpha & -\sin \alpha & 0 \\
\sin \alpha & \cos \alpha & 0 \\
0 & 0 & 1
\end{array}\right) \times\left(\begin{array}{ccc}
\cos \beta & 0 & \sin \beta \\
0 & 1 & 0 \\
-\sin \beta & 0 & \cos \beta
\end{array}\right) \times\left(\begin{array}{ccc}
\cos \gamma & -\sin \gamma & 0 \\
\sin \gamma & \cos \gamma & 0 \\
0 & 0 & 1
\end{array}\right)= \\
& \left(\begin{array}{ccc}
\cos \alpha \cos \beta \cos \gamma-\sin \alpha \sin \gamma & -\cos \alpha \cos \beta \sin \gamma-\sin \alpha \cos \gamma & \cos \alpha \sin \beta \\
\sin \alpha \cos \beta \cos \gamma+\cos \alpha \sin \gamma & -\sin \alpha \cos \beta \sin \gamma+\cos \alpha \cos \gamma & \sin \alpha \sin \beta \\
-\sin \beta \cos \gamma & \sin \beta \sin \gamma & \cos \beta
\end{array}\right)
\end{aligned}
$$

We give here two special cases which will be useful later :

$$
\begin{gathered}
R_{a, x}(\pi)=R_{a}(\pi, \pi, 0) \text { or } R_{a}\left(\frac{\pi}{2}, \pi,-\frac{\pi}{2}\right)=\left(\begin{array}{ccc}
1 & 0 & 0 \\
0 & -1 & 0 \\
0 & 0 & -1
\end{array}\right), \\
R_{a, y}(\pi)=R_{a}(0, \pi, 0) \text { or } R_{a}(\pi, \pi, \pi)=\left(\begin{array}{ccc}
-1 & 0 & 0 \\
0 & 1 & 0 \\
0 & 0 & -1
\end{array}\right) .
\end{gathered}
$$

\section{A.2.3 Inversion}

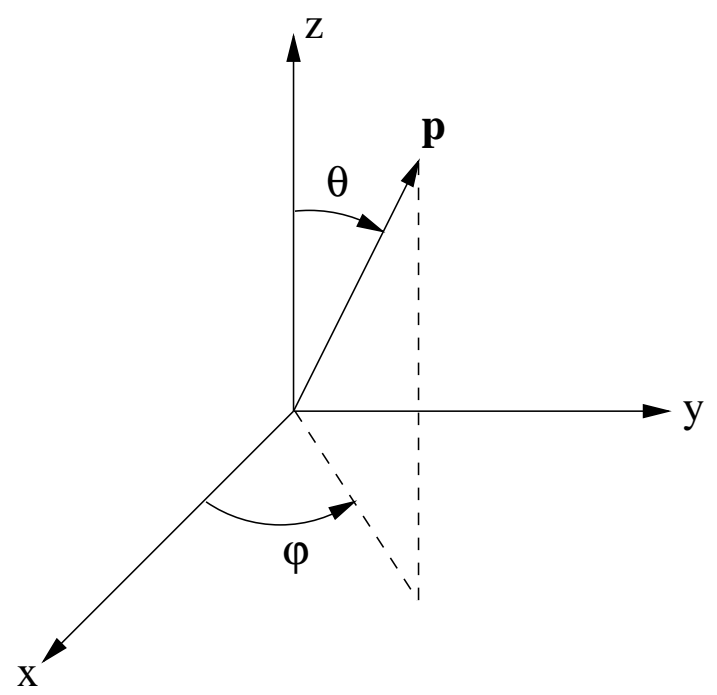

FIG. A.2: Definition of the spherical angles $(\theta, \varphi)$ in a right-handed coordinate system. The polar angle $\theta$ is going from the $z$-axis to the vector $\vec{p}$ in the $(z, \hat{p})$ plane. The azimuthal angle $\varphi$ is counterclockwise, i.e. going from the $x$-axis to the projection of the vector $\vec{p}$ onto the $x-y$ plane, as seen from the positive $z$-axis. We have $0 \leq \varphi \leq 2 \pi$ and $0 \leq \theta \leq \pi$.

Let us consider the special case where the system is simply a vector $\vec{p}$ in an arbitrary direction specified by the spherical angles $\theta$ and $\varphi$. In addition to the coordinates $x, y, z$ fixed in space, let us introduce a "moving" coordinate system $\left(x^{\prime}, y^{\prime}, z^{\prime}\right)$ with $\vec{p}$ along the positive $z^{\prime}$-axis. A rotation of the coordinates $\left(x^{\prime}, y^{\prime}, z^{\prime}\right)$ relative to $(x, y, z)$ is defined by three Eulerian angles $\alpha, \beta$, $\gamma$, whereas the direction of $\vec{p}$ is defined by two spherical angles $\alpha \equiv \varphi$ and $\beta \equiv \theta$. In other words, $(\theta, \varphi)$ determine 
the new $z^{\prime}$-axis but leaves indefinite the position of the $x^{\prime}$ and $y^{\prime}$ axes, which depends also on the third Eulerian angle $\gamma$. It is the choice of the angle $\gamma$ that fixes the $x^{\prime}$ and $y^{\prime}$ axes with respect to the fixed coordinate system $(x, y, z)$. It is thus necessary to agree on some definite choice of the angle $\gamma$. Two conventions are used in the literature :

- the Jacob-Wick convention with $\gamma=-\varphi$;

- the Jackson convention with $\gamma=0$.

Under inversion, $\vec{p} \rightarrow-\vec{p}$. The vector $\vec{p}$ is defined by the two angles $\theta$ and $\varphi$, and the transformation $\vec{p} \rightarrow-\vec{p}$ is brought about by the changes $\varphi \rightarrow \varphi+\pi, \theta \rightarrow \pi-\theta$. What about the transformation for $\gamma$ ? By using the identities

$$
\begin{aligned}
R_{a}(\alpha+\pi, \pi-\beta,-\gamma) & =R_{a}(\alpha, \beta, \gamma) R_{a, x}(\pi), \\
R_{a}(\alpha+\pi, \pi-\beta, \pi-\gamma) & =R_{a}(\alpha, \beta, \gamma) R_{a, y}(\pi),
\end{aligned}
$$

it shows that inversion is thus the transformation :

$$
\gamma \rightarrow-\gamma \text { for } R_{a, x}(\pi), \text { or } \gamma \rightarrow \pi-\gamma \text { for } R_{a, y}(\pi)
$$

It means the following : the system initially parallel to the $z$-axis is brought anti-parallel to the $z$ axis by a rotation of $\pi$ either around the $x$-axis $(\gamma \rightarrow-\gamma)$ or the $y$-axis $(\gamma \rightarrow \pi-\gamma)$. One notices that the choice of the rotation either around the $x$-axis or around the $y$-axis is independent of the convention used for $\gamma$.

\section{A.3 Rotation Operator in Hilbert Space $\mathcal{H}$}

A rotation is represented in $\mathcal{H}$ by a unitary operator $U[R]$. A rotation by angle $\theta$ about an arbitrary axis whose direction is given by the unit vector $\hat{n}$ is given by the operator

$$
U\left[R_{a, \hat{n}}(\theta)\right]=e^{-i \theta \hat{\mathbf{n}} . \mathbf{J}},
$$

where $\mathbf{J}$ is the angular momentum operator. The general rotation $R_{a}(\alpha, \beta, \gamma)$ is then written as $[1,2,5]$

$$
U\left[R_{a}(\alpha, \beta, \gamma)\right]=U\left[R_{a, z}(\alpha)\right] U\left[R_{a, y}(\beta)\right] U\left[R_{a, z}(\gamma)\right]=e^{-i \alpha J_{z}} e^{-i \beta J_{y}} e^{-i \gamma J_{z}}
$$

The angular momentum eigenstates $|j m\rangle$ transform irreducibly under rotation because $\left[U[R], J^{2}\right]=0$. Thus, a representation is labeled by the total angular momentum $j$. The rotation of a state $|j m\rangle$ is given by

$$
U\left[R_{a}(\alpha, \beta, \gamma)\right]|j m\rangle=\sum_{m^{\prime}}\left|j m^{\prime}\right\rangle D_{m^{\prime} m}^{j}(\alpha, \beta, \gamma),
$$

where $D_{m^{\prime} m}^{j}(\alpha, \beta, \gamma) \equiv\left\langle j m^{\prime}\left|U\left[R_{a}(\alpha, \beta, \gamma)\right]\right| j m\right\rangle$ is the standard rotation matrix

$$
D_{m^{\prime} m}^{j}(\alpha, \beta, \gamma)=e^{-i m^{\prime} \alpha} d_{m^{\prime} m}^{j}(\beta) e^{-i m \gamma}
$$


and

$$
d_{m^{\prime} m}^{j}(\beta)=\left\langle j m^{\prime}\left|e^{-i \beta J_{y}}\right| j m\right\rangle .
$$

$d^{j}$ is a real matrix. The general expression of the $d^{\prime}$ s is due to Wigner (that's why it is sometimes called the Wigner d-matrix)

$$
\begin{aligned}
d_{m^{\prime} m}^{j}(\beta)= & \sum_{n} \frac{(-1)^{n} \sqrt{(j+m) !(j-m) !\left(j+m^{\prime}\right) !\left(j-m^{\prime}\right) !}}{\left(j-m^{\prime}-n\right) !(j+m-n) !\left(n+m^{\prime}-m\right) ! n !} \times \\
& \cos (\beta / 2)^{2 j+m-m^{\prime}-2 n}(-\sin (\beta / 2))^{m^{\prime}-m+2 n} .
\end{aligned}
$$

Some useful formulas are (from the properties of the Wigner's formula)

$$
d_{m^{\prime} m}^{j}(\beta)=(-1)^{m^{\prime}-m} d_{-m^{\prime},-m}^{j}(\beta)=(-1)^{m^{\prime}-m} d_{m m^{\prime}}^{j}(\beta)
$$

Finally, the orthogonality relation is

$$
\int_{0}^{2 \pi} \mathrm{d} \alpha \int_{0}^{\pi} \sin \beta \mathrm{d} \beta \int_{0}^{2 \pi} \mathrm{d} \gamma D_{m n}^{j *}(\alpha, \beta, \gamma) D_{m^{\prime} n^{\prime}}^{j^{\prime}}(\alpha, \beta, \gamma)=\delta_{m m^{\prime}} \delta_{n n^{\prime}} \delta_{j j^{\prime}}\left[\frac{8 \pi^{2}}{2 j+1}\right]
$$

\section{A.4 One-Particle Plane-Wave Helicity State}

In the relativistic theory, the orbital angular momentum $\vec{l}$ and the spin $\vec{s}$ of a moving particle are not separately conserved. Only the total angular momentum $\vec{j}=\vec{l}+\vec{s}$ is conserved. The component of the spin in any fixed direction (taken as the $z$-axis) is therefore also not conserved, and cannot be used to enumerate the polarization (spin) states of the moving particle. The states are labeled with the component of the spin along the direction of motion of the particle, which is called the helicity. There are two major advantages to do so. The first one is that it is invariant under ordinary rotations, so it is possible to construct states of definite angular momentum $\vec{J}$. Indeed, the quantization axis itself rotates with the system under rotation. The second one appears when we deal with two-particle states. In the center-of-mass frame of the two-particle system, the orbital angular momentum is always perpendicular to the direction of relative motion of the two particles. This means that the component of spin along the direction of relative motion is also the component of the total angular momentum of the two-particle system along the direction of relative motion.

The helicity state describing a particle with 4-momentum $p$ and helicity $\lambda$ is constructed from the rest state $|\vec{p}=0, \lambda\rangle \equiv|j m\rangle$, with spin component $\lambda=m$ along the $z$-axis, as follows :

$$
\begin{aligned}
|\vec{p}, \lambda\rangle & =U\left[\tilde{R}_{a}(\theta, \varphi)\right] U\left[L_{z}(p)\right]|\vec{p}=0, \lambda\rangle \\
& =U\left[L_{\vec{p}}(p)\right] U\left[\tilde{R}_{a}(\theta, \varphi)\right]|\vec{p}=0, \lambda\rangle
\end{aligned}
$$

where $(\theta, \varphi)$ are the spherical angles of $\vec{p}$ (see Fig A.2). It means either the particle is given a boost along $z\left(U\left[L_{z}(p)\right]\right)$ so that it acquires a momentum $\vec{p}_{z}$ and then is rotated $\left(U\left[R_{a}(\theta, \varphi)\right]\right)$, or it is rotated first so that the quantization axis is along the $\vec{p}$ direction and then boosted along $\vec{p}$. These two definitions of helicity state are of course equivalent. 
Here, we have not yet precise what is $\tilde{R}_{a}(\theta, \varphi)$. As seen before, because $\vec{p}$ is defined by two spherical angles $\theta$ and $\varphi$, we need to choose a value for the third angle $\gamma$ to completely define the rotation ${ }^{2}$. Two conventions exist in the literature :

- the Jacob-Wick convention [JW] : $\gamma=-\varphi[1,3,4,5,11,12]$;

$$
|\vec{p}, \lambda\rangle^{J W}=U\left[R_{a}^{J W}(\varphi, \theta,-\varphi)\right] U\left[L_{z}(p)\right]|\vec{p}=0, \lambda\rangle
$$

- the Jackson convention $[\mathrm{J}]: \gamma=0[2,9,10,13]$;

$$
|\vec{p}, \lambda\rangle^{J}=U\left[R_{a}^{J}(\varphi, \theta, 0)\right] U\left[L_{z}(p)\right]|\vec{p}=0, \lambda\rangle
$$

The choice of a convention is arbitrary but has to be used consistently.

An other important point is the question of phases that arises because the state $|\vec{p}, \lambda\rangle$ cannot be defined in a way that is continuous for all angles $\theta, \varphi$ (see footnote 2 in Reference [14] ). In the limit $\theta \rightarrow 0$ ( $\vec{p}$ parallel to the $z$-axis) and $\theta \rightarrow \pi$ ( $\vec{p}$ anti-parallel to the $z$-axis), the meaning of $|\vec{p}, \lambda\rangle$ has to be specified by an appropriate convention. These conventions are chosen so that one recovers the formalism of the canonical states $|j, m\rangle$ in the limit $\vec{p}_{z} \rightarrow 0$, that is to say we obtain the correct relations with the raising or lowering operators $J_{ \pm}=J_{x} \pm i J_{y}$.

The limit $\theta \rightarrow 0$ is in fact well-defined in the Jacob-Wick convention because $R_{a}(\varphi, 0,-\varphi)=I$, independent of $\varphi$. In the Jackson convention, this limit is not independent of $\varphi$ (see eq. (A.3) with $\alpha \equiv \varphi, \beta \equiv \theta=0$ and $\gamma=0$ ). We set, by definition, $|\vec{p}, \lambda\rangle$ equal to the limit of equation (A.20) for $\theta=0$ and $\varphi=0$, i.e. $\left|\vec{p}_{z}, \lambda\right\rangle^{J} \equiv U\left[R_{a}(0,0,0)\right] U\left[L_{z}(p)\right]|\vec{p}=0, \lambda\rangle$.

The limit $\theta \rightarrow \pi$ in not defined in both conventions. There are two ways to define these states for each convention. We bring $\vec{p}$ parallel to the $z$-axis to $\vec{p}$ anti-parallel to the $z$-axis either by atation around the $x$-axis or by a rotation around the $y$-axis. We set, independently of the convention for the third Eulerian angle $\gamma$ (see discussion in section A.2.3) :

- rotation of $\pi$ around the $\mathbf{x}$-axis $\left(U\left[R_{a, x}(\pi)\right]=e^{-i \pi J_{x}}\right)$ :

$$
\left|-\vec{p}_{z}, \lambda\right\rangle=(-1)^{-j} e^{-i \pi J_{x}}\left|\vec{p}_{z}, \lambda\right\rangle
$$

- rotation of $\pi$ around the $\mathbf{y}$-axis $\left(U\left[R_{a, y}(\pi)\right]=e^{-i \pi J_{y}}\right)$ :

$$
\left|-\vec{p}_{z}, \lambda\right\rangle=(-1)^{j-\lambda} e^{-i \pi J_{y}}\left|\vec{p}_{z}, \lambda\right\rangle
$$

As said before, the phase factor is introduced for convenience in such a way that for a particle at rest $(\vec{p}=0)$, we have

$$
\lim _{-\vec{p}_{z} \rightarrow 0}\left|-\vec{p}_{z}, \lambda\right\rangle=\lim _{\vec{p}_{z} \rightarrow 0}\left|\vec{p}_{z},-\lambda\right\rangle \text {. }
$$

Because both rotations (around $x$ or $y$ ) can be described in both conventions, one cannot say that the Jacob-Wick convention is only with $R_{y}(\pi)$ and the Jackson convention is only with $R_{x}(\pi)$ (however, most of the literature follows this order, except [10] ).

Conclusion : each convention has its own advantages and disadvantages. The Jacob-Wick conven-

\footnotetext{
${ }^{2}$ When we rotate (active rotation) $\vec{p}$ from $\vec{p}_{z}$ to $\vec{p}(\theta, \varphi)$, we rotate a full right-handed frame $\left(x^{\prime}, y^{\prime}, z^{\prime}\right)$ with $\vec{p}$ along the positive $z^{\prime}$-axis.
} 
tion attempts to minimize the discontinuities in the definition of $|\vec{p}, \lambda\rangle$ but is somewhat more complex in the geometrical construction of the coordinate systems. The Jackson convention contains more discontinuities but is very simple in the geometrical construction of the coordinate system. Indeed, in the Jackson convention, the positive $y^{\prime}$ direction is along $z \times z^{\prime}$ and the positive $x^{\prime}$ direction is along $\left(z \times z^{\prime}\right) \times z^{\prime}$. There are no such relations in the Jacob-Wick convention because of the additional rotation of $-\varphi$.

\section{A.5 Two-Particle Plane-Wave Helicity States in the Center-of-Mass Frame}

The state vector describing a non-interacting two-particle system is simply given by the direct product of two single-particle plane-wave state vectors :

$$
\left|\vec{p}_{1}, \lambda_{1} ; \vec{p}_{2}, \lambda_{2}\right\rangle=\left|\vec{p}_{1}, \lambda_{1}\right\rangle \otimes\left|\vec{p}_{2}, \lambda_{2}\right\rangle=U\left[\tilde{R}_{a}^{(1)}\left(\theta_{1}, \varphi_{1}\right)\right]\left|\vec{p}_{z 1}, \lambda_{1}\right\rangle \otimes U\left[\tilde{R}_{a}^{(2)}\left(\theta_{2}, \varphi_{2}\right)\right]\left|\vec{p}_{z 2}, \lambda_{2}\right\rangle
$$

We are, however, mostly interested in states of zero total linear momentum, for which say $\vec{p}_{1}=$ $-\vec{p}_{2}=\vec{p}$ with $\vec{p}$ of direction $(\theta, \varphi)$. Then $\theta_{1}=\theta, \varphi_{1}=\varphi$ and $\theta_{2}=\pi-\theta, \varphi_{2}=\varphi+\pi$ and the two rotations $\tilde{R}_{a}^{(1)}$ and $\tilde{R}_{a}^{(2)}$ may be replaced by a single rotation $\tilde{R}_{a}^{(1+2)}$ involving the total angular momentum $\mathbf{J}=\mathbf{J}_{1}+\mathbf{J}_{2}$. Let us emphasize that one can choose a different convention for the third Eulerian angle for $\tilde{R}_{a}^{(1)}$ and $\tilde{R}_{a}^{(2)}$. The state vector $\left|\vec{p}_{1}, \lambda_{1} ; \vec{p}_{2}, \lambda_{2}\right\rangle$ is given in the center-of-mass frame by

$$
\left|\vec{p}, \lambda_{1}, \lambda_{2}\right\rangle=U\left[\tilde{R}_{a}^{(1+2)}(\theta, \varphi)\right]\left|\vec{p}_{z}, \lambda_{1}\right\rangle \otimes \overline{\left|-\vec{p}_{z}, \lambda_{2}\right\rangle},
$$

where, because of the indefinite limit $\theta \rightarrow \pi$, the state vector $\overline{\left|-\vec{p}_{z}, \lambda_{2}\right\rangle}$ is written as

$$
\overline{\left|-\vec{p}_{z}, \lambda_{2}\right\rangle}=\begin{aligned}
& (-1)^{-j} e^{-i \pi J_{x}} \\
& (-1)^{j-\lambda} e^{-i \pi J_{y}}
\end{aligned}\left|\vec{p}_{z}, \lambda_{2}\right\rangle
$$

depending on the chosen convention for the "second" particle. The choice of the convention for the "second" particle tells us how the coordinate system associated with this particle is related to the coordinate system of the first particle.

\section{A.6 Angular Distribution}

In this section, we use the Jackson convention, because angular distribution may involve many coordinate systems and as far as one pays attention of the phases, there is no special trouble to deal with them. Let us consider the decay of a particle $A \rightarrow B+C$ where $B$ and $C$ are also unstable particles decaying into $B_{1}+B_{2}$ and $C_{1}+C_{2}$ respectively. The matrix element describing this decay is simply

$$
\mathcal{M}\left(\lambda_{B_{1}}, \lambda_{B_{2}}, \lambda_{C_{1}}, \lambda_{C_{2}}\right) \propto \sum_{\lambda_{B}, \lambda_{C}} A_{\lambda_{B}, \lambda_{C}}^{A \rightarrow B+C} \cdot A_{\lambda_{B_{1}}, \lambda_{B_{2}}}^{B \rightarrow B_{1}+B_{2}} \cdot A_{\lambda_{C_{1}}, \lambda_{C_{2}}}^{C \rightarrow C_{1}+C_{2}}
$$


with

$$
\begin{aligned}
A_{\lambda_{B}, \lambda_{C}}^{A \rightarrow B+C} & =\sqrt{\frac{2 J_{A}+1}{4 \pi}} D_{M_{A}, \lambda_{B}-\lambda_{C}}^{J_{A} *}\left(\varphi_{A}, \theta_{A}, 0\right) H_{\lambda_{B}, \lambda_{C}}^{A}, \\
A_{\lambda_{B_{1}}, \lambda_{B_{2}}}^{B \rightarrow B_{1}+B_{2}} & =\sqrt{\frac{2 J_{B}+1}{4 \pi}} D_{\lambda_{B}, \lambda_{B_{1}}-\lambda_{B_{2}}}^{J_{B^{*}}}\left(\varphi_{B}, \theta_{B}, 0\right) H_{\lambda_{B_{1}}, \lambda_{B_{2}}}^{B}, \\
A_{\lambda_{C_{1}}, \lambda_{C_{2}}}^{C \rightarrow C_{1}+C_{2}} & =\sqrt{\frac{2 J_{C}+1}{4 \pi}} D_{-\lambda_{C}, \lambda_{C 1}-\lambda_{C 2}}^{J_{C} *}\left(\varphi_{C}, \theta_{C}, 0\right) H_{\lambda_{C 1}, \lambda_{C 2}}^{C},
\end{aligned}
$$

where $M_{A}$ is the spin-projection of the particle $A$ along the (arbitrary) $z$-axis of the fixed coordinate system $(x, y, z)$. All other spin-quantization axes involved in the decay are no more arbitrary but are the direction of the momentum of the particles, i.e. the direction of particle $B, B_{1}$ and $C_{1}$.

As discussed above, the coordinate system for the "second" particle, here particle $C$ in the decay of particle $A$, is obtained by rotating the coordinate system of the first particle, $\mathrm{B}$, either by $\pi$ around its $x$-axis, i.e. the $x$-axis of particles $B$ and $C$ frames are parallel or by $\pi$ around its $y$-axis, i.e. the $y$ axis of particles $B$ and $C$ frames are parallel, depending on the chosen convention. One remark on the amplitude $A^{C \rightarrow C_{1}+C_{2}}$ : the first subscript of $D^{J_{C} *}$ is $-\lambda_{C}$ and NOT $\lambda_{C}$ because the quantization axis is along the direction of the momentum of particle $B$ so that the spin-quantization projection $M_{C}$ in the particle $C$ rest frame verifies $M_{C}=-\lambda_{C}$.

The unpolarized angular distribution is then given by averaging over initial spins and by summing over final spins :

$$
\frac{1}{\Gamma} \frac{\mathrm{d}^{3} \Gamma}{\mathrm{d} \Omega_{A} \mathrm{~d} \Omega_{B} \mathrm{~d} \Omega_{C}} \propto \frac{1}{2 s_{A}+1} \sum_{\lambda_{B_{1}}, \lambda_{B_{2}}, \lambda_{C_{1}}, \lambda_{C_{2}}}\left|\mathcal{M}\left(\lambda_{B_{1}}, \lambda_{B_{2}}, \lambda_{C_{1}}, \lambda_{C_{2}}\right)\right|^{2} .
$$




\section{Bibliography}

[1] J.D. Richman, An Experimenter's Guide To The Helicity Formalism, CALT-68-1148, June 1984.

[2] S.U. Chung, Spin Formalism, CERN Yellow Report 71 - 8, Geneva, 1971.

[3] M. Chaichian and R. Hagedorn, Symmetries In Quantum Mechanics: From angular momentum to supersymmetry, Inst. Phys., 1998. (Graduate Students Series in Physics).

[4] A.D. Martin and T.D. Spearman, Elementary Particle Theory, North-Holland, 1970.

[5] M.L. Perl, High Energy Hadron Physics, Wiley, 1974.

[6] A.R. Edmonds, Angular Momentum In Quantum Mechanics, Princeton Univ. Press, 1957.

[7] M.E. Rose, Elementary Theory Of Angular Momentum, Wiley, 1957.

[8] M. Bouten, On The Rotation Operators In Quantum Mechanics, Physica 42, 572, (1968).

[9] J. Werle, Relativistic Theory Of Reactions : model independent methods, North-Holland, 1966.

[10] V.B. Berestetskii, E.M. Lifshitz and L.P. Pitaevskii, Quantum Electrodynamics, Pergamon Press, 1982.

[11] H. Pilkuhn, The Interactions Of Hadrons, Wiley, 1967.

[12] M. Jacob and G.C. Wick, On the General Theory of Collisions for Particles with Spin, Ann. Phys. 7, 404, (1959).

[13] J. D. Jackson, Particle and Polarization for Two and Three Body Decays, Les Houches, C. deWitt and M. jacob ed, Gordon and Breach, New York, 1965.

[14] G.C. Wick, Angular Momentum States for Three Relativistic Particles, Ann. Phys. 18, 65, (1962).

[15] EvtGen Package, BABAR Analysis Document \# 522.

[16] C. Bourrely, E. Leader and J. Soffer, Polarization Phenomena In Hadronic Reactions, Phys. Rep. 59, 95, (1980).

[17] R. Kutschke, An Angular Distribution Cookbook, (1996). [http://www-cpd.fnal.gov/personal/kutschke/Angdist/angdist.ps ] 


\section{Annexe B}

\section{Développement de la distribution}

angulaire de $B \rightarrow \psi\left(\rightarrow \ell^{+} \ell^{-}\right) K^{*}(\rightarrow K \pi)$

\section{en moments}

Dans cet appendice, on dérive la distribution angulaire correspondante à la désintégration $B \rightarrow$ $\psi\left(\rightarrow \ell^{+} \ell^{-}\right) K^{*}(\rightarrow K \pi)$ en ondes partielles en partant de la distribution en base d'hélicité. Cette distribution est donnée (cf. eq. (2.58)) par :

$$
|\mathcal{M}|^{2}=\sum_{\alpha= \pm 1}\left|\sum_{\lambda=0, \pm 1} \sqrt{\frac{3}{4 \pi}} D_{-\lambda, 0}^{1 *}\left(\varphi_{K^{*}}, \theta_{K^{*}}, 0\right) \sqrt{\frac{3}{4 \pi}} D_{\lambda, \alpha}^{1 *}\left(\varphi_{\psi}, \theta_{\psi}, 0\right) H_{\lambda}\right|^{2} .
$$

En développant le module au carré, on obtient

$$
\begin{array}{r}
|\mathcal{M}|^{2}=\left(\frac{3}{4 \pi}\right)^{2} \sum_{\alpha= \pm 1} \sum_{\lambda, \lambda^{\prime}=0, \pm 1} D_{-\lambda, 0}^{1 *}\left(\varphi_{K^{*}}, \theta_{K^{*}}, 0\right) D_{-\lambda^{\prime}, 0}^{1}\left(\varphi_{K^{*}}, \theta_{K^{*}}, 0\right) \\
\times D_{\lambda, \alpha}^{1 *}\left(\varphi_{\psi}, \theta_{\psi}, 0\right) D_{\lambda^{\prime}, \alpha}^{1}\left(\varphi_{\psi}, \theta_{\psi}, 0\right) H_{\lambda} H_{\lambda^{\prime}}^{*}
\end{array}
$$

Les relations suivantes

$$
\begin{aligned}
D_{M^{\prime}, M}^{J *}(\Omega)= & (-1)^{M^{\prime}-M} D_{-M^{\prime},-M}^{J}(\Omega), \\
D_{M_{1}^{\prime}, M_{1}}^{J_{1}}(\Omega) D_{M_{2}^{\prime}, M_{2}}^{J_{2}}(\Omega)= & \sum_{\substack{\left|J_{1}-J_{2}\right| \leq J_{3} \leq J_{1}+J_{2}\\
}}\left\langle J_{1}, M_{1}^{\prime} ; J_{2}, M_{2}^{\prime} \mid J_{3}, M_{1}^{\prime}+M_{2}^{\prime}\right\rangle \\
& \times\left\langle J_{1}, M_{1} ; J_{2}, M_{2} \mid J_{3}, M_{1}+M_{2}\right\rangle D_{M_{1}^{\prime}+M_{2}^{\prime}, M_{1}+M_{2}}^{J_{3}}(\Omega),
\end{aligned}
$$


permettent de réexprimer $|\mathcal{M}|^{2}$ en

$$
\begin{aligned}
|\mathcal{M}|^{2}= & \left(\frac{3}{4 \pi}\right)^{2} \sum_{\alpha= \pm 1}(-1)^{\alpha} \sum_{\lambda, \lambda^{\prime}=0, \pm 1} H_{\lambda} H_{\lambda^{\prime}}^{*} \\
& \sum_{J_{L}, J_{R}=0,1,2}\left\langle 1, \lambda ; 1,-\lambda^{\prime} \mid J_{R}, M\right\rangle\left\langle 1,0 ; 1,0 \mid J_{R}, 0\right\rangle D_{M, 0}^{J_{R}}\left(\varphi_{K^{*}}, \theta_{K^{*}}, 0\right) \\
& \left\langle 1,-\lambda ; 1, \lambda^{\prime} \mid J_{L},-M\right\rangle\left\langle 1,-\alpha ; 1, \alpha \mid J_{L}, 0\right\rangle D_{-M, 0}^{J_{L}}\left(\varphi_{\psi}, \theta_{\psi}, 0\right)
\end{aligned}
$$

avec $M \equiv \lambda-\lambda^{\prime}$. En utilisant la relation qui relie les fonctions $D^{J}$ aux harmoniques sphériques :

$$
D_{M, 0}^{J}(\varphi, \theta, 0)=\sqrt{\frac{4 \pi}{2 J+1}} Y_{J, M}^{*}(\theta, \varphi), \text { et } Y_{J, M}^{*}(\theta, \varphi)=(-1)^{M} Y_{J,-M}(\theta, \varphi)
$$

on obtient

$$
\begin{aligned}
|\mathcal{M}|^{2}= & \left(\frac{3}{4 \pi}\right)^{2} \sum_{\alpha= \pm 1}(-1)^{\alpha} \sum_{\lambda, \lambda^{\prime}=0, \pm 1} H_{\lambda} H_{\lambda^{\prime}}^{*} \\
& \sum_{J_{L}, J_{R}=0,1,2}\left\langle 1, \lambda ; 1,-\lambda^{\prime}, \mid J_{R}, M\right\rangle\left\langle 1,0 ; 1,0 \mid J_{R}, 0\right\rangle \sqrt{\frac{4 \pi}{2 J_{R}+1}} Y_{J_{R},-M}\left(\theta_{K^{*}}, \varphi_{K^{*}}\right) \\
& \left\langle 1,-\lambda ; 1, \lambda^{\prime}, \mid J_{L},-M\right\rangle\left\langle 1,-\alpha ; 1, \alpha \mid J_{L}, 0\right\rangle \sqrt{\frac{4 \pi}{2 J_{L}+1}} Y_{J_{L}, M}\left(\theta_{\psi}, \varphi_{\psi}\right) .
\end{aligned}
$$

Au final, on a

$$
|\mathcal{M}|^{2} \equiv \sum_{L R M} T_{L R M} Y_{J_{R},-M}\left(\theta_{K^{*}}, \varphi_{K^{*}}\right) Y_{J_{L}, M}\left(\theta_{\psi}, \varphi_{\psi}\right)
$$

avec $L \equiv J_{L}, R \equiv J_{R}$. Les moments $T_{L R M}$ sont définis par

$$
T_{L R M}=\int|\mathcal{M}|^{2} Y_{J_{R},-M}^{*}\left(\theta_{K^{*}}, \varphi_{K^{*}}\right) Y_{J_{L}, M}^{*}\left(\theta_{\psi}, \varphi_{\psi}\right) \mathrm{d} \cos \left(\theta_{K^{*}}\right) \mathrm{d} \varphi_{K^{*}} \mathrm{~d} \cos \left(\theta_{\psi}\right) \mathrm{d} \varphi_{\psi}
$$

et valent

$$
\begin{aligned}
T_{L R M}= & 4 \pi\left(\frac{3}{4 \pi}\right)^{2} \sum_{\alpha= \pm 1}(-1)^{\alpha} \sum_{\lambda, \lambda^{\prime}=0, \pm 1} H_{\lambda} H_{\lambda^{\prime}}^{*} \\
& \sqrt{\frac{1}{2 J_{R}+1}}\left\langle 1, \lambda ; 1,-\lambda^{\prime}, \mid J_{R}, M\right\rangle\left\langle 1,0 ; 1,0 \mid J_{R}, 0\right\rangle \\
& \sqrt{\frac{1}{2 J_{L}+1}}\left\langle 1,-\lambda ; 1, \lambda^{\prime}, \mid J_{L},-M\right\rangle\left\langle 1,-\alpha ; 1, \alpha \mid J_{L}, 0\right\rangle .
\end{aligned}
$$

À noter que $T_{L R M}^{*}=T_{L R(-M)}$. Puisque les coefficients de Clebsch-Gordan $\langle 1,0 ; 1,0 \mid 1,0\rangle=0$ et 
Chapitre B. Développement de la distribution angulaire de $B \rightarrow \psi\left(\rightarrow \ell^{+} \ell^{-}\right) K^{*}(\rightarrow K \pi)$ en moments

$\sum_{\alpha}\langle 1,-\alpha ; 1, \alpha \mid 1,0\rangle=0$, on aboutit à l'expression suivante pour les moments $T_{L R M}$ :

$$
\begin{aligned}
T_{L R M}= & -2\left(\frac{9}{4 \pi}\right) \sum_{\lambda, \lambda^{\prime}=0, \pm 1} H_{\lambda} H_{\lambda^{\prime}}^{*} \\
& \sqrt{\frac{1}{2 J_{R}+1}}\left\langle 1, \lambda ; 1,-\lambda^{\prime}, \mid J_{R}, M\right\rangle\left\langle 1,0 ; 1,0 \mid J_{R}, 0\right\rangle \\
& \sqrt{\frac{1}{2 J_{L}+1}}\left\langle 1,-\lambda ; 1, \lambda^{\prime}, \mid J_{L},-M\right\rangle\left\langle 1,-1 ; 1,1 \mid J_{L}, 0\right\rangle .
\end{aligned}
$$

Les expressions explicites des moments $T_{L R M}$ en fonction des amplitudes d'hélicité sont données dans la table ci-dessous :

\begin{tabular}{|c|c|c||c|}
\hline$L$ & $R$ & $M$ & $4 \pi T_{L R M}$ \\
\hline \hline 0 & 0 & 0 & $2\left(\left|H_{0}\right|^{2}+\left|H_{1}\right|^{2}+\left|H_{-1}\right|^{2}\right)$ \\
0 & 2 & 0 & $\frac{2}{\sqrt{5}}\left(2\left|H_{0}\right|^{2}-\left|H_{1}\right|^{2}-\left|H_{-1}\right|^{2}\right)$ \\
2 & 0 & 0 & $\frac{1}{\sqrt{5}}\left(-2\left|H_{0}\right|^{2}+\left|H_{1}\right|^{2}+\left|H_{-1}\right|^{2}\right)$ \\
2 & 2 & 0 & $-\frac{1}{5}\left(4\left|H_{0}\right|^{2}+\left|H_{1}\right|^{2}+\left|H_{-1}\right|^{2}\right)$ \\
2 & 2 & 1 & $-\frac{3}{5}\left(H_{0} H_{-1}^{*}+H_{1} H_{0}^{*}\right)$ \\
2 & 2 & 2 & $-\frac{6}{5} H_{1} H_{-1}^{*}$ \\
\hline \hline
\end{tabular}




\section{Annexe C}

\section{Distribution angulaire de la désintégration $B \rightarrow \chi_{c 1} K^{*}(892)$ en base d'hélicité}

Cet appendice est consacré à la distribution angulaire de la désintégration d'un méson $B$ en $\chi_{c 1} K^{*}(892)$, où le $\chi_{c 1}$ est reconstruit dans sa désintégration radiative $J / \psi \gamma$ et le $J / \psi$ dans sa désintégration leptonique $\ell^{+} \ell^{-}(\ell=e$ ou $\mu)$ ) en prenant en compte la désintégration $\mathrm{du} J / \psi$ dans la distribution. L'amplitude totale s'écrit ${ }^{1}$

$$
\begin{gathered}
A\left(\alpha, \lambda_{\gamma}\right)=\sum_{\lambda_{\chi}, \lambda_{K^{*}}, \lambda_{\psi}}\left\langle\theta_{K^{*}}, \varphi_{K^{*}}, \lambda_{K}, \lambda_{\pi}\left|\mathcal{T}_{K^{*}}\right| 1, m_{K^{*}}=-\lambda_{K^{*}}\right\rangle\left\langle\theta_{\psi}, \varphi_{\psi}, \lambda_{\ell^{+}}, \lambda_{\ell^{-}}\left|\mathcal{T}_{\psi}\right| 1, m_{\psi}=\lambda_{\psi}\right\rangle \\
\left\langle\theta_{\chi}, \varphi_{\chi}, \lambda_{\psi}, \lambda_{\gamma}\left|\mathcal{T}_{\chi}\right| 1, m_{\chi}=\lambda_{\chi}\right\rangle\left\langle\theta_{B}, \varphi_{B}, \lambda_{\chi}, \lambda_{K^{*}}\left|\mathcal{T}_{B}\right| 0,0\right\rangle
\end{gathered}
$$

soit, à une constante globale près,

$$
A\left(\alpha, \lambda_{\gamma}\right)=\sum_{\lambda=0, \pm 1, \nu\left(\lambda_{\gamma}\right)} D_{-\lambda, 0}^{1 *}\left(\varphi_{K^{*}}, \theta_{K^{*}}, 0\right) D_{\nu+\lambda_{\gamma}, \alpha}^{1 *}\left(\varphi_{\psi}, \theta_{\psi}, 0\right) D_{\lambda, \nu}^{1 *}\left(\varphi_{\chi}, \theta_{\chi}, 0\right) H_{\lambda} H_{\lambda_{\psi}, \lambda_{\gamma}}^{\chi}
$$

où $\alpha \equiv \lambda_{\ell^{+}}-\lambda_{\ell^{-}}= \pm 1$ et $\nu=\lambda_{\psi}-\lambda_{\gamma}$ avec

$$
\nu=-1,0 \text { pour } \lambda_{\gamma}=+1, \quad \nu=0,+1 \text { pour } \lambda_{\gamma}=-1,
$$

l'hélicité du photon ne pouvant prendre que les valeurs +1 et -1 et $|\nu| \leq J_{\chi}=1$.

Les différents angles intervenant dans cette distribution angulaire sont définis comme suit : l'angle $\theta_{K^{*}}\left(\theta_{\chi}\right)$ est l'angle polaire de l'impulsion du kaon $(J / \psi)$ dans le repère au repos du $K^{*}\left(\chi_{c 1}\right)$ par rapport à l'impulsion du $K^{*}\left(\chi_{c 1}\right)$ dans le repère du méson $B$ au repos. L'angle azimutal $\varphi=$ $\varphi_{\chi}-\varphi_{K^{*}}$ est l'angle entre les deux plans des produits de désintégration du $\chi_{c 1}$ et du $K^{*}$, en allant du kaon vers le $J / \psi$ avec une rotation positive autour de l'axe $z_{\chi}$ (voir Fig. $2.7:$ la désintégration

\footnotetext{
${ }^{1}$ Les conventions utilisées sont les mêmes que celles utilisées pour $\psi K^{*}(892)$ : convention de Jackson pour les opérateurs de rotation finie et rotation autour de l'axe $x$ pour définir l'état $\left|-\vec{p}, \lambda_{2}\right\rangle$ (voir appendice A).
} 
du $J / \psi$ en paire de leptons n'est pas représentée)) :

$$
\sin (\varphi)=\left(\hat{p}_{\chi} \times \hat{c}\right) \cdot \hat{d}, \quad \cos (\varphi)=\hat{c} . \hat{d}
$$

où les vecteurs unités $\hat{c}$ et $\hat{d}$ sont définis par :

$$
\hat{c}=\left(\hat{p}_{K^{*}} \times \hat{p}_{K}\right) \times \hat{p}_{K^{*}}, \quad \hat{d}=\left(\hat{p}_{\chi} \times \hat{p}_{\psi}\right) \times \hat{p}_{\chi} .
$$

Les impulsions de chaque particule sont mesurées dans le repère au repos de leur mère respective. Enfin, l'anlge $\theta_{\psi}$ est l'angle polaire de l'impulsion du lepton positif dans le repère au repos du $J / \psi$ par rapport à l'impulsion du $J / \psi$ dans le repère au repos du $\chi_{c 1}$. L'angle azimutal $\varphi_{\psi}$ est l'angle allant du plan défini par l'impulsion du $\psi$ dans le repère au repos du $\chi_{c 1}$ et l'impulsion du $\chi_{c 1}$ dans le repère au repos du méson $B$ vers le plan défini par l'impulsion du lepton positif dans le repère au repos du $J / \psi$ et l'impulsion du $J / \psi$ dans le repère au repos du $\chi_{c 1}$ :

$$
\sin \left(\varphi_{\psi}\right)=\left(\hat{p}_{\psi} \times \hat{a}\right) . \hat{b}, \quad \cos \left(\varphi_{\psi}\right)=\hat{a} . \hat{b}
$$

où les vecteurs unités $\hat{a}$ et $\hat{b}$ sont définis par :

$$
\hat{a}=\left(\hat{p}_{\chi} \times \hat{p}_{\psi}\right) \times \hat{p}_{\psi}, \quad \hat{b}=\left(\hat{p}_{\psi} \times \hat{p}_{\ell^{+}}\right) \times \hat{p}_{\ell^{+}} .
$$

Les impulsions de chaque particule sont mesurées dans le repère au repos de leur mère respective.

La distribution angulaire en base d'hélicité est donnée, après sommation sur les états d'hélicité du photon et des leptons, par :

$$
\begin{gathered}
\frac{1}{\Gamma} \frac{\mathrm{d}^{5} \Gamma\left(B \rightarrow \chi_{c 1}\left(\rightarrow J / \psi\left(\rightarrow \ell^{+} \ell^{-}\right) \gamma\right) K^{*}(\rightarrow K \pi)\right)}{\mathrm{d} \cos \theta_{K^{*}} \mathrm{~d} \cos \theta_{\chi} \mathrm{d} \varphi \mathrm{d} \cos \theta_{\psi} \mathrm{d} \varphi_{\psi}}= \\
\frac{1}{\left|H_{1}\right|^{2}+\left|H_{0}\right|^{2}+\left|H_{-1}\right|^{2}} \frac{1}{\left|H_{1,1}^{\chi}\right|^{2}+\left|H_{0,1}^{\chi}\right|^{2}} \sum_{\lambda_{\gamma}= \pm 1, \alpha= \pm 1}\left|A\left(\lambda_{\gamma}, \alpha\right)\right|^{2}= \\
\frac{1}{\left|H_{1}\right|^{2}+\left|H_{0}\right|^{2}+\left|H_{-1}\right|^{2}} \frac{1}{\left|H_{1,1}^{\chi}\right|^{2}+\left|H_{0,1}^{\chi}\right|^{2}} \sum_{i=1}^{9} X_{i} h_{i}\left(\theta_{K^{*}}, \theta_{\chi}, \varphi, \theta_{\psi}, \varphi_{\psi}\right)
\end{gathered}
$$

où les expressions de $X_{i}$ et $h_{i}\left(\theta_{K^{*}}, \theta_{\chi}, \varphi, \theta_{\psi}, \varphi_{\psi}\right)$ sont données dans la table C.1. La distribution angulaire est normalisée par construction. Les expressions de $A\left(\alpha= \pm 1, \lambda_{\gamma}= \pm 1\right)$ sont données dans la table C.2. La distribution angulaire réduite du $J / \psi$ est

$$
\frac{1}{\Gamma} \frac{\mathrm{d} \Gamma}{\mathrm{d} \cos \theta_{\psi}}=\frac{3}{8}\left[2\left(1-\cos ^{2}\left(\theta_{\psi}\right)\right)+R_{1,1}^{\chi}\left(3 \cos ^{2}\left(\theta_{\psi}\right)-1\right)\right] .
$$


ТАВ. С.1: Observables et fonctions angulaires de la distribution angulaire $B \rightarrow \chi_{c 1}\left(\rightarrow J / \psi\left(\rightarrow \ell^{+} \ell^{-}\right) \gamma\right) K^{*}(\rightarrow K \pi)$ dans la base d'hélicité pour un méson $B$ initialement $B_{d}^{0}$ ou $B_{u}^{+}$.

\begin{tabular}{|c|c|c|}
\hline$i$ & $X_{i}$ & $h_{i}\left(\theta_{K^{*}}, \theta_{\chi}, \varphi, \theta_{\psi}, \varphi_{\psi}\right)$ \\
\hline 1 & $\left|H_{1}\right|^{2}$ & $\begin{array}{l}\frac{27}{128 \pi^{2}} \frac{1}{4} \sin ^{2}\left(\theta_{K^{*}}\right)\left[\left|H_{1,1}^{\chi}\right|^{2} \sin ^{2}\left(\theta_{\chi}\right)\left(1+\cos ^{2}\left(\theta_{\psi}\right)\right)+\left|H_{0,1}^{\chi}\right|^{2}\left(1+\cos ^{2}\left(\theta_{\chi}\right)\right) \sin ^{2}\left(\theta_{\psi}\right)\right. \\
\left.+\sin \left(\theta_{\chi}\right) \sin \left(2 \theta_{\psi}\right)\left\{\Re e\left(H_{1,1}^{\chi} H_{0,1}^{\chi *}\right) \cos \left(\theta_{\chi}\right) \cos \left(\varphi_{\psi}\right)+\Im m\left(H_{1,1}^{\chi} H_{0,1}^{\chi *}\right) \sin \left(\varphi_{\psi}\right)\right\}\right]\end{array}$ \\
\hline 2 & $\left|H_{-1}\right|^{2}$ & $\begin{array}{l}\frac{27}{128 \pi^{2}} \frac{1}{4} \sin ^{2}\left(\theta_{K^{*}}\right)\left[\left|H_{1,1}^{\chi}\right|^{2} \sin ^{2}\left(\theta_{\chi}\right)\left(1+\cos ^{2}\left(\theta_{\psi}\right)\right)+\left|H_{0,1}^{\chi}\right|^{2}\left(1+\cos ^{2}\left(\theta_{\chi}\right)\right) \sin ^{2}\left(\theta_{\psi}\right)\right. \\
\left.+\sin \left(\theta_{\chi}\right) \sin \left(2 \theta_{\psi}\right)\left\{\Re e\left(H_{1,1}^{\chi} H_{0,1}^{\chi *}\right) \cos \left(\theta_{\chi}\right) \cos \left(\varphi_{\psi}\right)-\Im m\left(H_{1,1}^{\chi} H_{0,1}^{\chi *}\right) \sin \left(\varphi_{\psi}\right)\right\}\right]\end{array}$ \\
\hline 3 & $\left|H_{0}\right|^{2}$ & $\begin{array}{c}\frac{27}{128 \pi^{2}} \cos ^{2}\left(\theta_{K^{*}}\right)\left[\left|H_{1,1}^{\chi}\right|^{2} \cos ^{2}\left(\theta_{\chi}\right)\left(1+\cos ^{2}\left(\theta_{\psi}\right)\right)+\left|H_{0,1}^{\chi}\right|^{2} \sin ^{2}\left(\theta_{\chi}\right) \sin ^{2}\left(\theta_{\psi}\right)\right. \\
\left.-\frac{1}{2} \Re e\left(H_{1,1}^{\chi} H_{0,1}^{\chi *}\right) \sin \left(2 \theta_{\chi}\right) \sin \left(2 \theta_{\psi}\right) \cos \left(\varphi_{\psi}\right)\right]\end{array}$ \\
\hline 4 & $\Re e\left(H_{1} H_{-1}^{*}\right)$ & $\begin{array}{l}\frac{27}{128 \pi^{2}} \frac{1}{2} \sin ^{2}\left(\theta_{K^{*}}\right)\left[\sin ^{2}\left(\theta_{\chi}\right) \cos (2 \varphi)\left\{\left|H_{1,1}^{\chi}\right|^{2}\left(1+\cos ^{2}\left(\theta_{\psi}\right)\right)-\left|H_{0,1}^{\chi}\right|^{2} \sin ^{2}\left(\theta_{\psi}\right)\right\}\right. \\
\left.+\Re e\left(H_{1,1}^{\chi} H_{0,1}^{\chi *}\right) \sin \left(\theta_{\chi}\right) \sin \left(2 \theta_{\psi}\right)\left\{\cos \left(\theta_{\chi}\right) \cos (2 \varphi) \cos \left(\varphi_{\psi}\right)-\sin (2 \varphi) \sin \left(\varphi_{\psi}\right)\right\}\right]\end{array}$ \\
\hline 5 & $\Im m\left(H_{1} H_{-1}^{*}\right)$ & $\begin{array}{l}-\frac{27}{128 \pi^{2}} \frac{1}{2} \sin ^{2}\left(\theta_{K^{*}}\right)\left[\sin ^{2}\left(\theta_{\chi}\right) \sin (2 \varphi)\left\{\left|H_{1,1}^{\chi}\right|^{2}\left(1+\cos ^{2}\left(\theta_{\psi}\right)\right)-\left|H_{0,1}^{\chi}\right|^{2} \sin ^{2}\left(\theta_{\psi}\right)\right\}\right. \\
\left.+\Re e\left(H_{1,1}^{\chi} H_{0,1}^{\chi *}\right) \sin \left(\theta_{\chi}\right) \sin \left(2 \theta_{\psi}\right)\left\{\cos \left(\theta_{\chi}\right) \sin (2 \varphi) \cos \left(\varphi_{\psi}\right)+\cos (2 \varphi) \sin \left(\varphi_{\psi}\right)\right\}\right]\end{array}$ \\
\hline 6 & $\begin{array}{l}\Re e\left(H_{1} H_{0}^{*}\right. \\
\left.+H_{-1} H_{0}^{*}\right)\end{array}$ & $\begin{array}{l}-\frac{27}{128 \pi^{2}} \frac{1}{4} \sin \left(2 \theta_{K^{*}}\right)\left[\sin \left(2 \theta_{\chi}\right) \cos (\varphi)\left\{\left|H_{1,1}^{\chi}\right|^{2}\left(1+\cos ^{2}\left(\theta_{\psi}\right)\right)-\left|H_{0,1}^{\chi}\right|^{2} \sin ^{2}\left(\theta_{\psi}\right)\right\}\right. \\
\left.+\Re e\left(H_{1,1}^{\chi} H_{0,1}^{\chi^{*}}\right) \sin \left(2 \theta_{\psi}\right)\left\{\cos \left(2 \theta_{\chi}\right) \cos (\varphi) \cos \left(\varphi_{\psi}\right)-\cos \left(\theta_{\chi}\right) \sin (\varphi) \sin \left(\varphi_{\psi}\right)\right\}\right]\end{array}$ \\
\hline 7 & $\begin{array}{l}\Im m\left(\left(H_{1} H_{0}^{*}\right.\right. \\
\left.\left.-H_{-1}\right) H_{0}^{*}\right)\end{array}$ & $\begin{array}{l}\frac{27}{128 \pi^{2}} \frac{1}{4} \sin \left(2 \theta_{K^{*}}\right)\left[\sin \left(2 \theta_{\chi}\right) \sin (\varphi)\left\{\left|H_{1,1}^{\chi}\right|^{2}\left(1+\cos ^{2}\left(\theta_{\psi}\right)\right)-\left|H_{0,1}^{\chi}\right|^{2} \sin ^{2}\left(\theta_{\psi}\right)\right\}\right. \\
\left.+\Re e\left(H_{1,1}^{\chi} H_{0,1}^{\chi *}\right) \sin \left(2 \theta_{\psi}\right)\left\{\cos \left(2 \theta_{\chi}\right) \sin (\varphi) \cos \left(\varphi_{\psi}\right)+\cos \left(\theta_{\chi}\right) \cos (\varphi) \sin \left(\varphi_{\psi}\right)\right\}\right]\end{array}$ \\
\hline 8 & $\begin{array}{l}\Re e\left(\left(H_{1} H_{0}^{*}\right.\right. \\
\left.-H_{-1} H_{0}^{*}\right)\end{array}$ & $-\frac{27}{128 \pi^{2}} \frac{1}{4} \sin \left(2 \theta_{K^{*}}\right) \sin \left(2 \theta_{\psi}\right) \Im m\left(H_{1,1}^{\chi} H_{0,1}^{\chi *}\right)\left[\sin (\varphi) \cos \left(\varphi_{\psi}\right)+\cos \left(\theta_{\chi}\right) \cos (\varphi) \sin \left(\varphi_{\psi}\right)\right.$ \\
\hline 9 & $\begin{array}{l}\Im m\left(\left(H_{1} H_{0}^{*}\right)\right. \\
\left.\quad+H_{-1} H_{0}^{*}\right)\end{array}$ & $-\frac{27}{128 \pi^{2}} \frac{1}{4} \sin \left(2 \theta_{K^{*}}\right) \sin \left(2 \theta_{\psi}\right) \Im m\left(H_{1,1}^{\chi} H_{0,1}^{\chi *}\right)\left[\cos (\varphi) \cos \left(\varphi_{\psi}\right)-\cos \left(\theta_{\chi}\right) \sin (\varphi) \sin \left(\varphi_{\psi}\right)\right.$ \\
\hline
\end{tabular}


TAB. C.2: Expressions de $A\left(\alpha, \lambda_{\gamma}\right)=\sum_{\lambda=0, \pm 1} H_{\lambda} g_{\lambda}\left(\alpha, \lambda_{\gamma}\right)$. À noter que $\left|A\left(\alpha, \lambda_{\gamma}\right)\right|^{2}=\sum_{\lambda}\left|H_{\lambda}\right|^{2}\left|g_{\lambda}\left(\alpha, \lambda_{\gamma}\right)\right|^{2}+$ $2 \sum_{\lambda^{\prime}<\lambda}\left(\Re e\left(H_{\lambda} H_{\lambda^{\prime}}^{*}\right) \Re e\left(g_{\lambda} g_{\lambda^{\prime}}^{*}\right)-\Im m\left(H_{\lambda} H_{\lambda^{\prime}}^{*}\right) \Im m\left(g_{\lambda} g_{\lambda^{\prime}}^{*}\right)\right)$.

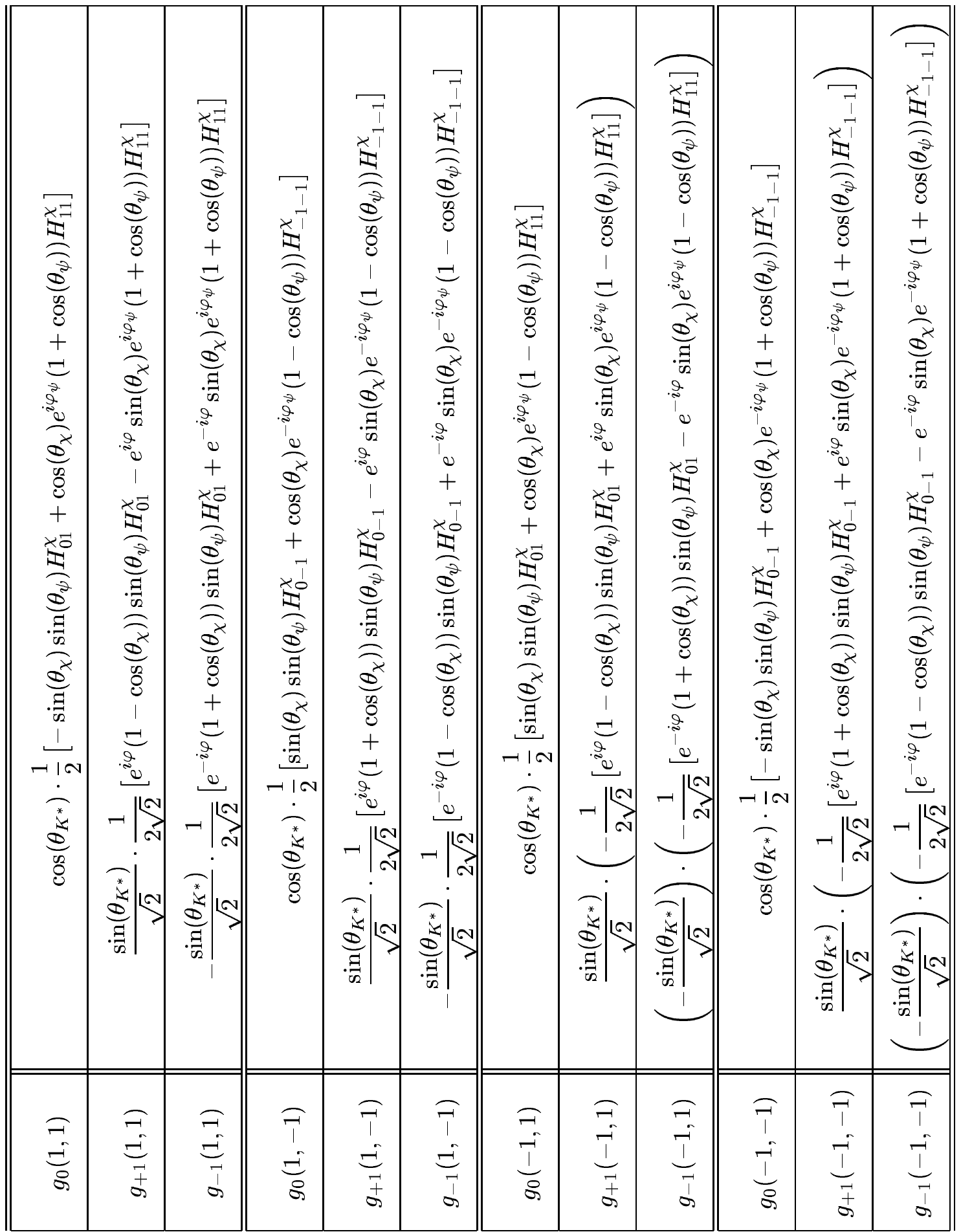




\section{Annexe D}

\section{Distribution angulaire de la désintégration $B \rightarrow J / \psi(K \pi)_{S+P+D}$}

Cet appendice donne la distribution angulaire de la désintégration $B \rightarrow J / \psi X, X \rightarrow K \pi$, où $X$ est de spin 0, 1 et 2 dans les bases d'hélicité et de transversité. Cette distribution est une extension de celle donnée au paragraphe 2.3.3 en incluant une onde $D$.

\section{D.1 Distibution angulaire en base d'hélicité}

La distrubtion angulaire en base d'hélicité est donnée par (les conventions utilisées sont celles choisies au chapitre 2) :

$$
\frac{\mathrm{d}^{4} \sigma\left(B \rightarrow J / \psi X, X \rightarrow(K \pi)_{S+P+D}\right)}{\mathrm{d} m \mathrm{~d} \cos \left(\theta_{X}\right) \mathrm{d} \cos \left(\theta_{\psi}\right) \mathrm{d} \varphi} \propto p q|\mathcal{M}|^{2}
$$

où

$$
|\mathcal{M}|^{2}=\sum_{\alpha= \pm 1}\left|\sum_{J_{X}=0}^{2} \sum_{\lambda\left(J_{X}\right)} \sqrt{\frac{2 J_{\psi}+1}{4 \pi}} \sqrt{\frac{2 J_{X}+1}{4 \pi}} H_{\lambda}^{J_{X}} B^{J_{X}} e^{i \lambda \varphi} d_{\lambda, \alpha}^{J_{\psi}}\left(\theta_{\psi}\right) d_{-\lambda, 0}^{J_{X}}\left(\theta_{X}\right)\right|^{2} .
$$

$p$ et $q$ sont les impulsions respectivement du système $(K \pi)$ dans le repère du méson $B$ au repos et du kaon dans le repère du système $(K \pi)$ au repos. $p$ et $q$ dépendent tous les deux de $m$, la masse du système $K \pi \cdot \lambda\left(J_{X}\right)$ prend les valeurs

$$
\lambda\left(J_{X}=0\right)=0 ; \quad \lambda\left(J_{X} \geq 1\right)=0, \pm 1
$$

Les amplitudes d'hélicicté sont

$$
H_{\lambda}^{J_{X}}=\langle\psi(\lambda) X(\lambda)|\mathcal{H}| B\rangle, \quad B^{J_{X}}=\langle K \pi|\mathcal{T}| X\rangle=B_{B W}^{J_{X}}(m) F^{J_{X}}
$$

où $B_{B W}^{J_{X}}(m)$ est une Breit-Wigner relativiste (cf. eq. (2.101)). On obtient

$$
\frac{\mathrm{d}^{4} \sigma}{\mathrm{d} m \mathrm{~d} \cos \left(\theta_{X}\right) \mathrm{d} \cos \left(\theta_{\psi}\right) \mathrm{d} \varphi} \propto p q \frac{3}{16 \pi}\left[P W_{h}+S W_{h}+S P W_{h}+D W_{h}+S D W_{h}+P D W_{h}\right],
$$


où

$$
\begin{aligned}
P W_{h}= & 3\left|B^{1}\right|^{2}\left[h_{1}\left|H_{0}^{1}\right|^{2}+h_{2}\left(\left|H_{1}^{1}\right|^{2}+\left|H_{-1}^{1}\right|^{2}\right)+h_{3} \Re e\left(H_{1}^{1} H_{-1}^{1 *}\right)+h_{4} \Im m\left(H_{1}^{1} H_{-1}^{1 *}\right)\right. \\
& \left.+h_{5} \Re e\left(H_{1}^{1} H_{0}^{1 *}+H_{-1}^{1} H_{0}^{1 *}\right)+h_{6} \Im m\left(H_{1}^{1} H_{0}^{1 *}-H_{-1}^{1} H_{0}^{1 *}\right)\right] \\
S W_{h}= & h_{7}\left|B^{0}\right|^{2}\left|H_{0}^{0}\right|^{2} \\
S P W_{h}= & h_{8} \Re e\left(B^{1}\left(H_{1}^{1}+H_{-1}^{1}\right) B^{0 *} H_{0}^{0 *}\right)+h_{9} \Im m\left(B^{1}\left(H_{1}^{1}-H_{-1}^{1}\right) B^{0 *} H_{0}^{0 *}\right) \\
& +h_{10} \Re e\left(B^{1} H_{0}^{1} B^{0 *} H_{0}^{0 *}\right) \\
D W_{h}= & 5\left|B^{2}\right|^{2}\left[h_{11}\left|H_{0}^{2}\right|^{2}+h_{12}\left(\left|H_{1}^{2}\right|^{2}+\left|H_{-1}^{2}\right|^{2}\right)+h_{13} \Re e\left(H_{1}^{2} H_{-1}^{2 *}\right)+h_{14} \Im m\left(H_{1}^{2} H_{-1}^{2 *}\right)\right. \\
& \left.+h_{15} \Re e\left(H_{1}^{2} H_{0}^{2 *}+H_{-1}^{2} H_{0}^{2 *}\right)+h_{16} \Im m\left(H_{1}^{2} H_{0}^{2 *}-H_{-1}^{2} H_{0}^{2 *}\right)\right] \\
S D W_{h}= & h_{17} \Re e\left(B^{2}\left(H_{1}^{2}+H_{-1}^{2}\right) B^{0 *} H_{0}^{0 *}\right)+h_{18} \Im m\left(B^{2}\left(H_{1}^{2}-H_{-1}^{2}\right) B^{0 *} H_{0}^{0 *}\right) \\
& +h_{19} \Re e\left(B^{2} H_{0}^{2} B^{0 *} H_{0}^{0 *}\right) \\
P D W_{h}= & h_{20} \Re e\left(B^{1} H_{0}^{1} B^{2 *} H_{0}^{2 *}\right)+h_{21} \Re e\left(B^{1} B^{2 *}\left(H_{1}^{1} H_{1}^{2 *}+H_{-1}^{1} H_{-1}^{2 *}\right)\right) \\
& +h_{22} \Re e\left(B^{1} B^{2 *}\left(H_{1}^{1} H_{-1}^{2 *}+H_{-1}^{1} H_{1}^{2 *}\right)\right)+h_{23} \Im m\left(B^{1} B^{2 *}\left(H_{1}^{1} H_{-1}^{2 *}-H_{-1}^{1} H_{1}^{2 *}\right)\right) \\
& +h_{24} \Re e\left(B^{1}\left(H_{1}^{1}+H_{-1}^{1}\right) B^{2 *} H_{0}^{2 *}\right)+h_{25} \Im m\left(B^{1}\left(H_{1}^{1}-H_{-1}^{1}\right) B^{2 *} H_{0}^{2 *}\right) \\
& +h_{26} \Re e\left(B^{1} H_{0}^{1} B^{2 *}\left(H_{1}^{2 *}+H_{-1}^{2 *}\right)\right)+h_{27} \Im m\left(B^{1} H_{0}^{1} B^{2 *}\left(H_{-1}^{2 *}-H_{1}^{2 *}\right)\right) .
\end{aligned}
$$

Les fonctions angulaires $h_{i}(i=1, \ldots, 27)$ sont données par les équations (D.20) à (D.46).

\section{D.2 Distibution angulaire en base de transversité}

La distribution en transversité s'obtient en effectuant les transformations décrites par les équations (2.64) et (2.69) :

$$
\frac{\mathrm{d}^{4} \sigma}{\mathrm{d} m \mathrm{~d} \cos \left(\theta_{X}\right) \mathrm{d} \cos \left(\theta_{t r}\right) \mathrm{d} \varphi_{t r}} \propto p q \frac{3}{32 \pi}\left[P W_{t}+S W_{t}+S P W_{t}+D W_{t}+S D W_{t}+P D W_{t}\right]
$$

où

$$
\begin{aligned}
P W_{t}= & 3\left|B^{1}\right|^{2}\left[f_{1}\left|A_{0}^{1}\right|^{2}+f_{2}\left|A_{\|}^{1}\right|^{2}+f_{3}\left|A_{\perp}^{1}\right|^{2}+f_{4} \Im\left(A_{\|}^{1 *} A_{\perp}^{1}\right)\right. \\
& \left.+f_{5} \Re\left(A_{\|}^{1} A_{0}^{1 *}\right)+f_{6} \Im\left(A_{\perp}^{1} A_{0}^{1 *}\right)\right] \\
S W_{t}= & f_{7}\left|B^{0}\right|^{2}\left|A_{0}^{0}\right|^{2} \\
S P W_{t}= & f_{8} \Re\left(B^{1} A_{\|}^{1} B^{0 *} A_{0}^{0 *}\right)+f_{9} \Im\left(B^{1} A_{\perp}^{1} B^{0 *} A_{0}^{0 *}\right) \\
& +f_{10} \Re\left(B^{1} A_{0}^{1} B^{0 *} A_{0}^{0 *}\right), \\
D W_{t}= & 5\left|B^{2}\right|^{2}\left[f_{11}\left|A_{0}^{2}\right|^{2}+f_{12}\left|A_{\|}^{2}\right|^{2}+f_{13}\left|A_{\perp}^{2}\right|^{2}+f_{14} \Im\left(A_{\|}^{2 *} A_{\perp}^{2}\right)\right. \\
& \left.+f_{15} \Re\left(A_{\|}^{2} A_{0}^{2 *}\right)+f_{16} \Im\left(A_{\perp}^{2} A_{0}^{2 *}\right)\right] \\
S D W_{t}= & f_{17} \Re\left(B^{2} A_{\|}^{2} B^{0 *} A_{0}^{0 *}\right)+f_{18} \Im\left(B^{2} A_{\perp}^{2} B^{0 *} A_{0}^{0 *}\right) \\
& +f_{19} \Re\left(B^{2} A_{0}^{2} B^{0 *} A_{0}^{0 *}\right),
\end{aligned}
$$




$$
\begin{aligned}
P D W_{t}= & f_{20} \Re\left(B^{1} A_{0}^{1} B^{2 *} A_{0}^{2 *}\right)+f_{21} \Re\left(B^{1} B^{2 *} A_{\|}^{1} A_{\|}^{2 *}\right) \\
& +f_{22} \Re\left(B^{1} B^{2 *} A_{\perp}^{1} A_{\perp}^{2 *}\right)+f_{23} \Im\left(B^{1} B^{2 *}\left(A_{\perp}^{1} A_{\|}^{2 *}-A_{\|}^{1} A_{\perp}^{2 *}\right)\right) \\
& +f_{24} \Re\left(B^{1} A_{\|}^{1} B^{2 *} A_{0}^{2 *}\right)+h_{25} \Im\left(B^{1} A_{\perp}^{1} B^{2 *} A_{0}^{2 *}\right) \\
& +h_{26} \Re\left(B^{1} A_{0}^{1} B^{2 *} A_{\|}^{2 *}\right)+f_{27} \Im\left(B^{1} A_{0}^{1} B^{2 *} A_{\perp}^{2 *}\right) .
\end{aligned}
$$

Les fonctions angulaires $f_{i}(i=1, \ldots, 27)$ sont données par les équations (D.47) à (D.73). 


$$
\begin{aligned}
& h_{1}=\sin ^{2}\left(\theta_{\psi}\right) \cos ^{2}\left(\theta_{X}\right) \\
& h_{2}=\frac{1}{4}\left(1+\cos ^{2}\left(\theta_{\psi}\right)\right) \sin ^{2}\left(\theta_{X}\right) \\
& h_{3}=-\frac{1}{2} \sin ^{2}\left(\theta_{\psi}\right) \cos (2 \varphi) \sin ^{2}\left(\theta_{X}\right) \\
& h_{4}=\frac{1}{2} \sin ^{2}\left(\theta_{\psi}\right) \sin (2 \varphi) \sin ^{2}\left(\theta_{X}\right) \\
& h_{5}=\frac{1}{4} \sin \left(2 \theta_{\psi}\right) \cos (\varphi) \sin \left(2 \theta_{X}\right) \\
& h_{6}=-\frac{1}{4} \sin \left(2 \theta_{\psi}\right) \sin (\varphi) \sin \left(2 \theta_{X}\right) \\
& h_{7}=\sin ^{2}\left(\theta_{\psi}\right) \\
& h_{8}=\frac{\sqrt{3}}{2} \sin \left(2 \theta_{\psi}\right) \cos (\varphi) \sin \left(\theta_{X}\right) \\
& h_{9}=-\frac{\sqrt{3}}{2} \sin \left(2 \theta_{\psi}\right) \sin (\varphi) \sin \left(\theta_{X}\right) \\
& h_{10}=2 \sqrt{3} \sin ^{2}\left(\theta_{\psi}\right) \cos \left(\theta_{X}\right) \\
& h_{11}=\frac{1}{4} \sin ^{2}\left(\theta_{\psi}\right)\left(3 \cos ^{2}\left(\theta_{X}\right)-1\right)^{2} \\
& h_{12}=\frac{3}{16}\left(1+\cos ^{2}\left(\theta_{\psi}\right)\right) \sin ^{2}\left(2 \theta_{X}\right) \\
& h_{13}=-\frac{3}{8} \sin ^{2}\left(\theta_{\psi}\right) \cos (2 \varphi) \sin ^{2}\left(2 \theta_{X}\right) \\
& h_{14}=\frac{3}{8} \sin ^{2}\left(\theta_{\psi}\right) \sin (2 \varphi) \sin ^{2}\left(2 \theta_{X}\right) \\
& h_{15}=\frac{\sqrt{3}}{8} \sin \left(2 \theta_{\psi}\right) \cos (\varphi) \sin \left(2 \theta_{X}\right)\left(3 \cos ^{2}\left(\theta_{X}\right)-1\right) \\
& h_{16}=-\frac{\sqrt{3}}{8} \sin \left(2 \theta_{\psi}\right) \sin (\varphi) \sin \left(2 \theta_{X}\right)\left(3 \cos ^{2}\left(\theta_{X}\right)-1\right) \\
& h_{17}=\frac{\sqrt{15}}{4} \sin \left(2 \theta_{\psi}\right) \cos (\varphi) \sin \left(2 \theta_{X}\right) \\
& h_{18}=-\frac{\sqrt{15}}{4} \sin \left(2 \theta_{\psi}\right) \sin (\varphi) \sin \left(2 \theta_{X}\right) \\
& h_{19}=\sqrt{5} \sin ^{2}\left(\theta_{\psi}\right)\left(3 \cos ^{2}\left(\theta_{X}\right)-1\right) \\
& h_{20}=\sqrt{15} \sin ^{2}\left(\theta_{\psi}\right) \cos \left(\theta_{X}\right)\left(3 \cos ^{2}\left(\theta_{X}\right)-1\right) \\
& h_{21}=\frac{3 \sqrt{5}}{2}\left(1+\cos ^{2}\left(\theta_{\psi}\right)\right) \sin ^{2}\left(\theta_{X}\right) \cos \left(\theta_{X}\right) \\
& h_{22}=-\frac{3 \sqrt{5}}{2} \sin ^{2}\left(\theta_{\psi}\right) \cos (2 \varphi) \sin ^{2}\left(\theta_{X}\right) \cos \left(\theta_{X}\right) \\
& h_{23}=\frac{3 \sqrt{5}}{2} \sin ^{2}\left(\theta_{\psi}\right) \sin (2 \varphi) \sin ^{2}\left(\theta_{X}\right) \cos \left(\theta_{X}\right) \\
& h_{24}=\frac{\sqrt{15}}{4} \sin \left(2 \theta_{\psi}\right) \cos (\varphi) \sin \left(\theta_{X}\right)\left(3 \cos ^{2}\left(\theta_{X}\right)-1\right) \\
& h_{25}=-\frac{\sqrt{15}}{4} \sin \left(2 \theta_{\psi}\right) \sin (\varphi) \sin \left(\theta_{X}\right)\left(3 \cos ^{2}\left(\theta_{X}\right)-1\right) \\
& h_{26}=\frac{3 \sqrt{5}}{2} \sin \left(2 \theta_{\psi}\right) \cos (\varphi) \sin \left(\theta_{X}\right) \cos ^{2}\left(\theta_{X}\right) \\
& h_{27}=-\frac{3 \sqrt{5}}{2} \sin \left(2 \theta_{\psi}\right) \sin (\varphi) \sin \left(\theta_{X}\right) \cos ^{2}\left(\theta_{X}\right)
\end{aligned}
$$




$$
\begin{aligned}
& f_{1}=2 \cos ^{2}\left(\theta_{X}\right)\left[1-\sin ^{2}\left(\theta_{t r}\right) \cos ^{2}\left(\varphi_{t r}\right)\right] \\
& f_{2}=\sin ^{2}\left(\theta_{X}\right)\left[1-\sin ^{2}\left(\theta_{t r}\right) \sin ^{2}\left(\varphi_{t r}\right)\right] \\
& f_{3}=\sin ^{2}\left(\theta_{X}\right) \sin ^{2}\left(\theta_{t r}\right) \\
& f_{4}=\sin ^{2}\left(\theta_{X}\right) \sin \left(2 \theta_{t r}\right) \sin \left(\varphi_{t r}\right) \\
& f_{5}=-\frac{1}{\sqrt{2}} \sin \left(2 \theta_{X}\right) \sin ^{2}\left(\theta_{t r}\right) \sin \left(2 \varphi_{t r}\right) \\
& f_{6}=\frac{1}{\sqrt{2}} \sin \left(2 \theta_{X}\right) \sin \left(2 \theta_{t r}\right) \cos \left(\varphi_{t r}\right) \\
& f_{7}=2\left[1-\sin ^{2}\left(\theta_{t r}\right) \cos ^{2}\left(\varphi_{t r}\right)\right] \\
& f_{8}=-\sqrt{6} \sin \left(\theta_{X}\right) \sin ^{2}\left(\theta_{t r}\right) \sin \left(2 \varphi_{t r}\right) \\
& f_{9}=\sqrt{6} \sin \left(\theta_{X}\right) \sin \left(2 \theta_{t r}\right) \cos \left(\varphi_{t r}\right) \\
& f_{10}=4 \sqrt{3} \cos \left(\theta_{X}\right)\left[1-\sin ^{2}\left(\theta_{t r}\right) \cos ^{2}\left(\varphi_{t r}\right)\right] \\
& f_{11}=\frac{1}{2}\left(3 \cos ^{2}\left(\theta_{X}\right)-1\right)^{2}\left[1-\sin ^{2}\left(\theta_{t r}\right) \cos ^{2}\left(\varphi_{t r}\right)\right] \\
& f_{12}=\frac{3}{4} \sin ^{2}\left(2 \theta_{X}\right)\left[1-\sin ^{2}\left(\theta_{t r}\right) \sin ^{2}\left(\varphi_{t r}\right)\right] \\
& f_{13}=\frac{3}{4} \sin ^{2}\left(2 \theta_{X}\right) \sin ^{2}\left(\theta_{t r}\right) \\
& f_{14}=\frac{3}{4} \sin ^{2}\left(2 \theta_{X}\right) \sin \left(2 \theta_{t r}\right) \sin \left(\varphi_{t r}\right) \\
& f_{15}=-\sqrt{\frac{3}{8}} \sin \left(2 \theta_{X}\right)\left(3 \cos ^{2}\left(\theta_{X}\right)-1\right) \sin ^{2}\left(\theta_{t r}\right) \sin \left(2 \varphi_{t r}\right) \\
& f_{16}=\sqrt{\frac{3}{8}} \sin \left(2 \theta_{X}\right)\left(3 \cos ^{2}\left(\theta_{X}\right)-1\right) \sin \left(2 \theta_{t r}\right) \cos \left(\varphi_{t r}\right) \\
& f_{17}=-\sqrt{\frac{15}{2}} \sin \left(2 \theta_{X}\right) \sin ^{2}\left(\theta_{t r}\right) \sin \left(2 \varphi_{t r}\right) \\
& f_{18}=\sqrt{\frac{15}{2}} \sin \left(2 \theta_{X}\right) \sin \left(2 \theta_{t r}\right) \cos \left(\varphi_{t r}\right) \\
& f_{19}=2 \sqrt{5}\left(3 \cos ^{2}\left(\theta_{X}\right)-1\right)\left[1-\sin ^{2}\left(\theta_{t r}\right) \cos ^{2}\left(\varphi_{t r}\right)\right] \\
& f_{20}=2 \sqrt{15} \cos \left(\theta_{X}\right)\left(3 \cos ^{2}\left(\theta_{X}\right)-1\right)\left[1-\sin ^{2}\left(\theta_{t r}\right) \cos ^{2}\left(\varphi_{t r}\right)\right] \\
& f_{21}=6 \sqrt{5} \sin ^{2}\left(\theta_{X}\right) \cos \left(\theta_{X}\right)\left[1-\sin ^{2}\left(\theta_{t r}\right) \sin ^{2}\left(\varphi_{t r}\right)\right] \\
& f_{22}=6 \sqrt{5} \sin ^{2}\left(\theta_{X}\right) \cos \left(\theta_{X}\right) \sin ^{2}\left(\theta_{t r}\right) \\
& f_{23}=3 \sqrt{5} \sin ^{2}\left(\theta_{X}\right) \cos \left(\theta_{X}\right) \sin \left(2 \theta_{t r}\right) \sin \left(\varphi_{t r}\right) \\
& f_{24}=-\sqrt{\frac{15}{2}} \sin \left(\theta_{X}\right)\left(3 \cos ^{2}\left(\theta_{X}\right)-1\right) \sin ^{2}\left(\theta_{t r}\right) \sin \left(2 \varphi_{t r}\right) \\
& f_{25}=\sqrt{\frac{15}{2}} \sin \left(\theta_{X}\right)\left(3 \cos ^{2}\left(\theta_{X}\right)-1\right) \sin \left(2 \theta_{t r}\right) \cos \left(\varphi_{t r}\right) \\
& f_{26}=-3 \sqrt{10} \sin \left(\theta_{X}\right) \cos ^{2}\left(\theta_{X}\right) \sin ^{2}\left(\theta_{t r}\right) \sin \left(2 \varphi_{t r}\right) \\
& f_{27}=-3 \sqrt{10} \sin \left(\theta_{X}\right) \cos ^{2}\left(\theta_{X}\right) \sin \left(2 \theta_{t r}\right) \cos \left(\varphi_{t r}\right)
\end{aligned}
$$




\section{Annexe E}

\section{Développement en moments des fonctions $\varphi_{l}^{i \rightarrow j}\left(\vec{\omega}^{\prime}\right)$}

Cet appendice traite du développement en moments de la fonction $\varphi_{l}^{i \rightarrow j}\left(\vec{\omega}^{\prime}\right)$ (cf. eq. (5.21)) sans faire d'hypothèse sur la résolution. En définissant les fonctions $\mathcal{Y}_{\vec{\nu}}\left(\vec{\omega}^{\prime}\right)$ par :

$$
\mathcal{Y}_{\vec{\nu}}\left(\vec{\omega}^{\prime}\right)=\sqrt{2 \pi} \cdot Y_{L, M}\left(\theta_{\psi}, \varphi_{\psi}\right) \cdot Y_{R,-M}\left(\theta_{K^{*}}, 0\right)
$$

où $Y_{l m}$ sont les harmoniques sphériques, $\left(\theta_{\psi}, \varphi_{\psi}, \theta_{K^{*}}\right)$ sont les angles d'hélicité correspondant aux angles de transversité $\vec{\omega}$ et où $\vec{\nu}$ symbolise le triplet $\{L, R, M\}$. La fonction $\varphi_{l}^{i \rightarrow j}\left(\vec{\omega}^{\prime}\right)$ peut être développée sur cette base :

$$
\varphi_{l}^{i \rightarrow j}\left(\vec{\omega}^{\prime}\right)=\sum_{\vec{\nu}} \Phi_{l, \vec{\nu}}^{i \rightarrow j} \cdot \mathcal{Y}_{\vec{\nu}}\left(\vec{\omega}^{\prime}\right)
$$

Les coefficients $\Phi_{l, \vec{\nu}}^{i \rightarrow j}$ sont donnés, grâce à l'orthonormalité des fonctions $\mathcal{Y}_{\vec{\nu}}\left(\vec{\omega}^{\prime}\right)$, par :

$$
\Phi_{l, \vec{\nu}}^{i \rightarrow j}=\int \varphi_{l}^{i \rightarrow j}\left(\vec{\omega}^{\prime}\right) \cdot \mathcal{Y}_{\vec{\nu}}^{*}\left(\vec{\omega}^{\prime}\right) \mathrm{d} \vec{\omega}^{\prime}=\iint f_{l}(\vec{\omega}) \cdot T^{i \rightarrow j}\left(\vec{\omega}, \vec{\omega}^{\prime}\right) \cdot \mathcal{Y}_{\vec{\nu}}^{*}\left(\vec{\omega}^{\prime}\right) \mathrm{d} \vec{\omega} \mathrm{d} \vec{\omega}^{\prime}
$$

La notation $\Phi_{l, \vec{\nu}}^{i \rightarrow j}$ a été introduite puisque $\left(\mathcal{Y}_{000}=1 / \sqrt{8 \pi}\right)$

$$
\begin{aligned}
\Phi_{l,(\vec{\nu} \equiv 000)}^{i \rightarrow j} & =\iint f_{l}(\vec{\omega}) \cdot T^{i \rightarrow j}\left(\vec{\omega}, \vec{\omega}^{\prime}\right) \cdot \mathcal{Y}_{(\vec{\nu} \equiv 000)}\left(\vec{\omega}^{\prime}\right) \mathrm{d} \vec{\omega} \mathrm{d} \vec{\omega}^{\prime} \\
& =\frac{1}{\sqrt{8 \pi}} \cdot \iint f_{l}(\vec{\omega}) \cdot T^{i \rightarrow j}\left(\vec{\omega}, \vec{\omega}^{\prime}\right) \mathrm{d} \vec{\omega} \mathrm{d} \vec{\omega}^{\prime} \\
& =\frac{1}{\sqrt{8 \pi}} \cdot \Phi_{l}^{i \rightarrow j}
\end{aligned}
$$

d'après l'équation (5.24).

Afin d'obtenir la fonction $\varphi_{l}^{i \rightarrow j}\left(\vec{\omega}^{\prime}\right)$, il convient de déterminer les coefficients $\Phi_{l, \vec{\nu}}^{i \rightarrow j}$ définis par l'équation (E.3). Ces coefficients sont obtenus à partir de données simulées. La difficulté de cette évaluation provient du fait que l'équation (E.3) comporte une double intégration sur les variables $\vec{\omega}$ and $\vec{\omega}^{\prime}$. Le passage de l'intégration analytique à l'évaluation par Monte Carlo requiert un certain 
soin. Dans l'évaluation Monte Carlo, $N_{g e n}^{i}$ angles $\vec{\omega}_{\alpha}$ sont générés dans le canal $i$ et $N_{a c c}^{i \rightarrow j}$ angles $\vec{\omega}_{\beta}^{\prime}$ sont reconstruits dans le canal $j$. Il n'est pas possible d'effectuer une sommation indépendante sur les angles générés d'une part, et sur les angles reconstruits d'autre part. Afin de traiter ce point, on introduit une nouvelle variable $\vec{\Omega}$ définie par :

$$
\vec{\Omega} \equiv\left(\vec{\omega}, \vec{\omega}^{\prime}\right)
$$

de sorte que la double intégration sur $\vec{\omega}$ et $\vec{\omega}^{\prime}$ se transforme en une simple intégration sur $\vec{\Omega}$. Cela nécessite d'étendre les définitions de $f_{l}$ et $\mathcal{Y}_{\vec{\nu}}$ :

$$
\begin{aligned}
f_{l}(\vec{\Omega}) & \equiv f_{l}(\vec{\omega}) \\
\mathcal{Y}_{\vec{\nu}}(\vec{\Omega}) & \equiv \mathcal{Y}_{\vec{\nu}}\left(\vec{\omega}^{\prime}\right)
\end{aligned}
$$

L'équation (E.3) devient alors :

$$
\Phi_{l, \vec{\nu}}^{i \rightarrow j}=\int f_{l}(\vec{\Omega}) \cdot T^{i \rightarrow j}(\vec{\Omega}) \cdot \mathcal{Y}_{\vec{\nu}}^{*}(\vec{\Omega}) \mathrm{d} \vec{\Omega}
$$

On considère $N_{g e n}^{i}$ angles générés $\vec{\omega}_{\alpha}$ dans le canal $i$, distribués selon une densité de probabilité normalisée $h(\vec{\omega})$ (notée aussi $h(\vec{\Omega})$ ) arbitraire et conduisant à $N_{a c c}^{i \rightarrow j}$ angles reconstruits $\vec{\omega}_{\beta}^{\prime}$ dans le canal $j$. La densité de probabilité normalisée $H^{i \rightarrow j}(\vec{\Omega})$ est définie à partir de $h(\vec{\omega})$ par :

$$
H^{i \rightarrow j}(\vec{\Omega}) \equiv \frac{h(\vec{\Omega}) \cdot T^{i \rightarrow j}(\vec{\Omega})}{\int h(\vec{\Omega}) \cdot T^{i \rightarrow j}(\vec{\Omega}) \mathrm{d} \vec{\Omega}}
$$

L'évaluation de (E.3) conduit à :

$$
\begin{aligned}
\Phi_{l, \vec{\nu}}^{i \rightarrow j} & =\int f_{l}(\vec{\Omega}) \cdot T^{i \rightarrow j}(\vec{\Omega}) \cdot \mathcal{Y}_{\vec{\nu}}^{*}(\vec{\Omega}) \mathrm{d} \vec{\Omega} \\
& =\int f_{l}(\vec{\Omega}) \cdot T^{i \rightarrow j}(\vec{\Omega}) \cdot \mathcal{Y}_{\vec{\nu}}^{*}(\vec{\Omega}) \cdot \frac{1}{H^{i \rightarrow j}(\vec{\Omega})} \times H^{i \rightarrow j}(\vec{\Omega}) \mathrm{d} \vec{\Omega} \\
& =\left\{\int h\left(\vec{\Omega}^{\prime}\right) \cdot T^{i \rightarrow j}\left(\vec{\Omega}^{\prime}\right) \mathrm{d} \vec{\Omega}^{\prime}\right\} \times E\left[f_{l} \cdot \mathcal{Y}_{\vec{\nu}}^{*} \cdot \frac{1}{h}\right] .
\end{aligned}
$$

L'espérance $E\left[f_{l} \cdot \mathcal{Y}_{\vec{\nu}}^{*} \cdot \frac{1}{h}\right]$ est estimée à partir de Monte Carlo en utilisant « $N$ angles générés $\vec{\Omega}_{\alpha}$ ", ce qui en réalité correspond à $N_{a c c}^{i \rightarrow j}$ angles générés et reconstruits $\left(\vec{\omega}_{\beta}, \vec{\omega}_{\beta}^{\prime}\right)$. Ainsi :

$$
\begin{aligned}
E\left[f_{l} \cdot \mathcal{Y}_{\vec{\nu}}^{*} \cdot \frac{1}{h}\right] & \asymp \frac{1}{N_{a c c}^{i \rightarrow j}} \sum_{\beta=1}^{N_{a c c}^{i \rightarrow j}} f_{l}\left(\vec{\Omega}_{\beta}\right) \cdot \mathcal{Y}_{\vec{\nu}}^{*}\left(\vec{\Omega}_{\beta}\right) \cdot \frac{1}{h\left(\vec{\Omega}_{\beta}\right)} \\
& =\frac{1}{N_{a c c}^{i \rightarrow j}} \sum_{\beta=1}^{N_{a c c}^{i \rightarrow j}} f_{l}\left(\vec{\omega}_{\beta}\right) \cdot \mathcal{Y}_{\vec{\nu}}^{*}\left(\vec{\omega}_{\beta}^{\prime}\right) \cdot \frac{1}{h\left(\vec{\omega}_{\beta}\right)}
\end{aligned}
$$


L'intégrale $\int h(\vec{\Omega}) \cdot T^{i \rightarrow j}(\vec{\Omega}) \mathrm{d} \vec{\Omega}$ est, en utilisant l'équation (5.23) :

$$
\begin{aligned}
\int h(\vec{\Omega}) \cdot T^{i \rightarrow j}(\vec{\Omega}) \mathrm{d} \vec{\Omega} & =\iint h(\vec{\omega}) \cdot T^{i \rightarrow j}\left(\vec{\omega}, \vec{\omega}^{\prime}\right) \mathrm{d} \vec{\omega} \mathrm{d} \vec{\omega}^{\prime} \\
& =\int h(\vec{\omega}) \cdot \epsilon^{i \rightarrow j}(\vec{\omega}) \mathrm{d} \vec{\omega} \\
& =\left\langle\epsilon^{i \rightarrow j}\right\rangle .
\end{aligned}
$$

En revenant à l'équation (E.14), $\Phi_{l, \vec{\nu}}^{i \rightarrow j}$ devient :

$$
\begin{aligned}
\Phi_{l, \vec{\nu}}^{i \rightarrow j} & \asymp\left\langle\epsilon^{i \rightarrow j}\right\rangle \times \frac{1}{N_{a c c}^{i \rightarrow j}} \sum_{\beta=1}^{N_{a c c}^{i \rightarrow j}} f_{l}\left(\vec{\omega}_{\beta}\right) \cdot \mathcal{Y}_{\vec{\nu}}^{*}\left(\vec{\omega}_{\beta}^{\prime}\right) \cdot \frac{1}{h\left(\vec{\omega}_{\beta}\right)} \\
& =\frac{1}{N_{a c c}^{i \rightarrow j} /\left\langle\epsilon^{i \rightarrow j}\right\rangle} \sum_{\beta=1}^{N_{a c c}^{i \rightarrow j}} f_{l}\left(\vec{\omega}_{\beta}\right) \cdot \mathcal{Y}_{\vec{\nu}}^{*}\left(\vec{\omega}_{\beta}^{\prime}\right) \cdot \frac{1}{h\left(\vec{\omega}_{\beta}\right)} \\
& =\frac{1}{N_{g e n}^{i}} \sum_{\beta=1}^{N_{a c c}^{i \rightarrow j}} f_{l}\left(\vec{\omega}_{\beta}\right) \cdot \mathcal{Y}_{\vec{\nu}}^{*}\left(\vec{\omega}_{\beta}^{\prime}\right) \cdot \frac{1}{h\left(\vec{\omega}_{\beta}\right)} .
\end{aligned}
$$

À noter que le lien entre les angles générés et reconstruits, exprimé par la fonction $T^{i \rightarrow j}\left(\vec{\omega}, \vec{\omega}^{\prime}\right)$, est transféré dans les moments $\Phi_{l, \vec{\nu}}^{i \rightarrow j}$ par le fait que la fonction $f_{l}$ est évaluée avec les angles générés $\vec{\omega}_{\beta}$ alors que $\mathcal{Y}_{\vec{\nu}}^{*}$ est évalué avec les angles reconstruits $\vec{\omega}_{\beta}^{\prime}$. De même, l'efficacité de reconstruction présente dans $T^{i \rightarrow j}\left(\vec{\omega}, \vec{\omega}^{\prime}\right)$ est décrite par le fait que la somme s'étend jusqu'à $N_{a c c}^{i \rightarrow j}$ et non $N_{g e n}^{i}$. Les coefficients $\Phi_{l, \vec{\nu}}^{i \rightarrow j}$ et donc les fonctions $\varphi_{l}^{i \rightarrow j}\left(\vec{\omega}^{\prime}\right)$ sont indépendants des amplitudes.

En résumé, la densité de probabilité normalisée observée pour le canal reconstruit $j$ s'écrit :

$$
\begin{aligned}
g_{o b s}^{j}\left(\vec{\omega}^{\prime} ; \vec{A}\right) & =\frac{\int g(\vec{\omega} ; \vec{A}) \times\left\{\sum_{i=1}^{4} F_{i} \cdot T^{i \rightarrow j}\left(\vec{\omega}, \vec{\omega}^{\prime}\right)\right\} \mathrm{d} \vec{\omega}}{\sum_{l=1}^{6} K_{l} \cdot\left\{\sum_{i=1}^{4} F_{i} \cdot \Phi_{l}^{i \rightarrow j}\right\}} \\
& =\frac{\sum_{l=1}^{6} K_{l} \cdot \sum_{i=1}^{4} F_{i} \cdot \sum_{\vec{\nu}} \Phi_{l, \vec{\nu}}^{i \rightarrow j} \cdot \mathcal{Y}_{\vec{\nu}}\left(\vec{\omega}^{\prime}\right)}{\sum_{l=1}^{6} K_{l} \cdot \sum_{i=1}^{4} F_{i} \cdot \Phi_{l}^{i \rightarrow j}} .
\end{aligned}
$$




\section{Bibliographie}

[1] R. Feynman, The Character of Physical Law, Penguin Books, (1965).

Trad. française : La nature des lois physiques dans La nature de la physique, Éditions du seuil, (1980).

[2] Y. S. Kim et M. E. Noz, Theory and Applications of Poincaré Group, Reidel, Dordrecht, (1986).

B. Delamotte, Un soupçon de théorie des groupes : groupe de rotations et groupe de Poincaré, Cours du DEA Champs, Particules, Matières. [http://www. lpthe.jussieu.fr/DEA/]

[3] R. G. Sachs, The Physics of Time Reversal, University of Chicago Press, (1987).

[4] T. D. Lee et G. C. Wick, Space Inversion, Time Reversal, and Other Discrete Symmetries in Local Field Theories, Phys. Rev. 148, 1385, (1966).

[5] C. Jarlskog, dans CP Violation, ed. C. Jarlskog, World Scientific, Singapore (1989).

[6] T. D. Lee et C. N. Yang, Question of Parity Conservation in Weak Interactions, Phys. Rev. 104, 254, (1956).

Errata : Phys. Rev. 106, 1371, (1957).

[7] C. S. Wu et al., Experimental Test of Parity Conservation in Beta Decay, Phys. Rev. 105, 1413, (1957).

[8] M. Goldhaber, L. Grodzins et A. W. Sunyar, Helicity Of Neutrinos, Phys. Rev. 109, 1015, (1958).

[9] L. D. Landau, On the conservation laws for weak interactions, Nucl. Phys. 3, 127, (1957).

[10] O. Darrigol, From c-Numbers to q-Numbers : the Classical Analogy in the History of Quantum Theory, Univerty of California Press, (1992).

[11] M. Bunge, Philosophy of Physics, Reidel, Dordrecht, (1973).

Trad. française : Philosophie de la Physique, Éditions du seuil, (1975).

[12] J. H. Christenson, J. W. Cronin, V. L. Fitch et R. Turlay, Evidence for the $2 \pi$ Decay of the $K_{2}^{0}$ Meson, Phys. Rev. Lett. 13, 138, (1964).

[13] R. F. Streater et A. S. Wightman, PCT, Spin and Statistics, and All That, W. A. Benjamin, Inc., New York, (1964).

[14] Sous la direction de E. Klein et M. Spiro, Le temps et sa flèche, coll. Champs, Flammarion, (1996).

[15] M. Gardner, The Ambidextrous Universe, Penguin Books, (1982).

Trad. française : L'univers ambidextre, Éditions du seuil, (1985).

[16] A. D. Sakharov, Violation Of CP Invariance, C Asymmetry, And Baryon Asymmetry Of The Universe, JETP Lett. 5, 24, (1967). 
[17] B. Aubert et al., BaBar Collaboration, Observation of $C P$ Violation in the $B^{0}$ Meson System, Phys. Rev. Lett. 87, 091801, (2001).

[18] K. Abe et al., Belle Collaboration, Observation of Large CP Violation in the Neutral B Meson System, Phys. Rev. Lett. 87, 091802, (2001).

[19] P. K. Kabir, Appendice A de : The CP Puzzle, Academic Press, (1968).

[20] B. Winstein, $C P$ violation in neutral-B-meson decays in the standard and superweak models, Phys. Rev. Lett. 68, 1271, (1992).

B. Winstein et L. Wolfenstein, The Search for Direct CP Violation, Rev. of Mod. Phys. 65, 1113, (1993).

J. M. Soares et L. Wolfenstein, Testing the superweak theory of CP violation in neutral $B$ decays, Phys. Rev. D46, 256, (1992).

[21] KTeV Collaboration, A. Alavi-Harati et al., Observation of direct CP violation in $K_{(S, L)} \rightarrow \pi \pi$ decays, Phys. Rev. Lett. 83, 22, (1999).

NA48 Collaboration, A. Lai et al., A precise measurement of the direct $C P$ violation parameter $R e\left(\epsilon^{\prime} / \epsilon\right)$, Eur. Phys. J. C 22, 231, (2001).

[22] G. C. Branco, L. Lavoura et J. P. Silva, CP Violation, Oxford University Press, Oxford (1999).

[23] I. I. Bigi et A. I. Sanda, CP Violation, Cambridge University Press, Cambridge (2000).

[24] J. D. Richman, dans Les Houches Summer School Proceedings, 68, eds. R. Gupta et al., NorthHolland,(1999).

[25] Y. Nir, Proceedings of the $27^{\text {th }}$ Annual SLAC Summer Institute on Particle Physics (1999).

Y. Nir, Proceedings of the $20^{\text {th }}$ Annual SLAC Summer Institute on Particle Physics (1992). SLAC Report 412.

[26] BaBar Physics Book, eds. H. Quinn \& P. Harrison, SLAC Report 504, Chapitre 1.

[27] I. I. Bigi et A. I. Sanda, Comments on the sign of CP asymmetries, Phys. Rev. D60, 033001, (1999).

[28] Particle Data Group, K. Hagiwara et al., Phys. Rev. D66, 010001, (2002).

[29] I. I. Bigi et A. I. Sanda, CP Violation In Heavy Flavor Decays : Predictions And Search Strategies, Nucl. Phys. B281, 41, (1987).

[30] R. Aleksan, I. Dunietz, B. Kayser et F. Le Diberder, CP Violation Using Non CP Eigenstate Decays Of Neutral B Mesons, Nucl. Phys. B361, 141, (1991).

[31] I. I. Bigi et al., dans CP Violation, ed. C. Jarlskog, World Scientific, Singapore (1989).

[32] C. Quigg, Gauge Theories of the Strong, Weak and Electromagnetic Interactions, Benjamin/Cummings, Menlo Park, (1983).

T. P. Cheng et L. F. Li, Gauge Theory of Elementary Particle Physics, Oxford University Press, Oxford, (1984).

E. Predazzi et E. Leader, An Introduction to Gauge Theories and Modern Particle Physics, Cambridge University Press, Cambridge, (1996).

J. Donoghue, E. Golowich et B. R. Holstein, Dynamics of the Standard Model, Cambridge Univerity Press, Cambridge, (1992). 
[33] M. E. Peskin et D. V. Schroeder, An Introduction to Quantum Field Theory, Addison-Wesley, Menlo Park, (1995).

[34] R. N. Cahn et G. Goldhaber, The Experimental Foundations of Particle Physics, Cambridge University Press, Cambridge, (1989).

[35] W. Grimus et M. N. Rebelo, Automorphisms in gauge theories and the definition of $C P$ and $P$, Phys. Rep. 281, 239, (1997).

[36] R. D. Peccei, dans CP Violation, ed. C. Jarlskog, World Scientific, Singapore (1989).

[37] M. Kobayashi et T. Maskawa, CP Violation In The Renormalizable Theory Of Weak Interaction, Prog. of Theor. Phys. 49, 652, (1973).

[38] K. Huang, Quarks, Leptons E Gauge Fields, $2^{\text {nd }}$ edition, World Scientific, Singapore (1992).

[39] S. L. Glashow, J. Iliopoulos et L. Maiani, Weak Interactions With Lepton - Hadron Symmetry, Phys. Rev. D2, 1285, (1970).

[40] F. Halzen et A. D. Martin, Quarks and Leptons : An Introductory Course in Modern Particle Physics, Wiley, New York, (1984).

[41] S. L. Glashow et S. Weinberg, Natural Conservation Laws For Neutral Currents, Phys. Rev. D15, 1958, (1977).

E. A. Paschos, Diagonal Neutral Currents, Phys. Rev. D15, 1966, (1977).

[42] Super-Kamiokande Collaboration, Y. Fukuda et al., Solar B-8 and he p neutrino measurements from 1258 days of Super-Kamiokande data, Phys. Rev. Lett. 86, 5651, (2001).

SNO Collaboration, Q. R. Ahmad et al., Measurement of the charged current interactions produced by B-8 solar neutrinos at the Sudbury Neutrino Observatory, Phys. Rev. Lett. 87, 071301, (2001).

[43] Z. Maki, M. Nakagawa et S. Sakata, Remarks On The Unified Model Of Elementary Particles, Prog. of Theor. Phys. 28, 870, (1962).

[44] B. Kayser, dans CP Violation, ed. C. Jarlskog, World Scientific, Singapore (1989).

[45] N. Cabibbo, Unitary Symmetry And Leptonic Decays, Phys. Rev. Lett. 10, 531, (1963).

[46] C. Jarlskog, Commutator Of The Quark Mass Matrices In The Standard Electroweak Model And A Measure Of Maximal CP Violation, Phys. Rev. Lett. 55, 1039, (1985).

C. Jarlskog, A Basis Independent Formulation Of The Connection Between Quark Mass Matrices, CP Violation And Experiment, Z. Phys. C29, 491, (1985).

[47] C. Jarlskog et R. Stora, Unitarity Polygons And CP Violation Areas And Phases In The Standard Electroweak Model, Phys. Lett. B208, 268, (1988).

[48] L. L. Chau at W.-Y. Keung, Comments On The Parametrization Of The Kobayashi-Maskawa Matrix, Phys. Rev. Lett. 53, 1802, (1984).

[49] C. Jarlskog, Flavor Projection Operators And Applications To CP Violation With Any Number Of Families, Phys. Rev. D36, 2128, (1987).

[50] L. Wolfenstein, Parametrization Of The Kobayashi-Maskawa Matrix, Phys. Rev. Lett. 51, 1945, (1983).

[51] F. J. Gilman, K. Kleinknecht et B. Renk, dans Particle Data Group, K. Hagiwara et al., Phys. Rev. D66, 010001, (2002). 
[52] A. J Buras, M. E. Lautenbacher et G. Ostermaier, Waiting for the top quark mass, $K^{+} \rightarrow \pi^{+} \nu \bar{\nu}$, $B_{s}^{0}-\bar{B}_{s}^{0}$ mixing and CP asymmetries in B decays, Phys. Rev. D50, 3433, (1994).

[53] A. J. Buras et R. Fleischer, dans Heavy Flavours II, eds. A. J. Buras et M. Lindner, World Scientific, Singapore (1998).

[54] G. C. Branco et L. Lavoura, Wolfenstein Type Parametrization Of The Quark Mixing Matrix, Phys. Rev. D38, 2295, (1988).

[55] C. O. Dib et al., Standard Model Predictions For CP Violation In $B^{0}$ Meson Decay, Phys. Rev. D41, 1522, (1990).

[56] A. J. Buras, W. Slominski et H. Steger, $B^{0} \bar{B}^{0}$ Mixing, $C P$ Violation And The B Meson Decay, Nucl. Phys. B245, 369, (1984).

[57] J. S. Hagelin, Mass Mixing And CP Violation In The $B^{0} \bar{B}^{0}$, Nucl. Phys. B193, 123, (1981).

[58] T. Inami et C. S. Lim, Effects Of Superheavy Quarks And Leptons In Low-Energy Weak Processes $K_{L} \rightarrow \mu \bar{\mu}, K^{+} \rightarrow \pi^{+} \nu \bar{\nu}$ And $K^{0} \bar{K}^{0}$, Prog. of Theor. Phys. 65, 297, (1981). Erratum : 65, 1772, (1982).

[59] M. Gronau, J. L. Rosner et D. London, Weak coupling phase from decays of charged $B$ mesons to $\pi K$ and $\pi \pi$, Phys. Rev. Lett. 73, 21, (1994).

M. Gronau et al., Decays of B mesons to two light pseudoscalars, Phys. Rev. D50, 4529, (1994).

M. Gronau et al., Electroweak penguins and two body B decays, Phys. Rev. D52, 6374, (1995).

[60] M. Neubert, Rescattering effects, isospin relations and electroweak penguins in $B \rightarrow \pi K$ decays, Phys. Lett. B424, 152, (1998).

[61] M. Bauer, B. Stech et M. Wirbel, Exclusive Semileptonic Decays Of Heavy Mesons, Z. Phys. C29, 637, (1985).

M. Bauer, B. Stech et M. Wirbel, Exclusive Nonleptonic Decays Of $D, D_{S}$, And B Mesons, Z. Phys. C34, 103, (1987).

[62] M. Gronau, Large penguin effects in the CP asymmetry of $B_{d}^{0} \rightarrow \pi^{+} \pi^{-}$, Phys. Lett. B300,163, (1993).

[63] I. Dunietz et al., How To Extract CP Violating Asymmetries From Angular Correlations, Phys. Rev. D43, 2193, (1991).

[64] A. S. Dighe, I. Dunietz et R. Fleischer, Extracting CKM phases and $B_{s} \bar{B}_{s}$ mixing parameters from angular distributions of non-leptonic B decays, Eur. Phys. J. C6, 647, (1999).

[65] R. Sinha, New Physics in $B \rightarrow J / \psi K^{*}$, hep-ph/0207007 v2.

[66] Y. Grossman, A. L. Kagan et Z. Ligeti, Can the $C P$ asymmetries in $B \rightarrow \psi K_{S}$ and $B \rightarrow \psi K_{L}$ differ ?, Phys. Lett. B538, 327, (2002).

[67] BaBar Physics Book, eds. H. Quinn \& P. Harrison, SLAC Report 504, Chapitres 2 et 10.

[68] M. Neubert et B. Stech, dans Heavy Flavours II, eds. A. J. Buras et M. Lindner, World Scientific, Singapore (1998). [hep-ph/9705292]

[69] A. Le Yaouanc, L. Oliver, O. Pène et J.-C. Raynal, Factorization versus duality in nonleptonic decays, a quark model approach, Phys. Rev. D52, 2813, (1995). 
[70] J. D. Bjorken, Slac Summer Institute 1990, SLAC-PUB-5389.

J. D. Bjorken, Nucl. Phys. B11 (Proc. Suppl.), 325, (1989).

[71] M. J. Dugan et B. Grinstein, QCD Basis For Factorization In Decays Of Heavy Mesons, Phys. Lett. B255, 583, (1991).

[72] A. J. Buras, M. Jamin, M. E. Lautenbacher et P. H. Weisz, Two loop anomalous dimension matrix for $\Delta S=1$ weak nonleptonic decays I : $\mathcal{O}\left(\alpha_{s}^{2}\right)$, Nucl. Phys. B400, 37, (1993).

M. Ciuchini, E. Franco, G. Martinelli et L. Reina, The $\Delta S=1$ effective Hamiltonian including next-to-leading order QCD and QED corrections, Nucl. Phys. B415, 403, (1994).

[73] G. Buchalla, A. J. Buras et M. E. Lautenbacher, Weak Decays Beyond Leading Logarithms, Rev. of Mod. Phys. 68, 1125, (1996).

[74] J. M. Gérard et W. S. Hu, CP Violation In Inclusive And Exclusive Charmless B Decays, Phys. Rev. D43, 2909, (1991).

[75] M. A. Shifman, A. I. Vainshtein et V. I. Zakharov, Light Quarks And The Origin Of The $\Delta I=1 / 2$ Rule In The Nonleptonic Decays Of Strange Particles, Nucl. Phys. B120, 316, (1977).

[76] E. Pilon, École de Gif 1995.

[77] O. Haan et B. Stech, Violation Of The $\Delta I=1 / 2$ Rule In Non-Leptonic Decays, Nucl. Phys. B22, 448, (1970).

D. Fakirov et B. Stech, F And D Decays, Nucl. Phys. B113, 315, (1978).

B. Stech, dans CP Violation, ed. C. Jarlskog, World Scientific, Singapore (1989).

[78] C. Reader et N. Isgur, Factorization And Heavy Quark Symmetry In Hadronic B Meson Decays, Phys. Rev. D47, 1007, (1993).

[79] H. Y. Cheng, Y. Y. Keum et K. C. Yang, Angular distribution analysis of $B \rightarrow J / \psi K^{*}$ and resolving discrete ambiguities in the determination of $\phi_{1}$, hep-ph/0112257.

[80] H.-Y. Cheng, Y.-Y. Keum et K.-C. Yang, $B \rightarrow J / \psi K^{*}$ decays in QCD factorization, Phys. Rev. D65, 094023, (2002).

[81] M. Gourdin, A. N. Kamal et X. Y. Pham, Difficulties In Explaining Recent Data On $B \rightarrow J / \psi+$ $K\left(K^{*}\right)$ Decays With Commonly Used Form-Factors Within The Factorization Approach, Phys. Rev. Lett. 73, 3355, (1994).

[82] M. Neubert, V. Rieckert, B. Stech et Q. P. Xu, M. Neubert et B. Stech, dans Heavy Flavours, eds. A. J. Buras et M. Lindner, World Scientific, Singapore (1992).

[83] N. Isgur, D. Scora, B. Grinstein et M. B. Wise, Semileptonic B And D Decays In The Quark Model, Phys. Rev. D39, 799, (1989).

N. Isgur et D. Scora, Polarization In $\bar{B} \rightarrow D^{*} e^{-} \bar{\nu}_{e}$ And $D \rightarrow \bar{K}^{*} e^{+} \nu_{e}$, Phys. Rev. D40, 1491, (1989).

[84] R. Casalbuoni et al., Light vector resonances in the effective chiral Lagrangian for heavy mesons, Phys. Lett. B292, 371, (1992).

R. Casalbuoni et al., Effective Lagrangian for heavy and light mesons : Semileptonic decays, Phys. Lett. B299, 139, (1993).

A. Deandrea et al., Two body nonleptonic decays of B and $B_{s}$ mesons, Phys. Lett. B318, 549, (1993). 
[85] A. Ali et T. Mannel, Exclusive rare B decays in the heavy quark limit, Phys. Lett. B264, 447, (1991). Erratum : Phys. Lett. B274, 526, (1992).

A. Ali, T. Ohland et T. Mannel, Radiative rare $B$ decays into higher $K$ resonances, Phys. Lett. B298, 195, (1993).

[86] W. Jaus, Semileptonic Decays Of B And D Mesons In The Light Front Formalism, Phys. Rev. D41, 3394, (1990).

W. Jaus et D. Wyler, The Rare Decays Of $B \rightarrow K \ell \bar{\ell} A$ nd $B \rightarrow K^{*} \ell \bar{\ell}$, Phys. Rev. D41, 3405, (1990).

[87] R. Aleksan, A. Le Yaouanc, L. Oliver, O. Pène et J.-C. Raynal, Critical Analysis Of Theoretical Estimates For $B$ To Light Meson Form-Factors And The $B \rightarrow \psi K\left(K^{*}\right)$ Data, Phys. Rev. D51, 6235, (1995).

[88] H.-Y. Cheng et B. Tseng, Extraction of $a_{1}$ and $a_{2}$ from $B \rightarrow \psi K\left(K^{*}\right), D\left(D^{*}\right) \pi(\rho)$ decays, Phys. Rev. D51, 6259, (1995).

[89] P. Ball et V. M. Braun, Exclusive semileptonic and rare B meson decays in QCD, Phys. Rev. D58, 094016, (1998).

[90] D. Melikhov et B. Stech, Weak form factors for heavy meson decays : An update, Phys. Rev. D62, 014006, (2001).

[91] H. Y. Cheng, C. Y. Cheung et C. W. Hwang, Mesonic form factors and the Isgur-Wise function on the light-front, Phys. Rev. D55, 1559, (1997).

[92] K. C. Yang, Weak decay process of $B \rightarrow \rho l \bar{\nu}_{l}: A$ varying external field approach in QCD sum rules, Phys. Rev. D57, 2983, (1998).

[93] M. Gourdin, Y. Y. Keum et X. Y. Pham, Factorization and SU(2) heavy flavor symmetry for B meson decays producing charmonium, Phys. Rev. D52, 1597, (1995).

[94] F. M. Al-Shamali et A. N .Kamal, Nonfactorization in Cabibbo-favored B decays, Phys. Rev. D59, 054020, (1999).

F. M. Al-Shamali et A. N .Kamal, Factorization and nonfactorization in B decays, Phys. Rev. D60, 114019, (1999).

[95] H. Y. Cheng, Nonfactorizable contributions to nonleptonic weak decays of heavy mesons, Phys. Lett. B335, 428, (1994).

H. Y. Cheng, Hadronic weak decays of heavy mesons and nonfactorization, Z. Phys. C69, 647, (1996).

H. Y. Cheng, Can $B \rightarrow J / \psi K\left(K^{*}\right)$ decays be described by factorization ?, Phys. Lett. B395, 345, (1997).

[96] J. Soares, "Nonfactorizable" terms in hadronic B meson weak decays, Phys. Rev. D51, 3518, (1995).

[97] A. N. Kamal et A. B. Santra, Nonfactorization and color suppressed $B \rightarrow \psi(\psi(2 S))+K\left(K^{*}\right)$ decays, Z. Phys. C72, 91, (1996).

F. M. Al-Shamali et A. N. Kamal, Nonfactorization and final state interactions in $\left(B, B_{s}\right) \rightarrow \psi P$ and $\psi V$ decays, Eur. Phys. J. C4, 669, (1998).

[98] A. Ali et C. Creub, An analysis of two-body non-leptonic $B$ decays involving light mesons in the standard model, Phys. Rev. D57, 2996, (1998).

[99] A. Ali, G. Kramer et C. D. Lu, Experimental tests of factorization in charmless non-leptonic twobody B decays, Phys. Rev. D58, 094009, (1998). 
[100] H. Cheng et B. Tseng, Nonfactorizable effects in spectator and penguin amplitudes of hadronic charmless $B$ decays, Phys. Rev. D58, 094005, (1998).

[101] A. J. Buras et L. Silvestrini, Generalized factorization in non-leptonic two-body B decays : A critical look, Nucl. Phys. B548, 293, (1999).

[102] A. J. Buras, QCD factors $a_{1}$ and $a_{2}$ beyond leading logarithms versus factorization in nonleptonic heavy meson decays, Nucl. Phys. B434, 606, (1995).

[103] M. Beneke et al., QCD factorization for exclusive, non-leptonic B meson decays : General arguments and the case of heavy-light final states, Nucl. Phys. B591, 313, (2000).

[104] X. S. Nguyen et X. Y. Pham, On the $B \rightarrow J / \psi+K^{*}$ decay, hep-ph/0110284 v4.

[105] J. G. Körner et G. R. Goldstein, Quark And Particle Helicities In Hadronic Charmed Particle Decays, Phys. Lett. 89B, 105, (1979).

[106] L. Wolfenstein, Final State Interactions And CP Violation In Weak Decays, Phys. Rev. D43, 151, (1991).

[107] A. N. Kamal, Two Body Final State Interactions With Examples, Int. J. of Mod. Phys. A7, 3515, (1992).

[108] M. Bander, D. Silverman et A. Soni, CP Noninvariance In The Decays Of Heavy Charged Quark Systems, Phys. Rev. Lett. 43, 242, (1979).

L. L. Chau et H. Y. Cheng, B Decays Without Final State Charm Particles And CP Noninvariance, Phys. Rev. Lett. 59, 958, (1987).

[109] G. Kramer, W. F. Palmer et H. Simma, $C P$ violation and strong phases from penguins in $B^{ \pm} \rightarrow$ $V V$ decays, Nucl. Phys. B428, 77, (1994).

G. Kramer, W. F. Palmer et H. Simma, $C P$ violation and strong phases from penguins in $B^{ \pm} \rightarrow$ $P P$ and $B^{ \pm} \rightarrow V P$ decays, Z. Phys. C66, 429, (1995).

[110] Y. Y. Keum et H. N. Li, Nonleptonic charmless B decays : Factorization vs. perturbative QCD, Phys. Rev. D63, 074006, (2001).

[111] M. Ciuchini, E. Franco, G. Martinelli et L. Silvestrini, Charming penguins in B decays, Nucl. Phys. B501, 271, (1997).

[112] R. N. Cahn et M. Suzuki, Final-state interactions in nonleptonic weak decays of $D$ and $B$ mesons, hep-ph/9708208 v1.

[113] N. G. Deshpande et J. Trampetic, CP Asymmetries In Penguin Dominated $b \rightarrow s$ Transitions, Phys. Rev. D41, 2926, (1990).

H. Simma et D. Wyler, Branching Ratios And CP Violating Asymmetries In Exclusive Charged B Meson Decays, Phys. Lett. B272, 395, (1991).

[114] J. F. Donoghue, E. Golowich, A. A. Petrov et J. M. Soares, Systematics of soft final state interactions in B decay, Phys. Rev. Lett. 77, 2178, (1996).

[115] M. Suzuki et L. Wolfenstein, Final state interaction phase in B decays, Phys. Rev. D60, 074019, (1999).

[116] M. Suzuki, Final state interaction in heavy hadron decay, hep-ph/0001170. 
[117] A. N. Kammal et A. B. Santra, Probing factorization in color suppressed $B \rightarrow \psi(2 S)+K\left(K^{*}\right)$ decay, Phys. Rev. D51, 1415, (1995).

[118] S. U. Chung, Helicity Coupling Amplitudes In Tensor Formalism, Phys. Rev. D48, 1225, (1993). Erratum : Phys. Rev. D56, 4419, (1997).

[119] C. P. Jessop et al., CLEO Collaboration, Measurement of the decay amplitudes and branching fractions of $B \rightarrow J / \psi K^{*}$ and $B \rightarrow J / \psi K$ decays, Phys. Rev. Lett. 79, 4533, (1997).

T. Affolder et al., CDF Collaboration, Measurement of the decay amplitudes of $B^{0} \rightarrow J / \psi K^{* 0}$ and $B_{s}^{0} \rightarrow J / \psi \phi$ decays, Phys. Rev. Lett. 85, 4668, (2000).

B. Aubert et al., BaBar Collaboration, Measurement of the $B \rightarrow J / \psi K^{*}(892)$ decay amplitudes, Phys. Rev. Lett. 87, 241801, (2001).

$\mathrm{K}$. Abe et al., Belle Collaboration, Measurements of branching fractions and decay amplitudes in $B \rightarrow J / \psi K^{*}$ decays, Phys. Lett. B 538, 11, (2002).

[120] S. J. Richichi et al., CLEO Collaboration, Study of $B \rightarrow \psi(2 S) K$ and $B \rightarrow \psi(2 S) K^{*}(892)$ decays, Phys Rev. D63, 031103(R), (2001).

F. Abe et al., CDF Collaboration, Observation of $B^{+} \rightarrow \psi(2 S) K^{+}$and $B^{0} \rightarrow \psi(2 S) K^{*}(892)^{0}$ decays and measurements of $B$-meson branching fractions into $J / \psi$ and $\psi(2 S)$ final states, Phys. Rev. D 58, 072001, (1998).

[121] Z. Song et K. T. Chao, Problems of QCD factorization in exclusive decays of B meson to charmonium, hep-ph/0206253 v2.

[122] G. Valencia, Angular Correlations In The Decay $B \rightarrow V V$ And CP Violation, Phys. Rev. D39, 3339, (1989).

[123] G. Kramer et W. F. Palmer, Branching ratios and $C P$ asymmetries in the decay $B \rightarrow V V$, Phys. Rev. D45, 193, (1992).

G. Kramer et W. F. Palmer, Polarization and $C P$ asymmetries in the decays $B \rightarrow K^{*} \psi, K^{*} \omega$ and $K^{*} \rho$, Phys. Lett. B279, 181, (1992).

G. Kramer et W. F. Palmer, Direct $C P$ asymmetries in the decays $B \rightarrow V V$ from an effective weak Hamiltonian, Phys. Rev. D46, 2969, (1992).

[124] D. M. Asner et al., CLEO Collaboration, Analysis of $B \rightarrow J / \psi K$ and $B \rightarrow J / \psi K^{*}$ Decays, CLEO CONF 96-24.

[125] A. S. Dighe, I. Dunietz, H. J. Lipkin et J. L. Rosner, Angular distributions and lifetime differences in $B_{s} \rightarrow J / \psi \phi$ decays, Phys. Lett. B369, 144, (1996).

[126] Stephen Pappas, communication privée.

[127] C. W. Chiang et L. Wolfenstein, Observables in the decays of B to two vector mesons, Phys. Rev. D61, 074031, (2000).

[128] K. Abe, M. Satpathy et H. Yamamoto, Time-dependent angular analyses of B decays, hepex/0103002.

[129] C. W. Chiang, Angular distribution of charming $B \rightarrow V V$ decays and time evolution effects, Phys. Rev. D62, 014017, (2000).

[130] H. J. Lipkin et A. I. Sanda, Isospin Invariance, CP Violation And B Anti-B Mixing, Phys. Lett B201, 541, (1988). 
[131] C. Bourrely, E. Leader et J. Soffer, Polarization Phenomena In Hadronic Reactions, Phys. Rep. 59, 95, (1980).

[132] A. Kotanski, Application Of Transversity Amplitudes To The Kinematical Analysis Of Two-Body Processes At High Energies, Acta Phys. Pol. 30, 629, (1966).

A. Kotanski, Transversity Amplitudes And Their Application To The Study Of Collisions Of Particles With Spin, Acta Phys. Pol. B1, 45, (1970).

[133] J. L. Rosner, Determination Of Pseudoscalar Charmed Meson Decay Constants From B Meson Decays, Phys. Rev. D42, 3732, (1990).

[134] S. U. Chung, A General formulation of covariant helicity coupling amplitudes, Phys. Rev. D57, 431, (1997).

V. Filippini, A. Fontana et A. Rotondi, Covariant Spin Tensors In Meson Spectroscopy, Phys. Rev. D51, 2247, (1995).

V. Filippini, Covariant Tensor In Spin-Parity Analysis, Nucl. Phys. A655, 99c, (1999).

[135] T. Hurth et al., Present and future CP measurements, J. Phys. G27, 1277, (2001).

[136] K. J. Sebastian, H. Grotch et F. L. Ridener, Jr., Multipole Amplitudes In Parity Changing One Photon Transitions Of Charmonium, Phys. Rev. D45, 3163, (1992).

F. L. Ridener, Jr., K. J. Sebastian et H. Grotch, Radiative Angular Distributions From Charmonium States Directly Produced By Anti-P P Annihilatio, Phys. Rev. D45, 3173, (1992).

[137] C. Bailin et al., Angular Distributions In The Reactions $P \bar{P} \rightarrow \chi_{(1,2)} \rightarrow \gamma \psi \rightarrow \gamma e^{+} e^{-}$, Phys. Lett. B195, 85, (1987).

[138] M. Oreglia et al., A Study Of The Reaction $\psi^{\prime} \rightarrow \gamma \gamma J / \psi$, Phys. Rev. D25, 2259, (1982).

[139] R. G. Newton, Scattering Theory of Waves and Particles, McGraw-Hill, New York, (1966).

J. R. Taylor, Scattering Theory, New York, Wiley, (1972).

R. Omnès, Introduction à l'étude des particules élémentaires, Ediscience, Paris, (1970).

[140] K. Gottfried et J. D. Jackson, On The Connection Between Production Mechanism And Decay Of Resonances At High-Energies, Nuovo Cimento 33, 309, (1964).

J. D. Jackson, Remarks On The Phenomenological Analysis Of Resonances, Nuovo Cimento 34, 1644, (1964).

A. Barbaro-Galtieri, dans Advances in Particle Physics, eds. R. L. Cool et R. E. Marshak, Wiley, (1968).

R. D. Tripp, dans Proceedings of the International School of Physics, Enrico Fermi, XXXIII Course, Strong Interactions, Academic Press, New York, (1966).

[141] H. Pilkuhn, The Interactions of Hadrons, North-Holland Publishing, Amsterdam, (1967).

[142] S. U. Chung et al., Partial Wave Analysis In K Matrix Formalism, Ann. Phys., 4, 404, (1995).

[143] J. M. Blatt et V. F. Weisskopf, Theoretical Nuclear Physics, Wiley, New York, (1952).

[144] F. von Hippel et C. Quigg, Centrifugal-Barrier Effects In Resonance Partial Decay Widths, Shapes, And Production Amplitudes, Phys. Rev. D5, 624, (1972).

[145] E. P. Wigner, Phys. Rev. 98, 145, (1955).

R. H. Dalitz, Ann. Rev. Nucl. Sci., 13, 339, (1963). 
[146] D. Aston et al., A Study Of $K^{-} \pi^{+}$Scattering In The Reaction $K^{-} P \rightarrow K^{-} \pi^{+} N$ At $11 \mathrm{GeV} / \mathrm{c}$, Nucl. Phys. B296, 493, (1988).

[147] S. Spanier, Parametrization of the Dynamics of Partial Wave Amplitudes, BABAR Analysis Document \# 303 version 2, (2001).

[148] B. Kayser, CP violation and quantum mechanics in the B system, hep-ph/9509386.

[149] A. Pompili et F. Selleri, On a possible EPR experiment with $B_{d}^{0} \bar{B}_{d}^{0}$ pairs, Eur. Phys. J. C14, 469, (2000).

[150] Z. Z. Xing, Time dependence of coherent $P^{0} \bar{P}^{0}$ decays and $C P$ violation at asymmetric $B$ factories, Phys. Rev. D53, 204, (1996).

[151] T. Nakada, Future Prospects for Studying CP Violation in B-Meson Decays, hep-ex/9609015. G. J. Feldman et. al., Report of the B-Factory Group : I. Physics and Techniques, SLAC-PUB-4838.

[152] PEP II : An Asymmetric B Factory, Conceptual Design Report, SLAC-R-418, LBL-5379 (1993).

[153] B. Aubert et. al., BaBar Collaboration, The BaBar detector, Nucl. Inst. Meth. A479, 1, (2002).

[154] M. Sullivan, B-Factory Interaction Region Design, SLAC-PUB-7563.

Y. Nosochkov et. al., Detector Solenoid Compensation in the PEP-II B-Factory, SLAC-PUB-6890.

[155] A. Drescher et al., The argus electron-photon calorimeter III. Electron-hadron separation, Nucl. Inst. Meth. A237, 464, (1985).

[156] R. Sinkus et T. Voss, Particle identification with neural networks using a rotational invariant moment representation, Nucl. Inst. Meth. A391, 360, (1997).

[157] G. C. Fox et S. Wolfram, Observables For The Analysis Of Event Shapes In $e^{+} e^{-}$Annihilation And Other Processes, Phys. Rev. Lett. 41, 1581, (1978).

[158] H. Albrecht et al., Argus Collaboration, Search For Hadronic $b \rightarrow u$ Decays, Phys. Lett. B241, 278, (1990).

H. Albrecht et al., Argus Collaboration, Exclusive Hadronic Decays Of B Mesons, Z. Phys. C48, $543,(1990)$.

[159] G. Zech, Comparing statistical data to Monte Carlo simulation - parameter fitting and unfoldin, DESY 95-113, (June 1995).

W. T. Eadie et al., Statistical Methods in Experimental Physics, North-Holland, Amsterdam, (1971).

G. Cowan, Statistical Data Analysis, Oxford University Press, Oxford, (1998).

[160] B. Aubert et al., BaBar Collaboration, Measurement of the $B \rightarrow J / \psi K^{*}(892)$ decay amplitudes, Phys. Rev. Lett. 87, 241801, (2001).

[161] R. Cahn, Unbinned Maximum Likelihood in the Presence of Background, May 2002.

[http://www.slac.stanford.edu/ cahn/internal/likelihood_with_bkgd.ps]

[162] N. Kuznetsova, J. D. Richman et A. Ryd, Search for $B \rightarrow\left(K, K^{*}\right) \ell^{+} \ell^{-}$, BABAR Analysis Document \# 164 version 8 appendice A, (2001).

[163] W. Dunwoodie, note on $S$ wave, (2003).

[http://www.slac.stanford.edu/ wmd/kpi_swave/kpi_swave_fit.note] 
[164] B. Aubert et al., BaBar Collaboration, A study of time dependent CP-violating asymmetries and flavor oscillations in neutral B decays at the $\Upsilon(4 S)$, Phys. Rev. D66, 032003, (2002).

[165] J. Cohen-Tanugi, thèse de l'université Paris VII (2001).

[166] B. Aubert et al., BaBar Collaboration, Measurement of the CP-violating asymmetry amplitude $\sin 2 \beta$, Phys. Rev. Lett. 89, 201802, (2002).

[167] B. Aubert et al., BaBar Collaboration, Measurement of $B^{0}-\bar{B}^{0}$ flavor oscillations in hadronic $B^{0}$ decays, Phys. Rev. Lett. 88, 221802, (2002).

[168] B. Aubert et al., BaBar Collaboration, Measurement of the $B^{0}$ and $B^{+}$meson lifetimes with fully reconstructed hadronic final states, Phys. Rev. Lett. 87, 201803, (2001).

[169] J. Beringer, BTagger - A Multivariate Tagging Algorithm with Categories Based on the Physics of the $B_{t a g}$ Decay, BABAR Analysis Document \# 317 version 4, (2002).

[170] D. G. Cassel, CP Physics at $e^{+} e^{-}$Colliders, CLNS 97/1538.

[171] F. Fernando-Martinez et al., Vertexing performances and systematic checks with fully reconstructed $B$ events, BABAR Analysis Document \# 130 version 3, (2001).

[172] J. Stark, thèse de l'université Paris VI (2001).

[173] Y. Grossman et H. R. Quinn, Removing discrete ambiguities in CP asymmetry measurements, Phys. Rev. D56, 7259, (1997).

[174] M. Suzuki, Large violation of s-quark helicity conservation in $B \rightarrow J / \psi K^{*}$, Phys. Rev. D64, 117503 (2001). 
Abstract : this thesis presents the full-angular time-dependent analysis of the vector-vector channel $B_{d}^{0} \rightarrow J / \psi\left(K_{S}^{0} \pi^{0}\right)^{* 0}$. After a review of the $C P$ violation in the $B$ meson system, the phenomenology of the charmonium- $K^{*}(892)$ channels is exposed. The method for the measurement of the transversity amplitudes of the $B \rightarrow J / \psi K^{*}(892)$, based on a pseudo-likelihood method, is then exposed. The results from a $81.9 \mathrm{fb}^{-1}$ of collected data by the BABAR detector at the $\Upsilon(4 S)$ resonance peak are $\left|A_{0}\right|^{2}=0.565 \pm 0.011 \pm 0.004,\left|A_{\|}\right|^{2}=0.206 \pm 0.016 \pm 0.007,\left|A_{\perp}\right|^{2}=0.228 \pm 0.016 \pm 0.007$, $\delta_{\|}=-2.766 \pm 0.105 \pm 0.040$ and $\delta_{\perp}=2.935 \pm 0.067 \pm 0.040$. Note that $\left(\delta_{\|}, \delta_{\perp}\right) \rightarrow\left(-\delta_{\|}, \pi-\delta_{\perp}\right)$ is also a solution. The strong phases $\delta_{\|}$and $\delta_{\perp}$ are at $\gtrsim 3 \sigma$ from $\pm \pi$, signing the presence of final state interactions and the breakdown of the factorization hypothesis. The forward-backward analysis of the $K \pi$ mass spectrum revealed the presence of a coherent $S$-wave interfering with the $K^{*}(892)$. It is the first evidence of this wave in the $K \pi$ system coming from a $B$ meson. The particularity of the $B_{d}^{0} \rightarrow J / \psi\left(K_{S}^{0} \pi^{0}\right)^{* 0}$ channel is to have a time-dependent but also an angular distribution which allows to measure $\sin 2 \beta$ but also $\cos 2 \beta$. The results from an unbinned maximum likelihood fit are $\sin 2 \beta=-0.10 \pm 0.57 \pm 0.14$ and $\cos 2 \beta=3.32_{-0.96}^{+0.76} \pm 0.27$ with the transversity amplitudes fixed to the values given above. The other solution for the strong phases flips the sign of $\cos 2 \beta$. Theoretical considerations based on the $s$-quark helicity conservation favor the choice of the strong phases given above, leading to a positive sign for $\cos 2 \beta$. This sign of $\cos 2 \beta$ is the one predicted by the Standard Model.

Keywords : $C P$ Violation; BABAR; Charmonium- $K^{*}(892) ; \sin 2 \beta$; $\cos 2 \beta$; Angular and/or timedependent Analysis ; Standard Model ; CKM ; Factorization ; Final State Interactions ; Transversity ; Helicity ; Partial Waves ; $K \pi$ Mass Spectrum ; $S, P, D$ Waves ; Weak and Strong Phases. 
Résumé: Les travaux présentés dans cette thèse décrivent l'analyse angulaire complète dépendante du temps du canal vecteur-vecteur $B_{d}^{0} \rightarrow J / \psi\left(K_{S}^{0} \pi^{0}\right)^{* 0}$. Après un rappel consacré à la violation de $C P$ dans le système des mésons $B$ est traitée la phénoménologie des canaux vecteur-vecteur du type charmonium- $K^{*}(892)$. La méthode pour la mesure des amplitudes de transversité des canaux $B \rightarrow J / \psi K^{*}(892)$, basée sur l'utilisation d'un pseudo-maximum de vraisemblance, est ensuite exposée. Les résultats obtenus avec $81.9 \mathrm{fb}^{-1}$ de données collectées par le détecteur BABAR au pic de la résonance $\Upsilon(4 S)$ sont $\left|A_{0}\right|^{2}=0.565 \pm 0.011 \pm 0.004,\left|A_{\|}\right|^{2}=0.206 \pm 0.016 \pm 0.007$, $\left|A_{\perp}\right|^{2}=0.228 \pm 0.016 \pm 0.007, \delta_{\|}=-2.766 \pm 0.105 \pm 0.040$ et $\delta_{\perp}=2.935 \pm 0.067 \pm 0.040$. À noter que $\left(\delta_{\|}, \delta_{\perp}\right) \rightarrow\left(-\delta_{\|}, \pi-\delta_{\perp}\right)$ est aussi une solution. Les phases fortes $\delta_{\|}$et $\delta_{\perp}$ sont à $\gtrsim 3 \sigma$ de $\pm \pi$, signant ainsi la présence d'interactions dans l'état final et la rupture de l'approximation de factorisation. L'analyse de l'asymétrie avant-arrière en fonction de la masse $K \pi$ a révélé la présence d'une onde $S$ cohérente qui interfère avec le $K^{*}(892)$. C'est la première mise en évidence de la présence de cette onde dans le système $K \pi$ provenant de la désintégration d'un méson $B$. La particularité du canal $B_{d}^{0} \rightarrow J / \psi\left(K_{S}^{0} \pi^{0}\right)^{* 0}$ est d'avoir une distribution qui dépend de la différence des temps propres $\Delta t$ des deux mésons $B$ provenant de la désintégration $\Upsilon(4 S) \rightarrow B_{d}^{0} \bar{B}_{d}^{0}$ mais aussi des trois angles de transversité, permettant de mesurer $\sin 2 \beta$ mais aussi $\cos 2 \beta$. Les résultats obtenus avec un ajustement par maximum de vraisemblance sont $\sin 2 \beta=-0.10 \pm 0.57 \pm 0.14$ et $\cos 2 \beta=3.32_{-0.96}^{+0.76} \pm 0.27$ avec les amplitudes de transversité fixées aux valeurs précédentes. L'autre solution pour les phases fortes conduit à la même valeur de $\sin 2 \beta$ mais change le signe de $\cos 2 \beta$. Des considérations théoriques basées sur la conservation de l'hélicité du quark $s$ favorisent le choix des phases fortes données ci-dessus conduisant au signe positif pour $\cos 2 \beta$. Ce signe de $\cos 2 \beta$ est celui prédit par le Modèle Standard.

Mots clés: Violation de $C P$; BABAR; Charmonium- $K^{*}(892) ; \sin 2 \beta ; \cos 2 \beta$; Analyse angulaire et/ou temporelle ; Modèle Standard ; CKM ; Factorisation ; Interaction dans l'état final ; Transversité ; Hélicité ; Ondes Partielles ; Spectre en masse $K \pi$; Ondes $S, P$ et $D$; Phases fortes et faibles. 